\title{
To Swerve and Neglect: De-Policing Throughout Today's Front-Line Police Work
}

Gregory Roy Brown

A thesis submitted to the Faculty of Graduate and Postdoctoral Affairs in partial fulfillment of the requirements for the degree of

Doctor of Philosophy

in

Sociology

Carleton University

Ottawa, Ontario

C2019 Gregory Brown 


\begin{abstract}
This mixed methods study investigates how American and Canadian front-line police officers are responding to policing's new visibility, which implicates citizengenerated mobile device and CCTV footage and concomitant online interconnectivity and social media discourse, and to intensified scrutiny of officers' actions by a more critical public audience. Quantitative and qualitative data was collected from 3,660 rank-and-file officers at 23 police agencies across Canada and throughout the State of New York.
\end{abstract}

This study finds that a substantial majority of today's rank-and-file officers in the 23 jurisdictions across both countries (72\%) are intentionally reducing, or eliminating, proactive interactions in the community, in response to officers' perceptions that such discretionary initiatives are unnecessarily risky. Little variation was found across location and agency variables (country, region, and police agency size) or across individual officers' demographic variables (gender and race/ethnicity). For individual officers, the decision to practice de-policing and any subsequent intensification in an officer's de-policing practices, is associated with the accumulation of negative policecitizen interactions over an officer's years of front-line police service. Influence from the rank-and-file police subculture also plays a significant role in contributing to these widespread risk-averse practices.

For many officers, de-policing is connected with attitudes toward, and avoidance of, individuals perceived as presenting with mental health issues and/or a 'nontraditional' sexual orientation, and, even more strongly, in relation to persons perceived by front-line officers as visible racialized minorities. Implications of the methodology 
and findings are discussed, including those in relation to the author's role as a 'pracademic' researcher, to the current and future situation of policing in North America, and to the author's efforts to enter into public debates regarding today's policing. 


\section{ACKNOWLEDGMENTS}

This dissertation is dedicated to my father, Roy Gilbert Brown, P.Eng., M.Sc. (Queens 1962), who passed away on 28 May 2019 after a courageous battle with cancer. Some of Chapter Six was written while he rested in hospital and I was at his bedside. My Dad, who was known for his remarkable work ethic, approved of my efforts in this regard and he encouraged me to keep working hard at my journey within academia. $\mathrm{He}$ was well versed in the other six chapters, having read them over the past few months after he retired from 60 years of work in structural steel engineering. My Dad told me over the years that he was glad he had declined a scholarship to pursue a $\mathrm{PhD}$ in structural engineering at Lehigh University, as he decided instead to work as a practitioner in the field and to marry my mother and have children. However, he also related that he would have liked to have returned to graduate school at some point later in life - a journey upon which he never embarked. Dad, I know you are proud of my accomplishments, as I am of all of your achievements, and this doctoral dissertation is dedicated to you - an incredible father, role model, and friend!

It is not lost on me that this dissertation is considerably longer than most and, in this regard, I am grateful to my co-supervisors, committee members, other colleagues in academia, and the examiners at my oral defence for their commitment to, and efforts in, reviewing this voluminous material. I should explain that the reason I undertook such an expansive research project was, in large part, because of advice I received from several accomplished academics (who shall remain anonymous) that explained that this would likely be the only time in my academic career when I would have the time, the funding, and the relatively uninterrupted focus to take on such an extensive project. I am 
especially indebted to my wife Lisa and to Ben, Kyle, Meredith, and Will for accommodating my absences and long hours of commitment to this project. I am appreciative for the generous funding provided by Carleton University, the Social Sciences and Humanities Research Council, and the Fulbright Foundation for Educational Exchange Between Canada and the United States of America, which allowed me to carry out this research.

I am profoundly grateful to my co-supervisors, Aaron Doyle and Leslie-Anne Keown. You have both been exceptional mentors and your guidance, encouragement, and friendship have made this an incredibly rewarding experience. I also extend my heartfelt appreciation to George Rigakos and Michael Mopas, who have been supportive and instructive committee members throughout this challenging process. Thank you for your commitment and your dedication. I also acknowledge the advice and support provided along this journey by some special people - James Sheptycki (York University), Robert Worden (University at Albany), Dr. Shelley Jordan, and Ed Keeley (retired Deputy Chief and co-chair of the Canadian Association of Chiefs of Police Ethics Committee).

To the 3660 front-line police officers that trusted me with your innermost thoughts and experiences, which made this unique contribution to knowledge around important 'real world' issues in today's public-police relationship possible, I am grateful not only for your participation, but also for your service to our communities. Finally, to the police agency and police labour organization leaders that authorized the participation of their front-line officers in this project, I appreciate your understanding of the importance of academic research into contemporary issues in today's policing. 


\section{TABLE OF CONTENTS}

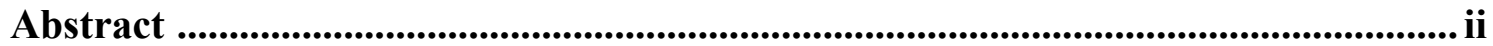

Acknowledgements ...................................................................................................... iv

Table of Contents ............................................................................................. vi

List of Tables .............................................................................................................. viii

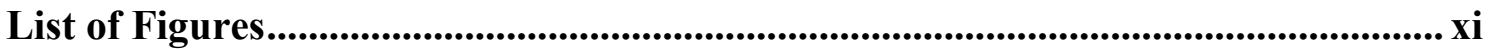

List of Images ......................................................................................................................... xii

List of Appendices ................................................................................................................ xiii

Acronyms and Abbreviations .......................................................................................... xiv

Chapter One: Situating the Project ................................................................................. 1

1.1 Chapter Introduction ................................................................................... 1

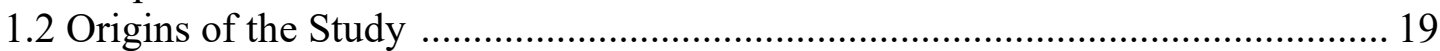

1.3 Relevance, Originality, Research Objectives, and Research Questions .............. 28

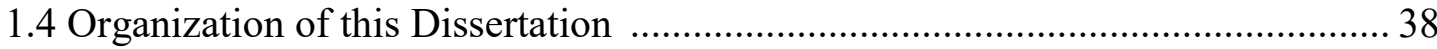

Chapter Two: Literature Review and Historical Context ........................................... 40

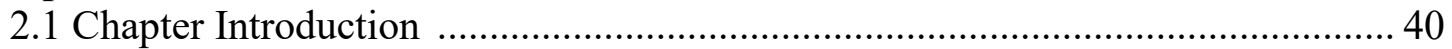

2.2 A Survey of the Literature and Historical Context (1977 to 2019) ...................... 44

2.3 Recent Empirical Studies Focused on the Phenomenon of De-Policing ............. 74

Chapter Three: Establishing Conceptual and Theoretical Frameworks ................ 83

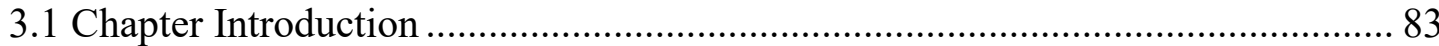

3.2 Today's Techno-Social Landscape for Front-Line Policing ............................... 87

3.3 Today's Socio-Political Landscape for Front-Line Policing ................................. 94

3.4 Establishing a Theoretical Framework ............................................................. 104

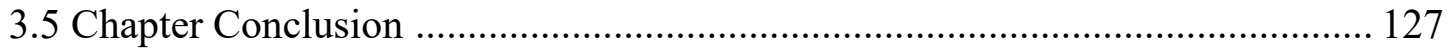

Chapter Four: Methodology .............................................................................................. 129

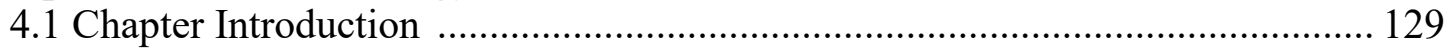

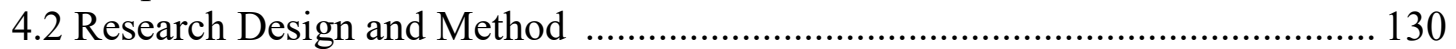

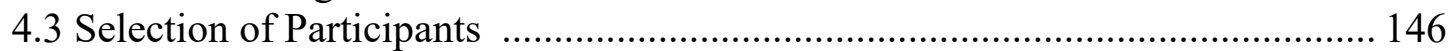

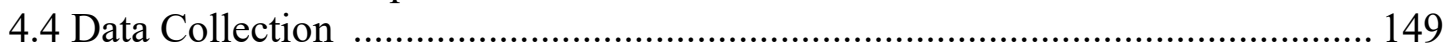

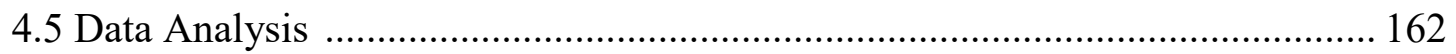

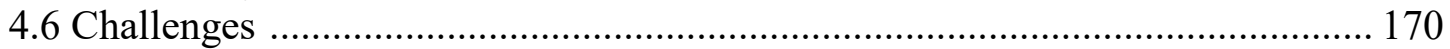

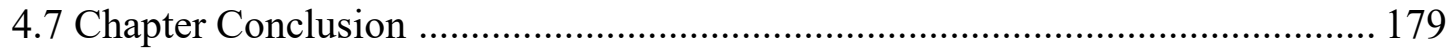

Chapter Five: Analysis, Results, and Findings .................................................... 180

5.1 Chapter Introduction ............................................................................. 180

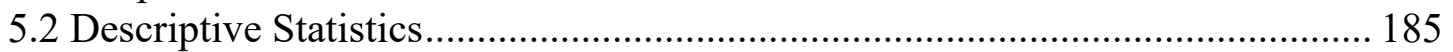

5.3 Bivariate Analysis - The Practice of De-Policing ..............................................2. 253

5.4 Official Police Data Received Pursuant to FOI Applications.............................. 277

Chapter Six: Discussion .................................................................................................... 292

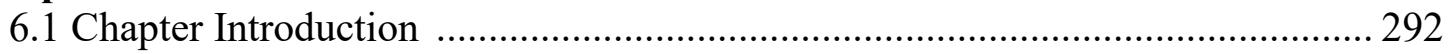

6.2 'Lazy' Officers Versus Today's De-Policers ………………………................ 296

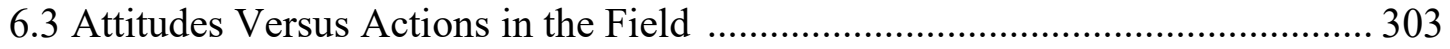


6.4 Canadian and American Policing Contexts .................................................. 307

6.5 Career Stage and the De-Policing Continuum ................................................ 312

6.6 Front-Line Police Subculture and De-Policing ............................................... 333

6.7 De-policing and Police-Community Relations .............................................. 354

6.8 De-Policing Implications Related to Race/Ethnicity ........................................ 381

6.9 Non De-Policers ..................................................................................... 402

6.10 Researcher Positionality, Participants' Trust, and Data Integrity ................... 414

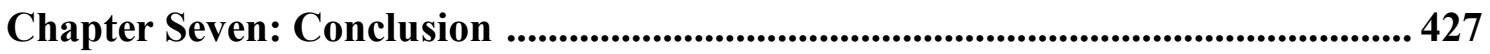

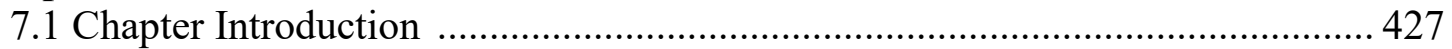

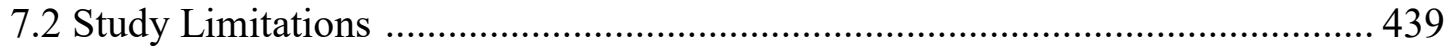

7.3 Suggested Areas for Further Research ......................................................... 440

7.4 Solutions to Address Problematic De-Policing................................................. 447

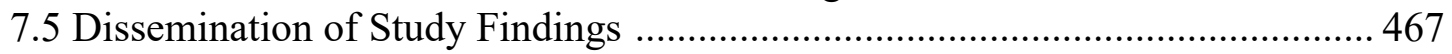

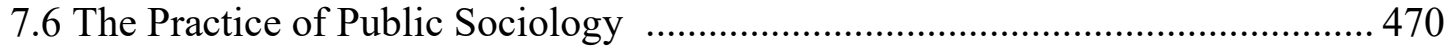

Appendix A: Consent Form (2012-2013 Study) ........................................................... 481

Appendix B: Consent Form (2015-2106 Study) ................................................... 483

Appendix C: Data Site Information .................................................................... 487

Appendix D: Explanation Letter to Prospective Agencies (English) ...................... 542

Appendix E: Explanation Letter to Prospective Agencies (Français)..................... 544

Appendix F: Research Ethics Board Clearance....................................................... 546

Appendix G: Survey Questionnaire (English)....................................................... 547

Appendix H: Survey Questionnaire (Français) ..................................................551

Appendix I: Bivariate Analysis for Questions 16, 24, and 26 ............................... 555

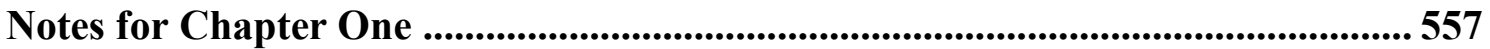

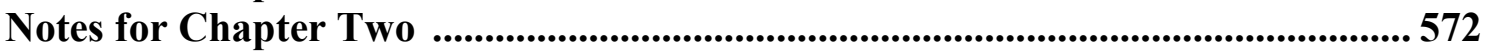

Notes for Chapter Three ............................................................................................ 577

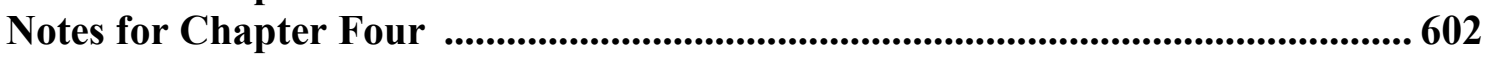

Notes for Chapter Five ......................................................................................6 618

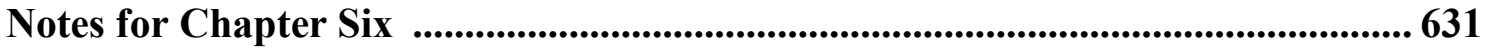

Notes for Chapter Seven .................................................................................................. 662

Notes for Appendices .......................................................................................................... 668

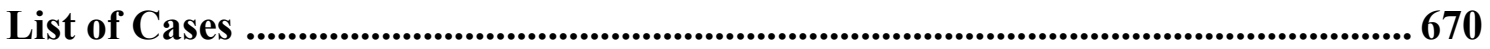

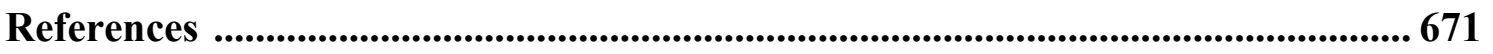




\section{LIST OF TABLES}

Table 1: Population of the State of New York (2010) ............................................ 155

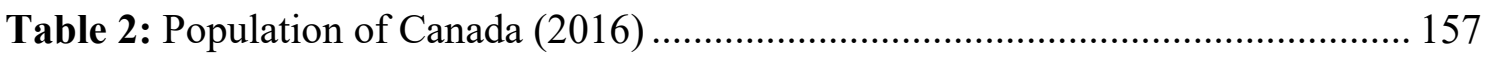

Table 3: Study participants by police agency ………….......................................... 158

Table 4: Sample characteristics (total sample) ………………………................... 160

Table 5: Awareness of video recording by a member of the public ........................... 186

Table 6: Circumstances of video recording by a member of the public .................... 187

Table 7: Assessment of potential for video recording of on-duty activities ................ 188

Table 8: Discussion about potential for video recording of police conduct

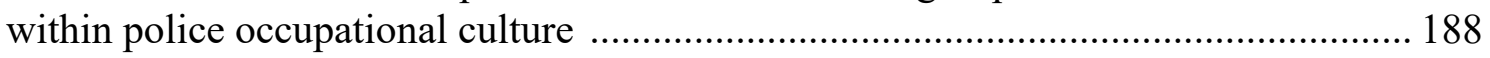

Table 9: Discussion about 'viral' video recordings of police occurrences within police occupational culture ................................................................... 188

Table 10: Level of officers' awareness of potential video recording of their on-duty actions

Table 11: Officers contemplating not doing something because of a concern that those actions could be video recorded

Table 12: Officers not doing something because of a concern that those actions could be video recorded

Table 13: Change in frequency of officers' use of force because of video recording 200

Table 14: Change in level of force used by officers because of video recording _..... 201

Table 15: Officers' employment of measures to avoid video recording of actions ..... 202

Table 16: Actions taken when there is potential for video recording …………......... 207

Table 17: Perception of public criticism of police (today versus start of career) ....... 213

Table 18: Officers contemplating not doing something because of a concern that those actions could be criticized by the public

Table 19: Officers not doing something because of a concern that those actions could be criticized by the public

Table 20: Officers' perceptions of risk (of criticism and/or complaint) as related to 'demographic' characteristics of individuals with whom they interact

Table 21: Specific 'demographic' characteristics identified as presenting an increased risk of criticism and/or complaint 224

Table 22: Avoidance of interaction with those presenting specific characteristics ..... 230

Table 23: Familiarity with the concept of de-policing (as described in the survey) ... 237 
Table 24: Participants who have heard of, or witnessed, de-policing where they work

Table 25: Familiarity with the researcher's policing background

Table 26: Influence on participants' responses due to knowledge of the researcher's policing background 248

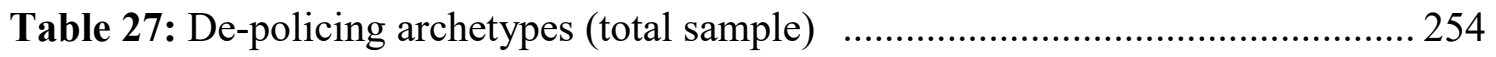

Table 28: De-policing archetypes by participating agency .................................... 255

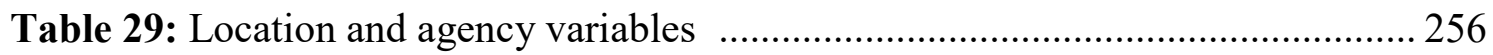

Table 30: Type of front-line police work and officers' policing environment ........... 258

Table 31: Front-line officers' characteristics 262

Table 32: 'New' and 'experienced' front-line officers and collapsed de-policing categories (by agency)

Table 33: De-policing archetypes and officers' perceptions of elevated risk (overall) 264

Table 34: 'No' respondents to Q. 25 in the survey and de-policing archetypes 265

Table 35: De-policing archetypes and officers' perceptions of elevated risk (specific categories identified) 266

Table 36: De-policing archetypes and familiarity with the concept of de-policing .... 268

Table 37: De-policing archetypes and participants hearing of, or witnessing, de-policing 270

Table 38: Participants who have (and have not) thought abouth changing behaviours(s). 272

Table 39: Nature of concerns by de-policing archetype 273

Table 40: De-policing archetypes and officers' awareness of video recording potential (on scale of 1 to 10 ) 275

Table 41: 'New' versus 'experienced' officers and officers' awareness of video recording potential (on scale of 1 to 10 ) 275

Table 42: De-policing archetypes and influence of the researcher's 'pracademic' biography on research participants' answers in the questionnaire 276

Table 43: CPS officer-generated calls 2006-2016 ('check on welfare' and ‘ suspicious person') 279

Table 44: HRP 'street check' data 2008-2017 ....................................................... 281

Table 45: OPS officer-initiated calls 2009-2017 .................................................... 282

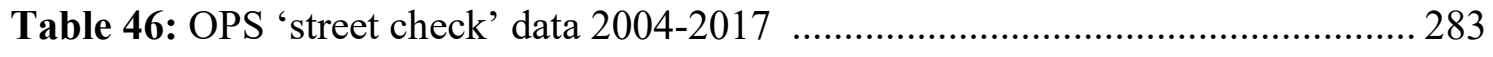

Table 47: RPD ‘directed patrol' 2010-2017 ........................................................... 285 
Table 48: TPS data (vehicle stops, suspicious events, public intoxication, cause disturbance) 286

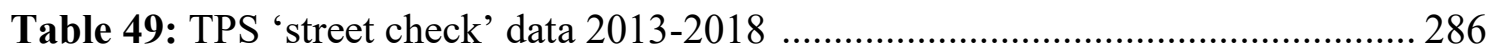

Table 50: PRPS ‘street check' data 2013-2018 ...................................................... 286

Table 51: VPD ‘street check’ data 2008-2017 .......................................................... 286

Table 52: WPS data ('GRASP' checks, spot checks, consume liquor in public) ....... 289

Table 53: EPS 'street check' data 2012-2017 ........................................................ 292

Table 54: Question 16 - De-policing and video recording of police actions .............555

Table 55: Question 24 - De-policing and public criticism of police actions .............. 556

Table 56: De-policing and avoidance of interaction with those perceived to present elevated risk of criticism/complaint (frequency of responses) .......................556

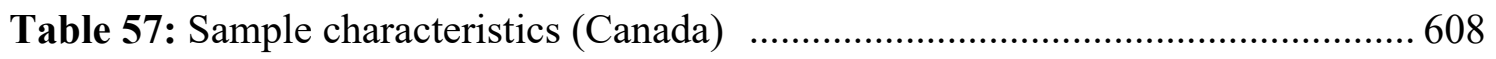

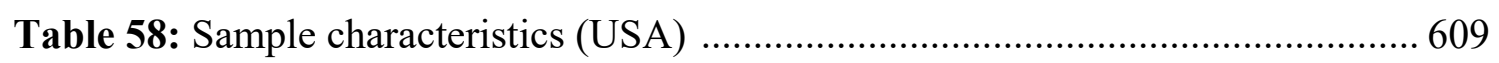

Table 59: Concerns around interacting with 'minorities' (by police agency percentages of qualitative responses)

Table 60: All CPS officer-generated calls data 629 


\section{LIST OF FIGURES}

Figure 1: Chicago Police Department (CPD) 'street checks' (2014-2016) ................... 14

Figure 2: Calgary Police Service (CPS) self-initiated calls 2006-2016 ('check on

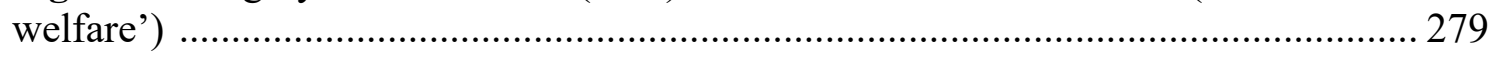

Figure 3: CPS self-initiated calls 2006-2016 ('suspicious person') .......................... 281

Figure 4: Halifax Regional Police (HRP) ‘street checks’ 2008-2017 ...................... 282

Figure 5: Ottawa Police Service (OPS) officer-initiated calls 2009-2017.................. 282

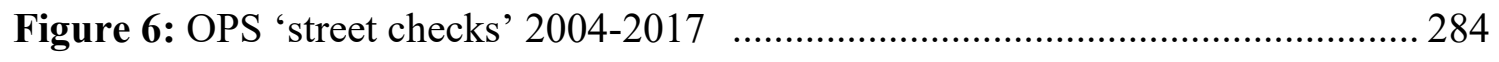

Figure 7: Rochester Police Department (RPD) 'directed patrol' 2010-2017 ............ 285

Figure 8: Vancouver Police Department (VPD) street checks 2008-2017

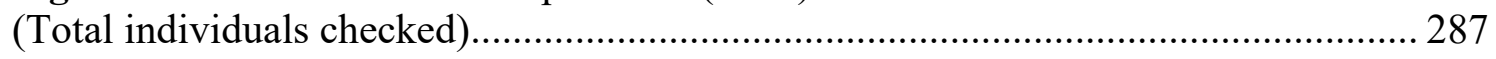

Figure 9: VPD street checks 2008-2017 (gang affiliation) ...................................... 287

Figure 10: VPD street checks 2008-2017 (suspected drug dealer) .......................... 288

Figure 11: Winnipeg Police Service (WPS) 'GRASP' checks 2013-2017 ............... 289

Figure 12: WPS 'spot checks' 2008-2017 ............................................................ 290

Figure 13: WPS citation for consuming liquor in a public place 2007-2018 ............ 290

Figure 14: Homicide rates in Chicago 1985-2016 .................................................. 561

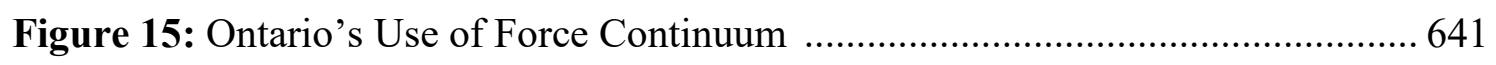




\section{LIST OF IMAGES}

Image 1: Toronto Police Service (TPS) cruiser (Bond 2019) ................................... 8

Image 2: Chicago Police Department (CPD) cruiser (Bedi 2017) .............................. 8

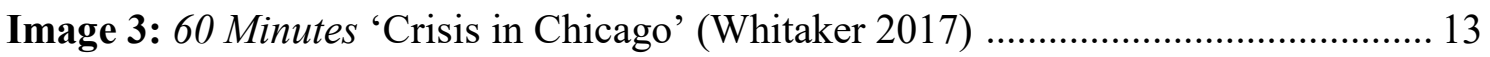

Image 4: CNN 'Chicago's deadliest year in 2 decades' (Flores 2017) ....................... 16

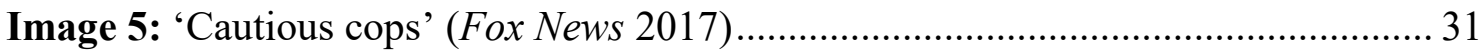

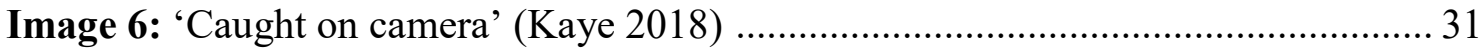

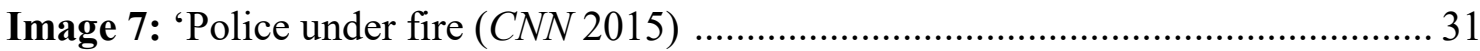

Image 8: 'Thousands dead: Few prosecuted' (Kindy \& Kelly 2015) ......................... 31

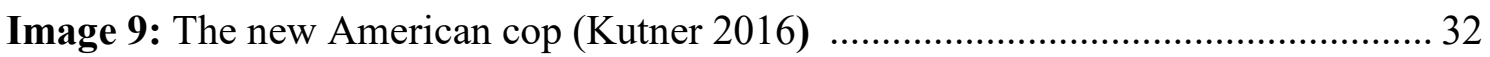

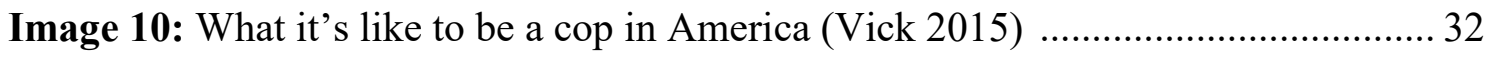

Image 11: 'Protect and serve? De-policing in America's urban neighbourhoods'

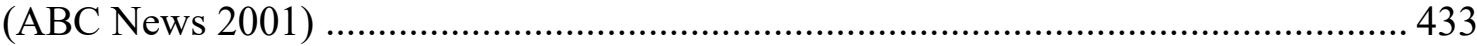

Image 12: 'The Ferguson Effect: FBI report reveals law enforcement pullback' (Wallace 2017)

Image 13: Abdi protests (James Park/Post Media) 3 November 2017 in Laucius (2017) 517

Image 14: Abdi protests (Patrick Doyle/Reuters) 31 July 2016 in Reuters (2016b) 517

Image 15: Yatim protests (Philip Cheung/Globe \& Mail 2013) ................................ 536

Image 16: Yatim protests (Philip Cheung/Globe \& Mail 2013) ................................ 536

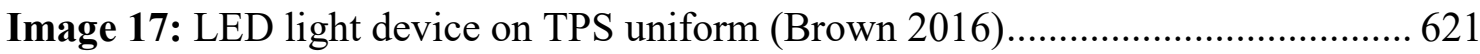

Image 18: TPS officer wearing LED light device on uniform (McQuigge 2017) ...... 621

Image 19: Video display in Syracuse Police briefing room (Brown 2016) 637 


\section{LIST OF APPENDICES}

Appendix A: Consent Form (2012-2013 study) .................................................. 481

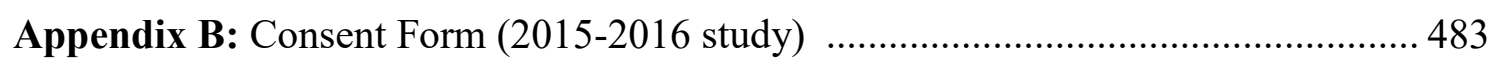

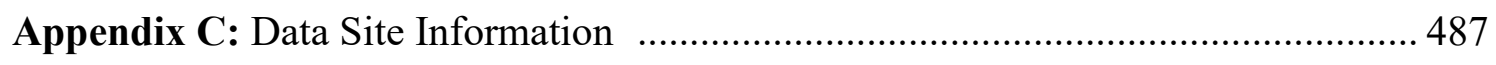

Appendix D: Explanation Letter to Prospective Agencies (English) ....................... 542

Appendix E: Explanation Letter to Prospective Agencies (Français) ........................544

Appendix F: Research Ethics Board Clearance .............................................. 546

Appendix G: Survey Questionnaire (English) ................................................... 547

Appendix H: Survey Questionnaire (Français) .................................................. 551

Appendix I: Bivariate Analysis for Questions 16, 24, and 26 ................................. 555 


\section{ACRONYMS AND ABBREVIATIONS}

$\begin{array}{ll}\text { ALB } & \text { Albany Police Department } \\ \text { BUF } & \text { Buffalo Police Department } \\ \text { BWC } & \text { body-worn camera } \\ \text { CACP } & \text { Canadian Association of Chiefs of Police } \\ \text { CAL } & \text { Calgary Police Service } \\ \text { CBP } & \text { community-based policing } \\ \text { COD } & \text { Codiac Detachment (RCMP New Brunswick - greater Moncton region) } \\ \text { COR } & \text { Cornwall Community Police Service } \\ \text { CPD } & \text { Chicago Police Department } \\ \text { CYA } & \text { 'cover your ass' } \\ \text { DEA } & \text { Drug Enforcement Administration (United States) } \\ \text { DEL } & \text { Delta Police Department } \\ \text { DWB } & \text { 'driving while Black' } \\ \text { DOJ } & \text { Department of Justice (United States) } \\ \text { EDP } & \text { emotionally-disturbed person } \\ \text { FART } & \text { '[Take the] first available right turn' } \\ \text { FBI } & \text { Federal Bureau of Investigation (United States) } \\ \text { FIDO } & \text { 'Fuck it, drive on' (or 'Forget it, drive on') } \\ \text { GAT } & \text { Service de police de la Ville de Gatineau } \\ \text { GOA } & \text { 'gone on arrival' } \\ \text { HAL } & \text { Halifax Regional Police } \\ \text { HAM } & \text { Hamilton Police Service } \\ \text { KIN } & \text { Kingston Police Force } \\ \text { LAPD } & \text { Los Angeles Police Department } \\ \text { LEO } & \text { law enforcement officer } \\ \text { MHA } & \text { Mental Health Act (Ontario) } \\ \text { NEW } & \text { New Westminster Police Department } \\ \text { NC/NC } & \text { 'no contact = no complaint' } \\ \end{array}$




$\begin{array}{ll}\text { NIA } & \text { Niagara Regional Police Service } \\ \text { NYPD } & \text { New York Police Department } \\ \text { OIPRD } & \text { Office of the Independent Police Review Director (Ontario) } \\ \text { OPP } & \text { Ontario Provincial Police } \\ \text { OPS } & \text { Ottawa Police Service } \\ \text { POR } & \text { Port Moody Police Department } \\ \text { PRPS } & \text { Peel Regional Police Service } \\ \text { PSA } & \text { Police Services Act (Ontario) } \\ \text { PSS } & \text { Professional Standards Section ('internal affairs') } \\ \text { RCMP } & \text { Royal Canadian Mounted Police } \\ \text { RED } & \text { Red Deer Detachment (RCMP Alberta) } \\ \text { RID } & \text { Ridge Meadows Detachment (RCMP British Columbia) } \\ \text { ROC } & \text { Rochester Police Department } \\ \text { SAS } & \text { Saskatoon Police Service } \\ \text { SIU } & \text { Special Investigations Unit (Ontario) } \\ \text { SPVM } & \text { Service de police de la Ville de Montréal } \\ \text { STR } & \text { Stratford Police Service } \\ \text { SYR } & \text { Syracuse Police Department } \\ \text { TOR } & \text { Toronto Police Service } \\ \text { VPD } & \text { Vancouver Police Department } \\ \text { VPU } & \text { Vancouver Police Union } \\ \text { YPBA } & \text { Yonkers Police Benevolent Association } \\ \text { YON } & \text { Yonkers Police Department } \\ \text { YRPS } & \text { York Regional Police Service } \\ \text { WIN } & \text { Windsor Police Service } \\ \text { WPA } & \text { Winnipeg Police Association } \\ \text { WPS } & \text { Winnipeg Police Service } \\ & \end{array}$




\section{CHAPTER ONE}

\section{SITUATING THE PROJECT}

Our ignorance of just what goes on between police and citizens poses one of the central issues in policing today. How can we make the police accountable to the citizenry in a democratic society and yet not hamstring them in their legitimate pursuit of law and order?

— Albert Reiss (1968: 19)

\subsection{Chapter Introduction}

James Comey (2015b, emphasis added), then Director of the United States of America's Federal Bureau of Investigation (FBI), in a 23 October 2015 speech

delivered to an assembly at the University of Chicago Law School, observed,

Something deeply disturbing is happening all across America....Most of America's 50 largest cities have seen an increase in homicides and shootings this year, and many of them have seen a huge increase...Police and civilian leaders need to demand answers. Academic researchers need to hit this hard. I've been part of a lot of thoughtful conversations with law enforcement, elected officials, academics, and community members in recent weeks. I've heard a lot of theories - reasonable theories...but I've also heard another explanation, in conversations all over the country. Nobody says it on the record, nobody says it in public, but police and elected officials are quietly saying it to themselves. And they're saying it to me, and I'm going to say it to you. And it is the one explanation that...makes the most sense to me. Maybe something in policing has changed.

In today's YouTube world, are officers reluctant to get out of their cars and do the work that controls violent crime? Are officers answering 9-1-1 calls but avoiding the informal contact that keeps bad guys from standing around, especially with guns? I spoke to officers privately in one big city precinct who described being surrounded by young people with mobile phone cameras held high, taunting them the moment they get out of their cars. They told me, 'We feel like we're under siege and we don't feel much like getting out of our cars.'

I've been told about a senior police leader who urged his force to remember that their political leadership has no tolerance for a viral video. So the suggestion, the question that has been asked of me, is whether these kinds of things are changing police behaviour all over the country. And the answer is, I don't know. I don't know whether this explains it entirely, but I do have a strong sense that some part of the explanation is 


\section{a chill wind blowing through American law enforcement over the last}

year. And that wind is surely changing behaviour. ${ }^{1}$

Comey's (2015a, 2015b) remarks, which, I suggest, can be related to concerns expressed by Reiss (1968) more than 50 years ago (as presented in this chapter's epigraph), touched off a firestorm, of sorts, across American policing's leadership and within the nation's political sphere. As (former United States’ Attorney General Alberto) Gonzales and Cochran (2017: 303) observe, "Not everyone agreed with the FBI Director. President Obama countered Director Comey’s speech, saying he saw no evidence that police officers were policing less aggressively, and [suggesting that] Comey was cherry picking the data." This contrarian position was endorsed by then Attorney General Loretta Lynch (McCarthy 2015) and her immediate predecessor, former Attorney General Eric Holder, opined, “I frankly don’t think police officers are laying down on the job...I don't think they're taking a knee" (Reilly 2015). There was also criticism of Comey from some American police executives - with chiefs from Seattle and Oakland publicly disagreeing with Comey’s analysis (Reuters 2016a). On 27 October 2015, Josh Earnest, the White House press secretary, speaking on behalf of the Obama administration, challenged the observations of Comey (America's 'highestranking law enforcement officer') in advancing, "The fact is the evidence does not support [Comey's] claim that somehow our law enforcement officers across the country are shirking their duties and failing to fulfill their responsibility to serve and protect the communities in which they are assigned" (Asher-Shapiro 2015; Mallin 2015).

However, one week later, Chuck Rosenberg, who headed the United States’ Drug Enforcement Administration (DEA), entered the public conversation, advising that, in his assessment, the observations of Comey were "spot on" (Frankel 2015; Graham 
2015; Levine \& Taylor 2015). Rosenberg explained that he has been told by various police chiefs across the country,

With cops now under intense scrutiny and videos of their interactions often posted online, officers are concerned 'rightly or wrongly that [they] become the next viral video...I rely on the chiefs and the sheriffs who are saying that they have seen or heard behavioural changes among the men and women of their forces...The manifestation of it may be a reluctance to engage' (Levine \& Taylor 2015, emphasis added).

Rosenberg advanced that while "cops are more reluctant to engage, in certain instances, he doesn't think such an effect is playing out among DEA agents... [that] are not typically policing's first responders - [as are] the [local and state front-line] police [officers] who "handle domestic assaults at 3 in the morning"” (Frankel 2015). Political reaction to Rosenberg's endorsement of Comey's observations was swift, with Earnest challenging "the second administration official to make that kind of claim without any evidence" and suggesting, "You might ask [Rosenberg] if there's any evidence to substantiate the claim he's made" (Graham 2015).

On 11 May 2016, Comey reiterated his de-policing observations (as I characterize them), advancing that, while he could offer "no statistical proof," he understands, after speaking with many police officials across the USA, "There's a perception that police are less likely to do the marginal additional policing that suppresses crime - the getting out of your car at 2 in the morning and saying to a group of guys, 'Hey, what are you doing here?'” (Lichtblau 2016; Reuters 2016a). In this regard, Comey observed, "Officers are deterred from more active policing because they fear being filmed and ending up online ... [with] changes in the way police may be acting and in the way communities may be acting" (Reuters 2016a). He explained that, as FBI Director, he continues to "hear from police officials in private conversations that 
police officers are pulling back from aggressive confrontations with the public because of viral videos" (Lichtblau 2016). Comey elaborated that what is going throughout American front-line police work relates to the "effect of marginal pullbacks by lots and lots of police officers... a change in the way police are doing their work and in the way communities are interacting with police... What I'm hopeful of, and I've seen some signs of it, is that academics will dive into this" (FBI National Press Office 2016).

James Pasco Jr., Executive Director of the National Fraternal Organization of Police (an American police labour organization with more than 330,000 members), suggested that Comey "ought to stick to what he knows...He's basically saying that police officers are afraid to do their jobs with absolutely no proof' (Lichtblau 2016). In response to further criticism from the White House, again discounting Comey's anecdotal de-policing observations because of an absence of empirical support for his claims, retired New York Police Department (NYPD) Commissioner Ray Kelly commended Comey for "telling it like it is...If you talk to police officers in other jurisdictions, not only in New York where I am, they will tell you that they are backing off' (Berman 2016; Reuters 2016a). Further, during a 28 February 2017 speech, then United States Attorney General Jeff Sessions added his voice to America's nascent depolicing dialogue, explaining, "We've heard from law enforcement leaders, including the FBI Director and many police chiefs...[that] many of our men and women in law enforcement are becoming more cautious. They're more reluctant to get out of their squad cars and do the hard but necessary work of proactive, up-close policing" (Sessions 2017, emphasis added). 
In these contemporary policing circumstances, Reiss' observations (as featured in this chapter's epigraph) remain as relevant today as they were in 1968 - in considering challenges in the public-police relationship in 2019 and in relation to the research question investigated in this dissertation's empirical study -How are front-line police officers in Canada and the United States responding to recent techno-social and socio-political modifications to the policing landscape - such as the 'YouTube Effect', advanced by Comey, and the 'intense scrutiny' of policing by today's public audience, as observed by Rosenberg? ${ }^{2}{ }^{3}$ The work of the eminent Yale sociologist, who, in 1965, led "the largest-ever observational study of policing" (Reiner 2017: 239), is considered by many scholars as the gold standard in social-scientific research examining police contact with citizens (Mastrofski, Reisig \& McCluskey 2002; Sherman 2007; Waring \& Weisburd 2002) and Reiss' seminal book-length contribution, The Police and the Public (1971), introduced "a rich array of data on the day-to-day working milieu of the policeman" into popular, scholarly, and policy discourses of that era (Hudson 1973: $1568)^{4}$

My ambition, in the pages that follow, is to present, in the tradition of Reiss and other distinguished policing researchers (several of whom I have had the privilege to meet along this academic journey), a rigorous empirical and sociological contribution to contemporary understandings of 'what is going on between police and citizens' - to loosely paraphrase Reiss (1968: 19) - with a specific focus on today's experiences, attitudes, and behaviours of front-line police officers in Canada and the United States. ${ }^{5}$ In this vein, I argue that, in recent years and across various forums, extensive and influential discussions have been engaged around the public's concerns with police 
actions in the field. However, relatively insignificant consideration has been given to the other constituent in the public-police relationship - the police - and, more specifically, how rank-and-file officers are impacted by today's widely-publicized controversial interactions with citizens, such as events that occurred in Ferguson, Missouri throughout the summer of 2014 (Deuchar, Fallik \& Crichlow 2018; Fallik, Deuchar \& Crichlow 2018; Wolfe \& Nix 2016; Nix \& Wolfe 2017). Similarly, Marier and Moule (2018) counsel, "While much research has examined how public perceptions of the police influence citizen attitudes and behaviours, we hope that criminologists will [examine] the other half of this exchange, as well."

In the specific context of this study's primary research question, I observe that little attention has been directed at trying to understand how today's rank-and-file officers are responding to the increased visibility of, and intensified societal engagement with, their work (exceptions within the extant literature are detailed in Chapter Two). In other words, most of us likely have a fairly clear understanding around how many of our fellow citizens have reacted to contemporaneous and controversial representations of front-line police work, which now come to the public's attention through the contemporary phenomenon of 'policing's new visibility' (e.g., see Brown 2013, 2016; Brucato 2015a, 2015b; Campeau 2015, 2016a; Farmer 2016; Goldsmith 2010; Haggerty 2012; Haggerty \& Sandhu 2014; Lautt 2012; Newell 2019; Sandhu 2016, 2019; Sandhu \& Haggerty 2015, 2017; Schneider 2016) but little awareness around how front-line officers are adapting to the policing landscape in which they now perform their sworn duties to serve and protect us - the community (Deuchar et al. 2018; Nix \& Wolfe 2017). ${ }^{6}$ 
In contributing to scholarly and popular understandings in this regard, this dissertation reports on the first (and, to my knowledge, the only) large-scale, multisite, binational, and mixed methods empirical research into front-line officers' behavioural adaptations to what many across the rank-and-file consider to be inherent risks within today's policing environments throughout Canada and the USA - with findings of widespread de-policing practices across this study's research population. I define the term de-policing, as was exemplified in the observations of Comey and Sessions earlier in this chapter, as the practice by which an officer intentionally reduces, or eliminates, proactive police activities, in response to the officer's perception that discretionary initiatives and interactions with citizens are inherently and unnecessarily 'risky' (in that they hold the potential for substantial occupational risks), within the context of recent transformations in the front-line policing landscape - in terms of the aforementioned techno-social and socio-political developments throughout contemporary Western democratic societies. In this regard, the reader should understand de-policing as specific and proactive risk-averse practices among some officers - as contrasted with the "workaverse' practices of some police officers that are simply 'lazy'. These distinctions are elaborated in subsequent chapters.

Consider that when we contact 9-1-1 and request police assistance, help from the responding officer(s) is what most people, at least those residing in Western democracies like Canada and the United States, expect we will receive. Along these lines, I suggest that most of us operate on the assumption, consistent with the tenets of the 'social contract' (in the tradition of Hobbes, Rousseau, and Locke) and historical policing practices, that rank-and-file representatives of our public policing agencies - 
apparently deployed with some strategic rationale throughout our communities - will respond to our distressing circumstances expeditiously, professionally, and with some genuine concern for our wellbeing. ${ }^{7}$ In other words, police officer(s) will 'serve and protect' us - the public. This understanding, around the central function of public policing, and variations - such as 'protect and serve', is often presented as the fundamental organizational ethos of many Western police agencies, in the form of foundational mottos, mission statements, expressions of key principles, declarations of core values, etc., and is frequently extolled, in writing, on police patrol vehicles - as demonstrated with the Toronto Police Service and Chicago Police Department cruisers depicted below in Image 1 and Image $2 .^{8}$

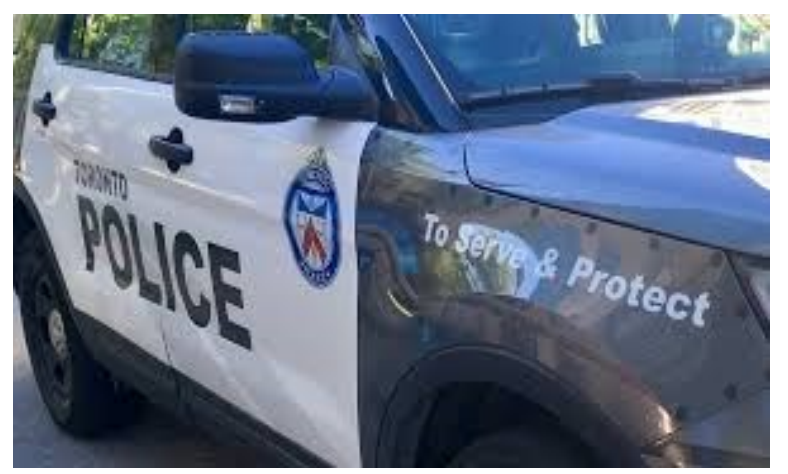

Image 1: TPS cruiser (Bond 2019)

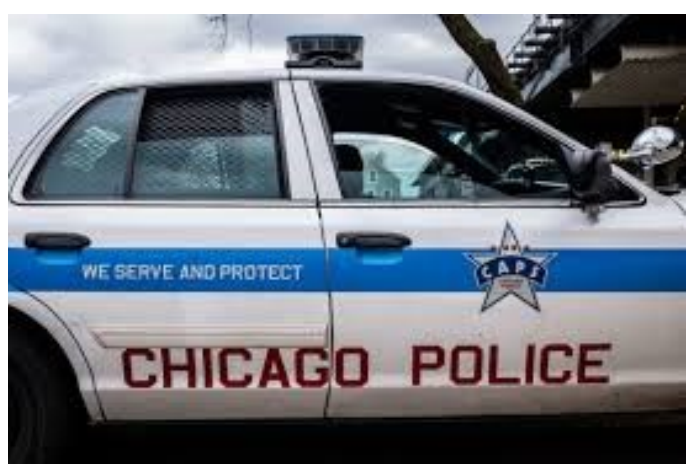

Image 2: CPD cruiser (Bedi 2017)

In this vein, Bonkiewicz (2017: 164, citing Bittner 1970; Goldstein 1987) observes,

Law enforcement agencies and their jurisdictions invest a substantial amount of resources to train, equip, and maintain a police force. In return, police supervisors and citizens expect that police officers - specifically patrol officers - will use their time productively to reduce crime, improve community safety, and foster partnerships with community members.

Along these lines and through the widespread endorsement of the 'communitybased policing' (CBP) doctrine across most Canadian and American police agencies over the past five decades - and, therefore, the repudiation of the reactive emphasis of previous models of front-line police operations (e.g., see Bayley 1988, 1994; Greene \& 
Mastrofski 1988; Kelling \& Coles 1996; Manning 1988, 1993; Reiss 1992; Rosenbaum 1996, 1998; Skogan 2003, 2006b, 2019; Skolnick \& Bayley 1988; Uchida 2005;

Weisburd \& Braga 2019) - we, as a society, have come to expect that front-line officers will not only attend to dispatched calls resulting from citizens' calls for assistance (reactive policing), but that they will also dedicate their discretionary time to selfinitiated practices in pursuit of the ideals of $\mathrm{CBP}$ - one of the tenets of which is proactive policing. As Sherman (2007: 1110) explains, it was Reiss (see Bordua \& Reiss 1966; Reiss \& Bordua 1967),

[who] first contrasted the most common form of police work, 'reactive', with 'proactive' [policing]...These terms spread like wildfire after they were first published in the mid-1960s...First among academic social scientists, then among police officials themselves...This [proactive] concept was not only new to police work. It was also new to the English language. ${ }^{9}$

In 1988, Skolnick and Bayley suggested that "the continuing shift [to CBP] represents a major effort to restructure the role of police... by replacing the traditional reactive, rulebound, highly hierarchical [model] with a more responsible, decentralized, proactive [model]" (Correia 2000: 219).

Some scholars have characterized reactive emergency order maintenance practices in front-line police work as 'fire-brigade policing' (e.g., see Bowling, Iyer, Reiner \& Sheptycki 2016; Joyce \& Wain 2010; Reiner 1985, 2012; Taylor 1981). In this regard, Reiner (1985: 221) explains, 'fire-brigade policing' involves "police actions that are geared to responding to emergency calls for service." He later observes, "The label 'fire-brigade policing' originated in the 1970s and was invariably used as a pejorative term [in the context of the new CBP model]. It referred to policing styles that were seen as damaging to a consensual police-public relationship" (Reiner (2012: 7). Taylor 
(1981: 147) advances that this style of policing "not only leads to people generally meeting the police in conflict, but necessarily negates 'community/preventive' policing in any meaningful way." In this regard, Joyce and Wain (2010: 84-85) find,

Fire-brigade (or reactive) policing involved redirecting patrol work to respond to events after they had taken place rather than seeking to prevent them from occurring...However, there were several problems with firebrigade policing, in particular the way in which the police service failed to cultivate intimate relationships with the communities in which they operated. This posed particular problems in multi-racial, inner-city areas...[and these problems] resulted in a shift away from reactive methods in favour of community policing.

As discussed, several prominent law enforcement officials, and a handful of scholars, media commentators, and politicians, as detailed throughout the literature review in Chapter Two, have suggested recently that as yet unascertained numbers of front-line officers have now retreated from proactive engagements with individuals in the community and have reverted to the practice of reactive (or 'fire-brigade') police work - the result of officers' risk-versus-reward assessments related to discretionary policing activities/interactions. As I explained, in defining the terminology earlier in this chapter, I refer to such intentional and proactive risk-avoidance practices as de-policing.

It is ironic that Comey presented his initial concerns about de-policing in Chicago. This is because, since the 9 August 2014 fatal police shooting of Michael Brown in Ferguson, Missouri, the City of Chicago has been at the forefront of discussions, across media and throughout policing, around (1) the belief that today's risk aversion among the rank-and-file is impacting negatively on front-line police work, including encouraging officers to practice de-policing behaviours, and (2) the popular 'theory', which is often intertwined with observations of de-policing, which has come to be known as the 'Ferguson Effect.' 10 Those advancing the 'Ferguson Effect' hypothesis 
suggest that correlations exist between the rise in violent crime rates observed in many American cities throughout this 'post-Ferguson era' (as some have characterized the period following the fatal police shooting of Michael Brown in August 2014 to the present) (e.g., see Campbell, Nix \& Maguire 2017; Chang, Neugebauer \& Birmingham 2017; Culhane, Boman \& Schweitzer 2016; Deuchar et al. 2018; Fallik et al. 2018; Lindsey 2015; Torres et al. 2018) and the de-policing phenomenon - the apparent retreat of large numbers of front-line officers from proactive police work (e.g., see Campbell et al. 2018; Maguire, Nix \& Campbell 2017; Nix \& Pickett 2017; Nix \& Wolfe 2016, 2018; Nix, Wolfe \& Campbell 2018; Pyrooz, Decker, Wolfe \& Shjarback 2016; Shjarback, Decker, Wolfe \& Pyrooz 2017, Shjarback, Pyrooz, Wolfe \& Decker 2017; Wallace, White, Gaub \& Todak 2018; Wolfe \& Nix 2016). In this regard, Dewan (2017), in a New York Times piece titled 'Deconstructing the Ferguson Effect', wrote, "The police, vilified and facing a hostile public, [are] unable to do their jobs, leaving criminals to run amok. It [is] called the Ferguson Effect. Though based on thin evidence and met with fierce rebuttals, the theory keeps coming up."

Given the limited scope of this dissertation, I have not engaged in any empirical investigation around the validity of the so-called 'Ferguson Effect'. Rather, the present study is focused exclusively on the phenomenon of de-policing throughout today's front-line police work. However, it is important that the reader appreciates that the term 'Ferguson Effect' is often conflated, in media and across some of the extant scholarly literature, with the behavioural adaptations of some front-line officers to their contemporary policing landscape, which I refer to as de-policing - absent any association to purported increases in crime rates. For example, Gately (2015) describes 
the 'Ferguson Effect' as, "The argument that police, fearful of being disciplined or even indicted for misconduct, have pulled back on enforcement amid protests and fierce public criticism of law enforcement after several prominent incidents of police killings and other violence against civilians caught on tape over the past year." I approach depolicing and any 'Ferguson Effect' as two discrete concepts - in this regard, what Gately describes above is, by my definition, de-policing rather than the 'Ferguson Effect. ${ }^{11}$ It is certainly possible that the practice of de-policing by rank-and-file officers could result in a 'Ferguson Effect' - the absence of a proactive front-line police deterrent influence and, therefore, an emboldening of the 'criminal element' in which some individuals are induced to engage in violence - but such a linkage will have be the product of another researcher's work.

In saying this, based on my review of the literature to date, in relation de-policing and, tangentially, any potential 'Ferguson Effect', Chicago could be considered as the best possible case study - or perhaps more appropriately, given the tremendous violence over the past few years, the worst-case de-policing scenario. Along these lines, in their reporting on the 'Crisis in Chicago,' during an episode aired across the United States and Canada on 1 January 2017, the popular television newsmagazine 60 Minutes focused not on the incessant violence traumatizing several Chicago neighbourhoods, but rather on the challenges confronting the rank-and-file of the Chicago Police Department (CPD) and front-line officers' reactions to their perceptions of antagonistic and 'risky' policing environments in several neighbourhoods throughout that city. ${ }^{12}$ 


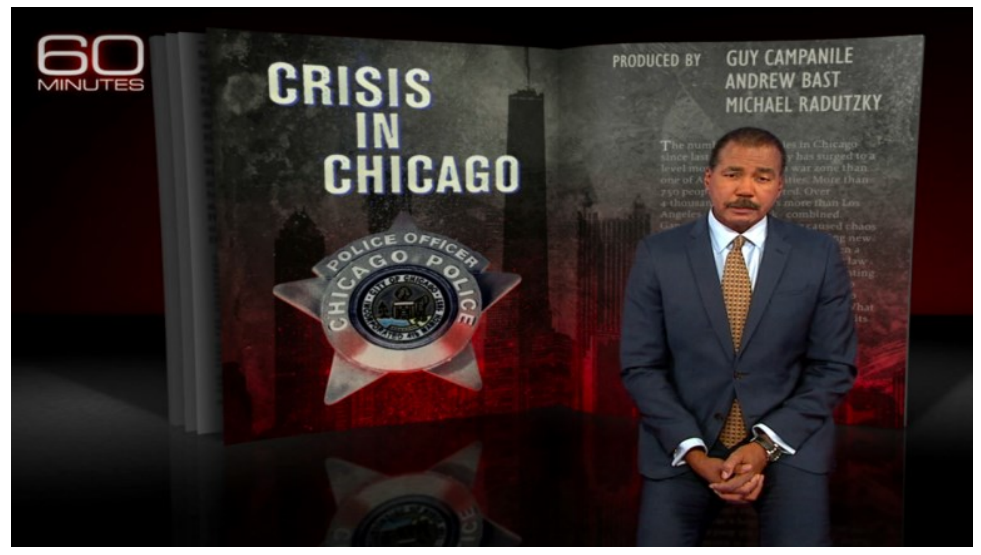

Image 3: 60 Minutes 'Crisis in Chicago' (Whitaker 2017)

The introduction to the episode advanced,

Gangs, guns and drugs have caused chaos in Chicago for years. But something new caught our attention. There's been a drop in the kind of police work that law enforcement says is critical to preventing crime. Usually stops and arrests go up when violence is rising. So, we went to Chicago to look for an explanation. What we found was a police department on its heels as the city suffered its worst bloodshed in 18 years...We were astonished by data we obtained from inside the police department. It revealed that as killings rose, police activity fell. In August of 2015, cops stopped and questioned 49,257 people. A year later those stops dropped to 8,859 , down 80 percent. At the same time arrests were off by a third, from just over 10,000 to 6,900 (Whitaker 2017, emphasis added). ${ }^{13}$

The interchange between Bill Whitaker, the episode's host, and Brian Warner, a CPD

officer who was wounded in a 2011 shooting (and who now counsels CPD officers

struggling with mental health challenges), is informative:

Whitaker: You talk to cops every day.

Warner: [I] do.

Whitaker: What's the morale?

Warner: Lowest it's ever been.

(Warner is a former Chicago cop [and] he explained what a dozen beat cops told us offcamera: They had stepped back.)

Warner: You have a 9-1-1 call; you go to your 9-1-1 call. But aggressive patrol when you're out looking for people breaking the law. That's not happening as much as it was.

Whitaker: You say they're not being as proactive? 
Warner: No. They're not. And how could you ask them to be? And why would you expect them to be?

Whitaker: Because it's their job. They signed on to do that.

Warner: It's my job to go to work and listen to your 9-1-1 calls and respond. That's the basic [responsibility] of my job. So, if you want me to do the basics that's what I am doing now.

Crime Lab researchers at the University of Chicago analyzed official CPD data from 2014 through the end of 2016 and they presented an interesting (some would characterize it as a troubling) finding in relation to the number of 'street checks' of 'suspicious' persons by CPD officers during this period (Kapustin, Ludwig, Punkay, Smith, Speigel \& Welgus 2017). I have reproduced their analysis, in this regard, in a graphic representation (below). As Ford (2017) observes, “Chicago experienced a confluence of events in late 2015 [that included the public release of a video recording of the fatal police] shooting of Laquan McDonald, [which instigated] protests throughout the city and heightened scrutiny [of front-line CPD officers].”

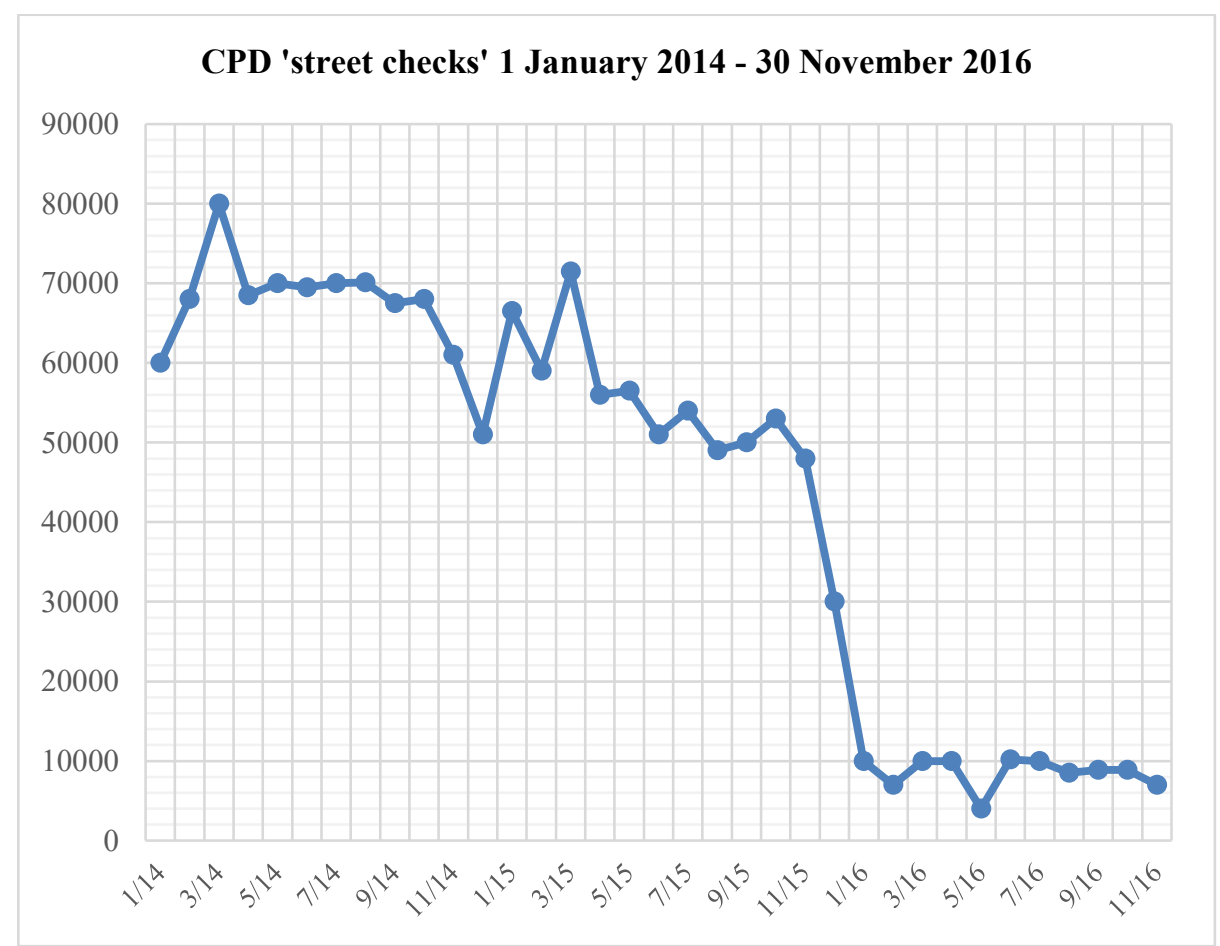

Figure 1: CPD 'street checks' 2014-2016 
Whitaker (2017) showed this information to former CPD superintendent Garry McCarthy, who was terminated in the wake of controversy after the release of the Laquan McDonald video recording, and Whitaker asked, 'How can a police officer who has taken a vow to protect and serve defend stepping back from taking proactive action?' ${ }^{14}$ McCarthy responded, 'Officers are under attack...That's how they feel in this environment, and they're not going to put themselves and their families in jeopardy.'

On 8 October 2015, the Washington Post reported on what was supposed to be a private, closed-door meeting, involving "more than 100 of the nation's top law enforcement officers and politicians" - including then Attorney General Loretta Lynch (A. Davis 2015). ${ }^{15}$ "The unifying and controversial [conclusion of attendees was that] officers in American cities have pulled back and have stopped policing as aggressively as they used to, fearing that they could be the next person in a uniform featured on a career-ending viral video" (A. Davis 2015). At the meeting, Chicago Mayor Rahm Emanuel told Lynch, "[Officers] have pulled back...they don't want to be a news story themselves; they don't want their career ended early, and it's having an impact" (A. Davis 2015). In an interview the following week, Emanuel explained, "Officers themselves [are] telling me about how the news over the last 15 months [has] impacted their instincts - do they stop, or do they keep driving? 'When I stop here, is it going to be my career on the line?"' (Sanchez 2015).

On 2 January 2017, American cable news network $C N N$ discussed the apparent depolicing taking place in Chicago across all of that day's programming. Appearing with host Anderson Cooper was David Axelrod - a Chicago native and resident, CNN's 
senior political commentator, former senior advisor to President Obama, and founder and director of the University of Chicago's Institute of Politics (Flores 2017).

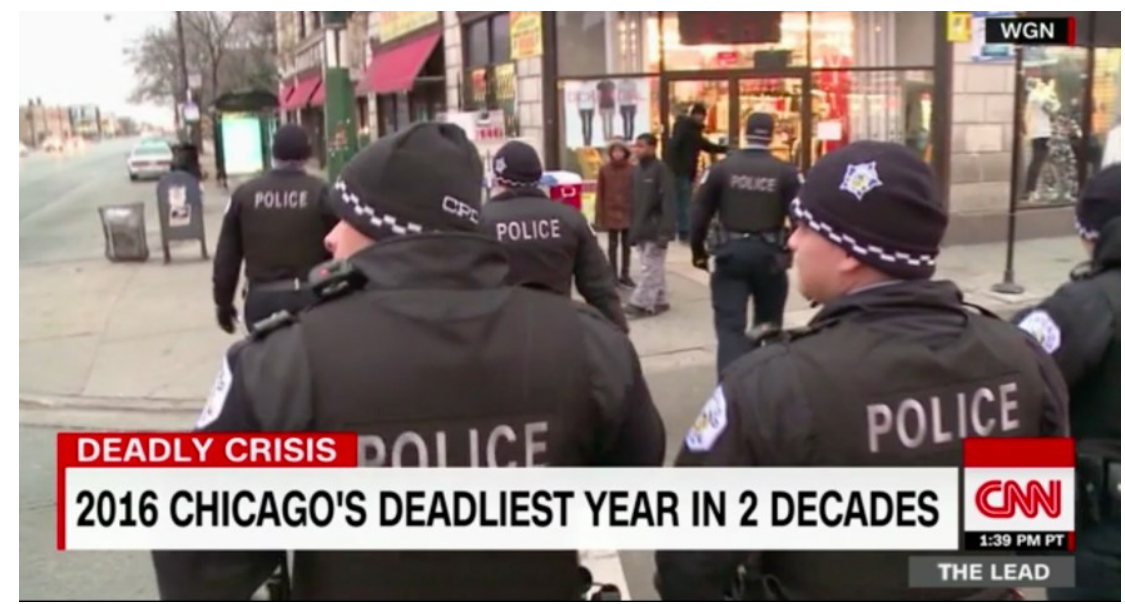

Image 4: $C N N$ Chicago's deadliest year in 2 decades (Flores 2017)

Their discussion around the recent CPD street check and arrest data, is instructive:

Cooper: What is behind these numbers?

Axelrod: Well, one thing that is behind them for sure is there has been a real slowdown of police action since the shooting of Laquan McDonald erupted as an issue in Chicago... All of the ramifications and reverberations from that have left the police department demoralized and really standing down in many interactions; arrests are way down, stops are way down, because police officers do not want to become engaged.

In investigative pieces published in the Chicago Tribune, addressing de-policing among front-line CPD officers, Gorner (2016b, 2016c) presented the thoughts of a 20year CPD veteran and front-line patrol supervisor who now wants to leave what the officer had hoped would be a lengthy and rewarding police career,

Like many others he works with, this cop believes his proactive style of policing has become a relic of the past because of real fears over lawsuits, firings, even indictments...'The bad element knows that policemen aren't willing to do the job the way they did it (before),' said the veteran cop, a supervisor who's getting his resume in order... '[The criminals] are right in tune with how police are [now] policing.'

Officers of different ranks - from patrol officers to high-level supervisors - had a consistent message...They have grown frustrated, worried that their interactions with uncooperative suspects will be video-recorded, 
posted online and misunderstood. They fear an unfounded citizen complaint could cause their firing or other harsh punishment. They also chided the news media for nonstop negative coverage that they believe is fueling the poor public perception of law enforcement. 'There's much more scrutiny, there's much more second-guessing,' said one veteran sergeant assigned to citywide duties. 'It's almost like we're the bad guys.'

Given the uncertainty and concern, some officers say police have stepped back on their aggressiveness. Some said they're only responding to 9-1-1 calls over the radio, not relying on their street smarts to act on their own. 'Unfortunately, it's more reactionary than proactive,' one patrol officer who primarily patrols parts of the North Side said of police work. 'You have to wait for the crime to be committed.' Dennis Rosenbaum, a professor of criminology at the University of Illinois at Chicago, said it has become increasingly difficult for police to be proactive on the street amid so much criticism. 'It's much easier to stay below the radar, respond to calls, engage in what we call reactive policing,' said Rosenbaum. 'For an individual officer on the street, they need to know that somebody has their back and if they feel that they're going to get thrown under the bus when they do something, why would you proactively get out of your car or stop a group of kids who ... seem to be up to no good?'

In the Canadian context - although my perception is that there has been

considerably less discussion around the phenomenon of de-policing across Canada's

policing, governance, media, and academic communities than south of the border,

Canada's national broadcasting agency (the $C B C$ ) presented an in-depth and hour-long examination into the issues (and controversies) engaged with 'policing's new visibility' in a 4 November 2016 episode of The Fifth Estate, titled 'Police shootings: Caught on camera' (Kelly 2016). ${ }^{16}$ Also, Chris Lewis, a former Commissioner of the Ontario Provincial Police (2010 to 2014) and now CTV News' national public safety analyst, who appears frequently across Canadian television discussing contemporary policing occurrences and issues, wrote an opinion piece for the Canadian trade publication Blue Line Magazine, titled 'Is de-policing alive and not so well?', in which he advances,

Proactive policing should be a big part of what officers do. It shouldn't simply be responding to radio calls. Conducting high visibility patrols, 
checking out suspicious people in suspicious circumstances, interacting with vulnerable people and preventing crime and victimization - these are all critical roles for police officers. However, conversations I've had with officers from a variety of police services suggest that members are only doing what they have to more and more due to a variety of factors. These include increasing oversight, public criticism and officer morale (Lewis 2018b: 30, emphasis added).

Similarly, in a 15 February 2018 'op/ed' published across CTV News' Toronto affiliate, responding to my de-policing study (being reported in this dissertation) and mediated discussions on this study's preliminary information (e.g., see Crawford 2018; CTV News 2018; Yogaretnam 2018b), Lewis (2018a, emphasis added) addressed depolicing as "a concerning situation in North American policing" and he advanced,

With some real and many perceived police wrongdoings becoming viral postings on social media sites, apparently some officers have chosen to avoid the potential for conflict altogether...many officers feel the balance has tipped to the point where everything that they do is overly scrutinized...I personally think the malaise identified by Mr. Brown [also implicates] declining officer morale...I communicate with officers from all over North America and in the 40 years I've been talking to cops, I've never seen it worse...Police morale can be negatively impacted by antipolice rhetoric and protests; violent acts against police; and the seemingly never-ending swirl of mainstream and social media criticism...I agree with Mr. Brown that the current situation is not good...[and] without public and government support, many police officers will only do what they have to do and no more. None of us want that - not the officers or the public they serve. We want them to professionally keep us safe and secure. I truly believe that's what the overwhelming majority of our police officers want as well.

Further, Mike McCormack, president of the Toronto Police Association - the labour organization representing roughly 5,500 front-line Toronto Police Service (TPS) officers, has discussed, on several occasions, via media to public audiences, the phenomenon of de-policing throughout the rank-and-file of the TPS. For example, in responding to the 2016 conviction of TPS Constable James Forcillo in the fatal police shooting of Sammy Yatim (Forcillo was convicted of attempted 
murder but acquitted of second-degree murder) and concurrent antipathy

demonstrated by some Toronto residents toward the TPS, McCormack interpreted the mindset of TPS front-line officers - in relation to them remaining diligent in their police work,

Clearly this [verdict] sends a chilling message to our members and our goal is to keep our members engaged, [to] keep them out there doing their job, but when you have an officer who is vindicated for the first three shots and then who said [consistent with] my training I made a mistake and the he's put in this position it sends a chilling message to our members and that's going to be a challenge for our front-line members to deal with...It has definitely impacted the psyche of front-line officers (CBC News 2016b, emphasis added). ${ }^{17} 18$

\subsection{Origins of the Study}

In a previous study, completed in 2013, I explored the phenomenon of 'policing's new visibility' vis-à-vis behavioural modifications in how front-line officers use force in certain interactions with citizens (Brown 2013, 2016). Several participants in that study, from both the Ottawa Police Service (OPS) and the Toronto Police Service (TPS), disclosed, in their interviews, that they are engaged in the practice of de-policing - in response to the visibility of their work in the field and the influence of social media critiques of police actions. ${ }^{19}$ For example, a veteran front-line TPS officer explained,

With everyone with a camera phone, including the drug dealers and the gangsters, and their girlfriends and mothers, etcetera, you would have to be retarded not to be concerned about what could happen on a call where there was resistance and use of force was required. So, you are fighting with the guy and you go to a use of force option that is not allowed by the 'rules' [sarcastic tone accompanied by eye rolling] and say he gets hurt bad. Now you've got SIU and they've got a video that shows exactly what he was doing and exactly what you did, and what you did is 'technically' against policy and therefore illegal. So yeah, that is a big concern for me. A big concern! I don't want to lose my job over something like that or over some piece of shit drug dealer who puts up a fight. These camera phones everywhere is a game-changer and anyone who tells you they are not 
concerned or they haven't changed how they do some things is full of shit (Brown 2013: 212). ${ }^{20}$

In research commissioned by leadership of the OPS in 2015 , a colleague and I interviewed 57 front-line OPS officers in relation to a traffic stop race data collection study being conducted by that police agency (Brown \& Primeau 2015). ${ }^{21}$ Some officers disclosed, in their interviews, that they are practicing de-policing to avoid being accused of 'racial profiling'. For example, a veteran OPS officer shared,

Officers are hesitating doing [traffic stops] because they don't want to be the guy who is found to have pulled over more [minorities] than the stats say they should be pulling over...Like driving through [a high-crime area] and thinking, I already pulled over 3 [visible minorities] today so I can't do anymore...This is helping the people who hate the police and who are breaking the law... What we are training officers to do is to think about staying out of trouble rather than to do proactive police work (Brown \& Primeau 2015: 18).

Similarly, another OPS officer, who reported that s/he has not changed proactive traffic stop practices, has observed that officers, who work in areas of the city where there are higher concentrations of visible minorities, are avoiding discretionary traffic and street stops of persons who are perceived to be visible minority because,

They are wary of getting a reputation from their stats as being a racial profiler. A lot of officers would rather not get involved than have to deal with the fallout from the [visible minority] community if their numbers are interpreted the wrong way (Brown \& Primeau 2015: 18).

The de-policing practices described by many participants in these two earlier studies coincided with some of my own experiences, while working as a front-line patrol supervisor with the OPS from 2010 to $2013 .{ }^{22}$ In considering these personal observations and given: (1) the limited literature discussing de-policing; (2) the dearth of empirical research examining front-line officers' behaviours in relation to the current techno-social and socio-political environments in which they do their work in our 
communities; and (3) the importance of these new policing realities for sociological understandings of contemporary policing, I decided that my doctoral research would focus on the investigation of risk-averse practices (specifically de-policing) among today's rank-and-file. My first thoughts, in relation to the phenomenon that has now become known as de-policing, emerged in the aftermath of a controversial Ottawa court ruling in 2011 - in which the presiding judge (Justice Dianne Nicholas of the Ontario Court of Justice) suggested that the involved OPS officers had engaged in the practice of 'racial profiling' during a 10 August 2010 traffic stop, search and seizure, and arrest of two Black males.

In this regard, one officer, in his court testimony, indicated that the men provoked his suspicion because they were in 'a high crime area' known for street-level drug transactions and because they avoided making eye contact (Hurley 2012). A search of the vehicle (without warrant) resulted in the seizure of crack cocaine, cash, and trafficking paraphernalia (Tatum 2011). During the court proceedings on 23 June 2011, Justice Nicholas questioned the rationale for stopping the two men, inquiring 'How many White women do you stop [in that area] just because they're driving a car' (Hurley 2012)? The judge "inferred from the evidence before the court that it sounded like the two [defendants] had been racially profiled" and, in light of this conclusion, the prosecutor withdrew the charges (Tatum 2011) ${ }^{23}$ Considerable traditional and social media commentary ensued, which included public disclosure of the names of the officers involved, and both of the arrested parties initiated civil lawsuits alleging false arrest, false imprisonment, and violations of their constitutional rights (Hurley 2012). 
This 'real life' occurrence bears remarkable likeness to the hypothetical scenario introducing a 2017 journal article, co-authored by Alberto Gonzales (a former United States Attorney General), which addressed de-policing in today's front-line police work:

You are a police officer working the night shift in a major U.S. city. In the dark hours of the early morning, you come across a group of young males in a part of the city known for criminal activity. When they see your patrol car, the young men stop what they are doing and look away quickly. All of your training, as well as the instincts that you have developed over years patrolling these same streets, tells you to stop and at least attempt to start a conversation with the group to determine whether criminal activity is afoot and perhaps prevent it. There is, however, a nagging thought in the back of your head. Isn't it possible - or perhaps likely - that someone in the group or nearby will have a video device and record the encounter? What if the crowd attempts to provoke a confrontation and then records it? What if the recording is posted to the Internet or sent to the media? Should such thoughts temper your judgment in this situation? Would they make you hesitate to get out of the car? (Gonzales \& Cochran 2017: 299).

In returning to the 'real world,' I was working an overnight shift in July 2011, shortly after media reports began circulating about Justice Nicholas' 'racial profiling' finding. I had read, heard, and watched some of the traditional media coverage. ${ }^{24}$ In the early morning hours of that busy summer night, I found myself driving through a neighbourhood plagued by gang violence, shootings, and street-level drug trafficking and I encountered an oncoming Mercedes Benz sedan with a Québec licence plate, which disclosed (from the vehicle registration system practiced in Québec) that the car was a rental. Occupying the Mercedes Benz were three young Black men, who made a point of not looking in my direction (despite my police cruiser being the only other moving object in the otherwise quiet neighbourhood at 4 a.m.) and, after the vehicle had passed, I observed the back-seat passenger shifting seats with the front-seat passenger and the vehicle's windows being closed. 
As with the fictional officer depicted in Gonzales and Cochran's (2017) scenario, these observations, in the context of my 11 years of front-line patrol experience (to that point in my career) and my background in both drug investigations and major case investigations (of which more than just a few drug trafficking files and violent crime occurrences I investigated were in that very neighbourhood), were setting my suspicions (or 'street-policing' intuitions) into motion. I, like all police officers in Ontario, had been trained (through courses at the Ontario Police College and subsequent in-service training) that the core function of a front-line officer is to be alert to, and suspicious of, things that seem 'out of place.' Throughout my career, I was told innumerable times that police officers should - as part of their mandate, duties, and to fulfill the 'serve and protect' expectations of most citizens - demonstrate an inherently-suspicious 'working personality’ (Skolnick 1966; Van Maanen 1973, 1974, 1975), which, if one is skilled (and lucky), can develop into the highly-coveted 'cop's sixth sense' that characterizes exceptional and legendary 'street cops.'

So, while a high-end rental vehicle from Montréal in that neighbourhood, at that hour; passengers who inexplicably shifted seats upon seeing a passing police cruiser; and occupants who purposely avoided eye contact with an officer, signified 'suspicious' to me - and all of my policing experiences and instincts portended that these were persons that should be investigated - I had not observed the violation of any statute. ${ }^{25} \mathrm{I}$ will not insult the intelligence of the reader by denying that many (what are often considered the 'most effective') 'street cops' can be resourceful in developing lawful authorities to stop a vehicle and make inquiries of occupants - for example, conducting sobriety checks; inspecting a vehicle's mechanical fitness; and/or examining licence, 
registration, and insurance documents to ensure these requirements are 'in order'. Under Canadian law, random stops of vehicles for such purposes are lawful (R.v Ladouceur [1990]). However, since that 5-4 decision of the Supreme Court of Canada in 1990, reliance on such provisions to otherwise investigate 'suspicious' circumstances have been assessed more and more disapprovingly as contrived (and inappropriate) 'pretext stops' by Canadian courts, media, and concerned citizens across the public sphere. For example, Justice David Doherty of the Ontario Court of Appeal defined a 'pretext stop' (in Brown v. Durham Regional Police Force [1998]) as the use by the police of legitimate powers to stop a vehicle, but in doing so, they have an additional improper purpose, which involves "the infringement of a person's constitutional rights... Officers who stop persons intending to conduct unauthorized searches, or who select persons to be stopped based on their sex or colour...all act for an improper purpose."26

In various discussions with Canadian prosecutors, defence lawyers, judges, and police/court media reporters over the last few years, I have been told that today it is the case that officer's observational claims that lead to searches, seizures, and arrests - such as 'I thought I saw a bottle that was possibly open alcohol in the vehicle' or 'I smelled a faint odour of marihuana emanating from the vehicle' - are now being received with considerable (and increasing) skepticism in the courts and, not insignificantly, in the 'court of public opinion.' In any event, I would be very disappointed with myself if my 'gut feeling' (or street-policing 'sixth sense') had anything to do with the skin colour of those three young men in the Mercedes Benz, rather than the behavioural cues I was observing, however, as a sociologist, I am acutely aware that bias is something that can function unconsciously in all of us - as was articulated in the Ontario Court of Appeal's 
landmark decision in R. v. Brown (2003). In that ruling, the justices found, "The attitude underlying racial profiling is one that may be consciously or unconsciously held. That is the police officer need not be an overt racist. His or her conduct may be based on subconscious racial stereotyping" (R. v. Brown [2003]).

Regardless, as I considered stopping the Mercedes Benz, summoning other officers (given that there were three persons in the vehicle), and then investigating the occupants' circumstances, I paused and engaged in what can be considered a spontaneous risk assessment (or perhaps cost-benefit analysis). On the one hand, these might be Montréal 'gang bangers' and my self-initiated and discretionary (proactive) police work might result in interdicting serious criminal activity for the benefit of those citizens I was sworn to serve and protect. Alternatively, given my perceptions around a prevailing 'anti-police' socio-political climate - particularly involving public concerns, and frequent criticisms, around police relations with those from minority communities and today's techno-social realities of 'policing's new visibility', I appreciated that initiating such a proactive interaction could cause me significant professional and personal grief.

My mind turned to the recent 'racial profiling' controversy (resulting from Justice Nicholas' observations) and a potential scenario in which these three men in the Mercedes Benz: (1) greeted me with camera phones ablaze - video recording my words, gestures, and actions, which would be subject to after-the-fact interpretation by an unknown audience; (2) made allegations that I stopped them because of 'racial profiling' (sometimes referred to in the literature as 'driving while Black'); (3) refused to cooperate with my requests; and (4) created such a commotion that citizens in the 
vicinity attended our location and began agitating in opposition to police. Such situations can deteriorate precipitously, as I have experienced first-hand, and can result, in an immediate sense, in arrests that become police use of force events (with the potential for injuries - to arrested parties and/or officers) and, after the fact, in police oversight and human rights tribunal complaints against officers, internal investigations, negative media exposure; public ridicule and shaming, damage to (professional and personal) reputation, civil lawsuits, and disciplinary sanctions and/or criminal convictions - including, in some cases, dismissal from the police agency and even incarceration.

I am not particularly proud to report that my decision, in that moment, was to practice de-policing. Weighing all of the potential outcomes, I decided, albeit hastily, that the risk to me (of professional and personal consequences) was too great to justify taking the action that my police experience and instincts suggested was prudent in the circumstances. As a result of my decision - I was not video recorded engaging in behaviour that could be interpreted, by some in the community and/or media, as problematic; I was not accused of 'racial profiling'; and I was not the subject of a formal complaint, lawsuit, or disciplinary hearing. But, these thoughts, which translated into behaviour in that moment, and similar situations I encountered thereafter, caused me much concern. Hence, why I chose this topic to study in my dissertation research.

The reader should understand that, while, as a front-line supervisor, I was not mandated, nor necessarily expected, to respond to 9-1-1 calls from the public or to engage in discretionary patrol work - i.e. traffic stops; proactive 'suspicious person' or 'street' checks; and/or interacting with persons I came upon that were intoxicated or in 
the midst of a mental health crisis - I found myself on several occasions engaging in the same risk analysis process described in the preceding paragraphs. And, I often concluded, in those moments, that de-policing would be the most prudent course of action - versus the risk of intervening in a situation that could become controversial or contentious, in terms of ex post facto interpretations of my decisions and/or actions by some across the public sphere and/or those involved in police oversight investigations. Earlier in my front-line policing career (spanning 1985-1995), a perhaps 'marginal' traffic stop or street check of a person (based more on an officer's 'suspicion' of criminal conduct rather than the 'official' statutory authorities relied on to justify the proactive intervention) that turned up evidence of a crime, might have been 'unofficially' commended by the presiding judge, who, in acquitting the accused person(s), still offered encouraging praise for the officer's 'good faith' police work and diligence in serving and protecting the community. In a conversation 25 years ago with one of Ottawa's premier criminal defence lawyers (who shall remain anonymous), after the evidence I discovered during a vehicle search had been ruled inadmissible because I had not established reasonable grounds to the court's satisfaction, s/he expressed, 'Hey, it was great street policing, but, for court, it just wasn't enough. But, keep up the good work, I want a cop like you in my neighbourhood.'

Now, in 2019, it seems, in my assessment, that front-line officers face much greater stakes - in terms of the potential fallout from proactive interventions developing from their subjective perceptions of 'suspicious' circumstances. This apparent new reality for rank-and-file police work and the potential consequences of the contemporary policing landscape, for me, and for the officers I was responsible to supervise, became something 
I assessed as urgently requiring empirical investigation. In this project's infancy, I consulted informally with several officers on my platoon and received near-unanimous feedback suggesting that this 'pull back' (or de-policing) is both a recent and a widespread phenomenon, which is not talked about outside of the front-line police subculture - it is just the 'new reality', with the rank-and-file adapting to 'cover their ass' and make it unscathed to a transfer away from front-line duties and/or to retirement. In this regard, Gonzales and Cochran (2017: 306) find, "It is apparent that...citizenrecorded videos, posts of videos on the Internet, and aggressive confrontations with police officers - are now simply a reality of modern policing that police officers must come to terms with."

\subsection{Relevance, Originality, Research Objectives, and Research Questions}

With any empirical research, in addition to deliberations around the nature of the inquiry and the study's methodology, another matter that invites reflection from the reader is the 'so what' (or relevance) question. In this regard, it is widely accepted across the discipline of sociology that advancing understandings of policing is important, given the social, legal, cultural, and political significance of police in contemporary Western systems of governance and social control (Klockars 1985; Neocleous 2000; Newburn \& Reiner 2012; Reiner 2010; Reiss 1971). For example, Reiner (1992a: 762) finds that "policing is at the heart of the functioning of the state, and central to an understanding of legal and political organization." Along these lines, I suggest that the particular importance of policing arises because of both this institution's unique role, as that which is mandated to maintain order throughout civil society, by force if necessary, and the substantial authority granted by the state in support of that 
role. Police are the formal institutionalization of the policing function in our society, individuals collectively invested with awesome powers to encroach on citizen's fundamental rights, to restrict individual's liberty, and to resort to violence, when necessary, in order to coerce compliance in resolving conflicts and guaranteeing certain behaviour from the public (Bittner 1970; Brodeur 2007, 2010; Brown 2014; Dunham \& Alpert 2005, 2010; Ericson 1989; Goldsmith 2005; Manning 1977, 1997, 2005b, 2010; McLaughlin 2007; Walsh \& Conway 2011; Westley 1970).

In furtherance of this responsibility, rank-and-file police work is the most direct mechanism through which the power of the state over the citizenry is applied and these front-line officers are the most visible embodiment of the state's exercise of control and authority (Bayley 2005; Bridenball \& Jesilow 2008; Ericson 1982; Luna 2000; Mawby 2002; Neocleous 2000; Paperman 2003). Therefore, understanding the mindset and the behaviour of these officers in their interactions with citizens (or absence thereof) is crucial in ascertaining the state of, and challenges within, today's public-police relationship. In this regard, in a recent opinion piece, titled 'Canada's citizens aren't the enemy and neither are the cops,' National Post columnist Christie Blatchford (2015a: 3) observed that in "the shrinking world...woven by instant communication, 24/7 news and the power of the web... [ it would be] a useful thing if the police and citizens in Canada could manage to remember that they're not at war."

Along these lines, I suggest that it is not controversial to advance that we, as a society, clearly need better understandings of how we are policed by the organizations and the individuals we collectively entrust with that responsibility. Given their awesome powers, the police occupy a position of formal trust in which they are expected to 
operate within our laws and to meet public expectations of proper conduct (Goldsmith 2005; Mawby 2002; Seron, Pereira \& Kovath 2004). The distrust of, and loss of confidence in, the police that accompanies public exposures of officers' misbehaviour present serious challenges for the institution of policing, internally, and, in my view, more significantly, externally - particularly in terms of legitimacy in the crucial sociopolitical relationship between front-line officers and the public they are mandated to serve and protect (Jackson \& Sunshine 2007; Jefferis, Butcher \& Hanley 2011; Lersch \& Mieczkowski 2005; Sindall, Sturgis \& Jennings 2012). ${ }^{27}$ As the editorial board of the National Post (2012: A16) observed, "In an age where any citizen can capture officer misconduct on his smartphone; a single video upload can serve to erode the trust of millions of Canadians overnight."

I also suspect that I would find little disagreement in my observation that we, as a society, and as thoughtful and responsible individual citizens therein, need better understandings around how police (both the institutions of policing and, in particular individual officers - at which level this study focuses) are responding to these "unsettled times," as the current policing landscape has been characterized by Campeau (2015: 670), or today's "age of sociopolitical disruption," as Williams and colleagues (2019) describe the common assessment throughout police occupational culture vis-àvis officers' everyday interactions within the community. Today, discourses around (1) police officers' conduct in the field - particularly involving use of force on 'minority' suspects and interactions, in general, with 'minority communities'; (2) policing's accountability, legitimacy, transparency, and visibility; and (3) the public's confidence in, and trust of, police, are ubiquitous in media, across the public sphere, and throughout 
academia - now a key concern of police administrators, sociologists, criminologists, policing scholars, legislators, the judiciary, and policy makers (Gallagher, Maguire, Mastrofski \& Reisig 2001; Hough 2003, 2007; Hough, Jackson, Bradford, Myhill \& Quinton 2010; Lee \& McGovern 2013; Punch 2009; Reiner 2006, 2010; Tyler 2004, 2006b). The following images (screen captures and photographs I produced from an assortment of recent news broadcasts, newsmagazine cover pages, and page one of the 11 April 2015 edition of the Washington Post) are illustrative representations and not, by any stretch, an exhaustive accounting, that demonstrate the contemporary public sphere interest in the circumstances of front-line policing today - via American media to broad audiences across both the United States and Canada. ${ }^{28} 29$

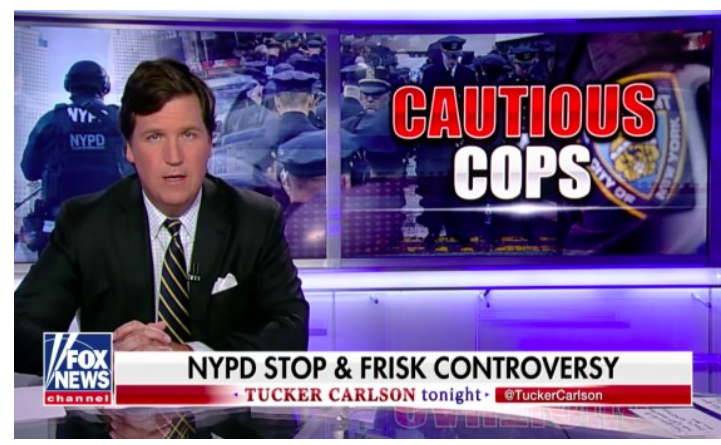

Image 5: 'Cautious cops' (Fox News 2017)

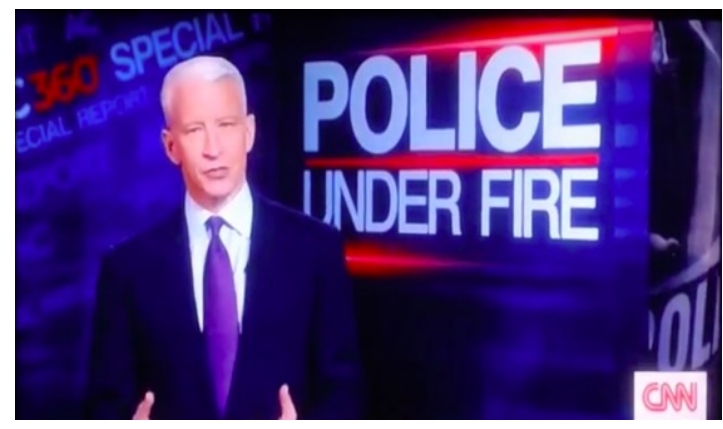

Image 7: 'Police under fire (CNN 2015)

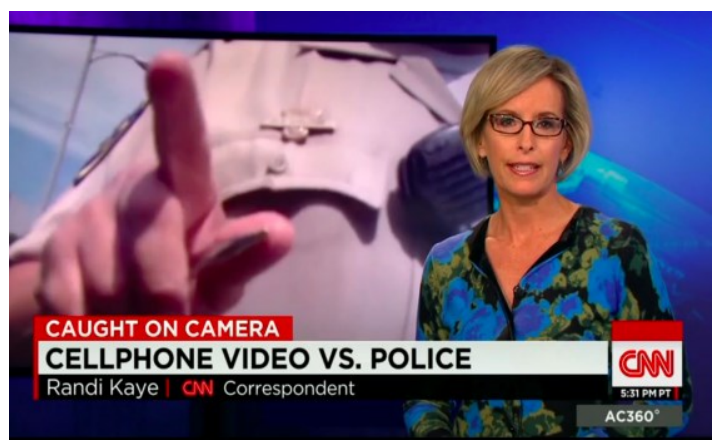

Image 6: 'Caught on camera' (Kaye 2018)

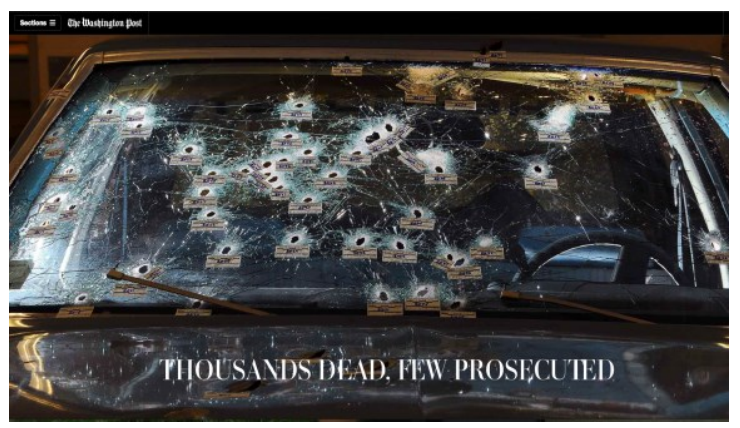

Image 8: 'Thousands dead: Few prosecuted. (Kindy \& Kelly 2015) 


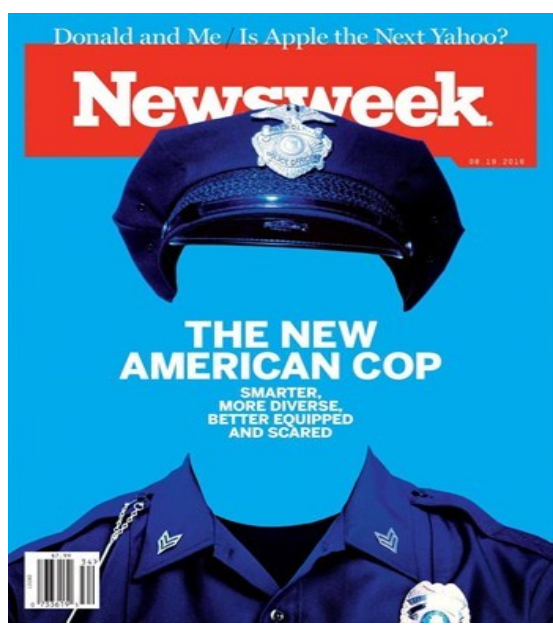

Image 9: The new American cop (Kutner 2016)

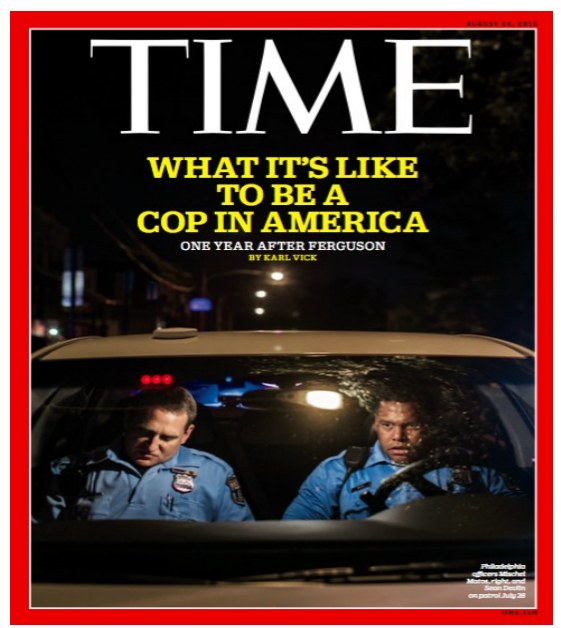

Image 10: What it's like to be a cop in America (Vick 2015)

In these contemporary circumstances, Campeau (2015: 670 citing Swidler 1986, 2001) observes, "Police departments are currently experiencing transition - a time of disruption, which sociologists consider to be a prime moment to observe social patterns; both habitual and renegotiated routines." In a similar vein, in his introductory comments at the 2015 American Society of Criminology conference, Division of Policing chair Dennis Rosenbaum (2015: 1) advanced, "This is a defining moment in the history of policing, marked by a nationwide crisis of legitimacy. The reverse side of crisis is opportunity...over the next decade we can expect high-impact research that will influence the direction of policing for years to come.”

In this vein, I argue that the issues explored in this dissertation are significant with substantial relevance to both the discipline of sociology and to contemporary social, political, and cultural understandings around the policing of Western democracies in 2019. If the amount of interest to date, across the Canadian public sphere, as expressed through various media outlets across the country, and broadly throughout Canadian policing, following the disclosure of preliminary study information in a 12 February 2018 front-page story featured in the Ottawa Citizen (see 
Yogaretnam 2018b), is any indicator, I submit that this research can be properly characterized as substantially relevant to both constituents in the public-police relationship - the public and the police - and to the academic community. Media coverage of this research and my invited presentations to various policing audiences are elaborated in subchapter 7.4.

As mentioned earlier, I advance that this original research addresses the dearth of scientific data in discussions around de-policing in today's front-line police work and fills the knowledge gaps identified by some scholars over the past few years. For example, Oliver (2017: 437), who stresses the exploratory nature of his qualitative study, which examined de-policing (as 'rhetoric or reality?') among a small sample of American police officers $(\mathrm{N}=25)$, identifies a need for further research because, While the topic of de-policing is often discussed in political rhetoric, media reporting, and reports on police behaviour, there exists little empirical analysis of the phenomenon at the police officer (individual) level...Among [this study's] findings is a strong belief that de-policing is a real and growing phenomenon...Despite the widespread allegations of depolicing [in several American cities] there is relatively little known about this phenomenon. ${ }^{30}$

Along the same lines, Nix, Pickett, Baek and Alpert (2019: 530, citing Morin, Parker, Stepler \& Mercer 2017; Nix et al. 2018; Shjarback et al. 2017; Weitzer 2015) observe, "Mounting concerns about increased police-civilian tensions in countries such as the United States and the potential for de-policing have rendered survey research examining officers' attitudes, fears, and behaviours even more important.” These American policing researchers also find, "Police surveys are vital to enhancing our understanding of officers' attitudes, beliefs, customs, and ultimately, behaviours perhaps more than ever given the current climate of distrust between citizens and police 
in the United States" (Nix et al. 2019: 543). Similarly, Nix and Wolfe (2017: 100) find that while most public discussion and research attention focuses on community perceptions of policing practices, "little if any attention is ever devoted toward understanding how such widespread negative publicity can impact individual officers' orientations toward their job and confidence in their authority."

In also promoting further study, Campeau (2015: 683), a Canadian researcher, finds that the resilience of traditional core descriptors of police culture "waver at the individual [officer] level" in "unsettled times" and she encourages further empirical investigation to determine what adaptations individual officers are relying on today in making sense of their occupational lives, which Campeau concludes differ across individuals and deviate from what classic police culture accounts would suggest. Continuing with Canadian researchers, in his recent work on police 'image work,' in this era of video recording and the posting of footage to YouTube, Schneider (2015: 241) suggests, "How police respond to user-generated content and what this tells us about social power remain a developing area of inquiry.” In the same vein, Farrar (2013), observes that the effects of video recording technologies on police behaviours have been virtually ignored across social sciences research and Todd (2015:1-2) identifies an "apparent lack of research [into the] increasingly relevant issue[s]" emerging from "the impact of surveillance on the police population." ${ }^{31}$ Similarly, Murphy (2014: 108) suggests, "The impact of increasing accountability and scrutiny on police officers is something that is often overlooked by researchers and policy-makers. Indeed, surveys of police perceptions of the level and impact of public visibility and 
internal/external accountability mechanisms may have value." Finally, for these present purposes, Marier and Moule (2018, citing Nix \& Wolfe 2016; Nix \& Pickett 2017) find,

Policing researchers have long argued that officer perceptions of public antipathy or hostility toward them influence several aspects of police culture...Given the current climate of police protests and criticism directed toward police, understanding normative consequences of perceived public antipathy and criticism of the police is a meaningful and timely topic for criminological inquiry.

\section{Research Objectives}

The principal objective of this research was to determine whether the shifting topography in today's front-line policing landscape is, or is $\boldsymbol{n o t}$, influencing in the field conduct among today's rank-and-file police officers in Canada and/or the United States. In this regard, to contribute to the existing limited scholarship and broadening public sphere discourse on de-policing, the present study sought to establish, through original empirical research, whether de-policing behaviours among contemporary front-line officers are a 'real world' - as opposed to theoretical, conjectural, or anecdotal phenomenon with tangible outcomes, and, if so, how this is happening (where, when, by whom, and to what degree) and why.

Further, given the unique opportunity to access such a significant data set, in terms of both funding and the time necessary to complete such a project, this study also sought, as a secondary research objective, to inquire into the existence (or not) of a researcher influence on the nature of data acquired in empirical studies involving rankand-file officers, as contrasted with investigative, administrative, or managerial/ command police personnel. In other words, this study strived to determine whether (or not) front-line officers participating in external academic research inquiring into their police practices, such as the present study, provide different data to a 'pracademic' 
(Sherman 2013) or 'outside insider' researcher (Brown 1996; Thomas 2014) than they otherwise would to an academic researcher with no experience working as a police officer - an 'outside outsider' ${ }^{32}$

\section{Research Questions}

Hoogenboom (2009: 48) suggests, "In police research we must start asking different questions - questions that are related to the changing realities of policing." I had the opportunity to meet with the Dutch professor in 2015 and we discussed his call for a shift in scholarly work examining today's policing, to more 'insightful' and 'comprehensive' empirical studies, which delve into the 'seismic shifts' in today's socio-political and techno-social policing landscape and the implications thereof for 'on-the-ground' police work (Hoogenboom, personal communication, 2 March 2015). After having spent much of the last eight years reading through the sociological and criminal justice literature on police, I find myself in agreement with Hoogenboom's assessment and, in this regard, this dissertation seeks to answer just such a question related to the changing realities of 'on-the-ground' policing within the current policing environment - through the study's primary research question.

More specific subsidiary questions were also developed, which operationalized the study's specific areas of inquiry and focused the project's scope - as follows:

(1) Have the occupational behaviours of front-line police officers changed in reaction to techno-social developments across contemporary society, as related specifically to 'policing's new visibility', and, if so, in what ways and to what extent?

(2) Have the occupational behaviours of front-line police officers changed in reaction to socio-political developments across today's public sphere, specifically involving 
heightened scrutiny by a contemporary public that is now more engaged in, and critical of, matters involving the policing of society, and, if so, in what ways and to what extent? (3) Are risk-averse practices in front-line police work (such as de-policing) a specific adaptation to the contemporary policing landscape, vis-à-vis techno-social and sociopolitical external influences, and, if so, in what ways and to what extent?

In furtherance of the study's secondary research objective - achieving an understanding around whether there exists researcher influence on data acquired in empirical policing studies, the following subsidiary question was conceived:

(4) Do the circumstances of the researcher, in terms of 'insider/outsider' positionality (such as an 'outside insider' or 'pracademic' versus a researcher with no policing experience), matter in the conduct of front-line police studies, as relate to issues of trust and data integrity - with participants being influenced to provide, or not provide, forthright and complete data?

\section{Researcher's Original (Working) Thesis}

Given, (1) my own experiences with de-policing during my front-line police career; (2) my exposure to many of the mediated discussions addressing de-policing over the past few years, at least in Canada and the United States; (3) my reading of much, if not all, of the limited literature on the subject; and (4) data originating from my two previous studies with rank-and-file officers (Brown 2013, 2016; Brown \& Primeau 2015), I was expecting to confirm, in the present study, that de-policing behaviours are an actual, or bona fide, phenomenon and not just simply anecdotal fictions or exaggerated 'canteen' talk within the rank-and-file police subculture (e.g., see Fielding 1994; Van Hulst 2013; Waddington 1999). At the project's outset, I anticipated that 
some front-line officers have modified aspects of their behaviour in response to 'policing's new visibility' and elevated criticism of police by today's increasingly inquisitive and skeptical, perhaps cynical, public audience, which is "more attuned to policing than ever" (Huggins, Wright \& Murphy 2014: 3) and demonstrate an increasing willingness to challenge police practices. These observations, within policing, are consistent with declining trust in major institutions (Birchall 2011b), as described in the 'risk society thesis' (Beck 1992a, 1992b, 1994; Beck, Giddens \& Lash 1994; Giddens 1990, 1991) and the reconfiguration of social and institutional relations in 'network society' (Castells 2009, 2010, 2011, 2012). ${ }^{33}$

In relation to the manifestation of risk-averse policing, as a specific adaptation in front-line police work to the technological, social, cultural and political developments examined in this study, I expected to discover that de-policing practices do, in fact, exist and to a degree that has been underappreciated to date both within policing, the public sphere, and academia - consistent with Oliver's (2017: 454) conclusion in his study, "De-policing is a real phenomenon...[and] possibly far more pervasive that what the literature has suggested to date... academics, police chiefs, and police supervisors may remain largely unaware of the size and scope of the problem." However, my initial expectations, around de-policing were modest in comparison to what I discovered in this study.

\subsection{Organization of this Dissertation}

Before delving into the relevant conceptual scaffolding and theoretical framework that inform this dissertation (Chapter Three) and the methodology relied on in this empirical study (Chapter Four), it is important to present, for the reader's consideration, 
some frames of reference - through a review of the extant literature and historical circumstances related to the phenomenon of de-policing, which follow in Chapter Two. The substantive components of this dissertation comprise Chapter Five, which presents analysis of study data and results, and Chapter Six, which discusses the study's findings in further detail and within nuanced theoretical and conceptual contexts. Finally, Chapter Seven presents the dissertation's conclusion, which addresses study limitations, includes recommendations for further study to advance understandings around today's identified de-policing phenomenon, and discusses the practice of public sociology and the dissemination of this study's findings. 


\section{CHAPTER TWO}

\section{LITERATURE REVIEW AND HISTORICAL CONTEXT}

\subsection{Chapter Introduction}

As described in Chapter One, this empirical study has investigated the phenomenon of de-policing across today's rank-and-file at various police agencies throughout Canada and across the State of New York (USA). This chapter situates the present study within the extant literature addressing de-policing, both in relation to recent research specifically focused on the topic (subchapter 2.3) and more generally, within other scholarly, media, and governmental dicussions touching on the concept of de-policing (subchapter 2.2). These materials also inform the reader's subsequent consideration of the dissertation's conceptual scaffolding and theoretical frameworks, as presented throughout Chapter Three, within historical context.

Throughout Chapter One, a limited selection of previous scholarly, media, and police practitioner/governmental contributions were presented to provide some context as the reader was introduced to the present study, which inquires into whether police are responding to anxieties, occasioned because of today's techno-social and socio-political front-line policing environments, by becoming risk averse and, therefore, practicing depolicing. ${ }^{1}$ While the literature presented in Chapter One was not intended to be exhaustive, as this study developed from initial conceptualization, I discovered that the totality of materials directly addressing this important social, political, occupational, and cultural phenomenon (and particularly approaching it through rigorous empirical research) are surprisingly sparse - especially given that sporadic discussion around the concept of de-policing in front-line police work has appeared from time to time across 
scholarly, mediated, and practitioner discourse dating back to 1977. In corroborating my assessment around the dearth of scholarly contributions addressing de-policing, Newell (2019: 61-62, citing Sandhu \& Haggerty 2017) finds, “There is little...social research examining how police officers understand and perceive the impact of bystander video on their work (for important exceptions see Brown 2016; Farmer \& Sun 2016; Huey, Walby \& Doyle 2006; Sandhu 2016, 2019; Sandhu \& Haggerty 2015, 2017).” Similarly, Murphy (2014: 108) observes, "The impact of increasing accountability and scrutiny on police officers is something that is often overlooked by researchers and policy-makers.”

In their article addressing de-policing, Rushin and Edwards (2017: 732) argue that it "is safe to say that the de-policing hypothesis did not start with Director Comey's statements, [which were] just the latest iteration of a long history of scholars, politicians and pundits worrying that scrutiny and oversight handcuff police officer effectiveness." In this regard, my survey of the literature suggests that the earliest scholarly discourse around de-policing (as I would now define the observed behaviours) was presented by Muir (1977) in Police: Streetcorner Politicians - his seminal book-length examination of front-line Oakland Police Department officers' conduct in the field and concurrent analysis of their occupational culture. Throughout, Muir (1977: 65) raised concerns that some rank-and-file officers were practicing "the avoidance response," with those he categorized as 'avoiders,' in his typology of front-line officers, adapting to challenges in their work environment by withdrawing from proactive work and discretionary interactions with citizens - "doing 'nothing'...making just enough arrests to keep the sergeant off [their] back", "avoiding hot situations," and "when you see a job that looks 
like it might blow up, you sometimes want to say, 'So what?' and move on.”2 As I also address later in the context of the present study (within Chapter Six), Muir (1977: 66) advanced that the community ultimately suffers when "policemen take a walk when the situation [gets] hot." He argued that the absence of effective formal social control, through engaged front-line officers working diligently in the community (those I would categorize as 'non de-policers', which is elaborated throughout Chapters Five and Six), facilitates an environment in which certain "persons stood to profit from the policeman's absence - the strong-arm, the bully, the vicious" (Muir 1977: 66).

Throughout the intervening 42 years, there have been some further (albeit, as described, surprisingly limited) contributions addressing the onset of risk-averse practices, across policing's rank-and-file, as an adaptation to officers' perceptions of increased visibility, elevated public scrutiny and criticism, heightened oversight, and lack of community and/or organizational support - not only in the scholarly literature (e.g. Crank \& Caldero 2010; Heaton 2010; Kennedy 1997; MacDonald 2005; McNamara \& Burns 2008; Miller 2007; Natapoff 2006; Novak, Smith \& Frank 2003; Shi 2005, 2009; Sklansky 2008; Stone, Fogelson \& Cole 2009; Toch 2012), but also appearing within mediated discussions (e.g. Celona, Cohen \& Golding 2014; Dunphy 2005; Fenton \& George 2015; Gorner 2016a, 2016b, 2016c; Hall 2009; Knapp 2016; O'Donnell 2018; Scrivener 2010; Spangenthal-Lee 2011; Tizon \& Forgrave 2001) and across policing's administration and governance (e.g. Association of Chief Police Officers 2008; Berry 2010; Better Regulation Commission 2006; Federal Bureau of Investigation 2016; Flanagan 2008; Home Office 2008, 2009; Risk and Regulation Advisory Council 2009). ${ }^{34}$ 
Many of these contributions to the discourse find, as did Muir (1977), that the development of perceptions of risk across policing's rank-and-file, vis-à-vis technosocial and/or socio-political strains in the front-line work environment, can culminate in officers intentionally avoiding what they perceive as potentially 'risky' situations in their day-to-day work in the community. Like Newell (2019), Heaton (2010: 75) concludes that this "subject matter remains under-developed conceptual territory." In this regard, Wolfe and Nix (2016: 3) find that de-policing "is an important issue, and empirical research is the necessary next step.” Similarly, Pyrooz and colleagues (2016: 3) note that the de-policing discussion has been "long on anecdotes and speculation and short on data." In this vein, Shjarback and colleagues (2017: 50) assess that "surprisingly, little empirical research has been conducted on de-policing and whether police behaviour changes in response to high-profile events." Likewise, Torres and colleagues (2018: 359) find that officers' "apprehensiveness, or the perceived inability to perform one's duties out of fear or anxiety" is significantly understudied among policing researchers. Along the same lines, Rushin and Edwards (2018) assess, "The empirical research related to de-policing is under-developed."

Similarly, Oliver $(2017: 456,438)$, in what he argues is "the first [study] to present direct empirical evidence that the phenomenon of de-policing is more reality than rhetoric" observes, "Despite the importance of the topic, there is little research focused directly on de-policing. While there is abundant literature on such issues as racial profiling, our understanding of de-policing lacks empirical analysis." In what follows in subchapter 2.2, I present, in approximately chronological order (which I consider to be the most logical, albeit perhaps somewhat unorthodox, format for such a 
presentation), the most germane contributions across the literature - those that address de-policing (and closely related contexts) in more depth than simply a superficial reference and that provide the reader with an understanding of the historical context in which this empirical study considers the identified de-policing phenomenon. A more thorough presentation of the few empirical studies that have specifically focused on depolicing is then engaged in subchapter 2.3.

\subsection{A Survey of the Literature and Historical Context (1977 to 2019)}

Following Muir's (1977) initial contribution, Dunn (1996: 185), writing about his experiences as a rookie officer in the Los Angeles Police Department (LAPD) in the tumultuous front-line policing landscape following the 1991 Rodney King occurrence and the subsequent criminal trials of involved LAPD officers explained, "Some [veteran LAPD officers] are so angry and frustrated by events surrounding the Rodney King video that they have all but given up on doing any effective police work. Many of them will only respond to radio calls and that they do slowly." The following year, Klinger (1997) observed that the level of 'vigour' that the rank-and-file employ in their various police duties depends on a multiplicity of factors - including, not insignificantly, officers' perceptions as to the nature of the citizenry being policed. In locations where officers view the public through a generalized negative (or adversarial) lens they often patrol their assigned communities with less vigour and withdraw from discretionary interactions and interventions (Klinger 1997). Similarly, Kelling (1997: 21) spoke of his field observations of front-line policing in terms of officers not engaging in proactive work, particularly in crime-ridden communities. He reported,

Everywhere I went - in the toughest neighbourhoods in [, for example,] Chicago or New Haven I learned about it...from poor citizens who were 
suffering and demanding that police do something about [crime] and who were constantly frustrated by the police whisking in and whisking out. That is a social disaster, how we have turned police into an emergency response system. It was at the cost, as I have said, of de-policing our cities.

In this vein and a few years later, Tizon and Forgrave (2001) observed front-line officers serving with the Seattle Police Department (SPD) were practicing "passive law enforcement" by "looking the other way in Black neighbourhoods," because of concerns about being accused of biased policing practices through racism complaints by members of the public (see also Murakami's 2002 reporting on the SPD, racial profiling allegations, and SPD officers' de-policing responses). During a subsequent U.S. Department of Justice (hereinafter referred to as DOJ) inquiry into practices of the SPD (often referred to as a 'consent decree'), an SPD officer disclosed to federal investigators, "Maybe 20 or fewer [SPD] officers in patrol are doing proactive work right now" (Spangenthal-Lee 2011). ${ }^{5}$

In this same temporal period, a handful of academics contributed scholarly discussions around the practice of de-policing in the specific contexts of race/ethnicity and the American public-police relationship. These scholars (e.g., see Cooper 2002, 2003, 2006; Kennedy 1997) argued that 'minority' neighbourhoods in some cities were receiving significantly less discretionary police attention than 'non-racialized' communities - resulting from entrenched racial bias within the occupational culture of rank-and-file policing. In this regard, Cooper (2002: 359) advanced, “As a general matter, de-policing is a response to popular controversy over police tactics toward racial minorities." For example, in the aftermath of rioting in Cincinnati following the fatal police shooting of a Black man (Timothy Thomas) by a White officer in 2001, it was suggested across scholarly and mediated discourses that the rank-and-file of the 
Cincinnati Police Department (CPD) were responding to widespread public criticism and elevated scrutiny of their work in the field by practicing de-policing (Cooper 2002, 2006; Fisher 2014; Leo 2001; Martin 2013; Miller 2001; MacDonald 2005; Roberg et al. 2015; Shi 2005, 2009). Leo (2001) explained officers' actions in the following terms, "Shell-shocked or resentful, [CPD] police overlook a lot of suspicious behaviour. They stop trying to prevent low-level crime and simply react to 9-1-1 calls."

In his analysis of significant reductions in front-line police activity in Cincinnati following the 2001 Thomas occurrence, Shi (2005: 19) found that the "de-policing probably does not result from orders from above but rather from officer's own assessments of risk.” Along these lines, Cooper (2003) emphasized that individual officer discretion is at the core of de-policing - engaging considerations for each officer around reactive versus proactive front-line police activities. Keith Fangman, the president of the labour union representing CPD rank-and-file at the time, embodied Cooper's (2003) observations in his counsel to front-line CPD officers, "If you want to make 20 traffic stops a shift and chase every dope dealer you see, go right ahead. Just remember that if something goes wrong, or you make the slightest mistake in that split second, it could result in having your worst nightmare come true for you and your family" (McLaughlin \& Prendergast 2001). In his assessment of the situation, Kent Ryan, Cincinnati's public safety director observed, "The term is de-policing...The guys got demoralized, tired of being beat up by everyone from the media to the federal government. They just backed all the way off' (Fisher 2014). In this regard, Martin (2013) advances,

As images of risk, uncertainty, and danger in the inner city are reified by the media, the public and the police, the withdrawal of emergency support 
creates as self-fulfilling prophecy. Poor neighbourhoods in need of increased control are defined as too dangerous to enter and/or are resented as 'problem populations,' becoming victims of de-policing (Cooper 2002) ...In the days immediately following the shooting of Thomas, police patrols were conspicuously absent in Black neighbourhoods around the city. In part, this absence appeared to be a form of resistance on the part of police frustrated with citizen protests that had demonized them before the national media.

In the same way that locations and/or persons can be over-policed, with more police attention than others, they can also be de-policed, a situation in which officers intentionally and proactively refrain from attending particular locations, engaging with certain persons, and/or involving themselves in specific types of police interventions (e.g., see Brown 2005; Cooper 2002, 2006; Desmond \& Valdez 2012; Kennedy 1997; Miller 2007; Natapoff 2006; Shi 2009; Sklansky 2008; Walker, Spohn \& DeLone 2000; Withrow 2011). These concepts are often discussed, particularly in the American context, in relation to police interactions, or absence thereof, with racial/ethnic 'minority' communities and in the context of allegations, or actual instances, of 'racial profiling' in policing practices (e.g., see Brown 2004; Cooper 2002, 2003, 2006; Kennedy 1997; Miller 2007; Rojek, Rosenfeld \& Decker 2004; Shi 2009; Smith \& Alpert 2002; Susswein 2008; Walker et al. 2000; Withrow 2011) and these considerations are elaborated in subchapter 6.8 within Chapter Six.

At around the same time as the de-policing conversation was developing in the aftermath of the contentious circumstances in Cincinnati, Davis and colleagues (2002, 2005), in their research into repercussions arising from a DOJ 'consent decree' with the Pittsburgh Police Department (PPD), found that a significant number of front-line officers in that city had responded to the external scrutiny and critique of their work by adopting a less proactive, less aggressive, and less energetic style of police work - with 
$42 \%$ of the study's PDP research participants $(\mathrm{N}=129)$ reporting "major changes in how they deal with citizens," including less (and more circumspect) interaction, and " $79 \%$ of officers indicated they were now less proactive [in their police work]" (Davis et al. 2005: 22). These researchers reported, "We heard officers were hesitant to intervene in situations involving conflict" (Davis et al. 2005: 16) and "We were told that 'a lot of cops are afraid to do their jobs,' with officers often unwilling to go above or beyond minimal requirements of their assignments" (Davis et al. 2002: 51). Similarly, in their research with LAPD officers in 2003 (following the LAPD entering into a 'consent decree' with the DOJ), Stone and colleagues (2009: 19) found "widespread belief in depolicing [practices] among LAPD officers." These same researchers observe, "In every instance where the [DOJ] has entered into a consent decree with a state or local government to address an alleged pattern and practice of police misconduct, concerns have been raised that the consent decree would lead to de-policing" (Stone et al. 2009: 19).

In this same period, but in the U.K., Goold $(2003,2004)$ conducted an empirical study examining how the increasingly-pervasive CCTV presence in many cities and towns had impacted on front-line officers' behaviour. His work can be viewed as an extension of the research of Newburn and Hayman (2001), who had assessed the reactions of police and inmates to CCTV monitoring of police custodial suites in the U.K. Goold (2004: 179) observed, "Many police officers now find themselves faced with the prospect of being watched and possibly recorded as they go about their daily routine on the streets. How this change affects the exercise of police discretion and the way the police respond to incidents in view of the cameras was one of the key concerns 
of this project." He found that, as a result of the introduction of cameras in public areas across the study's six data sites (towns in southern England), over two-thirds of the officers in the study $(\mathrm{N}=50)$ were 'more careful' when out on patrol, were anxious about being watched and the possibility that their activities could be scrutinized after the fact, and many officers had come to the conclusion that the potential for video recording of their actions in the field made it essential for them to 'go by the book,' or at least to create the appearance of doing so (Goold 2003, 2004). Goold (2003: 202, 197) suggested, "The spread of CCTV may also have sparked a transformation in the very nature of police work, and the move towards a society in which ordinary police officers, like Bentham's prison guards, are no longer able to avoid the public gaze" and, along these lines, he observed, "During the course of the study it became increasingly clear that officers at all of the towns were worried about the possibility of camera footage being used in support of complaints against them or their colleagues." In terms of specific behavioural adaptations to their visibility, Goold (2004: 185) found, "The concern about how CCTV might be used... translated into more circumspect behaviour on the part of many [officers], particularly when it came to using force or making arrests within view of the cameras."

In a 2005 Los Angeles Times 'op/ed' written by an LAPD officer, who regularly wrote a column for the National Review (under the pseudonym Jack Dunphy), the author observed,

It has never been more dangerous to be a Los Angeles police officer. The danger lies not only in the risk of being shot, stabbed or otherwise injured. We train and mentally prepare for those times when we are called to put our lives on the line...I accept these risks each day as I put on the uniform of my profession. What is much more difficult to accept, and all but impossible to prepare for, is the risk to my family's security if I survive an encounter by 
means that deviate even slightly from the way things are taught in the controlled environment of the Police Academy. The decisions I make in the blink of an eye, decisions that will either save my life or get me killed, will be scrutinized by people with no understanding of what it feels like to be attacked...[and] the decision to prosecute me or to fire me from the LAPD may rest in the hands of people whose allegiances to political constituencies outweigh their commitment to fairness...The rise in officer morale that accompanied William Bratton's appointment as chief of the LAPD has now ebbed. Officers are again beginning to follow a 'drive-and-wave' policy as they did under former Chief Bernard Parks. They are again avoiding confrontations with those who most deserve to be confronted. As [dismissed LAPD officers] John Hatfield and Steven Garcia discovered too late, sometimes fighting crime doesn't pay (Dunphy 2005).

Similarly, Warner (2005), also a serving police officer, in his book titled,

101 Reasons Why You Should Not Become a Cop, advanced that reason \#93 was

de-policing. In this regard, Warner (2005: 83) observes,

De-policing is a phenomenon with sporadic attention over the last ten years. The phenomenon has also been referred to as tactical disengagement of detachment. The result of de-policing is a reduction in proactive policing...the cause of [which] is a conscious or unconscious belief that performing one's duties will result in greater risk of negative sanctions, that is, citizen complaints resulting in an internal investigation [or] public scrutiny...The adage one hears from line officers related to de-policing is 'More work, more problems - no work no problems.' 6

One year later, Canadian researchers Huey, Walby and Doyle (2006: 155), in their field observations of 'Cop Watch' activists and rank-and-file officers of the Vancouver Police Department (VPD), found, in certain instances, officers are "pulling back from doing their jobs" because of concerns around having their front-line interactions with citizens video recorded by a bystander. Similarly, in Black \& Blue: Understanding Modern Law Enforcement in Your America, Bradley (2007: 36), reporting on his observations of officers' risk-averse practices, described "patrol and specialized units avoid[ing] a specific area or police activity (such as traffic stops), because of politics, lack of administrative support, or excessive citizen complaints against police.” 
Miller (2007: 259), similar to earlier discussions of de-policing within the context of race/ethnicity (e.g. Cooper 2002, 2003, 2006; Kennedy 1997), advanced that frontline officers can react adversely to perceptions of increased risk (to them), which can materialize with the introduction of race data collection and/or racial profiling policies, by practicing de-policing - which he defines as "the practice of withdrawing from citizen encounters that would trigger an organizational record of the encounter. The assumption is that de-policing would be evident if a decrease in encounters between police and racial minorities is observed following the implementation of a profiling policy." In this vein, McNamara and Burns (2008: 68) suggested that de-policing is "a law enforcement strategy in which police avoid accusations of racial profiling by ignoring traffic violations and other petty crime committed by members of visible minorities, in a sense, de-policing is the opposite of racial profiling." Along the same lines, Wynn (2008: 20-22), in his book on police leadership, observed, "Operationally, [disgruntled officers] may practice 'FIDO policing.' The concept of 'F--- It, Drive On' policing is a manner of withholding discretionary effort... [Similarly, such officers] may practice what has become known as ' $N C N C$ policing'. The acronym, which stands for 'No Contact, No Complaint' [suggests] if I don't do anything, I can't get into trouble." Similarly, Sklansky (2008: 165) advanced that "the most notorious manifestations of [the problems of cynicism and shirking by police officers is] the 'drive-and-wave' approach to patrolling.”

A year later, The Edmonton Journal published a story titled, 'Heard this police acronym? FIDO: Forget it, Drive on', in which rumoured de-policing behaviour among front-line Edmonton Police Service (EPS) officers was discussed (Hall 2009). Loftus 
(2009), in that same year, in her research on police occupational culture, addressed 'a mood of resistance' in the work of officers who resent the implications of socio-political shifts in society. Similarly, in their book on police ethics, Crank and Caldero (2010: 307) presented a hypothetical high-performing 'star' front-line officer, who, in the aftermath of a public scandal involving her being video recorded conducting an unlawful search, responded to the ensuing critique and professional consequences with the mindset, "Full investigation, heavy degradation...Permanent record. Newspapers called me a racist "cause the asshole was Black. End of game. After that it was all FIDO (Fuck it Drive on)...[I've only got] twelve years to early retirement."

In a political context, Chris Grayling (U.K. Secretary of State for Justice from 2012 to 2015 and Leader of the House of Commons from 2015 to 2016) suggested that front-line British officers were practicing risk-averse police work in the community and he demanded a shift toward "more 'in your face' street policing" (Porter 2010). Also in the U.K., Heaton (2010) presented a conceptual overview of risk aversion in British policing. ${ }^{7}$ He observed that the current socio-political and judicial climate has led "to the exercise of [law enforcement] being carried on in a detrimentally defensive frame of mind" and is "tend[ing] to lead to defensive policing" (Heaton 2010: 77). He also discussed a Home Office (2008) 'green paper' that was endorsed by the Association of Chief Police Officers, which found, "An intrusive modern media, national and local politics, along with a growing societal culture of needing someone to be accountable as factors that have created risk aversion [in policing]" (Heaton 2010: 81).

In the same year, in an article titled Policing's New Visibility, Australian criminologist Andrew Goldsmith (2010: 923) observed, "As technology changes there 
are different ways and many more opportunities for [police] to be caught behaving badly if they choose to behave badly." He proposed that today's video recording capabilities in the hands of the citizenry could result in "a more reflective approach [among police officers] to the use of...potentially controversial policing practices" (Goldsmith 2010: 926). Along the same lines and framed specifically within the context of police occupational culture, Cockcroft (2013: 101) suggested that contemporary social and cultural changes impacting on policing might have "implications for the ways in which police engage with people from specific social groups."

A year later, Canadian researchers Haggerty and Sandhu (2014: 11) argued that today's "routine presence of cameras is changing the dynamics of policing on the ground." They observed, "Changes in the police's visible field have put them in the midst of a new and quite different crisis of legitimacy" with the proliferation of video recordings of police activities in the field now "revealing to ordinary audiences unpalatable police behaviours historically hidden from view" (Haggerty \& Sandhu 2014: 10). In his Canadian study, with use-of-force trainers serving with police agencies in British Columbia and Manitoba as research participants, Murphy (2014: 45), within a chapter titled 'Officer hesitancy and timidity,' observed,

A consequence of the increased visibility and scrutiny of police actions is that officers are hesitant or unwilling to use force in situation in which they are legally justified to do so. In some cases, officers simply avoid potential use of force situations, commonly referred to as FIDO ('Fuck it and drive on'). In other instances...some officers hesitate despite the presence of clear justification and necessity...related to fear of departmental reprisals or other consequences of their actions (e.g. civilian review; negative media attention; legal repercussions). The preoccupation with personal consequences that might arise out of taking action against a suspect can have a significant impact on performance. 
In that same year, Scrivener (2014) published a piece in The Toronto Star, discussing front-line officers' de-policing practices, titled 'When police are guilty of FIDO Forget it, Drive on.' In a similar vein, on 29 December 2014, The New York Post published a front-page story titled, 'Arrests plummet 66\% with NYPD in virtual work stoppage' (Celona et al. 2014). ${ }^{8}$

Todd (2015: 14) investigated the impact that civilian surveillance has on officers' use of force and she found that today's video recording of police by the public and the potential for subsequent uploading of footage to the Internet has "had marked effects on [officers]." She discovered, "Due to the unprecedented lack of [public] trust in policing, officers feel they must manage their public image through their actions - even if this means limiting some actions considered justified" and further that "police behaviour is [now] adapted in order to prevent controversy and to avoid backlash from the general public" (Todd 2015: 64, 75). In a chapter titled 'Citizen monitoring contributing to the FIDO effect,' Todd (2015: 80-81) reported on her research with officers serving in metro Vancouver police agencies,

In order to avoid the public scrutiny that occasionally results from difficult calls for service, police officers are known to deliberately disregard potential use of force situations. This phenomenon is frequently referred to, within policing circles, as FIDO (Fuck It, Drive On). FIDO is speculated to stem from the heightened visibility of police officers. As an increasing number of controversial use-of-force incidents are publicized on the Internet, police officers feel the need to bypass situations that could potentially tarnish their professional and personal reputations. Instead of attending the call, officers drive on, in the hopes that the incident will resolve itself, or, that the crime/incident occurring is best left to run its course without intervention as it is not worth taking the chance on a possible career ending incident. In other words, it seems some officers weigh the risk of involvement in protecting the community versus their career. The officers interviewed expressed particular concern over attending certain calls, for example incidents involving a racial minority group because of the rise in citizen monitoring. ${ }^{10}$ 
As discussed in Chapter One, Davis (2015) reported on a 2015 meeting involving leadership of American policing, in which various police chiefs observed that "patrol officers [technically] still do their jobs - clocking in and policing their beats, however, fewer take extra steps such as confronting a group loitering on a sidewalk late at night that might glean intelligence or lead to arrests, for fear that any altercations that ensued would be uploaded to the Internet." NYPD Commissioner William Bratton "called it the 'YouTube Effect' that has emerged for officers post-Ferguson" (Davis 2015). During the meeting, "Chuck Wexler, a former top officer in Boston and head of the Police Executive Research Forum, [advised Attorney General Loretta] Lynch, 'Perhaps the most difficult [problem] to calibrate, but the most significant,' he said, 'is this notion of a reduction in proactive policing"” (Davis 2015). However, "police chiefs and elected leaders from Baltimore, Chicago, New York and St. Louis were more blunt: 'We have allowed our police department to get fetal and it is having a direct consequence,' Chicago Mayor Rahm Emanuel told Lynch” (Davis 2015).

In contrast to the conclusions of Todd (2015) and the American police chiefs (as described in the preceding paragraph) and perhaps even contradicting their own previous (albeit) anecdotal observation that "the routine presence of cameras is changing the dynamics of policing on the ground" (Haggerty \& Sandhu 2014: 11), Sandhu and Haggerty (2015: 4), in reporting on their study that examined Edmonton police (and those I would characterize as 'quasi-police') officers' attitudes towards their current 'high visibility status,' found “...the crisis narrative is overstated and undernuanced. While cameras are undeniably altering the police's visible field, the consequences of this change are complicated, somewhat contradictory, and rather 
uncertain. The police have complex relationships with cameras and are finding ways to adapt to being on camera." The authors suggest that today's "officers have nuanced relationships to cameras, sometimes fearing them, occasionally embracing them, and often simply seeing them as a banal and inevitable part of contemporary life" (Sandhu \& Haggerty 2015: 4). They characterize [officer's] orientations within three typologies - 'camera shy,' 'habituated' and officers that use today's visibility to their 'strategic advantage' (Sandhu \& Haggerty 2015: 4). ${ }^{11}$

'Camera-shy' officers "are anxious and annoyed about being filmed on the job" and, "in sum, [these] officers occasionally fear the presence of cameras" (Sandhu \& Haggerty 2015: 5-6). In this regard, Sandhu and Haggerty (2015: 6) find that "the anxieties articulated by such officers correspond most closely to the 'crisis narrative' about how cameras are changing police work," however, such officers did not represent the majority position of study participants. 'Habituated' officers have "simply learned to live with...the reality of policing on camera" and so many study participants expressed this view that the authors wrote, "We could fill this article with quotes presenting variations on this theme, but there are only so many ways one can say: 'I don't care about cameras' or 'I never think about them'” (Sandhu \& Haggerty 2015: 6-7). The third typology was characterized as 'strategic advantage,' because for these officers, cameras "can be empowering [in that] they create opportunities to gather evidence, challenge false accusations, manage interpersonal encounters, and produce preferred representations of situations" (Sandhu \& Haggerty 2015: 7). In concluding, Sandhu and Haggerty (2015: 8) counselled, "By focusing almost exclusively on the prospect that 
cameras are creating a crisis in policing, we can lose sight of the full range of police behaviour now being recorded and publicized."

In contrast to the findings of Sandhu and Haggerty (2015), Randy Sutton (2015), a retired and highly-decorated lieutenant who served as a police officer for 33 years in New Jersey and Las Vegas and is the national spokesperson for the American Council on Public Safety, wrote an 'op/ed' piece in The New York Post, titled 'The dangers of de-policing: Will cops just stand down,' in which he observed, 'In precinct locker rooms, police association meeting halls and whispered conversations between partners, the topic is the same: de-policing - that is, the conscious decision by cops to provide only minimum service." He described a situation in which,

Concerned about overzealous prosecution or runaway civil liability, cops are understandably considering a logical option: de-policing. They'll handle calls, write a ticket or two - but do nothing proactive...Proactive policing is the standard by which good police supervisors, administrators and even the officers themselves judge the effectiveness and competency of police service...It's the desire to actively pursue criminal elements and to serve and protect neighbourhoods that motivates much of law enforcement. Undermine that pride and make them the enemy because of personal or political agendas and you will create a perfect storm that leads to depolicing (Sutton 2015).

In another dimension related to 'policing's new visibility,' following on the seminal body-worn camera (BWC) study with front-line officers in Rialto, California, which found a 59\% reduction in officers' use of force after the introduction of BWCs (Ariel, Farrar \& Sutherland 2014) - in a study of police BWCs with rank-and-file of the Mesa (Arizona) Police Department, Ready and Young (2015) found that officers were more 'risk-averse' when experiencing the increased visibility afforded by the camera, with officers wearing BWCs (versus those in the experiment's control group) conducting significantly fewer street 
stops, 'stop-and-frisks', and arrests of citizens due to the potential for after-thefact scrutiny around the rationale for those interactions with members of the public. More recently, Wallace and colleagues (2018: 481) tested the idea that "BWCs may generate de-policing through camera-induced passivity" by implementing a randomized controlled trial with front-line officers in Spokane (Washington). The term 'camera-induced passivity' describes the researcher's hypothesized form of de-policing, in which, "given the potential for BWCs to generate increased surveillance of officer actions, officers reduce their activity to avoid exposure to scrutiny and discipline" (Wallace et al. 2018: 483). Study results did not support the de-policing thesis, with officers assigned BWCs differing significantly in only one measure in the study - those with BWCs actually became slightly more proactive during the study relative to their peers (engaging in $8 \%$ more officer-initiated calls). In sum, very few empirical studies, to date, have examined the effects of BWCs on rank-and-file officers' work in the field, including their willingness to be proactive (Braga, Sousa, Coldren \& Rodriguez 2018; Gaub, Choate, Todak, Katz \& White 2016; Lum, Koper, Merola, Scherer \& Reioux 2015; Yokum, Ravishankar \& Coppock 2017).

Similar to Sandhu and Haggerty (2015), and relying on data from the same empirical study, Sandhu, in a 2016 publication, discussed his interpretation as to how officers are responding to cameras and he referenced 'speculative theories' (in scholarly discourse) arguing that video recording "will deter police misconduct...might improve police accountability...[or] that police might respond to cameras by engaging in a riskaverse style of policing." In this regard, he observed, "Unfortunately, little empirical 
data is available to assess these theories" (Sandhu 2016: 78). In this publication, Sandhu (2016: 82, 79) reported that while "a minority of [participants in his study] admitted that they sometimes respond to cameras and photographers with efforts to avoid being recorded" most officers are apparently learning to adapt to cameras by engaging in 'camera-friendly policing,' which involves the management of one's 'presentation of self' "in an effort to control how they will be perceived by photographers and viewers of recorded footage.” In another publication in 2019, using the same 2013 data set, Sandhu (2019: 223, citing MacDonald 2015; Simon 2012; Wall \& Linnemann 2014; Wilson \& Seriesier 2010) elaborated on his 'camera-friendly policing' findings, suggesting that while most officers "expressed resentment towards cameras and photographers," responses to their new visibility are not being "shaped by an effort to avoid being recorded as is sometimes argued," but rather, they are reflective of efforts by officers "to optimize the on-camera experience by producing footage which will discredit complaints and reinforce a favourable assessment of the police institution."

Sandhu and Haggerty authored another article published in 2017, again using the 2013 data set, discussing 'policing on camera,' in which they critiqued Mathiesen's (1997) analysis of synoptic surveillance (in neglecting to consider how those who are subjected to scrutiny relate to their monitoring) and wondering whether "public figures [in the context of the article this would include today's 'high-visibility' police officers] modify their behaviour as a result" (Sandhu \& Haggerty 2017: 79)? The authors observe, "The synoptic scrutiny of the police represents a tremendous change in policing. To date, however, there has been little empirical research examining how individual police officers relate to their increased camera visibility. Discussions about 
policing on film have instead tended to be highly speculative, focusing on the ostensible challenges cameras pose for policing" (Sandhu \& Haggerty 2017: 80, citing Goldsmith 2010). In revisiting their three identified typologies ('camera shy,' 'habituated,' and 'strategic advantage') the authors suggest, "The defining aspect of [the camera-shy] orientation is a series of anxieties about the risks and annoyances of working in an environment where officers are regularly recorded or have the realistic prospect of being recorded...Their anxieties tended to revolve around how cameras alter the dynamics of policing, and how they might impact an officer's reputation" (Sandhu \& Haggerty 2017: 81-82). These contemporary realities have led to a recognition among some officers that they need to be more 'camera-friendly,' which is denounced by 'camera-shy' officers as a capitulation to 'political correctness' (Sandhu \& Haggerty (2017: 85). The authors report that during field work in 2013, "On several occasions we observed officers delay engaging suspicious individuals based on their concern about being recorded. These delays often had racial overtones" (Sandhu \& Haggerty (2017: 86). Sandhu and Haggerty (2017: 86) reiterated that, in their research, much more common than the 'camera-shy' orientation were officers who perform their police duties with "resigned acceptance of [their] camera visibility."

Writing about significantly different observations, Heather MacDonald (2016), following up on her 'op-ed' published in The Wall Street Journal a year earlier (MacDonald 2015), presented anecdotal observations originating from her "having spoken with police officers across the [USA]",

Officers are backing off of proactive policing in reaction to the hostility they were encountering in urban areas...Officers were continuing to answer 9-1-1 calls with alacrity, but in that large area of discretionary policing - getting out of a squad car at 1 a.m., for example, to question someone who appears 
to have a gun or may be casing a target - many officers were deciding to simply drive on by rather than risk a volatile, potentially career-ending confrontation that they were under no obligation to instigate...I dubbed this latest outbreak of de-policing and the resulting emboldening of criminals the "Ferguson Effect," picking up on a term first used by St. Louis police chief Sam Dotson...Activists and academics denounced pedestrian stops and public order policing... as racially biased and oppressive. As a result, officers were doing a lot less of such discretionary enforcement...It is in predominantly Black neighbourhoods where police worry that a videoed use of force will land them on TV in the role of racist cop of the week and where officers tell each other 'If you get out of your car, you're crazy, unless there's a radio call,' in the words of an [LAPD] cop...In short, it is in high-crime Black neighbourhoods where the police are backing off the most under the relentless charge that they are racist. And it is in high-crime neighboruhoods where a fall-off in proactive policing is going to produce the biggest negative impact. It is in those neighbourhoods where informal social controls - above all families - have most broken down and where policing most critically takes up the slack.

The FBI's Office of Partner Engagement conducted research in the wake of the murder of 64 police officers across the USA in 2016, in which researchers interviewed front-line officers and command staff from 13 of the police agencies where an officer was killed. This study found,

Recent [socio-political changes] and a 'turnstile justice system'...have had the effect of 'de-policing' in law enforcement agencies across the country...Departments - and individual officers - have increasingly made the conscious decision to stop engaging in proactive policing. The intense scrutiny and criticism law enforcement has received in the wake of several high-profile incidents has caused officers to (1) 'become scared and demoralized' and (2) avoid interacting with the community...In communities where law enforcement's community relationship is poor, officers are more likely to be purely reactive (Federal Bureau of Investigation 2016).

Along the same lines, Rosenfeld (2016: 2), who pioneered research into the socalled 'Ferguson Effect' in a study commissioned by the DOJ, observed that the dominant interpretation of today's front-line policing across the USA advances "[that] criticism of the police stemming from widely publicized and controversial incidents of the use of force against minority citizens [have caused] police to disengage from 
vigorous enforcement activities. ${ }^{" 12}$ In this vein, Wolfe and Nix (2016: 1) observe that some academics and media commentators have "pointed the finger of blame at depolicing" for "increasing violent crime rates in several U.S. cities," which, as explained in Chapter One, is often referred to as the 'Ferguson Effect.' Also, as discussed earlier, some working in this area of policing scholarship have conflated the practice of depolicing with the 'Ferguson Effect,' which creates, in my view at least, confusion in understanding the important distinction between a practice (de-policing) and a potential symptom of the practice (i.e. a 'Ferguson Effect' on violent crime rates). Wolfe and Nix (2016: 1) provide a good example in their study's introductory remarks,

Although the Ferguson Effect on crime rates remains an open question, there may also be a Ferguson Effect on other aspects of police officers' jobs, such as willingness to partner with community members...The Ferguson Effect hypothesis suggests that officers are conscious of the negative publicity surrounding their profession, understand that their actions could be recorded by the public at any given time, and become less willing to do their job as a way to avoid being accused of racial profiling or excessive force. In turn, this de-policing leads to increases in crime...Although the Ferguson Effect on crime rates is an empirical question awaiting research scrutiny.

Regardless of uncertainty around terminology, Wolfe and Nix (2016), through survey research in 2015 with 567 sheriff's deputies serving with a police agency in the southeast (USA), sought to establish whether (or not) consequences of the practice of de-policing by front-line officers is showing-up (in ways that can be measured through empirical study) in on-the-ground outcomes that are not immediately associated with official indicators like the violent crime rate - in the case of this specific study, in officers' willingness to engage in community partnerships, as contemplated with community-based policing and officer proactivity (Nix \& Wolfe 2016; Braga, Kennedy, Waring \& Piehl 2001; Gill, 
Weisburd, Telep, Vitter \& Bennett 2014). The researchers' hypothesis advanced that with relentless negative media critique and "a social climate whereby the legitimacy of [policing] is being challenged" it might be the case that such a situation "may diminish officers' motivation on the job" and, as a consequence, officers would be less willing to collaborate in community partnerships (Wolfe \& Nix 2016: 1-4). In this regard, they find that "some officers indicated being less motivated to perform their duties" and that the present negative policing environment in the USA was associated with a reduced willingness among frontline deputies to engage in community partnerships - although this effect seems to be confounded by perceptions of 'organizational justice' (Wolfe \& Nix 2016: 7). ${ }^{13}$

In their introductory observations, discussing studies by Pyrooz, Decker, Wolfe and Shjarback (2016) and Morgan and Pally (2016), Nix and Wolfe (2016: 14) clarify some of the confusion (as above) by observing, "Together, these studies suggest that there is no Ferguson Effect on national crime rates; however, negative publicity stemming from events like Ferguson and Baltimore do appear to have an effect on police behaviours [i.e. de-policing]. ${ }^{114}$ Later, these authors clarify further, in finding, "Research has revealed that there are important 'Ferguson-related' effects on officers' orientations toward their job and de-policing behaviours" (Nix \& Wolfe 2016: 18). With similar considerations (and it appears the same data set) underlying their previous study (Wolfe \& Nix 2016), Nix and Wolfe (2016) examined whether organizational justice is a factor that influences officers' sensitivity to negative publicity and societal backlash against police officers and, in this regard, they observe,

We know very little about what is associated with officers' sensitivity to such 'Ferguson Effects.' In other words, what is it that makes a police 
officer more or less likely to feel affected by negative publicity and public discontent stemming from Ferguson?...What can supervisors do to help prevent their officers from being adversely impacted by negative publicity stemming from high-profile incidents like that in Ferguson" (Nix \& Wolfe 2016: 12)?

Ultimately Nix and Wolfe (2016) find that officers perceiving organizational fairness (those that feel fairly and respectfully treated by their supervisors) may be, at least partially, shielded from the effects of negative media reporting and public animosity surrounding their occupation - including being unmotivated and feeling that citizens' attitudes toward police have worsened.

In a publication the following year, Nix and Wolfe (2017: 91) observe that "in today's world of social media, negative press is seemingly inescapable and may resonate strongly with officers now more than ever. To date, this remains a largely unanswered empirical question." In this study (apparently relying on the same data set as that in Wolfe and Nix 2016 and Nix and Wolfe 2016), the researchers found that post-Ferguson publicity that has been highly critical of police, which officers can interpret as indicative of a lack of public support for their work in the field, has resulted in both reduced motivation and reduced levels of 'self-legitimacy' - the confidence officers have in the authority vested in them (Bottoms \& Tankebe 2012; Bradford \& Quinton 2014). ${ }^{15}$ In this regard, Nix and Wolfe $(2017: 100,103)$ argue, "Officers with lower self-legitimacy may de-identify with their agency and its goals (e.g. communityoriented policing) and, in turn, withdraw from interacting with community members and other effective policing strategies" and they suggest, "In our mind, it is important to take a step back from obviously unfortunate incidents and see how such issues may impact law enforcement officers themselves. Unfortunately, it appears that such negative 
publicity has a deleterious relationship with officers' sense of self-legitimacy. This is bad for law enforcement agencies and the communities they serve."

Pyrooz and his colleagues (2016: 1) observe that de-policing is one of the hypotheses advanced for the apparent change in the post-Ferguson trajectory of crime trends in the USA, which they suggest can occur

where negative publicity and public protest regarding police behaviour leads officers to withdraw from enforcing the law for fear of criticism and lawsuits. From this perspective, police officers throughout the United States may have become hesitant to be proactive out of concerns for being subjected to negative media scrutiny for racial profiling or the use of excessive force.

They caution that their "analysis cannot speak to...the extent to which de-policing or a crisis in police legitimacy [may] have occurred post-Ferguson, and if so, the impact it may have had on crime rates," however the researchers find, in relation to the notion of a 'Ferguson Effect' on crime rates, "no systematic and widespread change in crime trends among the [81] cities [with populations exceeding 200,000] in our study beyond robbery rates" (Pyrooz et al. 2016: 5). ${ }^{16}$

Morgan and Pally (2016) analyzed official Baltimore Police Department (BPD) data related to reported criminal occurrences and arrests from 2010 through 2015 and they found that de-policing, in the form of significantly fewer arrests, was certainly taking place across the rank-and-file of the BPD since the death of Michael Brown in Ferguson on 9 August 2014 and then, to an even more significant degree, following the death of Freddie Gray in Baltimore on 12 April 2015 - which the researchers find is "consistent with the widely discussed conjecture that the Baltimore police pulled back from some policing" (Morgan \& Pally 2016: 3). The researchers determined that arrests "decreased substantially" for many less serious crimes (those largely implicating police 
discretion) during the post-Ferguson period and that, "It is likely, but very far from verified, that these changes reflect evolving police practice, and that these practices evolved in response to the Ferguson protests and other attention to police conduct" (Morgan \& Pally 2016: 48). Rates of arrest, particularly for those less serious crimes that often involve officer discretion (i.e. disorderly conduct and driving offences), declined even more in the 'post-Gray period' - by an additional $49 \%$ and $44 \%$ for the two offences specified, "consistent with the widely discussed claim that the Baltimore police pulled back from some routine policing activity in response to a perceived lack of support" (Morgan \& Pally 2016: 50)

In their research, Farmer and Sun (2016: 240) assess that "while recording the police has increased with recent technological advances, little empirical research has examined its impact on policing and police-community relations." In interviews with 10 officers working in a small police agency in the mid-Atlantic (USA), they found that officers "have become used to citizens recording them, as it has become a routine expectation of the job" and that "annoyance was the most common feeling that police officers recounted regarding citizens recording them" (Farmer \& Sun 2016: 240, 248). Through a 2016 nationwide survey of 7917 officers from 54 American police and sheriff's departments (of all ranks and excluding only those whose primary responsibilities involve court security, jail operations, or civil processes), which received extensive media coverage across the USA and a significant amount in Canada and internationally, Morin and his colleagues at the Pew Research Center (2017) obtained data that supported various findings, including several that relate to the 
contemporary phenomenon of de-policing. ${ }^{17}$ In introducing the study, the Pew

researchers observe,

Police work has always been hard. Today police say it is even harder...[The study] comes at a crisis point in America's relationship with the men and women who enforce its laws, precipitated by a series of deaths of Black Americans during encounters with the police that have energized a vigorous national debate over police conduct and methods. Within America's police and sheriff's departments, the survey finds that the ramifications of these deadly encounters have been less visible than the public protests, but no less profound (Morin et al. 2017: 1).

In summary, the study found: (1) while $83 \%$ of the American public think they understand the risks and challenges their police officers face, $86 \%$ of officers think that citizens do not; (2) $86 \%$ of officers believe that controversy around recent high-profile fatal encounters between police and Black citizens has made police work harder today; (3) $76 \%$ of officers observe that officers are now more reluctant to use force, even when it is appropriate to do so; (4) $75 \%$ of officers perceive that interactions between police and Black citizens have become more tense; (5) 93\% of officers are now more concerned about their personal safety than they were before 2014 ; and (6) $72 \%$ of officers report that they are now less willing to proactively stop and question suspicious persons. Further, 54\% of White officers report they nearly always or often feel frustrated by their police work, whereas $41 \%$ of Black officers report the same, with Hispanic officers falling somewhere in the middle (Morin et al. 2017). Overall, these researchers find, "These fatal encounters and the public outcry they have generated have affected police work in fundamental ways" (Morin et al. 2017: 60). As specifically relate to the phenomenon of de-policing, findings in this Pew study "raise the possibility that many officers are responding to the [high-profile] incidents by 'de-policing' - that 
is, by not fully carrying out their law enforcement responsibilities out of fear of becoming involved in a high-profile incident" (Morin et al. 2017: 65).

Similarly, Maguire and colleagues (2017: 740) observe that some American police leaders "have suggested that placing police under heightened levels of scrutiny has resulted in the emergence of a 'Ferguson Effect' in which [rank-and-file] officers are now less willing to engage in proactive enforcement activities because they are afraid to get in trouble" and some front-line officers have taken this "one step further, [arguing] that fear of being disciplined [internally], losing their jobs or their pensions, or being prosecuted or sued is leading police officers to hesitate in dangerous situations." In the same vein, Gonzales and Cochran (2017: 301) advance that de-policing has occurred because of "highly publicized incidents of police use of deadly force against minority citizens, including but not limited to the Ferguson incident, causing police officers to disengage from their duties, particularly proactive tactics that prevent crime." Along these lines, they suggest,

De-policing occurs whenever a police officer decides not to get out of a patrol car for fear that the encounter will be recorded for public release, and the recording may portray the officer in a negative light...Many situations arise [within front-line police work] in which the decision to take action is largely or entirely a matter of discretionary decisions by the individual officers...In light of the decentralized nature of these decisions, it seems reasonable to assume that the risk of confrontation and highly visible exposure might cause at lease a fair number of police officers to hesitate to take actions that they might have taken in the past (Gonzales \& Cochran 2017: 307).

Lee, Weitzer and Martinez (2018: 198, citing Simonson 2016) reached a similar conclusion, observing,

News coverage, especially when it includes video recordings or public protests after an incident, can also affect police officers themselves...They may alter their behaviour in response to public criticism of the police or if 
they feel they are being monitored. Officers may avoid certain types of encounters altogether or may consciously temper their treatment of the citizens they interact with.

Nix and Wolfe (2018: 263) observe, "A growing body of evidence suggests that regardless of whether crime has meaningfully increased post-Ferguson, officers have indeed been psychologically affected by public scrutiny in the wake of the incident (and related incidents over the past [four] years)." Data for their empirical study originated from surveys completed in 2015 by 114 alumni of a southeastern (USA) police training institute geared toward mid-level police managers and their study findings suggest that, "Similar to line-level officers...management cops feel less motivated on the job, less willing to engage in proactive policing, and less satisfied with their careers in law enforcement (Morin et al. 2017; Nix \& Wolfe 2016). The microscope of scrutiny on the police appears to influence their attitudes and behaviours" (Nix \& Wolfe 2018: 272). Specifically, it was found that $31 \%$ of study participants are less motivated at work; $30 \%$ reported being less proactive on the job than they were in the past, "which is a clear sign that a non-trivial portion of the sample has engaged in de-policing;" $60 \%$ find policing has become less enjoyable; and $46 \%$ of these management-level officers believe that situations, such as the fatal police shooting in Ferguson, will result in depolicing and therefore, higher crime rates, which the researchers find to be "alarming because it suggests that many police managers in the sample believe line-level officers will engage in de-policing" (Nix \& Wolfe 2018: 267). However, these researchers determined that "those officers who felt that their communities perceive the police as more legitimate were less sensitive to the ill effects [of negative publicity and public criticism of police]" (Nix \& Wolfe 2018: 270). 
As discussed earlier in the context of de-policing in Cincinnati, and similarly in the aftermath of rioting in Baltimore following the 2015 death of Freddie Gray, some observers have claimed that de-policing of predominantly Black neighbourhoods has been adopted as a common practice by front-line Baltimore Police officers. Brad Heath's reporting in USA Today (2018) is representative of these perspectives,

Just before a wave of violence turned Baltimore into the nation's deadliest big city, a curious thing happened to its police force: officers suddenly seemed to stop noticing crime. Police officers reported seeing fewer drug dealers on street corners. They encountered fewer people who had open arrest warrants. Police questioned fewer people on the street. They stopped fewer cars. In the space of just a few days in spring 2015 - as Baltimore faced a wave of rioting after Freddie Gray, a Black man, died from injuries he suffered in the back of a police van - officers in nearly every part of the city appeared to turn a blind eye to everyday violations. They still answered calls for help. But the number of potential violations they reported seeing themselves dropped by nearly half. It has largely stayed that way ever since. 'What officers are doing is they're just driving looking forward. They've got horse blinders on,' says Kevin Forrester, a retired Baltimore detective.

Police typically learn about crime in one of two ways: either someone calls for help, or an officer sees a crime himself and stops to do something. The second category, known among police as an 'on-view,' offers a sense of how aggressively officers are doing their job. Car stops are a good example: Few people call 9-1-1 to report someone speeding - instead, officers see it and choose to pull someone over. Or choose not to. Millions of police records show officers in Baltimore respond to calls as quickly as ever. But they now begin far fewer encounters themselves. From 2014 to 2017, dispatch records show the number of suspected narcotics offenses police reported themselves dropped 30 percent; the number of people they reported seeing with outstanding warrants dropped by half. The number of field interviews - instances in which the police approach someone for questioning - dropped 70 percent.

Police officials acknowledge the change. 'In all candor, officers are not as aggressive as they once were, pre-2015. It's just that fact,' says acting Police Commissioner Gary Tuggle, who took command of Baltimore's police force in May. 'The cops are being less proactive at the same time violence is going up,' says Peter Moskos, a John Jay College of Criminal Justice professor and former Baltimore officer who reviewed USA Today's data and analysis. 'Cops are doing as requested: lessening racial 
disparity, lessening complaints, lessening police-involved shootings. All those numbers are just great right now, and if those are your metrics of success, we're winning. The message has clearly gotten out to not commit unnecessary policing.' Anthony Barksdale, a retired Baltimore police commander, says the message to officers was unmistakable. 'These guys have family members who tell them "Don't go to work and chase people for a city that doesn't care about you," he says. 'If I'm riding down the street and I see an incident, I see it, but you know what? It's not worth it. That's what these cops are thinking' (Heath 2018).

In another mediated contribution to the contemporary de-policing discourse, titled

'Why cops are standing down all across America,' published in The New York Post, Eugene O'Donnell (2018), a former NYPD officer and now a professor of law and police studies at John Jay College of Criminal Justice, observed,

Under sustained assault by the City Council, pundits and self-styled civilliberties advocates, a new generation of New York City cops is being conditioned to avoid showdowns with civilians, especially where coercion or force is called for. We could have saved the costly investment in body cameras by explicitly telling the cops two words: 'Do nothing!' Officers who do more engagement than what is absolutely required have been threatened by HQ that they'll find themselves on a list of 'troublemakers.'

In many parts of the country, the police have gone to ground. Racked by gun violence and pervasive fear, Detroit managed to get through 2016 without police shooting anyone - even as murders continued to rise. Police paralysis is great news for the elite critics of the cops but terrible news for the people dragooned in that town - it likely signals that the police are taking the long and winding road around trouble of all kinds...In Chicago, the street cops' ethos is 'go fetal, stay fetal.'

...Rearranging policing so that it revolves around fault-finding is going to have grave consequences in communities where the police are needed most. But all over the country, policing is now about avoiding all but the most essential, life-and-death interactions. Two recent videos of public officials being pulled over, one in New Jersey and the other in Baltimore, were seen as triumphs for the officers who remained calm and professional in the midst of a shower of abuse and name-dropping. The truth, however, is that the proliferation of video recording of cops' every interaction only will increase officers' timidity. That will look like selfrestraint in some cases, but overall it'll put law enforcement on the defensive and dull cops' instincts. String together a series of adversarial videos, and almost every officer can find him or herself fast-tracked to 
trouble. The new-era calculation for the police is: Conflict is the quickest path to extinction (O’Donnell 2018).

Further, Steve Pomper, a retired Seattle police officer, wrote a book titled De-

Policing America: A Street Cops' View of the Anti-Police State, which does not report

on findings from an empirical study but rather presents the author's anecdotal

observations of, and perspective on, the current state of American front-line policing. A

passage captures the essence of the claims advanced by Pomper (2018: 1-2),

Officer Michelle Malken leans back in the seat of her patrol car. Michelle has worked a dozen years all on the streets. This cop knows what she's doing. She observes a young man step into the crosswalk against the Don't Walk sign. The jaywalker locks his cold eyes on hers, maybe a challenge or a dare, and strolls past. Is he looking for a confrontation? Is he fingering the cellphone camera in his pocket ready to record the event?

Michelle should confront him, but will she? Just last week two fellow officers got jammed up over a simple littering incident. A man waiting in line for a nightclub show threw a food wrapper into the street in front of two passing officers. What should have been a simple, 'Please pick up your litter, sir,' turned into a melee when the man became combative after refusing police orders to pick up his trash. Officers eventually subdued and arrested the man. Of course, news media reported that the police used excessive force and arrested a man for 'only littering.'

...Should Michelle enforce the law? Yes, she should - in a perfect world. But cops do not work in a perfect world. Still, many people believe cops should be perfect. Well, they're not. They're what anthropologists call human beings. The question should she becomes, will she? The answer has become, why would she?..The officer subdues her instinct to enforce the law, chooses the better part of valour, and drives to Starbucks. After all, why should she chance having to use force or arresting the man for only jaywalking? De-policing.

De-policing is...the provocative terms used to describe a phenomenon where cops reduce or avoid proactive patrol. Though it's become a hot top, it's been around for a while...Today, everyone knows de-policing. Are police officers in Seattle and across America de-policing? Yes. In this hostile, antipolice climate, who can blame them? 
Also, in 2018, Marier and Moule (2018) observed that since 2014 (postFerguson) police across the USA have faced increased scrutiny, public condemnation, and criticism, which has included protests in urban communities, the proliferation of the Black Lives Matter social movement, and more DOJ investigations. In this current policing landscape (elaborated in the discussion throughout Chapter Three), some prominent members of the policing community have made claims that police work in the field may be compromised when officers perceive substantial public hostility toward them, "[including] officers' hesitancy to enforce the law due to fears of public and legal scrutiny" (Marier \& Moule 2018). These authors suggest that the current situation is consistent with the classic ethnographic accounts of police culture from the 1960s and 1970s, which found links between officers' perceptions of public antipathy, police occupational norms (officers' approaches to police work), and police culture - or, in other words, that "police cultural norms are a function of transactional relationships with the public" (Marier \& Moule 2018). As Marier and Moule (2018, citing Muir 1977; Niederhoffer 1967; Skolnick 2011) observe, "Policing researchers have long argued that officer perceptions... of hostility toward them influence several aspects of police culture, including isolation from the public, work-group solidarity, cynicism toward the public, and favourability toward coercive strategies."

In describing the rationale for their study, which involved surveying 12,376 sworn officers from 98 police agencies across the USA, Marier and Moule (2018) advance that "limited empirical research has examined these [historical] claims." They find that officers' perceptions of public antipathy have a direct, positive association with 
the four aspects of traditional police culture that were tested - social isolation, police solidarity, cynicism toward the public, and coercion and the authors advance,

This study presents compelling evidence that officers' sense of hostility from the public significantly influences police culture. Officers' approach to public service is significantly influenced by their perceptions of public sentiment, underscoring claims made by police ethnographers (e.g. Skolnick 2011), law enforcement leaders (e.g. Byers 2014), and editorialists (e.g. MacDonald 2015). Tension between officers and their communities is not merely incidental to police work; rather, the degree of such tension predicts cultural adaptations that may further damage policepublic relationships. These theoretical arguments are echoed in the popular wisdom of the 'Ferguson Effect,' which suggests that criticism of the police has a tangible influence on police culture and practice. Police officers are not immune to public antagonism, and they appear to respond with a degree of reciprocity (Marier \& Moule 2018).

Finally, and along the same lines, Weitzer (2018: 29, citing Ariel et al. 2014;

Brown 2016; Goold 2003; Jennings, Fridell \& Lynch 2014) observes, "Patrol

officers today are aware that their actions may be [video] recorded and widely

distributed. And some recent studies suggest that this new visibility may be having some constraining effect on officer behaviour."

\subsection{Recent Empirical Studies Focused on the Phenomenon of De-Policing}

Campeau (2015) drew on participant observation over 18 months and interviews with 100 officers serving with a mid-sized, urban police agency in Ontario to gather empirical data in her examination of how officers are negotiating meaning in today's 'unsettled' occupational environment, prompted by heightened levels of police oversight, which for her study, was understood "as increasing accountability and public visibility" (Campeau 2015: 675). Campeau's analysis focused on the impact of today's oversight on two occupational practices (or officer 'ideal types'), which are often cited in the police occupational culture literature - group solidarity and mission/action (other 
traditional police cultural representations include societal isolation, machismo, and conservatism), and she found, at the individual officer level, that cultural ideas about managing risk in today's front-line policing landscape include disengagement and second-guessing (what I would characterize as de-policing practices).

Two study participants were presented as representative of cultural scripts about risk - “communicated as 'staying out of trouble' and 'FIDO' [that] prompts [officers] to pursue alternative strategies for action, which entail avoiding scenarios that would typically warrant their attention" (Campeau 2015: 681).${ }^{18}$ Campeau (2015: 681) also observed "several examples of [officers] disengaging...throughout the ride-along stage of this research." With today's "intense accountability" and policing's new visibility, Campeau (2015: 683-684) finds that officers "are less likely to eagerly engage in activity that may be deemed exciting or dangerous in light of the greater risk of being caught doing something that can be construed as a violation of policy" and that traditional police culture representations do not capture how "officers understand their work" today.

As discussed in Chapter One, Oliver (2017) conducted interviews with 25 American police officers as part of a qualitative exploratory study into de-policing. His objective was to "gain an understanding of de-policing from the police officers' perspective in order to understand if de-policing is merely rhetoric created by pundits or reality as demonstrated by the lived experience of police officers (Oliver 2017: 438). Oliver (2017) observed that 23 of the 25 research participants in the study were familiar with the terminology 'de-policing' and he found that the phenomenon is bona fide and growing, it is more widespread than many within policing appreciate, and its causes are 
unique to each individual officer, but include: officers' concerns with being accused of racial profiling; being sued; being adversely impacted by new laws and policies (for example, being mandated to record demographic information of those persons stopped by police); being investigated, and/or disciplined, by 'internal affairs'; and/or being criticized by the public. Oliver (2017) finds that, in the American context, de-policing practices are often a response to race-centred issues and, while typically individual or small group responses to an external event, de-policing has the potential to spread throughout departments to other officers, who share vicariously in the phenomenon.

Shjarback, Pyrooz, Wolfe and Decker (2017: 49) observe that today's front-line officers "find themselves in the 'social media era of policing' where many of their actions are potentially captured on video, uploaded to websites, livestreamed, and viewed by millions of people." Further, post-Ferguson, "unprecedented" public, media, and governmental scrutiny; civil unrest; social media "outbursts"; and a galvanized Black Lives Matter movement have,

Led to widespread speculation [by politicians, journalists, scholars, and policing leadership] that officers are de-policing - retreating from active police work in reaction to the negative publicity that has been placed on police agencies across the country...In short, the de-policing argument suggests that officers are withdrawing from proactive styles of law enforcement as a way to avoid getting caught up in a controversial use-offorce incident...Rigorous empirical inquiry has not kept pace with contemporary accusations of de-policing (Shjarback et al. 2017: 42).

Shjarback and colleagues (2017) define 'de-policing' as "a pullback or withdrawal from proactive or discretionary police work" and their study examined whether such behaviour was occurring among officers serving in the 118 municipal police agencies across the State of Missouri - which the authors suggest could be considered 'ground zero' for de-policing - given that Ferguson and St. Louis are located within the state. 
They found that statewide, there were over 100,000 fewer vehicle stops made in 2015 than there were in 2014, which cannot be attributed to normal operational fluctuations (Shjarback et al. 2017). The researchers concluded that de-policing was most certainly happening in Missouri in the post-Ferguson period and they found "consistent evidence of a racialized de-policing effect" (Shjarback et al. 2017: 50). They advance, "Interestingly, de-policing was most likely to occur in minority communities. Departments serving jurisdictions with larger African-American populations conducted fewer stops and searches and made fewer arrests in 2015 than in 2014...This pattern might be expected given that the national conversation and debate about policing in recent years has been focused on race" (Shjarback et al. 2017). The authors find that these de-policing behaviours involve officers' assessments of risk and 'selfpreservation,' in terms of avoiding situations that increase the risk of discipline, demotion, public ridicule, injury, or death (Wallace et al. 2018, citing Shjarback et al. 2017). Shjarback and colleagues (2017: 50) suggest, "Perhaps the topics of de-policing and self-preservation can be linked to Muir's (1977) seminal explanation of officers engaging in "avoidance behaviour" and Worden's (1995b) categorization of officers 'laying low' and they counsel that "future work should continue to survey or interview officers about their motivations/rationale for engaging in de-policing behaviour."

Also, in 2017, Rushin and Edwards (2017: 736), in their article titled simply 'Depolicing' in the Cornell Law Review, observed that the extant literature suggests that "external scrutiny may contribute to de-policing - but the evidence is limited at best." Their study used the 61 DOJ oversight investigations of American police departments, spanning 1995 to 2015 , as a proxy for the presence of intensified public scrutiny and 
they assessed, through statistical modeling, whether there were any associated changes in crime rates in each of those jurisdictions. The study found "that external regulation of American law enforcement agencies through [DOJ investigations] appears to be associated with a temporary uptick in some reported crime rates" but the authors conceded that their study "only provides a limited insight into the world of de-policing. It leaves many important questions unanswered" (Rushin \& Edwards 2017: 768). The researchers observe that post-Ferguson, with debates about policing "raging on" and police officers remaining under unprecedented public scrutiny, "Critics have worried that this additional oversight may cause police to be less aggressive, thereby increasing crime. This article demonstrated that such a de-policing phenomenon is not entirely implausible" (Rushin \& Edwards 2017: 776).

Deuchar, Fallik and Crichlow (2018:11, citing Wolfe \& Nix 2016) observe, "While some evidence has emerged regarding the impact of [high-profile media events depicting the deaths of young Black males by police, such as the 2014 fatal police shooting in Ferguson, Missouri] on citizens, researchers rarely consider how they impact police." Therefore, the focus of their research "was to prioritize the voices and perspectives" of officers and, in this regard, Deuchar and colleagues (2018: 4) find that the accumulation of these events "have taken a toll on law enforcement" and that "perhaps the biggest impact of these events has been on de-policing or withdrawal of law enforcement duties. Though it is unlikely that police will stop responding to crime altogether, de-policing can take on several forms including fewer arrests and traffic stops." The 20 officers participating in the study (serving with a police agency in a southern American state) described "the way in which the post-Ferguson media 
attention had led to a situation where many officers were less inclined to be proactive in their approaches, particularly in relation to communities of colour and with respect to use-of-force" (Deuchar et al. 2018: 8). Deuchar, while conducting field observations, himself witnessed "the phenomenon of de-policing," in situations where "officers were not always proactive at intervening even when it was evident that young Black men were engaging in drug deals" (Deuchar et al. 2018: 9). Deuchar and his colleagues (2018: 12) found, "Regardless of rank or years of experience, there was a general feeling [among the study's research participants] that a process of de-policing had gradually begun to take root in the county divisions where these officers were based."

Chanin and Sheats (2018: 1-2, citing Brehm \& Gates 1999) observe that police examples feature prominently in the organizational and occupational theory literature suggesting that workers "may respond to unfavourable changes to their professional environment by 'dissent shirking,' or using their discretionary authority in way inconsistent with established rules, norms, and community expectations" and that "the recent and highly publicized increase in violent crime across many of [America's] biggest cities has injected de-policing into the national conversation." In their assessment of extant theory, which suggests that officers might push back against changes brought about in their work environment, Chanin and Sheats (2018: 5) suggest that the more risk of personal consequences that officers perceive "the less likely a street-level agent will engage in the kind of proactive, aggressive policing thought to expose him or her to increased risk." Similar to Rushin and Edwards' (2017) methodology, Chanin and Sheats (2018) examined data from DOJ investigations of American police departments but they focused on arrest rates for minor offences, 
typically implicating substantial officer discretion (i.e. liquor law violations, disorderly conduct, and public drunkenness). Their study rationale suggests that "officer behaviour is a more reliable indicator of de-policing than are changes to an outcome, like crime, which have been tied to a variety of exogenous social and ecological factors and may or may not reflect shifts in police activity" and that "de-policing will likely manifest in the discretionary response to low-level, misdemeanour offences" (Chanin \& Sheats 2018: 7-8). The researchers found, through reviewing 20 years of arrest records from 10 jurisdictions investigated by the DOJ, that "clearly" DOJ investigations were not "causally related to statistically significant declines in arrest rates" (Chanin \& Sheats 2018: 14). In reconciling anecdotal reports of officers' de-policing behaviours and this study's statistical findings, Chanin and Sheats (2018, citing Ajzen \& Fishbein 2005; Engel \& Worden 2003) suggest it is possible that there could be inconsistencies between officer's attitudes/verbal expressions and their physical behaviours.

Finally, Nix, Wolfe and Campbell (2018) advance that under the present conditions of 'extreme' scrutiny, some commentators are suggesting that there exists a 'war on cops' being waged across the USA and several of these observers are now finding that, in response, front-line officers are de-policing (i.e. avoiding proactive stops). The authors advance, "In sum, regardless of whether crime rates have changed or de-policing is actually occurring on a broad scale, it is apparent that recent criticism of their profession has adversely affected police officers' perceptions. Officers appear to be feeling strain related to national-level challenges to their legitimacy and may believe de-policing is a common response" (Nix et al. 2018: 40). They also suggest that 
"regardless of whether the war on cops is objectively real, police officers might nevertheless perceive it to be real and de-police in response" (Nix et al. 2018: 38).

Their empirical study involved surveying 210 command-level officers, from a southeastern state in the USA, about their attitudes concerning any purported 'war on cops' and/or 'de-policing' phenomenon, with the objective of determining whether these officers believe a 'war on cops' actually exists (as some have suggested) and, in turn, "if they believe that de-policing is a common coping mechanism among officers" (Nix et al. 2018: 35). To measure command-level officers' perceptions of their rankand-file officers' de-policing practices, the researchers asked study participants to indicate their level of agreement with three statements (using a five-point Likert scale measure) - (1) 'In today's world, it is in police officers' best interest to avoid making proactive stops', (2) 'My officers avoid proactive stops because it might lead to a useof-force situation', and (3) 'My officers avoid proactive stops because a citizen might capture the stop on video and upload it to the Internet' (Nix et al. 2018: 41).

Analysis of study data found that only $14 \%$ of this sample of command-level police officers agreed that de-policing is a common practice among today's front-line officers, however Nix and colleagues (2018: 49) alert the reader that "it is possible that there was a degree of social desirability when answering the questions on our survey. Despite the promise of anonymity, it may have been difficult for some respondents to be truthful in reporting issues concerning the de-policing behaviours of fellow police officers." The authors also caution that given the 'newness' of today's apparent depolicing phenomenon, "the consequences of the perceptions observed in this study may 
not have manifested yet in actual [measurable] increases in crime or de-policing" (Nix et al. 2018: 48).

Nix and colleagues (2018) propose general strain theory (Agnew 1992) as an available theoretical rationale to inform the study of police officers' perceptions about, and their attitudes toward, the prevailing policing environment and the behavioural practice of de-policing, which I discuss further in Chapter Three. In this regard, these researchers propose that today's policing environment can be perceived as a threat to officers' wellbeing and a psycho-social strain experienced by the rank-and-file. Therefore, de-policing can serve as a (perhaps 'antisocial') coping and a selfpreservation mechanism (Nix et al. 2018). Along these lines, Nix and colleagues (2018: 48) encourage that "future studies should consider the role of strain - and officers' emotional responses - in explaining their attitudes toward de-policing." 


\section{CHAPTER THREE}

\section{ESTABLISHING CONCEPTUAL AND THEORETICAL FRAMEWORKS}

\subsection{Chapter Introduction}

This dissertation considers the identified de-policing phenomenon in relation to recent technological, social, political, and cultural transformations across Western democracies - specifically, (1) 'policing's new visibility,' which should be understood within the broader conceptual framework that I refer to as today's techno-social landscape for front-line policing, and (2) the pervasive public scrutiny directed at, and frequent critique of, front-line officers' work in the field, which I discuss within the conceptual framework that I refer to as the contemporary socio-political landscape for front-line policing. This chapter assembles the necessary conceptual scaffolding to enable a better appreciation of the nuanced contours of today's de-policing practices (elaborated within subchapters 3.2 and 3.3) before the reader proceeds to consider information (in subchapter 3.4) about the contemporary front-line police subculture, which is this dissertation's theoretical framework - informing the discussion of study findings in Chapter Six. The sociological study of occupations through the theoretical framework of culture has deep roots that include Durkheim, Geertz, Parsons, and Weber and, specifically in relation to police, Chen (2016: 11, citing Cockcroft 2013) observes, "Research about police culture has been driven and theoretically supported by studies of sociologists that aim to comprehend police behaviour through the lens of the occupational culture perspective."1

In transitioning into consideration of the two conceptual frameworks, it is worthwhile to revisit the study's three principal areas of inquiry: (1) Have today's front- 
line officers changed their behaviours in response to transformations in the technosocial environment in which they do their work in the field? (2) Have policing's rankand-file modified behaviours in response to today's socio-political forces of change? And, more specifically, (3) Have police responded to anxieties, occasioned because of the remodeled policing landscape in which they now operate, by becoming risk averse in some of their practices, as has been suggested in some recent scholarship addressing the contemporary phenomenon of de-policing? Along these lines, Bayley and Shearing (1996), in assessing the situation of the police entering the new millennium, argued that transformations occurring in the policing of Western democratic societies are on a scale unprecedented since the introduction of the public police in the nineteenth century. In concurring with, and amplifying upon, the theoretical elaborations of Jones and Newburn $(1998,2002)$ - around the epochal nature of change in today's policing and the determinants of these changes - Stenning and Shearing (2005) find that modifications to traditional police practices are now largely the product of external social, cultural, and political catalysts, rather than of 'change forces' internal to, or developing from within, police agencies. ${ }^{2}$ Cockcroft (2013: 33) lends support to these observations in arguing that there is "a need to situate [today's police] culture within the ever-changing social and cultural contexts of police work."

In this vein, this dissertation reports on the de-policing practices of rank-andfile Canadian and American officers in relation to two external 'change forces' (Stenning \& Shearing 2005), which, I argue (with empirical support from study data and corroborative contributions from other scholars) have impacted substantially on today's front-line policing landscape. The first relates to the implications of recent 
developments in, and the ubiquity today of, mobile video recording and networked communications technologies (e.g. smartphones, Facebook, Twitter, Instagram, Snapchat, WhatsApp, Tumblr, and YouTube) and associated socio-cultural practices, such as 'citizen journalism' and 'participatory culture'. In this regard, in addressing current "transformations in policing of a magnitude at least as great as occurred with the introduction of the New Police in the early nineteenth century," Jones and Newburn (2002: 129) particularize "the impact of new technologies upon policing."

In his analysis of the conviction of Toronto Police Constable James Forcillo (in relation to the 2013 shooting death of Sammy Yatim), Globe and Mail columnist Marcus Gee (2016: A5) suggests that the historic "carte blanche," or, "at least broad discretion" afforded to front-line officers, "is changing." He observes, "One impetus for change is the emergence of on-the-scene video that gives us an up-close look at police encounters. Another is the push for greater scrutiny of police and greater accountability for officers" (Gee 2016: A5). As described in Gee's (2016) last sentence, the second external 'change force' (Stenning \& Shearing 2005) impacting on front-line police work today involves political, social, and cultural shifts across a not insignificant proportion of the broad citizenry throughout Western democracies - as relates to increasingly prevalent antagonism between the policed and the police and concurrent challenges to the fundamental nature of the public-police relationship. I argue that these sociopolitical transformations are intertwined with the aforementioned techno-social developments, which, through today's extensive video-recorded documentation of officers' actions in the field, have drawn the attention of a public audience that is now more inquisitive, skeptical, and informed (Cockcroft 2013; Ivkovic 2008; Loftus 2009) 
and influenced by contemporary cultural sensibilities around, and increasing disenchantment with, various police practices - such as those involving racial bias and violence (e.g., see Comack \& Silver 2008; Elias 1994; Greer \& McLaughlin 2010b; Haggerty \& Sandhu 2014).

For 'real world' demonstrations of how these 'externalities' (Stenning \& Shearing 2005) have become such significant issues in today's policing, and by implication, important considerations for the sociological study of today's front-line police work, one needs simply to open a newspaper, watch a television news broadcast, or access one of a multitude of online posts, tweets, blogs, or websites discussing policing. With remarkable frequency, occurrences featuring controversy around rankand-file police conduct come to our attention and these highly-publicized (and now often very much visible) events substantially influence our understandings of front-line police work. Recent incidents - such as fatal police interactions with Sammy Yatim (Toronto), Robert Dziekański (Vancouver), Walter Scott (North Charleston, SC), Freddie Gray (Baltimore), Laquan McDonald (Chicago), Philando Castile (Falcon Heights, MN), John Crawford II (Beaver Creek, OH), James Boyd (Albuquerque), Tony Robinson (Madison, WI) Oscar Grant (Oakland), Terrence Crutcher (Tulsa), Tamir Rice (Cleveland), Samuel DuBose (Cincinnati), Alton Sterling (Baton Rouge), and Eric Garner (New York) are dramatically illustrative (and are, sadly, not an exhaustive accounting).

As described throughout Chapter One, this study focuses specifically on investigating outcomes of today's front-line policing environment in the everyday work of rank-and-file officers - as distinguished from, for example, changes in governance, 
managerial decision-making, or administrative policy - and the study's data originates from, and interrogates the perspective of, the officers who perform their work in our communities and, for all intents and purposes, in front of today's vast public audience. The following three subchapters assemble the conceptual scaffolding and theoretical framework that will allow the reader to consider this dissertation's empirical presentation within proper social, political, technological, and cultural contexts.

\subsection{Today's Techno-Social Landscape for Front-Line Policing}

The extraordinary enlargement in the field of the socially visible has emerged as a defining characteristic of contemporary Western society - transforming various aspects of social, cultural, and political relations (e.g., see Ball, Haggerty \& Lyon 2012;

Bauman \& Lyon 2013; Castells 2004, 2009, 2010, 2012; Etzioni 2010; Goldsmith 2010; Lyon, Doyle \& Lippert 2012; Norris \& Armstrong 1999; Thompson 2005). ${ }^{3}$ Bogard (2006: 76), for example, finds, "The powers of monitoring and recording have expanded exponentially in postmodern societies, to the point where virtually every space, interior and exterior, has become a space of observations" and, also along these lines, Chan (2008: 224) observes, "Routine, intense and even intrusive surveillance technology has become an accepted part of the landscape in Western democracies." Similarly, Whitaker (1999: 140) argues that new technologies "render individuals 'visible' in ways that Bentham could not even conceive...they are visible to multiple gazes coming from many different directions looking for different things.” Also, in this vein, Andrejevic (2007: 239) observes that "we are becoming habituated to a culture in which we are all expected to monitor one another." 
Finn (2012: 77) finds that the rise of surveillance technologies and practices is now "woven into the fabric of everyday life in our society" and Goold, Loader and Thumala (2013: 9) note that "CCTV cameras have disappeared into the background of urban life and become socially invisible." Within these circumstances of universal, continuous, and comprehensive surveillance (Goold et al. 2013; Lyon 2007; McGrath 2012), Doyle (2011:285) finds that the wide-ranging surveillance that characterizes today's Western democratic societies has been enabled by “sweeping social and technological changes." Today, those who exercise power (such as the police) are those that are the focus of the 'new visibility' (Antony \& Thomas 2010; Goldsmith 2010; Thompson 2005) - a social transformation that has been facilitated by the technologies that are now an inextricable dimension of contemporary Western urban life (Castells 2000, 2004, 2009, 2010, 2012). In this regard, I argue (see also Brown 2013, 2016) that three recent techno-social developments have facilitated a recent and pronounced intensification in the public's exposure to performances of front-line policing - the rending of the 'sacred canopy' (Manning 2008a) that formerly concealed much police conduct from the critical scrutiny of the citizenry (Bonilla \& Rosa 2015; Doyle 2003; Ericson 1989; Goldsmith 2010; Hirschfield \& Simon 2010; Intravia, Wolff \& Piquero 2018; Lautt 2012; Martin 2005; Toch 2012; Walker \& Archbold 2014). ${ }^{4}$

These are: (1) the ubiquity of mobile devices equipped with video recording functionality (Bock 2016; Brown 2013, 2016; Brucato 2015a, Roche 2017; Wilson \& Serisier 2010; Yesil 2011) ${ }^{6}$ and CCTV surveillance systems (Goold 2003, 2004; Goold et al. 2013; Koskela 2003; Lippert \& Walby 2012; Lyon 2001; Norris, McCahill \& Wood 2002 $)^{78}(2)$ the entrenchment of an awareness of the capacity to engage in, and 
the effectiveness of, citizen journalism across much of the population - in terms of ordinary people now having, in their immediate possession at most times, the ability to document and therefore, to intervene in, events unfolding within their field of vision (Allan 2013; Antony \& Thomas 2010; Bowman \& Willis 2003; Doyle 2006a; Farmer \& Sun 2016; Farmer et al. 2015; Koskela 2006, 2009, 2011; Kurasawa 2008; Newell $2014,2015,2019)^{9}{ }^{10}$ and (3) the proliferation of user-created content; autonomous 'new' (or 'social') media and concomitant online interactivity across Western societies (Bonilla \& Rosa 2015; Jenkins 2008; Kahn \& Kellner 2006; Shirky 2008; Trottier 2015; Yesil 2011) $)^{11}$ - which allows much of today's public to bypass the traditional gatekeeping function of mainstream media, overcome long-established institutional power imbalances, submit substantive contributions to post-event narratives through direct dissemination to an engaged and participatory public audience, and to access an unprecedented volume of information from a multiplicity of sources (Deuze 2005; Diamond \& Plattner 2012; Lievrouw 2011; Loader 2008; Newell 2014; Waisbord 2014)..$^{12} 13$

As a result of these techno-social developments, the historical 'invisibility' of police officers' actions in the field (Ericson 1989, 1995; Goldsmith 2010; Goldstein 1960; Haggerty 2012; Newburn \& Hayman 2001; Smith 2009; Waddington 2014) ${ }^{14}$ has been supplanted by a 'new visibility' (Thompson 2005; Brighenti 2007, 2010a, 2010b) of front-line police work (Brown 2013, 2016; Goldsmith 2010; Haggerty 2012; Haggerty \& Sandhu 2014; Sandhu 2016, 2019; Sandhu \& Haggerty 2015, 2017). ${ }^{15}$ In this regard, Haggerty (2012: 241) observes,

The police are often referred to as a 'low visibility' profession, one where officers work outside of the limelight of public scrutiny and away from the 
direct oversight of supervisors. It is clear, however, that this image has been inaccurate for some time. Officers are [now] monitored by practices that operate both internally and externally to the police organizations.

As a result, today's policing occurrences often play out in settings within video recording range of bystanders' mobile devices and/or CCTV systems.

In this vein, Jeffries (2011: 73) finds, "Cell phone camera surveillance of police officers is exposing behaviour that some police officers have gotten away with for years" and Ariel and colleagues (2015: 509) conclude, "Cameras have become the most effective weapon that ordinary people have to protect against and to expose police abuse." Further, Trottier (2015: 212) observes that "as a result of social convergence, social media enables a diffuse kind of visibility for police work...platforms like Facebook are spaces where users can not only view information about crime and policing but also discuss these issues, submit what they believe to be evidence and call for social action beyond these sites." In alignment, Pyrooz and colleagues (2016: 2) suggest, "The massive social media response following the events in Ferguson may have precipitated de-policing through contagion, the viral spread of information across social media." Shjarback and colleagues (2017: 43) explain, in this regard, "The process of social media contagion allows coverage of such incidents to spread quickly and to exert an impact in other jurisdictions. For example, social media may allow the actions of police officers in Chicago to affect citizen responses to the police in an entirely different city or state."16

The tremendous proliferation of these recording and communication technologies and associated social and cultural practices enable tangible documentation and explicit exposure (now often in high-definition resolution and featured across mainstream and 
social media platforms) of significantly more police work in the field, including instances of officer misconduct, than was possible only a few years ago (Chan 2003; Farmer \& Sun 2016; Goldsmith 2010; Greer \& McLaughlin 2010b; Haggerty 2012; Koskela 2009; Robinson 2012; Walker \& Archbold 2014). ${ }^{17}{ }^{18}$ Sandhu (2019) observes, in my view, accurately, that the number of cameras monitoring police officers today is likely rivaled only by the number of cameras watching politicians and celebrities. In his discussion of contemporary visibility, Brighenti (2007: 330) refers to circumstances of 'super-visibility,' which is "a condition of paradoxical double bind that forbids you to do what you are simultaneously required to do by a whole ensemble of social constraints." In this vein and in the specific context of front-line policing, Haggerty and Sandhu (2014: 11) find, "Officers can consequently find themselves in catch-22 situations where they are required to use circumscribed violence while contemplating the consequences of having that violence captured on video."

Situated within what Lyon (2013) assesses as our current 'culture of surveillance'; Andrejevic (2005) characterizes as the recent phenomenon of "citizen-to-citizen surveillance'; Ericson (2007) describes as the new 'lateral surveillance' that shapes, and is shaped by, today's 'politics of uncertainty'; and what Koskela (2011, in amplifying on Andrejevic's 2007 discussion of the emergent 'culture of monitoring'), addresses in the context of 'camwitnessing' - I argue that the nature of today's public audience observing front-line police actions can be considered on a continuum - ranging from one citizen's dispassionate disinterest in how officers conduct their work to, at the other end of the spectrum, another's keen attentiveness to, and inherent distrust of, police behaviour. In relation to the latter perspective, Finn (2012: 76-77), who relied on the 
2009 fatal police shooting of Oscar Grant to inform his study, found that the multiple citizen-generated video recordings of that event exemplify what is now the widespread social practice of seeing our world 'surveillantly' and are "indicative of a pervasive cultural trend of citizens documenting police activity." ${ }^{, 19}$ In this regard, Farmer and colleagues (2015: 356) advance, "Using personal electronic devices to record policepublic encounters has surfaced as an important social phenomenon that could potentially shape public perceptions of the police and police-community relations."

Ian Scott, who, from 2009 until 2014 served as director of Ontario's Special Investigations Unit (SIU), a civilian oversight agency that investigates potentiallycriminal police actions, finds that the "meteoric rise" of these techno-social developments has "had a huge impact [on the police]" and they have "become a game changer" in both police work and in oversight of the police (Perkel 2013). Along these lines, Kelly (2014) observed five years ago that policing was on "the cusp of a paradigm shift" instigated by "accountability by camera" and, similarly, Haggerty and Sandhu (2014: 11) assessed that the pervasiveness of video recording devices throughout Western societies "is changing the dynamics of policing on the ground." As presented in Chapter Two, over the past few years a number of scholars have observed that today's omnipresent potential for video-recorded documentation of police actions in the field is changing aspects of how front-line officers do their jobs (e.g., see Braga et al. 2018; Brown 2013, 2016; Farmer et al. 2015: Goold 2003, 2004; Haggerty \& Sandhu 2014; Murphy 2014; Potere 2011; Sandhu 2016, 2019; Sandhu \& Haggerty 2015; 2017; Schaefer 2012; Shjarback et al. 2017; Todak 2017; Todd 2015). 
As elaborated in the following subchapter (3.3), with significant challenges around trust and confidence in the police across various geographic and cultural contexts - particularly in relation to 'minority' communities (e.g., see Tyler \& Wakslak 2004; Weitzer 2002, 2018; Weitzer \& Tuch 2002, 2006) and mistrust of the police prevailing across a seemingly-increasing proportion of the American and Canadian populations, the ability to see, with one's own eyes, what the police are doing (or what the police did, in the case of a retrospective analysis) is now the preferred source of information for many citizens in making assessments of police actions and this audiovisual scrutiny informs people's ideas around police accountability and policing's legitimacy (Bradford, Jackson \& Hough 2014; Jeffries 2011; Levi 2013; National Research Council 2004; Stenson \& Silverston 2014; Tyler, Braga, Fagan, Meares, Sampson \& Winship 2007). This contemporary reality can be juxtaposed with the observations of Skolnick and Fyfe (1993: 19), who, writing, 25 years ago, observed, "In the absence of videotapes or other objective recording of gratuitous violence, [police] brutality rarely causes public controversy and is extremely difficult to prove."

As Brucato (2015b: 40, citing Hood 2006) advances, "Rousseau held that public servants ought to perform 'in the eyes of the public,' and 'to permit no office-holder to move about incognito'." Along these lines, Han (2012) observes that with today's new transparency, "to see is to know" and Bock (2016: 17) finds, "Modern Western culture has long favoured vision as a form of knowledge and the ability to see something for oneself is especially valued." In this regard, some now argue that policing's legitimacy resides (in the literal sense) in the eyes of the public. For example, Levi (2013: 157) finds that the legitimacy of the police is today determined by "whether the policed see 
the police as legitimate." Such is the new techno-social landscape for today's front-line policing and the new reality for today's rank-and-file officer performing their work in the community. In this regard, a recent report from the DOJ identified citizen journalism directed at police as one of the principal challenges facing American policing in the coming years (Gallagher, Kim, Markovic \& Spence 2016).

\subsection{Today's Socio-Political Landscape for Front-Line Policing}

Goldsmith (2010: 914-915) concludes that the increased visibility of police work in the field, "brought about through developments in visual recording technologies in the past two decades" and particularly of "less flattering or illegal practices," carries with it the potential for more police-related scandals, the potential for collective damage to the institution of policing in our society, and the diminishing of police power in the socio-political hierarchy. Also, in this vein, Cave and Oliver (2015) find, "Raw video has thoroughly shaken American policing. Grainy images of questionable police behaviour, spread through social media, have led to nationwide protests, federal investigations and changes in policy and attitudes on race." Several other scholars and media commentators have also observed that 'policing's new visibility' has largely facilitated this new era of public engagement with, and criticism of, policing and today's heightened public consciousness around police use of force (inappropriate applications of which are often characterized as 'brutality') and discriminatory practices (often termed 'racial profiling') (Bonilla \& Rosa 2015; Friedersdorf 2014; Lee et al. 2018; Lersch \& Mieczkowski 2005; Potere 2012; Weitzer 2015). ${ }^{20}$

In concurring with various scholars (e.g., see Bonilla \& Rosa 2015; Evans 2015; Ivkovic 2008; Jackson, Bradford, Stanko \& Hohl 2012; Mawby 2010; Miller \& Davis 
2008; Walker 2005), I argue that intertwined with and augmenting, or perhaps amplifying, the recent techno-social developments in the front-line policing landscape is the engagement of interested, inquiring, and, much more so than in the past, informed and growing public audiences across the USA and Canada that demonstrate an increasing willingness to scrutinize, criticize, and challenge socio-political institutions, including, of particular relevance to this study, Canadian and American police. ${ }^{21} 22$ As Evans (2015: 228, citing Ericson \& Haggerty 1997) notes, "The entrenched distrust endemic to [today's] risk society extends to the agents of the state, [and includes] equipment and systems which treat both citizens and police officers as cunning, corrupted, untrustworthy, risky, [and] guilty."23

In this vein, today, to a much more pronounced degree than at any time since the tumultuous socio-political upheavals of the 1960s and 1970s, many citizens question, and offer critiques regarding, police actions that come to their attention and they participate, in large numbers, in critical discourses around police conduct (Campeau 2015; Doyle 2007, 2011; Mawby 2010; Murphy 2012; National Academies of Sciences, Engineering \& Medecine 2018; Oliver 2017; Reiner 2010; Savage 2013). In this regard, Kutner (2016: 1) assesses that "the tension between law enforcement and large swaths of citizens has not been this high since the 1960s and 1970s" and Terrill and Paoline (2017: 211), similarly, in the context of the policing of American society, conclude that "the US [is currently] experiencing the most widespread civil unrest since the 1960s." Along these lines, Smith (2005: 494) finds, "[There exists today] an evolving public mindset that views police operations as a phenomenon not only to be closely observed but, as well, to be potentially called into question in less-than-favourable outcomes. The 
upshot of the foregoing is that the police find themselves increasingly subject of a watchful, and frequently, critical public eye.”

Collins and Klahm (2019) observe that "a perceived increase in instances of police excesses has gripped the public narrative in recent years and has given rise to an increase in discourse pertinent to police ethics and accountability.” The National Academies of Science, Engineering, and Medecine (2018: 17) find a "heightened discontent with policing" throughout the USA and observe that "the emergence of the Black Lives Matter group...suggests heightened concerns of specific non-White communities to the policies and practices of the police." In this regard, Collins and Klahm (2019: 263) advance, "The crisis of confidence in police and in the legitimacy of their actions that has characterized the post-Ferguson era illustrates a vital lesson for those involved in the field: it is a tough time for policing in the United States. Indeed, a recent Gallup poll found that confidence in policing has eroded to a 22 -year low." In the Canadian context, Huggins and colleagues (2014: 3) find that today's public audience is "more attuned to policing than ever" and, in the U.K., Loftus (2010: 2) observes, "Political sensitivity around policing has changed remarkably since the classic [ethnographic] studies [of police occupational culture]." Therefore, as is now obvious to most of us, given the frequency of our exposure to such events with today's technosocial realities, controversial police-citizen interactions can have considerable deleterious effects on public confidence in police officers (Flanagan \& Vaughn 1995; Jefferis, Kaminski, Holmes \& Hanley 1997; Maxson, Hennigan \& Sloane 2003) and, as a result, how the public views the police is a major concern within policing, its governance, and broadly across society - given the significance of the public-police 
relationship in Western democratic systems of social control (Jefferis et al. 2011; Luna 2000).

Campeau (2015: 674) finds that there is today, as a result of "society's changing accountability standards" and what Savage (2013) refers to as 'civilianness' in police oversight (demands for greater accountability and external monitoring), a "bright spotlight that is now being shone on an organization that has historically hidden behind a 'blue curtain," which functions as a "disruptive influence" on police (both the sociopolitical institution and also in terms of individual officers). Jackson and Sunshine (2007: 218-219) present similar findings and conclude that the once "iconic status" of the police has been tarnished as a result of changing citizen's perceptions, vis-à-vis officers' integrity, trustworthiness, and fairness and that there now exists a "tentative and brittle" relationship between the citizenry and the police that is "renegotiated case by case." This is the result of deteriorations in public confidence that have developed, cumulatively, from the exposure of scandals involving excessive force, racial profiling, dishonesty, corruption, and other police misconduct (Bradford 2011; Friedersdorf 2014; Jackson \& Sunshine 2007; Loader \& Mulcahy 2003; Reiner 2010; Roberts \& Hough 2005; Rosenbaum, Schuck, Costello, Hawkins \& Ring 2005; Sturges \& Cooke 2008). In this regard, Jackson and Sunshine (2007: 218) find, "Public confidence in policing has become shorthand for trust, legitimacy and consent, and there is considerable evidence that public confidence has been falling steadily over the past few decades."

It is important to appreciate that this kind of negative public sentiment toward police - involving suspicion, distrust, and demands for more scrutiny and accountability - is a relatively new development. As Sir Ian Blair (Metropolitan Police Commissioner 
from 2005 to 2008) explains, "Until recently [policing was] broadly left alone to get on with the job that [the public] have given it" (BBC News 2005). ${ }^{24}$ Along these lines, it was traditionally understood that police officers enjoyed the affective attachment and largely unconditional support from the vast majority the citizenry, within the "thin blue line' framing of their crucial socio-political function in society - as those standing between order and the ever-present forces of chaos (Reiner 2010). In this regard, Loader (1997) observes that historically "The idea of policing...brings to mind (and stomach) sensations of order, authority and protection; it makes it possible for people to believe that a powerful force for good stands between them and an anarchic world, that the state is willing to defend its citizens." In other words, as advanced by Giddens (1991) and then Loader and Mulcahy (2003) it was generally understood that police were considered by much of society as an idealized force for good, providing law-abiding citizens with a sustained 'ontological security.' As a result, front-line officers were given "considerable leeway" in their conduct (Lawrence 2000: 59), by a largely deferential public (Hirschfield \& Simon 2010; Reiner 2010). In this vein, Reiner (2010: 253) suggests that the misconduct of officers in the past was not revealed, as it is today, because of "the much more deferential culture of the social strata at the receiving end of policing, and the [sympathetic treatment of the police by traditional] media and the educated middle class. ${ }^{25} 26$

This explains why even high-profile police wrongdoings of that earlier era, which were exposed rarely, but from time to time, by television news cameras to vast public audiences (such as the police brutality exhibited during the 1968 'Chicago Police riot'), did not result in the kind of broad societal uneasiness and vociferous condemnation 
from many citizens that such behaviours would most certainly now attract. Doyle (2006a: 208) assists with his observation that today such events would be considered by a more educated public, which is more critical and skeptical of major institutions (including police and media) and who have the ability to access "alternative sources of knowledge besides the [traditional] media." Loftus (2010:2, citing Loader \& Mulcahy 2003) finds that "officers now deal with a less compliant and more demanding populace," while Reiner (2010: 253), in addressing police misconduct today, observes, "It is plausible that what appears to be a growing amount of police malpractice is largely just a greater likelihood of it coming to light. ${ }^{327}$ In concurring with the contributions of Doyle (2006a), Loftus (2010), and Reiner (2010) in this paragraph, I submit that contentious policing events that occur in 2019 are clearly situated not only in new technological, but also much different cultural, political, and social contexts than in the past - now scrutinized and evaluated by a more engaged and activist public with unprecedented access to information. I argue that the combination of this contemporary information landscape (enabled through 'policing's new visibility') and the entrenchment of more widespread societal skepticism (wariness, distrust, and/or suspicion) into our collective cultural consciousness are key factors in the declining trust in both the institution of policing and in the conduct of its front-line officers working in our communities. ${ }^{28}$

Perhaps the reader will, at this juncture in the discussion, be wondering about the proverbial 'elephant in the room' - that being the seminal event represented in the fatal police shooting of Michael Brown on 9 August 2014 in Ferguson, Missouri. In this regard, while Sunshine and Tyler (2003b: 515) observe that throughout American 
history "the relationship between the police and the public has been tumultuous," it is axiomatic to observe that the socio-political landscape for today's front-line police work across the USA (and other Western democracies, including Canada, the U.K., and Australia) was drastically altered as a result of the fallout from that occurrence, which many scholars and other observers suggest has been the catalyst for the current circumstances that constitute the public-police relationship in 2019 across many Western democracies (e.g., see R. Davis 2015; Deuchar, Crichlow \& Fallik 2019; Duechar et al. 2018; Fallik et al. 2018; MacDonald 2015, 2016; Silver \& Pickett 2015; Torres et al. 2018). Nix and colleagues (2018: 34) are representative of these observations, with their finding that the Ferguson incident sparked nationwide protests for weeks thereafter, massive media coverage, critical mediated commentary, "and renewed the historic debate concerning excessive police force against minority citizens" (Michael Brown was a Black male and Darren Wilson, the Ferguson Police officer, is a White male). ${ }^{29} 30$

The Ferguson occurrence came three weeks after the controversial death of Eric Garner during his interaction with NYPD officers, which was video recorded by bystanders, and what has followed, in the five years since, have been a tragic series of visible, contentious, and high-profile fatal police use of force interactions with primarily Black citizens in various locations across the USA (it is worth recalling that the Ferguson occurrence was, almost inexplicably, given the ubiquity today of video recording devices in urban environments, not documented in any footage). While controversial policing events are nothing new in the American experience (Antony \& Thomas 2010; Brucato 2015a; King 2013; Kutner 2016; Lawrence 2000; National 
Advisory Commission on Civil Disorders 1968; Nix \& Wolfe 2016, 2017; Reiss 1968; Stone et al. 2009; Sunshine \& Tyler 2003a, 2003b; Weitzer 2002, 2015), these 'postFerguson' occurrences have been reported across traditional media platforms with what many observe has been unprecedented volume, duration, scope, and intensity; and they have spawned street protests; episodes of civil disorder (e.g. rioting in Ferguson, Baltimore, and New York City); heightened scrutiny of officers' conduct in the field, by not only citizens but also by police oversight agencies and a relentless traditional and online media; and social movements demanding policing reforms (e.g. Black Lives Matter, Campaign Zero, Cop Block, Copwatch) (Bishopp, Piquero, Piquero \& Worrall 2018; Bishopp, Piquero, Worrall \& Piquero 2018; Braga et al. 2018; Sousa, Miethe \& Sakiyama 2017; Wilson 2015). ${ }^{3132}$

Many are characterizing the present circumstances in the American public-police relationship as nothing short of a 'crisis' (e.g., see Braga et al. 2018; Collins \& Klahm 2019; Fallik et al. 2018; Maguire et al. 2017; Morin et al. 2017; National Academies of Sciences, Engineering \& Medicine 2018; Nix et al. 2018; Todak 2017; Torres et al. 2018; Wallace et al. 2018; Weisburd \& Braga 2019; White \& Fradella 2016), which, I suggest, has been validated by the empaneling of no less than a full-blown presidential task force to address the prevailing issues - the President's Task Force on $21^{\text {st }}$ Century Policing initiated by President Obama. ${ }^{33}$ Weitzer (2015: 475, citing Pyrooz et al. 2016; Shjarback et al. 2017; Weitzer 2002) advances that the creation of this task force "signals to the public that recent incidents are much more serious than the sum of their parts" and he observes that similar to the contagion effect observed with pervasive social media discourse around police misconduct, 
A series of incidents that occur in a compressed time period and gain massive traction in the media can tarnish the image not only of the police in the cities where the incidents took place but can also damage the reputation of the police nationwide...This contamination-by-association is occurring today in a cumulative manner - with each incident pollinating subsequent ones - in part because activists and the media are drawing connections between them.

In this vein, Nix and Wolfe (2017: 100), consistent with the previous findings of

Loader and Sparks (2015), observe that the profound negative attention toward controversial policing occurrences in the USA "has transcended the specific jurisdictional boundaries where the events occurred and appear to represent a general undercurrent of discontent with U.S. policing. A similar trend has also been observed recently in countries outside of the U.S." Wolfe and Nix (2016: 2) also find,

[The] trend [toward nontrivial portions of the public wanting changes to their policing] is not idiosyncratic to the U.S. Similar undercurrents of discontent regarding police actions have resulted in wide-spread negative media attention and public protest in [London,] England in 2011 [e.g., the police shooting of Mark Duggan], [Queensland,] Australia in 2004 [e.g., the death of Mulrunji Doomadgee while in police custody], and [Tel Aviv,] Israel in 2015 [the video recorded police beating of Ethiopian-Jewish soldier Damas Pakada], to name a few.

In looking outside the American context, Loader (2016: 13) finds that police in Wales and England today are working "in a climate in which the practices, performance, and legitimation claims of the police are routinely subject to external scrutiny and public contestation: from politicians, journalists, civil society groups, and across social media" and Murray and Harkin (2017) find a similar situation prevailing across Scottish policing. In Canada, Murphy (2012), a policing scholar at Dalhousie University, observes that the Canadian public is now more negative toward police than ever before and he finds that today Canadians are making increasingly loud demands for greater accountability from the police in how they do their work. In the same vein, 
Toronto Star columnist Rosie DiManno (2017) observes, "With the confluence of so many recent events involving on-duty and off-duty cops, the crisis of confidence in policing has become acute. But it's no longer just a handful of activists and journalists decrying police delinquency and monkey-business. The public is demanding: What the hell?" ${ }^{34}$ Campeau (2016a :2), in her study of Canadian front-line police subculture, finds that recent controversies in, and unprecedented public attention to, police actions in the field are "symptomatic of a broader shift toward what we might call an 'unsettled moment' in the policing landscape."

It should be apparent (even without the benefit, as yet, of reviewing the present study's data or the previous research findings of some other scholars) that the current socio-political landscape would almost certainly have some impact on the attitudes and behaviours of some of today's front-line officers. It is well established across the literature that officers' perceptions about any number of factors can have a significant effect on officer morale and how officers do their work, including, in this context, public sentiment toward police and media's characterization of police, (e.g., see Association of Chief Police Officers 2008; Berry 2010; Bradford \& Quinton 2014; Byers 2014c; Deuchar et al. 2018; Heaton 2010; MacDonald 2015; Marier \& Moule 2018; Nix \& Pickett 2017; Pickett \& Ryon; Skolnick 2011). For example, Todak (2017: 251) finds that today's front-line officers experience considerable anxiety around "having one's mistakes made public" given the prevalence of public video recording and online dissemination of police actions in the field, which can engender significant scrutiny and critique across the public sphere. 
Specifically, in relation to this post-Ferguson period, Nix and Wolfe (2018: 263, citing Pyrooz et al. 2016; Campbell et al. 2018) note that "scholars have since referred to [the aftermath of] Ferguson as an 'exogenous shock' or 'environmental jolt' to law enforcement.” In this vein, Torres and colleagues (2018: 358, citing Nix et al. 2018) find that "officers have perceptually bought into the idea that negative publicity [has] brought on a 'war on cops'." Similarly, Nix and Pickett (2017: 25, citing Campbell et al. 2017; Meyer 1982) conclude, "Ferguson undoubtedly was an environmental jolt to policing...a sudden and unprecedented event with an impact that is disruptive and potentially inimical ...The scrutiny has been so extreme that some contend there is currently a 'war on cops'...In response, it is argued that officers are de-policing (i.e. avoiding proactive stops)." As a 'real life' demonstration of the sentiment being expressed by some of today's officers in response to their present techno-social and socio-political policing landscape, in an open letter published on 18 September 2015, Patrick Lynch, the president of the labour organization representing 24,000 rank-andfile NYPD officers, wrote,

To all armchair judges: If you have never struggled with someone who is resisting arrest or who pulled a gun or knife on you when you approached them for breaking a law, then you are not qualified to judge the actions of police officers putting themselves in harm's way for the public good... No one should ever jump to an uninformed conclusion based upon a few seconds of video (Speiser 2015).

\subsection{Establishing a Theoretical Framework}

Campeau (2015: 672, citing Manning 2005c) observes that, "police studies [have] been criticized for investigating a plethora of topics without theorizing these." This will not be the case with the present study and, in this regard, unlike the complexities involved in arriving at an empirical understanding of the phenomenon of de-policing 
and investigating this practice within the front-line police subculture (with all of the attendant challenges in securing honest and reliable data from rank-and-file officers - as discussed throughout Chapter Four and Chapter Six), I found arriving at a sociological theory that is well suited to inform this study's findings was actually a relatively straightforward matter. In fact, once I got immersed in data analysis, it became apparent that police occupational culture, or, more accurately and specifically, as I will explain shortly, the front-line subculture, not only provides the ideal theoretical framework to develop a comprehensive and nuanced understanding of de-policing, but also presents a classic sociological perspective, as was the case with many of the pioneering studies of police behaviour - such as, for example, the influential and, as some might argue, unresolved, micro-sociological works of Reuss-Ianni (1983) on the 'street cop' culture, Ericson's (1982) examination of the officer's 'recipe of rules,' and Skolnick's (1966) revelations about the officer's 'working personality., ${ }^{35}$ As Waddington (1999: 287) finds, "The notion that the police possess a distinctive occupational subculture lies at the centre of much research and theorizing about policing and police work" and, in this vein, Reiner (2010) assesses that police culture, as a theoretical framework, developed as a means of understanding police practices and as a way to critique officers' behaviour and attitudes. Manning (1995: 472) defines occupational culture as "a reduced, selective, and task-based version of culture that is shaped by and shapes the socially relevant worlds of the occupation." In this vein, Schein (2010), the eminent cultural theorist, finds that, just as aspects of our complex personal makeups influence our actions and constrain our behaviour, culture can also both guide and inhibit behaviours of cultural group members. 
Along these lines, Sklansky (2007: 23) assessed that Westley $(1953,1970)$, in his ground-breaking investigation of police conduct in Gary, Indiana in the early 1950s, found, "The key to understanding the [behaviour of] police was to understand their shared mentality - their subculture. [And it was] this set of premises [that] became the central motif of police studies in the 1960s and 1970s." In this regard, police cultural theory functioned (as it is intended to in the present study) as "a heuristic...a tool for thinking about [and producing a better understanding] of an empirically-identified phenomenon" (Scollon, Scollon \& Jones 2011: 3). More recently, Loftus (2009: 18, citing Chan 1997) advanced that the key to understanding contemporary policing "is to appreciate the influence of police culture and the wider context in which it takes place" and, in this vein, it can be said that occupational culture is a genre of sociological theory with a rich tradition in informing the empirical study of police officers' conduct in the field. Bacon (2014: 103) observes, "Research and reflection have consistently recognized the significant role that the 'occupational culture' of the police plays in shaping their decisions and actions." In fact, Newburn and Reiner (2012: 811) find, "The impact of the informal culture of the rank-and-file is the most common explanation of police working practices found in the research literature."

Workman-Stark (2017, citing Crank 1998; Skolnick 1994) suggests that police culture is most often understood as a set of beliefs shared by police officers that develop as an adaptation to 'unfriendly' (or hostile) working conditions, which are reinforced through processes of socialization and group solidarity. Cordner (2017: 11, citing Cockcroft 2013; Crank 2014) finds that the two interrelated considerations of police socialization and police culture "have continued to interest contemporary scholars. 
Modern theories of police behaviour tend to assign great significance to the police culture, critics blame it for rationalizing police misconduct and deviance, and reformers regard it as a major obstacle to be overcome in pursuit of police transparency and accountability." Along these lines, Terrill, Paoline and Gau (2016: 65) advance that "another piece of the puzzle in understanding officer-citizen relations is police culture." Reiner (2017: 236-237), who explains that he has been involved with police culture as a theoretical framework since beginning his $\mathrm{PhD}$ in 1969, observes,

The idea of police culture has become a pivotal element in both academic and policy discussion of policing...[Developing from the previous] era in which 'the law' was often used as a synonym for police, [researchers came to recognize that] law was at best one factor shaping police practice, alongside a variety of social, political, and economic processes that was the project of empirical research to analyse. In the array of possible explanations of police conduct and misconduct, the ideas and perspectives the world view - of rank-and-file police officers on the ground was widely seen as pivotal. This is the germ of the idea of police culture...

Of course, throughout the decision-making process during the writing of this dissertation, my background within the police rank-and-file subculture and familiarly with much of the police occupational culture literature informed my deliberations on potentially-applicable theories as I engaged with the present study's data and results, and then as I considered what to focus on in discussing study findings. ${ }^{36}$ This progression led me to conclude that the de-policing phenomenon, identified through this study's data as commonplace among front-line officers, can be best considered, on a theoretical plane, as a subcultural adaptation to the techno-social and socio-political challenges that are presented across today's policing landscape and experienced, firsthand, by the rank-and-file as they perform their police work in the community (more 
extensive discussion of the specific relationship between this study's findings and today's front-line police subculture is forthcoming in Chapter Six).

In another dimension related to theory, the reflections of C. Wright Mills (1959) contributed to the broad theoretical orientation of this research project; particularly his observation that among the most compelling aspects of the 'sociological imagination' is the linking of 'personal troubles' with public issues, through the lenses of social and political theory. Lyon (2006) lauds Mills' approach in the linking of individual concerns with broader sociological issues, which Lyon endorses as consistent with the traditions of the best social and political theories. In a similar vein, Burawoy (2012: $\mathrm{x}$ ) advances that what is required, in addition to a 'sociological imagination,' is the "'political imagination' to turn personal troubles into public issues." Mills' thoughts on the linking of 'personal troubles' with contemporaneous socio-political issues has dual applicability in this study.

First, the recognition of the significance of de-policing came to my attention as a 'personal trouble' (quite literally), through my own experiences performing police work in the field and interacting within the rank-and-file subculture - as a front-line officer confronting the same risk considerations in today's policing landscape that many study participants identified and have responded to through the practice of de-policing (as I, myself, did on a number of occasions). Also, while the vast majority of individuals across Western democracies are never involved (as an active participant) in a controversial interaction with a front-line officer, the conduct of officers in those situations transcends the interests of those directly involved in such 'personal troubles' - with broad implications for both of the constituencies participating in perhaps the 
most important socio-political arrangement in advanced societies - the policed and the police (as discussed throughout Chapter One). Along these lines, Lyon (in a lecture I attended at the University of Victoria on 7 June 2013) implored sociologists to think in "more socio-cultural terms" about the implications of various contemporary phenomena - such as, for example, today's widespread societal surveillance and 'new visibility.' Similarly, McLaughlin (2007: ix) encourages, "it has never been more important to forge a critical police studies that is capable of conceptualizing policing developments against socio-cultural, economic and political transformations.” This study has been guided by such sage advice.

In reflecting my philosophical orientation around the role of theory within empirical sociological studies, I advance that Garland (2004: 163) presented a particularly well-phrased contribution in addressing "the place and purpose of theory," with his finding that "theory and research achieve maximum synergy when a focused empirical inquiry throws light upon a more abiding matter of philosophical or sociological importance." Similarly, I concur with the guidance of Rigakos and Frauley (2011: 252), who advance, "Explanation is necessarily theoretical, and theoretical work is necessary for social scientific inquiry...theoretical elaboration is tethered to an ongoing concern with the thing to be described and explained." I also subscribe to the practice advanced by Castells (2011: 6), who counsels, "I use theories, any theory, in the same way that I hope my theory will be used by anyone; as a toolbox to understand social reality. So I use what I find useful and I do not consider what is not directly related to the purpose of my investigation." Haggerty and Ericson (2000: 608, citing Deleuze \& Foucault 1977), in endorsing the practices of scholars in this regard 
(including such sociological luminaries as Deleuze, Guattari, and Foucault), argued, "Our approach is entirely in keeping with their philosophy...to approach theory not as something to genuflect before, but as a tool kit from which to draw selectively in light of the analytical task at hand." Finally, I find considerable merit in the observations of Goold (2004: 211-212), who finds that "given how much has been written in recent years about [developments such as societal surveillance, technology-enabled increased visibility, insecurity, etc.], there is a danger that such issues have been largely removed from their real-life contexts and have become over-theorized" and he suggests, for example, that "unless we make more of an effort to understand how changes in the technology of surveillance actually affect institutions like the police, the theoretical literature will become increasingly divorced from reality."

Cordner (2017: 14, citing Crank 1998) observes that the wide-ranging theoretical apparatus of police culture "covers a lot of intellectual and emotional territory" and, in terms of the outcomes of this occupational peculiarity, it has been found to be "carried in the minds of street cops who work together" and it is "often reflected and reinforced through customs, traditions, and a variety of informal and formal practices." 37

Throughout the abundant literature on the topic, it is often advanced that because of the precarious role of the public police in democratic societies - being simultaneously mandated to enforce social control across the population, yet being constrained in the exercise of how that is to be accomplished - and the distinctive challenges inherent to police work (i.e. danger, the exercise of authority, and conflict) police officers operate in an environment of uncertainty, risk, and strain, which spawns a reactionary, insular, and protective occupational culture (Blake 1981; Goldsmith 1990; Greene, Bergman \& 
McLaughlin 1994; Kappeler, Sluder \& Alpert 2010; Manning \& Van Maanen 1978;

Reiner 2010, 2015; Terrill, Paoline \& Manning 2003). ${ }^{38} 39$ As articulated by Murphy

and McKenna (2007: 5, citing Van Maanen 1984),

Police culture is explained as a functional, even necessary cultural response to the broad, complex and uncertain nature of doing police work; especially managing the discretionary exercise of coercive police powers in uncertain and potentially risky situations...Culture in this sense is understood as a set of social solutions devised by a group of people to understand and respond to a common set of real and perceived problems.

Paoline (2003: 200) defines police occupational culture as "the accepted practices and underlying attitudes and values that construct and transmit norms of how to be a police officer and how to do policing." Along the same lines, O'Neill and Singh (2007: 2) find, "Police occupational culture can best be considered as the "way things are done around here' for the officers, not always 'by the book,' but not always without it either. Police have socially constructed ways of viewing the world, their place in it, and the appropriate action to take in their jobs." In amalgamating these and various other scholar's definitions of police culture, which Punch (2007: 107) finds "is more of a complex matrix than an easily defined, one-dimensional concept" and, similarly, which Campeau (2015: 671) observes is "meant to encompass a complex system of values and attitudes that define the normative social world of police," I argue that police occupational culture can be best understood as the shared values and attitudes, common understandings, agreed upon informal 'craft rules,' and principles of conduct that are situationally applied and that inform officers' actions in the field.

In what follows in this subchapter, I present an overview explaining the most salient aspects of police occupational culture theory, in relation to its applicability in informing the present study of front-line officers' risk-averse behavioural adaptations 
their contemporary external policing environment. In this regard, Reiner (1985: 85) observes, "An understanding of how police officers see the social world and their role in it - 'cop culture' - is crucial to an analysis of what they do and their broad political function." This presentation is facilitated through discussions focused on the following sub-themes, which I argue are subsumed within the overarching theoretical construct that is police occupational culture: (1) the 'traditional' conception of police culture; (2) more contemporary conceptions of police culture; (3) police culture or subcultures; (4) rank-and-file officer typologies; and (5) front-line police subcultural socialization. This material allows the reader to consider the various facets of the study's underlying theoretical framework as they advance through the discussion presented in Chapter Six.

\section{The 'Traditional' Conception of Police Culture}

As Waddington (1999: 288, citing Manning 1989) observes, "In common with general sociological and anthropological definitions of culture, the [traditional police culture] refers to the accepted practices, rules, and principles of conduct that are situationally applied, and generalized rationales and beliefs." In this regard, Holdaway (1983: 2) finds that "a residual core of beliefs and values, of associated strategies and tactics relevant to policing, remains a principal guide for the day-to-day work of the rank-and-file officer." This occupational culture implicates "a layer of informal occupational norms and values operating under the apparently rigid hierarchical structure of police organizations" (Chan 1997: 43). As articulated by Paoline and Terrill (2014: 6, citing Van Maanen 1974), it is important to understand that a key theoretical assumption advanced by proponents of the 'monolithic' police occupational culture perspective holds that, 
[Police] culture originates and is maintained and transmitted by lower level participants during their time on patrol. It is assumed that because all police start at this introductory level that this is where culture takes place. Once officers leave patrol they still carry some degree of their cultural values with them, but the heart of this perspective focuses on the streetlevel police officer. Moreover, culture is viewed as less of a function of what you bring to the profession, in terms of your background, orientations, and demographics, and much more about how the police occupation itself shapes you.

The core referents of this traditional concept of the singular police occupational culture, as they were most famously enumerated by Reiner (1985) and since, broadly adopted across the literature, can be considered to encompass the following generalized descriptors of the prototypical police officer: cynical; suspicious; distrusting and stereotyping of outsiders; pessimistic; secretive; conservative; authoritarian; masculine; sexist, macho; socially isolated (outside of policing); prejudiced; action oriented; pragmatic; emphasizing physical prowess; presenting an 'us versus them' mentality; believing policing is a mission; prioritizing group solidarity; and valuing loyalty (e.g., see Bittner 1970, 1974; Manning 1977, 1989, 1995, 1997, 2007; Parnaby \& Leyden 2011; Punch 2007, 2009; Reiner 1992b, 2010, 2015; Skogan \& Frydl 2004; Worden 1995b; Young 1991). ${ }^{40}$ As such, police culture is often characterized pejoratively - as both a contributing factor in, and a shield against the exposure of, problematic conduct by officers (brutality, corruption, racism, discriminatory practices, etc.) (e.g., see Brown 1988; Chan et al. 2003; Foster 2003; Paoline 2004; Reiss 1971; Rubinstein 1973; Terrill et al. 2003). In this regard, Chan (1996: 110) found, "Police culture has become a convenient label for a range of negative values, attitudes and practice norms among police officers." 
It has been theorized that within this distinctive occupation, police officers, through a unique process of socialization involving shared experiences with other officers, are encouraged to internalize and adhere to certain 'traditional' philosophical orientations and in-the-field practices of their immediate peer group - their occupational culture ${ }^{41}$ The pressure to conform to these cultural expectations is exacerbated given the importance of loyalty and trustworthiness within the rank-and-file subculture and, as will be discussed in detail within Chapter Six, the career-stage progression of the officer - from the newly-hired recruit attending the police academy to the veteran front-line officer in pre-retirement mode - is relevant to the argument I advance that this socialization (acculturation or perhaps indoctrination) process implicates the accumulation of both, in-the-field experiences, and ongoing and frequent interactions among peers within the close-knit work unit (the 'squad', 'platoon,' etc.). As Worden (1995b: 50) observes, within the overarching culture, the individual officer's belief system is "comprised of common beliefs, attitudes, values, and other subjective outlooks" and Terrill and colleagues (2016: 65, citing Paoline 2003) suggest that this commonality within "the primary work environments [is thought to] produce collective coping mechanisms and outcomes.” In this regard, Skolnick (2008: 35) argues that "being a police officer is a defining identity."

Today, scholars focused on police occupational culture are divided around the applicability of 'traditional' deterministic representations of universal cultural values and standardized behaviours across the population of police officers. Sklansky (2007: 20) has observed that this narrow understanding of police culture has resulted in "cognitive burn-in" among some researchers (applying the analogy of the permanent 
impression left on the screen of older televisions if an image was paused for an extraordinary length of time). Along the same lines, Campeau (2015: 671) finds, "The conceptual template of Reiner's ['ideal type'] list now serves as a collective imprint in how we think about the topic and this diverts our attention from new directions and emerging trends." This 'traditional' genre of police culture scholarship "largely downplayed the scope for variation in police behaviour and orientation" (Cockcroft 2013: 79), despite, Reiner (2010: 116), himself, conceding that "police culture - like any other culture - is not monolithic, and is embodied in individuals who enjoy autonomy and creativity." ${ }^{42}$

\section{More Contemporary Conceptions of Police Culture}

Some scholars, despite acknowledging that police occupational culture has changed in some respects over time - certainly since the classic sociological studies of the 1960s and 1970s - find that there remains a solid core from the 'traditional' understandings and fundamental elements of this culture (e.g., see Chan 1996, 1997; Foster 2003; Loftus 2008, 2009, 2010; Myhill \& Bradford 2013; Paoline 2003; Sklansky 2007; Van Hulst 2013). For example, Foster (2003: 208) relates, "Despite the dangers of generalizing about police culture (as a singular and homogenous entity), police researchers...often find it difficult to move away from the general (and seemingly all powerful) characteristics of the dominant police culture, in part because these elements appear to be common for most, if not all, officers."

That being said, other policing researchers have challenged what they contend are overly simplistic conceptions of police culture and the sociological orthodoxy of those contributions, with their assumptions of an immutable 'traditional' police culture 
operating within an overarching and universally-accepted informal occupational ethos ideas that are now critiqued as cliché (e.g., see Campeau 2015; Chan 2007a, 2007b; Christenson \& Crank 2001; Cockcroft 2013; Cordner 2017; Ingram, Paoline \& Terrill 2013; Kapeller, Sluder \& Alpert 2005; O’Neill \& Singh 2007; Reiner 2017; Terrill et al. 2003; Terrill et al. 2016). Along these lines, Campeau (2017) assesses, "Perhaps the most confounding message about police culture is found in academic literature, which reveals a seemingly contradictory conclusion that it has both changed and remained the same."

Terrill and colleagues (2016: 65) find, in contrast to "characterizations of police officers immersed in a monolithic police culture, there is also a body of competing research illustrating the diversity of coping mechanisms officers employ" and this research has "uncovered substantial diversity among officers' attitudes and role orientations" (e.g., see Campeau 2015; Chan 1996, 1997; 2007b; Chan et al. 2003; Cochran \& Bromley 2003; Jermier, Slocum, Fry \& Gaines 1991; Paoline 2001, 2004; Paoline et al. 2000). For example, Campeau (2015: 669) observes that some scholars today conceive of the theoretical construct of police occupational culture, in its current socio-political context, as "a resourceful tool on which [officers] rely to make sense of situations they navigate in everyday life" rather than the more deterministic 'traditional' interpretations. Loftus $(2009,2010)$ observes that research on police has often acknowledged the important role that the informal occupational norms, values, and assumptions associated with the rank-and-file play in shaping these officers' everyday decisions and behaviours and, in advocating for new research initiatives to determine 
how today's officers experience their new realities in a transformed policing landscape, she found,

Contemporary understandings of police culture have been shaped by the findings of classic ethnographies...that reflect police environments and culture of an earlier and different social, economic and political context. Many of the studies were conducted over twenty years ago...[and] we are left, therefore, with an account of police culture which largely predates many of the significant changes which have since taken place...changes in the internal and external policing landscape have altered the character of the differing 'publics' that the police come into contact with. The classic 'police culture' paradigm which has been much invoked to describe and explain a range of police attributes is by now somewhat exhausted, and new lines of research and reflection are needed to track the shifts in the wider field of policing (Loftus 2007: 184).

Similarly, Cockcroft (2007: 91) counsels, “The pitfalls of investigating police culture without taking into account socio-political factors leads to a reading of police work that situates it in a sociological cul-de-sac devoid of sufficiently broad cultural appreciation - wider culture does not simply 'end' at the front door of the police station." 43 In this vein, Workman-Stark (2017: 30) assesses that, "in recent years there is a growing consensus that police culture is in transition, although just how far it has come is still debated. Certainly, social media and the increased political sensitivities around policing have influenced the working practices of police officers." Paoline (2004), in presenting his thoughts on the future direction of police culture research, relied on the earlier work of Fielding (1988b: 185), who stated, "If occupational culture is to serve as an empirically satisfactory concept as well as a theoretically necessary one, the sense of its internal variations and textures must be brought out..." On this point, Reiner (2017: 238-239) advances,

As Weber stressed, an ideal type is a purely conceptual model for illuminating reality but is never or hardly ever actually embodied in particular people. Partly this is because of detailed contextual differences, 
but fundamentally it is because of human subjectivity and a degree of autonomy in interpretation and action. Officers bring different personalities and initial orientations to situations; although, the structural weight of the problems they face then tends to shape some commonalities in response.

Reiner's (2010: 116) scholarship is also instructive for this dissertation's understandings around the theoretical construct of front-line police subculture - including his finding, "Cultures are complex ensembles of values, attitudes, symbols, rules, recipes, and practices, emerging as people react to the exigencies and situations they confront, interpreted through the cognitive frames and orientations they bring with them from prior experiences. Cultures are shaped, but not determined, by the structural pressures of actors' environments."

\section{Culture or Subculture?}

Paoline (2004: 205) observes, "Research on police culture has generally fallen within one of two competing camps - one that depicts culture as an occupational phenomenon that encompasses all police officers and one that focuses on officer differences. ${ }^{44}$ The latter conceptualization of police culture suggests subcultures (or at least segmentation) that bound or delimit the occupational culture.” Bacon (2014: 105, citing Geertz 1973; Goodenough 1976; Taylor 1981) finds that "the term 'culture' is usually reserved for large, ordered social systems...whereas 'subculture' is used to refer to the smaller micro-cultures that exist within the macro-culture." In this regard, ReussIanni's (1983) seminal Two Cultures of Policing: Street Cops and Management Cops disaggregated the 'traditional' conception of a single and unifying police culture, irrespective of rank and/or role within the police organizational structure, into a more nuanced understanding around the existence of multiple and discrete police subcultures. Since, this finding has been corroborated by many scholars (e.g., see Boivin, Faubert, 
Gendron \& Poulin 2018; Ericson 2007b; Glomseth \& Gottschalk 2009; Loftus, Goold \& MacGiollabhui 2016; Manning 1995, 1997, 2005, 2007c, 2014; Punch 2007; ReussIanni \& Ianni 2005). ${ }^{45}$ This accords with my understanding, based on experiences throughout my own policing career and observations in the field during police research projects.

In this regard, it seems clear (at least to me) that there exists a broad and overarching police occupational culture, with a limited number of fundamental tenets regardless of role or rank within the structure of the police organization (from the new recruit in training at the police academy to the Chief of Police) - for example, a belief in the importance of law and order in society and in the imperative to back-up a fellow officer in need of assistance in dangerous circumstances. Along these lines, Cordner (2017: 12) finds, "Certainly there is some sort of overlay of police occupational culture that exerts a degree of influence on police thinking and behaviour everywhere." However, while concurring with his observation, I argue that there are also significant variations in many of the values, attitudes, understandings, assumptions, informal 'craft' rules, and principles of conduct based on the specific type of work an officer performs within policing (Ericson 2007). As Kappeler and colleagues (2010: 269) find, "Members of a subculture, while sharing many values and beliefs of the larger culture, also have a separate and distinct set of values that set them apart." In my estimation, there exist at least four discrete subcultures within policing, as the institution is typically constituted today - front-line (uniform officers performing primary response and patrol work in the community), investigative (plain-clothes detectives), administrative, and command. This adds one subculture to earlier identifications of three - command, 
middle management, and lower echelons (Chan 1996, 1997; Manning 1993); however others have also previously identified that detectives present as a distinctive subculture (e.g., see Ericson 1981, 1993; Hobbs 1988; Innes 2003; Waddington 1999; Young 1991). ${ }^{46}$ In addition, I advance that Reiner (2010: 116) lends support to my position, with his observation, “There are particular variants - 'subcultures' - that can be discerned within broader police culture, generated by distinct experiences associated with specific structural positions (ranks, specialisms, areas, etc.)."

\section{Rank-and-File Officer Typologies}

Also, in contrast to the notion of a singular police occupational culture within the 'traditional' characterization, other research has advanced that understanding officer typologies forms the basis for a more accurate representation of policing. This approach advances that officers practice certain diverse, yet identifiable, styles of performing their police work as related to individual variations in attitudes -including those officers whose attitudes do not conform to 'traditional' conceptions of police culture (e.g., see Broderick 1977; Brown 1988; Engel \& Worden 2003; Muir 1977; Paoline 2001, 2004;

Paoline et al. 2000; White 1972; Wilson 1968; Worden 1989, 1995b). Paoline (2004:

207) provides illuminating analysis on this point,

...Descriptions of a 'single' [or 'traditional'] police culture have focused on the widely shared attitudes, values, and norms that serve to manage strains created by the nature of police work (i.e., the occupational street environment)...Although research on the police culture has stressed the central tendencies among officers, other research (i.e., typologies) notes variation...[While] all typology studies identify a group of officers that carry many of the outlooks of the traditional conceptualization of police culture...these studies also identify contrasting groups that do not equally share many of the attitudes, values, and norms commonly associated with the police culture. Typology studies of police contrast the notion that all officers see the world through the same lens and suggest that officers might cope with the strains of their occupation differently. 
Wilson (1968), in his comparative study examining varieties of behaviour at eight American police departments in the early 1960s (Wilson's data sites included Albany and Syracuse, New York, as did the present study), found three distinct philosophies across front-line police work (at the generalized organizational level but also found to influence the individual officer) - the 'watchman,' the 'legalistic,' and the 'service' styles. In Muir's (1977: 292) discussion of Wilson's study, he observed that "sometimes whole departments institutionalized [a] pattern of avoidance" and that 'watchman' officers' policing practices involved "ignoring infractions, tolerating deliberately illegal acts, expecting less orderly conduct from certain groups, and steering clear of private disputes." In his own work, Muir (1977: 144-145) assessed whether differences in American officers' attitudes led to differences in their behaviour (manifested in their styles of police work) and he found the existence of four distinct officer typologies related to "reactions to the paradoxes of power recurring in street situations" - the 'avoider,' the 'reciprocator,' the 'enforcer,' and the 'professional.' Similarly, Reiner (1978) determined six rank-and-file archetypes within policing across the UK - the 'Bobby,' the 'new centurion,' the 'social worker,' the 'Federation activist,' the 'professional,' and the 'uniform carrier.'

Worden's (1995b: 49-50) analysis of typology studies found that 'conventional wisdom' (both academic and popular), which advanced that police officers maintain a distinctive belief system within a homogenous occupational culture, no longer conformed to the 'attitudinal heterogeneity' discovered in more recent studies. Worden (1995b) identified five types of front-line officers, based on their attitudinal orientations - 'tough-cops,' 'clean-beat crime-fighters,' 'avoiders,' 'problem-solvers,' and 
'professionals.' Of particular relevance to the present study, Worden (1995b: 60, citing

Van Maanen 1974) found that 'avoiders,'

Have a cynical perspective and a conflicted morality...Unable to cope with the characteristic exigencies of police work, they excuse themselves from their duties, subscribing to very narrow conceptions of the police role. They prefer to do as little police work as possible, only that amount of work necessary to meet the minimum expectations of supervisors; otherwise, they adopt what has elsewhere been called a 'lay-low-and-don't-make-waves' approach to policing.

Paoline (2004: 212, citing Reiner 1978), in describing Worden's (1995b) identified typologies, observed, “As the label implies, 'avoiders' are just doing their time, avoiding as much police work as possible... Whereas 'tough- cops' and 'clean-beat crime-fighters' have a mission (i.e., law enforcement), the 'avoiders" mission is to do just enough to wear the uniform." In their research within American policing, Mastrofski and colleagues (2002: 106) discerned four distinct types of rank-and-file officers - 'professionals,' 'tough cops,' 'reactors,' and 'avoiders', with the latter two typologies comprising $40 \%$ of the research population. Relevant to this dissertation's findings in relation to de-policing, 'reactors' were found to prefer reactive rather than proactive work and were selective with any efforts at the latter type of interaction with citizens, while 'avoiders' were found to be "reluctant to engage in any kind of encounter" (Mastrofski et al. 2002: 102).

In his American research, Paoline (2004: 218-220) identified seven "analytically distinct groups of officers" based on their attitudinal similarities and differences and he argued that "officers are responding to and coping with aspects of their occupational world in different ways" and that these findings "call into question some of the assumptions associated with a monolithic police culture.” Paoline's (2004) identified 
officer types are the 'lay-lows,' 'old-pros,' 'traditionalists,' 'anti-organizational streetcops,' 'Dirty Harry enforcers,' 'peacekeepers,' and 'law enforcers.' Similar to Worden's (1995b) 'avoiders,' Paoline's (2004: 224) ‘lay-low's,' “have a narrow role orientation that focuses on traditional unavoidable law enforcement functions, rejecting other roles that usually entail more ambiguous and time-consuming functions (i.e., order maintenance and community policing)." Finally, and highly relevant to the present study, Paoline (2004: 231) urged, "Future research should continue to explore variation among officers' cultural outlooks. Such research should work toward identifying and examining additional attitudinal dimensions from the ones examined here, in finding out more about police officers' views of their occupational and organizational environments."

\section{Front-Line Police Subcultural Socialization}

The existence of a discrete police occupational culture (in any of the various iterations presented across the literature - including the front-line subculture), by its very definition, implies that officers hold values, attitudes, beliefs, understandings, and assumptions that distinguish them from other citizens (the policed) within the same society. As social beings, we (including police officers) all inhabit and interact within a socially-constructed world and we construct our identities in relation to groups with which we collaborate. In this vein, much has been written about the 'us versus them' division in officers' socio-political configurations (e.g., see Brough, Chataway \& Biggs, 2016; Cockcroft 2007, 2013; Paoline 2003; Reiner 2010; Van Maanen 1973, 1974, 1975, 1978, 1988; Westmarland 2008). ${ }^{47}$ Such mindsets among rank-and-file officers have been most famously articulated in the seminal scholarship of Van Maanen and 
Skolnick, who, in researching the external occupational (versus the internal organizational) policing landscape, made discoveries that included the front-line officer's 'working personality', the perceived risk presented to these officers by various 'symbolic assailants' within the community (Skolnick 1966), and the classification of the entire 'civilian population' into only three types of citizens' - 'assholes,' 'suspicious persons' and 'know nothings' (Van Maanen 1974, 1978). Along these lines, Campeau (2015: 669) observes,

Within police studies...the 'police culture' concept is dually invoked to describe both an overarching occupational ethos (e.g. cop code of silence, 'us versus them' mentality, and the thin blue line) and individual officer typologies (e.g. enforcers, optimists, and DirtyHarrys). At either level, culture is often depicted according to a series of police values or attitudes acquired through on-the-job socialization and is said to play a vital role in predictably explaining officer behaviour.

As Campeau $(2015,2017)$ has suggested, scholars discussing how enduring tenets of police occupational culture are revealed, transmitted, encouraged, acculturated, and largely preserved over time (with relatively minor and periodic modifications in response to transformations in the policing environment) often advance the concept of a socialization or indoctrination process, through which officers are influenced to participate in prevailing police cultural doctrine and to adopt the norms of their particular subcultural (or typological) variant (i.e. front-line police subculture) (e.g., see Bacon 2014; Chappell \& Lanza-Kaduce 2010; Loftus 2009, 2010; Oberfield 2012; Paoline 2003; Shearing \& Ericson 1991; Stoddard 2006). ${ }^{48}$ Most notable is Van Maanen, who wrote extensively on the phenomenon of 'police socialization' and analyzed officer behaviours that developed as a result of this acculturation process - in what are widely-acknowledged as classic sociological texts (e.g., see Manning \& Van 
Maanen 1978; Van Maanen 1973, 1974, 1975, 1978, 1984). Also, in this vein, Ellis (1991: 95) argues, "Like all members of distinctive and social professional groups, police undergo a process of 'socialization,' which is the inculcation of values, attitudes, expectations and role requirements thought necessary for appropriate or effective behaviour as a full-fledged group member.” In this regard, Charman (2017: 93) observes, "Where levels of discretion and autonomy are high and where informal adaptations to formal rules are an occupational characteristic (both seen particularly within policing), the influence of that socialization can be even more apparent." Murphy and McKenna (2007: 8) find,

Police officers learn the culture or are socialized into its collective values and understandings, through informal group interaction, both on and off the job. To become a police officer means learning and internalizing the culture and adhering to its score values and rules. Acceptance by one's peers as being trustworthy and reliable often depends on being aware, and accepting, this culture or code and subscribing to its various behavioural and attitudinal tenets.

Kappeler and colleagues (2010: 268, citing Skolnick 1994) address this process of socialization, as related to police behaviours, within what they categorize as the 'sociological paradigm' - in which "police learn how to behave and what to think from their shared experiences with other police officers." This is in contrast to the 'psychological paradigm' that advances the 'predisposition model' of behaviour - in which it is argued that those attracted to careers in policing already possess certain traditionally-valued attributes even before joining a police department (as discussed in more detail within this chapter's endnote 27). Haarr (2005: 434, citing Fielding \& Fielding 1987) also discusses 'the sociological conception of occupational socialization' as applied to policing, which "places emphasis on the formal and 
informal processes of socializing [police] recruits to the fundamental features of the occupational culture, including team membership, acceptance of particular values and beliefs, stereotypical thinking, trust, forming of relationships through joke telling, and the manner of dealing with racial/ethnic minorities and other members of the public." Along these lines, I concur with scholars' observations (e.g., see Chan et al. 2003; Cooper-Thomas \& Anderson 2002; Haarr 2001, 2005; MacAlister 2004; Obst \& Davey 2003; Rose \& Unnithan 2015; Sato 2003; Van Maanen 1973) that the socialization process begins with the hiring of the applicant by a police agency, which Van Maanen $(1974,1975)$ and Van Maanen and Schein (1979) characterize as 'anticipatory socialization', and then intensifies with entry into the agency's training program - the police academy. ${ }^{49}$

Bacon (2014: 110, citing Cain 1973; Innes 2003) observes that the 'apprenticeship' of new recruits involves "relaying and transferring the tacit and informal knowledge...of how to get the job done in 'the real world', and the recipe knowledge, working rules, and attitudes that will facilitate this." Van Maanen (1973: 411) finds that, within the acculturation environment of the police academy, the recruit "begins to absorb the [prevailing] subcultural ethos" as conveyed incessantly from those in positions of power (veteran officers serving as not only instructors but also highlyinfluential 'role models' in an imbalanced socio-political relationship) and begins to “think like a policeman." More specifically, Chappell and Lanza-Kaduce (2010: 200, citing Albuquerque \& Paes-Machado 2004; Kraska \& Cubellis 1997) find that the paramilitary training regimen of the police academy fosters the indoctrination of the well-documented 'us versus them' mentality. ${ }^{50}$ This occupational culture socialization 
continues after graduation from the police academy and is, in fact, intensified with deployment in the field under the guidance of a 'coach officer' (sometimes referred to as a 'field training officer' ['FTO'] in the USA or a 'tutor constable' in the UK) and frequent interactions with experienced rank-and-file officers in formal and informal social settings (Bennett 1984; Department of Justice 2003; Engelson 1999; Fielding 1988; Ford 2003; Getty et al. 2016; Haberfeld 2013; Sun 2002; Van Maanen 1973, 1974). As Getty and colleagues (2016: 6-7, citing Caldero and Crank 2011) find, "The FTO's influence is so important that new officers are going to role model the FTO's actions down to the last doughnut." Further discussion addressing officers' ongoing socialization within the front-line police subculture, including in relation to field training, is engaged throughout Chapter Six.

\subsection{Chapter Conclusion}

In transitioning into the presentation of the study's methodology, it is worthwhile to note that while Manning (2010: 100) has critiqued recent policing research for being preoccupied with whatever is currently en vogue within police circles and, therefore, "too little [focus is] on the context or culture of policing," on the other hand, Cockcroft (2013: 229) has recently observed, "Scholars continue to find that police culture provides a helpful tool with which to understand the complexities associated with 21 stcentury policing." One such example is Campeau (2015), who, in her study of front-line officers' attitudes and behaviours, mobilized police occupational culture theory to inform empirical results. In doing so, Campeau (2015) relied on insights from Swidler (1986), the eminent cultural sociologist, who argues that the best time to study culture is during moments where lives are 'unsettled.' It is during 'unsettled times,' such as those 
accompanying unprecedented societal scrutiny of police "or when damaging police videos surface online," that an officer's "ways of orienting oneself are revealed and contested" (Campeau 2015: 674). Further, Paoline and Terrill (2014: 6), in reviewing the extant literature, observe, "What we find in descriptions of police culture, from the occupational point of view, is a series of writings that attempt to characterize the various features and strains encountered in the policing environments, as well as the cultural responses endorsed in buffering such strains." This dissertation investigates the phenomenon of de-policing as individual risk-averse responses to the techno-social and socio-political strains characterizing these 'unsettled times' in today's front-line policing landscape and also considers these contemporary developments within the context of the occupational subculture of the rank-and-file. 


\section{CHAPTER FOUR}

\section{METHODOLOGY}

\subsection{Chapter Introduction}

As discussed in Chapter One, the primary goal of this dissertation research is to determine whether the shifting topography in the contemporary policing landscape is, or is not, influencing the behaviour(s) of today's rank-and-file officers as they perform their front-line police work in the field, throughout Canada and/or the USA. To inform previous scholarship and public sphere discourse in this regard, as presented throughout the preceding chapters, the present study seeks to establish, through original empirical research, whether risk-averse behavioural adaptations among front-line officers are a 'real world', as opposed to theoretical, conjectural, rhetorical, or anecdotal phenomenon, and, if so, how this is happening - where, when, by whom, and to what degree - and why. To obtain data in furtherance of studying the de-policing phenomenon, which has been purported to be widespread and cutting across multiple police agencies, I administered a survey instrument to the study's 3660 research participants at 23 different data sites (police agencies) throughout Canada and across the State of New York.

To not only understand the scope (or prevalence) of de-policing practices in today's front-line police work, but to also flesh out the detailed rationales for such riskaverse behaviours, this mixed methods study employes a hybrid quantitative/qualitative survey instrument. Finally, to determine if there is any empirically-verifiable evidence available, outside the reported perceptions and actions by this study's research participants, I obtained policing data through FOI requests, to ascertain whether any 
changes to police-initiated activities in the field, as one might expect would eventually accompany a widespread de-policing phenomenon, are yet being revealed in the official metrics routinely generated by most police agencies throughout Western democracies. The methodology involved in testing the study's primary research question is presented in this chapter, which is organized into five substantive sections: (4.2) research design and method; (4.3) selection of participants; (4.4) data collection; (4.5) data analysis; and (4.6) challenges.

\subsection{Research Design and Method}

\section{The Research Question}

The study's research question, as detailed in Chapter One, functioned throughout the project to provide focus and conceptual, theoretical, and methodological scaffolding, which informed considerations around research design, assumptions, choice of method, data collection, and analytical frameworks. Hesse-Biber (2010: 24) is instructive on this point, arguing that the research question itself should be the "pivotal" consideration in guiding the design of the research project. She applies the 'cart before the horse' metaphor in cautioning against the tendency to pursue "methods-centric" research - in which a "methods focus sometimes turns into the driving force" (Hesse-Biber 2010: 30). Along the same lines, Teddlie and Tashakkori (2010: 10-18) counsel, "Once researchers have decided what they are interested in studying...the specifics of their research question will determine the choice of the best tools to use." Mixed methods research, which is the approach relied on in this project, focuses on the research question in determining the specific methods employed with a given study (Bryman 2006; Johnson \& Onwuegbuzie 2004; Schulenberg 2007, 2014, 2016; Teddlie \& 
Tashakkori 2010). In this regard, Teddlie and Tashakkori (2010: 18) counsel, "We have always asserted that a mixed methods question is one that clearly calls for a mixed methods study."

\section{Research Design}

Yin (2014: 20) advances that the research design "links the data to be collected and conclusions to be drawn to the initial questions of the study - it provides a conceptual framework and an action plan for getting from questions to a set of conclusions." The design stage of research involves a series of important decisions about the project and Berg (2009: 41-42) is instructive, through his review of fundamental considerations that should be addressed when planning social scientific studies - What type of information will be gathered and what methods of data collection are best suited to the project's objectives? What overall approach will the research follow - qualitative, quantitative, mixed methods, or others? Where will the research be undertaken and among what group of people?

Through deliberation around the study's primary research question and the interrelated subsidiary areas for investigation, Berg's guidance was operationalized in the research design. The nature of the project dictated that the study's unit of analysis should be at the level of the research populations' individual lived perceptions, attitudes, experiences, observations, and actions - rather than, for example, an organizational or societal examination, or a study that relies on documents (i.e. police administrative records or media reports), archival records, physical artifacts, or third-party observations (i.e. ethnography or systematic social observation). In this regard, some policing researchers (e.g., see Deuchar et al. 2018; Fallik et al. 2018) have identified 
that often absent within other post-Ferguson scholarship examining officers' feelings, thoughts, and behaviours are the voices of the officers themselves. Along these lines, MacDonald (2016) encourages today's policing researchers to "get out there and talk to some officers" and Wolfe and Nix (2016: 3) advance that the "only way to determine whether ['de-policing' and any resulting 'Ferguson Effect'] exists is to ask officers themselves."

Therefore, to achieve the project's objectives, this original empirical research was executed as a mixed methods and multi-site study, with data originating directly from the research population. In this regard, the sampling frame involved only police officers that were, at the time of the survey's administration, performing front-line duties. Those rank-and-file officers that consented to participate in this research were asked to share information within the study's anonymity assurance protocols, principled ethics, and through the hybrid quantitative/qualitative data collection instrument.

\section{Assumptions}

All studies are informed and guided by the worldviews of the researcher(s) - the underlying philosophical assumptions and understandings about the world and the nature of knowledge, which unquestionably influence research design and the conduct of research projects (Bisman 2010; Creswell \& Plano-Clark 2007; Denzin 2004; Greene \& Hall 2010; Guba \& Lincoln 2005; Hesse-Biber \& Leavy 2004; Olson 2011; Schulenberg 2007; Teddlie \& Tashakkari 2003, 2010). As expressed by Wynn and Williams (2012: 788), "In the conduct of scientific research, the actions of researchers are guided by the systems of belief by which they generate and interpret knowledge claims about reality." These paradigmatic assumptions can be considered as lenses for 
viewing, perceiving, and experiencing the world, which can generate insights and reveal phenomena that would not be available through other perspectives (Greene 2007; Maxwell \& Mittapalli 2010). Paradigms (or systems of belief) are comprised of the tripartite linkage of ontology, epistemology, and methodology (Morgan 2007; Wynn \& Williams 2012).

Mauthner and Doucet (2003: 415-424) counsel that social sciences methods are not neutral techniques, but, rather, they are infused with epistemological understandings and these methodology scholars caution that the researcher must be aware of (and articulate) the subjectivities and assumptions underpinning the researcher's knowledge construction - including the researcher's social, emotional, and intellectual location and the researcher's interpersonal, political, and institutional contexts. Given my position as a former 28-year operational police officer, this dissertation contains explicit disclosure of relevant aspects of my biography, subjectivities, and assumptions and I can advise the reader that I have come to appreciate that researcher reflexivity is an important consideration in social scientific research (as discussed in the subsection below). In this regard I am particularly fond of an insightful observation offered by Greenhill (1981: 91), writing on The Value of Sociology in Policing from his positioning as a sociologist embedded within a police agency, "Nothing [is] surprising in police and sociologists evaluating each other; what is important is to encourage an awareness of the distorting effect on the perception of facts which evaluative prejudice can produce."

\section{Reflexivity}

Flyvbjerg (2001), in rejecting the application of natural sciences' standards for objectivity and validity and asserting that social scientific knowledge production is 
largely interpretive, finds that the practice of reflexivity is crucially important. In this regard, Mauthner and Doucet (2003: 424) caution that "research which relies on the interpretation of subject accounts can only make sense with a high degree of reflexivity and awareness about the epistemological, theoretical, and ontological conceptions of subjects and subjectivities that bear on research practices." In concurring with these observations and the general tenets around reflexivity in contemporary social scientific methodology, I argue that reflexive practices can mitigate methodological purists' concerns in relation to objectivity and reliability (Freeman, de Marrais, Preissie, Roulston \& St. Pierre 2007; Fox 2003; Lather 1994; Lenzo 1995; Whittemore, Chase \& Mandle 2001) and I subscribe to the position that researchers must be reflexive about underlying assumptions, which can influence their methodological choices and are inevitably built into the research design (Denzin \& Lincoln 2005; Findlay 2003; Guba \& Lincoln 2005; Mauthner \& Doucet 2003).

The practice of researcher reflexivity involves the critical examination of one's subjectivities and the identification of how they interact with, impact on, and transform the research process (Findlay 2003; Greene 2007; Guba \& Lincoln 2005; Kirby et al. 2006; Mason \& Dale 2011; Olson 2011; Teddlie \& Tashakkori 2003). Gallagher's (2004: 217) observation is instructive on this point, "Data does not 'speak for itself" nor does it 'emerge' in a vacuum. Who we are...influences the questions we ask, the responses we get, and the scholarship we produce." Along these lines, my understanding of reflexivity involves the proactive practice of introspection, at all stages of the research project, in reflecting on, and being responsive to, assumptions, worldviews, and/or tacit theories in the research design, in data collection and analysis, 
and in the interpretation and write-up of findings/results - which I have exercised throughout this project. In approaching reflexivity in this project, I was mindful of the researcher's responsibility to maintain active and ongoing vigilance throughout the research process and of the judiciousness in disclosing my operational policing biography (as detailed in Chapter One), to allow readers of this dissertation to understand, and make their own subjective assessments in regard to, my police practitioner background and experiences.

\section{Mixed Methods}

While acknowledging a lack of consensus among scholars, Creswell and PlanoClark (2007: 5) advance that mixed methods “...focuses on collecting, analyzing, and mixing both quantitative and qualitative data in a single study or series of studies. Its

central premise is that the use of quantitative and qualitative approaches in combination provides a better understanding of research problems than either approach alone." Similarly, Johnson, Onwuegbuzie and Turner (2007: 123) describe mixed methods as, "[Research that] combines elements of qualitative and quantitative research approaches...for the broad purposes of breadth and depth of understanding and corroboration." In the same vein, Hesse-Biber (2010: 2) observes, "Mixed methods research holds greater potential to address [complex social inquiry] by the dynamic interconnections that traditional research methods have not adequately addressed."

Robson (2011: 166) observes that in some instances "it is difficult to judge what has been gained by employing both [qualitative and quantitative] approaches" and, therefore, it is important for mixed methods researchers to be clear in articulating why they relied on mixed methods. I argue that a single source of data (either quantitative or 
qualitative), in the present study, would have been inadequate to produce a comprehensive understanding of the contemporary de-policing phenomenon and, therefore, a mixed methods approach was necessary to achieve greater completeness (Arnold 2014; Creswell \& Plano-Clark 2007; Denzin \& Giardina 2009; Hesse-Biber 2010; Johnson et al. 2007; Porta \& Keating 2008; Schulenberg 2014, 2016; Teddlie \& Tashakkori 2003, 2010). In this regard, mixed methods allow the researcher to simultaneously address a diverse range of confirmatory and exploratory questions, to deepen the understanding and explanation of a phenomenon, and to verify and generate theory in the same study (Danermark 2002; Teddlie \& Tashakkori 2003, 2010). HesseBiber (2010: 4) explains that this 'complementarity' of quantitative and qualitative data allows for an understanding of "the social story in its entirety."1 Similarly, Denzin and Giardina (2009: 19) conclude, "No one could refute the argument that the use of more than one method produces stronger inferences, answers research questions that other methodologies could not, and allows greater diversity of findings."

Mixed methods are widely employed, and respected as legitimate, across much of today's policing research community (e.g., see Alise \& Teddlie 2010; Engel \& Cohen 2014; Greene 2014; Jenkins 2015; Wood \& Bryant 2015; Wood, Cockcroft, Tong \& Bryant 2017). For example, Shulenberg (2007: 101), in her mixed methods study of police decision-making, concludes that "the use of mixed methods allows for the presentation of a larger spectrum of divergent views." Similarly, Wood and colleagues (2017: 3) find, "Policing, like other social interactions, is so context-dependent, with a multitude of nuanced variables, that statistically-based research will always need to be contextualized." 
In relation to such statistically-based research, involving the use of quantitative methods, Schulenberg (2007: 107) finds, "[This] numeric precision allows for a more extensive documentation of the data concerning a social phenomenon." I agree, however, similar to the argument advanced by Wood and his co-authors (2017) in the preceding paragraph, it is my position that, in the present study, the use of quantitative methods alone would not have allowed for further contextualization, consideration, explanation, and expansion of issues disclosed by participants in their 'Yes' or 'No', Likert-scale, and/or other forms of quantitative responses within the survey instrument. This dissertation would have presented only statistical information, which would have accurately represented the prevalence of the issues under investigation, but absent would have been the personal elaborations of research participants that can inform, compliment, and provide valuable context to quantitative data. Greene (2014: 202) counsels, "In our goal to pursue the more 'scientific' aspects of research on the police we have often settled for statistical results - absent contextual meaning - without the associated social interpretation."

As Johnson and Onwuegbuzie (2004: 21) counsel, mixed methods allow "words, pictures, and narrative [to] be used to add meaning to numbers." Woodside (2010: 263264) discusses such qualitative contributions, which can develop "thick descriptions of individual cases" through "learning the thinking, feeling, and doing processes of the informants, including an understanding of the informants' worldview of the topic under study in their own language." I recognize that such contextual information is valuable; however, on the other hand, an exclusively qualitative approach to the present study would have been unable to produce precise and statistically significant results, which 
clearly establish the scope of the current de-policing phenomenon and also present the opportunity for some degree of generalization to a larger context.

In concluding this subsection, I argue that the mixed methods employed in the present study allow for both (1) accurate scientific measurement of, and insightful penetration into, the innermost thoughts, experiences, and observations of individual rank-and-file police officers and also (2) the opportunity to explore and understand complexities in today's external front-line working environments and rank-and-file occupational subculture - an achievement which, as will be elaborated later in this chapter, is acknowledged by many policing scholars to be a fundamentally challenging task.

\section{The Potential for Greater Understanding with a Mixed Methods Multi-Site Study}

Flyvbjerg (2001: 81) observes, "The value of [any] study will depend on the validity claims which researchers can place on their study." In this regard, Stake (2005) advances that researchers' arguments can be strengthened when their declarations originate from a multiplicity of data sources, each making the subject under investigation visible in a different way and achieving redundancy of data. This validation of findings through multiple sources of data, is often referred to as 'triangulation' - the combining of several lines of inquiry directed at the same point and a convergence of the data, or, in other words, the verification of findings emerging from one source of data with those derived from another data source (Berg 2009; Denzin \& Lincoln 2005; Hesse-Biber 2010; Kadushin et al. 2008; Small 2011; Tashakkori \& Teddlie 2003; Woodside 2010). In sum, the use of mixed methods and multiple data sites presents the opportunity to apply 'replication logic,' allows for consistency across 
findings, and supports more substantial conclusions in relation to implications for, and significance to, applicable theoretical paradigms (Gray 2014; Yin 2018).

In other words, the availability of data from a variety of sources, as in the case of the present study, allows researchers to "obtain a better, more substantive picture of reality" (Berg 2009: 5). In this vein, I argue that through combining 23 well-chosen police agencies as data sites with multiple methods of data collection - quantitative, qualitative and other sources of corroboration for this study's findings - a comprehensive understanding around complexities in today's front-line police work, including the phenomenon of de-policing, has been achieved in the present study, which enables me, as the researcher, to advance validity claims with a high degree of confidence. Before transitioning into discussions focused on data collection and analysis, I advance that this chapter's thorough treatment of the study's methodological considerations - including assumptions, reflexivity, and method - has presented readers with the necessary backdrop as they move into examining and evaluating data and findings. In this regard, Alise and Teddlie (2010: 122), found, in their analysis of journal articles across the social and behavioural sciences, that authors "of theoreticallyoriented articles often explicitly discuss" such methodological considerations, however, "researchers reporting empirical studies seldom do so."

\section{Designing the Hybrid Quantitative/Qualitative Survey Instrument}

As Nix and colleagues (2017: 1, citing Reiner 2000a; Squires 2016) find, "Until recently, the majority of police research was ethnographic using participant observation ... [However] in recent years, policing scholars have increasingly used survey methods to gain insight into officers' attitudes and behaviours." Similarly, Schulenberg (2016: 
169) observes, "Designing and conducting survey research is both an art and a

science...One of the most common reasons for conducting surveys is to assess attitudes, behaviour, experiences, or beliefs."

As Biemer and Lyberg (2003: 28) counsel, "A well-specified set of research objectives is a critical component of the survey process and will facilitate many of the decisions involved in survey design," in addition to minimizing the risk of 'specification error' - those errors that arise when survey questions fail to ask respondents about what is essential to answer the research question(s). With guidance from the survey methods literature (i.e., Aldridge \& Levine 2001; Babbie 1992; Biemer \& Lyberg 2003; Blair, Czaja \& Blair 2014; Bulmer, Gibbs \& Hyman 2010; Dillman et al. 2002; Dillman, Smyth \& Christian 2014; Fowler 1995; Groves et al. 2009; Harrison 2006, 2007; Payne 1980; Rea \& Parker 1992; Redline \& Dillman 2002) and in order to obtain essential data from study participants, comprising both quantitative responses and qualitative elaborations, I designed a 30-question hybrid survey - in both English and French (see Appendices $\mathrm{G}$ and $\mathrm{H}$ respectively). ${ }^{2}$ As an example of the hybrid nature of this instrument, survey question 15 inquired whether the participant has experienced '.. an occasion while you were doing front-line police work when you have thought about not doing something, because of a concern that your actions could be video recorded, that you otherwise would normally do?' Participating officers were invited to provide a quantitative response, by indicating 'Yes' or 'No' in the corresponding box on the survey document, and, for those participants that answered in the affirmative, they were invited to explain (qualitatively) by writing text within the allotted space on the survey document. ${ }^{3}$ 
As Aldridge and Levine (2001: 95) counsel, "Question design calls our sociological imagination into play in a number of ways. We need to frame questions that are meaningful, sensitive, precise, searching and salient to our respondents. We need to construct the questions in such a way that respondents will want to answer them as fully and truthfully as they can." While designing and then subsequently refining the survey instrument, through several iterations, I relied on guidance from a variety of survey methods scholars (as enumerated in the preceding paragraph). Such is the common practice in police survey research - as Nix and colleagues (2017: 13) observe, "Policing scholars typically use Dillman et al. (2014) as a guide." This body of survey design literature was instructive in many respects, including determining the nature, wording, number, and ordering of survey questions. For example, Redline and Dillman's (2002) idea of the 'respondent friendly' questionnaire (for self-administered surveys) served as a reminder to keep questions simple to understand - in order to ensure that all respondents interpret questions the same way and to make the survey easy for respondents to navigate. Also, the reader likely will have noticed that the 'Yes' and 'No' response boxes on the questionnaire document alternate positions from question to question - in order to counter the 'primacy effect' that is inherent to paper (and Internet) surveys, which Harrison (2007) advocates as good survey design practice.

Perhaps most importantly, the reviewed survey methods literature assisted with my efforts to minimize (or ideally eliminate) survey bias, which can be considered as error resulting from survey design. Throughout the process, I was live to issues of response bias and/or social desirability bias (the latter is discussed in more detail later in this chapter) and I worked toward designing questions that were worded in such a way 
that they would not influence a positive or negative response - through neutral phrasing, avoiding emotive language, being single-focused, and relatively jargon-free. ${ }^{4}$ I used language that should be familiar to most front-line officers and, in the case of certain terminologies (e.g. 'de-policing', 'risk-averse policing', and 'F.I.D.O.'), I presented explanations, directly within the survey document, preceding the participant's consideration of the relevant question(s).

In their recent analysis of police officer surveys and response rates, Nix and cowriters (2017: 1-2) find,

Unfortunately, methodological research analyzing surveys of police officers is rare. As a consequence, the guidance available to researchers seeking to conduct police surveys is shockingly thin and often inconsistent. In turn, the strategies, norms, and best practices for designing, conducting, and evaluating police surveys remain unclear. Although the broader literature on survey research methods is a useful starting place (e.g. Babbie 1992, Groves et al. 2009, Dillman et al. 2014), there are unique difficulties to surveying police officers [and] research methods texts in criminal justice provide...virtually no guidance about surveying police officers specifically.

In this regard, given all of the considerations with the rank-and-file police subculture, including, but not limited to, insularity and distrust of 'outsiders', which will be elaborated later in this chapter's discussion of challenges in researching front-line policing, and the individuals that would be comprising this study's research population, I decided that the mode of survey administration would be nonprobability convenience sampling through in-person 'roll call' administration - as opposed to mail, telephone, or online. This decision was made after assessing considerations of statistical power, comprehensiveness, data quality, and the logistics of, and realities involved with, this research population - in terms of shift work, officer's time off, temporary assignments, etc.). ${ }^{5}$ It is well documented that in-person administration achieves the highest survey 
response rates - over those administered by telephone, mail, or the Internet (Groves et al. 2009; Nix et al. 2017; Paoline \& Terrill 2014; Tourangeau \& Plewes 2013).

Based on the guidance in the literature, I determined that it would be best if the surveys were self-administered through distribution of paper questionnaires to potential research participants during roll call at the start of shift. Biemer and Lyberg (2003: 104) suggest that self-administered questionnaires are the preferred mode for the collection of 'sensitive information' and their counsel, in this regard, was helpful. Through this protocol, research participants were able to record their answers individually and privately directly on the questionnaire document and then return it to me at the end of the roll call - after which I deposited all of the completed surveys from that session into an envelope, in no particular order and after some shuffling of the documents, that was then sealed. This process allowed me to segregate the completed surveys by police agency and to provide participants with transparent assurances of confidentiality and anonymity.

As contrasted with other data-collection regimens, such as 'face-to-face' interviews, in which participants present their answers to the researcher, the approach I took in this study encourages participants to be more comfortable in answering survey questions candidly and completely, allows them to be more confident in the study's anonymity assurances, and mitigates against social desirability bias (Krumpal 2013; Tourangeau \& Yan 2007). Aldridge and Levine (2001: 103) find, “A major challenge for all overt forms of social research is the social desirability problem." Social desirability bias relates to the 'sensitivity' of the question(s) being asked of the respondent and Fowler (1995: 29) suggests, "Questions tend to be categorized as 
'sensitive' if a 'Yes' answer is likely to be judged by society as undesirable behaviour." In this regard, Krumpal (2013: 2027-2028) observes, "To conform to social norms, respondents may present themselves in a positive light, independent of their actual attitudes and true behaviours respectively." Therefore, social desirability bias can be defined as a situation in which survey respondents, due to self-presentation concerns, tend to provide socially-approved answers to questions about their behaviour(s) - overreporting virtuous activities and under-reporting their 'vices' (or undesirable activities) (Aldrige \& Levine 2001; Krumpal 2013; Ong \& Weiss 2000).

Tourangeau and Yan (2007: 860) raise the spectre of the 'threat of disclosure' within the context of social desirability bias - suggesting a potential adverse impact on participants' honesty resulting from "concerns about the possible consequences of giving a truthful answer should the information become known to a third party. A question is sensitive in this sense if it raises fears about the likelihood or consequences of disclosure of the answers to agencies or individuals not directly involved in the survey." Along the same lines, Krumpal (2013: 2041-2041) counsels,

The degree of social desirability bias in sensitive surveys depends on the perceived items' sensitivity, the degree of privacy in the interview situation, the fraction of the population who have behaved in the socially undesirable manner, and on specific aspects of the survey design...Social desirability bias could be reduced by appropriately tailoring the survey design...by increasing the anonymity of the question-and-answer process (e.g. selfadministered interviews), [and] by decreasing the respondent's concerns in admitting to some taboo (e.g. via confidentiality assurances).

As discussed, such procedures were considered and then employed in the present study. As Nix and colleagues (2017: 5) counsel, "Researchers must take added precaution to ensure anonymity in order to increase participation - as well as honesty among those 
who do participate. This is especially important if the survey pertains to sensitive topics such as police use of force or misconduct."

Relations between police and 'minorities' is also a difficult topic within policing research and, as a researcher working toward greater understanding in this area, addressing issues implicating race/ethnicity (and certain other 'demographic' categories) in the present study was a challenge. My view, as a social scientist, which accords with my fundamental personal beliefs, is that the use of 'demographic' descriptors (i.e. race, ethnicity, gender, age, sexual orientation, physical and/or mental health challenges, etc.) to characterize human beings and as a measurement category in empirical studies is presumptively inappropriate and can often be problematic.

However, in the context of the present study, I determined that exploring issues in today's front-line policing, vis-à-vis officers' relations with 'minorities' and the impact, if any, on individual officer's thoughts and/or actions, warranted the use of demographic variables within the survey questionnaire. ${ }^{6}$

\section{Piloting the Hybrid Quantitative/Qualitative Instrument}

Biemer and Lyberg (2003: 32) suggest, "Several iterations of the [various] steps of the design process may be necessary before the final design is determined." This proved to be the case with the present study and, in relation to the survey questionnaire itself, there were numerous tweaks that resulted from guidance I took from the methodology literature, discussions with colleagues, and feedback from piloting of the instrument. In terms of the latter, throughout the fall of 2015, I had opportunities to meet with many rank-and-file serving with the Ontario Provincial Police and the New York State Police and 20 front-line officers from each agency agreed to complete 
various draft forms of the questionnaire and to provide me with critiques (oral and/or written) - feedback that allowed me to refine the survey instrument to ensure the questions were more fair, balanced, and clear, than otherwise would be the case.

\subsection{Selection of Participants}

\section{Front-Line Police Officers}

During the early conceptualization of this project, I concluded that sampling should be done exclusively with police officers deployed in front-line capacities. These individuals are those that are operational, as opposed to administrative, investigative, or assigned to support functions, and that, at the core of their duties, wear a uniform, patrol our communities, and routinely engage with citizens in a wide variety of contexts. As such, these are the officers who account for the vast majority of police-citizen interactions (Bayley 2005; Krupanski 2012; Paoline \& Terrill 2014; Walker \& Katz 2008) and therefore, they are in the best position to provide the most relevant insights into the impact, if any, of techno-social and/or socio-political developments in today's policing landscape on rank-and-file attitudes and behaviours in front-line police work.

As discussed, given the realities of front-line police work, it was clear that nonprobability convenience sampling would, by necessity, be the strategy for this study. Nonprobability convenience sampling is not random sampling, in the strict sense of the statistician's concept of randomness, which I suggest might be, for all intents and purposes, impossible in a study such as this. Rather, sampling in the present study involved soliciting potential respondents that would be readily available to the researcher, upon my attendance at shift briefings. As will be discussed in more depth later in this chapter, the results of this study, therefore, can be considered 'suggestive' - 
as with sampling that is not, strictly-speaking, random, there can be a number of intervening factors that can impact on who is sampled (Northey, Tepperman \& Albanese 2012). In other words, with non-random sampling, as was the case in the present study, the results are typically not considered generalizable to a wider population, in accordance with strict statistical conventions.

\section{Ethics Clearance for Research with Human Subjects}

As this research involved interaction with human subjects, ethics clearance was required and sought from Carleton University’s Research Ethics Board (REB), to ensure that this study met ethical standards as outlined in the Tri-Council Policy Statement: Ethical Conduct for Research Involving Humans (second edition) and Carleton University Policies and Procedures for the Ethical Conduct of Research. The REB found that this project met prescribed standards and ethics clearance was granted on 29 January 2016 (refer to Appendix F). A renewal was issued by the REB on 25 May 2016 (that expired on 31 May 2017).

\section{Expansiveness of the Study}

The objective in soliciting a broad range of data sites for the study was to enable participation from a wide variety of front-line officers - those from different regions across Canada; from the United States (from cities across the State of New York); and from large, medium, and small police agencies. Participation of rank-and-file officers from three detachments of the RCMP was secured, which allowed for representation from officers not working within municipal and regional police agencies. ${ }^{7}$ Fyfe, Klinger and Flavin (1997) find that there can be issues in data sampling that are related to the particularities of host agencies selected for police studies, and, they counsel that these 
issues can be moderated through employing wider lenses in policing research, which also enhance the generalizability of the study. These accomplished policing scholars observe,

Since 1968, police research...typically has been conducted in a few progressive jurisdictions that have closely regulated officers' conduct and that, presumably, have had little to fear from publication of what researchers might find...As a consequence, and regardless of the sophistication of the methodologies they have employed, police researchers generally have provided information concerning the operations of a small, self-selected, and therefore, non-representative sample of police agencies" (Fyfe et al. 1997: 468-469).

Wortley (2006: 5) finds, "The few Canadian studies that have been conducted... have been plagued by methodological issues including small sample size and a reliance on newspaper coverage." As Nix and colleagues (2017: 2) caution, “A smaller sample size results in greater sampling error and lower statistical power, which reduces the precision of resultant survey estimates and increased the risk of Type 2 errors (false negatives)." Along these lines, in their chapter on non-random sampling, Biemer and Lyberg (2003: 310) argue, "Samples of somewhat larger sizes can be quite accurate since sampling error decreases as the sample size increases." Similarly, Smith (2013: 2), in his discussion around determining sample size, observes, "How many responses do you really need? This simple question is a never-ending quandary for researchers. A larger sample can yield more accurate results - but excessive responses can be pricey." Given the availability of significant research funding, the present study had, as a key objective, the acquisition of a substantial sample, which was achieved through the solicitation of thousands of front-line officers $(\mathrm{N}=3660)$ serving with 23 police agencies from coast to coast across Canada and across the breadth of the State of New York. ${ }^{8}$ 


\subsection{Data Collection}

\section{Launching the Study}

In practical terms, to recruit large numbers of the rank-and-file as potential research participants, a researcher must have institutional permission to attend on the property of police facilities to meet with front-line officers, individually or in groups. ${ }^{9}$ The endorsement of this study by senior command of the 23 police agencies involved in this project and the granting of permission to meet potential study participants on police property, was secured most often through initial telephone contacts between myself and a high-ranking officer and then follow-up contacts throughout 2015 and 2016, in order to coordinate logistics and to answer any questions, for example, the type of time commitment involved in the survey administration. ${ }^{10}$ With only three exceptions the Service de police de la Ville de Montréal (SPVM), the Peel Regional Police Service (PRPS), and the York Regional Police Service (YRPS) - all of the agencies solicited for participation, at least initially, expressed support for this study. ${ }^{11}$ Specific challenges, which developed during data collection planning for Winnipeg and Vancouver and during data collection in Yonkers, are discussed further later in this chapter.

After the project's data sites were finalized, an information document was created and approved by the REB, which explained to senior police administration, police labour organization leadership, and front-line officers themselves (the research population and potential individual research participants) the nature of the study (see Appendices D and E). This document requested that rank-and-file officers give consideration to participating in the study by consenting to, and completing, to the degree they felt comfortable, a survey questionnaire, to be administered at a future date 
when I attended at their workplace. This document was circulated at each data site prior to my arrival and I made a point to bring additional copies with me in order to provide the information to potential participants that may have not received the material in advance.

In all but a few situations, I discussed the proposed research with leadership of the police labour organization in each location. However, in a small number of jurisdictions, such discussions were left to senior command of the police agency. These were in circumstances where they suggested, for reasons known to them, that conversation would be best handled by them. In two of the three locations where police labour organizations expressed reservations around participation of their membership in the study - the Winnipeg Police Association (WPA) and the Vancouver Police Union (VPU) - there were ultimately, in my view, issues that developed, which could have impacted on potential study participants' voluntariness and informed consent. Therefore, I decided that involving front-line officers of the Winnipeg Police Service (WPS) and the Vancouver Police Department (VPD) would not be appropriate. In the case of challenges experienced with leadership of the Yonkers Police Benevolent Association (YPBA), during my data collection in Yonkers, I found that through a modification in sampling technique, I could obtain data from the research population, one at a time, and at locations away from Yonkers Police Department (YPD) facilities. This assuaged my concerns with coerced non-participation, which was occurring within the research setting, resulting from the dictates of the YPBA, and allowed individual YPD officers to make their own determinations regarding consent to participate (or not participate) in the study. These circumstances are elaborated later in this chapter. 


\section{Sampling Procedure}

As discussed, nonprobability convenience sampling was used in this study. To collect data, I typically attended the police facility a few minutes prior to the scheduled shift briefing in order to meet with the shift supervisor and to discuss how the survey would be administered to potential study participants. These were the individuals that had been designated by the police agency to facilitate my attendance on the roll call and were typically a sergeant or lieutenant. Often that supervisor would introduce me to the officers present or would invite me to introduce myself to the group. Purposefully, in considering mitigation of any potential survey bias, and consistent with the guidance of veteran American policing researchers Paoline and Terrill (2014) and members of my committee, I kept such introductions brief - my name, university affiliation, and I advised that I am 'a researcher interested in contemporary front-line policing.' I then provided the officers with a general overview of the project. ${ }^{12}$ At this time, I also ensured that each of the potential participants had read and understood the preliminary information sheet (Appendices D and E) and, consistent with the practice recommended by Paoline and Terrill (2014: 98), I "informed the officers that no individual or group of officers was being singled out for participation in this survey...[and then] discussed important issues of informed consent, confidentiality, and anonymity." ${ }^{.13}$ In this regard, in their chapter addressing lessons learned in the survey process with patrol officers, Paoline and Terrill (2014: 95-96) counsel,

We chose to survey a population of police officers assigned to street-level patrol functions, rather than selecting a sample of them...Since we were attending roll call sessions to survey groups of officers, across every shift, we decided to capture the population of all officers instead of a select sample. From a manpower perspective, we realized this would be worth the investment. From a practical standpoint, given the sensitive nature of the 
survey topics (i.e. police culture and the use of force), we did not want select officers to be suspicious as to why they might have been randomly chosen over others.

Prior to officers beginning to answer the survey questions, I reminded them that their participation was voluntary and that the survey collection, handling, and data analysis protocols in the study ensures them of confidentiality (in that no one other than myself would be viewing their individual survey documents) and anonymity (through the use of alpha-numeric coding rather than officer's names, badge numbers, etc.). I also reminded roll call attendees that the survey was intended only for current front-line officers. No incentives were offered in return for participation. I explained that the surveys were to be completed, if the officer wished to participate, immediately and that time had been allotted in the shift briefing for this purpose. At this point, I typically introduced a humourous remark along the lines of, 'It has been the experience of many police researchers that surveys leaving with officers to be completed later, seldom return, and those that do, often have to be handled with latex gloves after spending a shift rolling around the floor of a police cruiser.' I found this often changed the tone in the room - from perhaps stand-offish skepticism to more light-hearted curiosity (according to my subjective perceptions). After I had collected all of the surveys, completed or otherwise, I made a point of 'shuffling' them before placing them into an envelope, which I sealed in the presence of the study participants before leaving the briefing room. ${ }^{14}$

In addition to survey administration at shift briefings, which accounted for more than $98 \%$ of the 3660 surveys considered in this study, I was, from time to time, able to meet with uniformed officers in parking lots, on the street, and at other random 
locations. Through such chance encounters, a small number of surveys were received from officers that were not sampled during regular shift briefings in police facilities (e.g. those working overtime assignments, those who were on leave, and/or those on other uniform duty assignments). For example, in relation to the Buffalo Police Department (BPD), I was able to meet with officers from the BPD Strike Force (gang interdiction) and BPD Housing Unit (officers assigned to work in public housing projects) during my ten days of data collection in that city. ${ }^{15}$ In addition to some additional completed surveys that originating from these spontaneous interactions with front-line officers away from police facilities, I received unexpected 'windfall' information from officers that sought me out, on or off police property, to discuss their views and observations in more detail than they could present in the survey questionnaire. Some of this supplemental information is included throughout the discussion in Chapter Six.

\section{Data Sites (Contextual Information)}

\section{Policing in the United States of America (and the State of New York)}

The population of the United States of America is approximately 326,000,000 (United States Census Bureau 2017). There are more than 750,000 sworn police officers deployed across the country, of which 12\% are women (Bureau of Justice Statistics 2016; Federal Bureau of Investigation 2016). The Bureau of Justice Statistics (2016) reports, "Law enforcement in the United States is made up of about 18,000 federal, state, county, and local agencies. Each agency has varying legal and geographic jurisdictions, ranging from single-officer departments to those with more than 30,000 
officers." In reporting on a major American policing study in 2017, researchers at the Pew Research Centre observed,

In 2013, more than a quarter (27\%) of full-time [American] police officers were racial or ethnic minorities. Some $12 \%$ of full-time local police officers were Black; another $12 \%$ were Hispanic; and $4 \%$ were Asian, Pacific Islander, American Indian or multiracial. By comparison, $12 \%$ of the U.S. adult population was Black; $15 \%$ was Hispanic; and $8 \%$ was some other racial or ethnic minority in 2013. Women remain underrepresented in the field, making up just $12 \%$ of full-time officers in 2013; [while] women are $51 \%$ of the U.S. adult population (Morin et al. 2017: 11).

Total operating expenditures for police agencies across the United States exceeded $\$ 100$ billion in 2016 (McCarthy 2017). In 2016, the American national violent crime rate was 386.3 occurrences per 100,000 persons (Federal Bureau of Investigation 2016). ${ }^{16}$

The State of New York has a population of 19,400,000 (United States Census Bureau 2017) and is policed by roughly 61,000 officers, of which $14 \%$ are female, serving with 418 law enforcement agencies (Federal Bureau of Investigation 2015). ${ }^{17}$ Within the state, New York City (NYC) has a population of 8,600,000 and the New York Police Department (NYPD), the largest municipal police agency in the United States, has a complement of about 35,400 officers (Federal Bureau of Investigation 2015). After NYC, the most populous cities in the state are Buffalo $(257,000)$, Rochester $(209,000)$, Yonkers $(201,000)$, Syracuse $(143,000)$, Albany $(98,000)$, and New Rochelle $(80,000)$ (United States Census Bureau 2017). In 2016, the violent crime rate for the State of New York was 376.2 occurrences per 100,000 persons. The average median household income for the state is $\$ 59,269$ and the average per capita income is $\$ 33,236$ (both in 2015 dollars) (United States Census Bureau 2017). Approximately $14.7 \%$ of the state's population is living in poverty (United States Census Bureau 2017). 
The table below reports on the race/ethnicity of the state's population (United States Census Bureau 2017). ${ }^{18}$

Table 1: Population of the State of New York (2010)

\begin{tabular}{|c|c|c|}
\hline Population of the State of New York (2010) & $\mathbf{1 9 , 3 7 8 , 1 0 2}$ & $\mathbf{1 0 0} \%$ \\
\hline White & $11,304,247$ & $58.3 \%$ \\
\hline Hispanic/Latino & $3,416,922$ & $17.6 \%$ \\
\hline Black/African American & $2,783,857$ & $14.4 \%$ \\
\hline Asian & $1,406,194$ & $7.3 \%$ \\
\hline Two or More Races & 326,034 & $1.7 \%$ \\
\hline Other Race & 81,620 & $0.4 \%$ \\
\hline American Indian/Alaska Native & 53,908 & $0.3 \%$ \\
\hline Native Hawaiian/Other Pacific Islander & 5,320 & $0 \%$ \\
\hline
\end{tabular}

\section{$\underline{\text { Policing in Canada }}$}

The population of Canada is approximately 35,200,000 (Statistics Canada 2017c) and there are roughly 69,000 sworn police officers deployed across the country, of which 21\% are women (Statistics Canada 2017e). In 2016, total operating expenditures for Canadian police agencies were $\$ 14.2$ billion (Statistics Canada 2017e). Front-line policing in Canada is provided through various arrangements, dependent upon location. Most is performed by officers employed by municipal (city and town) agencies, including regional municipalities such as Halifax, Peel, York, Durham, and Niagara. In 2016, at this municipal policing level, there were 38,812 officers employed with 144 agencies and 36 First Nations' self-administered police agencies - with 50 of these agencies serving cities, towns, and regional municipalities with populations greater than 100,000 (Statistics Canada 2017e). The Royal Newfoundland Constabulary (409 officers), the Ontario Provincial Police (6097 officers), and the Sûreté du Québec (5476 officers) provide primary-response policing for communities without municipal police agencies (generally smaller towns, villages, and outlying rural locations) in these three 
provinces and they are also responsible for patrolling provincial highways (Statistics Canada 2017e). Those Canadian provinces without a provincial police agency (British Columbia, Alberta, Saskatchewan, Manitoba, New Brunswick, Prince Edward Island, and Nova Scotia) have primary-response policing, in various locations throughout those provinces, performed by members of the RCMP, through contractual arrangements with cities, towns, and villages and the provincial government (Statistics Canada 2017e). ${ }^{19}$

Statistics Canada does not produce a violent crime rate that is directly comparable to that of the United States and therefore, to create an approximately similar numeric representation for Canada, I relied on data from Canadian criminal offence categories that are relatively equivalent to those that comprise the American rate. ${ }^{20} \mathrm{~A}$ total of 103,243 of such occurrences were reported to Canadian police in 2016, which represents 286.8 incidents per 100,000 persons. This can be considered an imprecise equivalent to the American national violent crime rate (of 386.3 incidents per 100,000 persons). ${ }^{21}$

Statistics Canada (2017d) does produce a metric called the violent Crime Severity Index (CSI) to characterize "the overall volume and severity of violent crime." The national violent CSI for 2016 was 75.3 , which is based on the approximately 381,000 'violent' occurrences reported to police, which are defined, within the broad Canadian criteria, as "those violations in the Criminal Code identified as crimes against the person [which includes minor assaults, firearms offences, threatening, harassment, etc.], as opposed to property crimes and other violations, such as offences against the administration of justice" (Statistics Canada 2017d). This 2016 violent CSI value is presented (within Appendix C), when available, for Canadian census metropolitan areas 
of relevance to this study. ${ }^{22} 23$ The highest violent CSI (in 2016) was recorded for Winnipeg at 149.6 while the lowest was recorded for the Niagara region at 37.6 (Statistics Canada 2017d).

The table below reports on the race/ethnicity of the Canadian population as reported in the national census. Statistics Canada (2017b) considers 'visible minorities' as "persons, other than Aboriginal peoples, who are non-Caucasian in race or non-white in colour.”

Table 2: Population of Canada (2016)

\begin{tabular}{|c|c|c|}
\hline Population of Canada (2016) & $\mathbf{3 4 , 4 6 0 , 0 6 5}$ & $\mathbf{1 0 0 \%}$ \\
\hline White & $26,785,480$ & $77.7 \%$ \\
\hline Total 'Visible Minority' Population & $7,674,580$ & $22.3 \%$ \\
\hline South Asian & $1,924,635$ & $5.6 \%$ \\
\hline Chinese & $1,577,060$ & $4.6 \%$ \\
\hline Black & $1,198,540$ & $3.5 \%$ \\
\hline Filipino & 780,125 & $2.3 \%$ \\
\hline Latin American & 447,325 & $1.3 \%$ \\
\hline Arab & 523,235 & $1.5 \%$ \\
\hline Southeast Asian & 313,260 & $0.9 \%$ \\
\hline West Asian & 264,305 & $0.7 \%$ \\
\hline Korean & 188,710 & $0.5 \%$ \\
\hline Japanese & 92,920 & $0.3 \%$ \\
\hline \hline Other 'Visible Minority' & 132,090 & $0.4 \%$ \\
\hline Multiple 'Visible Minorities' & 232,375 & $0.7 \%$ \\
\hline Aboriginal & $2,130,520$ & $6.2 \%$ \\
\hline
\end{tabular}

\section{The Study's Data Sites and Research Participants}

The table below presents the 23 participating agencies (column 1) and their estimated front-line officer complement, or potential sampling frame, which was provided to me by officials within each agency (column 2). That figure represents the approximate population of front-line officers that could be available to participate in this study. Columns 3,4 , and 5 report on the number of survey questionnaires administered, 
received, and subsequently included in the study. ${ }^{24}$ Column 6 presents the proportion of each agency's front-line population included in this study.

Table 3: Study participants by police agency

\begin{tabular}{|c|c|c|c|c|c|}
\hline $\begin{array}{l}\text { participating } \\
\text { agency }\end{array}$ & $\begin{array}{c}\text { potential } \\
\text { front-line } \\
\text { participants }\end{array}$ & $\begin{array}{c}\text { surveys } \\
\text { administered }\end{array}$ & $\begin{array}{l}\text { surveys } \\
\text { received }\end{array}$ & $\begin{array}{l}\text { surveys } \\
\text { included } \\
\text { in the } \\
\text { study }\end{array}$ & $\begin{array}{l}\text { \% of research } \\
\text { population } \\
\text { included in the } \\
\text { study }\end{array}$ \\
\hline Albany & 200 & 158 & 156 & 156 & $78.0 \%$ \\
\hline Buffalo & 500 & 325 & 317 & 317 & $63.4 \%$ \\
\hline Calgary & 1173 & 362 & 362 & 358 & $30.5 \%$ \\
\hline Cornwall & 48 & 36 & 36 & 36 & $75.0 \%$ \\
\hline Delta & 75 & 58 & 58 & 58 & $77.3 \%$ \\
\hline Gatineau & 175 & 148 & 148 & 148 & $84.6 \%$ \\
\hline Halifax & 250 & 210 & 210 & 206 & $82.4 \%$ \\
\hline Hamilton & 406 & 295 & 295 & 289 & $71.2 \%$ \\
\hline Kingston & 107 & 78 & 78 & 78 & $72.9 \%$ \\
\hline New Westminster & 65 & 49 & 49 & 49 & $75.4 \%$ \\
\hline Niagara Regional & 280 & 200 & 199 & 191 & $68.2 \%$ \\
\hline Ottawa & 450 & 383 & 382 & 382 & $84.9 \%$ \\
\hline Port Moody & 24 & 21 & 21 & 20 & $83.3 \%$ \\
\hline Rochester & 480 & 288 & 287 & 281 & $58.5 \%$ \\
\hline RCMP Codiac & 88 & 71 & 71 & 69 & $78.4 \%$ \\
\hline RCMP Red Deer & 80 & 75 & 75 & 75 & $93.8 \%$ \\
\hline $\begin{array}{c}\text { RCMP Ridge } \\
\text { Meadows }\end{array}$ & 85 & 70 & 70 & 66 & $77.6 \%$ \\
\hline Saskatoon & 259 & 180 & 180 & 175 & $67.6 \%$ \\
\hline Stratford & 37 & 29 & 29 & 29 & $78.4 \%$ \\
\hline Syracuse & 225 & 170 & 170 & 168 & $74.7 \%$ \\
\hline Toronto & 2500 & 314 & 312 & 310 & $12.4 \%$ \\
\hline Yonkers & 362 & 74 & 51 & 51 & $14.1 \%$ \\
\hline Windsor & 210 & 150 & 150 & 148 & $70.5 \%$ \\
\hline Total & 8079 & 3746 & 3706 & 3660 & \\
\hline
\end{tabular}

For the purpose of categorizing participating police agencies, for analysis of variables and subsequent discussion, those with fewer than 100 front-line officers are considered 'small' (Cornwall, Delta, New Westminster, Port Moody, RCMP Codiac, RCMP Red Deer, RCMP Ridge Meadows, and Stratford); those with between 100 and 399 front-line officers are considered 'medium' (Albany, Gatineau, Halifax, Kingston, 
Niagara, Saskatoon, Syracuse, Yonkers, and Windsor); and those with 400 and more front-line officers are considered 'large' (Buffalo, Calgary, Hamilton, Ottawa, Rochester, and Toronto). There are no uniform criteria for such categorizations across the literature, however these agency-size categories are consistent with those applied by the Canadian Association of Police Governance and in recent research publications (e.g., see Kiedrowski, Melchers, Riddell \& Petrunik 2015; Wyatt \& Bell 2014).

\section{Participant Response Rate}

This study's participant response rate can be calculated by dividing the number of completed surveys received by the researcher $(\mathrm{N}=3706)$ by the total number of surveys administered to potentially eligible research participants $(\mathrm{N}=3746)$ (Nix et al. 2017; Smith et al. 2002). This represents a response rate of $\mathbf{9 8 . 9 \%}$ for the present study. Nix and colleagues (2017: 3), who found that "there is no prior research on response rates in policing studies," examined the response rates to 497 police surveys, as reported in 390 journal articles published in fifteen scholarly journals from 2008 to 2017. Their findings report that the average response rate was $64.3 \%$, but there was a great deal of variation, (ranging from $5.2 \%$ to $100 \%$ ). In-person surveys achieved substantially higher response rates (79.4\% versus $48.6 \%$ for surveys distributed through other means) and overall response rates have declined over time, though primarily among surveys not administered in-person (Nix et al. 2017). They suggest that response rates are an important consideration "Because researchers, reviewers, and editors often rely on response rates to estimate the quality of data from surveys" (Nix et al. 2017: 2, citing Byrne 2000; Carley-Baxter et al. 2009; Peytchev 2013; Pickett 2017). 
In this regard, I submit that any potential concerns with sampling error, in terms of systematic sampling bias, which can occur when certain subgroups in the study's target population are underrepresented in the sampling frame (Groves et al. 2009), are mitigated with this study's design and sample size. ${ }^{25}$ Similarly, nonresponse error, which is "the proportion of eligible sample elements for which data are not collected" (Groves et al. 2009: 59) and, which is often a significant concern in 'sensitive' surveys (Fox \& Tracy 1986), is not an issue in this study - given the response rate of $98.9 \%$.

Of note, a widely-publicized 2017 Pew Research Centre study, Behind the Badge (see Morin et al. 2017) that interrogated some of the same front-line policing issues as the present study, received 7,917 completed online surveys from the 57,062 officers invited to participate (serving with 54 police and sheriff's departments across the United States) - for a response rate of 13.9\%. ${ }^{26}$ Paoline and Terrill (2014: 95), in providing guidance on surveying patrol officers, observe,

The administration of the patrol officer survey, across seven police agencies (of which many had separate patrol stations) was an arduous task... While it would have been more efficient and inexpensive to utilize an electronic mail survey from our home universities, we feared the response rate would have suffered. Instead, our research team chose to visit each of the sites, over an extended period of time, to administer the survey face to face.

\section{Study Sample Characteristics}

The following table presents information describing the study's research participants (collectively) - in relation to the total sample (Canada and the USA). ${ }^{27}$

Table 4: Sample characteristics (total sample)

\begin{tabular}{|c|c|c|c|}
\hline \multicolumn{2}{|c|}{} & $\mathbf{N}$ & $\mathbf{\%}$ \\
\hline \hline \multirow{3}{*}{ Gender } & Male & 2997 & $82.0 \%$ \\
\cline { 2 - 4 } & Female & 660 & $18.0 \%$ \\
\cline { 2 - 4 } & Total & 3657 & $100 \%$ \\
\cline { 2 - 4 } & Missing & 3 & \\
\cline { 2 - 4 } & White & 2910 & $80.0 \%$ \\
\hline
\end{tabular}




\begin{tabular}{|c|c|c|c|}
\hline & Black & 222 & $6.1 \%$ \\
\hline & Hispanic & 110 & $3.0 \%$ \\
\hline & Asian & 102 & $2.8 \%$ \\
\hline & Aboriginal & 68 & $1.9 \%$ \\
\hline & Mixed & 98 & $2.7 \%$ \\
\hline & Other & 126 & $3.5 \%$ \\
\hline & Total & 3636 & $100 \%$ \\
\hline & Missing & 24 & \\
\hline \multirow{9}{*}{ Age } & $21-25$ & 163 & $4.5 \%$ \\
\hline & $26-30$ & 736 & $20.2 \%$ \\
\hline & $31-35$ & 833 & $22.8 \%$ \\
\hline & $36-40$ & 762 & $20.9 \%$ \\
\hline & $41-45$ & 561 & $15.4 \%$ \\
\hline & $46-50$ & 380 & $10.4 \%$ \\
\hline & Older than 50 & 214 & $5.9 \%$ \\
\hline & Total & 3649 & $100 \%$ \\
\hline & Missing & 11 & \\
\hline \multirow{8}{*}{ Years of police service } & $1-5$ & 990 & $27.1 \%$ \\
\hline & $6-10$ & 1128 & $30.9 \%$ \\
\hline & $11-15$ & 673 & $18.4 \%$ \\
\hline & $16-20$ & 450 & $12.3 \%$ \\
\hline & $21-25$ & 210 & $5.8 \%$ \\
\hline & 26 or more & 200 & $5.5 \%$ \\
\hline & Total & 3651 & $100 \%$ \\
\hline & Missing & 9 & \\
\hline \multirow{8}{*}{ Years of front-line policing } & $1-5$ & 1076 & $29.5 \%$ \\
\hline & $6-10$ & 1370 & $37.5 \%$ \\
\hline & $11-15$ & 659 & $18.1 \%$ \\
\hline & $16-20$ & 346 & $9.5 \%$ \\
\hline & $21-25$ & 128 & $3.5 \%$ \\
\hline & 26 or more & 71 & $1.9 \%$ \\
\hline & Total & 3650 & $100 \%$ \\
\hline & Missing & 10 & \\
\hline \multirow{3}{*}{ Agency location (country) } & Canada & 2686 & $73.4 \%$ \\
\hline & USA & 974 & $26.6 \%$ \\
\hline & Total & 3660 & $100 \%$ \\
\hline \multirow{4}{*}{ Agency location (region) } & western Canada & 801 & $21.9 \%$ \\
\hline & eastern Canada & 1885 & $51.5 \%$ \\
\hline & USA & 974 & $26.6 \%$ \\
\hline & Total & 3660 & $100 \%$ \\
\hline \multirow{4}{*}{ Size of police agency } & Small ( $<100$ front-line) & 691 & $18.9 \%$ \\
\hline & Medium (100-399 front-line) & 1320 & $36.1 \%$ \\
\hline & Large (400+ front-line) & 1649 & $45.0 \%$ \\
\hline & Total & 3660 & $100 \%$ \\
\hline
\end{tabular}




\begin{tabular}{|c|c|c|c|}
\hline \multirow{7}{*}{$\begin{array}{c}\text { Policing environment } \\
(* \text { more than } 1 \text { response }=\text { mixed })\end{array}$} & Rural & 126 & $3.5 \%$ \\
\hline & Suburban & 401 & $11.0 \%$ \\
\hline & Urban & 2623 & $72.0 \%$ \\
\hline & Commercial & 28 & $0.8 \%$ \\
\hline & Mixed* & 467 & $12.8 \%$ \\
\hline & Total & 3645 & $100 \%$ \\
\hline & Missing & 15 & \\
\hline \multirow{7}{*}{$\begin{array}{c}\text { Type of police work } \\
\left({ }^{*} \text { more than } 1 \text { response }=\text { mixed }\right)\end{array}$} & Primary Response/General Patrol & 3162 & $86.5 \%$ \\
\hline & Community-Based & 109 & $3.0 \%$ \\
\hline & Foot/Bicycle Patrol & 87 & $2.4 \%$ \\
\hline & $\begin{array}{l}\text { Other (gang intervention, traffic } \\
\text { enforcement, school liaison, tactical, } \\
\text { canine, etc.) }\end{array}$ & 74 & $2.0 \%$ \\
\hline & Mixed* & 223 & $6.1 \%$ \\
\hline & Total & 3655 & $100 \%$ \\
\hline & Missing & 5 & \\
\hline
\end{tabular}

\section{Data Site Information}

Spanning 16 February 2016 to 11 December 2016, I spent 122 days in the field, administering survey questionnaires to the rank-and-file at the study's 23 data sites and discussing contemporary policing issues with many front-line officers, police command staff, police labour representatives, police governance leaders, elected officials, and members of the public. Appendix C presents information, if such information exists, on each data site in relation to: (1) population, violent crime rate, economic conditions, and the racial/ethnic composition of the community, (2) number of sworn officers, number of officer engaged with front-line duties, and select local policing circumstances/issues - including specific signal incidents for each location, and (3) the dates and locations of survey administration and any particularly noteworthy events that I encountered during data collection at each site. ${ }^{28}$

\subsection{Data Analysis}

Olson (2011: 77) articulates, "The objective of the analytical phase [of the research project] is to sift through all of the data... and use the links that run through 
these data sources to construct an answer to the research question." The counsel of various social sciences methods scholars were considered throughout decision-making around how the present study's data would be analyzed (e.g., Berg 2009, Charmaz 2004, Creswell \& Plano-Clark 2007, Denzin 2004, Denzin \& Lincoln 2005, Glaser 1978; Glaser \& Strauss 1967; Kirby et al. 2006, Hesse-Biber \& Leavy 2006, Noaks \& Wincup 2006, Olson 2011, Onwuegbuzie \& Teddlie 2003). Given the study's mixed methods approach, both quantitative and qualitative analytical frameworks were necessary, which presented the opportunity to realize both exploratory and confirmatory modes of analyses (Onwuegbuzie \& Teddlie 2003).

\section{Organizing the Study's Data}

In situations where study participants declined (or neglected) to provide any response to a question in the survey, no value was entered during inputting of survey data from the paper questionnaire documents into the quantitative data management software. This 'missing' data accounts for differences in the numbers of respondents for various questions. In this regard, some interesting observations emerged. For example, 24 participants did not record an answer to Question 3 (identifying their race/ethnicity). ${ }^{29}$ Also, 36 participants that answered 'Yes' to Question 25 (inquiring into an officer's perception of elevated risk related to characteristics of individuals with whom the officer interacts) left the follow-up question (Question 25a) blank, which asked the respondent to identify the specific characteristic(s) they find are 'risky'. In the same vein, 21 participants that answered 'Yes' to Question 25 did not record any answer to Question 26, which asked the respondent whether they do anything to avoid interacting with persons they perceive as presenting an elevated risk. ${ }^{30} \mathrm{I}$ argue that these 
latter two circumstances, which will be discussed in more detail throughout Chapter Six, likely demonstrate that, for some officers, the practice of de-policing is a socio-political and/or workplace 'hot potato' - most particularly as relates to race/ethnicity. This may represent a tangible demonstration of social desirability bias - with some in the sample population being reluctant to admit to what many across contemporary society likely consider 'unacceptable' behaviour for police officers.

In relation to Question 3 of the survey, research participants were invited to identify their race/ethnicity with a write-in response. No pre-selected categories were presented, for two reasons - (1) to not restrict how each participant would choose to self-identify and, (2) because across the social sciences literature there is nothing approaching consensus on race/ethnicity classifications. ${ }^{31}$ For the purposes of the present study's data analysis, I decided that it was best to assign participants' Question 3 responses within seven race/ethnicity categories - 'White', 'Black', 'Hispanic', 'Asian', 'Aboriginal', 'Mixed', or 'Other'. I suggest that this simplified approach best addresses the variances throughout the literature and harmonizes the Canadian and American differences in race/ethnicity categories, in terms of official census data. In situating a small number of participant's race/ethnicity identifications (those that did not specifically record one of the seven keywords corresponding to the study's categories), I had to apply subjective interpretation on a case-by-case basis. ${ }^{32} 33$

In relation to the attribution of qualitative data in the chapters that follow, it is my view that reporting on a participant's gender, age, race/ethnicity, years of overall and/or front-line policing experience, type of police work, and/or policing environment could inadvertently identify a specific participant as the source of information, which would 
compromise the study's anonymity assurances and violate principles of ethical research. Nix and colleagues (2017: 5) caution policing researchers in this regard, observing, "For example, a female Lieutenant with 16 years of service in Division 'A' could easily be identified if there are only one or two officers who fit those demographics." ${ }^{34}$ Therefore, for the sake of consistency and to ensure study participants' anonymity, the qualitative information presented in the following chapters, will be attributed only through the participant's assigned alpha-numeric coding (by their police agency and their assigned participant number within that agency). ${ }^{35}$

\section{Data Saturation and Quantification of the Qualitative Data}

Given the comprehensive nature of this investigation and the size of the sample, I was hoping to achieve redundancy in the study's mixed methods data. In this regard, the concept of data saturation, which is often considered in qualitative studies, is the point when additional data fails to yield new information (Bryman 2012; Guest, Bunce \& Johnson 2006; Mason 2010; Morse 1995; Sandelowski 1995; Strauss \& Corbin 1998). In terms of qualitative data saturation in the present study, while it was clearly achieved much sooner than with the analysis of the last survey, I advance that it is advantageous to be able to present an element of quantification of the study's qualitative data. There are various opinions on this and, in this regard, Mason (2010) observes, "There is an obvious tension between those who adhere to qualitative research principles, by not quantifying their samples - and those who feel that providing guidance on sample sizes is useful."

In my view, it is one thing for a researcher to report that a certain type of information (a theme) was disclosed by 'some participants' or 'many participants', but 
quite another matter to present to readers, that will be assessing the validity of the study's findings, the precise number of participants that provided such information. For example, does a researcher's characterization of 'some' represent $0.5 \%, 5 \%$, or $50 \%$ of the sample? Similarly, does 'many' mean $20 \%$ or $80 \%$ ? In other words, I believe that offering precision in the presentation of results, through the quantification of qualitative data, assists with better understanding of study findings. Some scholars (see, e.g. Bryman \& Bell 2003; Guest et al. 2006; Mason 2010; McEvoy \& Richards 2006; Silverman 2006) observe that, within qualitative social scientific research, it is a common practice to adopt a degree of 'quasi-quantification'. In this regard, consistent with Silverman's (2006: 296-301) guidance, I present basic tabulations in the presentation of the present study's qualitative data, to provide, for the reader, a basis for appreciating the strengths of the patterns identified in analysis and as a form of measurement, to verify the representativeness of the themes I identify as significant, emerging from data analysis.

\section{Randomness of the Sample and Generalizability}

As has been discussed at a few points throughout this chapter, a limitation inherent to the present study's data collection regimen is that the sample was not random, which statisticians often advance is a situation that constrains the generalizability of a study's findings. I accept this premise, in the context of strict statistical conventions, however, I argue that, given this study's considerable sample size, its proportion of the sampling frame, and the collection of data from research populations at 23 discrete sites, I am in a position to assert, with a high degree of confidence, but, as stipulated, not with exacting statistical conviction, that this sample is 
almost certainty representative of the population of Canadian and American front-line police officers. Hayle, Wortley \& Tanner (2016: 329), in advancing a similar argument in their study of Toronto street youth, wrote,

Admittedly, our sample was not random, and we cannot estimate to any degree of confidence how representative, exactly, it may be of the wider street youth population in Toronto. As a result, we endeavoured to compensate for the non-representativeness of the sample... [by] striv[ing] to collect as large a sample of street youth as possible - and indeed, to our knowledge, it is the largest sample of street youth ever produced by Canadian researchers.

As presented earlier in this chapter, Paoline and Terrill (2014) followed a similar strategy in their sampling of front-line officers and Nix and colleagues (2017: 14-15, citing Blair et al. 2014; Shadish, Cook \& Campbell 2002;) are also instructive on this point,

Larger sample sizes are important for increasing statistical power. Additionally, most police surveys are conducted with nonprobability samples. However, when relying on nonprobability samples, greater sample heterogeneity (e.g. recruiting officers from different agencies) is critical for reducing sampling bias and increasing generalizability of findings.

\section{Quantitative Data Processing and Analysis}

As defined by Biemer and Lyberg (2003: 215), "[Quantitative] data processing is a set of activities aimed at converting the survey data from its raw state as the output from data collection to a cleaned and corrected state that can be used in analysis, presentation, and dissemination." Through manual inputting, the study's quantitative data was replicated from the 3660 paper survey documents into survey data management software (acquired, under licence, from Snap Surveys). Within this software's organizing, processing, and analytical functionality, the raw quantitative responses of research participants were arranged in a more coherent, or user-friendly, 
state to facilitate subsequent analysis. Upon completion, the data file, in its entirety, was then ported into statistical analysis software (IBM SPSS version 25, under licence).

For this study's statistical analysis, an alpha of $0.05(\alpha=0.05)$ was used as the level of confidence - or, in other words, as the cut-off for significance determinations. That being said, as the reader will observe throughout Chapter Five, almost all results were found to be statistically significant, even at $p \leq .01$. Pearson's chi square tests $\left(\chi^{2}\right)$ and Cramer's V were employed to examine bivariate relationships - to identify whether statistically-significant associations exist between specific aspects of the identified depolicing phenomenon and the variables considered in this study.

\section{Qualitative Data Analysis}

The study's qualitative data was reproduced verbatim (into MS Word word-

processing software) from research participants' handwritten submissions, exactly as recorded on the 3660 paper surveys. Each respondent's qualitative contribution was 'stamped' with that participant's unique alpha-numeric code - consisting of a prefix for the police agency (i.e. BUF for the Buffalo Police Department) and the number assigned by the Snap Surveys software for each participant's questionnaire - beginning sequentially at 01 for each agency. For example, BUF36 represents the participant corresponding to the $36^{\text {th }}$ Buffalo survey entered into the software from among the pile of 317 Buffalo surveys.

Participants' qualitative data was considered in relation to each survey question. While the study invited participants to respond to 30 questions, not all were ultimately relevant to the limited scope of this dissertation (however, this information will be integral to future research and publications). After qualitative data was inputted into 
analytical documents organized by question number, I examined each on a question-byquestion basis, using a two-stage system of initial analysis and reflection and then formal coding and organization, prioritization, and categorization in relationship to the study's research questions and identified emergent themes.

During the first stage of this analysis process, I allowed my thoughts to be directed by the data itself and, in doing so, I was able to detect emerging patterns, key concepts, and linkages to existing literature and theoretical frameworks - those that were considered in the study's conceptualization and those that emerged for potential consideration during data analysis. As Saldaña (2013: 146) counsels, I avoided a strict (or inflexible) coding regimen because otherwise "preconceptions of what to expect... may distort [the researcher's] objective and even interpretive observations of what is ‘really' happening." In this regard, I followed the guidance of Olson (2011: 72-73), in first working toward recognizing and establishing in vivo codes from the words of participants themselves, and then subsequently developing preliminary codes, through analysis of the materials in their totality. Such an approach is consistent with that employed in a recent study by Huey and Broll (2015: 240), who, in their analysis, followed Glaser's (1978) concept-indicator model, “looking first for key concepts and then for words and phrases that functioned as indicators of that concept."

The next stage of qualitative analysis involved more in-depth coordination and analysis in the context of the study's specific research questions and any potentiallyapplicable theoretical/conceptual frameworks. This second, and more formal, analytical process involved coding organized bits of data, based on similarities and applicability of salient themes (Charmaz 2004; Kirby et al. 2006; Miles \& Huberman 1994; Noaks \& 
Wincup 2006; Olson 2011; Schulenberg 2007). Through the use of axial coding, clear themes became readily apparent and these were organized in the grouping of patterned data into memos. Scott and Medaugh (2017) advance, "Axial coding requires researchers to continually modify and reshape their emerging conceptual framework as more data are examined. It results in a coding framework from which to synthesize and organize data into more coherent, hierarchically-structured categories.” These formed the basis for the presentation of qualitative study data throughout Chapter Five and the focused discussions around certain study findings throughout Chapter Six.

\subsection{Challenges}

\section{Barriers to Accessing Data in Policing Research}

The methodological problems that "confound efforts to study the clandestine behaviour of the police" (Holmes \& Smith 2008: 9) are well known to policing scholars (see, e.g. Brown 2013, 2016, 2017; Brown \& Primeau 2015; Dawson \& Williams 2009; Loftus 2009; Marks 2004; Marks, Wood, Ali, Walsh \& Witbooi 2010; Reiner 2000a; Rosenbaum 2010). In assessing the historical circumstances of access barriers to policing research, Perez and Shtull (2002: 170) observe that "outside meddling" by academics was unwelcomed within the "universally accepted idea that nobody understands police work that has not been on the streets" and, as a result, researchers were often unable to "penetrate the walls of...a closed [policing] fortress" (Peak 1997: 142). Similarly, Loftus (2009: 201-202), in her research on police occupational culture, finds, "The problems of access and trust, which are integral to most social science research, are particularly intensified in police research...Researchers have long 
documented the challenges and issues associated with gaining access to the police for academic study.” Along the same lines, Nix and his colleagues (2017: 1) find,

Over the 50-year history of police research, the characteristics of the researchers involved (e.g. 'inside insiders' versus 'outside outsiders'), their agendas (e.g. supportive versus critical), and the methods used have varied considerably... [however] one constant has been the enormous formal and informal challenges to gaining access to police officers for research purposes.

As discussed earlier, the first step in any study seeking to involve front-line officers (through surveys, interviews, observation, etc.) is obtaining 'official' approval from senior administration of the police agency. With the gatekeeping positioning of police leadership over access to data, including a monopoly on decisions to grant or deny permission to speak with on-duty personnel at police facilities, what amounts to a unilateral and unaccountable 'veto power' can present as an insurmountable challenge, in the form of an impenetrable obstacle, to front-line policing research (e.g., see Bradley, Nixon \& Marks 2006; Brown 2016, 2017; Dawson \& Williams 2009; Loftus 2009; Paoline \& Terrill 2014; Peak 1997; Reiner 2000a). In this regard, KutnjakIvkovic (2014: 328) finds, "Police chiefs may be reluctant to open their doors to researchers because of their fear that the results will create serious problems for them." Similarly, Rosenbaum (2010: 144) observes, "Police executives find themselves facing intense external scrutiny and litigation. In this context, the last they want is a group of critical researchers. ${ }^{.36}$

In addition to obtaining cooperation from police leadership, it is often necessary for the policing researcher to secure endorsement from the police labour organization that represents the potential rank-and-file research population (e.g., see Fisk \& SongRichardson 2017; Marks 2007; Morabito 2014; Nix et al. 2017; Reiner 2000a; Skogan 
2008, 2015; Walker 2008; Wilson \& Buckler 2010; Wortley \& Marshall 2005). In situations where leadership of the police labour organization opposes officers' participation in research that is being supported by the police administration, researchers can find themselves in an ethical predicament - related to issues around voluntariness and consent. My uncomfortable experiences in this regard are elaborated later in this chapter.

The experiences of other policing researchers (e.g., see Davis et al. 2005; Fox \& Lundman 1974; Goold 2004; Ericson 1982; Loftus 2009; Marks 2004; Paoline \& Terrill 2014) corroborate my own observations in previous studies (see Brown 2013, 2016, 2017; Brown \& Primeau 2015), in terms of discovering that earning the trust of frontline officers, as potential research participants, can present even more significant challenges than those encountered in obtaining 'official' approval - particularly for research seeking to examine the innermost thoughts and workings of policing's rankand-file occupational subculture. In this regard, both Ericson (1982) and Marks (2004) found that officers at the lower ranks are the most resistant to being investigated by academic researchers. In the present study, penetrating these barriers to trust presented as a significant consideration in the research design, given that the research involved inquiring into the largely private (and personal) views and behaviours of individual front-line officers - including actions that could be regarded, by some, as police misconduct. As will be elaborated in Chapter Six, I suggest that it was by establishing a mutual trust relationship with front-line officers I met during this study, and, thereby accessing data that is likely inaccessible to some other policing researchers, that this 
project makes a unique contribution to sociological inquiry and to the limited scholarship in this important area of contemporary social relations.

In a related consideration, Nix and colleagues (2017: 5) observe, "While administrative approval may be required simply to gain access to the officers, it may be seen by line-level officers as a reason not to respond." Similarly, Loftus (2009: 203), in corroborating the previous findings of Ericson (1982), suggests, "Being granted formal access by senior officers can in fact heighten the suspicions of the lower ranking members of the police organization. Although I had been granted access by senior officers, accomplishing the trust of the rank and file was something I needed to negotiate on a daily basis." Canter (2004: 118) also found, "Academics [often] make the mistake of assuming that because something has been authorized by senior police officers there will inevitably be support for it from the rank and file."

As mentioned previously, it is axiomatic to observe that police occupational culture - particularly at the rank-and-file level - involves a code of silence among officers, an inherent suspicion of 'civilians', and a distrust of academics (Blake 1981; Brannick \& Coghlan 2007; Chan 2007a, 2007b; Chappel \& Lanza-Kaduce 2010; Ericson 1982; Loftus 2009, 2010; O’Neill et al. 2007; Reiner 2000; Rosenbaum 2010; Skolnick 1966; Wortley 2006). This mindset and worldview impact significantly on the ability of researchers to acquire meaningful data; a situation that is even more pronounced in studies inquiring into certain aspects of policing - particularly those probing the inside workings of occupational culture, interpersonal issues, and conflict. For example, in their study of front-line Pittsburgh officers' responses to federal oversight, including officers disclosing their practices of de-policing, Davis, Henderson 
and Ortiz (2005: 9) found, "In each administration of the survey, some officers expressed suspicions that the answers might be used against them in spite of the [study's] promise of anonymity." ${ }^{\prime 37}$ This presents a good illustration of the concept of social desirability bias and, in this regard, Kutnjak-Ivkovic (2014: 328) observes, "Because of the nature of the beast, collecting data in a direct way could be troublesome; police officers...may be reluctant to discuss their experiences and engagement in police misconduct, even when researchers promise them confidentiality and/or anonymity."

Given my former practitioner experience and my ongoing interactions, over the past eight years, with many Canadian police leaders through, for example, my presentations for, and discussions with, the Canadian Association of Chiefs of Police, I anticipated that my 'insider, or more accurately my 'pracademic', positioning might facilitate less challenging experiences with securing access to research participants. As discussed, 'insiders' can, in certain instances, overcome traditional research obstacles, including gatekeeper exclusions (Labaree 2002). While no police leader that I spoke with during the present study overtly suggested that they were approving access because of my 'pracademic' status, I believe it would be naïve to think it was not instrumental in at least a few, if not several, instances. I say this with confidence because in my recent research, involving twenty Canadian police leaders (chiefs and deputy chiefs), I found,

[Fifteen of the twenty] police commanders expressed anxieties related to...the granting of access into police organizations (to personnel and/or to internally-held materials) for external research [and] eight commanders expressed unequivocal reservations around studies that might delve into contentious areas of policing. Further, [twelve] advanced apprehensions around research proposals submitted by academics with a 'critical' or 'radical' theoretical orientation and/or with track records of scholarship that has been unfavourable to police (Brown 2017: 532-533). 
In this regard, I submit that while being situated within the general research setting of operational policing presents some potential fodder for skeptics to pose challenges related to objectivity, which I argue have been addressed through this study's rigorous protocols and reflexivity practices, it also presents unique opportunities at entry into a secretive subculture, access to honest and unreserved data that is not normally available to academic researchers, and insights that informed both the study design and then analysis. Greenhill (1981: 91), from his position as a sociologist working within a police agency, is instructive; his positioning being "an unusual one in relation to both sociology and police, [but permitting] certain insights which are perhaps less accessible from other perspectives." During my 2012-2013 study, many rank-and-file officers that participated in interviews (from Toronto Police and Ottawa Police) told me that they would not have participated in surveys and/or interviews, or alternatively would have outright lied or provided 'superficial', 'politically-correct', 'neutral', 'meaningless', 'bare minimum', or 'by-the-book' answers to the questions, had they not determined, in their own minds, that they could trust me and my objectives in conducting the research (Brown 2013, 2016).

\section{Researcher 'Positionality' and 'Insider', 'Outsider', and 'Pracademic' Research}

As discussed earlier, and elsewhere (Brown 2013, 2016, 2017), I address my policing research with the benefit of considerable experience as an operational police officer. In this dissertation, the inclusion of first-person observations, which I have presented for context at certain junctures, emerges from observations during my police work and my continued associations within policing. I view this aspect of the research as being consistent with the traditions of the classic police ethnographies - those 
important studies that examined police occupational culture, often with a micro-social focus (e.g., see Banton 1964; Barker 1999; Bayley 1985; Bayley \& Mendelsohn 1969; Bittner 1970; Cain 1973; Chan 1997; Ericson 1981, 1982; Holdaway 1983; Loftus 2009; Manning 1977, 2003; Muir 1977; Niederhoffer 1967; Reiner 1978; Reiss 1971, 1983; Reuss-Ianni 1983; Rubinstein 1973; Sheptycki 1993; Skolnick 1966; Van Maanen 1973, 1974; Waddington 1999; Weisburd \& Braga 2006a, 2006b; Westley 1970; Wilson 1968; Young 1991). By adopting the position that my 'pracademic' observations are relevant and that my 'pracademic' insights allow for a more informed analysis, my history within the police occupational culture becomes instrumental to theorizing the type of policing research undertaken in this study. In other words, since being a 'street cop' is fundamental to this research approach, it can be situated as a positive contribution to the study, rather than as presenting methodological dilemmas or as the foundation for challenges to the study's validity.

Given my 'pracademic' positioning in this project, a succinct presentation of some particularly germane scholarly positions on 'insider/outsider' research should be instructive for the reader. In this regard, Denzin (2004: 464) describes a research model that "begins and ends with the biography and the self of the researcher. The events and troubles that are written about are the ones the writer has already experienced and witnessed firsthand." Similarly, Flyvbjerg (2001: 83) finds, “The most advanced form of understanding is achieved when researchers place themselves within the context being studied. Only in this way can researchers understand the viewpoints and the behaviour which characterizes social actors." 
Historically, 'insider' research has been treated as suspect - a position taken by some academics arguing that such circumstances present methodological challenges based on perceived lack of objectivity - in the context of paradigmatic disputes and debates on "knowledge creation and transfer between practitioners and academics" (Brannick \& Coghlan 2007: 61, citing Rynes, Bartunek \& Daft 2001). The outsider doctrine, in what some scholars consider to be a dichotomous contrast to that of the insider doctrine, can be characterized as exemplifying the tenets of positivism and notions of the scientific ideal in the neutral, distanced, and detached researcher. Such a philosophical understanding coincides with Simmel's (1950) argument that "only the neutral outsider can achieve an objective account of human interactions" (Mercer 2007: $5)$.

In their advocacy for insider research and the repudiation of Morse's (1998) suggestion of an 'inherent incompatibility', Brannick and Coghlan (2007: 72) find, "In our view, insider research is not problematic in itself and is respectable research in whatever paradigm it is undertaken." Stanfield (2011: 286), in rejecting the insider/outsider dichotomy in sociological research, argues, "Only those researchers emerging from the life worlds of their 'subject' can be adequate interpreters of such experiences.” Similarly, in describing Merton's observations, Labaree (2002: 100) offers,

The insider-outsider position [is] an epistemological principle centered on the issue of access...In this framework, the insider is an individual who possesses intimate knowledge of the community and its members due to previous and ongoing association with that community and its members. The general assumption is that this intimate knowledge offers insights that are difficult or impossible to access by an outsider. 
Merton (1972: 15) characterized the outsider as "[having] a structurally imposed incapacity to comprehend alien groups, statuses, cultures and societies... [because the researcher]...has neither been socialized in the group nor has engaged in the run of experience that makes up its life." In assessing the value of 'insiderness', Labaree (2002: 103) observes that such researchers can have privileged access to information otherwise unobtainable and this positionality is, therefore, valuable in some research initiatives - through revealing "the unseen reality" of certain phenomena, exposing otherwise "hidden truths the public is unaware of", and presenting "a unique understanding that is not otherwise achievable by an outsider." As discussed earlier, some scholars (e.g., see Brown 2013, 2016, 2017; Brown \& Primeau 2015; Ericson 1982; Hsiung 1996; Labaree 2002; Loftus 2009; Marks 2004; Rosenbaum 2010) find that research benefits are realized because a researcher's insider status contributes to establishing initial levels of trust with participants, which can result in the sharing of more information in more open exchanges with the researcher. In our recent conversation, Canadian policing researcher and Carleton University psychology professor Craig Bennell (personal communication, 5 February 2018) advised me that he has found it beneficial to rely on former police practitioners (properly trained in research techniques) to interview front-line research participants because they are more trusted than academics and, therefore, can elicit more honest and comprehensive data.

As should be clear from the way this research project was conducted, I subscribe to the body of scholarship that supports the value of 'pracademic' (or 'insider') research and appreciate, in the final analysis, the significant advantages this positionality afforded me in the particular circumstances of this study. C. Wright Mills (1959: 195- 
196) perhaps most eloquently described my views, in his address to prospective social scientists,

The most admirable thinkers within the scholarly community you have chosen to join do not split their work from their lives...Scholarship is a choice of how to live as well as a choice of career... What this means is that you must learn to use your life experience in your intellectual work: continually to examine and interpret it.

\section{Specific Challenges Encountered in this Study}

As discussed earlier in this chapter, I experienced challenges while attempting to secure the participation of rank-and-file officers with SPVM, PRPS, and YRPS. These circumstances require no further discussion, however, the situations I encountered in relation to the VPD, VPU, WPA and YPBA, do require some elaboration and the reader's consideration. ${ }^{38}$ Van Maanen's (1978: 311$)$ experience is instructive in this regard, of which he wrote, "Access is continually problematic for the field researcher. Entry into the police system is no guarantee that one will be allowed to remain."

\subsection{Chapter Conclusion}

Engel and Cohen (2014: 392), in their policing research, advance, "We agree with Piquero (2009: 376) that 'there should be a high priority on focused theoretical and research efforts that use multiple methods to generate a careful description and understanding of police-citizen encounters, as well as myriad factors that influence both police and citizen decisions'." As described throughout this chapter, I argue that the methodology employed in the present study has set the stage for just such a 'careful description and understanding of police-citizen encounters' in the specific context of police decision-making and behaviours - vis-à-vis 'policing’s new visibility', unprecedented public critique of policing, risk, and the concept of de-policing. 


\section{CHAPTER FIVE}

\section{ANALYSIS, RESULTS, AND FINDINGS}

\subsection{Chapter Introduction}

Within the previous chapter, study participants' individual descriptors and police agency information were reported. Here, in subchapter 5.2, quantitative data, in terms of participants' 'Yes' or 'No', Likert-type scale, and multiple-option responses to survey questions, are presented through descriptive statistics - with specific relevance to this dissertation's focus on the phenomenon of risk-averse behavioural adaptations across the rank-and-file (data for this dissertation emerges from a broader study investigating several aspects of today's front-line policing in Canada and the USA). The reporting of descriptive statistics within this subchapter is most logically facilitated through thematic groupings of study participants' responses to relevant questions in the survey instrument - those that contribute to an empirical understanding of the extent and nature of depolicing throughout today's front-line police work (with this sample population).

The eight principal (or broadly overarching) themes identified are: (1) officers' awareness of 'policing's new visibility'; (2) officer's thoughts about, and behavioural adaptations to, 'policing's new visibility'; (3) officer's use of force practices in relation to 'policing's new visibility'; (4) officer's resistance to 'policing's new visibility'; (5) officer's perceptions as to the nature of today's public, vis-à-vis policing; (6) officers' perceptions of risk for criticism/complaint based on 'demographic' characteristics presented by individuals with whom officers interact in the community and, therefore, avoidance of interaction with such individuals; (7) officer's familiarity, and experiences, with the concept, and/or the practice, of de-policing; and (8) influence (if any) of my 
'pracademic' background on study participants' responses to survey questions.

Following, in subchapter 5.3, the results of bivariate statistical analysis are introduced specifically in relation to: (1) de-policing and variables of interest related to front-line officers; (2) rank-and-file avoidance of interactions with certain persons within the community; and (3) influence of the researcher's biography on the nature of data received from front-line officers in empirical studies of behaviour(s) and the rand-andfile police occupational subculture.

After deliberation around how to present the study's qualitative data, and in order to maintain consistency with fundamental tenets of mixed methods research, I decided to address both the study's quantitative and qualitative data within an integrated results and findings chapter rather than in separate treatments. As described in Chapter Four, during the process of qualitative data analysis, on a question-by-question basis (for those survey questions that invited the participant to provide a written elaboration), clear patterns emerged and it became apparent that these qualitative responses were inextricably intertwined with the study's quantitative results. Further, many of the identified themes (that materialized during analysis of qualitative data) were observed to be relevant to the literature reviewed in preparation for, and throughout, this research project and to various conceptual/theoretical frameworks being considered to inform this study's discussion (as described throughout Chapter Three).

Therefore, in this chapter, qualitative information provided by participants (their handwritten elaborations to survey questions) is presented within the eight principal themes identified in relation to the study's quantitative data. As a result, throughout subchapter 5.2, and in relation to each of the six themes for which qualitative data was 
collected, the most prevalent codes, which I refer to as 'subthemes' (or further refinements within the study's principal themes) are reported through representative and verbatim observations of study participants. ${ }^{1}$ Given the large amount of data in this study and the limited scope of this dissertation, which specifically examines de-policing throughout today's front-line police work, subtheme data presented in subchapter 5.2 is focused on this particular topic - with a particular emphasis on de-policing behaviours disclosed within officers' responses to Questions 16, 24, 26 and 28. In saying this, sufficient representative written elaborations, ranging between two and twelve depending on the breadth of issues disclosed within each subtheme (cogent subthemes within the subtheme, if you will), the homogeneity of responses, and relevance in relation to the study's principal theme of de-policing and its research questions - are also presented within each of the other identified subthemes (those not specifically related to de-policing thoughts and behaviours) in order to give the reader an accurate and comprehensive understanding of participants' reported thoughts and behaviours on all of the considerations engaged in this empirical research. ${ }^{2}$

Further, as the reader will discover when considering participants' qualitative elaborations that have been categorized within subtheme 6 for Question 16 (and then recurring in various subthemes for other survey questions - particularly subtheme 4 for Question 24), an unanticipated finding emerged during data analysis, which I argue constitutes a similar phenomenon to that of de-policing, as the term has been defined for the purposes of this dissertation. This behavioural adaptation to the contemporary external policing landscape, among a not-insignificant number of today's rank-and-file, does not involve the avoidance of proactive police work (as with de-policing), but rather 
the avoidance of aspects of reactive police work - such as avoiding certain types of dispatched calls and declining to take action in relation to particular situations (and the persons involved therein) that constitute violations of various statutes (including criminal offences). Also, as addressed within Chapter Four, for each thematicallysimilar grouping of qualitative responses, the reader is presented with specific counts of study participants ('quantification') in order to be in a position to evaluate the prevalence of each identified subtheme across this research population. The reader should also understand that survey responses written in French $(\mathrm{N}=144)$ have been translated to English (by a court-certified professional translator contracted for this task) and, therefore, these should be considered accurate but not necessarily verbatim.

In subchapter 5.4, I present what I argue can be considered corroboration of this dissertation's de-policing thesis, through findings from sources external to this empirical study. In this regard, it is impossible to prove to any scientifically-acceptable standard (at least in the positivist understanding) a correlation, much less causation, between the nature of 'on-the-ground' interactions between police officers and citizens (or the absence thereof - as is the case with de-policing) and decreases, or, for that matter, increases, in a variety of police metrics - as generated by police agencies and purported to relate to officers' work in field. This is due to the limitations within research methods (of any type, including mixed methods) to identify, isolate, and/or control for the multitude of, and complexities within, variables implicated in each such social interaction (or non-interaction). This being said, I, nonetheless decided it would be worth some extra effort to try to assess, in a general, perhaps suggestive (and possibly persuasive), but certainly not scientific sense, whether, or not, today's de- 
policing by front-line officers, as observed in this dissertation's results, is yet having any demonstrable impact in the community - as one might expect and as might now be revealed through official records generated by most police agencies in today's information age.

In this sense, while traditional and online media has previously presented limited and largely anecdotal information on de-policing (as discussed throughout Chapter One and Chapter Two) and, more recently, some media contributions have discussed dramatic drops in arrests, citations, traffic stops, street checks, and so forth, I was, at the time of this writing, unable to locate anything reported that could be considered a compelling analysis of official police records in the specific context of de-policing. Therefore, I sought out to obtain this kind of data through the filing of Access to (or Freedom of) Information requests (the terminology and the specific legislation, varies by jurisdiction but hereafter it is referred to as FOI), pursuant to existing statutory provisions and through official channels. This was pursued with a selection of six police agencies that participated in this study, chosen by random draw (Buffalo, Calgary, Halifax, Ottawa, Rochester, and Toronto). I also requested information from two police agencies that did not participate in the study (Winnipeg and Vancouver) to investigate whether there are any noteworthy differences between agencies that participated in the study versus those that declined to do so (to be fair it was the police labour organizations in those two cities that were the catalysts behind the non-participation). In addition to this FOI-generated police data, also reported in subchapter 5.4 is information recently obtained by journalists in relation to officers' proactive street checks activities in Edmonton (Wakefield 2018) and Toronto and Peel Region (Doucette 2018). ${ }^{3}$ The 
reader should also recall the information around, and the graphical representation of, dramatic declines in investigative street checks of 'suspicious' persons by Chicago Police officers, as presented in Chapter One.

\subsection{Descriptive Statistics}

\section{Theme 1: Front-line officers' awareness of 'policing's new visibility'}

Questions 10 through 14 in the survey instrument were conceived in order to elicit data from study participants that would allow for a comprehensive understanding as to the extent of the penetration of 'policing's new visibility' into the consciousness of today's front-line officer and, further, to work towards determining the degree to which this contemporary techno-social phenomenon has infiltrated the occupational subculture of policing's rank-and-file. It was thought that the development of such a large data set in this regard would further inform, and test the validity of, my previous empirical investigation, which addressed front-line behavioural adaptations to 'policing's new visibility' - focused particularly on officers' use of force practices (Brown 2013, 2016). Based on data gathered from the present study's research participants $(\mathrm{N}=3611)$, it is apparent that the video recording of front-line officers, as they perform their police work in public view within the community, is essentially a universal experience that occurs across a wide variety of circumstances - from dynamic life-and-death use of force occurrences, to interactions with resisting suspects during arrest, to simply dropping by a coffee shop on a work break, or grabbing a 'to go' sandwich in between calls for service. Only 45 officers (1.2\% of the study population) indicated that they are not aware of an occasion during which they were video recorded by a citizen. It should be noted that almost all of these 45 officers are those with limited time in front-line 
police service (including, for example, one respondent from the Buffalo Police Department who completed the survey during 'roll call' on their first shift) and therefore not many opportunities, as yet, to be involved in occurrences during which they can observe that they are being video recorded by a citizen.

Almost six in ten respondents (59.1\% of the study population) observe that video recording capabilities are essentially always present in the locations where they routinely perform their front-line police work and it is apparent that most of policing's rank-and-file are acutely aware of this pervasive video recording potentiality across their external working environments $(83.5 \%$ of respondents report a level of visibility awareness at 7 of 10 or greater - with almost $1 / 3$ of officers reporting the maximum level at 10 of 10). Discussion about this contemporary video recording potential is a frequent topic of conversation across the rank-and-file occupational subculture, as are discussions about video recorded policing occurrences that 'go viral' across social and traditional media. Almost half of the study's participants observe that these types of conversations come up 'fairly often' and almost one in five officers suggest discussions of 'viral' policing videos are 'often the primary topic of conversation' during interactions with peers. Descriptive statistics relating to Questions 10 through 14 are reported in the following tables (along with specific question wordings as presented in the survey instrument).

Question 10 Has there been an occasion while you were on duty when you became aware that a member of the public (not a media photographer) was video recording you using a mobile phone or similar device?

Table 5: Awareness of video recording by a member of the public

\begin{tabular}{|c|c|c|}
\hline & $\mathbf{N}$ & Valid \% \\
\hline Yes & 3611 & 98.8 \\
\hline No & 45 & 1.2 \\
\hline
\end{tabular}




\begin{tabular}{|c|c|c|}
\hline Missing & 4 & \\
\hline Totals & 3660 & 100.0 \\
\hline
\end{tabular}

Question 10(a) asked study participants, 'If yes [to Question 10], on how many occasions has this happened in your career?' Responses, both numeric and written, ranged dramatically - from ' 1 ', to ' 100 ', to ' 1,000 ', to 'thousands' and significant numbers of officers reported 'Hundreds', 'Too many to count', 'Almost every shift', 'Daily', 'At least once per week', 'Every shift', etc. There were 668 non-numeric responses submitted. The resounding message from this study's research population was that the overwhelming majority of today's rank-and-file officers perceive that they are routinely video recorded (and/or subject to an ever-present potential to be video recorded) as they perform their front-line police work in the community.

Question 10(b) If 'Yes' [to Question 10], in what circumstances has the video recording taken place? ${ }^{4}$

Table 6: Circumstances of video recording by a member of the public

\begin{tabular}{|c|c|c|c|}
\hline & $\mathbf{N}$ & $\begin{array}{c}\text { \% of sample 'Yes' to } \\
\mathbf{Q . 1 0}(\mathbf{N}=\mathbf{3 6 1 1})\end{array}$ & $\begin{array}{c}\text { \% of entire sample } \\
\text { population (N=3660) }\end{array}$ \\
\hline While making an arrest & 3467 & 96.0 & 94.7 \\
\hline $\begin{array}{c}\text { While talking with } \\
\text { another officer }\end{array}$ & 2050 & 56.8 & 56.0 \\
\hline $\begin{array}{c}\text { While on downtime/ } \\
\text { resting between calls }\end{array}$ & 1601 & 44.3 & 43.7 \\
\hline $\begin{array}{c}\text { While stopping for a } \\
\text { meal/coffee }\end{array}$ & 1294 & 35.8 & 35.4 \\
\hline $\begin{array}{c}\text { While talking with a } \\
\text { member of the public }\end{array}$ & 2879 & 80.2 & 78.7 \\
\hline Other & 1231 & 34.1 & 33.7 \\
\hline
\end{tabular}

A total of 758 study participants presented written elaborations to their selection of the category 'Other'. By a large measure, the 'Other' circumstance most identified in respondents' write-in responses were officers being video recorded during vehicle (traffic) stops $(\mathrm{N}=318)$. Following were officers being video recorded while conducting 
suspicious person (street) checks $(\mathrm{N}=149)$; while on public order duties (policing protests and demonstrations) $(\mathrm{N}=78)$; and while driving a police vehicle (not obeying rules of the road, parking in 'no parking' zones, talking or texting on a mobile phone, eating, etc.) $(\mathrm{N}=31)$. No other categories were prominent enough to merit inclusion within this presentation of results.

Question 11 What is your assessment as to the overall potential for video recording of your on-duty activities in the area where you do your police work?

Table 7: Assessment of potential for video recording of on-duty activities

\begin{tabular}{|c|c|c|}
\hline & N & Valid \% \\
\hline Non-existent & 10 & 0.3 \\
\hline Minimal & 149 & 4.1 \\
\hline Exists in some places but not in others & 477 & 13.0 \\
\hline Quite prevalent & 861 & 23.5 \\
\hline Video is everywhere & 2159 & 59.1 \\
\hline Missing & 4 & \\
\hline Totals & 3660 & 100.0 \\
\hline
\end{tabular}

Question 12 How much do you and your police colleagues speak about the potential for video recording of police conduct?

Table 8: Discussion about potential for video recording of police conduct within police occupational culture

\begin{tabular}{|c|c|c|}
\hline & N & Valid \% \\
\hline It is never a topic of conversation & 97 & 2.6 \\
\hline It is discussed on an occasional basis & 1515 & 41.5 \\
\hline It comes up fairly often & 1742 & 47.7 \\
\hline It is often the primary topic of conversation & 298 & 8.2 \\
\hline Missing & 8 & \\
\hline Totals & 3660 & 100.0 \\
\hline
\end{tabular}

Question 13 How much do you and your police colleagues speak about video recordings of policing occurrences that 'go viral' (i.e. widely circulated and discussed in social and traditional media)?

Table 9: Discussion about 'viral' video recordings of police occurrences within police occupational culture

\begin{tabular}{|c|c|c|}
\hline & $\mathbf{N}$ & Valid \% \\
\hline It is never a topic of conversation & 51 & 1.4 \\
\hline It is discussed on an occasional basis & 1162 & 31.8 \\
\hline
\end{tabular}




\begin{tabular}{|c|c|c|}
\hline It comes up fairly often & 1732 & 47.4 \\
\hline It is often the primary topic of conversation & 707 & 19.4 \\
\hline Missing & 8 & \\
\hline Totals & 3660 & 100.0 \\
\hline
\end{tabular}

Question 14 On a scale of 1 to 10 (with the value 1 representing it never entering your mind and the value 10 representing it is something that is always on your mind), while you are on duty, what level of awareness do you have about the potential for video recording of your actions?

Table 10: Level of officers' awareness of potential video recording of on-duty actions

\begin{tabular}{|c|c|c|}
\hline & $\mathbf{N}$ & Valid \% \\
\hline 1 & 24 & 0.7 \\
\hline 2 & 43 & 1.2 \\
\hline 3 & 70 & 1.9 \\
\hline 4 & 89 & 2.4 \\
\hline 5 & 197 & 5.4 \\
\hline 6 & 180 & 4.9 \\
\hline 7 & 437 & 12.0 \\
\hline 8 & 899 & 24.7 \\
\hline 9 & 532 & 14.6 \\
\hline 10 & 1172 & 32.2 \\
\hline Missing & 17 & \\
\hline Totals & 3660 & 100.0 \\
\hline
\end{tabular}

Theme 2: Front-line officer's thoughts about, and behavioural adaptations to, 'policing's new visibility'

Within the survey instrument, Questions 15 and 16 were designed to elicit data around the impact (if any) of 'policing's new visibility' on study participants' thought processes, as they carry out their front-line police work, and to determine whether this recent techno-social phenomenon has impacted on participants to such a degree that they have modified their in-the-field practices in response. Not only are many (six in ten) of today's front-line officers thinking about not doing something (that they otherwise normally would do), because they are concerned about video recording of their on-duty conduct, but the video recording potential accompanying 'policing's new 
visibility' also involves, for $57.3 \%$ of this study's participants, actual behavioural modifications as an adaptation to today's techno-social policing environment.

Descriptive statistics relating to these two survey questions are presented in the following tables (along with the specific question wording) and qualitative subthemes, identified through data analysis, and representative examples thereof (in the form of study participants' written elaborations) are also presented in relation to each of these questions. The reader should understand that the ordering of these qualitative responses is alphabetical (by police agency) and not indicative of importance or frequency (this holds true for the balance of the chapter).

Question 15 Has there been an occasion while you were doing front-line police work when you have thought about not doing something, because of a concern that your actions could be video recorded, that you otherwise would normally do?

Table 11: Officers contemplating not doing something because of a concern that those actions could be video recorded

\begin{tabular}{|c|c|c|}
\hline & N & Valid \% \\
\hline Yes & 2218 & 60.7 \\
\hline No & 1439 & 39.3 \\
\hline Missing & 3 & \\
\hline Totals & 3660 & 100.0 \\
\hline
\end{tabular}

Study participants answering 'Yes' were invited to provide a written explanation for their affirmative response and 1501 front-line officers contributed qualitative data in this regard (therefore, of the 2218 'Yes' quantitative respondents, $68 \%$ presented a written elaboration). Eight (8) subthemes emerged during analysis of this data (these written contributions). These are presented, as follows, in order of prevalence with which they were raised by study participants: (1) officers' considerations around use of force ( $N=447)$; (2) officers' avoidance of, or second-guessing engaging in, proactive/ discretionary activities ( $\mathrm{N}=312)$; (3) officers' apprehensions around misinterpretation of 
their actions and/or uninformed public critique ( $\mathrm{N}=284)$; (4) officers' concerns around language used in interactions $(\mathrm{N}=269)$; (5) officers' concerns/hesitation because of video recording or video recording potential $(\mathrm{N}=155)$; (6) officers' 'non-duty' behaviours $(\mathrm{N}=42)$; $(7)$ officers that discount any video recording influence on their actions in the field $(\mathrm{N}=32)$; and (8) officers that have thought about modifications in behaviour but have not yet changed actions in response to their thoughts $(\mathrm{N}=15) .{ }^{5}$ Many participant's responses to Question 15 were more consistent with answers one would expect for Question 16 - in that participants reported actual behavioural modifications in response to 'policing's new visibility' rather than reporting thinking about not doing something they otherwise would do. Therefore, some responses to Question 15 that describe a behaviour (rather than a thought) are presented within the Question 15 subthemes (below) in order to provide an accurate representation of the range of participants' written elaborations to Question $15 .^{6}$

Subtheme 1: Officers' considerations around use of force $(\mathrm{N}=447)$

Thought about degrees of use of force needed versus what would be likely 'perceived' to be needed (DEL46)

Use of force requires a lot of thought as it never appears 'graceful' (GAT95)

Any hands-on actions are now potentially video [recorded] and [subject to] criticism by media and public so I am very careful now; this is a serious officer safety issue as someone is going to get hurt from hesitating or not doing what they should be doing to handle a situation (NEW14)

Often catch myself hesitating before going hands on - is it worth my career? (OTT275)

More friendly-looking arrests (STR21)

Subtheme 2: Officers' avoidance of, or second-guessing engaging in, proactive/ discretionary activities $(\mathrm{N}=312)$

The potential of videotaping has raised the threshold for arrests. More disruptive behaviour is tolerated (BUF107) 
Second guessing traffic stops or street checks on visible minorities to avoid accusations of racism (CAL12)

Most officers feel under the microscope by the public to the point of questioning if most things are worth doing or generally having a FIDO attitude (CAL229)

Proactive work; we don't do it any more (OTT326)

The more you do the more likely you are to get into trouble (ROC19)

Engaging with visible minorities for anything other than a serious crime (STR20)

I frequently do not stop suspicious people because of the current climate of racial profiling and the optics of the interaction if recorded (TOR34)

The consideration is why engage and have a perceived negative outcome on video when I don't have to (TOR200)

I still get paid if I do nothing (TOR306)

Even if $100 \%$ in the right, why open yourself to the liability that comes with proactive police work? (YON38)

\section{Subtheme 3: Officers' apprehensions around misinterpretation of their actions and/or} uninformed public critique $(\mathrm{N}=284)$

Not my actions that are questionable, but their interpretation by people who are uninformed (ALB99)

Fear of video going viral and being judged by the public. Even though I know my actions are justified, the possibility of being recorded on duty and having it publicized is extremely stressful (CAL352)

Happens all the time now. I think first about how things will look on video before I do anything (OTT70)

Many times and many situations. I think, can this get misinterpreted and get me in trouble? If the answer if yes, I don't do it even if it is something I should be doing in my job (STR05)

Subtheme 4: Officers' concerns around language used in interactions $(\mathrm{N}=269)$

If someone is being uncooperative or verbally abusive, I have not said what's on my mind in case it's unprofessional and caught on camera (CAL189)

The way we speak to people at times using street language (CAL253)

I try to use more professional language, which over all is a good thing (CAL306)

Language - telling the public to 'fuck off' has been drastically reduced (OTT129) 
Subtheme 5: Officers' concerns/hesitation because of video recording or video recording potential $(\mathrm{N}=155)$

I always act within the scope of my authority. But the thought is always there about 'Monday morning quarterbacking' (BUF144)

Makes you second guess what you're thinking to do. Is it valid, how would the public view it? Places extra stress on officers (HAL25)

Plenty of times where I've hesitated because of cameras and not wanting to be the next viral hit (ROC103)

You lose your ability to do what comes natural. Instinct is lost (SAS89)

Subtheme 6: Officers' 'non-duty' behaviours $(\mathrm{N}=42)$

Smoke a cigarette, have a snack, go for a piss behind a tree when nature calls (COR02)

Getting food at a restaurant. Resting parked somewhere (COR19)

During downtime doing things not police-related; eating in public places, texting in the car, talking on the phone in the car, taking photos for personal use (TOR83)

Joking with colleagues (TOR272)

Subtheme 7: Officers that discount any video recording influence on their police actions in the field $(\mathrm{N}=32)$

You have to choose your words wisely and try to avoid hands on if possible, but if it needs to happen then it will happen to make sure myself and co-workers go home (ALB108)

Times have changed and you cannot conduct yourself as was done as explained by the veterans (BUF54)

If you do nothing inappropriate there is no reason for concern (OTT307)

At this point I assume that everything I do on the street will be captured in some sort of recording device (TOR24)

Subtheme 8: Officers that have thought about modifications in behaviour but have not yet changed actions in response to their thoughts $(\mathrm{N}=15)$

Thought about changing some things but have not yet acted on my thoughts (CAL81)

The way I speak with people or use force may change in a negative way as I get more experience (CAL348)

I have thought about it however I act professional (NIA152) 
Few officers answering Question 15 in the negative (with their quantitative response) $(\mathrm{N}=1439)$ provided written elaborations, which is not unexpected given that they were not asked to do so. Those that choose to do so regardless $(\mathrm{N}=49)$, reported observations that are generally consistent with the following:

Fuck it. I do my job the best I can and if someone wants to Monday morning $Q B$ me good for you (ALB94)

I'm here to do my job. I really don't care about being filmed, I'm gonna do what has to be done (BUF11)

I could get injured or worse if all I am thinking about is who is recording me. I would rather go home alive and lose my job than be killed (CAL166)

Not yet (DEL09, NEW01, SAS30)

I am always professional and I do my job well. I have no concern for my actions being recorded by anyone. (HAL88)

Question 16 Has there been an occasion while you were doing front-line police work when you have not done something, because of a concern that your actions could be video recorded, that you otherwise would normally have done?

Table 12: Officers not doing something because of a concern that those actions could be video recorded

\begin{tabular}{|c|c|c|}
\hline & N & Valid \% \\
\hline Yes & 2091 & 57.3 \\
\hline No & 1557 & 42.7 \\
\hline Missing & 12 & \\
\hline Totals & 3660 & 100.0 \\
\hline
\end{tabular}

Study participants answering 'Yes' were invited to provide a written explanation for their affirmative response and 1589 front-line officers contributed qualitative data in this regard (therefore, of the 2091 'Yes' quantitative respondents, 76\% presented some form of qualitative elaboration). While an Albany Police officer (ALB117) observed that today's video recording potential impacts front-line officers' behaviour in all aspects of police work, ten (10) subthemes emerged during analysis of other participants' more detailed written elaborations for Question 16. These are presented, as 
follows, in order of frequency with which they were cited by study participants: (1) changes in officers' use of force behaviours ( $\mathrm{N}=537)$; (2) officers' avoidance of proactive police work/discretionary interactions ( $\mathrm{N}=447)$; (3) changes in language used in interactions $(\mathrm{N}=237)$; (4) concerns with public perceptions/misinterpretations of police actions (including apprehensions around the integrity of citizen's video recordings)/uninformed public critique ( $\mathrm{N}=192)$; (5) officers being less assertive/more tolerant/more lenient ( $\mathrm{N}=152)$; (6) officers neglecting to make arrests in circumstances where arrests are warranted ( $\mathrm{N}=83)$; (7) officers' indecisiveness/hesitation/secondguessing ( $\mathrm{N}=77)$; (8) officers' 'non-duty' behaviours ( $\mathrm{N}=58)$; (9) officers' inability to use discretion due to video recording $(\mathrm{N}=36)$; and (10) officers that discount any influence of video recording on their police actions in the field/elevated professionalism $(\mathrm{N}=23)^{7}$

Three further observations emerged during data analysis for Question 16. First, it became apparent that more participants' qualitative elaborations were related to two or more identified subthemes - in comparison to how often this was observed across responses for Question 15. Also, as relates to the frequency of the identified behavioural modifications, while some officers indicated that their adaptations to 'policing's new visibility' are 'occasional', 'intermittent', or occur 'often', the overwhelming majority of study participants that addressed frequency in their written elaborations reported that their behavioural modifications are permanent and occur in all applicable situations that arise in their police work. Further, some study participants provided detailed accounts of specific occurrences. To include these would be tantamount to naming the officer as the 
source of the information and, therefore, these were excluded. Similarly, in some

instances, certain information has been redacted to safeguard participant anonymity.

Subtheme 1: Changes in officers' use of force behaviours $(\mathrm{N}=537)$

I think we have all sacrificed safety because we don't want to lose our jobs (ALB156)

Any force at all, or pretty much any officer/violator contact. It's brutal to know that people are recording to find a reason to criticize you. It's not the recording, but the attitude in it. It's old, and it kills your will to give a shit (CAL272)

We were in a struggle trying to arrest a male. I was going to use my Taser on him as he was assaultive. I stopped because there were a lot of people watching (NEW37)

Use of force authorized [by law] is often perceived as excessive by the public so I don't do what I am supposed to do (OTT14)

[I was] assaulted by a prisoner in cells. Location video recorded. Prevented me from providing free dental surgery to him (SAS41)

Take a less 'hands on' approach with individuals who need a more hands on approach. Not safe. But society is constantly taping and judging our actions without a real understanding of what is going on (SYR46)

$\underline{\text { Subtheme 2: Officers' avoidance of proactive police work/discretionary interactions }}$

$(\mathrm{N}=447)$

This country [USA] recently seems to be extremely anti-police and wanting to send officers to jail. If I do the bare minimum and just take the report they cannot fire me or send me to jail. This mindset while unfortunate is true. I used to think I could help people and be a positive impact (BUF29)

Less proactive work, less arrests, just let people get 'away' with things now (BUF113)

Again, paid either way. I get into more trouble doing my job than when I don't do it...Not worth the risk. FIDO all the time now (CAL121)

I don't go out of my way to get involved with something unless I am generally dispatched (HAM86)

Any form of proactive policing - the less I do, the less chance of a civilian complaint (KIN74)

Doing proactive work on Blacks in my area who are running drugs and guns, leave them alone now (OTT30)

Doing street checks on gang members or suspicious persons, nonexistent today (SAS51)

Generally disengaging with public now, seldom do anything but just dispatched calls (TOR179) 
I have seen many individuals and vehicles that I believe should be investigated proactively as they were very likely committing offences, particularly possession relating to drugs, weapons, and stolen property, and I have let them drive or walk on (TOR258)

Unless necessary, if race might become an issue, any interaction generally gets avoided (TOR284)

Subtheme 3: Changes in language used in interactions $(\mathrm{N}=237)$

Language - telling the public to 'fuck off' has been drastically reduced. And 'street justice' as opposed to arrest is no longer a practice (OTT129)

I watch the language or swearing or topics I discuss while at coffee in the public. People nearby may listen/record (RED21)

Having a conversation with people can be seen as racial profiling when this is just a person you want to investigate for their behaviour (TOR291)

Using 'street language' to gain control of a situation - very effective strategy at one time but now unavailable due to video (WIN06)

Subtheme 4: Concerns with public perceptions/misinterpretations of police actions (including apprehensions around the integrity of citizen's video recordings)/uninformed public critique $(\mathrm{N}=192)$

Police deal with the worst people and the work can be ugly at times. Public may not have stomach for what is on video (BUF238)

During the course of an arrest with an incredibly strong and violent offender it took multiple officers to subdue the subject. There were cell phones everywhere taping and I didn't jump is as I normally would have done due to fears of perceptions (CAL07)

Rather than do my job as trained I do my job as how I think my actions would be judged. Rather than acting I am looking for alternatives to putting myself at risk (CAL231)

Public perception and video editing allow for misrepresentations of what I do (TOR20)

This police service has 'trained' officers at the TPS College that you (the police officers) could do everything right in the course of duty, but still lose your job due to public perceptions (as in the public thinks the officer did the wrong thing) and the service will NOT back the police officer (TOR56)

Subtheme 5: Officers being less assertive/more tolerant/more lenient $(\mathrm{N}=152)$

Sometimes when people are recording it forces officers to have to deal with disrespect and take more bullshit than should be allowed (ALB96)

People not obeying commands and instead of getting the job done we waste time just giving commands over and over again (BUF05) 
I tend to show more tolerance with our 'clients' so I don't wind up in trouble when some people watch the video (GAT69)

Although the justification was there, I have made further attempts to de-escalate instead of going hands on (RID09)

Subtheme 6: Officers' neglecting to make arrests in circumstances where arrests are warranted $(\mathrm{N}=83)$

A drunk male was kicking over garbage cans and normally we would get out and stop him but because he was so violent and people were video recording we let him walk instead of getting his info. (BUF95)

For example, I remember once arresting a man who was screaming and being filmed. We let him go to show the video we weren't using too much force. Cover your ass! (COD26)

Political correct concerns - dealing with visible minorities - arresting would excite crowd and cause numerous complaints (HAM284)

Going hands on [during] an arrest. I hate to admit it but I have not arrested people who should have been arrested because I was more concerned with how the confrontation would look on video than with doing my job (OTT59)

Fighting with a drunk during bar close, pre-recording he would have been arrested (OTT264)

Making a disorderly conduct arrest for public disturbance. Due to video and public perception do not arrest for these anymore (ROC154)

Drove past the suspect, a Native male, so not to have the racist label (SAS49)

Use more discretion and not arrest for petty crimes (SYR65)

Don't enforce any statute or law that wouldn't be worth the headache to explain later when you are being called a racist (TOR192)

Some occasions where I would have arrested a drunk/belligerent person but didn't to avoid the possible fallout (WIN43)

\section{Subtheme 7: Officers' indecisiveness/hesitation/second-guessing $(\mathrm{N}=77)$}

Sometimes I find myself questioning the legal justification for arrest and deciding it is not worth getting involved (GAT70)

Happens all the time now. I think first about how things will look on video before I do anything (OTT70)

Taking a resisting subject into custody and I was afraid to use enough force because of the recording; it made me second guess myself (ROC152) 
Second guessing the law and my actions all the time now (TOR118)

\section{Subtheme 8: Officers' 'non-duty' behaviours $(\mathrm{N}=58)$}

Taking a break, eating in cruiser, checking phone messages/email (HAM116)

Not smoking in [police] vehicle or talking on phone/texting (OTT295)

Illegally parking [police] vehicle to get lunch (TOR73)

Meeting for coffee with other officers during down time (TOR188)

Stopped eating lunch/meals in public (WIN49)

Subtheme 9: Officers' inability to use discretion due to video recording $(\mathrm{N}=36)$

Use of discretion that would be in the favour of person recording (HAM233)

Giving an impaired driver the option of a ride home instead of jail, we can't do this now with video everywhere (NIA183)

When going 10-11 (roadside stop) - issuing warning not a ticket for offence is more problematic with video (RED13)

Would normally have issued a verbal warning. Due to the recording and the crowd gathered a citation was issued to document the interaction (ROC96)

Destroying small amounts of drugs (marihuana) on scene at arrest to save subject further charge and build trust (WIN113)

Subtheme 10: Officers that discount any influence of video recording on their police actions in the field/elevated professionalism $(\mathrm{N}=23)$

Things that NEED to be done by everyone are done. But extra work is mostly not going to happen (ALB29)

When dealing with 'regular clients' if video is around a more professional approach is required instead of being candid (CAL164)

At the beginning of the video recordings phenomenon I did, but now it happens so frequently I am accustomed to it and don't worry about changing my behaviour for the camera (GAT40)

Fighting with suspect on crack - public doesn't necessarily know he's on crack - they just see 3 cops on 1 guy and scream police brutality, worried about how it looked but did job anyway (ROC259)

I got sucker punched on a call by the caller. I wanted to punch her back however 'gently' got her into cuffs as passersby were recording on their phones - no one stopped to assist, just record. When it comes down to it, if something NEEDS to get done in order for me to get home, I'm going to do it regardless of camera (SYR61) 
Few officers answering Question 16 in the negative (with their quantitative response) $(\mathrm{N}=1557)$ provided written elaborations, which is not unexpected given that they were not asked to do so. Those that choose to do so regardless $(\mathrm{N}=31)$, reported observations that are consistent with the following: Treat everyone the same and act professionally at all times (ALB05) Sometimes the public won't understand, but you still need to do your job (CAL211) I always act like I'm being recorded (CAL249, HAL12) I do my job ethically and professionally, I don't take insults etc. personally (NIA112) Nothing to hide, job we do you can't live in fear or you are going to get hurt (OTT23) I only do actions that I can properly justify (TOR138) Accountability, part of the mindset (WIN118)

\section{Theme 3: Officer's use of force practices in relation to 'policing's new visibility'}

Within the survey instrument, Questions 17 and 18 were conceived to gather data around the impact (if any) of 'policing's new visibility' on study participant's use of force in engagements with citizens. In terms of specific behavioural modifications (as compared to practices when the participant started their police career), approximately half of today's front-line officers are moderating their use of force practices - in relation to both frequency $(47.3 \%)$ and degree $(50.1 \%)$. Descriptive statistics relating to these questions are presented in the following tables.

Question 17 In terms of frequency (as compared to when you started your police career), does the potential for video recording of your actions influence when you use physical force?

Table 13: Change in frequency of officers' use of force because of video recording

\begin{tabular}{|c|c|c|}
\hline & N & Valid \% \\
\hline Yes, I use force less often now & 1727 & 47.3 \\
\hline Yes, I use force more often now & 9 & 0.3 \\
\hline No, I use force the same & 1913 & 52.4 \\
\hline
\end{tabular}




\begin{tabular}{|c|c|c|}
\hline Missing & 11 & \\
\hline Totals & 3660 & 100.0 \\
\hline
\end{tabular}

Question 18 In terms of degree (as compared to when you started your police career), does the potential for video recording of your actions influence the level of physical force you use (when you are in a situation in which force is required)?

Table 14: Change in level of force used by officers because of video recording

\begin{tabular}{|c|c|c|}
\hline & $\mathbf{N}$ & Valid \% \\
\hline Yes, I use less force now & 1827 & 50.1 \\
\hline Yes, I use more force now & 0 & 0.0 \\
\hline No, I use force the same & 1822 & 49.9 \\
\hline Missing & 11 & \\
\hline Totals & 3660 & 100.0 \\
\hline
\end{tabular}

Although no space was allocated on the hardcopy survey document for written elaborations to Question 17 and Question 18, some participants $(\mathrm{N}=23)$ recorded observations in the margin adjacent to these questions. The following are largely representative:

Use force less often - only because less proactive work is being done (ALB94)

[I] use force the same, but I'm new (HAM245)

I use less force now if I can't just walk away (OTT08)

Video my whole career, I don't know any different (SAS07)

If you don't adapt use of force from the 'old days' you probably don't have a job anymore (SAS84)

For now, I know a lot of senior officers have pulled back (SAS144)

\section{Theme 4: Officer's resistance to 'policing's new visibility'}

To explore issues around rank-and-file officers' resistance to, and/or their countervailing strategies to mitigate, visibility, vis-à-vis today’s public audience, Questions 19 and 20 in the survey instrument were designed to elicit data in furtherance of such understandings. This data allows for a finding that, while not a majority, a substantial proportion of front-line officers today are engaging in practices to reduce the 
influence of 'policing's new visibility' on their in-the-field work practices and/or those of their colleagues. Almost four in ten study participants (37.6\%) report taking actions to avoid having their on-duty conduct video recorded, while almost half (47.0\%) take some form of action in circumstances in which they believe their actions are being (or potentially could be) video recorded. Descriptive statistics relating to these two questions are presented in the tables that follow.

Question 19 Do you do anything to avoid having your on-duty actions video recorded?

Table 15: Officers' employment of measures to avoid video recording of actions

\begin{tabular}{|c|c|c|}
\hline & N & Valid \% \\
\hline Yes & 1372 & 37.6 \\
\hline No & 2277 & 62.4 \\
\hline Missing & 11 & \\
\hline Totals & 3660 & 100.0 \\
\hline
\end{tabular}

Study participants answering 'Yes' were invited to provide a written explanation for their affirmative response and 1046 front-line officers contributed qualitative data in this regard (therefore, of the 1372 'Yes' quantitative respondents, 76\% presented some form of written elaboration). Ten (10) subthemes emerged during analysis of the data (these written contributions) for Question 19. These are presented, as follows, in order of prevalence with which they were cited by study participants: (1) using countermeasures to distort/defeat video recording ( $\mathrm{N}=246)$; (2) officers changing locations/leaving the area $(\mathrm{N}=206)$; (3) avoiding situations that may involve risk of police actions being video recorded ( $\mathrm{N}=205)$; (4) instructing/requesting person(s) to cease video recording ( $\mathrm{N}=143)$; (5) avoiding locations that may involve the potential for video recording ( $\mathrm{N}=134)$; (6) behaving more professionally ( $\mathrm{N}=48)$; (7) expediting police-citizen interactions to minimize exposure to video recording $(\mathrm{N}=47)$; (8) concerns around video recording related to 'undercover' police work ( $N=45)$; (9) 
concerns around video recording related to the officer's personal life $(\mathrm{N}=37)$; and (10)

recording the recorder(s) $(\mathrm{N}=15)$.

Subtheme 1: Using countermeasures to distort/defeat video recording $(\mathrm{N}=246)$

If I'm working patrol in a car I'll take a car without a camera if possible (ALB94)

If a partner or someone else is making an arrest or dealing with a confrontational individual who is unable to be dealt with by words alone I have stood in the way of people encouraging that individual so the camera does not see anything that will likely be perceived by some in the public as an issue (CAL330)

I do not like being recorded, I turn on my flashlight in the camera or I stand to block the video (GAT102)

If at night I will shine my flashlight on strobe at the person, while continuing my duties. If the person is in my face I will arrest for obstruction until the issue is settled (RED07)

Delay the start of BWC [police body-worn camera] recording (ROC11) ${ }^{9}$

Don't activate camera system as often, use less emergency lights, etc. [this defeats the dashcam which goes on when the cruiser's emergency lights are activated] (SAS152)

Warn other officers and shield other officers with my body, vehicle, lights (SYR154)

A lot of our platoon now wears lights on our vest/chest to hinder our faces from being seen $(\mathrm{TOR} 21)^{10}$

Use positioning of our vehicles, officers' bodies, and lights to block recordings while we are trying to do our work (YON21)

Subtheme 2: Changing locations/leaving the area $(\mathrm{N}=206)$

If being recorded do my best to get to an area or angle where it is difficult to be seen (CAL36)

I try to either block the view of the person with the video or move my interaction away to somewhere out of view (NEW14)

Drive away - walk away (ROC265)

Try to engage people I have to away from locations where there are cameras or where people might be able to film (STR17)

Move locations/ask party to stop recording (SYR79)

Move location or use vehicles/flashlights to provide some visual cover to what we are trying to do without someone making a biased recording (TOR76) 
If going to be use of force - try to move to other location before tipping hand that force will be used (TOR310)

Subtheme 3: Avoiding situations that may involve risk of police actions being video recorded $(\mathrm{N}=205)$

Less traffic stops, less street stops, less work in general. We answer calls now, that's about it (ALB77)

I avoid calls where recording might happen. Do just what I have to, nothing extra, that's where officers tend to get into shit (COR25)

Less interaction = less chance of being on YouTube! (OTT06)

I will avoid street checks or entering specific establishments (POR11)

I respond to calls but I'm not as proactive as I used to be due to higher possibility of confrontation (ROC37)

I have greatly decreased my proactive policing efforts (ROC173)

I don't make stops/proactive work unless necessary (SYR141)

Less self-initiated and less interaction with the public (TOR10)

Let the minor offences slide (TOR192)

Limit interactions with the public (YON38)

Subtheme 4: Instructing/requesting person(s) to cease video recording $(\mathrm{N}=143)$

I tell people to shut it off or fuck off (CAL07)

I ask to stop filming or I will go to a place where it is more difficult for anyone to film (GAT32)

[I] ask people not to record [but it] rarely works (HAM229)

I sometimes tell people that if they continue to record I will have to seize their phone for evidence because it will have footage that could be evidence on it (OTT58)

I am not shy to threaten someone who is trying to record me, 'Turn it off or you are going to jail', I consider it an obstruction and some things police get into is nobody's public business (YON08)

Subtheme 5: Avoiding locations that may involve the potential for video recording $(\mathrm{N}=134)$

More aware of what part of the city I am in (pro police - anti police) (BUF57) 
Avoid high risk areas such as bar districts because the risk of negative interaction with public is higher (HAM185)

Spend less time patrolling or parked in areas with lots of CCTV (NIA114)

I am careful where I park to do reports/notes. I choose isolated areas. The same for meal/coffee breaks (OTT117)

If I'm not on a call I tend to avoid parking in the public view (TOR258)

Subtheme 6: Behaving more professionally $(\mathrm{N}=48)$

Always try to do anything possible not to go 'hands on' (BUF207)

Try to remain professional so that there's nothing interesting to record (CAL189)

I make sure to be polite, respectful, and 'by the book' (GAT07)

I avoid yelling/screaming/appearing to have aggressive body language in hopes of avoiding drawing attention (NIA68)

I try to appear more 'nice' (ROC142)

Don't act like an asshole. Explain what you are doing (SAS43)

Subtheme 7: Expediting police-citizen interactions to minimize exposure to video recording $(\mathrm{N}=47)$

I terminate interactions with the public as soon as possible (ALB37)

Be brief, drive by, ignore (ALB120)

If cameras are around an arrest there is a conscious effort to get out of the public eye as quickly as possible (BUF27)

If their only interest is videotaping I will just leave the scene prematurely and let them solve their own problems (BUF215)

Ask them to not record - if they continue try to wrap scene up ASAP(BUF264)

$\underline{\text { Subtheme 8: Concerns around video recording related to 'undercover' police work }}$ $(\mathrm{N}=45)$

I am [race redacted] and in this region this is distinctive. I want to do undercover work in the future so I try to prevent my image from being available anywhere (especially if it is going to be linked with my job in policing) (COD46)

Turning my back to cameras if I see I'm being recorded - mainly because I don't want my face shown for safety reasons and future potential for $u / c$ work (NEW05) 
As a visible minority officer who wants to get into undercover investigation work I am very concerned about my facial image being accessible to anyone in the public on online sites. If you get identified that could be fatal. So, I keep my face turned when I see people are recording, use my light in their cameras, tell them to fuck off or move my call away from them to minimize my face time on camera (TOR91)

Subtheme 9: Concerns around video recording related to the officer's personal life $(\mathrm{N}=37)$

Turn my back to the recorder. Regardless of my actions, I do not want to have my face recorded while in uniform (HAL02)

I advise the person recording that posting the video could put myself or my family in danger if I'm seen (recognized) in public out of uniform (OTT157)

Lights and positioning. I don't want my image recognized off duty and I want to do undercover work (TOR135)

For those of us from a distinctive culture (i.e. [redacted]) we are easy to find on the Internetlink of professional and personal lives [is] not welcome! (TOR234)

Subtheme 10: Recording the recorder(s) $(\mathrm{N}=15)$

Make the other party aware that they are being recorded as well and then they back off or stop recording (CAL300)

If I notice someone with a cell phone is recording me, it is not rare that I will then record them in return with my device (GAT39)

Few officers answering Question 19 in the negative (with their quantitative response) $(\mathrm{N}=2277)$ provided written elaborations, which is not unexpected given that they were not asked to do so. Those that choose to do so regardless $(\mathrm{N}=62)$, reported observations that are generally consistent with the following: As long as I do what's right, I don't worry very much about video (BUF11) On the contrary, video can demonstrate how I do my best work (GAT105) Hiding or trying to stop someone [video recording] makes it look worse (HAM218) I am sound in the decisions I make. I don't use excessive force and don't have anything to hide (RID11)

Impossible due to the prevalence of cameras (TOR237) 
Try to be as professional as possible and 'hope' the cameras will save my ass (TOR267)

I treat every call as if I am being recorded (WIN87)

Too much video everywhere to avoid (YON25)

Question 20 On occasions when you think your on-duty actions could be (or are being) video recorded, do you do anything because of that video recording potential?

Table 16: Actions taken when there is potential for video recording

\begin{tabular}{|c|c|c|}
\hline & N & Valid \% \\
\hline Yes & 1720 & 47.0 \\
\hline No & 1936 & 53.0 \\
\hline Missing & 4 & 100.0 \\
\hline Totals & 3660 & \\
\hline
\end{tabular}

Study participants answering 'Yes' were invited to provide a written explanation for their affirmative response and 1412 front-line officers contributed qualitative data in this regard (therefore, of the 1720 'Yes' quantitative respondents, $82 \%$ presented some form of written elaboration). While a Saskatoon officer (SAS100) reported, I change my behaviour, more detailed contributions from other study participants resulted in the identification of eleven (11) subthemes, which emerged during analysis of the qualitative data (these written contributions) for Question 20. These are presented, as follows, in order of frequency with which they were cited by study participants: (1) articulating actions/using camera to advantage/staging the narrative $(\mathrm{N}=241) ;(2)$ avoiding cameras/defeating efforts to record/leaving the location as soon as possible $(\mathrm{N}=234)$; (3) changing behaviour - language $(\mathrm{N}=227)$; (4) changing behaviour - less proactive/ignoring certain circumstances $(\mathrm{N}=198)$; (5) elevating the level of professionalism $(\mathrm{N}=148)$; (6) changing behaviour - more kind/more patient $(\mathrm{N}=105)$; (7) instructing/requesting person(s) to cease video recording $(\mathrm{N}=97)$; (8) warning fellow officers ( $\mathrm{N}=91)$; (9) changing behaviour - using less force $(\mathrm{N}=60)$; (10) documenting the occurrence thoroughly $(\mathrm{N}=31)$; and (11) wearing my uniform hat $(\mathrm{N}=20)$. 
Subtheme 1: Articulating actions/using camera to advantage/staging the narrative

$(\mathrm{N}=241)$

Speak loud and state all the things going on that the camera might not see, ex. 'stop fighting me' 'stop resisting' (BUF230)

Tend to over-explain grounds for arrest/stop etc. far beyond what I would do without obvious cameras (DEL27)

Loud, clear articulation and commands and get call over fast regardless of whether it's the right outcome (OTT133)

I voice my commands louder, more frequent, so that subject AND camera person can hear (OTT150)

Articulate suspect actions that led to an arrest because they might not be articulated in the 'viral video' (ROC78)

If having to use force I voice 'stop resisting, etc.' to create witnesses on my side (SAS160)

Try to remain more calm, use camera to my advantage, positive witness (TOR39)

I verbalize my actions more, I don't get upset that I'm being recorded, remain professional (TOR140)

Subtheme 2: Avoiding cameras/defeating efforts to record/leaving the location as soon as possible $(\mathrm{N}=234)$

Blind lens with light, turn back to camera, move location and tell person with camera to depart (DEL39)

Block view so other officers can't be recorded. Act extremely professional and over polite with the public so that I don't get charged [disciplined] for anything - we can get screwed for anything (HAL53)

I speed up the call to get it over with quickly. Sometimes this is not what the call requires but the longer the recording goes on the more chance for something to go wrong and generate a complaint or viral YouTube (OTT38)

Try to move the interaction to another location out of view, if not possible I am very careful with language and actions (OTT68)

Use lights to blind cameras and warn other officers of people recording us (OTT300)

Get call over with ASAP regardless of if should take longer, get off camera ASAP before something happens (TOR12)

Often say other person's name/address often so they cannot put the video on YouTube [because of YouTube's privacy restrictions in relation to what can be included in submissions] (TOR82) 


\section{Subtheme 3: Changing behaviour - language $(\mathrm{N}=227)$}

Don't joke around with other officers because it could be on the news or YouTube (BUF32)

Keep conversations to a minimum and try not to curse! (HAL66)

Constantly watching my language as incivility tends to be the start of most viral videos (OTT352)

Spend longer talking/de-escalating, finding solutions other than arrest/use of force (RED47)

I make sure my language is tactful and polite rather than maybe talking normal. In certain areas of the city polite and tactful is not always useful. Sometimes using swear words when speaking will gain more compliance (SAS132)

Modify language to sound like a politically correct robot (STR24)

I call assholes, 'Sir' (WIN09)

Subtheme 4: Changing behaviour - less proactive/ignoring certain circumstances

$(\mathrm{N}=198)$

FIDO (ALB83)

Keep interactions brief, ignore interactions with potential to go bad (ALB120)

I do the bare minimum. I will not be made an example of and lose my career (BUF27)

Try not to get into anything and just answer calls for service (BUF150)

I do less of everything. Minimal effort now (BUF285)

Sometimes you let a subject get away with more liberties (CAL168)

Sometimes I take more time to consider the legal requirements for me interacting with someone and when there is any doubt I do not get involved (GAT70)

As mentioned, fewer patrols in certain areas - unless dispatched - stay clear of an area (KIN70)

Depending on the situation easier to ignore than be proactive (WIN58)

I now only perform the basis expectations of my job. Video always being present makes me worry about anything I do over and above the expectations to respond to calls sent to me (I worry on dispatched calls too but I can't not do those so it's a risk we all have to assume now on the front lines) (YON30) 


\section{Subtheme 5: Elevating the level of professionalism $(\mathrm{N}=148)$}

Follow procedures $100 \%$ to the letter, no variance (BUF107)

I try to ensure that my actions look controlled and deliberate as opposed to a reaction and losing my temper (COR14)

Act like the poster boy for politically correct policing (DEL13)

I act in a professional manner whether I am recorded or not. If police do our jobs properly and professionally, the 'footage' the public captures can only help us - and are usually of little interest to potential troublemakers (SAS147)

Subtheme 6: Changing behaviour - more kind/more patient $(\mathrm{N}=105)$

I will take time to speak to the recorder after the incident to answer any questions they may have (CAL164)

I am more patient when there is video, and more slow in going to physical force (GAT82)

Conduct myself in a very passive manner (NIA16)

Make sure I'm 100\% nice and pleasant so if things go viral I can't be criticized. Unfortunately, even if recording party is a complete asshole (NIA65)

I am more patient and give people more opportunities to comply. Sometimes that is good, sometimes it puts us at risk (TOR81)

I take on more risk and sacrifice officer safety to appear more passive and less confrontational. Often this involves putting my hands in my pockets (TOR255)

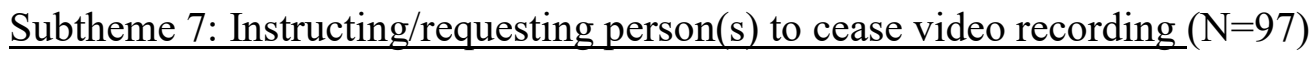

Tell person to stop or I move my location to somewhere out of view (COR36)

Kindly ask them to shut the recording off (HAM105)

Depending on the situation and the person doing the recording sometimes I will yell at them to stop and threaten to arrest them for obstructing if they are obnoxious (NEW14)

Try and use appropriate words to tell the filmer to 'fuck off' (OTT129)

\section{Subtheme 8: Warning fellow officers $(\mathrm{N}=91)$}

Let other cops know, be very professional (CAL316)

Warn other officers and point out locations of cameras/citizens with phones filming (HAM146) 
I have to make sure my officers present themselves in a better camera light (ROC225)

Make sure all other officers on scene are made aware of the fact that they are being recorded (WIN148)

Subtheme 9: Changing behaviour - using less force $(\mathrm{N}=60)$

Delay using force until absolutely necessary even if I believe I'm potential creating a greater risk (ALB73)

In some cases I'll avoid going hands on when normally I would (CAL21)

I try to verbally diffuse a situation longer rather than going right to physical arrest (SAS29)

Any force by police now is considered excessive in the eyes of the public (TOR247)

Subtheme 10: Documenting the occurrence thoroughly $(\mathrm{N}=31)$

[I do] more paperwork to cover myself. Also, sometimes [I] make an arrest to cover myself (BUF18)

Take precise notes and articulate my actions in notebook (CAL302)

I videotape on my own phone to make sure nothing is edited (COR33)

Very detailed report regardless of the type of call (GAT04)

Better notes, more elaborate in articulation of my grounds and actions (TOR92)

Subtheme 11: Wearing my uniform hat $(\mathrm{N}=20)^{11}$

Put on my forage cap to avoid signing over hours (HAM79)

Make sure my hat is on (TOR17)

Few officers answering Question 20 in the negative (with their quantitative response) $(\mathrm{N}=1936)$ provided written elaborations, which is not unexpected given that they were not asked to do so. Those that choose to do so regardless $(\mathrm{N}=25)$, reported observations that are generally consistent with the following: Waste of time, trying to stop the taping causes more drama (BUF33) I do every call as if I am being recorded (HAM224)

Good cops don't need to worry (ROC01) 
I assume we/I am recorded 24/7 (RID39)

\section{Theme 5: Officer's perceptions as to the nature of today's public (the broad citizenry) vis-à-vis policing}

Within the survey instrument, Questions 22 through 24 were designed to obtain data that would allow for an understanding regarding front-line officers' thoughts about today's public - their perceptions in relation to the citizens with whom the rank-and-file interact (or, in the case of de-policing, the citizens with whom officers are expected to interact as part of their duties) throughout their day-to-day activities within the community. More specifically, in the context of this study, information was sought regarding officers' perceptions about the publics' engagement with, and criticism of, officers' actions in the field. An objective was to determine the impact (if any) of public criticism on study participants' thought processes and whether this consideration has affected the front-line officer to such a degree that $\mathrm{s} /$ he has modified their behaviour in the field in response.

Study results reveal that today's front-line officers are in near-universal agreement that people in the community they police are now more critical in their assessments of those providing policing services. Not only are many officers $(60 \%)$ thinking about not doing something (that they otherwise would do) because they are concerned about the potential for public criticism, but, for $58 \%$ of study participants, their perceptions of a more critical socio-political policing landscape have instigated behavioural modifications to their conduct in the field. Descriptive statistics relating to these survey questions are presented in the following tables.

Question 22 As compared to when you started your policing career, what is your perception as to the degree of criticism of the police by the general public today in the community where you work? 
Table 17: Perception of public criticism of police (today versus start of career)

\begin{tabular}{|c|c|c|}
\hline & $\mathbf{N}$ & Valid \% \\
\hline The public is more critical of police & 3421 & 93.7 \\
\hline The public is less critical of police & 7 & 0.2 \\
\hline The public's criticism of police is the same & 222 & 6.1 \\
\hline Missing & 10 & \\
\hline Totals & 3660 & 100.0 \\
\hline
\end{tabular}

Although no space was allocated on the hardcopy survey document for written elaborations to Question 22, some participants $(\mathrm{N}=44)$ recorded observations in the margin adjacent to these questions. The following are largely representative:

Much more! (DEL46)

Far more critical! (HAM180)

$100 \%$ worse than when I started (NIA119)

It's more than I thought it would be (OTT89)

In my 15 years the public has gone from a few critics to the majority of people now not trusting the police in Ottawa (OTT121)

People use to help the police, now they just record us!! (RID53)

10x more critical (TOR77)

Question 23 Has there been an occasion while you were doing front-line police work when you have thought about not doing something, because of a concern that your actions could be criticized by some members of the public, that you otherwise would normally do?

Table 18: Officers contemplating not doing something because of a concern that those actions could be criticized by the public

\begin{tabular}{|c|c|c|}
\hline & N & Valid \% \\
\hline Yes & 2207 & 60.3 \\
\hline No & 1451 & 39.7 \\
\hline Missing & 2 & \\
\hline Totals & 3660 & 100.0 \\
\hline
\end{tabular}

Study participants answering 'Yes' were invited to provide a written explanation for their affirmative response and 1337 front-line officers contributed qualitative data in this regard (therefore, of the 2207 'Yes' quantitative respondents, 61\% presented some 
form of written elaboration). While a Buffalo Police officer (BUF95) observed that they thought about not doing something on many occasions, seven (7) more elaborate subthemes emerged during data analysis for Question 23. These are presented, as follows, in order of frequency with which they were cited by study participants: (1) avoidance of proactive police work/avoiding discretionary interactions ( $\mathrm{N}=408) ;(2)$ concerns over use of force $(\mathrm{N}=265)$; (3) concerns around potential consequences from scrutiny and negative public sentiment $(\mathrm{N}=221)$; (4) concerns over language $(\mathrm{N}=122)$; (5) overthinking/tentativeness/hesitancy ( $\mathrm{N}=99)$; (6) officers concerned about criticism but no behavioural change as yet/will never change behaviour because of criticism $(\mathrm{N}=65)$; and (7) concerns over the public's reaction to 'non-duty' behaviours $(\mathrm{N}=49)$. As was described in relation to Question 15, the reader should understand that many participant's responses to Question 23 were more consistent with answers one would expect to Question 24 - in that participants reported an actual behavioural modification in response to potential public critique within their answer to Question 23 (which was sometimes repeated in the answer to Question 24), rather than the respondent only thinking about not doing something they otherwise would do.

Subtheme 1: Avoidance of proactive police work/discretionary interactions $(\mathrm{N}=408)$

Traffic stops/clearing corners/stopping suspicious persons - anything proactive (BUF151) Always a concern when you're a White officer in a Black community (BUF230)

I think most cops are constantly weighing if something is worth doing to their own cost to self (CAL229)

I think about this all the time - sometimes it's not worth the risk to be proactive (CAL352)

Interacting with minorities $=$ racial profiling (HAM47)

The chance of getting into trouble from complaints by doing extra makes me be less proactive (OTT136) 
Can't get into trouble for doing nothing (ROC19)

Unfortunately the 'FIDO' consideration is becoming more prevalent (STR26)

No longer push the boundaries, if no grounds, no contact (TOR140)

Arresting a 'minority' for a minimal criminal offence (WIN58)

\section{Subtheme 2: Concerns over use of force $(\mathrm{N}=265)$}

I've thought about not using pepper spray during crowd control/fight calls (ALB141)

Anytime a cop has to use force, the majority of the public sees the force as excessive (BUF150)

It is a frequent consideration regarding lethal force. My present thought is that lethal encounters are becoming publicly unpalatable and therefore I do much thinking on when I would act (CAL336)

Use of force with anyone who is not White (OTT68)

Split second decisions about use of force are now second guessed and that is dangerous (OTT72)

Knowing that I may be scrutinized I can be hesitant to go hands on sooner (ROC178)

Subtheme 3: Concern around potential consequences from scrutiny and negative public sentiment $(\mathrm{N}=221)$

Policing in the U.S. has changed dramatically in that the public has become more critical (BUF157)

Where we work the public hates us, especially White cops (BUF285)

Public ignorance causes police grief - even when police actions are appropriate (NIA08)

Everything is criticized. I find we as a police community are scared of getting in trouble and try to avoid things that risk this happening (TOR01)

The way the public criticizes police today, there are many things that come up daily that we are not doing anymore because of a legitimate fear of what can happen to us because of that criticism (TOR30)

\section{Subtheme 4: Concerns over language $(\mathrm{N}=122)$}

The public criticizes everything. It's always a thought process as to how to even speak to someone (BUF61)

Stifled a lecture to a shitty motorist (CAL35) 
It is to the point now where even in general conversation with each other police must be careful not to swear or risk another public complaint (HAM151)

How people should be spoken to versus how they now have to be spoken to it today's politically correct reality (STR24)

Verbally shouting grounds for arrest/investigation rather than just doing it, so everyone observing can hear (TOR242)

\section{Subtheme 5: Overthinking/tentativeness/hesitancy $(\mathrm{N}=99)$}

The thought will creep into my head that I could end up like the Baltimore cops (CAL252)

Doubt settles into officers little by little, despite the legality of our actions in front of the public, that cannot comprehend what is involved with police work (GAT94)

It's always in the back of my mind - and I think a lot more about lawful authority, common law powers, articulation, etc. (HAL168)

I am constantly fearful of the criticism and lynching of cops that happens today in the media and society (HAM250)

Hesitation. Always considering the possibility of getting in trouble for trying to do my job (WIN52)

Subtheme 6: Officers concerned about criticism but no behavioural change as yet/will never change behaviour because of criticism $(\mathrm{N}=65)$

Thoughts of doing nothing do occur but I try to do what's right/lawful (BUF301)

There is always a concern because the media and armchair $Q B$ 's are ready to criticize but it doesn't stop me from doing my job (HAM87)

Never not done my duty but have reassessed ways of doing things (KIN66)

Many occasions it has crossed my mind, however never prevented the actual execution (ROC44)

Subtheme 7: Concerns over the public's reaction to 'non-duty' behaviours $(N=49)$

Having fun. I am human, I like to laugh, joke and have fun, but think about public perception that it may be taken as unprofessional (CAL175)

Taking a break, going into a Tim Horton's [a coffee shop] or meeting colleagues for breakfast (HAM273)

Parking (fire lanes), eating donuts in public, driving more appropriately (SAS13) 
Stop for a meal (sick of peoples' comments, e.g. 'Oh working hard I see, I pay your salary', etc.) (WIN11)

Few officers answering Question 23 in the negative (with their quantitative response) $(\mathrm{N}=1451)$ provided written elaborations, which is not unexpected given that they were not asked to do so. Those that choose to do so regardless $(\mathrm{N}=26)$, reported observations that are generally consistent with the following:

I will always do what I have to but I have noticed other officers worry about it (ALB36)

I do the job as it needs to be done regardless of public opinion (BUF78)

Not yet (DEL09, NEW01)

No, but I believe if I received a complaint for an action I did I would hesitate next time (DEL45)

Regardless you have a job and a mission to uphold (ROC47)

But I regularly think about how justified use of force is criticized by the public (SYR97)

I expect criticism from everyone including the public and senior administration. Getting none is a bonus (WIN87)

Question 24 Has there been an occasion while you were doing front-line police work when you have not done something, because of a concern that your actions could be criticized by some members of the public, that you would normally have done?

Table 19: Officers not doing something because of a concern that those actions could be criticized by the public

\begin{tabular}{|c|c|c|}
\hline & N & Valid \% \\
\hline Yes & 2108 & 57.7 \\
\hline No & 1546 & 42.3 \\
\hline Missing & 6 & 100.0 \\
\hline Totals & 3660 & \\
\hline
\end{tabular}

Study participants answering 'Yes' were invited to provide a written explanation for their affirmative response and 1372 front-line officers contributed qualitative data in this regard (therefore, of the 2108 'Yes' quantitative respondents, 65\% presented some form of written elaboration). Nine (9) subthemes emerged during analysis of the qualitative data (these written contributions) for Question 24. These are presented, as 
follows, in order of prevalence with which they were cited by study participants: (1) avoidance of proactive police work/avoiding discretionary interactions ( $\mathrm{N}=477)$; (2) changes in officers' use of force behaviours $(\mathrm{N}=357)$; (3) officers' neglecting to make arrests in circumstances where arrests are warranted/more lenient in enforcement $(\mathrm{N}=137) ;(4)$ concerns around potential consequences from scrutiny and negative public sentiment ( $\mathrm{N}=128) ;(5)$ concerns over language used in the field $(\mathrm{N}=124) ;(6)$ overthinking/tentativeness/hesitancy ( $\mathrm{N}=88)$; (7) concerns over the public's reaction to 'non-duty' behaviours ( $\mathrm{N}=54)$; (8) officers working 'by the book'/elevated professionalism ( $\mathrm{N}=25)$; and (9) officers concerned about criticism but no behavioural change/will never change behaviour because of criticism $(\mathrm{N}=21)$.

Subtheme 1: Avoidance of proactive police work/avoiding discretionary interactions $(\mathrm{N}=477)$

I come to work, answer my calls, and go home. I've almost completely stopped all proactive police work besides intoxicated driving (ALB141)

Car stops are much less frequent. Field stops are becoming non-existent (BUF275)

Because of people filming and criticizing police, I do not get involved with street gangs or outlaw motorcycle members (Hell's Angels) as much as I should (GAT08)

Stopping someone with suspected [psychological] issues to see if they're ok. It could go south so I'll just wait until dispatched (HAM86)

I don't initiate traffic stops or conversation with visible minorities due to social media and public misunderstanding (KIN74)

In my area I should be working on the gang members but that gets twisted into racial profiling and harassment. As you will see, shootings in Ottawa are way up because we can't do our job anymore because of political correctness and people having no idea what policing is about (OTT58)

Street stops, step outs on drug corners, traffic stops. All way down for me and others I work with (ROC03)

I do not talk to Black males because there is too much risk for a complaint (TOR12) 
I won't stop suspicious persons on street checks anymore despite the obvious crime prevention benefit; it's not worth the hassle and potential consequences to the individual officer (TOR99)

Do far less than I did even 5 years ago, if you don't do anything you cannot be criticized (TOR280)

Proactive policing is a relic of the past generation of YPD. To do it today you are risking your career (YON19)

\section{Subtheme 2: Changes in officers' use of force behaviours $(\mathrm{N}=357)$}

Using a high level of force during arrest - striking/punching (ALB44)

Being a White male working in the inner city dealing with minorities, I am screwed if I ever hurt someone (even if justified) (BUF207)

I know if I go hands on, regardless of the context, I will be criticized therefore I just avoid using force as much as possible even though it's warranted (BUF215)

I tend to choose a means of using force by my hands vs. a weapon, i.e. Taser because it might be perceived badly [even] knowing the weapon is safer for me and the offender (CAL330)

Use of force has changed, you have to be careful of how it looks more than whether it is effective or not (GAT90)

Public has no idea what is involved in using force to arrest someone so we don't do what we are supposed to and often that turns out worse for us and the suspect (STR12)

I have allowed myself to be bitten/punched/kicked/spit on because using force to prevent it is no longer acceptable (WIN120)

Subtheme 3: Officers' neglecting to make arrests in circumstances where arrests are warranted/more lenient in enforcement $(\mathrm{N}=137)$

Groups drinking/smoking weed in public left alone because public would riot (ALB94)

I no longer stop people for little things or rush to calls. I feel the less I deal with potential suspects then the less I can get in trouble. Basically, people are getting away with more crime because I feel it is not worth my job to stop them anymore (BUF173)

If the arrest might involve a visible minority, I ignore it or let them go (GAT83)

I've allowed criminals to yell and verbally abuse me - while in the past they would have been arrested for breach of peace at minimum (KIN61)

Physically contain someone. I let them run away instead (NIA124)

Affecting arrests of youth, homeless, mental health persons, we don't do these anymore unless absolutely necessary (OTT376) 
Public order (drinking, loitering) crimes are not enforced as much anymore (ROC37)

No longer address certain street level drug sales and suspects on their drug corners (ROC112)

Arresting an intox[icated] male outside a bar that could end up in a fight, too risky (SAS29)

Arresting people in crowded areas where people are hostile to police - knowing it will be misrepresented, don't do it! (TOR269)

Subtheme 4: Concerns around potential consequences from scrutiny and negative public sentiment $(\mathrm{N}=128)$

Public criticism can affect more than just individual officers (i.e. families) (ALB132)

Why be a headline on the news when the savages don't respect you anyway? (BUF77)

We are getting bad enough attention through media; we don't need any more fuel on the fire (COR23)

A snapshot of an incident could have lifelong impact. Body cameras are necessary to level the playing field and provide a lens from beginning to end of every interaction with the public (RID62)

Everything is criticized. I find we as a police community are scared of getting in trouble and try to avoid things that risk this happening (TOR01)

The public does not have enough education to make informed decisions about how criminal situations are dealt with and officers and their families will suffer solely based on public perceptions (TOR56)

Subtheme 5: Concerns over language used in the field $(\mathrm{N}=124)$

Swearing. I work in an area where the majority of our clients only respond to swearing. The general public don't get that (CAL149)

Asking certain questions with people of a visible minority class can be an issue when the [subject] is bringing race into the incident (POR14)

Primarily changing my language to avoid 'street talk' that the public does not understand sometimes needs to be used with certain people to have them understand (RID28)

Using blunt language to address an issue or force especially with minorities (STR29)

Subtheme 6: Overthinking/tentativeness/ hesitancy $(\mathrm{N}=88)$

Situation in Baltimore had negative effect on my actions; while in Ferguson cameras would have helped officer involved (BUF132)

You make sure to cover your ass more now, too many critics today (CAL283) 
My decision-making is made more according to public opinion rather than basic principles of policing (GAT26)

It's a lot of money to risk losing if I get fired! (OTT54)

It may even be something positive - the constant oversight and criticism makes you reserved. There is a concern that even positive actions may be misperceived so they are avoided altogether (OTT156)

Because of the recent backlash against police I feel officers hesitate at times to make decisions (SYR109)

I am less likely to put myself in a compromising position because of public safety, the safety of my job comes first (TOR296)

$\underline{\text { Subtheme 7: Concerns over the public's reaction to 'non-duty' behaviours }}(\mathrm{N}=54)$

Getting a food discount [a free or discounted meal at a restaurant] (BUF190)

Idling vehicles, coffee breaks, speeding, bad language (CAL79)

I don't sit in coffee shops or use foul language (ROC175)

More conscious of how/where I spend my downtime while on duty (ROC271)

Driving to a call while being recorded - I slow down (SAS118)

Subtheme 8: Officers working 'by the book'/elevated professionalism $(\mathrm{N}=25)$

I make sure that I'm doing things by the book when someone is looking like they're going to complain (COD33)

[I am] more patient with uncooperative parties being arrested (TOR89)

Subtheme 9: Officers concerned about criticism, but no behavioural change/will never change behaviour because of criticism $(\mathrm{N}=21)$

Not yet! (HAM192, SYR128)

Not really - it's just being a little more reserved (NEW03)

Hasn't happened yet but I would consider it (TOR113)

Few officers answering Question 24 in the negative (with their quantitative response) $(\mathrm{N}=1546)$ provided written elaborations, which is not unexpected given that 
they were not asked to do so. Those that choose to do so regardless $(\mathrm{N}=20)$, reported observations that are generally consistent with the following:

Do your job and follow the rules, be able to justify actions and you'll be fine (BUF20)

You just have to do the job, knowing people will hate you for it (BUF33)

Always on the mind but never prevents me from doing my job (POR06)

\section{Theme 6: Officers' perceptions of risk for criticism/complaint based on characteristics presented by individuals with whom officers interact and, therefore, avoidance of interaction with such individuals}

Questions 25 and 26 within the survey instrument, were designed to elicit data from study participants that would allow for an understanding as to whether (or not) officers perceive that there are certain 'demographics' (certain characteristics in persons that are apparently identifiable or that someone might be able to perceive about an individual they are observing) within the community where the officer works that present an elevated risk of criticism and/or the initiation of a complaint about an officer's work, and, if so, whether officers take measures to avoid interacting with such persons. Among this study's participants, $83 \%$ of officers perceive that in the community where they work there is an elevated risk of certain persons being critical of, or making a complaint about, their police work - as related specifically to one (or more) 'demographic' characteristic(s) that the participant discerns as identifiable. Six in ten (59.9\%) study participants report that their perceptions around these 'risky' persons do not translate into risk-avoidance behavioural adaptations - in terms of avoiding interaction with individuals presenting the identified 'demographic' characteristic(s). However, it is important to appreciate that conversely, four in ten $(40.1 \%)$ of today's front-line officers report that they do, in fact, proactively avoid interaction with these 
'risky' individuals (those that the officer perceives as belonging to the 'risky' 'demographic').

Citizens perceived as 'visible minorities' (those interpreted by study participants as being of a race, ethnicity, or culture different than 'White') are considered as more 'risky' than those citizens that appear as 'White', by eight in ten officers (79.2\%). Citizens perceived as experiencing a 'mental health issue' are considered riskier for an officer to interact with than other citizens, by four in ten officers (42.6\%). Lesser perceptions of elevated risks for professional consequences to rank-and-file officers were reported by study participants as relate to those citizens perceived to be of a 'sexual orientation' other than heterosexual (LGBTQ+), women, persons 'with a physical disability', or any of a variety of 'other' characteristics (as identified by participating officers). Descriptive statistics relating to Questions 25 and 26 are presented in the following tables.

Question 25 Do you feel that there is an elevated risk of criticisms of, or complaints about, your front-line police work that is related to particular characteristics presented by individuals with whom you interact in policing occurrences?

Table 20: Officers' perceptions of risk (of criticism and/or complaint) as related to 'demographic' characteristics of individuals with whom they interact

\begin{tabular}{|c|c|c|}
\hline & N & Valid \% \\
\hline Yes & 3027 & 83.0 \\
\hline No & 621 & 17.0 \\
\hline Missing & 12 & \\
\hline Totals & 3660 & 100.0 \\
\hline
\end{tabular}

The reader should understand that participants answering 'No' to this question were instructed to proceed directly to Question 27 - bypassing questions 25(a) and 26.

Question 25(a) If 'Yes' [to Question 25], please indicate which characteristic(s) you feel present an elevated risk of criticism or complaint about your police work, and please consider elaborating. 
Table 21: Specific 'demographic' characteristics identified as presenting an increased risk of criticism and/or complaint ${ }^{12}$

\begin{tabular}{|c|c|c|c|}
\hline & $\mathbf{N}$ & $\begin{array}{c}\text { \% of sample 'Yes' } \\
\text { to Q. 25 (N=3027) }\end{array}$ & $\begin{array}{c}\text { \% of entire sample } \\
\text { population (N=3660) }\end{array}$ \\
\hline Race/Ethnicity & 2897 & 95.7 & 79.2 \\
\hline Persons with mental health issues & 1560 & 51.5 & 42.6 \\
\hline Sexual orientation & 955 & 31.5 & 26.1 \\
\hline Gender & 776 & 25.6 & 21.2 \\
\hline Persons with a physical disability & 280 & 9.3 & 7.7 \\
\hline Other & 165 & 5.5 & 4.5 \\
\hline
\end{tabular}

Study participants responding 'Yes' to Question 25 were invited to select none, one, or more of the five 'demographic' characteristics presented for their consideration within Question 25(a) of the survey instrument, which were: (1) 'gender', (2) 'race', (3) (persons with) 'mental health issues', (4) ‘sexual orientation', (5) (persons with a) 'physical disability', and/or to specify 'Other' distinguishing characteristic(s) that they find relevant to this topic. Respondents were also encouraged to provide written elaborations explaining the rationale for their selection(s).

Of the 165 officers that selected the 'Other' category in Question 25(a), 81\% $(\mathrm{N}=133)$ presented written explanations for their response. The 'Other' characteristic most identified by study participants was criminals $(\mathrm{N}=30)$. This was followed by: youth/students $(\mathrm{N}=28)$; the homeless $(\mathrm{N}=18)$; the poor/persons of lower socio-economic class $(\mathrm{N}=15)$; drunks/substance abusers/drug addicts $(\mathrm{N}=11)$; and political activists $(\mathrm{N}=10)$.

In terms of written explanations for their affirmative responses to Question 25(a), 1622 study participants contributed data in this regard (therefore, of the 3027 'Yes' quantitative respondents, $54 \%$ presented some form of qualitative elaboration). ${ }^{13}$ Fourteen (14) subthemes emerged during analysis of these written contributions. These are presented, as follows, in order of frequency with which they were cited by study 
participants: (1) Black persons ( $\mathrm{N}=419)$; (2) visible minorities $(\mathrm{N}=282)$; (3)

assumptions across the public sphere regarding police behaviour/attitudes $(\mathrm{N}=174)$; (4) non-specified race/ethnicity/culture $(\mathrm{N}=142)$; (5) persons experiencing mental health challenges $(\mathrm{N}=136)$; (6) 'playing the race card' $(\mathrm{N}=121)$; (7) media and public critique $(\mathrm{N}=99) ;(8)$ Indigenous persons $(\mathrm{N}=63)$; (9) 'all of the above' (all five characteristics presented for consideration within the question) $(\mathrm{N}=59)$; (10) persons from the LGBTQ+ community (N=57); (11) 'Black Lives Matter' $(\mathrm{N}=51)$; (12) women $(\mathrm{N}=41)$; (13) police occurrences in the USA penetrating into Canadian consciousness $(\mathrm{N}=17)$; and (14) such are irrelevant considerations in the officer's police work $(\mathrm{N}=16)$.

\section{Subtheme 1: Black persons $(\mathrm{N}=419)$}

African Americans in the hood don't like White people (BUF43)

I patrol an area that's $90 \%$ Black and 10\% White. Race is an issue on every call (BUF228)

It's all about race now. A Black person can commit a crime but that becomes forgotten in all the talk about race profiling. The fact that the crime was committed seems irrelevant, it's all about the actions of the police in catching the person who committed the crime (COR15)

Getting involved in arresting or speaking with a Black person exposes an officer to a lot of risk (GAT90)

I am a Black officer and I get DWB [Driving While Black] accusations all the time and [accused of] racial profiling of my own race! (HAL57)

Blacks and Aboriginals especially critical and getting public sympathy anytime the police have to deal with them (HAM131)

White officer in an area where mostly Middle Eastern and Black is in for a lot of criticism and complaints (OTT112)

Cop on Black interaction is always seen as negative - release the crime stats and you would know why we investigate Black males more frequently (TOR50)

I think I have been called a racist at least 1,000 times as a result of simply doing my job as a cop with no conscious or, I think, subconscious bias. You can catch someone with the evidence right on them but still, the reason you are arresting them is because they are Black (TOR76)

In my job we don't care anymore that the public cannot understand that if one population that is $2 \%$ of the city's population commits over $50 \%$ of the crime in the city that the police should 
maybe pay extra attention to such people. But, we are fine to let things get out of control until the pendulum swings the other way and people demand we address young Black males who are responsible for most of the shooting and killing (TOR106)

A big White cop arresting a Black male is a perfect recipe for dismissal (WIN120)

For White officers race is a big issue, a lot of them avoid Black citizens when possible (YON06)

\section{Subtheme 2: Visible minorities $(\mathrm{N}=282)$}

Avoid visible minorities and I am one! Too much criticism of police from that demographic (99\% of the time unfounded) (CAL163)

In my opinion, it is often those who are criminals that are always criticizing police and it is the fact that many criminals are visible minorities (GAT79)

Particularly people of minority races more so than gender (women) or the mentally ill, who are a moderate risk, whereas minorities are a high risk (HAL11)

It's all about race (and I am a minority female). We look at behaviour not colour but no matter how guilty, if you arrest a minority it's always a concern that you will be judged a racist in court or in the media (OTT58)

Because of this I do not engage with visible minorities unless they are involved in a serious crime call. I do not pull them over, I do not ticket them, I pretend I don't see them (STR20)

Anyone from a visible minority is much more a threat to a police career than anyone else (TOR219)

If a person is a visible minority, we're more likely to be accused of racism regardless of our actions being appropriate (YON39)

Subtheme 3: Assumptions across the public sphere regarding police behaviour/attitudes

$(\mathrm{N}=174)$

Being a White male, it has become unacceptable for me to use force against anything other than White males (CAL277)

Anything we ever do is because the person is Black, Aboriginal, or whatever race other than White (COR36)

The public today tends to prejudge us from what they think are racial and social prejudices among officers. We live these public prejudices regularly (GAT39)

Being a White male I'm often accused that I'm dealing with them [race, mental health issues, sexual orientation] due to their characteristics not because of the event that lead to police being involved (HAL33)

Police are the targets - the new racism is the blue uniform (ROC53) 
The culture believes we only stop Black males, but in an area dominated by Blacks, there isn't anybody else to stop (ROC69)

We are all racist against everyone we have to arrest. It seems to be overlooked that the people we deal with are criminals who have broken the law (TOR28)

The public will make on site assumption that police are in misconduct when dealing with above [race and mental health issues] (TOR292)

\section{Subtheme 4: Non-specified race/ethnicity/culture $(\mathrm{N}=142)$}

As a White officer any action taken against any other race has an elevated risk of criticism (BUF40)

Certain races always feel picked on or targeted, and file complaints with more frequency and unnecessarily (CAL35)

People of different ethnicities seem to always complain about police even when they have done the crime (GAT55)

I am a visible minority and I get people of other races telling me I'm racist for stopping them (NEW25)

If you are not White then anything you do wrong that we investigate is because you are not White (not because you are a criminal) (OTT18)

Race is the big issue. For us, it is Blacks and Arabs. We are criticized for stopping them 'disproportionately' but guess what the police stats tell us, they are responsible for virtually all of the street crimes (gangs, shootings, drug dealing)! But, if that's what the public wants, no problem, let them run around the city shooting each other and we will stand on the sidelines (OTT272)

\section{Subtheme 5: Persons experiencing mental health challenges $(\mathrm{N}=136)$}

Minority races and people with obvious mental problems (homeless, drug addicts) (HAL24)

Public feels every [psychiatric distress] case should be solved by de-escalation (KIN29)

Members of public quick to complain about treatment of [those in psychiatric distress]. But they call about them thinking sometimes you have a 'magic wand' to fix them (NIA48)

Higher CYA [cover your ass] risks with minorities, EDPs [emotionally-disturbed people] on the street, and LGBT (OTT239)

\section{Subtheme 6: 'Playing the race card' $(\mathrm{N}=121)$}

Many Black people use excuse of me being White for the interaction, as if they weren't Black it wouldn't happen (BUF302) 
I am a visible minority so it's hard to admit but even I avoid visible minorities to avoid race card' complaints (CAL10)

Black/Brown people always go straight to saying you are dealing with them because of their race (CAL189)

Race is brought up all the time and seldom is it relevant to what is going on. Play the race card to make the officer 'timid' and worried about a complaint (DEL39)

It's all about the race card by the people and political correct[ness] by the police. And, I am Black. It is embarrassing to see how people behave when they get caught doing something wrong (traffic, crime, or doing something suspicious). The police respond to your behaviour not your skin colour! (OTT121)

Often hear 'You wouldn't treat a White suspect this way' (ROC166)

You can commit a crime but somehow the police are at fault for catching you - 'It's just because I am Black/Indian/ '(STR29)

\section{Subtheme 7: Media and public critique $(\mathrm{N}=99)$}

Black people are becoming untouchable due to media/social pressure (BUF285)

I think the media has played a large role in elevating the risk of criticism especially in regard to race (CAL113)

The public complains more about how police do their jobs due to media outlets criticizing the job as a whole (NEW06)

Much media attention on policing/racial profiling make me more sensitive to non-White interactions (NIA118)

Police are all racists according to much of the public and the media. So, minorities now get a free pass from many of us. If that's what the public wants we will see how they feel when a minority does something to them and the police tell them there's nothing they can do (OTT79)

The media has portrayed all White officers as racist (ROC154)

I am a Black officer and I get accused of being anti-Black as much as the White guys I work with, it's a convenient excuse for those who break the law and those who support them and today it gets a lot of traction with media and politicians (TOR30)

\section{Subtheme 8: Indigenous persons $(\mathrm{N}=63)$}

Lots of 'they're just misunderstood' or 'they're victims too' even when dealing with long-term Aboriginal criminals (CAL27)

We spend $75 \%$ of our police time dealing with Natives, they are the quickest to complain or judge (SAS45) 
There is BLM, we have ALM (Aboriginals). Very political (SAS76)

A significant number of the Aboriginals we are forced to deal with because of their behaviours (and then the public calls about them) accuse us of targeting them because of race, when we actually stay away from any proactive policing of them to stay away from career-damaging accusations of racial profiling or use of force (there are many who will resist arrest when drunk) (SAS135)

We have a poor relationship with Aboriginals so unless I have to engage them on a call it is smart to avoid anything proactive with them (SAS168)

Subtheme 9: All of the above (all of the five characteristics presented in the question) $(\mathrm{N}=59)$

Because I am female, I don't worry about gender, but every other 'touchy' subject I do (CAL175)

All of the above mean risk of complaints for a cop (CAL231)

All the above, public can't wait to get police on video in a 'gotcha moment' (NIA31)

If something bad happens, there is always an excuse generated by the public in regards to one of the above listed categories (ROC281)

\section{Subtheme 10: LGBTQ+ persons $(\mathrm{N}=57)$}

I will go out of my way to treat visible minorities and people with different sexual orientations with kid gloves otherwise I might face a complaint or aggravation (CAL338)

Hot button groups for political correctness and complaints - visible minorities, mentally ill (homeless) and LGBTQ activists (HAL02)

Particularly certain minority races, e.g. Blacks, Aboriginals and activist-types in the gay community (OTT227)

Blacks, Aboriginals and LGBT much more critical and likely to complain regardless of legitimacy of the police work being done (OTT256)

\section{Subtheme 11: 'Black Lives Matter' $(\mathrm{N}=51)$}

'Black Lives Matter' movement has made policing harder for Caucasian officers in our district because the population is predominantly African American (BUF223)

People making race an issue when it has nothing to do with race (i.e. Black Lives Matter movement) (CAL36)

The Black Lives Matter movement has been vocal in widespread criticism of police which has carried over to the general public (HAM179) 
The BLM movement affects interactions with Black members of the public (RID07)

The Ferguson Effect. False narrative of Black Lives Matter (ROC10)

Subtheme 12: Women/gender $(\mathrm{N}=41)$

Women and minorities and I am both! (CAL291)

As a woman I find I don't get the same gender criticism of my actions (HAL168)

$\underline{\text { Subtheme 13: Police occurrences in the USA penetrating into Canadian consciousness }}$ $(\mathrm{N}=17)$

Police involved shootings, particularly in the USA, involving Blacks, has heightened awareness of that group and others. Statistical data made available to the public has also been a driver (HAM220)

USA incidents brought over to Canada, public using race card in interactions (HAM225)

Situation in U.S. with Black people has spilled over to Canada (NIA12)

I feel the things happening in the [United] States with relation to race are coming up here (TOR130)

Subtheme 14: Such are irrelevant considerations in the officer's police work $(\mathrm{N}=16)$

Front line police officers often are forced to deal with people that no else can or will (CAL173)

People think we 'pick on' homeless people for the sole reason that they're homeless, as if I care where someone lives or doesn't. I interact with criminals doing crime and social disorder. Here, they happen to often be homeless (CAL272)

All the above - race card played too often, I treat everyone the same. If I stop you it's because an offence has happened not because you're female or Black (WIN55)

Question 26 Do you do anything to avoid interacting with persons who present the characteristics you have identified (in the previous question)?

Table 22: Avoidance of interaction with those presenting specific 'demographic' characteristics

\begin{tabular}{|c|c|c|c|}
\hline & $\mathbf{N}$ & $\begin{array}{c}\text { Valid \% of sample 'Yes' to } \\
\mathbf{Q . 2 5}(\mathbf{N = 3 0 1 9})^{14}\end{array}$ & $\begin{array}{c}\text { Valid \% of entire sample } \\
\text { population }(\mathbf{N = 3 6 4 0})^{15}\end{array}$ \\
\hline Yes & 1460 & 48.4 & 40.1 \\
\hline No & 1559 & 51.6 & 42.8 \\
\hline No to Q. 25 & 621 & & 17.1 \\
\hline Missing Q. 25 & 12 & & \\
\hline Missing Q. 26 & 8 & & \\
\hline
\end{tabular}




\section{Totals}

3660

100.0

100.0

Study participants answering 'Yes' to Question 26 were invited to provide a written explanation for their affirmative response and 1196 front-line officers contributed qualitative data in this regard (therefore, of the 1460 'Yes' quantitative respondents, $82 \%$ presented some form of written elaboration). The reader should understand that many of these qualitative contributions contained information that was attributable to two or more of the nine (9) subthemes that emerged during analysis of data for Question 26. These are presented, as follows, in order of frequency with which they were cited by study participants, however, the reader should take note that all nine of these identified subthemes are intertwined with study findings in relation to officers' avoidance of perceived risk in their policing environment - in the context of both proactive and, as discussed earlier (in terms of a surprising discovery), reactive police work: (1) avoidance of proactive police work ( $\mathrm{N}=431)$; (2) avoiding interaction with Black persons ( $\mathrm{N}=420)$; (3) avoiding interaction with visible minorities ( $\mathrm{N}=236)$; (4) avoiding certain types of dispatched calls/delaying response to dispatched calls/letting certain things go ( $\mathrm{N}=185)$; (5) avoiding interaction with persons presenting with mental health challenges ( $\mathrm{N}=136)$; (6) avoiding interaction with persons of non-specified race/ethnicity/culture ( $\mathrm{N}=122)$; (7) avoiding interaction with Indigenous persons $(\mathrm{N}=74)$; (8) avoiding interaction with persons identified by the officer as associated with 'other' identifiable and distinguishing characteristics - i.e. the homeless, students, political activists, youths, Middle Eastern, Arabs, Muslims (N=52); and (9) avoiding interaction with persons presenting as LGBTQ+ $(\mathrm{N}=34)$. 


\section{Subtheme 1: Avoidance of proactive police work $(\mathrm{N}=431)$}

Try and not do anything except answer your calls (ALB125)

Go to calls - no proactive work (BUF151)

Less proactive policing, more likely to just respond to calls (NIA114)

Slower response and less proactive policing (OTT91)

Only deal with people when required. Radio calls only (OTT160)

I only respond when there is a call for service. I no longer engage in real proactive patrol (OTT253)

I respond to certain neighbourhoods only when dispatched (SYR61)

As a White police officer in a predominantly Black/Hispanic community, I have reduced my proactive activities to reduce exposure to complaints that I am racist. I just answer my dispatch calls now and avoid activities otherwise (SYR155)

NC/NC (no contact $=$ no complaint $)($ TOR95)

I will not do any proactive policing but [also] avoid any contact with minorities, due to propensity to make false complaints against police because of their own racism versus police (TOR278)

Less proactive than in the past. I'm not risking my career if I don't need to (TOR305)

FIDO - reactive policing - work like the firemen - answering calls only (WIN80)

\section{Subtheme 2: Avoiding interaction with Black persons $(N=420)$}

Most caution needs to be with Blacks, so many of us have learned to avoid interaction unless required on an official call (ALB35)

With the Black Lives Matter frenzy we all now try hard to avoid engaging with AfricanAmericans, not just criminals but everyone, which is sad but it is reality (BUF52)

Black community wants a hands-off policy towards policing in their community, I steer clear of these communities in my zone (BUF270)

You need to avoid Black males or you will rack up a pile of frivolous complaints (HAL65)

I am a Black female officer and I have decided, like my White colleagues, that the risk is too high for a police officer today to interact routinely with the Black community (ROC159)

Anything to do with a White officer and a Black citizen is an issue, anything! So, avoidance of Blacks is our new strategy (ROC215) 
I have reduced my proactive interactions with African Americans substantially over the last 5 years because of my community [this study participant is a Black officer] always playing the race card no matter what is the situation that caused the police to get involved. Many African Americans seem to believe that race should be a get out of jail free card (ROC224)

Because of above [Quite often White police officer/Black citizen contact results in false allegations of profiling] most of us have reduced such contacts to only on dispatched calls, no more community proactive policing (SYR166)

Avoid Blacks unless part of a dispatched call and even then keep to minimum because of their propensity to complain, even when they are 100\% guilty of the crime it's always the 'race card' (TOR204)

\section{Subtheme 3: Avoiding interaction with visible minorities $(\mathrm{N}=236)$}

Don't do proactive policing with visible minorities and if I know that visible minorities are on the call I will try to get off it or take my time getting there to lessen time exposed to danger of race allegations and complaints (DEL57)

You need to avoid visible minorities unless you want your career to be in trouble (GAT97)

To survive in this anti-police environment today you cannot do certain things with visible minorities: proactive street checks, traffic enforcement, arrests unless for serious in-progress call. Just leave them alone and don't impact your career (OTT340)

Unless it is essential on a dispatched call I avoid all 'visible minorities'; too risky (TOR200)

Avoid getting race card played against you by not interacting with non-White people (TOR293)

Subtheme 4: Avoiding certain types of dispatched calls/delaying response/letting certain things go $(\mathrm{N}=185)$

I drive slower to their [visible minorities] calls, only take action if they want to press charges (BUF183)

At times I don't address public disorder issues involving people of colour for fear of the 'the race card' and frivolous complaints (CAL21)

I am more likely to give a Black person a break on a ticket so they don't complain (CAL189)

Avoid them [visible minorities] if not on call. If on a call longer response time so they hopefully go away (COR19)

Because Black people always accusing us of racial profiling they are now ignored by police, regardless of their status - victim, witness, complainant (NIA65)

If I know the type of people on the call I will try to avoid being primary on the call. I don't need more complaints (OTT70) 
I no longer enforce minor quality of life or street level crimes (ROC05)

If I know the neighbourhood I am being sent to and its history of criticizing police I will not rush in and will wait for more officers to try to prevent false accusations from the people supporting the criminals in their neighbourhood. If you don't like us, why are you calling us? (TOR30)

Subtheme 5: Avoiding interaction with persons presenting with mental health challenges $(\mathrm{N}=136)$

Because of lack of training for mental health issues I avoid such people as much as possible even though I should be intervening and speaking with them (BUF34)

For self-preservation I have been taught by veteran officers to avoid visible minorities and those with mental health problems (CAL75)

I avoid people with mental health problems because they are unpredictable and the public always thinks the police did something wrong, we should be able to 'de-escalate' everything and everyone - even someone with a knife running at us yelling that they are going to kill us! (CAL320)

Racial minorities and [psychiatric] subjects - do not engage other than as required on a dispatched call (KIN04)

Those with mental issues who cause public scenes I avoid because of public criticism of how we deal with those, a lose for police almost every time (RED32)

Subtheme 6: Avoiding interaction with persons of non-specified race/ethnicity/culture $(\mathrm{N}=122)$

No proactive with minorities anymore, too dangerous for a police career (HAM135)

I avoid all persons who should be investigated by police [but] who are not White; too much risk (HAM194)

Minorities and I am a minority myself. The truth that nobody is willing to say is that minorities use their race as leverage to blame someone other than themselves for their crimes, they place themselves as victims even though they did the crime. So police are reluctant to be put in a position to be branded a racist even though it's not true, the public will judge without any facts (NIA32)

To survive in this job today you have to avoid any non-call contact with racial minorities; you can't defend yourself against an accusation of racial profiling so don't put yourself in that situation (OTT297)

Given the way things have been the last 5+ years officers need to avoid anyone of a minority race to CYA (TOR133) 
Interacting with above [persons of different race and ethnicity than White and European origin] takes on additional and unnecessary risks because there are more complaints, biased media coverage and uneducated public sympathy without them knowing the facts or anything about policing (e.g. allegations of racial profiling because we are arresting a criminal who happens to be Black) (WIN27)

With allegations of racial profiling I was trained to avoid certain minorities for my own protection (WIN88)

Subtheme 7: Avoiding interaction with Indigenous persons $(\mathrm{N}=74)$

I avoid Natives, they feel targeted by police even though they are committing a crime. I was trained to avoid such risks (CAL247)

Avoid race minorities, including First Nation's people (my own background) unless part of my call. Much more likely to complain for no reason other than they hate the police (OTT262)

Avoid Aboriginals = avoid complaints! (RED09)

With continual accusations of being a racist you learn to avoid any visible minorities, especially Natives, unless they are directly involved in a dispatched call you have to take (SAS41)

Subtheme 8: Avoiding interaction with persons of 'other' demographic categories $(\mathrm{N}=52)$

Except on a call I do not intervene with homeless people even when they are breaking the law (GAT126)

I avoid Black males and chronic drug users/drunks (HAL140)

Steer clear from Blacks and Muslims, which is 2/3 of [the people] where I work (OTT301)

I ignore disorderly drunks and, in general, minorities to stay clear of trouble as best as you can in this day and age (WIN85)

In this city [Windsor] you need to avoid Arabs, they dislike police officers (WIN100)

Subtheme 9: Avoiding interaction with persons presenting as LGBTQ+ $(\mathrm{N}=34)$

Avoid any proactive with Blacks or LGBT (OTT18)

Unless integral to a dispatched call I will avoid visible minorities and obvious non-straight people due to high risk of them complaining about 'harassment' (TOR260)

Few officers answering Question 26 in the negative (with their quantitative response) $(\mathrm{N}=1559)$ provided written elaborations, which is not unexpected given that 
they were not asked to do so. Those that choose to do so regardless $(\mathrm{N}=60)$, reported observations that are generally consistent with the following:

[I don't avoid,] but I do feel strongly I am at more risk when I deal with Black people (BUF173)

Although minorities (and as a minority female I know) do pose a much higher risk for officers, where I work there is no way to avoid interactions with minorities as they are a majority (CAL196)

In the end I don't give in to the temptation to avoid proactive police work (CAL276)

I treat everyone the same (CAL328, TOR221)

I am just beginning my career and feel a lot of these questions will apply to me as I continue to gain more experience and interact with the public more (DEL09)

Even with more risk for job consequences we still have a job to do. I have not reached the point yet where I FIDO, but many people I work with (both minorities and Whites) have reached it! (HAM140)

I will not avoid a situation. I stay true to myself and my job (OTT155)

Can't, the entire hood is Native (SAS27)

Police work is police work. I can't avoid people (SYR87)

I don't care, I have a job to do (TOR134)

\section{Theme 7: Officer's familiarity, and experiences, with the concept, and/or the practice, of de-policing}

Questions 27 and 28 within the survey instrument sought to obtain data from study participants to allow for an understanding of officers' familiarly with, and personal experiences in observing (and, if applicable, their own performance of), the practice of de-policing. Three of the most common terminologies describing this phenomenon (in my experience and across the extant literature) were presented to study participants to account for differences in jargon - 'F.I.D.O.' ('Fuck it, drive on' or 'Forget it, drive on'), 'de-policing', and the more generic term 'risk-averse police practices'. In this regard, the preamble to these two questions on page four of the survey 
document reads, 'Questions 27 and 28 relate to the concept of risk-averse police practices. Some within policing, the media, and some academics studying policing have discussed this concept, sometimes using different terminologies - such as 'de-policing' or 'F.I.D.O.'

It is clear that most of today's front-line officers are familiar with de-policing and their understanding of the concept (as described by one, or more, of the three terminologies presented) coincides with how de-policing is considered within this dissertation. Further, a majority of study participants report observing de-policing behaviours within their front-line subcultural work environment. Descriptive statistics relating to these two questions are presented in the following tables.

Question 27 Are you familiar with the concept of risk-averse policing/de-policing/ F.I.D.O.?

Table 23: Familiarity with the concept of de-policing (as described in the survey)

\begin{tabular}{|c|c|c|}
\hline & N & Valid \% \\
\hline Yes & 2267 & 62.0 \\
\hline No & 1390 & 38.0 \\
\hline Missing & 3 & \\
\hline Totals & 3660 & 100.0 \\
\hline
\end{tabular}

Study participants answering 'Yes' were invited to explain their affirmative response (through a written elaboration) and 1324 front-line officers contributed qualitative data in this regard (therefore, of the 2267 'Yes' quantitative respondents, $58 \%$ presented some form of written elaboration). Six (6) subthemes emerged during analysis of the qualitative data (these written contributions) for Question $27 .{ }^{16}$ These are presented, as follows, in order of prevalence with which understandings of risk-averse policing, de-policing, and/or 'F.I.D.O.' were cited by study participants: (1) avoidance of proactive police work/only performing reactive police work ( $\mathrm{N}=412)$; (2) ignoring 
certain violations of the law or types of dispatched calls $(\mathrm{N}=283)$; (3) 'Fuck it (Forget it), drive on' - many officers explained their understanding of de-policing using this specific acronym/phrasing ( $\mathrm{N}=221)$; (4) just do the bare minimum $(\mathrm{N}=188)$; (5) 'putting on blinders'/turning a 'blind eye' $(\mathrm{N}=101)$; and (6) officers policing in this way are exhibiting a lack of professionalism/not doing their job properly $(\mathrm{N}=85)$.

Subtheme 1: Avoidance of proactive police work/only performing reactive police work $(\mathrm{N}=412)$

When the public is trying to make every attempt to get officers fired, officers will do less proactive policing (BUF138)

It has been passed down from senior officers that it's not worth proactive policing (BUF146)

It is called the 'Ferguson Effect' in the U.S. Not as proactive as we once were (BUF157)

Reactive policing only. No proactive searching/looking for crime/shitheads (CAL35)

As is occurring in Chicago this year, officers choose not to take a proactive role as it may only expose them to complaints, legal risks, etc. (CAL331)

Minimizing your 'risk' potential through taking calls only (COR09)

Avoiding problematic people, situations, calls, proactive policing (DEL36)

Not doing things we would historically do based on the perception that these proactive things would open us up to criticism/liability/conflict (NIA157)

In Ottawa this means no more proactive work, less traffic stops, no street checks so more gang shootings, drug dealing, criminal getting away with things (OTT121)

If you are not dispatched to the call, and it is not a major safety risk that you see, who cares? (OTT136)

Unless you are directed or required to do something you don't do it - reactive not proactive (STR14)

No contact/no complaint. Disengaging from public interactions, stopping proactive policing (WIN38)

Subtheme 2: Ignoring certain violations of the law or types of dispatched calls $(\mathrm{N}=283)$

Small-scale criminal behaviours should be ignored to avoid having the chance of the selfgenerating call leading to an arrest or physical confrontation (CAL27) 
That officers will actively avoid certain calls or situations that could escalate and lead to use of force (CAL147)

Ignoring a clear offence, or avoiding actions that could lead to intervention by police (COD57)

Letting criminals deal with each other and 'driving on' unless forced to intervene (DEL27)

Conflict avoidance, staying out of shit. Avoid tough calls (KIN30)

Public criticism has caused most police officers to avoid certain calls or situations for fear of complaints (OTT83)

If you see something that is against the law - drive away (OTT153)

If no one is getting hurt and no one has called about something, drive away (TOR259)

Ignore - do nothing if not serious offence (WIN62)

\section{Subtheme 3: 'Fuck it (Forget it), Drive on' $(\mathrm{N}=221)$}

In addition to many responses of simply 'Fuck it, drive on' there were responses such as:

Fuck it drive on, keep your job (ALB83)

Fuck it drive on. Manage risk by avoiding risk (DEL44)

Fuck it drive on. If you don't have to get involved - don't! (OTT224)

Fuck it drive on. Happens more and more. Nobody else cares, why should we? (OTT293)

Fuck it, drive on. Taught when I was a recruit (RID28)

Why care? Fuck it and drive on or F.A.R.T. (first available right turn)! NC/NC! (TOR21)

Fuck it. Drive on. If you don't engage it - it can't hurt you (WIN67)

Subtheme 4: Just do the bare minimum $(\mathrm{N}=188)$

Only answer calls, see nothing, say nothing, do nothing (ALB128)

Why get shot? Why get charged for murder when you don't have too? (ALB137)

Only do what the radio tells you to do (ALB145)

Do the very minimum you have to do (BUF316)

Bare minimum work, risk manage every call (CAL39) 
Punch in, punch out. Get your pension not PSS [Professional Standards Section - an internal investigative/disciplinary entity, often known as 'internal affairs'] (OTT60)

Do the minimum, take your calls, don't get in shit (OTT79)

If no one is dead or being killed, fuck it! (OTT211)

Do less, risk less. Not engaging unless you have to (TOR17)

We get paid the same to drive by and the added benefit of no stress/conflict or second guessing (TOR144)

\section{Subtheme 5: 'Putting on blinders'/turning a 'blind eye' $(\mathrm{N}=101)$}

Blinders on, keep driving, wait for someone to phone it in (CAL191)

Reactive policing only. Blinders on between calls (HAM154)

Blinders, is it worth the risk? If it's not someone's immediate safety at risk - FIDO (OTT275)

Turning a blind eye to what should be acted upon (STR19)

Turn a blind eye to avoid potential bullshit (SYR51)

Subtheme 6: Officers policing in this way are exhibiting a lack of professionalism/not doing their job properly $(\mathrm{N}=85)$

Not crazy about it. It gives officers (lazy ones) excuse to do nothing (BUF90)

Fuck it drive on. I assure you, if someone told me to only respond to calls and not dig up my own shit; I would politely decline and advise that I'm not a fire fighter (CAL149)

Police laying down (ROC218)

$F I D O=$ find another job $($ SAS55)

Just ignore what is happening and drive on = cowardice!!! (TOR288)

Only a handful of officers answering Question 27 in the negative (with their quantitative response) $(\mathrm{N}=1390)$ provided written elaborations, which is not unexpected given that they were not asked to do so. Those that choose to do so regardless $(\mathrm{N}=9)$, reported observations that are generally consistent with the following:

Never heard of these terminology (ALB14)

I'm not sure what that refers to (HAM115) 
It is apparent that the cultural jargon describing the practice of de-policing varies across jurisdictions. In this regard, 'F.I.D.O.' seems to be primarily a Canadian terminology and the terms 'NC/NC' ('No contact = No complaint') and 'F.A.R.T.' ([Take the] first-available right turn [to avoid engaging]) are particularly popular with officers in, and around, the greater Toronto area - including those rank-and-file officers serving with the TPS, HPS, and NRPS. The phrase used in this dissertation's title, 'To Swerve and Neglect', was brought to my attention after a presentation I delivered in 2016 at a conference in Phoenix, which was attended by senior leadership of many of the larger police departments across the USA. The chief (of large police agency in Arizona) approached me and advised that he was unfamiliar with the terms 'depolicing', 'risk-averse policing', and 'F.I.D.O.', however, in his experience, officers in his police department and across the southwest region of the USA, use the phrase 'To Swerve and Neglect' to describe the contemporary de-policing phenomenon I had discussed in my presentation. This chief explained that this expression is a play on the organizational mottos that have been adopted by many Arizona police departments promising citizens that their officers will 'serve and protect' or 'protect and serve'.

Question 28 Have you ever heard of, or witnessed, risk-averse policing/de-policing/ F.I.D.O. being practiced where you work?

Table 24: Participants who have heard of, or witnessed, de-policing where they work

\begin{tabular}{|c|c|c|}
\hline & N & Valid \% \\
\hline Yes & 2112 & 58.1 \\
\hline No & 1521 & 41.9 \\
\hline Missing & 27 & \\
\hline Totals & 3660 & 100.0 \\
\hline
\end{tabular}

Study participants answering 'Yes' were invited to provide a written explanation for their affirmative response and 1512 front-line officers contributed qualitative data in 
this regard (therefore, of the 2112 'Yes' quantitative respondents, $72 \%$ presented some form of written elaboration). While a Hamilton Police officer (HAM68) and an Ottawa Police officer (OTT301) wrote that there are 'too many' instances of de-policing to list in responding to the question, nine (9) subthemes emerged during analysis of more elaborate qualitative data. These are presented, as follows, in order of prevalence with which they were cited by study participants: (1) I practice de-policing myself $(\mathrm{N}=409)$; (2) I have witnessed de-policing practiced by my colleagues (N=304); (3) Avoidance of proactive police work $(\mathrm{N}=280)$; (4) Avoidance of certain types of calls/certain situations $(\mathrm{N}=203)$; (5) Avoidance of interaction with certain persons (N=98); (6) Estimates of depolicing frequency/prevalence $(\mathrm{N}=91)$; (7) De-policing behaviour(s) as more officer laziness than a risk-mitigation strategy $(\mathrm{N}=41)$; (8) I have heard of de-policing, but not witnessed it $(\mathrm{N}=26)$; and (9) De-policing is unprofessional $(\mathrm{N}=11)$.

Subtheme 1: I practice de-policing myself $(\mathrm{N}=409)$

All the time now, I would say 95\% of people in this department do this now (ALB125)

Pull into parking lot after you get your coffee and wait to be dispatched somewhere (ALB137)

In the last 5 years it has become the normal. I've been on the streets for almost 25 years and where it used to be a few guys here and there, now almost everyone is now doing FIDO. You have to survive today with video and BLM etc. (CAL208)

It happens every day. Everything from not being proactive to frequently calling in sick to limit exposure to risks on the job. I do it myself, you have to today (COR13)

I think all of us are doing this now. To do police work like we used to is too dangerous today. It's not worth losing your job (COR25)

It honestly happens on a daily basis too many times to count. I think our patrol inspector encourages it (DEL25)

I have been practicing it for the last 3 years since things got worse in policing - it's safer for us (OTT30)

Almost everyone I work with, and myself, are de-policing to avoid personal consequences (OTT47) 
Widespread and I do it several times per shift (OTT76)

Why risk getting yourself in a situation that would jeopardize your career? Just do the basics and take your calls, avoid anything controversial (OTT268)

I do FIDO all the time when anyone of a visible minority is involved with something the police should get involved with. I got tired of being accused of being racist so if this is what the public wants they can have the results (STR20)

Every shift, I only go to the calls I'm sent to for the most part now (WIN115)

\section{Subtheme 2: I have witnessed de-policing practiced by my colleagues $(\mathrm{N}=304)$}

LT's [Lieutenants] telling officers just to answer calls (BUF96)

Officers generally just do the minimum to stay out of trouble with our department (BUF157)

I don't practice FIDO but I would approximate $75 \%$ of members on my shift do (CAL223)

Since I started in 2005, used to be for lazy cops, now it can be for anyone (CAL274)

Everyone I work with is doing it now (HAM73)

Most of the officers I work with have adopted this mode of policing for self-survival (NIA63)

It is a constant concern. Police officers are not performing the basic mandated duties anymore. I would say the majority of officer I work with do this (OTT35)

I have often observed members avoid public interactions even where they should be doing their duty in order to avoid friction and complaints (POR06)

I don't practice de-policing but most of the officers I work with have begun to do this over the past 5 years and now it is commonplace across the RPD (ROC94)

Many guys sit in a parking lot until their next dispatched call, 'can't get into trouble if you don't do anything mentality' (ROC173)

\section{Subtheme 3: Avoidance of proactive police work $(\mathrm{N}=280)$}

Proactive policing has decreased significantly. 'Ferguson Effect' (ALB132)

There is almost no proactive policing happening now, calls for service response only (CAL240)

I have been much less proactive since one of our Delta officers was charged with murder and the video was used to mischaracterize what he went through. We are all much more worried now and no one wants to lose their job over a controversy (DEL11)

Officers sit in a parking lot, no traffic/drug/proactive crime enforcement (NIA134)

I used to be proactive between calls but the last 5 years I do nothing but my calls (OTT18) 
Officers are no longer doing street checks, not stopping cars or stopping to talk to people they should be (known criminals, suspicious behaviours) (OTT55)

Take your calls - no more, no less. No proactive policing - common now with most officers (OTT110)

As a supervisor there is a marked decrease in officers being proactive especially with gang members who are usually of an ethnic minority in our city (OTT195)

No choice. Too much chance for false complaints, witch hunts about race. Safer to do reactive call- to-call policing (fire-house policing) and no risky proactive (optional) duties (OTT233)

It's easy. Just don't stop anyone, just take your dispatched calls. Minimal contacts means no complaints and no headaches. We call it no contact = no complaint (TOR95)

'Parking lot policing' do the call then go back to the parking lot (TOR304)

Field/street checks are being avoided, fewer vehicle stops, much less proactive work (WIN111)

\section{Subtheme 4: Avoidance of certain types of calls/certain situations $(\mathrm{N}=203)$}

Certain officers will avoid certain calls that have a high chance of violence (CAL147)

Next time there's a bum pissing on the patio of a restaurant where the staff gave me shit for picking on the last 'poor homeless guy' who was doing it, that staff can deal with him themselves (CAL272)

Checking call board and driving opposite direction of call, ignoring traffic violations (DEL17)

There are officers who seem to be afraid to go to calls where there will be controversy so they 'sit on calls' or do open signals etc. Avoid being first to adverse situations (HAM239)

Putting themselves on traffic stops on 'hot calls', slowly going to 'hot calls' (KIN33)

Cars circling calls, calls waiting in the queue because no one wants to take those types of calls (OTT131)

Members purposely not doing something like making a necessary forceful arrest because of fear of being prosecuted for it (RED67)

Officers avoid certain areas known for video recording police and criticizing police actions. Leads to higher violent crime in these areas because no police are on scene (TOR02)

Regular practice of driving slow to calls so the issue becomes GOA [gone on arrival] (TOR259)

Subtheme 5: Avoidance of interaction with certain persons $(\mathrm{N}=98)$

We avoid interactions with Blacks and Aboriginals unless we have to deal with them on a dispatched call for service (CAL09) 
Guys don't think checking these people (Blacks) is worth a potential complaint - they'd rather go home and forget it and get paid the same anyway (DEL48)

Many times I have not intervened and I have seen other officers not intervene when situations involve people of races that are not White (GAT59)

Members are worried about dealing with African descent neighbourhoods proactively out of fear of being labeled 'profiling' the community (HAL50)

Not stopping vehicles driven by racial minorities for fear of how it will be perceived (HAL75)

An area downtown well known for drug activity is avoided by some officers as area is mostly Black citizens (HAM246)

I find newer, younger officers avoid dealing with some high-risk populations, i.e. homeless, drug dealers, afraid of complaints (NEW49)

The public can no longer stomach police intervention with mentally disturbed people yet they leave it to us to fix (OTT274)

We have to avoid Blacks, Aboriginals, and MHA people (OTT311)

Ignore non-call situations with visible race minorities (OTT355)

Several incidents with native Canadians dropped/ignored simply to avoid being called racist (RED43)

The feeling your efforts are wasted on people who could care less about you (WIN04)

Subtheme 6: Estimates of de-policing frequency/prevalence $(\mathrm{N}=91)$

Most of the veteran officers I know do this regularly (CAL73)

Today this is common practice here and with other departments in this region (DEL39)

This is now a common practice and I estimate it started about 6-8 years ago (HAL32)

I think everyone on my platoon is not working on proactive policing anymore. It's just 'take your calls' (OTT58)

Estimate 50\% of officers here [RCMP Red Deer] are doing FIDO (RED75)

Many, many officers refuse to do proactive police work anymore!! (ROC265)

As a supervisor I can tell you this has become widespread practice now, even the new officers are learning about it and after a while of seeing issues first hand many of them are mostly just reactive because of the risks (SAS127)

We are all doing it now. I don't know how many of us will admit it to anyone else but it is the new reality in police work (STR12) 
In certain areas or neighbourhoods this is a common practice with TPS officers like me. Most of us are doing FIDO now (TOR203)

I am sure that more than half of TPS officers are practicing FIDO today (TOR19)

Across our division I would say 75\%+ do this routinely. It's about self-preservation (TOR28)

Subtheme 7: De-policing behaviour(s) as more officer laziness than a risk-mitigation strategy $(\mathrm{N}=41)$

Lazy, unprofessional police officers who would be the same type of employee in any other job (BUF69)

More to do with work ethic than risk averse (HAL125)

I've seen officers work harder to avoid doing work than just to do it in the first place (SAS55)

Subtheme 8: I have heard of de-policing, but not witnessed it $(\mathrm{N}=26)$

Heard of it but never witnessed with people I work with (NEW09)

People mention it. Haven't witnessed it being done yet (RED60)

Subtheme 9: De-policing is unprofessional $(\mathrm{N}=11)$

Cowardly and unprofessional (OTT192)

Yes, generally by members who are too timid and are afraid of the job. I've seen it from the beginning [of my career], wrong career choice made [by these officers] (RID22)

Most YPD officers do this and those of us who don't think it's disgraceful and disrespectful to us who are trying to change this department into a professional [unintelligible] (YON35)

Only a few officers answering Question 28 in the negative with their quantitative response) $(\mathrm{N}=1521)$ provided written elaborations, which is not unexpected given that they were not asked to do so. Those that choose to do so regardless $(\mathrm{N}=8)$, reported observations that are generally consistent with the following:

Not sure what it is to know if it's being practiced (HAM96)

I will never not be proactive in my job. I love this job and I became a police officer to help people and arrest bad guys (POR03) 
Later in this chapter, additional information is presented relating to officers that are, by their own admission (as disclosed in their responses to survey questions), practicing de-policing, but are unfamiliar with the terminologies used to describe the phenomenon in this study. Similarly, further discussion is engaged around the fact that some officers that are practicing de-policing (as detailed in their responses to one or more of survey questions 16, 24, and/or 26), reported that they have not heard of, or witnessed, de-policing - as described by the study's three terminologies. In this regard, clearly, if an officer is, themselves, 'doing' (actively practicing) de-policing, they are also witnessing the practice. In other words, these study participants seem not to appreciate that their behaviour(s), as they described in response to one or more of the survey's primary de-policing behavioural questions, constitute(s) the practice of depolicing. Therefore, in considering these two circumstances, it is apparent that the actual practice of de-policing is, in fact, more prevalent than the $58 \%$ of front-line officers who report (in their responses to Question 28 in the survey) an awareness of officers engaging in the practice where they work.

\section{Theme 8: Influence (if any) of the researcher's policing ('pracademic') background on an officer's response(s) in an empirical study}

Questions 29 and 30 within the survey instrument were designed to elicit data from study participants that would allow for an understanding as to whether (or not) a front-line officer's knowledge of an external researcher's 'pracademic' biography (specifically a background in rank-and-file police work) has any influence on how that anonymous study participant will answer confidential survey questions - within an empirical study examining the participant's (and his/her colleagues') attitudes, behaviours, and occupational subculture. In the present study, these two survey 
questions sought to contribute to an understanding as to whether knowledge that I had worked for many years as an operational police officer, before I entered academica, had any influence on how participants had answered the preceding questions (1 through 28) in the questionnaire. Many $(\mathrm{N}=548)$, at the time they completed the survey, had no knowledge of my policing background. All but 27 of the study's 3108 participants reporting awareness of my policing background answered Question 30. Of these, 56.5\% reported that my policing biography had no influence on how they had answered the preceding survey questions. However, 1339 study participants (37\% of this study's total research population) reported that my 'pracademic' background had influenced how they had answered the preceding 28 questions in the survey. In this regard, it is important that the reader recalls that study participants answered questions in the order they were presented within the four-page hardcopy survey document (with Question 29 and Question 30 presented last - at the bottom of page 4).

Question 29 Before answering the questions in this survey were you aware that the researcher conducting this study worked for many years as an operational police officer?

Table 25: Familiarity with the researcher's policing background

\begin{tabular}{|c|c|c|}
\hline & N & Valid \% \\
\hline Yes & 3108 & 85.0 \\
\hline No & 548 & 15.0 \\
\hline Missing & 4 & 100.0 \\
\hline Totals & 3660 & \\
\hline
\end{tabular}

Question 30 Has your knowledge of the policing background of this researcher influenced how you have answered questions 1 through 28 in this survey?

Table 26: Influence on participants' responses due to knowledge of researcher's policing background

\begin{tabular}{|c|c|c|}
\hline & N & Valid \% \\
\hline No & 1742 & 56.5 \\
\hline Yes & 1339 & 43.5 \\
\hline
\end{tabular}




\begin{tabular}{|c|c|c|}
\hline Missing & 27 & \\
\hline Totals & 3108 & 100.0 \\
\hline
\end{tabular}

Study participants answering 'Yes' were invited to provide a written explanation for their affirmative response and 706 front-line officers contributed qualitative data in this regard (therefore, of the 1339 'Yes' quantitative respondents, 53\% presented some form of written elaboration). Six (6) subthemes emerged during analysis of the qualitative data for this question. These are presented, as follows, in order of frequency with which they were cited by study participants: (1) My responses to survey questions were more honest ( $\mathrm{N}=259)$; (2) I have an elevated level of trust in this researcher/he has more credibility $(\mathrm{N}=117)$; (3) This researcher is someone I can relate to $(\mathrm{N}=108)$; (4) I would not have done the survey if not for the researcher's policing background/I put in more effort ( $\mathrm{N}=102)$; (5) This researcher is fair/unbiased $(\mathrm{N}=36)$; and (6) This researcher has more integrity/ethical treatment of data $(\mathrm{N}=31)$.

Subtheme 1: My responses to survey questions were more honest $(\mathrm{N}=259)$

There is no way I would be admitting to 'racial profiling' and not policing every demographic the same and not doing parts of my job that are expected if I did not trust this researcher to be ethical. A lot of academics are not trustworthy because they live in an artificial world and have anti-cop agendas that they have to justify to anti-cop administrations and students. I think a lot of their 'studies' are bullshit and their 'data' is made up. This guy has the 'street cred' plus the academic credentials so we can trust him to be fair and honest (not unethical) (ALB141)

Having a background in both policing and academics makes me trust you more and I have answered more honestly than I would otherwise (COR05)

Trust! I would not answer some of these questions honestly if they were being asked by someone else (HAM67)

I feel comfortable answering honestly because this researcher is from outside the HPS and the university 'establishment'. I trust him with the truth, which is sad because what does that say about other researchers? (HAM131)

If this were a 'traditional' university prof researching our work I know most of us would never have opened up about some of the 'sensitive' things to do with FIDO and the like, we talked about it before he arrived and we all felt because he was a respected cop we could trust him (NIA26) 
I would not trust someone who understands my job only from books. I answered honestly because of this (OTT21)

Having come out of university into [the Saskatoon Police Service] I am aware that many, or most, academics have a negative view of police. Some of my professors can't stand the police and so how would they ever do a fair study? This researcher seems neutral and has experiences in both worlds (police and academics) so I think he will be unbiased and just report on the results without slanting it to his anti- or pro-police views. Because of this I have answered honestly where I would have been much more cautious explaining my thoughts to someone else or with a less anonymous method (like a survey through SPS computer system) (SAS38)

I would not feel comfortable admitting to a regular Professor, like the ones I had at my [name of university redacted] experience (generally 'anti-cop' biased), that myself and many other [Syracuse Police Department] officers avoid Black males or females unless 100\% necessary for our protection from being called racists, even with a survey that is allegedly 'ethical' and 'anonymous' (SYR105)

I would not normally admit to the truthful answers I gave to this researcher because they would get me in a lot of trouble in my job but I trust him because of his background (SYR166)

Because of his background and his interest in understanding what's going on in police work today without a pre-ordained outcome I trust his motives and integrity and have answered much more candid than I would if this was someone else doing this (TOR30)

No way I would have admitted the truth in my answers if I didn't' feel I could trust the researcher. Getting exposed as not politically correct could have serious impact on police careers so not many people we trust with the truth of what's going on today (TOR144)

Would not give such honest answers if this was a different researcher - we can trust this guy, not many others (WIN72)

Subtheme 2: I have an elevated level of trust in this researcher/he has more credibility $(\mathrm{N}=117)$

No way I would be admitting to some of the information I shared unless I could trust him, which I do because he is not the average academic with an ideology and agenda (BUF275)

Knowing he's been there before is helpful and the fact that he took the time to come out and meet us in person went a long way to building my trust (CAL113)

I trust cops with 'street cred', not random sit in an office researcher (CAL123)

Trust! (COR16, HAL53, KIN15, NIA108, OTT16, RED42, TOR152)

I trust him to deal with these major issues in policing today, he has experience in both policing and academics so he has more credentials than those without both experiences (OTT366) 
Trust is important in these kinds of studies. He has ours and so I think the data will be honest (WIN78)

\section{Subtheme 3: This researcher is someone I can relate to $(\mathrm{N}=108)$}

He's been on the front lines and he knows what street cops deal with (ALB93)

I appreciate his years of experience and efforts to explain police-community relations today (BUF185)

I'm more comfortable answering the questions with someone who's experienced what I've experienced (COD25)

I appreciate the fact that this researcher already lived the reality of working in patrol (GAT21)

I feel his understanding of the nuances of the job make it more likely he will have a more indepth understanding of the complexities and challenges we face today (NIA157)

Important that the person doing the research on policing has intimate knowledge of police work/culture etc. (NIA179)

I've known of this researcher for over 20 years as a cop, he knows cops and will be able to get to the bottom of what is going on today, FIDO in proactive police work, which is a serious issue (OTT290)

He's not some life time academic. He's actually done the work I've done/doing, so I know he will understand more! (OTT367)

Subtheme 4: I would not have done the survey if not for the researcher's policing background/I put in more effort $(\mathrm{N}=102)$

If he didn't have police background I would not want to take survey (ALB120)

I took more time, gave more thought and gave honest answers. If this researcher were not who he is I would not have done so (HAM81)

I trust he understands our perspective and does not have a critical bias against police formed from years of brainwashing in academia. I would not do this survey or, if I had to, I would not answer honestly otherwise (HAM128)

I know the researcher from a case he did in this region and know he has integrity, I would not have done this survey if I didn't know I could trust the researcher (NIA31)

Showing up in person on night shift shows commitment and understanding of our job. I normally would not participate in something like this because I wouldn't trust answers wouldn't wind up with management (OTT91)

He was respected and trusted as a cop and I know he is not like other academics at Carleton [University - name redacted] so no issue with participating in this study (OTT173) 
Because of his background in both of his jobs he has more credibility with front-line cops and therefore I have given this more thought and honesty (TOR76)

I respect what he is doing and where he comes from for this research; this is one of the few studies I have chosen to participate in, usually I just delete them or decline. Attending in person is a very good idea for buy-in and getting honest cooperation (TOR80)

I would have never entertained this survey, and few of us would with the PBA directive, but he demonstrated he could be trusted and was formerly an officer himself (YON32)

\section{Subtheme 5: This researcher is fair/unbiased $(\mathrm{N}=36)$}

He's been through this, is trained as a researcher, and is not anti-police (CAL15)

Unlike some academics that study police (aka twist things to support their hatred of police) I trust that he will be fair and unbiased and base his findings on the data (CAL196)

He seems like he wants to get at the truth whatever that is without having an anti-police result already in his mind (DEL13)

He's done this job and while we all know there are a lot of things to criticize and to fix in policing he does not have an axe to grind against the profession (TOR19)

Seems like a fair and kind man. Overcame reluctance from our union because of this (YON16)

\section{Subtheme 6: This researcher has more integrity/ethical treatment of data $(\mathrm{N}=31)$}

When you trust who the researcher is and why they are doing it you can open up rather than worry how information will be distorted (DEL39)

I know his reputation from police work and know he will do his research ethically, [this is] not always how researchers behave as I have experienced before (GAT121)

I appreciate the ethics protocols and the integrity of this researcher, he gives off a vibe of trustworthiness (YON42)

Only a handful of officers answering Question 30 in the negative with their quantitative response $(\mathrm{N}=1742)$ provided written elaborations, which is not unexpected given that they were not asked to do so. Those that choose to do so regardless $(\mathrm{N}=11)$, reported observations that are generally consistent with the following:

But there may be more of an understanding and less judgmental (CAL154)

This issue is big enough and close enough to me that I would have answered as eagerly regardless of who did the study. I'd also talk way more and in depth if I ever got the chance (CAL272) 
Would be answered honestly regardless, however confident that results of survey will not be manipulated (HAM233)

\subsection{Bivariate Analysis - The Practice of De-Policing}

In developing an understanding around the phenomenon of de-policing in today's front-line police work, through this sample population of rank-and-file officers, the three survey questions that specifically interrogated de-policing behaviours - as differentiated from thoughts, sentiments, rhetoric, or observations - are 16, 24, and 26. In this regard, as the reader will recall, Question 16 queries 'Has there been an occasion while you were doing front-line police work when you have not done something, because of a concern that your actions could be video recorded, that you otherwise would normally have done?' Question 24 inquires 'Has there been an occasion while you were doing front-line police work when you have not done something, because of a concern that your actions could be criticized by some members of the public, that you otherwise would normally have done?' And, question 26 asks, 'Do you do anything to avoid interacting with persons who present the characteristics you have identified in Question 25?'

\section{De-policing archetypes}

A derived variable was created that combined data from these three questions to construct a continuum of de-policing (detailed bivariate analyses of these three depolicing behavioural questions are presented in Appendix I). This recoding of data resulted in the emergence of discrete categories of de-policing among the rank-and-file. Four archetypes emerged from analysis of study data, which identifies the existence of a continuum of de-policing across the present study's population of front-line officers. 
1. Non-depolicers (or perhaps 'duty-driven' officers) accounted for $\mathbf{2 8 . 3 \%}$ of study participants. These are front-line officers that do not practice any de-policing behaviours (those participants that did not respond 'Yes' to any of the three questions).

2. Limited de-policers (or perhaps 'aware' officers) accounted for $\mathbf{1 8 . 2 \%}$ of study participants. These are front-line officers that practice limited de-policing behaviours (those participants that responded 'Yes' to one of the three questions).

3. Moderate de-policers (or perhaps 'risk-averse' officers) accounted for $\mathbf{2 3 . 7 \%}$ of study participants. These are front-line officers that practice moderate de-policing behaviours (those participants that responded 'Yes' to two of the three questions). 4. Intensive de-policers (or perhaps 'avoidance-driven' officers) accounted for $\mathbf{2 9 . 9 \%}$ of study participants. These are front-line officers that practice intensive de-policing behaviours (those participants that responded 'Yes' to all three of the questions).

Table 27: De-policing archetypes (total sample)

\begin{tabular}{|c|c|c|}
\hline & N & Valid \% \\
\hline Non de-policers & 1024 & 28.3 \\
\hline Limited de-policers & 658 & 18.2 \\
\hline Moderate de-policers & 857 & 23.7 \\
\hline Intensive de-policers & 1081 & 29.9 \\
\hline Total & 3620 & 100.0 \\
\hline Missing & 40 & \\
\hline Totals & 3660 & \\
\hline
\end{tabular}

The ranges within each identified de-policing archetype, from highest proportion among participating police agencies to the lowest, are as follows:

Non-depolicers: RCMP Red Deer (44.6\% of study participants from this agency were categorized as non de-policers) and Buffalo Police Department (20.5\% of study participants from this agency were categorized as non de-policers). The average across all 23 participating agencies is $28.3 \%$ of officers categorized as non de-policers. 
Limited de-policers: Service de police de la ville de Gatineau (27.7\%) and RCMP Red Deer $(13.5 \%)$. The average across all 23 participating agencies is $18.2 \%$ of officers categorized as limited de-policers.

Moderate de-policers: Buffalo Police Department (30.8\%) and Stratford Police Service (7.1\%). The average across all 23 participating agencies is $23.7 \%$ of officers categorized as moderate de-policers.

Intensive de-policers: Niagara Regional Police Service (38.7\%) and RCMP Red Deer (17.6\%). The average across all 23 participating agencies is $29.9 \%$ of officers categorized as intensive de-policers.

The table below presents the de-policing archetype information by each participating police agency.

Table 28: De-policing archetypes by participating police agency

\begin{tabular}{|c|c|c|c|c|c|c|}
\hline & & & Non & Limited & Moderate & Intensive \\
\hline Agency & $\mathbf{N}$ & Missing & $\%$ & $\%$ & $\%$ & $\%$ \\
\hline Albany & 154 & 2 & 24.0 & 15.6 & 24.0 & 36.4 \\
\hline Buffalo & 312 & 5 & 20.5 & 18.9 & 30.8 & 29.8 \\
\hline Calgary & 354 & 4 & 26.0 & 15.3 & 29.7 & 29.1 \\
\hline Cornwall & 36 & 0 & 41.7 & 16.7 & 8.3 & 33.3 \\
\hline Delta & 58 & 0 & 43.1 & 20.7 & 17.2 & 19.0 \\
\hline Gatineau & 148 & 0 & 34.5 & 27.7 & 14.9 & 23.0 \\
\hline Halifax & 205 & 1 & 37.1 & 17.1 & 16.6 & 29.3 \\
\hline Hamilton & 287 & 2 & 31.0 & 15.7 & 26.1 & 27.2 \\
\hline Kingston & 77 & 1 & 24.7 & 24.7 & 26.0 & 24.7 \\
\hline New Westminster & 49 & 0 & 42.9 & 16.3 & 16.3 & 24.5 \\
\hline Niagara Regional & 186 & 5 & 24.2 & 15.1 & 22.0 & 38.7 \\
\hline Ottawa & 380 & 2 & 33.2 & 18.7 & 16.8 & 31.3 \\
\hline Port Moody & 20 & 0 & 35.0 & 25.0 & 20.0 & 20.0 \\
\hline RCMP Codiac & 67 & 2 & 43.3 & 17.9 & 16.4 & 22.4 \\
\hline RCMP Red Deer & 74 & 1 & 44.6 & 13.5 & 24.3 & 17.6 \\
\hline RCMP Ridge Meadows & 66 & 0 & 27.3 & 25.8 & 28.8 & 18.2 \\
\hline Rochester & 278 & 3 & 24.5 & 16.2 & 30.6 & 28.8 \\
\hline Saskatoon & 174 & 1 & 23.0 & 16.7 & 25.3 & 35.1 \\
\hline Stratford & 28 & 1 & 39.3 & 21.4 & 7.1 & 32.1 \\
\hline Syracuse & 168 & 2 & 24.7 & 21.1 & 21.1 & 33.1 \\
\hline
\end{tabular}




\begin{tabular}{|c|c|c|c|c|c|c|}
\hline Toronto & 307 & 3 & 21.8 & 19.9 & 23.8 & 34.5 \\
\hline Windsor & 144 & 4 & 25.7 & 17.4 & 26.4 & 30.6 \\
\hline Yonkers & 50 & 1 & 26.0 & 22.0 & 26.0 & 26.0 \\
\hline Totals & $\mathbf{3 6 2 0}$ & $\mathbf{4 0}$ & $\mathbf{2 8 . 3 \%}$ & $\mathbf{1 8 . 2 \%}$ & $\mathbf{2 3 . 7 \%}$ & $\mathbf{2 9 . 9 \%}$ \\
\hline
\end{tabular}

\section{De-policing archetypes in context with variables of interest}

The following tables present the bivariate analysis of the identified de-policing categories (or archetypes) in context with other variables of interest. The independent variables considered in the analysis are organized into subsections as follows: (i) geographic location and size of agency, (ii) type of front-line police work and policing environment, and (iii) individual officer characteristics.

\section{Geographic location and size of agency}

Only weak associations were found between both country (Canada and the USA) and region (eastern Canada, western Canada, and the USA) in relation to the identified de-policing archetypes. ${ }^{17}$ A weak association was also found in relation to size of police agency. However, perhaps not surprisingly, it is observed that front-line officers serving with 'small' police agencies (identified in this study as those with fewer than 100 frontline officers) are more likely to be situated toward the lower end of the de-policing continuum. The table below presents the analysis in relation to these variables.

Table 29: Location and agency variables

\begin{tabular}{|c|c|c|c|c|c|c|c|}
\hline \multicolumn{2}{|c|}{$\begin{array}{c}\text { Location and } \\
\text { Agency Variables }\end{array}$} & $\begin{array}{l}\text { Non de- } \\
\text { policers }\end{array}$ & $\begin{array}{c}\text { Limited } \\
\text { de- } \\
\text { policers }\end{array}$ & $\begin{array}{l}\text { Moderate } \\
\text { de-policers }\end{array}$ & $\begin{array}{c}\text { Intensive } \\
\text { de- } \\
\text { policers }\end{array}$ & $\chi^{2}$ & $\mathbf{V}$ \\
\hline \multirow{2}{*}{ Overall } & $\mathrm{N}$ & 1024 & 658 & 857 & 1081 & \multirow[b]{2}{*}{-} & \multirow{2}{*}{$\longrightarrow$} \\
\hline & Valid \% & $28.3 \%$ & $18.2 \%$ & $23.7 \%$ & $29.9 \%$ & & \\
\hline \multirow{2}{*}{ Country } & Canada & $30.1 \%$ & $18.2 \%$ & $22.2 \%$ & $29.5 \%$ & \multirow{2}{*}{$21.306^{*}$} & \multirow{2}{*}{.077} \\
\hline & USA & $23.2 \%$ & $18.1 \%$ & $27.7 \%$ & $30.9 \%$ & & \\
\hline \multirow{2}{*}{ Region } & $\begin{array}{l}\text { Eastern } \\
\text { Canada }\end{array}$ & $30.3 \%$ & $18.7 \%$ & $20.5 \%$ & $30.4 \%$ & \multirow{2}{*}{$31.771 *$} & \multirow{2}{*}{.066} \\
\hline & $\begin{array}{l}\text { Western } \\
\text { Canada }\end{array}$ & $29.7 \%$ & $17.0 \%$ & $26.2 \%$ & $27.2 \%$ & & \\
\hline
\end{tabular}




\begin{tabular}{|c|c||c|c|c|c||c|c|}
\hline & USA & $23.2 \%$ & $18.1 \%$ & $27.7 \%$ & $30.9 \%$ & & \\
\hline \hline \multirow{2}{*}{$\begin{array}{c}\text { Agency } \\
\text { Size }\end{array}$} & Small & $36.2 \%$ & $17.7 \%$ & $21.9 \%$ & $24.2 \%$ & & \\
\cline { 2 - 8 } & Medium & $27.9 \%$ & $18.6 \%$ & $21.9 \%$ & $31.5 \%$ & $34.445 *$ & .069 \\
\cline { 2 - 8 } & Large & $25.5 \%$ & $18.1 \%$ & $25.5 \%$ & $30.9 \%$ & & \\
\hline
\end{tabular}

$* \mathrm{p} \leq .01$

\section{Type of front-line police work and the officer's policing environment}

To examine if there are meaningful differences in de-policing practices that are related to the type of front-line police work engaged in by study participants, grouped variables were created in relation to study participants' principal role within rank-andfile policing (the type of work they do most often). The variables created for this analysis are explained in the table that follows:

\begin{tabular}{|c|c|}
\hline Variable for analysis & Categories as identified in Question 6 of the survey \\
\hline \hline Patrol $(\mathrm{N}=3125)$ & Primary response /general patrol \\
\hline Community $(\mathrm{N}=109)$ & Community-based unit \\
\hline Foot/Bike $(\mathrm{N}=85)$ & Foot/bicycle patrol \\
\hline Other $(\mathrm{N}=74)$ & $\begin{array}{c}\text { Gang intervention, traffic enforcement, school liaison, or } \\
\text { write-in answers - most prevalent were 'tactical' and 'canine' }\end{array}$ \\
\hline Mixed $(\mathrm{N}=223)$ & $\begin{array}{c}\text { Respondents who selected more than one category } \\
\text { in responding to this survey question }\end{array}$ \\
\hline
\end{tabular}

A weak association was found between the type of front-line police work being performed by study participants and the study's identified de-policing archetypes. However, it is interesting to consider that those officers who work more closely in contact with 'the community' (community officers) present with considerably higher proportions of intensive de-policers $(53.2 \%)$ than do patrol officers $(29.3 \%)$. Similarly, $61.2 \%$ of officers working on foot, or on bicycles, are situated as either moderate depolicers or intensive de-policers, whereas $52.7 \%$ of patrol officers are situated within these two de-policing archetypes. It is also noteworthy that officers working in frontline police work categorized as 'Other' (i.e. tactical, canine, gang intervention, traffic enforcement, school liaison) present considerably lower proportions of moderate and 
intensive de-policers (31.1\%) than do patrol officers (52.7\%), community officers (67.9\%), foot/bike officers (61.2\%), and officers doing various (mixed) rank-and-file duties (62.1\%), which will be discussed throughout Chapter Six - within the context of the rank-and-file police occupational subculture and front-line officers that subscribe to a 'mission orientation' in their fundamental beliefs, attitudes, and police work.

A weak association was also found between front-line policing environments and identified de-policing archetypes. It is interesting to note, however, that rank-and-file officers who work primarily in rural communities and commercial areas present considerably higher proportions of non de-policers ( $37.9 \%$ and $37.0 \%$ respectively) as compared to their urban and suburban colleagues (at $28.8 \%$ and $25.9 \%$ respectively). Similarly, rural and commercial officers present lower proportions of intensive depolicers (19.4\% and $11.1 \%$ respectively) as compared to their urban and suburban colleagues (at $28.1 \%$ and $32.9 \%$ respectively). The table below presents the analysis in relation to these variables.

Table 30: Type of front-line police work and officers' policing environment

\begin{tabular}{|c|c|c|c|c|c|c|c|}
\hline \multicolumn{2}{|c|}{$\begin{array}{l}\text { Type of police work being } \\
\text { performed and officer's } \\
\text { policing environment }\end{array}$} & $\begin{array}{c}\text { Non } \\
\text { de- } \\
\text { policers }\end{array}$ & $\begin{array}{l}\text { Limited } \\
\text { de- } \\
\text { policers }\end{array}$ & $\begin{array}{l}\text { Moderate } \\
\text { de- } \\
\text { policers }\end{array}$ & $\begin{array}{l}\text { Intensive } \\
\text { de- } \\
\text { policers }\end{array}$ & $\chi^{2}$ & $\mathbf{V}$ \\
\hline \multirow{2}{*}{ Overall } & $\mathrm{N}$ & 1024 & 658 & 857 & 1081 & \multirow[b]{2}{*}{ 一 } & \multirow[b]{2}{*}{ - } \\
\hline & Valid \% & $28.3 \%$ & $18.2 \%$ & $23.7 \%$ & $29.9 \%$ & & \\
\hline \multirow{5}{*}{$\begin{array}{l}\text { Type of } \\
\text { Front-Line } \\
\text { Police Work }\end{array}$} & Patrol & $28.8 \%$ & $18.4 \%$ & $23.4 \%$ & $29.3 \%$ & \multirow{5}{*}{$59.781 *$} & \multirow{5}{*}{.074} \\
\hline & Community & $17.4 \%$ & $14.7 \%$ & $14.7 \%$ & $53.2 \%$ & & \\
\hline & Foot/Bike & $18.8 \%$ & $20.0 \%$ & $30.6 \%$ & $30.6 \%$ & & \\
\hline & Other & $44.6 \%$ & $24.3 \%$ & $18.9 \%$ & $12.2 \%$ & & \\
\hline & Mixed & $23.8 \%$ & $13.9 \%$ & $30.5 \%$ & $31.6 \%$ & & \\
\hline \multirow{5}{*}{$\begin{array}{c}\text { Policing } \\
\text { Environment }\end{array}$} & Urban & $28.8 \%$ & $18.5 \%$ & $24.6 \%$ & $28.1 \%$ & \multirow{5}{*}{$52.482 *$} & \multirow{5}{*}{.070} \\
\hline & Suburban & $25.9 \%$ & $17.6 \%$ & $23.4 \%$ & $32.9 \%$ & & \\
\hline & Rural & $37.9 \%$ & $16.9 \%$ & $25.8 \%$ & $19.4 \%$ & & \\
\hline & Commercial & $37.0 \%$ & $29.6 \%$ & $22.2 \%$ & $11.1 \%$ & & \\
\hline & Mixed & $24.2 \%$ & $16.5 \%$ & $17.7 \%$ & $41.6 \%$ & & \\
\hline
\end{tabular}

$* \mathrm{p} \leq .01$ 


\section{Individual officer characteristics}

Contary to my original expectations, an officer's 'demographic' characteristics in terms of gender, race/ethnicity, and/or age, are not relevant to de-policing practices. This result is consistent with the results of Adams' (2015) research into police use of force practices. In analyzing variables that might be associated to differences in officers' use of force behaviours, Adams (2015: 539) found that multiple studies have determined that police use of force is "unrelated to an officer's personal characteristics, such as age, gender, and ethnicity.” Adams (2015) advances that the influence of the occupational subculture and the nature of the individual officer's experiences in the field are likely the key variables in explaining officers' differing use of force practices. Specifics in relation to individual officer characteristics in the present study are as follows:

Gender - There is no significant difference observed between male $(\mathrm{N}=2965)$ and female ( $N=653)$ police officers in the present study, in terms of the identified depolicing archetypes.

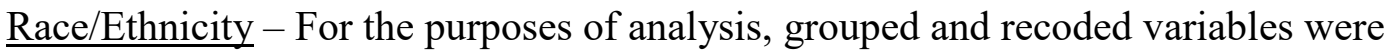
created for those study participants identifying as 'White' and those identifying as any race/ethnicity other than 'White' (coded as 'non-White'). A weak association is found between officer's race/ethnicity (as represented by the variables 'White' and 'nonWhite') in relation to the identified de-policing archetypes. Given the limited differentiation in this measurement, the race/ethnicities of research participants, as pertains to their de-policing practices, were examined in more detail. It is found that even when 'non-White' study participants' race/ethnicity is considered by more 
specifically-defined variables ('Black,' 'Hispanic,' 'Asian,' 'Aboriginal,' 'Mixed,' or 'Other') only a weak association exists in relation to the identified de-policing archetypes. It is interesting to consider, however, that all race/ethnicity categories other than 'White' recorded higher proportions of intensive de-policers that 'White' officers (except for 'Black' officers) and all race/ethnicity categories other than 'White' recorded higher proportions of moderate de-policers than 'White' officers (except for ‘Asian’ officers).

Age and Years of (overall) police experience - A moderate association is observed between both an officer's age and an officer's years of overall police experience (that includes police work not defined, for this study's purposes, as 'front-line' - i.e. investigative or administrative functions) and the identified de-policing archetypes. Years of front-line police experience - I advance (and elaborate in Chapter Six) that an officer's years of front-line police work experiences are more relevant, in relation to depolicing practices, than either an officer's age or years of (overall) police experience. The rationale for my argument is twofold. Today, in contrast to the typical practices in previous eras of policing, when most officers joined police agencies in their early $20 \mathrm{~s}$, new officers ('recruits' or 'rookies') begin their policing careers at all different ages ranging from persons in their early 20 s to those in their late $40 \mathrm{~s} .{ }^{18}$ Further, it is often the case that officers, during their policing career, will transfer voluntarily into (or are involuntarily assigned into) non-front-line positions (i.e. investigative or administrative roles) for varying durations, after which many will return to front-line positions. It is important to understand that all officers begin their policing career assigned to rank- 
and-file (uniform patrol) duties and generally spend several years in front-line roles before being considered for a different position within the police organization.

As demonstrated in the following table, the association between an officer's years of front-line police experience and the identified de-policing archetypes is a more pronounced substantive finding than the relationship found between an officer's age and an officer's years of overall police experience. It is in this area where we can begin, in quantitative terms, to understand the nature of de-policing in contemporary front-line police work and the concept of a continuum of de-policing within the rank-and-file occupational subculture (which is elaborated in Chapter Six). To examine, in further detail, the association between years of front-line police experience and the practice of de-policing, collapsed and recoded variables were created for study participants' years of front-line police experience - a variable 'New' (for those participants reporting 1 to 5 years of front-line experience) and a variable 'Experienced' (for those reporting 6 or more years of front-line experience). This analysis demonstrates an even more compelling substantive finding in the association between an officer's years of frontline police experience and the identified de-policing archetypes. It seems that many 'New' officers, at some point in their front-line career, will likely move along the depolicing continuum toward more robust de-policing behavioural practices. It is argued that this progression within the de-policing continuum is influenced through the officer's accumulation of 'time on the job' (as is the common jargon within police occupational culture) - including 'on the road' experiences and concurrent exposure to de-policing discussions and/or experiences within the front-line subculture. It is interesting to note that officers with more than 25 years of front-line experience present 
a considerably lower proportion of intensive de-policers and higher proportion of non-

depolicers as compared to their more-junior colleagues, excepting for the 'rookies'

(those with 5 or less years of front-line experience). ${ }^{19}$

Table 31: Front-line officers' characteristics

\begin{tabular}{|c|c|c|c|c|c|c|c|}
\hline \multicolumn{2}{|c|}{$\begin{array}{c}\text { Individual Officer } \\
\text { Variables }\end{array}$} & $\begin{array}{l}\text { Non de- } \\
\text { policers }\end{array}$ & $\begin{array}{c}\text { Limited } \\
\text { de- } \\
\text { policers }\end{array}$ & $\begin{array}{l}\text { Moderate } \\
\text { de- } \\
\text { policers }\end{array}$ & $\begin{array}{l}\text { Intensive } \\
\text { de- } \\
\text { policers }\end{array}$ & $\chi^{2}$ & $\mathbf{V}$ \\
\hline \multirow{2}{*}{ Overall } & $\mathrm{N}$ & 1024 & 658 & 857 & 1081 & \multirow[b]{2}{*}{ 一 } & \multirow[b]{2}{*}{ - } \\
\hline & Valid \% & $28.3 \%$ & $18.2 \%$ & $23.7 \%$ & $29.9 \%$ & & \\
\hline \multirow{2}{*}{ Gender } & Male & $27.8 \%$ & $18.3 \%$ & $23.9 \%$ & $30.0 \%$ & \multirow{2}{*}{2.020} & \multirow{2}{*}{ - } \\
\hline & Female & $30.5 \%$ & $17.6 \%$ & $22.5 \%$ & $29.4 \%$ & & \\
\hline \multirow{9}{*}{ Race } & White & $28.9 \%$ & $18.8 \%$ & $22.9 \%$ & $29.4 \%$ & \multirow{2}{*}{$10.490 * *$} & \multirow[b]{2}{*}{.054} \\
\hline & Non-White & $25.9 \%$ & $15.3 \%$ & $26.4 \%$ & $32.4 \%$ & & \\
\hline & White & $28.9 \%$ & $18.8 \%$ & $22.9 \%$ & $29.4 \%$ & \multirow{7}{*}{$39.799 *$} & \multirow{7}{*}{.061} \\
\hline & Black & $30.7 \%$ & $13.8 \%$ & $31.2 \%$ & $24.3 \%$ & & \\
\hline & Hispanic & $24.1 \%$ & $18.5 \%$ & $24.1 \%$ & $33.3 \%$ & & \\
\hline & Asian & $29.4 \%$ & $22.5 \%$ & $14.7 \%$ & $33.3 \%$ & & \\
\hline & Aboriginal & $19.1 \%$ & $16.2 \%$ & $28.0 \%$ & $36.8 \%$ & & \\
\hline & Mixed & $19.4 \%$ & $8.2 \%$ & $30.6 \%$ & $41.8 \%$ & & \\
\hline & Other & $24.8 \%$ & $14.4 \%$ & $25.6 \%$ & $35.2 \%$ & & \\
\hline \multirow{7}{*}{ Age } & $21-25$ & $55.6 \%$ & $19.1 \%$ & $22.2 \%$ & $3.1 \%$ & \multirow{7}{*}{$123.360 *$} & \multirow{7}{*}{.107} \\
\hline & $26-30$ & $29.2 \%$ & $16.7 \%$ & $26.3 \%$ & $27.7 \%$ & & \\
\hline & $31-35$ & $23.4 \%$ & $17.5 \%$ & $26.0 \%$ & $33.1 \%$ & & \\
\hline & $36-40$ & $25.8 \%$ & $16.0 \%$ & $22.3 \%$ & $35.9 \%$ & & \\
\hline & $41-45$ & $28.9 \%$ & $19.4 \%$ & $22.6 \%$ & $29.1 \%$ & & \\
\hline & $46-50$ & $27.3 \%$ & $22.5 \%$ & $20.3 \%$ & $29.9 \%$ & & \\
\hline & $51+$ & $33.0 \%$ & $21.5 \%$ & $20.1 \%$ & $25.4 \%$ & & \\
\hline \multirow{6}{*}{$\begin{array}{c}\text { Years of } \\
\text { (Overall) } \\
\text { Police } \\
\text { Experience }\end{array}$} & $1-5$ & $40.8 \%$ & $19.3 \%$ & $24.7 \%$ & $15.2 \%$ & \multirow{6}{*}{$207.879 *$} & \multirow{6}{*}{.139} \\
\hline & $6-10$ & $22.4 \%$ & $18.4 \%$ & $27.2 \%$ & $32.0 \%$ & & \\
\hline & $11-15$ & $22.9 \%$ & $16.5 \%$ & $19.6 \%$ & $41.0 \%$ & & \\
\hline & $16-20$ & $26.3 \%$ & $16.0 \%$ & $22.2 \%$ & $35.5 \%$ & & \\
\hline & $21-25$ & $22.5 \%$ & $20.1 \%$ & $18.2 \%$ & $39.2 \%$ & & \\
\hline & $26+$ & $27.8 \%$ & $20.6 \%$ & $20.6 \%$ & $30.9 \%$ & & \\
\hline \multirow{8}{*}{$\begin{array}{c}\text { Years of } \\
\text { Front-Line } \\
\text { Police } \\
\text { Experience }\end{array}$} & $1-5$ & $40.3 \%$ & $19.5 \%$ & $24.1 \%$ & $16.1 \%$ & \multirow{6}{*}{$237.650 *$} & \multirow{6}{*}{.148} \\
\hline & $6-10$ & $23.7 \%$ & $19.1 \%$ & $26.6 \%$ & $30.6 \%$ & & \\
\hline & $11-15$ & $22.9 \%$ & $14.8 \%$ & $19.1 \%$ & $43.1 \%$ & & \\
\hline & $16-20$ & $21.6 \%$ & $15.5 \%$ & $21.1 \%$ & $41.8 \%$ & & \\
\hline & $21-25$ & $20.6 \%$ & $18.3 \%$ & $16.7 \%$ & $44.4 \%$ & & \\
\hline & $26+$ & $31.9 \%$ & $21.7 \%$ & $29.0 \%$ & $17.4 \%$ & & \\
\hline & New & $\begin{array}{c}40.3 \% \\
\end{array}$ & $19.5 \%$ & $24.1 \%$ & $16.1 \%$ & \multirow{2}{*}{$174.955^{*}$} & \multirow{2}{*}{.220} \\
\hline & Experienced & $23.3 \%$ & $17.6 \%$ & $23.5 \%$ & $35.6 \%$ & & \\
\hline
\end{tabular}

$* \mathrm{p} \leq .01 \quad * * \mathrm{p} \leq .05$ 
To further examine the association between 'New' and 'Experienced' front-line officers and de-policing practices, collapsed and recoded variables were created by merging the non de-policing and limited de-policing archetypes into one variable ('Non + Limited') and also by merging the moderate and intensive de-policing archetypes into one variable ('Moderate + Intensive'). The table below demonstrates, by each participating police agency, the key considerations in understanding the relationship between de-policing behavioural practices and front-line policing experience.

Table 32: 'New' and 'experienced' front-line officers and collapsed de-policing categories (by agency)

\begin{tabular}{|c||c|c||c|c|}
\hline & $\begin{array}{c}\text { New front- } \\
\text { line officers }\end{array}$ & $\begin{array}{c}\text { Experienced } \\
\text { front-line } \\
\text { officers }\end{array}$ & $\begin{array}{c}\text { Non + } \\
\text { Limited } \\
\text { de-policers }\end{array}$ & $\begin{array}{c}\text { Moderate + } \\
\text { Intensive } \\
\text { de-policers }\end{array}$ \\
\hline Police Agency & $\mathbf{\%}$ & $\mathbf{\%}$ & $\mathbf{\%}$ & $\mathbf{\%}$ \\
\hline Albany & 42.3 & 57.7 & 39.6 & 60.4 \\
\hline Buffalo & 34.1 & 65.9 & 39.4 & 60.6 \\
\hline Calgary & 38.4 & 61.6 & 41.3 & 58.7 \\
\hline Cornwall & 27.8 & 72.2 & 58.4 & 41.6 \\
\hline Delta & 44.8 & 55.2 & 63.8 & 36.2 \\
\hline Gatineau & 43.5 & 56.5 & 62.1 & 37.9 \\
\hline Halifax & 31.6 & 68.4 & 54.1 & 45.9 \\
\hline Hamilton & 35.6 & 64.4 & 46.7 & 53.3 \\
\hline Kingston & 16.7 & 83.3 & 49.3 & 50.7 \\
\hline New Westminster & 44.9 & 55.1 & 59.2 & 40.8 \\
\hline Niagara Regional & 24.5 & 75.5 & 39.3 & 60.7 \\
\hline Ottawa & 17.5 & 82.5 & 51.9 & 48.1 \\
\hline Port Moody & 50.0 & 50.0 & 60.0 & 40.0 \\
\hline RCMP Codiac & 16.4 & 83.6 & 61.2 & 38.8 \\
\hline RCMP Red Deer & 37.3 & 62.7 & 58.1 & 41.9 \\
\hline RCMP Ridge Meadows & 54.5 & 45.5 & 53.1 & 46.9 \\
\hline Rochester & 19.6 & 80.4 & 40.6 & 59.4 \\
\hline Saskatoon & 32.0 & 68.0 & 39.6 & 60.4 \\
\hline Stratford & 17.2 & 82.8 & 60.7 & 39.3 \\
\hline Syracuse & 23.8 & 76.2 & 45.8 & 54.2 \\
\hline Toronto & 20.6 & 79.4 & 41.7 & 58.3 \\
\hline Windsor & 22.6 & 77.4 & 43.0 & 57.0 \\
\hline Yonkers & 22.0 & 78.0 & 48.0 & 52.0 \\
\hline Totals & $\mathbf{2 9 . 5 \%}$ & $\mathbf{7 0 . 5 \%}$ & $\mathbf{4 6 . 4 \%}$ & $\mathbf{5 3 . 6 \%}$ \\
\hline & & & &
\end{tabular}




\section{De-policing archetypes in the context of 'risky' demographic variables}

As $83 \%$ of study participants $(\mathrm{N}=3027)$ reported (by answering 'Yes' to

Question 25 in the survey questionnaire), it is clear that, today, many throughout policing's rank-and-file feel that there exists an elevated risk of criticisms of, or complaints about, their police work, which is related to officers' subjective interpretations of particular 'demographic' characteristics presented by individuals with whom officers interact in the communities where they perform their police work (e.g. persons who appear to be 'visible minority' or 'non-White', who are demonstrating behaviour suggestive of a mental health issue, and/or who are exhibiting a nonheterosexual orientation). The table below presents these risk perceptions, among frontline officers in this study, in the context of the identified de-policing archetypes.

Table 33: De-policing archetypes and officers' perceptions of elevated risk (overall)

\begin{tabular}{|c|c|c|c|c|}
\hline $\begin{array}{c}\text { De-policing archetypes and officer's } \\
\text { perceptions of risk (overall) }\end{array}$ & $\begin{array}{c}\text { Risk } \\
\text { Perceived }\end{array}$ & $\begin{array}{c}\text { No Risk } \\
\text { Perceived }\end{array}$ & Totals \\
\hline \multirow{2}{*}{ Non de-policers } & $\mathbf{N}$ & 665 & 357 & 1022 \\
\cline { 2 - 5 } & Valid \% & $65.1 \%$ & $34.9 \%$ & $100.0 \%$ \\
\hline \multirow{2}{*}{ Limited de-policers } & $\mathbf{N}$ & 516 & 140 & 656 \\
\cline { 2 - 5 } & Valid \% & $78.7 \%$ & $21.3 \%$ & $100.0 \%$ \\
\hline \multirow{2}{*}{ Moderate de-policers } & $\mathbf{N}$ & 736 & 119 & 855 \\
\cline { 2 - 5 } & Valid \% & $86.1 \%$ & $13.9 \%$ & $100.0 \%$ \\
\hline \multirow{2}{*}{ Intensive de-policers } & $\mathbf{N}$ & 1080 & 0 & 1080 \\
\cline { 2 - 5 } & Valid \% & $100.0 \%$ & $0 \%$ & $100.0 \%$ \\
\hline \multirow{2}{*}{ Count } & $\mathbf{N}$ & 2997 & 616 & 3613 \\
\cline { 2 - 5 } & Valid \% & $83.0 \%$ & $17.0 \%$ & $100.0 \%$ \\
\hline Missing & $\mathbf{N}$ & & & 47 \\
\hline Total & $\mathbf{N}$ & & & 3660 \\
\hline
\end{tabular}

$\chi^{2}=467.528 \quad \mathrm{p} \leq .01 \quad$ Cramer's $\mathrm{V}=.360$

A strong association is observed between an officer's subjective perception of this risk and proportions within the de-policing archetypes. The perception of risk is observed to increase in relation to the level of de-policing behaviour(s) - consistent with 
the concept of a de-policing continuum. For example, while $65 \%$ of non-depolicers report that they perceive an elevated likelihood of public criticism in interactions with members of (as they view them) 'risky' populations in society, intensive de-policers, situated at the other end of the de-policing continuum, are universal in such an understanding (100\%).

In relation to those study participants who reported 'No' to Question $25(\mathrm{~N}=621)$, the table below presents where these participants can be situated on the de-policing continuum. Of the study's research participants that indicated they do not perceive any elevated risk for criticisms of, or complaints about, their police work, which is related to 'demographic' characteristics of certain individuals in the community, it is interesting to consider that over half $(58.0 \%)$ of these officers are categorized as non de-policers (that make up only $28.3 \%$ of the research population).

Table 34: 'No' respondents to Q. 25 in the survey and de-policing archetypes

\begin{tabular}{|c||c|c|}
\hline $\begin{array}{l}\text { No perception of elevated risk presented by } \\
\text { certain individuals with whom officers interact }\end{array}$ & N & $\begin{array}{c}\text { \% of Q. 25 'No' } \\
\text { respondents }\end{array}$ \\
\hline \hline Non de-policers & 357 & 58.0 \\
\hline Limited de-policers & 140 & 22.7 \\
\hline Moderate de-policers & 119 & 19.3 \\
\hline Intensive de-policers & 0 & 0.0 \\
\hline Total & 615 & 100.0 \\
\hline Missing & 5 & \multicolumn{1}{|}{} \\
\hline Total & 621 & \\
\cline { 1 - 2 } & \multicolumn{1}{|c}{}
\end{tabular}

The following table presents bivariate analysis of the identified de-policing archetypes in the context of 'demographic' variables identified as particularly 'risky' by those study participants that answered 'Yes' to Question 25 in the survey ( $\mathrm{N}=3027)$. 
Table 35: De-policing archetypes and officers' perceptions of elevated risk (specific categories identified)

\begin{tabular}{|c|c|c|c|c|c|c|}
\hline $\begin{array}{c}\text { De-policing archetypes } \\
\text { and officer's perceptions } \\
\text { of risk (by specific } \\
\text { category) }\end{array}$ & $\begin{array}{c}\text { Non } \\
\text { de- } \\
\text { policers }\end{array}$ & $\begin{array}{c}\text { Limited } \\
\text { de- } \\
\text { policers }\end{array}$ & $\begin{array}{c}\text { Moderate } \\
\text { de- } \\
\text { policers }\end{array}$ & $\begin{array}{c}\text { Intensive } \\
\text { de- } \\
\text { policers }\end{array}$ & $\chi^{2}$ & V \\
\hline $\begin{array}{c}\text { Race/Ethnicity } \\
\text { ('visible minority') }\end{array}$ & $95.9 \%$ & $94.0 \%$ & $95.6 \%$ & $99.1 \%$ & $34.388^{*}$ & .107 \\
\hline Mental health issue(s) & $48.6 \%$ & $46.5 \%$ & $53.7 \%$ & $55.7 \%$ & $16.016^{*}$ & .073 \\
\hline $\begin{array}{c}\text { 'Non-traditional' } \\
\text { sexual orientation }\end{array}$ & $25.9 \%$ & $24.7 \%$ & $36.7 \%$ & $35.7 \%$ & $37.746^{*}$ & .113 \\
\hline Gender (female) & $35.4 \%$ & $36.2 \%$ & $32.8 \%$ & $10.1 \%$ & $217.406^{*}$ & .270 \\
\hline Physical disability & $13.8 \%$ & $11.9 \%$ & $12.6 \%$ & $3.1 \%$ & $79.037^{*}$ & .163 \\
\hline $\begin{array}{c}\text { 'Other' (as identified by } \\
\text { study participants) }\end{array}$ & $7.7 \%$ & $7.6 \%$ & $5.4 \%$ & $3.3 \%$ & $21.116^{*}$ & .084 \\
\hline
\end{tabular}

$* \mathrm{p} \leq .01$

Race/Ethnicity - A moderate association is observed between perceptions of risk, as related specifically to race/ethnicity (persons who are subjectively assessed by the officer as being 'visible minority' or 'non-White') and the present study's de-policing archetypes. This study finds that citizens who appear as 'visible minority' or 'nonWhite' (in the subjective perception of the front-line officer) are viewed by almost all study participants, across all identified de-policing archetypes (including $96 \%$ of non de-policers), as 'risky' - as relates to officers' understandings around the propensity for such individuals to criticize and/or file complaints about an officer's police work. Mental Health Issue(s) - In terms of persons who present with apparent mental health issues and officer's perceptions of risk, a weak association is observed in relation to depolicing archetypes. However, as the sheer numbers suggest, it is important to consider that substantial (and relatively similar) numbers of front-line officers across each depolicing archetype find this to be a 'risky' demographic (ranging from $46.5 \%$ to $55.7 \%$ ).

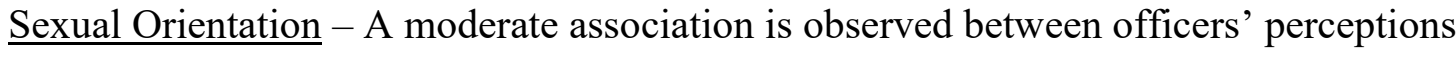
of risk, as related specifically to persons who present with a sexual orientation other 
than 'traditional' (heterosexual), and proportions within the study's identified depolicing archetypes. The reader should appreciate that substantial numbers of front-line police officers across each de-policing archetype (ranging from $24.7 \%$ of limited depolicers to $36.7 \%$ of moderate de-policers) find this to be a 'risky' demographic - with almost one-third of all study participants understanding that those perceived as 'nonheterosexuals' (LGBTQ+) pose an elevated risk to criticize and/or file a complaint about an officer's conduct.

Gender - In terms of gender (in this case female as compared to male) and officers' perceptions of risk, a moderate association is observed as relates to de-policing archetypes. It is interesting to note the circumstance with intensive de-policers in comparison to the other de-policing archetypes - with risk related to gender perceived by only $10.1 \%$ of intensive de-policers, whereas over $30 \%$ of officers in each of the other three de-policing categories find women to be a 'risky' demographic.

Physical Disabilities - A moderate association is observed between officers' perceptions of risk as related specifically to persons who present with apparent physical disabilities and de-policing archetypes. However, it is important for the reader to appreciate that more than $90 \%$ of study participants do not find this to be a 'risky' demographic. 'Other' - A small number of study participants (4.4\%) introduced other demographic characteristics (e.g. age, socio-economic status, persons with criminal histories, etc.) into the study through write-in responses to Question 25, which they recognize as presenting an increased risk for criticism of, and/or a complaint about, their front-line police work. 


\section{Study participants and familiary with the concept de-policing}

As discussed earlier in this chapter (in relation to officers' responses to questions 27 and 28) it became apparent during data analysis that a significant number of study participants did not understand (at the time of survey administration) what is involved with the concept (and/or practice) of de-policing - at least in relation to the three terminologies provided for their consideration in this study. The following table presents analysis related to participants' familiarity with the concept (as described in the study and specifically in relation to Question 27) in the contexts of de-policing archetypes and the variables 'New' versus 'Experienced' front-line officers.

Table 36: De-policing archetypes and familiarity with the concept of de-policing

\begin{tabular}{|c|c|c|c|c|}
\hline & $\begin{array}{c}\text { Know } \\
\text { concept }\end{array}$ & $\begin{array}{c}\text { Do not know } \\
\text { concept }\end{array}$ & $\chi^{2}$ & $\mathbf{V}$ \\
\hline Non de-policers & $45.2 \%$ & $54.8 \%$ & \multirow{4}{*}{$311.798^{*}$} & \multirow{4}{*}{.294} \\
\hline Limited de-policers & $55.5 \%$ & $44.5 \%$ & & \\
\hline Moderate de-policers & $61.8 \%$ & $38.2 \%$ & & \\
\hline Intensive de-policers & $81.7 \%$ & $28.3 \%$ & & \\
\hline New front-line officers & $47.4 \%$ & $52.6 \%$ & \multirow[b]{2}{*}{$137.698^{*}$} & \multirow[b]{2}{*}{.194} \\
\hline $\begin{array}{l}\text { Experienced front-line } \\
\text { officers }\end{array}$ & $68.1 \%$ & $31.9 \%$ & & \\
\hline
\end{tabular}

$* \mathrm{p} \leq .01$

Not unexpectedly, moderate associations were observed between an officer's familiarity with the concept of de-policing and both de-policing archetypes and years of front-line policing. It is apparent that, with more front-line experience, the likelihood of an officer becoming knowledgeable around the concept of de-policing increases. Also, in the context of the de-policing archetypes, officers situated higher on the continuum are found to be more likely to understand the concept $(81.7 \%$ of intensive de-policers versus $45.2 \%$ of non de-policers). 


\section{Study participants that have (apparently) not witnessed and/or practiced de-policing}

During data entry (and subsequent analysis) it became apparent that not insignificant numbers of study participants presented conflicting responses to different, but related, questions in the survey instrument. For example, some officers reported that they have not changed their behaviour because of a concern that their actions could be videorecorded (Question 16), yet they reported (in responding to Question 17 and/or Question 18) that they do, in fact, use force less often, or use less force than they otherwise would, because of the potential for videorecording of their actions. Further, it became apparent that a considerable number of study participants do not understand that the behaviours they are, themselves, practicing in the field (as disclosed in their affirmative responses and qualitative elaborations to questions 16, 24, and/or 26) coincide with the concept of de-policing - as articulated in the preamble wording to questions 27 and 28 within the hardcopy questionnaire document. For example (among many), an Ottawa Police officer (OTT119) answered Question 24 in the affirmative and reported, in their written elaboration to this question, that he/she avoids engaging public of different races for suspicious circumstances because of a concern that his/her actions could be criticized by some members of the public. However, this participant subsequently answered 'No' to both survey questions 27 and $28 .{ }^{20}$ In this regard, it is found that not-insignificant numbers of front-line officers that are, themselves, engaging in de-policing behaviours, apparently do not understand (at least in the context of Question 27 and/or Question 28 in this study's survey instrument) that this represents them having certainly witnessed de-policing - as this behaviour is defined within the present study. 
Put simply, if one is practicing de-policing they are also witnessing it being practiced. The table below presents analysis related to participants' hearing about, and/or witnessing, de-policing where they perform their police work - in the contexts of de-policing archetypes and variables 'New' and 'Experienced' front-line officers.

Table 37: De-policing archetypes and participants hearing of, or witnessing, depolicing

\begin{tabular}{|c|c|c|c|c|}
\hline & $\begin{array}{c}\text { Have heard } \\
\text { of, or } \\
\text { witnessed, } \\
\text { de-policing }\end{array}$ & $\begin{array}{c}\text { Have not heard of, } \\
\text { nor witnessed, de- } \\
\text { policing }\end{array}$ & $\chi^{2}$ & $\mathbf{V}$ \\
\hline Non de-policers & $37.1 \%$ & $62.9 \%$ & \multirow{4}{*}{$448.817 *$} & \multirow{4}{*}{.353} \\
\hline Limited de-policers & $51.1 \%$ & $48.9 \%$ & & \\
\hline Moderate de-policers & $58.1 \%$ & $41.9 \%$ & & \\
\hline Intensive de-policers & $81.9 \%$ & $18.1 \%$ & & \\
\hline New front-line officers & $42.1 \%$ & $57.9 \%$ & \multirow[t]{2}{*}{$159.935^{*}$} & \multirow[t]{2}{*}{.210} \\
\hline $\begin{array}{c}\text { Experienced front-line } \\
\text { officers }\end{array}$ & $64.8 \%$ & $35.2 \%$ & & \\
\hline
\end{tabular}

$* \mathrm{p} \leq .01$

Given that, by definition (in this study's criteria), non de-policers are those officers that are not practicing de-policing behaviour, it is perhaps not surprising that $63 \%$ of participants situated within the non de-policer archetype report not having heard of, or witnessing, de-policing where they work. However, limited de-policers, moderate de-policers, and, most profoundly, intensive de-policers are, through their own reported behaviours, not only witnessing de-policing but they are themselves, in fact, practicing de-policing. Therefore, it is interesting to consider that large numbers of limited depolicers $(\mathrm{N}=319)$, moderate de-policers $(\mathrm{N}=355)$, and even intensive de-policers $(\mathrm{N}=194)$ reported not having heard of, or witnessing, de-policing where they work. Given this data, it is clear that, for many study participants, their answers indicating unfamiliarity with the concept of de-policing (as defined by the three terms in this study - 'risk-averse policing', 'de-policing', or 'F.I.D.O.') do not coincide with their actual 
familiarity with de-policing practices (by virtue of their own behaviours - in themselves practicing de-policing).

In this regard, the reader should understand that there is, at a minimum, a $13.6 \%$ gap between study participants' actually witnessing de-policing where they perform their front-line police work (given that $71.7 \%$ of officers in this study are categorized based on their affirmative response to survey question 16, 24, and/or 26 - as limited, moderate, or intensive de-policers) and those $58.1 \%$ of participants reporting that they have heard of, or witnessed, the risk-averse practice (based on their affirmative response to survey question 28). As such, the actual proportion of officers having heard of, or witnessing de-policing practices where they work should be understood as a significantly-higher value (that is not possible to estimate with any precision) than that represented in the value (58.1\%) based on affirmative responses to survey question 28 .

\section{Non de-policers and the consideration of de-policing}

During data analysis, it became apparent that many front-line officers situated within this study as non de-policers give little consideration (or none) to contemporary circumstances of visibility and/or public critique in deciding on their operational actions in the field. The following table presents data related to participants (by de-policing archetype and collapsed variables 'New' and 'Experienced') that have (and have not) thought about changing behaviour(s) because of the potential for video recording by the public ('video') and/or because of the potential for public critique of their work ('criticism'). 
Table 38: Participants who have (and have not) thought about changing behaviour(s)

\begin{tabular}{|c||c|c||c|c|}
\hline & $\begin{array}{c}\text { Have thought } \\
\text { about (video) }\end{array}$ & $\begin{array}{c}\text { Have not } \\
\text { thought about } \\
\text { (video) }\end{array}$ & $\begin{array}{c}\text { Have thought } \\
\text { about (criticism) }\end{array}$ & $\begin{array}{c}\text { Have not } \\
\text { thought about } \\
\text { (criticism) }\end{array}$ \\
\hline Non & $5.9 \%$ & $94.1 \%$ & $6.1 \%$ & $93.9 \%$ \\
\hline Limited & $48.2 \%$ & $51.8 \%$ & $37.2 \%$ & $62.8 \%$ \\
\hline Moderate & $86.1 \%$ & $13.9 \%$ & $93.1 \%$ & $6.9 \%$ \\
\hline Intensive & $100 \%$ & $0 \%$ & $100 \%$ & $0 \%$ \\
\hline & \multicolumn{2}{|c|}{$\chi^{2}=2264.560 \mathrm{p} \leq .01$} & $\chi^{2}=2500.089 \mathrm{p} \leq .01$ \\
& Cramer's V $=.791$ & Cramer's V $=.831$ \\
\hline \hline 'New' & $50.0 \%$ & $50.0 \%$ & $48.0 \%$ & $52.0 \%$ \\
\hline 'Experienced' & $65.2 \%$ & $34.8 \%$ & $65.5 \%$ & $34.5 \%$ \\
\hline & \multicolumn{2}{|c|}{$\chi^{2}=73.114 \mathrm{p} \leq .01$} & \multicolumn{2}{|c|}{$\begin{array}{c}\chi^{2}=97.891 \mathrm{p} \leq .01 \\
\text { Cramer's V }=.142\end{array}$} \\
\hline
\end{tabular}

It is clear that among non de-policers, few give consideration to changing their inthe-field conduct in response to the techno-social and/or socio-political external environment(s) where they do their front-line police work. Data supports that a subcategory of non de-policers (operating largely independent from considerations around years of front-line experience) exists - comprised of officers that are fixed in their rejection of de-policing behaviours, to the point where they do not even give any thought to altering their conduct. The idea that such a philosophical outlook can be related to a 'mission orientation' within the psyche of certain officers, which has been advanced by various policing scholars as a core tenet of 'traditional' police occupation culture, is elaborated in the discussion engaged throughout Chapter Six.

\section{Nature of concerns across de-policing archetypes}

Among limited de-policers and moderate de-policers, in comparison to intensive de-policers, concerns are substantially more related to the potential for video recording of police actions in the field (as disclosed in officers' responses to Question 16) and/or criticism of police conduct by persons in the community (as disclosed in officers' 
responses to Question 24) rather than concerns related to elevated risk presented to front-line officers by those persons perceived to epitomize 'risky' 'demographic' characteristic(s) - in terms of the citizen's race/ethnicity, mental health issues, sexual orientation, gender, physical disabilities, or 'other' features (as disclosed in officers' responses to Question 26).

Table 39: Nature of concerns by de-policing archetype

\begin{tabular}{|c|c|c|c|c|c|c|}
\hline \multirow{2}{*}{} & \multicolumn{2}{|c|}{ 'Yes' Q.16 } & \multicolumn{2}{c|}{ 'Yes' Q. 24 } & \multicolumn{2}{c|}{ 'Yes' Q. 26 } \\
\cline { 2 - 7 } & $\mathbf{N}$ & $\mathbf{\%}$ & $\mathbf{N}$ & $\mathbf{\%}$ & $\mathbf{N}$ & $\mathbf{\%}$ \\
\hline Non de-policers & 0 & 0.0 & 0 & 0.0 & 0 & 0.0 \\
\hline Limited de-policers & 277 & 42.1 & 223 & 33.9 & 158 & 24.0 \\
\hline Moderate de-policers & 716 & 83.5 & 785 & 91.6 & 213 & 24.9 \\
\hline Intensive de-policers & 1081 & 100.0 & 1081 & 100.0 & 1081 & 100.0 \\
\hline Totals & 2074 & & 2089 & & 1452 & \\
\hline
\end{tabular}

As depicted in the representation of data in the table above, the distribution of 'Yes' responses for Question 26 is substantially different than those for Question 16 and Question 24. It is important to appreciate that limited de-policers and moderate depolicers (combined) account for only one-quarter (25.5\%) of participants that answered 'Yes' to Question 26, whereas intensive de-policers account for three-quarters (74.5\%) of front-line officers reporting this component of the contemporary de-policing phenomenon. Further, consistent with the concept of a de-policing continuum and the progression, among some front-line officers, toward additional/escalating/intensifying de-policing behaviours, study data illustrates that, in most instances, the difference between non de-policers and limited de-policers involves, for the latter, behavioural adaptations to either the potential for the video recording of, or public criticism of, their actions in the field, rather than avoidance of individuals in the community presenting with certain 'risky' 'demographic' characteristics (as interpreted by the officer). The differences are even more pronounced when considering data related to limited de- 
policers and moderate de-policers. It is observed that those front-line officers who progress along the de-policing continuum, from limited de-policers to moderate depolicers, do so, by and large, because of the development of risk-averse apprehensions related to either video recording potential or public criticism of their actions - rather than concerns about interacting with particularly 'risky' citizens. Intensive de-policers, however, demonstrate that they have developed anxieties toward populations they perceive as 'risky' and have modified their behaviour accordingly - by proactively working to avoid interaction with individuals perceived to represent the 'risky' characteristic(s).

\section{Awareness of video recording potential across de-policing archetypes}

While intensive de-policers comprise less than one-third (29.9\%) of the study population, they account for more than half (55.6\%) of research participants indicating (in response to Question 14 in the survey) that the potential for their conduct to be video recorded is something that is always on their mind (as represented by the value 10 out of a maximum value of 10). Of the 1163 front-line officers recording the value ' 10 ' in response to Question 14, the prevalence, by both de-policing archetype and variables 'New' and 'Experienced,' is consistent with what one would expect with the existence of a de-policing continuum. Only $14.4 \%$ of non de-policers recorded the value ' 10 'whereas $18.7 \%$ of limited de-policers, $29 \%$ of moderate de-policers, and fully $60 \%$ of intensive de-policers expressed this level of awareness around video recording potential in the environment in which they perform their front-line police work. While only one in five $(20.3 \%)$ officers with five or less years of front-line service recorded the value ' 10 ' in response to Question 14, over a third (37.2\%) of more experienced officers feel 
this way. The following tables present data related to study participants (by de-policing archetype and variables 'New' and 'Experienced') in relation to responses to Question 14 in the survey instrument.

Table 40: De-policing archetypes and officers' awareness of video recording potential (on scale of 1 to 10)

\begin{tabular}{|c|c|c|c|c|c|c|c|c|c|c|c|c|}
\hline & & 1 & 2 & 3 & 4 & 5 & 6 & 7 & 8 & 9 & 10 & TOTAL \\
\hline $\begin{array}{l}\text { Non de- } \\
\text { policers }\end{array}$ & $\mathbf{N}$ & 19 & 32 & 40 & 48 & 105 & 63 & 167 & 290 & 107 & 146 & 1017 \\
\hline $\begin{array}{c}\text { Limited de- } \\
\text { policers }\end{array}$ & $\mathbf{N}$ & 1 & 8 & 20 & 24 & 40 & 52 & 113 & 175 & 99 & 122 & 654 \\
\hline $\begin{array}{l}\text { Moderate de- } \\
\text { policers }\end{array}$ & $\mathbf{N}$ & 3 & 3 & 8 & 11 & 36 & 35 & 103 & 258 & 149 & 248 & 854 \\
\hline $\begin{array}{c}\text { Intensive de- } \\
\text { policers }\end{array}$ & $\mathbf{N}$ & 1 & 0 & 2 & 5 & 12 & 27 & 52 & 159 & 174 & 647 & 1079 \\
\hline Count & $\mathbf{N}$ & 24 & 43 & 70 & 88 & 193 & 177 & 435 & 882 & 529 & 1163 & 3604 \\
\hline Missing & $\mathbf{N}$ & & & & & & & & & & & 56 \\
\hline Total & $\mathbf{N}$ & & & & & & & & & & & 3660 \\
\hline
\end{tabular}

$\chi^{2}=846.529 \mathrm{p} \leq .01 \quad$ Cramer's $\mathrm{V}=.280$

Table 41: 'New' versus 'experienced' officers and officers' awareness of video recording potential (on scale of 1 to 10)

\begin{tabular}{|c|c|c|c|c|c|c|c|c|c|c|c|c|}
\hline & & 1 & 2 & 3 & 4 & 5 & 6 & 7 & 8 & 9 & 10 & TOTAL \\
\hline New & $\mathbf{N}$ & 8 & 19 & 29 & 47 & 77 & 67 & 157 & 295 & 155 & 217 & 1071 \\
\hline Experienced & $\mathbf{N}$ & 16 & 24 & 40 & 41 & 119 & 113 & 280 & 601 & 376 & 954 & 2564 \\
\hline Count & $\mathbf{N}$ & 24 & 43 & 69 & 88 & 196 & 180 & 437 & 896 & 531 & 1171 & 3635 \\
\hline Missing & $\mathbf{N}$ & & & & & & & & & & & 25 \\
\hline Total & $\mathbf{N}$ & & & & & & & & & & & 3660 \\
\hline
\end{tabular}

$\chi^{2}=129.799 \quad \mathrm{p} \leq .01 \quad$ Cramer's $\mathrm{V}=.189$

De-policing archetypes and the influence of the researcher's 'pracademic' background on officers' responses (data integrity and data comprehensiveness considerations)

During data analysis for Question 30, it became apparent (unexpectedly - at least to this researcher) that, for many front-line officers, a researcher's biography (visà-vis the 'traditional' university-based academic versus the 'pracademic' researcher with experience in front-line police work) is a significant consideration - as relates to 
issues around trust; agreement to participate in, and degree of participation within, the research project; honesty (of responses); and comprehensiveness (of data shared by the participants within the study). The following table presents data related to study participants - by de-policing archetype and variables 'New' and 'Experienced' - that were (or were not) influenced in how they answered the study's survey questions because of their understanding of my researcher biography (with substantial experience in rank-and-file police work).

Table 42: De-policing archetypes and influence of the researcher's 'pracademic' biography on research participants' answers in the questionnaire

\begin{tabular}{|c|c|c||c|c|}
\hline & Influenced & Not Influenced & $\chi^{2}$ & V \\
\hline Non de-policers & $21.1 \%$ & $78.9 \%$ & & \\
\hline Limited de-policers & $29.8 \%$ & $70.2 \%$ & \multirow{2}{*}{$485.627 *$} & .397 \\
\hline Moderate de-policers & $42.3 \%$ & $57.7 \%$ & & \\
\hline Intensive de-policers & $69.8 \%$ & $30.2 \%$ & & \\
\hline New front-line officers & $34.6 \%$ & $65.4 \%$ & & \\
\cline { 1 - 3 } $\begin{array}{c}\text { Experienced front-line } \\
\text { officers }\end{array}$ & $47.1 \%$ & $52.9 \%$ & $40.167 *$ & .114 \\
\hline
\end{tabular}

$* \mathrm{p} \leq .01$

As will be discussed further within Chapter Six, this research finding, grounded in empirical data, is significant - not only in the context of the present study, but also in terms of implications vis-à-vis researcher positionality, trust, and data integrity across policing research in general. In the present study, it is particularly interesting to note that $70 \%$ of intensive de-policers $(\mathrm{N}=755)$ reported information in their answers to survey question(s) that was influenced, in some fashion, by my 'pracademic' biography. As demonstrated through the various qualitative elaborations to Question 30, answers presented by many of this study's participants were different than those they would have reported in a study conducted by a non-'pracademic' researcher. It is also noteworthy that even among non de-policers, over $20 \%$ of those study participants 
presented different information than they otherwise would have reported, had the study been conducted by a non- 'pracademic' researcher $(\mathrm{N}=216)$.

\subsection{Official Police Data Received Pursuant to FOI Applications}

As described in this chapter's introduction, in furtherance of the present study's empirical results, I sought out further data (what can be considered perhaps confirmatory or corroborative information) through the filing of formal FOI applications from eight police agencies (by submitting the necessary documentation and, when requested, paying the required fees). ${ }^{21} 22$ The type of data I was seeking was, in general terms, that related to front-line officers' proactive/discretionary activities, as opposed to their actions on dispatched calls, and that which would be relatively consistent across police agencies (given differences in applicable laws and processes) over a 10-year time frame - if such information was available. The following are the types of official metrics I sought in the applications - understanding, in advance, that not all police agencies maintain the same type of data in relation to the activities of their officers: 1) Number of officer-initiated calls in the field (as compared to dispatched calls for service)

2) Number of officer-initiated traffic stops in the field

3) Number of officer-initiated street checks/person checks in the field

4) Number of contact reports (often called 'street checks' or 'contact cards') submitted by officers (or similar documents that officers complete to capture, for intelligence purposes, information emerging from their interaction with a citizen; e.g. name, physical descriptors, associates, vehicle, location, observed behaviour, etc.) 
5) Number of infraction citations (tickets/offence notices) issued by officers in the field for persons observed to be drinking alcoholic beverages in public locations

6) Number of arrests in the field for persons observed to be intoxicated in a public place (which is generally a violation of provincial liquor laws across Canada, however, such conduct is not an offence in the State of New York - unless the individual is also disorderly)

7) Number of arrests in the field for persons alleged to be causing a disturbance in a public place (a contravention of section 175 of Canada's Criminal Code, which is very similar to the offence of engaging in disorderly conduct under New York's Penal Law section 240.20)

Throughout the application process, I discovered that police agencies generate and maintain much different data related to the activities of their front-line officers - both in terms of comprehensiveness and categories of captured information. Most of the agencies, from which I sought information, were unable to produce metrics reporting on more than just a few of the seven categories of information I was seeking (as detailed in the preceding paragraph). The most relevant information received, pursuant to these FOI requests, is presented, as follows, in alphabetical order by police agency.

\section{$\underline{\text { Buffalo Police Department (BPD) }}$}

In a letter sent to me on 21 December 2017, BPD Captain Jeff Rinaldo denied my FOI request. The reason cited was that my application was not sufficiently detailed to enable BPD staff "to locate and identify the records sought" and further, "it appears likely that this department does not maintain some of the records that you seek." ${ }^{22}$ 


\section{$\underline{\text { Calgary Police Service (CPS) }}$}

Pursuant to my FOI application (file 17-G-1813), information was received on 20 January 2018 from Roxanne Wales, CPS Senior Disclosure Analyst. As excerpted from the document CPS CAD Events, 2006-2016, Onview calls by Subevent Type, PTRL Unit Type, the following table (and graphic representations) presents data, over an 11-year period (2006 to 2016), in relation to officer-generated 'check on welfare' occurrences reported by CPS officers in the field (for which the rationale is explained in a subsequent paragraph) and officer-generated 'suspicious person' occurrences. ${ }^{23}$

\section{Table 43: CPS self-initiated calls 2006-2016 (check on welfare and suspicious person)}

\begin{tabular}{|l|c|c|c|c|c|c|c|c|c|c|c|}
\hline & $\mathbf{2 0 0 6}$ & $\mathbf{2 0 0 7}$ & $\mathbf{2 0 0 8}$ & $\mathbf{2 0 0 9}$ & $\mathbf{2 0 1 0}$ & $\mathbf{2 0 1 1}$ & $\mathbf{2 0 1 2}$ & $\mathbf{2 0 1 3}$ & $\mathbf{2 0 1 4}$ & $\mathbf{2 0 1 5}$ & $\mathbf{2 0 1 6}$ \\
\hline Check on welfare & 347 & 336 & 356 & 327 & 371 & 383 & 337 & 406 & 425 & 355 & 379 \\
\hline Suspicious person & 9391 & 8718 & 9790 & 11141 & 10891 & 8192 & 6236 & 5215 & 4621 & 3842 & 4653 \\
\hline
\end{tabular}

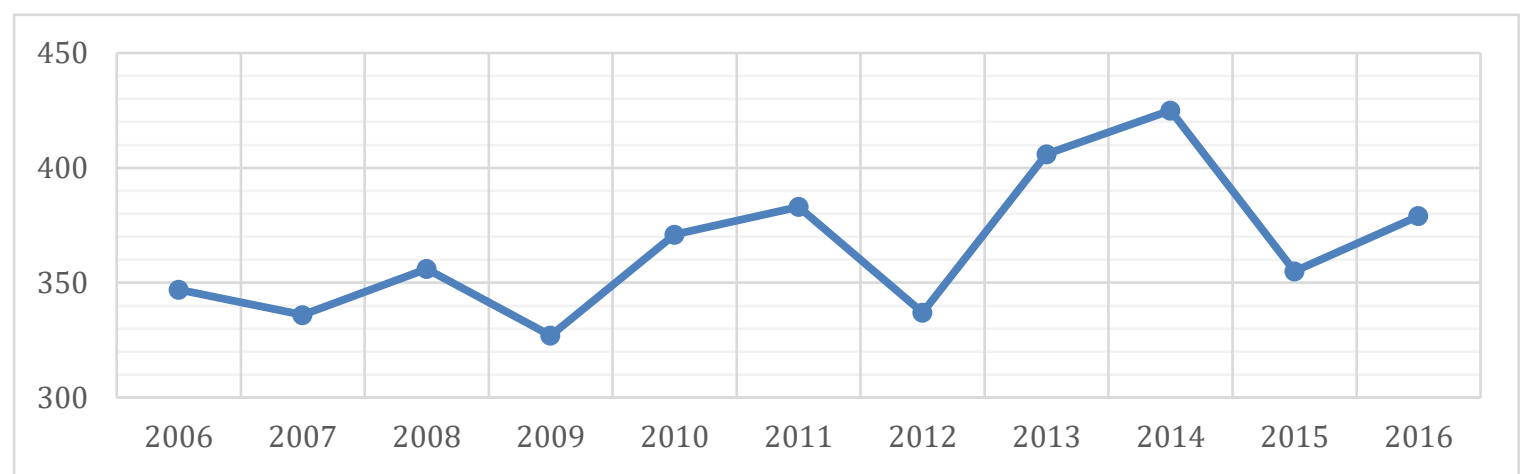

Figure 2: CPS self-initiated calls 2006-2016 ('check on welfare')

In relation to officer-generated 'check on welfare' calls, these are self-engaged activities by officers that come across information suggesting that an individual may be deceased or in urgent medical/psychiatric distress, which officers then typically followup on by attending immediately at the location reported by the complainant to check on the welfare of that individual. This information is included as comparison data (or could perhaps be considered within the context of a baseline measurement) - as such occurrences, while officer-initiated, are not really discretionary in nature and therefore 
not amenable to de-policing practices. In this regard, any officer who chose to ignore such information, reported directly to her/him by a concerned citizen, would be taking a substantial risk were he/she to be found out in not having followed-up on the information. Based on my policing experience, I suggest that this would amount to a prima facie case of serious professional misconduct and potentially could even be considered a criminal act - in circumstances where an officer's negligence resulted in someone being harmed. Further, because of the nature of these kinds of occurrences (involving potentially serious consequences for a citizen in distress) their frequency remains relatively consistent from year to year across the same population - in this case the roughly 1.2 million residents of the City of Calgary.

On the other hand, in this chapter I have not included data related to 'traffic stops' as, in my experience, these policing activities are not a measure of officers' discretionary/proactive practices across a police agency. In this regard, many police agencies have dedicated traffic officers (and, in larger agencies, traffic units) that are deployed, full-time or in periodic 'blitzes', exclusively in traffic enforcement duties, which can fluctuate considerably based on the resources allocated for such initiatives from time to time. As represented in the graph below, I argue that a more appropriate indicator of a potential de-policing effect on front-line police work would be officerinitiated activities inquiring into 'suspicious persons' - those kinds of street-level investigations by rank-and-file officers that develop from initial subjective determinations (by the officer) of 'suspicion', which are easier to overlook (or not respond to) than ignoring an observed traffic violation while specifically assigned to enforce such violations (as in the case of 'traffic stops'). 


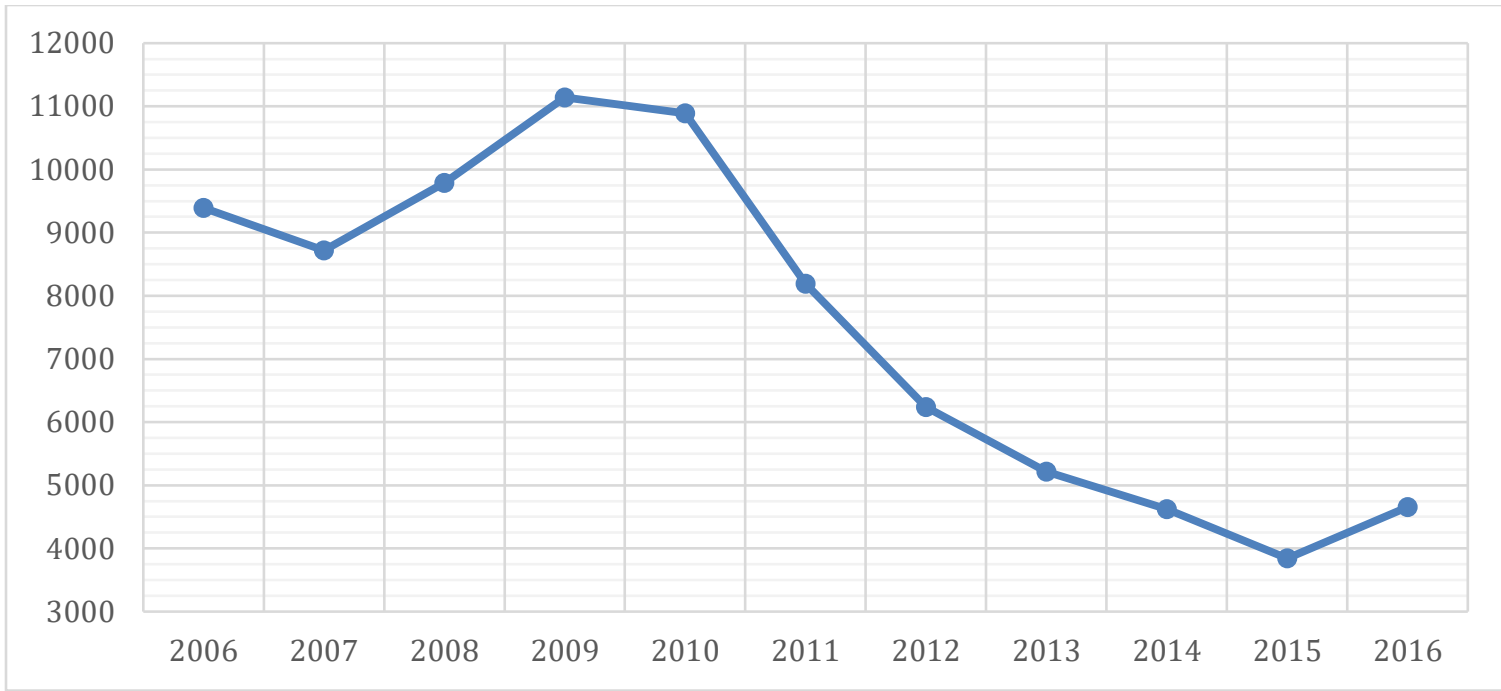

Figure 3: CPS self-initiated calls 2006-2016 ('suspicious person')

\section{$\underline{\text { Halifax Regional Police (HRP) }}$}

Some challenges, in terms of availability of data and retrieval of relevant information from databases, were encountered in attempting to acquire FOI data from the HRP through the formal application process. Fortunately, Christopher Giancomantonio, Research Coordinator for the HRP, is involved with the Canadian Society for Evidence-Based Policing and, through this mutual affiliation, I was able to at least receive some meaningful HPR data. Giancomantonio advised that HRP does not "hold data that could answer...how many 'self-initiated' [occurrences] officers undertake each year, with the exception of data on street checks" (personal communication, 8 March 2018). On 23 March 2018 I received the HRP data reporting on 'street checks', as represented below in table and graphic formats.

\section{Table 44: HRP 'street checks' 2008-2017}

\begin{tabular}{|c|c|c|c|c|c|c|c|c|c|c|}
\hline & $\mathbf{2 0 0 8}$ & $\mathbf{2 0 0 9}$ & $\mathbf{2 0 1 0}$ & $\mathbf{2 0 1 1}$ & $\mathbf{2 0 1 2}$ & $\mathbf{2 0 1 3}$ & $\mathbf{2 0 1 4}$ & $\mathbf{2 0 1 5}$ & $\mathbf{2 0 1 6}$ & $\mathbf{2 0 1 7}$ \\
\hline $\begin{array}{c}\text { Street } \\
\text { Checks }\end{array}$ & 7958 & 5312 & 4675 & 6305 & 7229 & 6893 & 6798 & 5631 & 5401 & 4853 \\
\hline
\end{tabular}




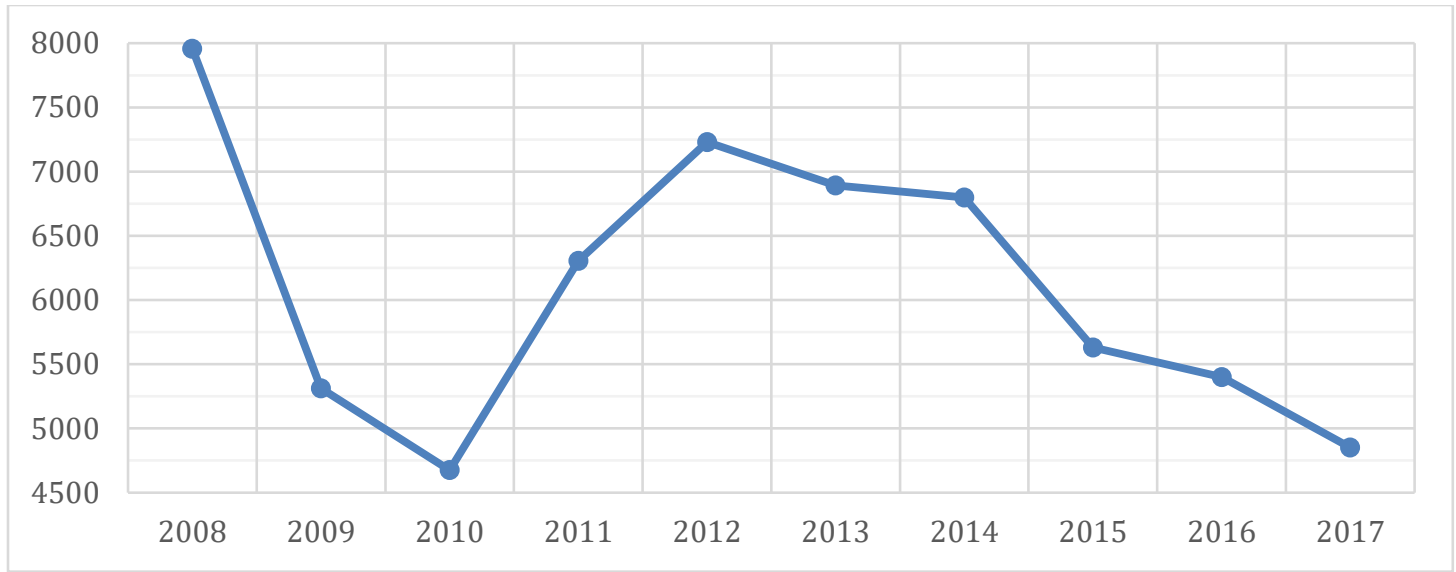

Figure 4: HRP 'street checks' 2008-2017

\section{Ottawa Police Service (OPS)}

Pursuant to my FOI application (file 18-020), information was received on 11 January 2018 from Carol Brunet, Freedom of Information Analyst. Brunet explained that the OPS can only provide historical data back to 2009 for 'officer-initiated calls' (as contrasted with dispatched calls). This data is presented below in table and graphic formats.

Table 45: OPS self-initiated calls 2009-2017

\begin{tabular}{|c|c|c|c|c|c|c|c|c|c|}
\hline & 2009 & 2010 & 2011 & 2012 & 2013 & 2014 & 2015 & 2016 & 2017 \\
\hline Calls & 107310 & 112969 & 101216 & 107567 & 109825 & 102709 & 102618 & 91622 & 86308 \\
\hline
\end{tabular}

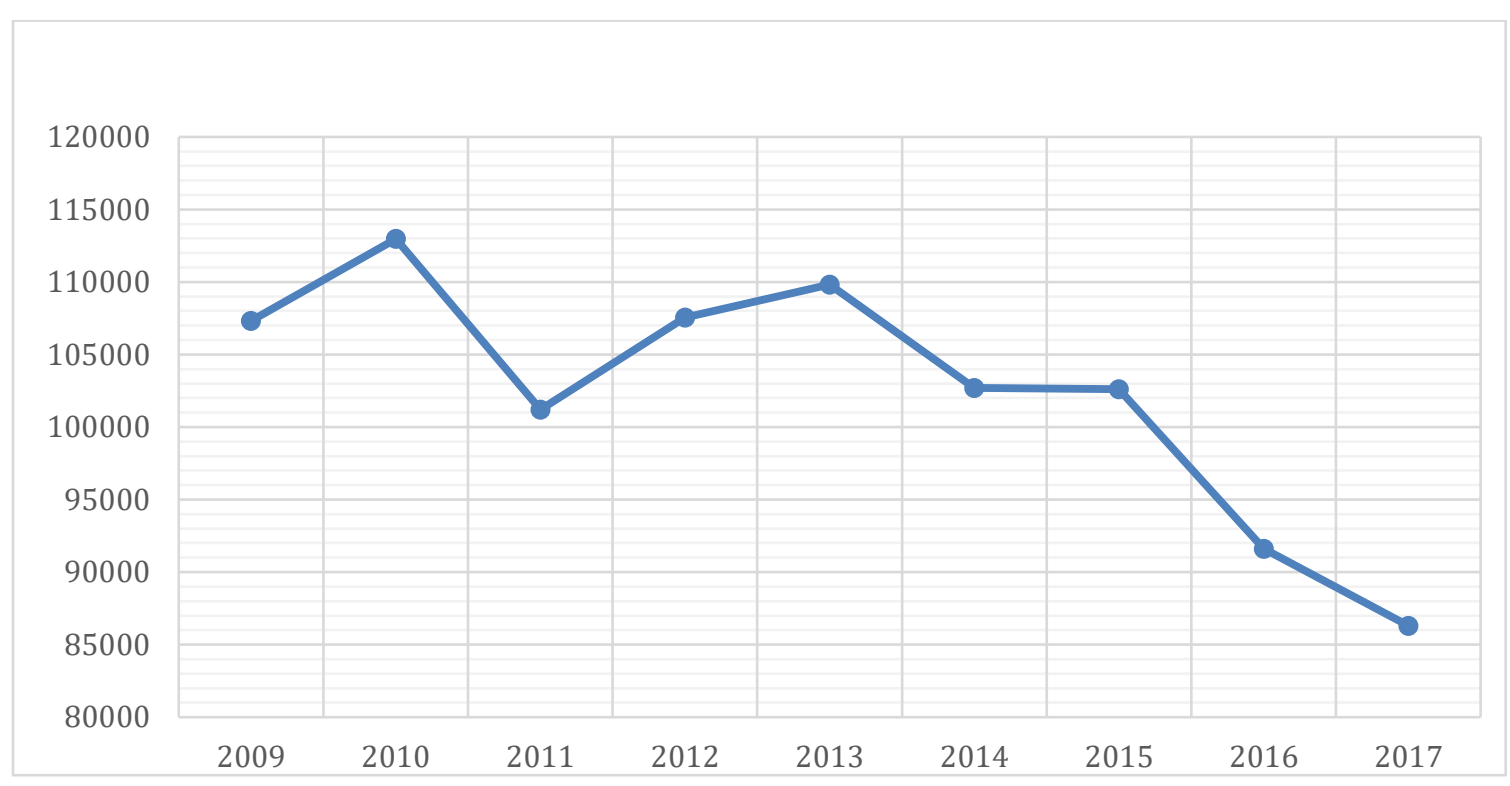

Figure 5: OPS officer-initiated calls 2009-2017 
After the public release of the OPS 2017 report on officer-initiated 'person' (or 'street') checks, disclosing that for the entire year OPS officers only engaged in seven (7) such documented citizen contacts, there was considerable mediated discussion across the Ottawa region (e.g., see Cosette 2018; Yogaretnam 2018a). Matt Skof, President of the Ottawa Police Association (the labour organization representing rankand-file OPS officers), observed that a number of factors, including "crippling legislation that prevents police from interacting with the public" have impacted on "traffic stops, street checks, [and] proactive interactions" (Yogaretnam 2018a). As Cosette (2018) reported, "Asked to explain how the number of annual checks could plummet from thousands to just seven, [OPS Deputy Chief Steve] Bell said, 'Officers are now reluctant to conduct checks... They feel they're inhibited from collecting a lot of information or having a lot of interactions with people.' The decline in the number of checks isn't limited to Ottawa, Bell added, describing it as part of a trend observed by police forces across the province."

As a follow-up to my original FOI application, further information regarding OPS officers' self-initiated 'street checks' was requested and received from Brunet on 30 January 2018. This data is presented below in table and graphic formats.

Table 46: OPS 'street checks' 2004-2017

\begin{tabular}{|l|l|l|l|l|l|l|l|l|l|l|l|l|l|}
\hline & 2004 & 2005 & 2006 & 2007 & 2008 & 2009 & 2010 & 2011 & 2012 & 2013 & 2014 & 2015 & 2016 \\
\hline Checks & 9251 & 9157 & 9158 & 8744 & 8018 & 8974 & 8240 & 6747 & 6950 & 5300 & 4405 & 3495 & 1748 \\
\hline
\end{tabular}




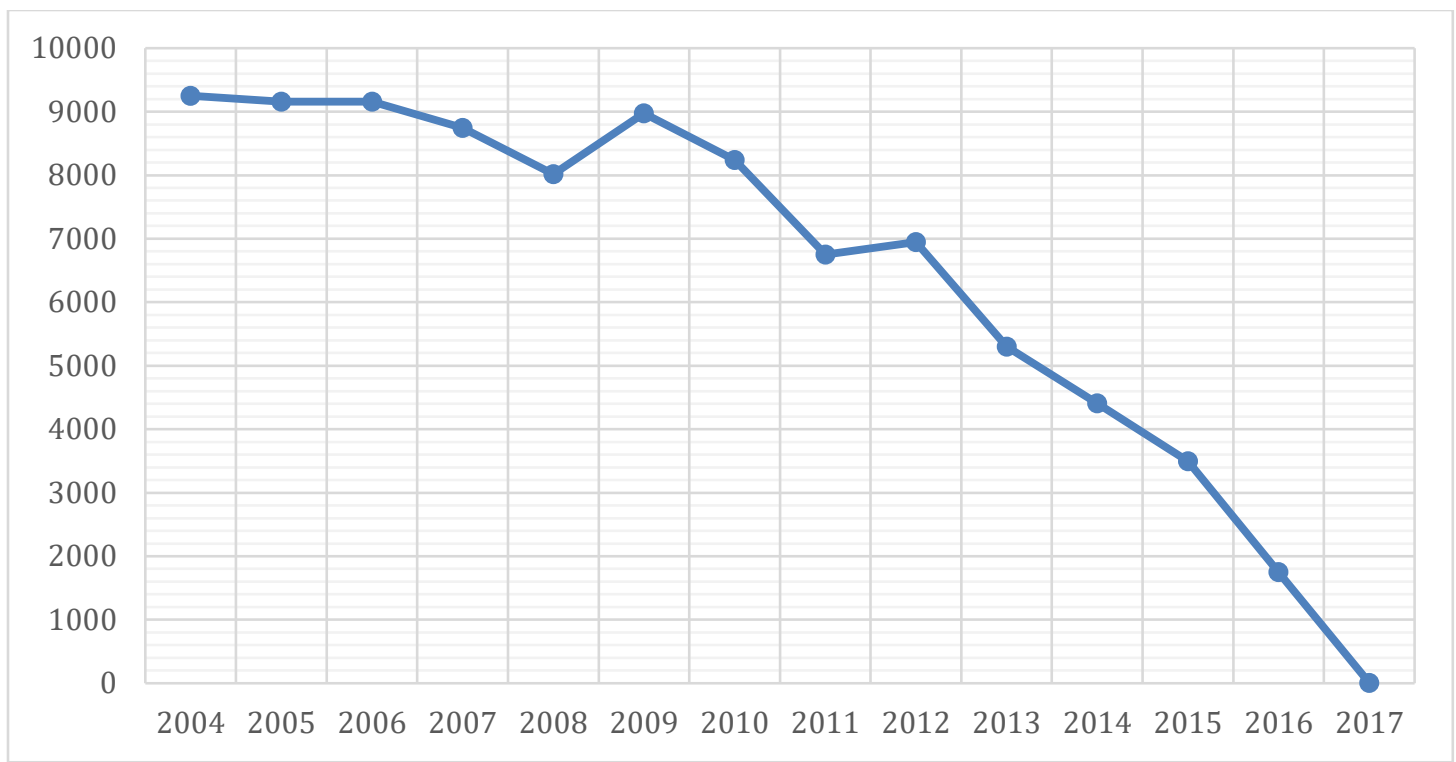

Figure 6: OPS 'street checks' 2004-2017

Between 28 March 2017 and 31 December 2017, OPS reported its approximately 1,350 officers engaged in 'regulated interactions' ('street checks') with just seven (7) individuals (Bordeleau 2018). As the official metrics demonstrate, this represents a massive reduction from even the 1748 in 2016 and a continuation of the steady and dramatic decline in this front-line policing practice since 2009, a year in which OPS officers submitted 8974 'street checks'.

\section{$\underline{\text { Rochester Police Department (RPD) }}$}

Pursuant to my FOI application (file 18-00111), information was sent to me on 13 March 2018 by Nicholas Petitti, Director of Business Intelligence. Petitti provided information on RPD calls for service from 2010 to 2017, including 'Directed Patrol.' Petitti (personal communication, 13 March 2018) advised that the category of self-initiated calls identified as 'Directed Patrol' is used "in practice to document general proactive efforts." This data is represented below in table and graphic formats. 
Table 47: RPD 'directed patrol' 2010-2017

\begin{tabular}{|c|c|c|c|c|c|c|c|c|}
\hline & $\mathbf{2 0 1 0}$ & $\mathbf{2 0 1 1}$ & $\mathbf{2 0 1 2}$ & $\mathbf{2 0 1 3}$ & $\mathbf{2 0 1 4}$ & $\mathbf{2 0 1 5}$ & $\mathbf{2 0 1 6}$ & $\mathbf{2 0 1 7}$ \\
\hline 'Directed Patrol' & 24037 & 16948 & 14065 & 12217 & 15499 & 13552 & 12849 & 10142 \\
\hline
\end{tabular}

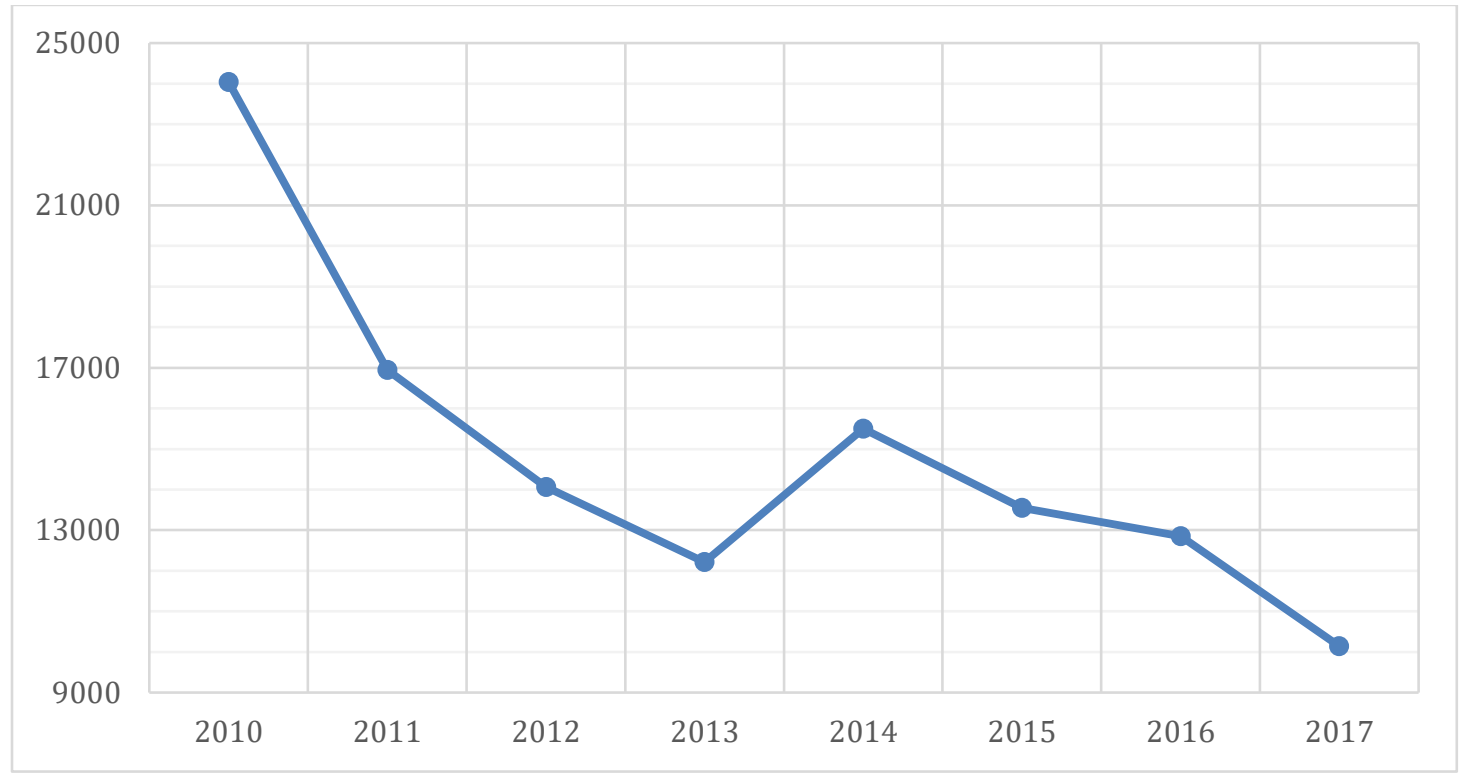

Figure 7: RPD 'directed patrol' 2010-2017

\section{$\underline{\text { Toronto Police Service (TPS) }}$}

Pursuant to my FOI application (file 17-5431), information was sent to me on 29

March 2018 by Analyst Carolyn Kelly of the TPS Access and Privacy Section, as

follows:

In corresponding with our Analytical unit, we are still having issues trying to address a way to respond to your request. Here are some of the issues they brought to my attention:

BIAU [the Business Intelligence and Analytics Unit] has no ability to query for 'officer-initiated calls' prior to our current database, so prior to 2014. From 2013 forward, we have no means of tying the occurrence to the communications side, which is the side that tracks who initiated the call. We can only provide this information from 2014 to date.

In written correspondence dated 24 September 2018, P. McGee (Coordinator of the TPS Access and Privacy Section) provided four separate tables containing data for 2014 through 2017 (inclusive). The data is presented (for present purposes) as follows. 
Table 48: TPS data (vehicle stops, suspicious events, public intoxication, cause disturbance)

\begin{tabular}{|l||c|c|c|c|}
\hline & Vehicle Stops & Suspicious Events & Public Intoxication & Cause Disturbance \\
\hline \hline $\mathbf{2 0 1 4}$ & 129595 & 210 & 92 & 28 \\
\hline $\mathbf{2 0 1 5}$ & 147491 & 263 & 102 & 15 \\
\hline $\mathbf{2 0 1 6}$ & 140288 & 252 & 37 & 16 \\
\hline $\mathbf{2 0 1 7}$ & 128860 & 263 & 34 & 19 \\
\hline
\end{tabular}

In an article published in the Toronto Sun on 29 September 2018, information related to police street checks, obtained from the TPS through the FOI process, was featured. ${ }^{25}$ This data is presented below in table format (Doucette 2018).

Table 49: TPS 'street check' data 2013-2018

\begin{tabular}{|l|c|c|c|c|c|c|}
\hline & 2013 & 2014 & 2015 & 2016 & 2017 & 2018 \\
\hline Street Checks & 196907 & 17001 & 0 & 0 & 25 & 1 \\
\hline
\end{tabular}

As part of the same journalist's investigative reporting, street check data was also obtained from the Peel Regional Police Service (PRPS), which borders on the City of Toronto (to the west). This is presented below in table format (Doucette 2018).

Table 50: PRPS 'street check' data 2013-2018

\begin{tabular}{|l|c|c|c|c|c|c|}
\hline & 2013 & 2014 & 2015 & 2016 & 2017 & 2018 \\
\hline Street Checks & 13167 & 14154 & 9513 & 1162 & 2 & 0 \\
\hline
\end{tabular}

$\underline{\text { Vancouver Police Department (VPD) }}$

Pursuant to my FOI application, on 19 May 2018 Darrin Hurwitz, Counsel in the VPD Access and Privacy Information Unit, provided information related to street checks by VPD officers from 2008 to 2017. This data is represented below in table and graphic formats.

Table 51: VPD 'street check' data 2008-2017

\begin{tabular}{|c||c|c|c|c|c|c|c|c|c|c|}
\hline & $\mathbf{2 0 0 8}$ & $\mathbf{2 0 0 9}$ & $\mathbf{2 0 1 0}$ & $\mathbf{2 0 1 1}$ & $\mathbf{2 0 1 2}$ & $\mathbf{2 0 1 3}$ & $\mathbf{2 0 1 4}$ & $\mathbf{2 0 1 5}$ & $\mathbf{2 0 1 6}$ & $\mathbf{2 0 1 7}$ \\
\hline \hline Total Individuals Checked & 9358 & 8987 & 12376 & 9879 & 9704 & 11412 & 11011 & 9645 & 8587 & 6322 \\
\hline Gang Affiliation & 309 & 294 & 225 & 236 & 214 & 141 & 154 & 148 & 127 & 83 \\
\hline Suspected Drug Dealer & 703 & 1204 & 1391 & 1001 & 1180 & 725 & 533 & 367 & 334 & 284 \\
\hline
\end{tabular}




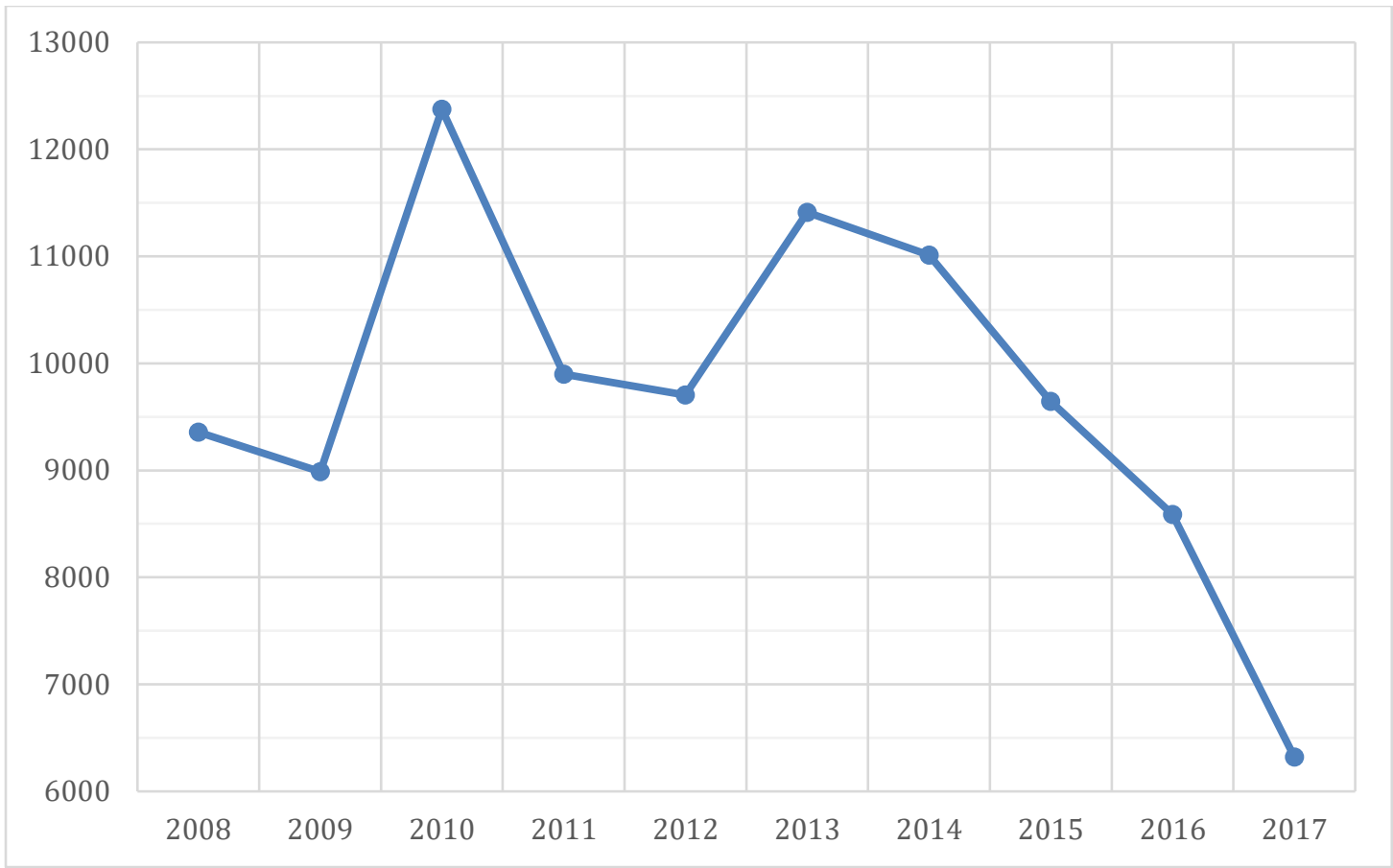

Figure 8: VPD street checks 2008-2017 (total individuals checked)

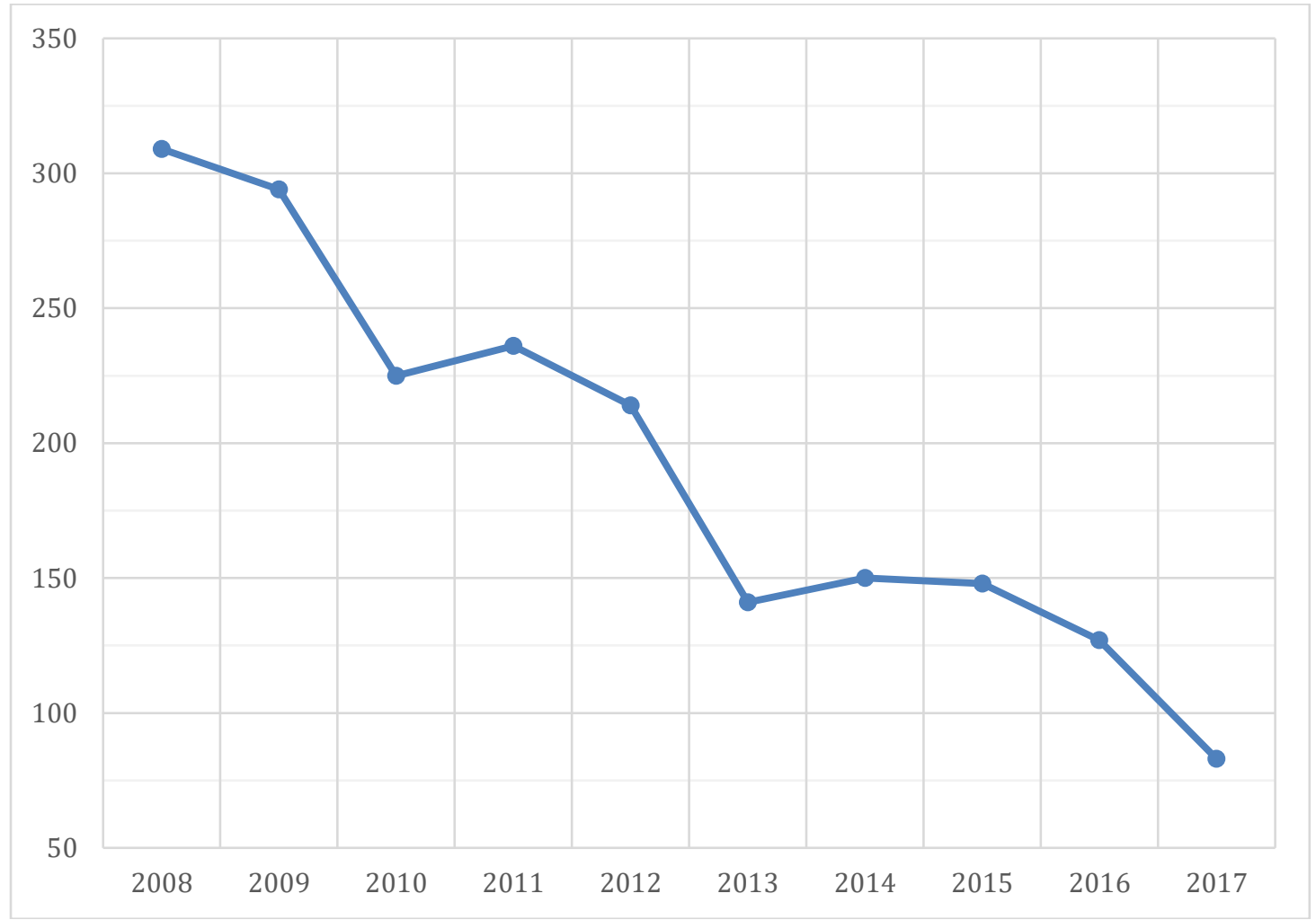

Figure 9: VPD street checks 2008-2017 (gang affiliation) 


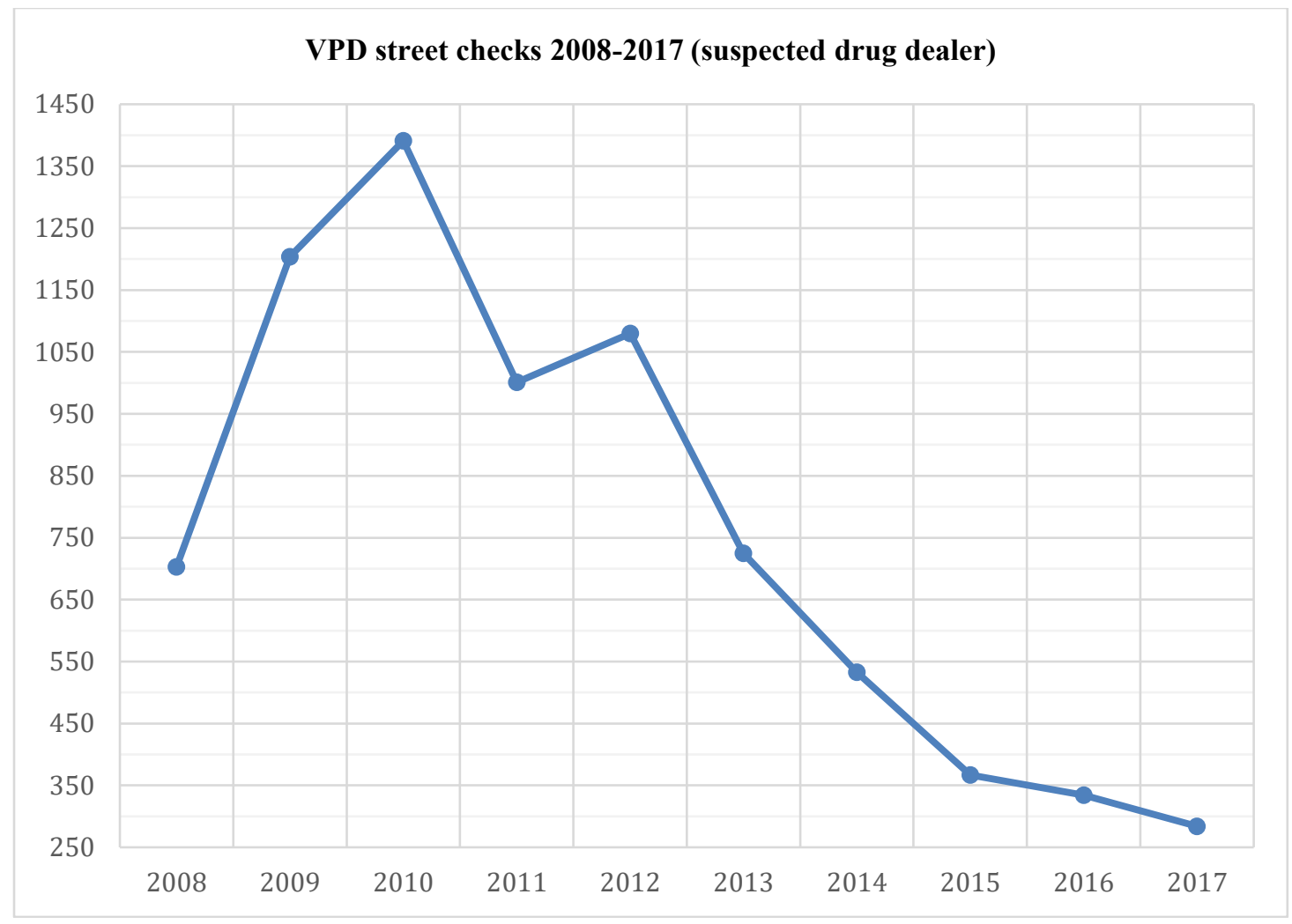

Figure 10: VPD street checks 2008-2017 (suspected drug dealer)

Winnipeg Police Service (WPS)

Pursuant to my FOI application (file 1803 253), information was sent to me on 28 June 2018 by Michele Trudel, WPS Freedom of Information and Protection of Privacy Act Coordinator. As explained in the materials, police-initiated occurrences within the WPS information management systems are difficult to distinguish - between 'on-view calls' (that demonstrate proactive police work) and 'dispatch/arrive' events, which are assigned through the WPS Communications Centre (that involve reactive police work). As a result, some of the WPS data is not useful in assessing discretionary versus reactive policing activities among WPS front-line officers. However, some of the data provided was suggested as useful for this purpose - 'GRASP' checks, spot checks, and citations issued for persons consuming alcoholic beverages in public locations. 
Table 52: WPS ('GRASP' checks, spot checks, consume liquor in public)

\begin{tabular}{|c||c|c|c|c|c|c|c|c|c|c|c|c|}
\hline & $\mathbf{2 0 0 7}$ & $\mathbf{2 0 0 8}$ & $\mathbf{2 0 0 9}$ & $\mathbf{2 0 1 0}$ & $\mathbf{2 0 1 1}$ & $\mathbf{2 0 1 2}$ & $\mathbf{2 0 1 3}$ & $\mathbf{2 0 1 4}$ & $\mathbf{2 0 1 5}$ & $\mathbf{2 0 1 6}$ & $\mathbf{2 0 1 7}$ & $\mathbf{2 0 1 8}$ \\
\hline $\begin{array}{c}\text { 'GRASP' } \\
\text { Checks }\end{array}$ & - & - & - & - & - & - & 1643 & 996 & 804 & 388 & 273 & - \\
\hline $\begin{array}{c}\text { Spot } \\
\text { Checks }\end{array}$ & - & 1918 & 2306 & 1529 & 665 & 1548 & 1361 & 973 & 1274 & 553 & 364 & - \\
\hline $\begin{array}{c}\text { Consume } \\
\text { Liquor }\end{array}$ & 268 & 345 & 344 & 306 & 182 & 421 & 619 & 392 & 239 & 181 & 124 & $6^{*}$ \\
\hline
\end{tabular}

*Note: Citations issued by WPS officers through March 2018.

As described by WPS Inspector Brian Miln (personal communication, 15 July 2018), “...GRASP stands for 'Gang Response and Suppression Plan.' This initiative identifies and monitors adult street gang members in Winnipeg who are bound by court orders. Officers monitor these offenders and conduct [proactive] checks to ensure they are abiding by their court-imposed conditions (an example may be a curfew)."

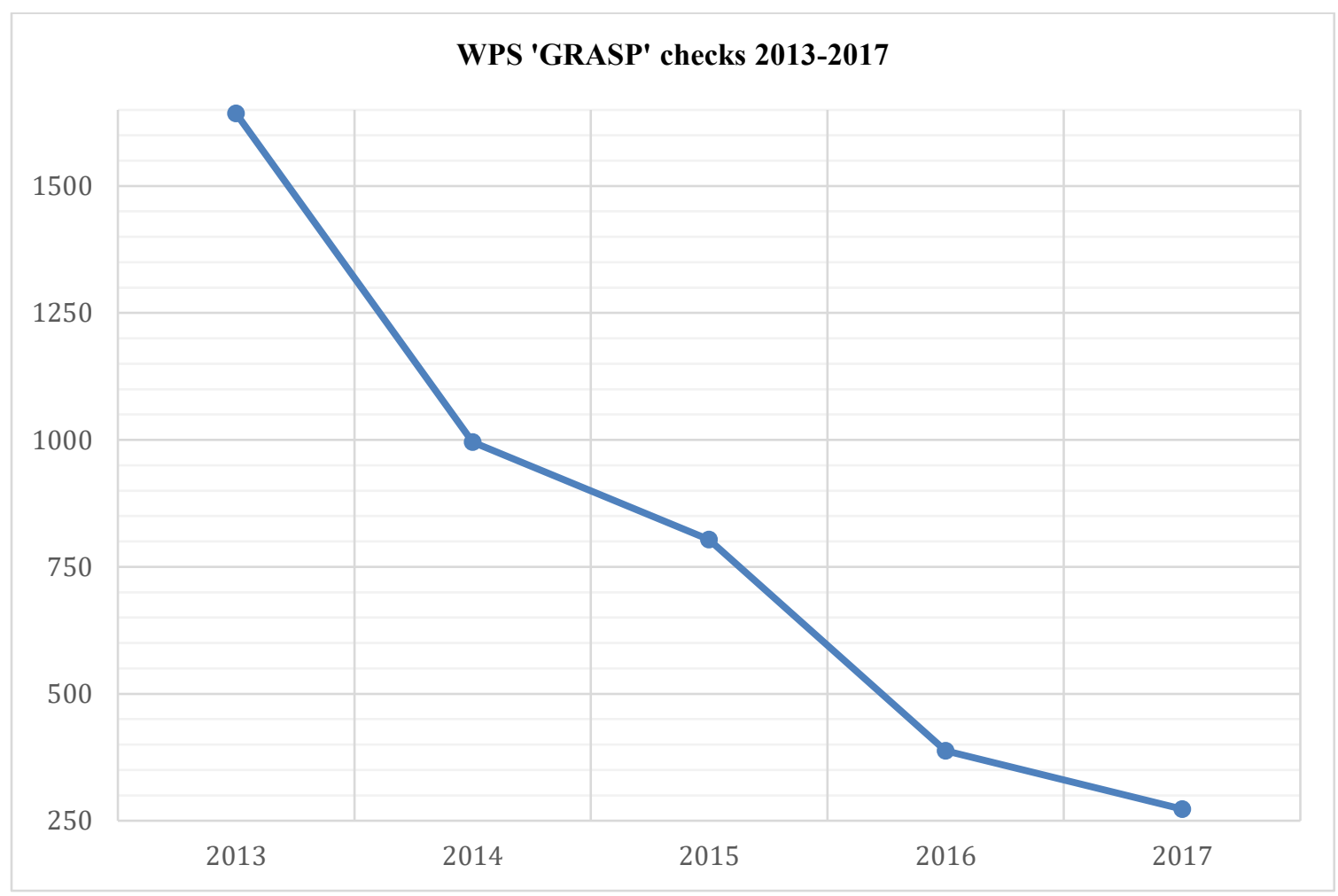

Figure 11: WPS 'GRASP' checks 2013-2017

As described by WPS Inspector Brian Miln (personal communication, 15 July 2018), a 'spot check' "...means anytime an officer [proactively] stops a person (who would usually be on foot) and requests to speak with them for an official or investigational purpose, but the person is not detained." 


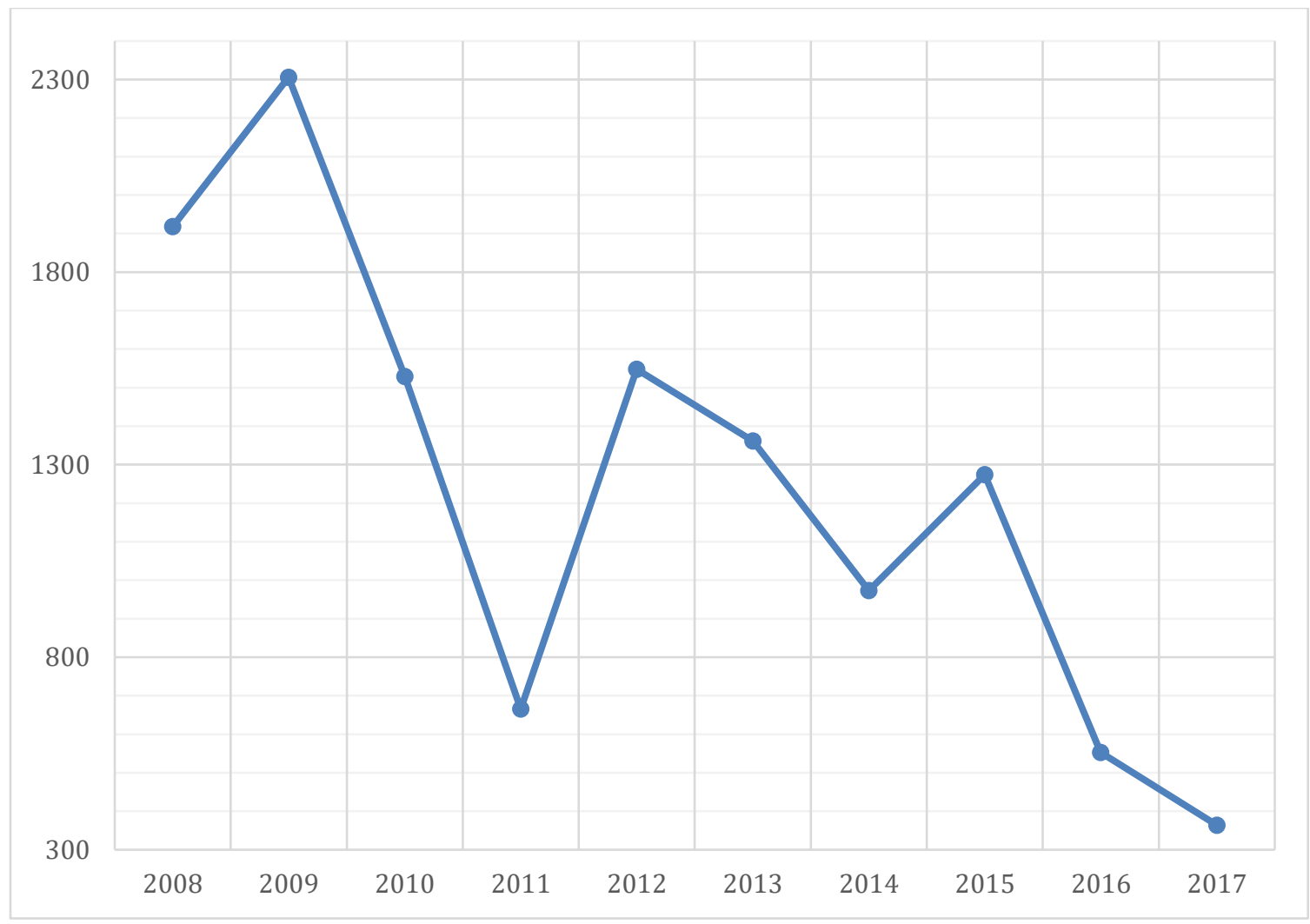

Figure 12: WPS spot checks 2008-2017

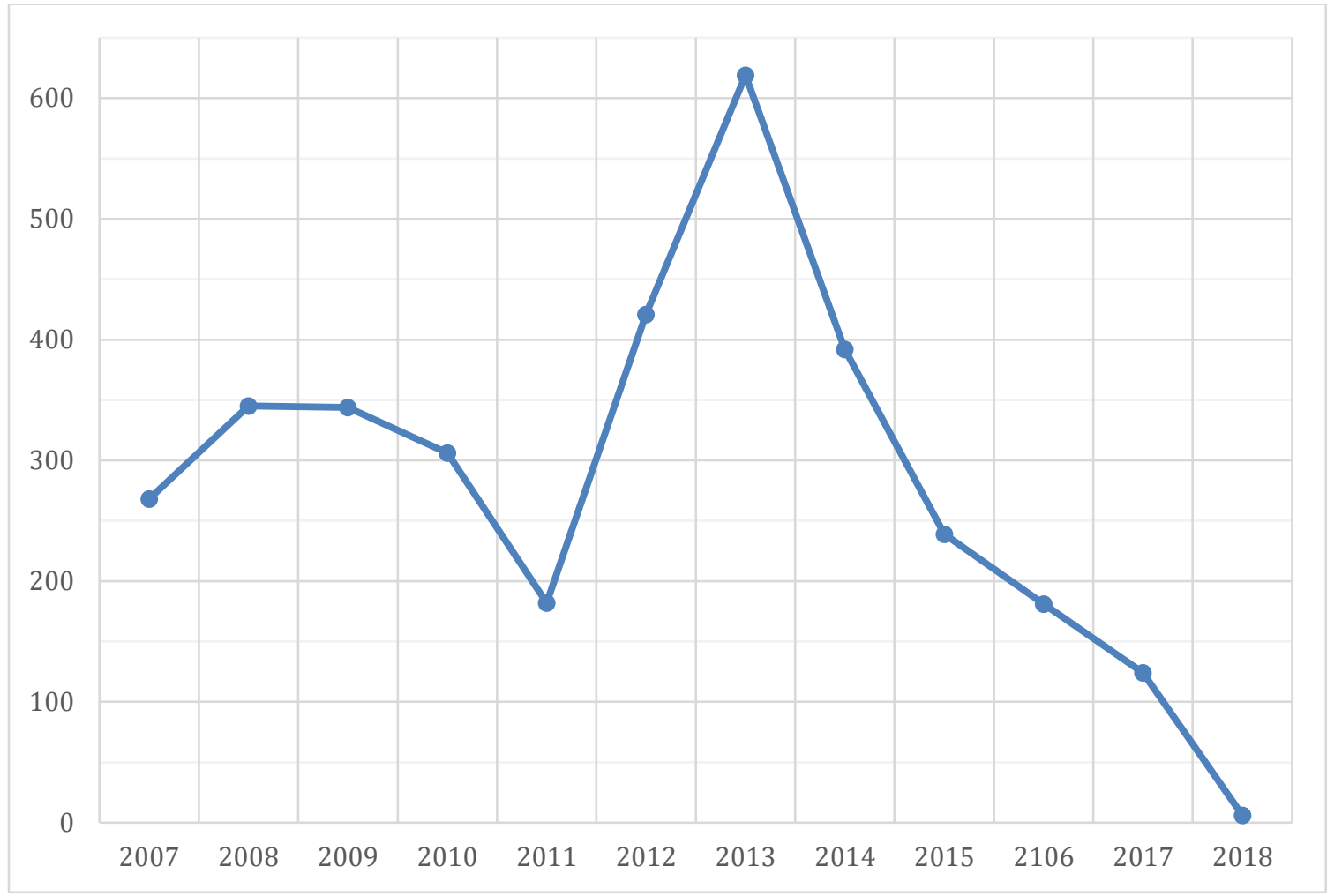

Figure 13: WPS citations for consuming liquor in a public place 2007-2018 


\section{$\underline{\text { Edmonton Police Service (EPS) }}$}

In a 23 June 2018 newspaper article in The Edmonton Journal, reporting on significant declines in proactive street checks by EPS officers, Chief Rod Knecht observed, "We have some officers who just aren't doing street checks anymore... They've sort of stepped back and said, 'I don't want to be investigated, I don't want to be hassled, I'm going to get in trouble, so I'm just not going to do these street checks anymore"” (Wakefield 2018). As part of the newspaper story, the official EPS data was presented to readers, which is reproduced below in table format (Wakefield 2018).

Table 53: EPS 'street check' data 2012-2017

\begin{tabular}{|c|c|c|c|c|c|c|}
\hline & 2012 & 2013 & 2014 & 2015 & 2016 & 2017 \\
\hline Street Checks & 27322 & 25897 & 27122 & 27155 & 22969 & 15909 \\
\hline
\end{tabular}




\section{CHAPTER SIX}

\section{DISCUSSION}

\subsection{Chapter Introduction}

I suggest that this study's results, as presented throughout Chapter Five, are, in and of themselves, convincing and compelling - through the sheer number of rank-and-file officers $(\mathrm{N}=2596)$ that report engaging in risk-averse behavioural adaptations to strains arising from today's techno-social and socio-political policing landscape. In this regard, just shy of three quarters of this study's participants (72\%) are practicing some form of de-policing; four in ten officers across the sample population are intentionally avoiding interaction with certain citizens, based on officers' understandings of particular individuals' 'demographic' characteristics (most significantly, those perceived as racial/ethnic minorities), and that engagements with such individuals present increased risks of professional consequences for officers (i.e. the filing of a complaint about an officer's conduct); and an astonishing three of every ten front-line officers working in our communities today, having sworn an oath to serve and protect, are engaging in intensive de-policing practices - essentially performing 'fire-brigade policing'. ${ }^{1}$ An Ottawa rank-and file officer is representative of this latter category of study participants, in reporting that, in this day and age,

[Officers] have no choice [but to practice de-policing]. [There is] too much chance for false complaints [and] witch hunts about race. [It is] safer to do reactive call-to-call policing (firehouse policing) and [to perform] no risky proactive (optional duties) (OTT233).

Therefore, given the relatively straightforward nature of the present study's data and results, this discussion chapter will not, beyond this paragraph, spend much time reiterating what I advance are the present study's most significant findings: 
(1) De-policing is a bona fide (and now empirically documented) phenomenon that is being practiced, in one form or another, by a substantial majority (72\%) of today's rankand-file officers in their everyday police work.

(2) Officers' de-policing attitudes and behaviours are essentially universal across Canadian and American front-line policing - with only minor variance across police agencies (regardless of size or region), by type of front-line police duties and the nature of officers' policing environments, and as relate to the 'demographic' characteristics of individual officers (i.e. gender, race/ethnicity, and age).

(3) The one variable found to be particularly relevant to the practice of de-policing is an officer's years of front-line experience (as differentiated from years of overall police service and age), which, I argue, is associated with career stage theory and the concept of a de-policing continuum (as elaborated later in this chapter). For many front-line officers, the genesis of risk-averse behavioural modifications to their police work can be related to two factors - the amassing of negative occurrences involving antagonistic police-citizen interactions in the field and concurrent encouragement from colleagues within the rank-and-file subculture advocating in favour of de-policing practices. Therefore, with the accumulation of time spent 'on the job' in front-line policing, rather than in other assignments away from the influence of these two factors (i.e. detective work or administration) officers have more exposure to experiences and interactions in the field and in the rank-and-file subculture that militate in favour of de-policing. (4) Policing's rank-and-file subculture indoctrinates, encourages, and facilitates practices of de-policing as a risk-averse and self-preservation response to the strains associated with techno-social and socio-political realities in today's front-line policing 
landscape. In this regard, police occupational culture theory informs the present study's findings.

(5) For many officers, de-policing behaviours implicate attitudes toward, and avoidance of, particular individuals within the community because of 'demographic' characteristics presented by such persons (in the subjective assessment of the officer). Officers perceive certain categories of persons as especially 'risky', in relation to criticizing, and/or registering a formal complaint about, the officer's conduct - most profoundly those citizens perceived as 'visible minorities'.

Further, the present study has also found that many of today's rank-and-file officers are not just avoiding proactive police work (this study's definition of de-policing) but they are also being selective in the reactive police work they do perform. This is done through, for example, responding intentionally slowly to, or avoiding altogether, certain types of dispatched calls and/or not making arrests in circumstances in which an arrest is warranted. As presented within Chapter Five, this research finding came as a surprise to me and, like de-policing in relation to proactive police work, it should be concerning to both constituents in today's public-police relationship. This collateral finding and my advocacy for further research, in this regard, is discussed further in Chapter Seven.

In the pages that follow, this chapter directs the reader's attention to aspects of depolicing beyond data, results, and findings in presenting a more complete picture of the widespread risk-averse phenomenon across today's front-line police work. This is accomplished through focused discussions that are largely structured around the most salient themes that emerged during data analysis and in relation to the study's primary research question. Before beginning, however, it should be acknowledged that nascent 
observations of today's de-policing phenomenon, as advanced by former FBI Director Comey and a few others (as presented throughout Chapter One and Chapter Two) and dismissed by some critics as 'cherry picking' of anecdotal information, were, despite the absence of an empirical foundation, quite accurate. Further, as with other areas throughout this dissertation, the reader will observe that there is considerable interconnectivity across much of the subject matter addressed throughout this chapter, which is indicative of the importance in explicating and understanding nuances in the identified de-policing phenomenon, the magnitude of which, for the first time, has been revealed in results from comprehensive and rigorous empirical research - including, where applicable, broader conceptual, theoretical, and collateral implications.

The structure of this chapter reflects this purpose, with further discussion, emerging from the study's findings, presented within discrete subchapters. I suggest that this format is ideal given the voluminous data, the analysis thereof, and the study's results, reported throughout Chapter Five, and given considerations of scope, comprehensiveness, and comprehensibility. Specifically, and in relation to scope, the purpose of this chapter is to introduce discussion on relevant considerations and instigate deliberation, among the readers, on the issues presented throughout this dissertation. Given that there are numerous book-length treatments addressing some of the subject matter (e.g. police occupational culture and issues in policing related to race/ethnicity) this chapter is, by necessity, relatively concise. The first three subchapters, following this introduction, provide important context before the reader is invited to consider more substantive issues related to the present study's findings. Subchapter 6.2 presents discussion addressing distinctions between the traditionally- 
characterized 'lazy' officer and today's front-line officers that are strategically and intentionally engaging in de-policing practices. Considerations of police officers' attitudes ('canteen talk'), vis-à-vis their actions in the field, are discussed in subchapter 6.3. And, reflections on Canadian versus American policing are presented throughout subchapter 6.4.

The concept of career stage and the present study's interrelated de-policing continuum construct are discussed in subchapter 6.5. Police occupational culture theory - the study's primary theoretical framework, specifically as it relates to key study findings, is addressed throughout subchapter 6.6. Following is a discussion centred around de-policing and police-community relations (subchapter 6.7). Implications related to race/ethnicity, in the context of today's socio-political policing landscape and officers' de-policing practices, are discussed throughout subchapter 6.8. Subchapter 6.9 discusses the nearly $30 \%$ of rank-and-file officers that are not engaging in any depolicing practices. Finally, subchapter 6.10 elaborates on issues discussed earlier within Chapter Four, in relation to researcher positionality and study participants' trust - vis-àvis data integrity in policing research.

\section{2 'Lazy' Officers Versus Today's De-Policers}

In discussing the contemporary de-policing phenomenon identified in the present study and situating these findings within previous scholarly literature and public sphere discourse, it is important to distinguish between what I would characterize as the traditional conception of the 'lazy' police officer from today's officer that is practicing de-policing. As described in preceding chapters, previous research has documented various typologies of officers, including the former having been characterized as, for 
example, 'uniform carriers' (Reiner 1978; Van Maanen 1974), 'lay lows' (Van Maanen 1973, 1974, 1975), and/or 'avoiders' (Mastrofski, Willis \& Snipes 2002; Muir 1977; Reiner 1978; Worden 1995b). Paoline (2004: 212) finds that these terminologies describe officers that are "just doing their time, avoiding as much work as possible." Muir (1977) described Officer Jim Garfield (a pseudonym), one of his Oakland Police study participants, as one such officer, who's familiar admonition was 'Don't get involved.' In the process of excising more and more tasks from his definition of 'necessary police work,'

Garfield began avoiding any dispute between residents of that part of the city he called 'asshole country,' those low-income public housing developments where the public peace was so frequently violated. From that point of retreat, the logic of his moral argument led him to exclude all activity in 'asshole country'...The social consequence of the avoidance response was that the citizens were thrown back on themselves to solve their own problems. As Garfield said with misplaced pride, 'I never had any more complaints' (Muir 1977: 87-88).

In this regard, as with probably every other occupation, there have almost certainly always been less motivated police officers that perform their work with reduced enthusiasm and lower productivity than their colleagues. In my early years in front-line patrol work (spanning 1985 to 1994) I came to know a veteran officer on the platoon to which I was assigned (that had a complement of approximately 50 rank-andfile officers) who had been nicknamed, by my partner, 'the workhorse' - for his less than spectacular efforts at engaging with anything other than the most mundane of dispatched calls. His 'unit history' (a computerized record of an officer's activities throughout the shift) was accessible through all of our in-car computers and this officer's absence of initiative was often the topic of derisive discussion among the $90 \%$ of my colleagues (in my unscientific estimation) that did not shy away from any type of 
reactive or proactive police work. In this vein, 'the workhorse' counselled me on several occasions, I get paid the same as you, so what's the point of busting your ass like you do? The job is a lot easier on you the way I do it. While this lethargic attitude and the behavioural manifestations thereof were relatively rare - certainly when compared to the proportion of officers that are currently practicing de-policing - 'the workhorse' was not alone on that platoon. In my conversations with the other 3 or 4 'lazy' officers on that platoon, I came to understand that, unlike with today's de-policing, these officers were not avoiding interactions with the citizenry as part of a considered and deliberate self- preservation strategy arising out of concerns with video recording potential, public criticism of an officer's actions, or a perception of elevated risk for professional consequences from engaging with 'visible minorities', but rather, their inaction was simply the result of deficient individual work ethic, which had developed in the latter stages of a police career spent largely assigned to front-line patrol work. Put simply, these veteran officers, on the threshold of retirement, would have almost certainly behaved similarly in any comparable occupation and work environment - exhibiting cynicism, low morale, low levels of job satisfaction, 'burn-out,' and lack of initiative/motivation (e.g., see Blum 2000; Crank 2003; Johnson 2012; McCarty \& Skogan 2012). Throughout those nine years working in front-line policing, I was transferred once to another platoon and had the opportunity to overlap on many shifts with officers assigned to the other OPS three platoons. As a result of those experiences, I came to appreciate that each of the five OPS platoons in that era had their own 'workhorses' and the proportion, which I estimate at roughly $10 \%$ of the front-line officers on each platoon, and occupational biography of those 'lazy' officers was largely 
consistent. And, significantly, there were certainly no 'lazy' officers with less than 10 years of front-line service.

As disclosed through the present study's results and as described in preceding chapters, I argue that what is happening now with de-policing is an entirely different phenomenon. I can comment on this, not just empirically through the findings of this study, but also, as with the previous paragraph, from my own experiences - in this context from working as a rank-and-file platoon supervisor from 2010 to 2013, after a 16-year absence from front-line police work. I observed during that three-year period that there still existed a handful of 'lazy' officers across the now six front-line OPS platoons, whose avoidance of many situations in the field was the result of generalized deficiencies in work ethic. These were the familiar grizzled 'old vets' that are doing only the minimal amount of police work as they near retirement. I also discerned that there still existed a relatively large cadre of 'mission-oriented' officers - those that view their police work as an important 'calling', in service of protecting a vulnerable society from evildoers, and therefore, those that will do their police work as they have always done it and without wavering (this category of non-depolicers is discussed in detail throughout subchapter 6.9).

However, I observed (and the results of the present study bear this out) that there are now significant numbers of front-line officers that have modified their conduct in the field because of what they perceive as negative experiences in the field and because of the prevailing influence of their rank-and-file occupational subculture, which encourages the practice of de-policing to mitigate against sources of strain and anxiety on the job. These are not the aforementioned 'lazy' front-line officers, but rather, these 
are officers whose behaviours are related to their perceptions of an adversarial, controversial, and dangerous (in terms of the potential for professional consquences rather than physical harm) contemporary extrernal policing landscape and their subjective analyses of risk presented therein - as relate to concerns with use of force, increased public scrutiny and critique, video recording, accountability, and interactions with certain 'demographics' within the population. As a Calgary officer observed in his/her written elaboration,

Since I started in 2005, [de-policing] used to be for lazy cops, now it can be for anyone (CAL274).

In the same vein, a Halifax officer advances,

[De-policing] is now a common practice and I estimate it started about 6-8 years ago (HAL32).

I concur with these officers' observations and, much to my surprise, unlike the case with traditional 'lazy' officers, I witnessed that today's de-policers include officers with limited 'time on the job' (years of front-line policing experience). ${ }^{3}$

Like the experiences I related with 'the workhorse', in relation to traditional 'lazy' officers, one of the officers that I supervised for the three years I was back doing frontline police work provides 'real world' illumination of the contemporary de-policing phenomenon. Constable Smith (a pseudonym) was, by all accounts, a conscientious, dedicated, and hard-working officer for the first few years of his/her police career - the kind of officer that would fit the 'mission-oriented' typology. After observing various instances of de-policing behaviours throughout my first few shifts, involving not only Constable Smith but many of the officers I was supervising, I spoke with Constable Smith, who was an officer with between 5 and 10 years of front-line policing experience and who served as an informal go-between for the platoon's constables in relations with 
their supervisors. Constable Smith explained that now most rank-and-file officers, after an initial 'honeymoon' phase as new recruits that typically lasts only a few years, eventually come to realize that they have no choice but to avoid certain risks inherent to today's front-line police work in the interest of 'career self-preservation'. Constable Smith advised that, as I was observing firsthand, most of our colleagues on the platoon have come to realize, through their own negative experiences in the field and the concomitant discussions that take place within the social relationships forged throughout the rank-and-file subculture, that it is safer to simply do the minimum of what is required of the front-line policing function. As Constable Smith explained, Answer your dispatched calls, not at 100 miles per hour, and issue a few traffic tickets per month. Nothing more [and] nothing less.

Given that I was going to be, from time-to-time, engaging in front-line policing interactions with the public, Constable Smith suggested that I keep in mind that an otherwise decorated police career, which was nearing a rewarding retirement pension, was simply one fuck-up away from being over and s/he observed that the more engaged an officer was in confrontations with members of the public and involved in controversial policing episodes (i.e. use of force and/or arresting persons of a race/ethnicity different than the officer) the higher the odds grew of getting into serious trouble - whether any resulting allegation of misconduct was factual or otherwise.

As a specific example, Constable Smith referenced the 2010 OPS policy change in relation to the arrest of individuals found to be intoxicated in public settings. ${ }^{4}$ In the wake of substantial public antagonism toward the OPS throughout 2010, over the alleged mistreatment of a Black female arrested for drinking alcohol while walking on a public street and being belligerent with officers (Duggal 2011), a number of new and 
mandatory considerations were introduced by OPS senior management - requiring that an officer be able to justify arrest (and detention in the OPS cellblock) versus other measures - such as driving the person home or to a social services facility. Included in the new policy were punitive disciplinary consequences for an officer who's decision was found ex post facto by senior OPS management not to have been in compliance with some aspect of the new directives. ${ }^{5}$ As Constable Smith interpreted the complex new policy, in virtually any arrest of a drunk individual by an officer, some misconduct could be contrived by police leadership, after the fact, in response to political/public pressure, which Constable Smith suggested would be most likely to occur in racialized police-citizen interactions (with Black and/or Indigenous individuals) given today's prevailing 'political correctness'.

As a result, as conveyed verbally through Constable Smith and corroborated by my own observations of various front-line officers over time, many officers made the decision, for their own career protection, that they would never arrest anyone for the offence of being drunk in a public location, because of the potential for public backlash and the risk of disciplinary consequences for the officer in today's highly-charged socio-political external policing landscape. In simple terms, it is easier and less risky for the officer to arrive at the disingenuous subjective determination that an obviouslyintoxicated individual does not meet one of the myriad criteria in the new policy and therefore not to intervene. This would include the chronic alcoholic 'street persons' that are routinely observed in states of inebriation nearing unconsciousness and in various conditions of disorder (i.e. without articles of clothing and/or vomiting, urinating, or 
defecating in public). Several officers explained to me that this was apparently the kind of police response that today's citizens want and expressed sentiments along the lines, If that is what the public wants, that is what the public is going to get. ${ }^{6}$

\subsection{Attitudes Versus Actions in the Field}

Waddington (1999), in his influential contribution to the scholarly literature on police occupational culture, cautioned about the potential for differences to exist between stories told by officers in 'backstage' locations within the front-line subculture (i.e. the 'canteen'), which he characterizes as a coping strategy to mitigate against occupational strain, and officers' actual behaviours in the field. ${ }^{7}$ I acknowledge this as a possibility and, in the following paragraphs, discuss what I observe to be unsettled territory in this regard - in terms of conflicting conclusions drawn from the limited studies to date, which have examined whether there are significant differences between officers' expressed attitudes versus their actions in the community. Charman (2017: 143) recently found that this "discussion has not been resolved and is highly complex" and I am hopeful that the present study will add some clarification to this undecided scholarly debate.

Reiss (1968: 16), in an observation that can be considered in agreement with the proposition advanced by Waddington (1999), noted, "As sociologists and social psychologists have often shown, prejudice and attitudes do not necessarily carry over into discriminatory actions." The policing studies, to date, that have failed to find a link between officers' attitudes and expected behaviours include DeJong's (2004) research into officers' comforting behaviours; Gould and Mastrofski's (2004) examination of officers' attitudes toward community policing and search behaviours; Terrill and 
Mastrofski's (2002) examination of severity of force; and Terrill and Paoline's (2015) study into allegations of improper force. ${ }^{8}$

On the other hand, various studies have found that officers' attitudes do, indeed, influence behaviours in the field. This body of work includes the influential study conducted by Mastrofski, Worden and Snipes (1995) that found officers with positive views toward community policing are significantly less likely to make arrests; Brown's (1988) research that found officers that define the police role as one of crime fighting and law enforcement are more aggressive on the street than are officers with broader role conceptions; Worden's (1989) research into officers' traffic stop behaviours; Friedrich's (1980) finding that officers exhibiting prejudice acted on those prejudices through the use of more force in interactions with citizens than their less prejudiced colleagues; and Worden's (1996) examination of officers' use of improper force. In this vein, Reiner (2010: 115) finds positions arguing that “[officer's] perspectives...bear no relation to their practices" are now difficult to sustain and, similarly, Charman (2017: 144, citing Van Hulst 2013) argues, "It might be naïve to consider that those who engage in 'talk' and 'stories' in the backstage arena of policing are doing so only to create a world of make-believe."

Specifically in relation to police occupational culture, Reiner (2000b: 87) recognizes the distinction between 'canteen talk' and 'cop culture' and he argues that "cop culture has developed as a patterned set of understandings that help officers to cope with and adjust to the pressures and tensions confronting the police." Ingram, Terrill and Paoline (2018: 780-781, citing Manning 2005b; Mastrofski 2004; National Research Council 2004; Waddington 1999) find that "work is still needed...in 
identifying the extent to which culture relates to police behaviour" and they refer to "the results of a few studies [that] have demonstrated an empirical link between cultural attitudes and officer behaviours." These studies include those conducted by McCluskey, Terrill and Paoline (2005) that investigated peer group aggressiveness and the use of coercion in police-suspect encounters; McCluskey and Terrill's (2005) research into complaints from the public and officers' use of coercion in interactions with suspects; Paoline and Terrill's (2005) inquiry into the impact of police cultural attitudes on officers' traffic stop search behaviours; and Terrill, Paoline and Manning's (2003) study of how alignment with prevailing cultural attitudes translates into differences in officers' coercive behaviours. In that study, Terrill and colleagues (2003) found that police use of force is a function of officers' attitudinal commitments to the tenets of the 'traditional' front-line police subculture. Further, in their recent study, assessing aspects of police culture and officer behaviour, Ingram and colleagues (2018) hypothesized that "workgroup culture will have a collective effect on behaviour" and they confirmed significant associations in several of the behavioural aspects they studied. For example, "The results show that officers who work in environments that subscribe to a culture of aggressive patrol practices...behaved similarly" (Ingram et al. 2018). Similarly, recent research by Terrill, Paoline and Gau (2016) finds that cultural attitudes can, and most certainly do, develop into behaviours on the streets.

In focusing on the present study, I advance that the data refutes the "canteen culture' premise, which suggests that rank-and-file officers express sentiments in private settings with peers but they do not act on these attitudes in their conduct in the field. I argue, with what I suggest is solid empirical support, that officers' risk-averse 
attitudes most certainly are being operationalized in their behaviour in the community in the form of de-policing. I feel confident is asserting this for two reasons. First, the sample size in this study $(\mathrm{N}=3660)$, which includes large numbers of front-line officers that provided not only quantitative responses but also qualitative elaborations, detailing their behavioural adaptations to the contemporary policing landscape, lends substantial confirmatory support. For example, $40 \%$ of the study's participants, in their quantitative responses, reported avoiding interaction with certain citizens $(\mathrm{N}=1460)$, but also, 1196 (or $82 \%$ ) of these officers provided a further written (qualitative) explanation confirming and amplifying on their affirmative quantitative response. I argue that it is reasonable to interpret such qualitative elaborations to supplement quantitative ('Yes' or 'No') responses as consistent with the participant being truthful and is suggestive of data integrity. In other words, phrased as a rhetorical question, is it likely that a research participant that answers a 'Yes' or 'No' question dishonestly is then going to expend additional efforts to elaborate (or in today's popular vernacular to 'double down') on that deceitful response through a disingenuous written elaboration in a study that is voluntary and anonymous? I suggest that the answer in the vast majority of instances would be 'No.' Second, the 'official' data obtained from various police agencies via FOI applicatons (as presented in subchapter 5.4), demonstrates quite clearly, in my view, that de-policing is undoubtedly happening and these widespread behaviours - not attitudes that are not acted upon - are now beginning to show up in internal organizational metrics related to discretionary policing activities. 


\subsection{Canadian and American Policing Contexts}

As discussed throughout Chapter Three, various scholars have characterized the current policing landscape in both Canada (Campeau 2016a, 2016b; Murphy 2012) and the USA (Braga et al. 2018; Campbell et al. 2018; Collins \& Klahm 2019; Fallik et al. 2018; Loader \& Sparks 2015; Maguire et al. 2017; National Academies of Sciences, Engineering \& Medicine 2018; Nix \& Pickett 2017; Nix \& Wolfe 2017, 2018; Nix et al. 2018; Pyrooz et al. 2016; Todak 2017; Torres et al. 2018; Wallace et al. 2018; Weitzer 2015; White \& Fradella 2016; Wolfe \& Nix 2016) as a 'crisis.' The disparities in the number of citations available in relation to the two bordering North American countries is, in my view, reflective of researchers" "reluctance to look outside [the USA and the UK] to policing in other societies," as Mawby has observed recently (2013: 13). As the the editor of Policing Across the World: Issues for the Twenty-First Century, he finds that the "lack of an international comparative dimension is surprising" (Mawby 2013: 13). The absence of scholarly contributions discussing differences between American and Canadian front-line policing was, frankly, unanticipated. I expected an abundance of such within the literature. Bayley's (2013: 3-4) observations on this are instructive,

The comparative study of the police is viewed as an exotic frill in the professional study of criminal justice. It is a marginal enterprise thought to be difficult, if not impossible, to do and yielding little of value...Part of the reluctance to study criminal justice, or anything else, internationally arises out of the perception that differences among phenomena are so great that analysis is impossible...The assumption seems to be that national boundaries impose discontinuities in human experience, qualitative differences that make it impossible to understand what is going on, let alone to learn something that might be useful at home.

I must confess that, despite my practitioner experience (including involvement with American law enforcement from time-to-time throughout my Canadian policing 
career), I previously subscribed to the taken-for-granted assumption that Canadian and American front-line policing are vastly different in many respects. As an example, Lipset (1990: 90-97), in his influential book Continental Divide: The Values and Institutions of the United States and Canada, advanced,

Efforts to distinguish Canada and the United States almost invariably point to the greater respect for law and those who uphold it north of the border...As Margaret Atwood notes, 'Canada must be the only country in the world where a policeman (the Mountie) is used as a national symbol'...Various studies of the attitudes of American police indicate that they commonly complain of being looked down on by the public. Conversely, the Royal Canadian Mounted Police celebrated its hundredth birthday in 1973 and was honoured across the country.

However, as a result of the present study, my previously-uninformed perspective has been adjusted, which I will explain further in the following paragraphs. In recent discussions with Professor Scott Phillips (State University of New York at Buffalo), in furtherance of our collaboration on a proposed comparative research project examining attitudes of rank-and-file American and Canadian officers to various realities in today's police work, he suggests that such presumed assumptions are likely the by-product of the "little, if any, rigorous comparative research" examining today's front-line police work in Canada and the USA. ${ }^{9}$

In saying this, as described in Chapter Four, obviously there are significant differences in terms of scale - with many more police agencies in the USA than Canada (more than 18,000 versus less than 300) and far more police officers deployed throughout the USA than Canada (more than 750,000 versus less than 70,000). For example, the Canadian population is approximately 35 million persons and the country is policed by 69,000 officers, whereas the State of New York, with a population of roughly 19.5 million persons, is policed by almost the same number of officers (more 
than 61,000). Other significant differences include that Canadian police officers enforce one criminal law statute (the Criminal Code) throughout the entire country, whereas across America there exist both federal and state criminal laws and, in the USA, the positions of many police chiefs and sheriffs are decided through civic elections whereas in Canada police chiefs are appointed as public servants by elected officials. The USA also has no equivalent to the RCMP - a national police force. Further, the American 'gun culture,' with over 300 million firearms in circulation as compared to less than 10 million throughout Canadian society (James \& Madhani 2018) and the extraordinarily high rates of gun and other violent crime experienced in the USA are not replicated in Canada. In terms of police shootings of citizens, between 2000 and 2017 across Canada there were approximately 19 police shooting deaths per year (Hopper 2018). In contrast, across the USA since 2015 there have been an average of 982 people shot and killed by police annually (Sullivan, Anthony, Tate \& Jenkins 2018).

However, one of the issues explored in the present study is whether there is a significant difference in the attitudes and behaviours of today's Canadian and American front-line officers, vis-à-vis de-policing. At the inception stage of this research project, given what I perceived were major differences in the policing landscapes between Canada and the USA and my assumption that the rank-and-file American officer is dramatically different than his/her Canadian counterpart, I expected to find significant distinctions in the Canadian and American data in all areas of the study's inquiry - with external technological, social, cultural, and political influences on risk-averse behaviours presenting as more pronounced with research participants from the study's five participating police agencies across the State of New York. However, as the study's 
data demonstrates, clearly this is not the case. On this point, the relevant portion of Table 29 is reproduced below, which reports on de-policing results by country. As presented in this table and as described throughout Chapter Five, there are only minimal differences observed in this study between Canadian and American front-line officers in terms of their de-policing attitudes and practices.

\begin{tabular}{|c|c|c|c|c|c|}
\hline \multicolumn{2}{|c|}{$\begin{array}{c}\text { Location and Agency } \\
\text { Variables }\end{array}$} & $\begin{array}{c}\text { Non de- } \\
\text { policers }\end{array}$ & $\begin{array}{c}\text { Limited de- } \\
\text { policers }\end{array}$ & $\begin{array}{l}\text { Moderate de- } \\
\text { policers }\end{array}$ & $\begin{array}{c}\text { Intensive de- } \\
\text { policers }\end{array}$ \\
\hline \multirow{2}{*}{ Country } & Canada & $30.1 \%$ & $18.2 \%$ & $22.2 \%$ & $29.5 \%$ \\
\cline { 2 - 6 } & USA & $23.2 \%$ & $18.1 \%$ & $27.7 \%$ & $30.9 \%$ \\
\hline
\end{tabular}

Perhaps this finding is not that surprising given recent similar observations. For example, Roziere and Walby (2019) studied the use of tactical (SWAT) teams in Canadian policing and, after observing that "[d]espite extensive social science analysis of [the militarization of policing] across the United States, the phenomenon in Canada has been overlooked," they found that assumed differences in SWAT team deployments between the two countries are contradicted in the empirical data. Also, during a 2018 radio interview, discussing police shootings of unarmed Black men in the USA, Robyn Maynard, author of the book Policing Black Lives, advanced, "We need to realize that this is very much a Canadian issue as well" (CBC Radio 2018). In the same vein, Canadians Akwasi Owusu-Bempah, a University of Toronto sociology professor, and Anthony Morgan, a Toronto civil rights lawyer, recently argued,

[A]s we see the U.S. becoming more racially divided, it's tempting to believe we're morally superior to the U.S. But in terms of policing and racial profiling, we are not as dissimilar as we might want to believe...[O]ur approaches to policing Black, indigenous and other racialized communities are remarkably similar... The dramatic racial lines along those who Canadian police forces target for carding and street checks is also eerily reminiscent of the practice known in New York City as stop and frisk. What few Canadians know or are willing to confront is that a Black person's chances of being carded in Toronto have actually 
been found to be higher than their chances of being stopped and frisked in New York City. The rate of over-representation of Black and indigenous people in instances of police use of lethal force and incarceration rates are two critical areas in which Canada and the U.S. are alarmingly similar. Controlling for variables such as differences in population size, demographic make-up and frequency of police use of force incidents, it becomes less and less clear whether policing interactions with Black people are better in Canada than they are in the US (Owusu-Bempah \& Morgan 2018).

The reader should understand that a specific discussion around front-line policing, race/ethnicity, the issue of racialization, and the identified de-policing phenomenon is forthcoming in subchapter 6.9.

In considering similarities between Canadian and American front-line policing, the reader should recall the discussion in subchapter 3.3 regarding 'the contagion effect,' which accompanies pervasive social and traditional news media discourses addressing various issues in today's policing (Loader \& Sparks 2015; Nix \& Wolfe 2016, 2017; Pyrooz et al. 2016; Shjarback et al. 2017; Weitzer 2015, 2018; Wolfe \& Nix 2016). In this regard, Nix \& Wolfe (2016: 14, citing Pyrooz et al. 2016) argue, It is important to emphasize that social media contagion has allowed Ferguson-type incidents to be experienced in agencies that have not experienced their own high-profile police shooting. Ultimately, social media has created a situation where citizens and officers alike can reap the negative effects of such incidents regardless of geographical proximity.

Similarly, Weitzer (2018: 25) discusses the phenomenon of 'contamination-byassociation,' in which each controversial policing occurrence 'pollinates subsequent ones" regardless of the location of the various events. As examples, an Ottawa officer, in discussing familiarity with the concept of de-policing, observes, [It is] all over social media, e.g. Chicago Police (OTT366) Another Ottawa officer reports, 
I do avoid interacting with Black males at times (proactive policing) with all of the issues in the States and public opinion here now (OTT276)

And a Calgary officer relates,

The thought will creep into my head that I could end up like the Baltimore cops (CAL252).

\subsection{Career Stage and the De-Policing Continuum}

Scholarly discussions involving workers' age and/or tenure, in relation to changes in attitudes and behaviours over time, often involve variations in, and/or particular aspects of, career stage theory - not only from the business and psychology literature presenting organizational research, but also in relation to the sociology of work and occupations. Both the fundamental tenets of this theory and the present study's findings of a strong association between years of front-line police experience and the prevalence of officers' risk-averse behavioural adaptations are important in terms of this study's determinations around the conceptual applicability of a de-policing continuum and the importance of front-line police subculture vis-à-vis today’s de-policing phenomenon.

\section{Career Stage Theory}

The concept of career can refer to the way individuals develop or change in their work in response to the accumulation of experiences over time (Hall 1976; Schein 1978). In the 1940s, sociologists Form and Miller (1949) researched commonalities they observed at various intervals throughout workers' careers, however Super (1953, 1957, 1963, 1980, 1984, 1990, 1992; also Super, Zelkowitz \& Thompson 1981) is the scholar most associated with pioneering theoretical developments in furtherance of understanding career stages, which should be understood as the evolutionary phases experienced throughout the course of one's working life (Dubar 1995; Selye 1974). Along these lines, Bedeian, Pizzolatto, Long and Griffith (1991) advance that career 
stage models build on three central assumptions: (1) individuals progress through distinct career stages, each having unique and distinct developmental tasks; (2) each stage is characterized by different work attitudes and behaviours; and (3) individuals in the same career stage tend to behave and respond in similar ways. As McElroy, Morrow and Wardlow (1999: 508) observe,

The most common means of studying attitudinal differences over individuals' careers is through the concept of career stage...Disagreements exist on the precise number and definition of career stages, though the basic premise is that employees pass through distinct stages over the course of their careers. In each successive stage, employees' expectations, attitudes, and goals are thought to differ.

As such, career stage theory can provide a useful framework for understanding commonplace developments throughout the span of workers' typical careers. In this regard, Munley (1977: 261) observes that since the 1950s, "Increased theoretical and empirical attention has been given to the developmental nature of how and why individuals... adjust [within their] occupations." Levinson's life course model (e.g., see Levinson 1986; Levinson, Darrow, Klein, Levinson \& McKee 1978) is also referenced often as a foundational theoretical contribution by researchers examining relationships between work attitudes, behaviours, and career stage. ${ }^{10}$ Similarly, Erikson's theoretical work has contributed to understandings around the developmental nature of career stages (Munley1977; Zaccaria 1965). Erikson $(1959,1963)$ advances that through various life stages each individual negotiates a range of sociocultural influences.

Super (1957) found four career stages, each distinguished by unique work attitudes and behaviours, which he argues every individual, essentially irrespective of occupation, passes through during their working life. ${ }^{11}$ This is the essence of all variations in career stage theory. As articulated by Griffin, Hogan and Lambert (2014: 
5, citing Cohen 1991; Greenhaus 1987), "Career stage theory holds that the effects of variables on work outcomes vary across the career stages of an individual." Like Erikson, Super argued that not everyone progresses through these career stages at fixed ages or in the same fashion and, in critiquing other scholars' linear theoretical schemes, Super (1984: 200) observes, "[Levinson] views the stages as rather firmly determined and as progressing in well-ordered sequence. My formulation has sought to make it clear that [each stage] of transition [are] very flexible."

Along these lines, Ornstein, Cron and Slocum (1989: 121) find that Super's model "is one determined by an individual's current circumstances and perceptions, whereas Levinson's is determined strictly by age." Dalton (1996; see also Dalton, Thompson \& Price 1977), as just one example, takes issue with reliance on age as the relevant variable in examining career stages and, throughout the literature, there is considerable debate on this point - with some researchers advocating for the use of age to define career stages, while others promote occupational tenure, organizational tenure, or positional tenure as the key variable (e.g., see Allen \& Meyer 1993; Aryee, Chay \& Chew 1994; Burke \& Mikkelsen 2006; Cohen 1991; Conway 2004; Griffin et al. 2014; McElroy et al. 1999; Morrow \& McElroy 1987; Noordin, Rahim, Ibrahim \& Omar 2011; Ornstein et al. 1989; Stumpt \& Rabinowitz 1981). I argue that positional tenure in front-line police work is the crucial consideration in the present study, rather than an officer's age or years of overall police work. As demonstrated through analysis of study data, it is clear that an officer's years of front-line experience in the field is substantially more germane, in arriving at an understanding of de-policing behaviours across today's front-line police work, than either an officer's age or years of overall police experience. 
The relevant portion of Table 31 is reproduced in the table below, as this is instructive in informing this discussion moving forward.

\begin{tabular}{|c|c||c|c|c|c|}
\hline \multicolumn{2}{|c|}{} & $\begin{array}{c}\text { Non de- } \\
\text { policers }\end{array}$ & $\begin{array}{c}\text { Limited } \\
\text { de-policers }\end{array}$ & $\begin{array}{c}\text { Moderate } \\
\text { de-policers }\end{array}$ & $\begin{array}{c}\text { Intensive } \\
\text { de-policers }\end{array}$ \\
\hline \hline & $1-5$ & $40.3 \%$ & $19.5 \%$ & $24.1 \%$ & $16.1 \%$ \\
\cline { 2 - 6 } & $6-10$ & $23.7 \%$ & $19.1 \%$ & $26.6 \%$ & $30.6 \%$ \\
\cline { 2 - 6 } & $11-15$ & $22.9 \%$ & $14.8 \%$ & $19.1 \%$ & $43.1 \%$ \\
\cline { 2 - 6 } \begin{tabular}{c} 
Years of Front- $\begin{array}{c}\text { Line Police } \\
\text { Experience }\end{array}$ \\
\cline { 2 - 6 }
\end{tabular} & $16-20$ & $21.6 \%$ & $15.5 \%$ & $21.1 \%$ & $41.8 \%$ \\
\cline { 2 - 6 } & $26+25$ & $20.6 \%$ & $18.3 \%$ & $16.7 \%$ & $44.4 \%$ \\
\cline { 2 - 6 } & $26+9 \%$ & $21.7 \%$ & $29.0 \%$ & $17.4 \%$ \\
\cline { 2 - 6 } & New & $40.3 \%$ & $19.5 \%$ & $24.1 \%$ & $16.1 \%$ \\
\cline { 2 - 6 } & Experienced & $23.3 \%$ & $17.6 \%$ & $23.5 \%$ & $35.6 \%$ \\
\hline
\end{tabular}

This approach coincides with recent developments in this theoretical genre, which reconceptualize career stage in recognition of today's more varied, fluid, and dynamic patterns of career development and many contemporary researchers, in their own applications, deviate from the theoretical rationales explicated by the early scholars in this field (Super, Levinson, Erikson, etc.) (Ornstein et al. 1989; Schein 1996). Accordingly, today's sociological and psychological studies in this domain often apply novel dimensions of career stage theory and continue to demonstrate differences in work-related attitudes (such as job satisfaction, motivation, cynicism, and turnover intention) and behaviours (including performance, absenteeism, turnover, commitment, burnout) in relation to workers' career stage transitions (Flaherty \& Pappas 2002) (e.g., see Adler \& Aranya 1984; Cohen 1991; Cron 1984; Cron \& Slocum 1986; Gau \& Paoline 2017; Griffin et al. 2014; Lambert \& Paoline 2012; McElroy, Rodriguez, Griffin, Morrow \& Wilson 1993; Morrow \& McElroy 1987; Pogson, Cober, Doverspike \& Rogers 2003). However, as Johnson and Lafrance (2016: 1580) have recently observed, "Little evidence exists... about the influence career stages have on the work activities of criminal justice agents." Among the exceptions are Johnson and Lafrance's 
(2016) own research into the influence of career stage on police officers' conduct; McElroy and colleagues' (1999) examination of police officers' work commitment; McGinnis' (1985) research into the development of officers' attitudes and behaviours among Canadian police; Cannizzo and Liu's (1995) study in which police officers' career stage was examined in relation to levels of perceived burnout; and Ho's (1997) research into police actions vis-à-vis use of deadly force. ${ }^{12}$ In the context of Johnson and Lafrance's (2016) observation around the dearth of scientific information about criminal justice occupational practices and career stage, I suggest that the present study can be considered a significant empirical contribution - through its use of mixed methods data to understand the influence of career stage on the everyday behaviours of rank-and-file police officers as they perform their front-line work in our communities.

Along these lines, Ho's (1997) comparison of 'rookie' versus 'veteran' officers is an example of career stage theory applied to police work as it should be understood in relation to the present study. Ho (1997: 128-129) noted that there exists a body of scholarship that has found differences in attitudes between 'rookies' and 'veterans' in terms of cynicism, job dissatisfaction, and professionalism (e.g., see Friedrich 1980; Fyfe 1981; Niederhoffer 1967; O'Connell, Holzman \& Armandi 1986; Robinette 1987; Sherman 1980) - with 'rookies' perceived as less cynical and more professional. In this vein, Ho (1997) studied whether police shooting behaviours would demonstrate that 'rookies' are less likely than 'veteran' officers to discharge their firearms when making decisions regarding using deadly force. To do so, Ho (1997) divided the Tallahassee (Florida) Police Department study participants into two groups - 'rookies' (officers that have five years' experience or less) and 'veterans' (officers that have six or more years 
of experience). Ho (1997) observed that the categorization of officers as either 'rookie' or 'veteran' is often arbitrary, but he based his determination on how those categories are understood by practitioners throughout the policing community.

Similar to Ho's reliance on a two-stage model, with 'rookies' transitioning to 'veteran' status, rather than the more traditional three-stage (e.g., Gould \& Hawkins 1978; Slocum \& Cron 1985) or four-stage models (e.g., Morrow \& McElroy 1987; Pogson et al. 2003; Super 1957, 1980), McElroy and colleagues (1999: 507-508) found that the two-stage model was most applicable and instructive in their examination of how officers' attitudes toward their work vary over the course of a police career - "with the trial stage [the recruit and early years of front-line work] being uniquely different from later career stages." In relation to the present study, the findings of Ho (1997) and McElroy and colleagues (1999) support the validity of a two-stage model and, along these lines, in the present study's examination of front-line police work I use the terminology 'new' (rather than 'rookies') and 'experienced' (rather than 'veterans') to describe these two career-stage variables. Similarly, the present study's findings also validate the observations of Johnson and Lafrance (2016: 1582), who, in endorsing the research findings of Van Maanen (1973) and Bayley and Bittner (1984), find that “experience [is] extremely important to the development of officers' attitudes and behaviours." 13 This aspect of career stage development is also elaborated in discussions of the de-policing continuum and front-line police subculture that follow this subsection.

Following on the scholarship of Erikson $(1959,1963)$ and Fagan and Ayers (1982), Burke and Mikkelsen (2006) observe that there is "increasing evidence" that 
police officers "pass through a series of psychosocial stages" as they progress through their career. In this vein, several studies involving rank-and-file officers have documented landmarks in the typical front-line policing career at which point officers' perceptions of, and attitudes toward, their work changes (e.g., see Barker 1999; Edelwich \& Brodsky 1980; Howard, Donofrio \& Boles 2004; McElroy et al. 1999; Morash \& Haarr 1995; Niederhoffer 1967). Further, Bonkiewicz (2017: 165) finds that patrol officers "pass through different career phases," which not only affects their perceptions of the job and their attitudes but also "ultimately, their performance."

In this regard, the use of five-year increments is a common practice in policing research to demarcate police officers' career stages or phases (Gau \& Paoline 2017). For example, McGinnis (1985) assigned officers in his study of career development in a Canadian police agency into four groups based on years of overall experience in policing -1 to 5 years, 6 to 10 years, 11 to 15 years, and $16+$ years. Similarly, Burke and Mikkelsen (2006), in their study of work experiences and psychological well-being among rank-and-file officers in Norway, used five-year increments to differentiate between officers at various career stages. However, they relied on officers' ages rather than their years of front-line experience (or 'positional tenure'). As discussed earlier, I advance that it is important to ensure that apples are being compared with apples and, in this respect, I argue that the present study's use of five-year increments representing research participants' front-line policing experience (not age or years of overall policing experience) is the proper approach in examining and understanding de-policing throughout today's front-line police work and within the front-line police subculture. 
In a recent study, Labaky (2013) advances that the 'training and adaptation' stage encompasses the first 5 years of an officer's career and involves a high degree of commitment to the altruistic ideals of policing and a positive attitude about police work and the community. These new officers are what Edelwich and Brodsky (1980) characterize as 'idealistic enthusiasts' - in that they believe that through their police work they can help the people they serve and protect and reduce social problems (Benner \& Phillips 1994; Harris 2014). In this regard, Labaky (2013: 6) observes, "Officers in this time frame have just entered the force with a hunger for crime fighting, hoping to make a difference in society.” Along the same lines, Barker (1999), in 18 years of ethnographic research within the LAPD, found that the first phase in an officer's career lasts about 3 years and "is characterized by academy training, initiation into department culture, and learning how to be a police officer. Subsequent stages can be characterized by growing disillusionment, fluctuating levels of organizational commitment, and ways to cope with discontent" (Bonkiewicz 2017: 168).

As Barker (1999) and Labaky (2013) both documented, in the career stages that follow the 'rookie' years, many officers experience a profound sense of disillusionment and discouragement. Labaky (2013) finds that for those officers transitioning into this 'disillusion phase,' which typically occurs at some point in the rank-and-file officer's career between 6 and 13 years of front-line service, reality has now fully set it and the belief in one's ability to make any meaningful difference in society has faded. This often results in reduced job satisfaction, diminished productivity, and increased workrelated stress and cynicism (Edelwich \& Brodsky 1980; Niederhoffer 1967). In what Labaky (2013: 9) finds is the fourth and final phase of the rank-and-file career, the onset 
of which usually occurs after 20 years of typical front-line policing experiences, "Officers have distanced themselves significantly from their job...The officer is now counting down the days to retirement and takes their job one day at a time."

\section{Career Stages and the Present Study}

As is clear from the present study's data, there are at least two career stages implicated in the observed risk-averse behavioural adaptations to today's techno-social and socio-political external policing landscape. In such a two-stage model (as applied to the present study), the first stage is captured by the variable 'New', which represents officers with five years or less front-line experience. ${ }^{14}$ Among this group of 'New' officers ( $\mathrm{N}=1076)$, over $40 \%$ report that they do not practice any form of de-policing and only $16 \%$ are categorized, within the study's criteria, as intensive de-policers. Within this model, the second career stage involves those officers with six or more years of front-line experience, which are categorized for this study's purposes as 'Experienced'. Among 'Experienced' rank-and-file officers ( $\mathrm{N}=2574)$, only 23\% report that they do not practice any form of de-policing and 36\% are categorized, within the study's criteria, as intensive de-policers. In comparison to non de-policers in the 'New' career stage, this represents a $73 \%$ decrease and, in comparison to intensive de-policers in the 'New' career stage, this represents a $121 \%$ increase.

\begin{tabular}{|c|c|c|c|c|}
\hline & $\begin{array}{c}\text { Non de- } \\
\text { policers }\end{array}$ & $\begin{array}{c}\text { Limited de- } \\
\text { policers }\end{array}$ & $\begin{array}{c}\text { Moderate de- } \\
\text { policers }\end{array}$ & $\begin{array}{c}\text { Intensive de- } \\
\text { policers }\end{array}$ \\
\hline 'New' officers & $40.3 \%$ & $19.5 \%$ & $24.1 \%$ & $16.1 \%$ \\
\hline $\begin{array}{c}\text { 'Experienced' } \\
\text { officers }\end{array}$ & $23.3 \%$ & $17.6 \%$ & $23.5 \%$ & $35.6 \%$ \\
\hline
\end{tabular}

In terms of the significant differences observed between 'New' and 'Experienced' officers in the present study, these results resemble those of the 2017 Pew Research 
Centre study involving officers from 54 police departments across the USA ( $N=7917)$

and examining officers' sentiments of frustration versus fulfillment. That study found,

Police officers who are new to the job are more likely than more seasoned officers to say their work makes them feel proud or fulfilled, and they are less likely to say they often feel frustrated...[For example,] 59\% of new officers say their work makes them feel fulfilled nearly always or often, a sentiment shared by $40 \%$ of officers who've been on the force for five years or more. And while $39 \%$ of new officers say they nearly always or often feel frustrated by their job, $53 \%$ of those with five or more years on the force say this (Morin et al. 2017: 32)

Illuminating the present study's results and those of the Pew researchers, in a spontaneous meeting with nine Calgary officers in the police station parking lot after shift briefing (an unanticipated, but fruitful, method of data collection as described earlier in subchapter 4.4), these officers elaborated on the answers they had provided within the survey instrument. With the contemporaneous concurrence of their colleagues, two CPS officers took turns explaining to me that most 'rookies' are exposed to de-policing as a potential and widespread self-preservation strategy, if not while attending the police academy, then most certainly within the first few months of field training - as de-policing is now a topic of frequent discussions within the rankand-file subcultural discourse.

[However, given that such officers] are full of piss and vinegar at that time in their career, it usually takes somewhere between 4 and 10 years for them to have a few episodes with the Professional Standards Section [about controversial interactions with citizens, often video recorded, and to] be accused of being racist and corrupt every shift. [Thereafter,] they begin to see the wisdom in de-policing some things cops used to do, if not everything but dispatched calls.

In considering the present study's data in relation to the more specific five-year increments used to represent years of front-line police service among the sample population, it is possible to find that, rather than two career stages (i.e. 'New' and 'Experienced'), there exist three, or perhaps even four, career stages of relevance to the 
identified de-policing phenomenon. Within a three-stage model, it is clear that significant differences exist among those officers with 26 and more years of front-line experience, when compared to their colleagues that have performed such work for between 6 and 25 years - particularly in relation to the proportion of intensive depolicers across those two groups of study participants. For example, among officers in the 21 - to 25 -year group, $44 \%$ report practicing intensive de-policing, whereas in the $26+$ year group only $17 \%$ of officers report this behaviour. This represents a clear distinction and a significant difference in the proportion of non de-policers is also observed between officers categorized within these two career stages. For example, among officers in the 21 - to 25 -year group, $21 \%$ report not practicing de-policing, whereas in the $26+$ year group $32 \%$ of officers are categorized as non de-policers.

\begin{tabular}{|c|c|c|c|c|}
\hline & $\begin{array}{c}\text { Non de- } \\
\text { policers }\end{array}$ & $\begin{array}{c}\text { Limited de- } \\
\text { policers }\end{array}$ & $\begin{array}{c}\text { Moderate de- } \\
\text { policers }\end{array}$ & $\begin{array}{c}\text { Intensive de- } \\
\text { policers }\end{array}$ \\
\hline 6-10 years & $23.7 \%$ & $19.1 \%$ & $26.6 \%$ & $30.6 \%$ \\
\hline 11-15 years & $22.9 \%$ & $14.8 \%$ & $19.1 \%$ & $43.1 \%$ \\
\hline 16-20 years & $21.6 \%$ & $15.5 \%$ & $21.1 \%$ & $41.8 \%$ \\
\hline 21-25 years & $20.6 \%$ & $18.3 \%$ & $16.7 \%$ & $44.4 \%$ \\
\hline 26 years + & $31.9 \%$ & $21.7 \%$ & $29.0 \%$ & $17.4 \%$ \\
\hline
\end{tabular}

However, some caution should be exercised in considering these results as the number of study participants represented in the $26+$ year category $(\mathrm{N}=71)$ were significantly fewer than those in the other five-year incremental categories. Nevertheless, this is interesting to consider and may represent, among some officers especially close to retirement, the distancing from all aspects of the job - including perhaps de-policing practices, which was referred to by Labaky (2013) and reported by some participants in the present study, for example, the following veteran front-line Buffalo and Saskatoon officers: 
I don't care about these things now. I feel bad for the younger officers today, but I do what I gotta do until I hit pension [information about the time frame redacted] (BUF315).

I lay low period. I don't get involved or avoid getting involved. I don't need to get in trouble for anything. I am just doing the basics until [year redacted] when I'm retiring (SAS53).

Niederhoffer's (1967) study documented a similar three-stage model in relation to officer cynicism as a developmental process related to career stages, "Progressively [rising] early in the career-course, peak[ing] around the mid-point, and then decreas[ing] until retirement" (Enciso, Maskaly \& Donner 2017: 86). Similarly, Barker (1999) found that officers begin their policing careers motivated to perform proactive work in the field, however,

Patrol officers became burned out from performing the same tasks for so long, and became disgruntled from experiencing injuries, lawsuits, citizen complaints, and being passed over for promotions or transfers. Finally, in the last career stage, Barker suggested that patrol officers began to disengage from the career, focusing more on outside interests and avoiding any risky situations that might jeopardize their retirement pensions (Johnson \& Lafrance 2016: 1594).

In considering study data in the context of a four-stage model, one could make the case that there is a transition period that relates specifically to intensive de-policing behaviours in the 6- to 10-year range of an officer's front-line career. In this regard, the proportion of officers categorized as intensive de-policers is observed to rise from $16 \%$ in the 1 - to 5 -year group to $31 \%$ in the 6 - to 10 -year group and then to $43 \%$ in the 11 - to 15- year group. Thereafter the value remains essentially constant until the significant drop observed in the 26+ year group (as discussed in the preceding paragraphs).

\begin{tabular}{|c|c|c|c|c|}
\hline & $\begin{array}{c}\text { Non de- } \\
\text { policers }\end{array}$ & $\begin{array}{c}\text { Limited de- } \\
\text { policers }\end{array}$ & $\begin{array}{c}\text { Moderate de- } \\
\text { policers }\end{array}$ & $\begin{array}{c}\text { Intensive de- } \\
\text { policers }\end{array}$ \\
\hline 1-5 years & $40.3 \%$ & $19.5 \%$ & $24.1 \%$ & $16.1 \%$ \\
\hline 6-10 years & $23.7 \%$ & $19.1 \%$ & $26.6 \%$ & $30.6 \%$ \\
\hline 11-15 years & $22.9 \%$ & $14.8 \%$ & $19.1 \%$ & $43.1 \%$ \\
\hline
\end{tabular}


However, it is interesting to note that the proportion of officers not practicing any form of de-policing remains essentially the same, as between the 6- to 10 -year group (24\%) and the 11- to 15-year group (23\%), and modest reductions in proportions of limited depolicers (-4\%) and moderate de-policers $(-8 \%)$ are observed - consistent with the migration of officers toward the practice of more intensive forms of de-policing $(+12 \%)$. I suggest that these career-stage observations are intertwined with, and can be understood in relation to, the existence of a de-policing continuum, which is elaborated in the following subsections.

\section{The De-Policing Continuum}

Various contributions throughout the literature document shifts in officers' attitudes and behaviours from the police academy, to field training, through the first years of front-line experiences, and then into later phases of the typical rank-and-file police career (e.g., see Foley, Guameri \& Kelly 2008; Garner 2005; Van Maanen 1973, 1974, 1975; White, Cooper, Saunders \& Raganella 2010). For example, researchers suggest that the excitement experienced by new officers sustains their motivation, as does the desire to prove themselves to other officers and the organization. However, it has been found that these extrinsic influences diminish as officers accumulate experiences in the field and, therefore, officers' behaviours often change over time (e.g., see Barker 1999; Bayley \& Bittner 1984; McElroy et al. 1999; Riksheim \& Chermak 1993; Sherman 1980; Skogan \& Frydl 2004). In this regard, Buckley and Petrunik (1995: 113) observe, “After training and gaining experience from their first few years on the job, officers may come to realize that the conceptions that they held about the job are more idealistic than realistic...In response to this, they may adapt to their career 
through a change of attitudes." Also, in this vein, Bonkiewicz (2017: 183, citing Brehm \& Gates 1993) finds,

As an officer passes through the stages of training, competence and confidence, and then disillusionment, coping, and retirement, it seems reasonable that their changing perceptions and attitudes affect their productivity, especially if their perceptions worsen...Patrol officers remain highly productive for only a few years before their productivity declines, [which] begin[s] immediately after officers' first year of service and worsen[s] across the length of their careers...By the 21-year career mark, a patrol officer will generate $88 \%$ fewer selectives [proactive initiatives and contacts with community members], $50 \%$ fewer traffic warnings, $58 \%$ fewer traffic citations, $41 \%$ fewer warrant arrests, and $57 \%$ fewer misdemeanour arrests compared to an officer with 1 year of service...[Officers in later stages of their police career] may become less proactive to avoid [certain] situations [and] more experienced officers also may recognize that proactive policing often results in more work.

Shane $(2010,2011)$ finds that many officers, over time, begin to curtail proactive work as they accumulate experiences, because they come to recognize that it leads to more work than is necessary in fulfilling the basic requirements of their job function. Johnson's (2012) recent research has corroborated an earlier body of scholarly work that found officers' job satisfaction consistently declines as tenure increases (e.g., see Burke 1989; Buzawa, Austin \& Bannon 1994; Dantzker 1992; Hunt \& McCadden 1985; Sheley \& Nock 1979; Zhao, Thurman \& He 1999). In a similar vein, Burke and Mikkelsen (2006) find that, "Over time, constables become hardened, less willing to trust others (particularly the public)." Similarly, Lafrance and Day (2013) observe that most officers begin their policing careers embracing the understanding that departmental rules and procedures are very important, however adherence to this doctrine declines precipitously with the accumulation of experiences 'on the job.' Finally, in terms of examples (and certainly not represented as an exhaustive accounting), Harris (2016: 226) finds consistency with the career stage perspective and 
the concept of a behavioural continuum in advancing, "An officer's involvement in problem behaviour can be conceived as a developmental trajectory, given it has a distinct beginning and end, and would likely be affected by important turning points in officer careers."

These research findings and those of other policing studies, whether they explicitly acknowledge reliance on the conceptual framework, or not, engage the concept of a continuum and, as discussed throughout this dissertation's earlier chapters, I advance that the de-policing behaviours of the front-line officers in the present study can be understood as existing on a continuum - ranging from those that unequivocally reject de-policing (a category that includes the 'mission-oriented officers, as discussed) to those that perform only minimal reactive police work ('fire-brigade policing'). In this vein and in the examples in the preceding paragraphs, Bonkiewicz (2017), Burke and Mikkelsen (2006), Harris (2016), Johnson (2012), Lafrance and Day (2013), and Shane (2010, 2011) all find that the identified attitudes and/or behaviours (productivity, trusting others, involvement in problem behaviour, job satisfaction, importance of departmental rules and procedures, and engaging in proactive police work) are not uniform across the research population. Rather, they exist at points on a continuum that range from one extreme of the attitude and/or behaviour to the opposite extremity.

Bonkiewicz's (2017) study functions as a particularly good example of a continuum in relation to police behaviours and this researcher also illuminates the interrelated consideration of career stage (as related to tenure). Similar to the depolicing behaviours reported in the present study, Bonkiewicz (2017) observes that there are extremes at either ends of the continuum in relation to patrol officers' 
productivity, with highly productive officers - usually 'rookies' - toward one pole and significantly non-productive officers - usually 'veterans' - toward the opposite end of the continuum, with a range of officer productivity behaviours situated at various points between the two extremities. Also, as relates to police officers' behaviours, the concept of a 'continuum of compromise' is often relied on as 'a useful framework for understanding the transition from 'honest cop' to 'compromised officer"” (Gilmartin \& Harris 1998: 25) and in illuminating the social-psychological model of unethical and unprofessional police behaviour (Sunahara 2004). ${ }^{15}$

\section{The Present Study and the De-Policing Continuum}

Johnson and Lafrance (2016: 1586) conducted research examining the issuance of citations for minor transgressions, impaired driving arrests, and illicit drug arrests, which they observe "result most often from proactive stops or investigations" rather than from dispatched calls. Similar to previous studies that found discretionary policing initiatives decline significant with experience (e.g., see Riksheim \& Chermak 1993; Sherman 1980; Skogan \& Frydl 2004), these policing scholars found that productivity, in all of the three areas measured, dropped precipitously among officers with ten or more years of (overall) police service (Johnson \& Lafrance 2016). Their findings are consistent with the conceptual framework of a de-policing continuum as it is understood within the present study - in that, in a general sense, it can be expected that for many rank-and-file officers (among those that ultimately decide that their best interest is served by engaging in de-policing behaviours) their risk averse practices will change (typically intensify) as they accumulate experiences in the field over time. Also instructive to the present study's discussion of a de-policing continuum are the 
observations of Oliver (2017: 455, citing Hawkins 2001; Martinussen, Richardsen \& Burke 2007; McCarty \& Skogan 2012; McCarty, Zhao \& Garland 2007; Schaible \& Gecas 2010), who found, "Unlike the findings on police burnout, de-policing does not appear to be present solely in veteran officers." Along these lines, in the present study it has been found that de-policing can manifest at any point in an officer's front-line career and in myriad forms.

In this vein, I advance that the conceptual framework of a de-policing continuum is useful in appreciating how, for most rank-and-file officers today, considerations of risk impact on their conduct in the field through a range of behavioural modifications to their police work - to various degrees and at varying times throughout the officer's years spent performing front-line police work. As discussed previously, an officer's years of front-line police experience has been identified as the key variable associated with the onset and then progression of de-policing practices among many participants in the present study. However, it is important to stipulate that the conceptual framework of a de-policing continuum is just that - a nuanced and imprecise proposition that is, nonetheless, instructive in informing readers' deliberations on the identified de-policing phenomenon. It is not a scientifically-validated formula that can produce a definitive answer after solving for the variables in operation (along the lines of cause and effect associations) with this complex development across today's front-line police work. And, therefore, it is not suggested that each rank-and-file officer will follow a known and certain progression in de-policing attitudes and behaviours throughout their front-line policing career - in the sense of a predictive capacity within the conceptual model. ${ }^{16}$ 
I argue it is the case that few individuals beginning their police career enter the profession with the understanding that they will immediately adopt risk-averse practices once deployed in the field. ${ }^{17}$ For example, consider the representative observations as reported by 'rookie' Calgary and Delta officers,

I am not doing [de-policing] yet, with only one year on the job, but most senior officers do it and discuss it with us often as 'veteran advice' (CAL117)

I am just beginning my career and feel a lot of these questions will apply to me as I continue to gain more experience and interact with the public more (DEL09).

Along these lines, the conceptual framework of a de-policing continuum contemplates that, after being mobilized in the community, front-line officers' attitudes and practices consistent with de-policing, can, and tend to (when considered in the aggregate), germinate and then progress in response to the accumulation of negative experiences in the field. On this point, the observations of a veteran Saskatoon officer are illuminating, As a supervisor I can tell you [de-policing] has become widespread now, even the new officers are learning about it and after a while of seeing issues first hand many of them are mostly just reactive because of the risks (SAS127).

This accumulation of negative experiences in the field implicates three key risk considerations in today's front-line policing landscape - (1) the 'new visibility' of officers' conduct in the field; (2) elevated public scrutiny of, and criticism directed at, the behaviours of rank-and-file officers; and (3) officers' subjective perceptions that certain individuals with whom they might interact in the community present as a particularly 'risky' demographic. The simultaneous influence from the shared experiences and counsel of peers within the front-line subculture, advocating in favour of risk-averse and self-protection practices, is also a key consideration in relation to the adoption of, and subsequent intensification in, de-policing behaviours (and this is elaborated in the next subchapter). 
As observed in the present study's results, the initial progression within the depolicing continuum involves, in many cases, the officer transitioning from being a non de-policer early in their rank-and-file career to an officer who has adopted at least one aspect of de-policing (as identified in the study's criteria) as a viable self-preservation strategy - in ceasing to perform certain discretionary aspects of proactive front-line police work because of concerns that their actions in the field could be video recorded and/or criticized by some members of today's public audience. For example, the riskaverse behavioural adaptations reported by a Halifax officer are representative of such developments,

For the first couple years on the road I thought there was no way I would do the FIDO thing like most of the more senior guys. We have an important job to do and [you] just can't decide to neglect parts of this job. But then I had people complain about me and they had a video showing I did nothing wrong in stopping the guy but it still caused me no end of grief. So I figured the senior guys are right, who needs the grief? [And] so I stopped stopping people not on a call. I haven't done proactive policing now for at least 3 years and guess what, no complaints and great performance evaluations (HAL03).

In other words, and in painting the typical de-policing developmental sequencing with a broad brush, as an officer accumulates negative experiences from acrimonious police-citizen interactions in the field, and with concurrent influence from colleagues within the rank-and-file subculture, progression within the de-policing continuum most often originates with apprehensions centred around the risk presented by video recording or critique of the officer's work by citizens. For example, and as typifies the circumstances related by numerous study participants, the officer with a few years of front-line experience often begins to practice one aspect of de-policing (i.e. modifying behaviours because of the potential for video recording) and then progresses further along the continuum later in their rank-and-file career toward moderate or intensive depolicing (i.e. additional modifications to the officer's conduct related to concerns about 
the potential for public criticism and/or perceptions of risk in interacting with persons in the community exhibiting certain 'demographic' characteristics). The progression in depolicing behaviours throughout the front-line career of the following Port Moody officer is emblematic,

For me it started with the video of the RCMP with the Polish guy at the airport [the Robert Dziekański occurrence in 2007]. After that shitstorm I realized it was probably wise to steer clear of video to protect a police career. Then, with the way the public jumps on us whenever there is any controversy, no matter whether it is true or invented, I realized steering clear of situations where the public could jump on me was wise and especially if there were minorities involved (POR19).

Along these lines, it is often the case that after the officer's initial progression within the de-policing continuum, further risk-averse behavioural adaptations can manifest in response to the officer's perceptions of additional strain accompanying today's challenging external policing landscape. Put simply, as the front-line officer perceives more risk, they become more susceptible to intensifying their de-policing practices and, therefore, to progressing within the de-policing continuum. In this regard, recall the discussion in Chapter Five around limited and moderate de-policers and their concerns being substantially more related to either the potential for video recording of their actions in the field or criticism of their conduct by persons in the community rather than concerns related to elevated risk presented to front-line officers by those persons perceived to epitomize 'risky' 'demographic' characteristics. As is clearly demonstrated in the study's results (refer to Table 39), the evolution from moderate depolicer to intensive de-policer most often implicates officers' perceptions around the risk for consequences presented by certain individuals in the community - associated to specific 'demographics' such as racial/ethnic minorities, the mentally ill, and/or those of non-traditional sexual orientations. ${ }^{18}$ 
This risk consideration, and the intentional and proactive avoidance of interaction with persons perceived as presenting the 'risky' characteristic(s), was found (through study participants' responses to survey Question 26) to be far more prevalent among those officers categorized as intensive de-policers than those engaging in risk-averse behaviours defined (within the study's criteria) as limited or moderate de-policing. Question 26 asked, 'Do you do anything to avoid interacting with persons who present the demographic characteristics you have identified [in Question 25(a)]?'

\begin{tabular}{|c|c|c|}
\hline \multirow{2}{*}{} & \multicolumn{2}{|c|}{ 'Yes' responses to Question 26 } \\
\cline { 2 - 3 } & $\mathbf{N}$ & $\mathbf{\%}$ \\
\hline Non de-policers & 0 & 0.0 \\
\hline Limited de-policers & 158 & 24.0 \\
\hline Moderate de-policers & 213 & 24.9 \\
\hline Intensive de-policers & 1081 & 100.0 \\
\hline Totals & 1452 & \\
\hline
\end{tabular}

In an off-site discussion with four front-line RCMP Ridge Meadows officers this key study finding was validated. Their unanimous observations were characterized by one of these veteran officers as follows,

It only takes a few years for most of us to recognize the dangers of video nowadays and that some vocal people like to judge and second guess what we do. The vocal minority that has an axe to grind against the police. If you are smart, you change how you do your job to avoid problems like these. You avoid video, you avoid doing anything that might put you in the centre of a controversy. You just take your calls. The last thing that you touch on in your survey that goes once after a few more years is the willingness to get involved with people because they belong to a group that is known to be anti-police and think they get picked on by us because we are all bigots against those groups. That is a crock but a bad situation with one of them can land you in a lot more hot water than a straight White middle-class male...[In this jurisdiction] I'm talking about your homeless with drug addiction and mental health issues, Blacks and East Indians, and LGBT. Get involved with these at your peril!

Similarly, the following qualitative elaboration of a veteran Toronto officer captures this final progression as contemplated within the conceptual framework of a de-policing continuum for many study participants that are categorized as intensive de-policers, 
I think the way this happens for a lot of us is that we start to get frivolous complaints after we are on the streets for a while and establishing our style of police work and then you have some videos that are now everywhere that come forward along with the complaints that people say prove that you have done something wrong and you are a bad officer. So you get gun shy about video and situations that might turn into a complaint more than other situations and you avoid them. And then comes the racial bullshit on top of these. I think I can be considered an expert witness on racial bullshit because I am a Black [gender redacted] and can tell you these accusations of racism by the public and BLM are exaggerated bullshit. So now you have a lot of coppers with a lot of veteran experience on the streets that don't engage with Blacks and other minorities and are worried about video and complaints and are doing NC/NC every shift looking forward to retirement day (TOR207).

Before moving into a discussion focused on the police front-line subculture and its influence on officers' de-policing practices, it merits repeating that the conceptual framework of a de-policing continuum is not a perfect model, otherwise we would expect to see all officers with many years of front-line experience practicing intensive de-policing. In this regard, the concept of a de-policing continuum represents broad trends and tendencies that assist in better appreciating how de-policing behaviours can develop in various forms among significant numbers of officers throughout their rankand-file policing career. Along these lines, Charman (2017: 158, citing Campeau 2015) observes,

Paoline's model [of police occupational culture] encompasses Chan's (1997) calls for a more dynamic and interactive account of police culture which presumes police officers to be active cultural agents in the creation and sustainability of their policing culture. Police culture can then be viewed as a 'resource' which police officers can utilize to guide their actions and to navigate the difficult terrain of policing citizens. Rather than presuming that police officers will utilize these cultural tools in the same way and to the same extent, we should instead view these cultural tools as being positioned along a continuum...

\subsection{Front-Line Police Subculture and De-Policing}

The discussion throughout this subchapter is focused on front-line officers' occupational subculture - in terms of both the present study's empirical findings of a significant relationship between this subculture's influence and the contemporary de- 
policing phenomenon and also in the context of its contribution, as this dissertation's primary theoretical framework, to a comprehensive and nuanced understanding of the identified risk-averse behaviours of today's rank-and-file officers. Similar to the approach outlined in the previous subchapter, this discussion will not revisit, to any considerable degree, the information presented throughout subchapter 3.4, however, it should be understood that those materials form part and parcel of this subchapter's analysis and discussion. In saying this, what I will remind the reader is that it is widely accepted among policing researchers that there exists a distinct front-line police subculture (e.g., see Boivin et al. 2018; Campeau 2015; Ingram et al. 2013; Manning 2014b; Paoline \& Gau 2018; Paoline \& Terrill 2014; Reuss-Ianni 1983) and that many policing scholars have observed that cultures within policing are central to the theorizing of, and/or research efforts seeking to better understand, officers' attitudes and behaviours in the field (e.g., see Bacon 2014; Chan 1996, 1997, 2004, 2007b, 2011; Cordner 2017; Ericson 1981, 1982, 2007b; Loftus 2009, 2010; Newburn \& Reiner 2012; Paoline 2003, 2004; Paoline \& Terrill 2014; Reiner 1985, 2010, 2017; Sklansky 2007; Terrill et al. 2016; Waddington 1999). For example, Bacon (2014: 103) observes, "Police researchers have argued...that [officers'] cultural elements...help officers determine how to exercise their discretionary powers and that they mediate the demands of the job with the exigencies of everyday life" and Westmarland (2008: 255) finds,

One of the reasons for the concentration [of research] on police cultures is...because of the importance of discretion in the police role. Since at least the 1960s when in-depth studies began to appear (Banton 1964; Skolnick 1966; and later, Van Maanen 1978), criminologists have argued that police culture is central to understanding or interpreting the use of police discretion [which is addressed in further detail in subchapter 6.7]. 
In relation to contributions advancing sociological or criminological theory, Paoline and Gau (2018: 671, citing Chan 1996; Fielding 1988b; Manning 2005c) observe, that despite some illuminating exceptions, "As an area of study, police culture has been criticized for its lack of theoretical development." Along the same lines, research into police mis/conduct has often been criticized for being atheoretical (Campeau 2015; Chappell \& Piquero 2004; Donner \& Jennings 2014; Harris 2016; Kane 2002; Pogarsky \& Piquero 2004). With the present study, I hope to make a contribution in advancing both theoretical and empirical understandings in relation to the association between front-line officers' risk-averse conduct, or misconduct depending on how one views the practice of de-policing - and the rank-and-file police subculture, which, in conjunction with negative police-citizen interactions in the field, that vary in their nature and quantity for each individual officer, has been found, in a collective sense, to influence many officers toward engaging in, and then subsequently intensifying their, de-policing practices.

Therefore, I suggest that the present study can be considered in the tradition of previous research that featured police occupational culture as an instructive theoretical framework. In this regard, Loftus (2010: 1) observes that many studies (e.g., Banton 1964; Cain 1973; Holdaway 1983; Punch 1979a; Reiner 1978; Rubinstein 1973;

Skolnick 1966; Smith \& Gray 1983; Westley 1970; Young 1991) "have highlighted the usefulness of 'police culture' in understanding the many facets of policing, including how officers learn the craft of the job, use their time and interact with different people." In relation to the studies enumerated in the previous sentence and others from an earlier era in policing, Sklansky (2007: 19) finds that assumptions about police subcultures in 
the 1950s, 1960s, and 1970s "make less sense today," as they "obscure differences between officers [and do not reflect] new complexities...dynamic processes...[and] emerging challenges in policing." ${ }^{19}$ Similarly, Loftus (2009: x-xi), while advocating for research initiatives to determine how today's police officers experience the new realities of their occupation in a changed, and changing world, finds that most studies involving police occupational culture are outdated and, as a result, "we are left...with an account of police culture which largely predates the transformations which have since taken place...in newly identified social fields of policing."

In this vein, Chan (2007b: 145-146) finds that a "tightening of accountability," through "constant scrutiny" of the police, and heightened sophistication in the surveillance of officers' practices, has led to significant "cultural change" within policing. Along the same lines, Campeau, in her recent research examining Canadian police subcultures and front-line officers' adaptations to today's policing challenges, observes that "“traditional' police culture notions do not capture how officers [now] understand their work...in [these] unsettled times" (2015: 684) and that 'traditional' representations of rank-and-file police culture "are being reinterpreted [and] reimagined in an age of increased oversight and accountability” (2016b). Shjarback (2016: 53-54), in assessing Paoline's contributions in this area, vis-à-vis the American rank-and-file police subculture, observes, "Paoline [has] performed perhaps the most complete and quantitatively rigorous examination[s] of the occupational culture/officer styles perspective with many of the [traditional] attitudinal dimensions previously identified in the literature...[However], studies have only recently begun to test culture's impact on police behaviour." 
In relation to Shjarback's last sentence, and in the intellectual tradition of Bhaskar and Harré, I argue that discovering behaviours, or more specifically, observed patterns in behaviour is "only the beginning of the process. What is then required [in the research process] is to locate the structures or mechanisms that have produced the [observed patterns in behaviour]" (Lewis-Beck, Bryman \& Liao 2003: 972). This is the rationale for the designation of contemporary front-line police subculture as the principal theoretical framework to inform the present study's empirical results and for this expansive, and hopefully illuminating, subchapter. I suggest that the identified depolicing phenomenon among today's front-line officers can be appropriately characterized as 'observed patterns of behaviour' and, in conjunction with individual officer's experiences in the field, the influence of the rank-and-file police subculture should be understood as a 'structure or mechanism' that contributes to the observed depolicing outcomes. This subchapter explains this latter consideration in relation to the present study's finding of widespread de-policing practices among today's population of Canadian and American front-line police officers.

As addressed throughout subchapter 3.4 and elsewhere in this dissertation, over the past 20 years, Paoline has refined a theoretical approach to police occupational culture that considers strains and anxieties resulting from the distinctive challenges and conflicts inherent to police work, which he observes officers can experience in both their internal and external policing environments. Paoline also considers officers' coping mechanisms and the resulting outcomes, which often implicate the subcultural endorsement of various behavioural adaptations to mitigate against the strains and anxieties experienced by individual rank-and-file officers (e.g., see Ingram, Paoline \& 
Terrill 2013; Ingram, Terrill \& Paoline 2018; McCluskey, Terrill \& Paoline 2005;

Paoline 2000, 2001, 2003, 2004; Paoline \& Gau 2018; Paoline \& Terrill 2005, 2014;

Terrill, Paoline \& Manning 2003). ${ }^{20}$ Scholars writing within the 'traditional' genre of

the police occupational culture literature have found that such adaptations can involve a

variety of attitudes and behaviours among officers, for example - distrustful and

suspicious of citizens, socially isolated (within the policing fraternity), secretive,

prioritizing solidarity (the 'thin blue line' mentality) and the adoption of 'lay low' or

'avoidance' approaches to front-line police work.

Kappeler and colleagues (2010) find that because of strains experienced in the external policing environment, officers tend to isolate themselves within peer groups and retreat from an outside social life. As a result, officers are "subjected to intense peer influence and control," which can involve the acceptance of various types of behaviour - including misconduct or other problematic activities (Kappeler et al. 2010: 265). In reviewing the literature in this regard, Paoline and Terrill (2014: 6) observe, "What we find in descriptions of police culture... is a series of writings that attempt to characterize the various features and strains encountered in the policing environments, as well as the cultural responses endorsed in buffering such strains." In this vein, Paoline and Gau (2018: 671) advance,

Since foundational work in the 1950s, researchers have described a variety of dimensions of the occupational culture of police. In an effort to integrate the disparate works, a theoretical model has been constructed depicting the ways in which the stressful features of the police work environment produce coping mechanisms and outcomes. While this model usefully organizes the vast literature on police culture, it has yet to be empirically tested...The implications are especially relevant given recent concerns over police-community relations and the renewed interest in the police occupational culture expressed by the President's Task Force on 21 st Century Policing. 
I suggest that the adoption of various de-policing practices across the majority of today's rank-and-file officers, as revealed in the results of the present study, represents a contemporary update to research findings from an earlier social, cultural, and political era in the policing of Western democratic societies. As demonstrated in the data presented throughout Chapter Five, this involves a range of individual officer's riskaverse behavioural adaptations to today's external techno-social and socio-political policing landscapes (addressed within the conceptual framework of a de-policing continuum), which are indivisibly linked to, and must be situated within the context of, today's rank-and-file police subculture. I respectfully advance that this position is consistent with the recent observations of other contemporary policing scholars (e.g., see Bacon 2014; Campeau 2015, 2016a; Cockcroft 2013; Ingram et al. 2013, 2018; Loftus 2009, 2010; Workman-Stark 2017) and, in particular, the present study corroborates, and elaborates on, the innovative scholarship of Paoline (and those with whom he has collaborated) in this area and can perhaps be considered as an empirical test of his theoretical approach to understanding today's police occupational culture.

In moving forward, recall that an officer's years of front-line experience (not age nor years of overall police experience) has been found to be the key consideration (or the most significant variable) associated to the development of risk-averse attitudes and behaviours within the study's research population and, in many instances, to an officer's progression within the de-policing continuum toward more intensive de-policing practices in their front-line police work. The accumulation of front-line experiences that can result in de-policing often implicates two interconnected mechanisms. The first involves the amassing of negative experiences during officers' interactions with citizens 
in the field (antagonistic, disagreeable, and/or problematic occurrences) - experienced individually in varying quantities, over various durations in officers' front-line tenure, in an innumerably diverse range of circumstances, and situated within the overarching context of today's techno-social and socio-political policing environments. In this vein, also recall that Stenning and Shearing (2005) find that modifications to police practices are now largely the product of external social, cultural, and political catalysts, rather than of 'change forces' internal to police agencies. I respectfully submit that de-policing outcomes resulting from negative experiences with citizens in the field, in terms of front-line officers' risk-averse perceptions, attitudes, and behavioural adaptations, have been thoroughly accounted for throughout Chapter Five and, therefore, require little in the way of further elaboration throughout this chapter. However, in saying this, issues related to race/ethnicity (racialization) are often a key subcurrent in conversations about the front-line police subculture, and this dissertation's discussion related to these, in the context of de-policing, is no exception. Further elaboration in this specific area is engaged throughout subchapter 6.8 .

The second mechanism that can contribute to the development of, and/or intensification in, de-policing behaviours, in considering the accumulation of experiences over an officer's tenure performing front-line police work, implicates the persuasive influence of the rank-and-file subculture on the attitudes and mis/conduct of individual officers. In this vein, Walker (2005: 153) finds that "much problematic police behaviour is rooted in the police culture." Similarly, Stoughton, Albert and Noble (2015) find, "Informal interactions between officers shape agency culture and affect officer actions at least as much, and often, more, than formal rules." Along the same 
lines, Ericson (2007b: 372) argues, "Clearly it cannot be contended that administrative rules control police decisions" and therefore to understand 'rules' that govern officers' decisions in the field one must turn "to the occupational culture of the police." It is interesting to recall that, in addressing the inappropriate use of force ('police brutality') in his seminal ethnographic investigation of front-line police work, Reiss (1968: 18) found, "Though the official police code does not legitimate this practice, police culture does." In speaking from my own experiences in the field, and, as is well documented throughout the literature, the police rank-and-file subculture condones and, in some instances, encourages certain practices that do not coincide with official policies, or public expectations, for that matter. Certainly, no official police policy today would mandate that officers de-police certain individuals or neighbourhoods or avoid any discretionary, proactive, or community engagement while performing their police work.

In other words, today's de-policing phenomenon should be understood in two dimensions, both existing within the overarching context of today's external technosocial and socio-political realities - (1) as an individual-level behavioural modification by front-line officers in response to their individual perceptions of risk in their unique policing circumstances and (2), given the substantial influence of policing's rank-andfile subculture, also as a subcultural adaptation, endorsed and practiced by many frontline officers in response to strains inherent to, and experienced in, their everyday police work. This latter dimension is the focus of the remainder of this subchapter, as a more elaborate discussion in this area will allow for a comprehensive understanding around how many of today's rank-and-file officers come to practice de-policing. 
In this regard, Westley $(1953,1970)$ is instructive with his observation that "the key to understanding the [behaviour of] police is to understand their shared mentality their subculture" (Sklansky 2007: 23). Similarly, Reiner, who defines cultures as "complex ensembles of values, attitudes, symbols, rules, recipes, and practices, emerging as people react to the exigencies and situations they confront" (2010: 1160), finds that, "An understanding of how police officers see the social world and their role in it - 'cop culture' - is crucial to an analysis of what they do" (1985: 85). ${ }^{21}$ Since Manning's (1977) early findings that the occupational culture of police relates to officers' assumptions about their social world (their policing environment), which, in turn, informs their conduct, the rank-and-file police subculture has been found in numerous studies to be significant in shaping front-line officers' everyday attitudes, decisions, and conduct (Loftus 2009, 2010) and as an important source of support and guidance for officers in coping with the strains of contemporary police work (Boivin et al. 2018; Ingram et al. 2018; Paoline 2003). In this vein, Paoline and Terrill (2014: 5) find that this subculture involves "the attitudes, values, and norms that are transmitted and shared among groups of individuals in an effort to collectively cope with the common problems and conditions [faced in their policing environment]." Specifically, in relation to de-policing behaviours among the rank-and-file, Cooper (2006: 140) found that the "cultural context" in which this practice operates is important in appreciating the nature of this contemporary policing phenomenon. Representative empirical contributions from study participants, in the form of written responses describing 'real world' events, illuminate the theoretical/conceptual observations of the scholars featured in this paragraph (and elsewhere throughout this dissertation): 
If the officers I work with didn't show me the light I would be off the job by now (terminated). I wasn't noticing how society was changing to where we could do no right no matter what. Other officers started to slow down and risk less but it took them showing me how the stuff I was doing could get twisted into racial profiling or abuse of authority to get me in line with the new program. (ALB143).

Learning from other officers' discipline cases to keep [my] work to a minimum. (ROC194).

My brothers and sisters that I work with every day watch out for me and I watch out for them. We stick together and help each other to avoid the kind of trouble that is too easy to get into with the way people are disrespecting of the police today. That means we all do our work the same way, which now mostly is about covering our own asses and staying out [of] any controversy [and] going home without any issues at the end of the day. (WIN80).

I resisted the FIDO mentality for a long time until officers I respect with time on the job changed what I thought my job was about when they dissected my calls and compared the downside risk of how they would handle the call versus how I would handle the call. This job now is about self-preservation so radio calls only. (OTT160).

I have only been on for a couple of years but I am giving [de-policing] more thought now because of what I see and what I am being told by my friends on the job. (YON18).

\section{Front-Line Police Subcultural Socialization}

In addition to appreciating the importance of occupational culture's influence on individual's attitudes and behaviours, a crucial consideration in many discussions on this topic involves arriving at an understanding of how certain values, attitudes, understandings, informal 'craft rules,' and principles of conduct come to be accepted by significant numbers of individuals that comprise the occupational culture within a work group - in this case the rank-and-file police subculture. In other words, why do certain attitudes and behaviours become the subcultural norms? However, while considering this question, it is important to stipulate that neither the front-line police subculture nor officers' practices of de-policing are monolithic. In fact, considerable diversity among individual front-line officers, in terms of both degrees of adherence to the dominant subcultural ethos and edicts around de-policing and officer's individualized risk-averse practices, was observed in the present study. ${ }^{22}$ 
Variations in de-policing practices among individual front-line officers working within the rank-and-file police subculture have been thoroughly discussed throughout this dissertation and addressed within the context of the conceptual framework of a depolicing continuum. They are exemplified, for example, in the $30 \%$ of study participants that do not practice any form of de-policing, which is considered in more detail throughout subchapter 6.9. It is noteworthy that included among the rank-and-file officers categorized as non-depolicers in the present study are significant numbers of 'experienced' front-line officers (those with more than five years of front-line experience). The observations of one such veteran Toronto officer are illustrative and representative of a not insignificant number of study participants that report not practicing any form of de-policing,

I don't give a shit who or how many of the officers I work with try to pressure me into doing their fucked-up FIDO approach to this job. I don't think its ethical and its not why I became a cop [redacted] years ago. I do this job to make a difference for those who need us not to cover my own ass. But they try to persuade you and warn those of us that don't subscribe to FIDO. And it is a popular topic in our division from the new recruits that learn it right out of [the police academy] to the officers close to [retirement] Whenever something happens that hits the media they say 'We told you so, if you don't change it's just a matter of time before it's you that's the one in shit' (TOR27).

However, with this diversity in subcultural adherence to, and individual officer's practices of, de-policing acknowledged, I advance that, in terms of the more common circumstances within today's front-line subculture, each officer, through a complex and informal process of socialization and throughout career-stage progressions within the rank-and-file work group, is encouraged to adopt the prevailing subcultural doctrines and the behavioural norms of their immediate peer group (Bacon 2014; Campeau 2015, 2017; Chan 1996, 1997, 2007; Chappell \& Lanza-Kaduce 2010; Charman 2017; Getty et al. 2016; Ingram et al. 2018; Kappeler et al. 2010; Loftus 2009, 2010; Manning 1977, 
1989; Manning \& Van Maanen 1978; Niederhoffer 1967; Paoline 2003, 2004; Paoline \& Terrill 2014; Reuss-Ianni 1993; Rubinstein 1973; Shearing \& Ericson 1991; Skolnick 1966; Van Maanen 1973, 1974, 1975, 1978, 1984). I argue that the de-policing socialization process involves two interrelated dimensions, which, not unexpectedly, are similar to the two interconnected mechanisms found to be associated with an officer's adoption of, or intensification in, risk-averse behavioural practices - (1) frequent interactions among front-line officers within the close-knit work group, often discussing each other's experiences in the field and how best to negotiate strains originating from particularly difficult occurrences and interactions and (2), the accumulation of challenging experiences in the field at the individual officer level, which resonate with common experiences being shared by other officers within the work group and, therefore, can give rise to the consideration of remedies (de-policing practices) being suggested by those within the subculture to mitigate against further problematic occurrences.

This commonality with the rank-and-file work environment has been found to produce "collective coping mechanisms and outcomes" (Terrill et al. 2016: 65, citing Paoline 2003) and Ingram and colleagues (2018: 784) observe, "Officers working in the same group are exposed to similar environmental characteristics, are interdependent on each other, and interact with each other on a regular basis, resulting in shared cultural prescriptions." Similarly, Bacon (2014: 104) finds that their subculture "provides officers with frames of reference, coping strategies, practical knowledge and 'commonsense' understandings about how to view their external environment and how and why policing should and can be done in any situation.” Marier and Moule (2018) observe 
that within the contemporary literature, "Existing evidence suggests that the occupational strains associated with policing contribute to widely shared coping, mechanisms, attitudes, and behaviours among officers." The common experiences of a group of six veteran Toronto officers (that I met, at their request, in the parking lot of a coffee shop near their divisional police station), expressed in the observations of the group's informal spokesperson, inform these understandings,

When I got out on the road as a recruit with my coach officer I heard a lot from him and the other guys about FIDO and NC/NC. He did it and about $90 \%$ of the guys did it, one way or another. I thought most of them were just burnt-out and had learned to despise certain people who mostly also hate the police, like the Blacks, homosexuals, [and] Natives and because of the wear and tear on you of year after year of no-win calls, second-guessing, complaints, lawsuits, and the public, [police] administration, politicians, and media disrespecting and misunderstanding what police work involves. But after some of my own issues on calls in the first year or two on the road in [redacted] and the advice of most of the guys I worked with day in and day out, junior and senior [in front-line tenure], I came to recognize [that] the guys were right. To survive in the job today and not wind up on the front page, suspended, fired, or in jail you need swallow your pride and forget your oath and do your job thinking FIDO in the back of your mind every shift. The few guys we work with that don't think this way are taking a huge risk for themselves and their families and they get no special gratitude from anyone for sticking their necks out.

The 'real world' experiences of these Toronto officers demonstrate several points that feature prominently throughout the police occupational culture literature (as also discussed throughout subchapter 3.4). For example, as articulated by Loftus (2010: 2), "Cultural mores are communicated and reinforced through on-the-job socialisation and arise as officers adapt to the demands of the police vocation...Police culture exerts considerable influence over the way officers think about and interact with their publics, often for the worse.” And, as Harris (2014) advances, front-line police work is clearly something learned through experiences in the field and not something that can be taught effectively (or realistically) in a classroom. Further, through an 'unsystematic' process of accumulating experiences and concurrent subcultural socialization, "Police officers determine which goals of policing are reasonable, which methods work in certain 
situations, and how to produce an operational style, which, in return, will guide them through the job in the long term" (Harris 2014: 286). Van Maanen (1973, 1974, 1975) and Skolnick (1966) referred to these experiences and influences, in describing the socializing of new patrol officers into the rank-and-file police subculture, as the development of an officer's 'working personality. ${ }^{, 23} 24$ In this vein, Kappeler and colleagues (2010: 268, citing Skolnick 1994) find that through the process of occupational socialization, "Police learn how to behave and what to think from their shared experiences with other police officers." This coincides with the observations of Buckley and Petrunik (1995: 111-112), who find,

Career orientations are influenced by the relationship between self and reference groups, groups with which one identifies in terms of values, norms and goals. A person's self emerges as a result of a social process involving interaction within these groups...Through socialization, individuals adopt the standpoint of their cultural group as a frame of reference which influences the way in which they perceive the world, think, form judgements, and control themselves in everyday life...One's attitudes, values and beliefs concerning the role of the police are subject to change during the course of one's career. What one's initial perception of policing was before training can often differ afterwards. Training, in fact, may serve to alter one's perspective in order to bring it into line with that shared by the same group. A similar process likely occurs when one has completed training and begins to work as a rookie police officer. The transition from rookie to veteran involves adapting to the perspectives of established officers.

In this regard, the rank-and-file subcultural socialization process has been found to intensify after training at the police academy, with the recruit officer's deployment in the field, under the guidance of a coach officer, and frequent interactions with other experienced front-line officers (Chan 2003; Department of Justice 2003; Fielding 1988; Ford 2003; Getty et al. 2016; Haberfeld 2013; Sun 2002; Tator \& Henry 2006; Van Maanen 1973, 1974, 1975; Van Maanen \& Schein 1979). 
Bacon (2014: 110, citing Cain 1973; Innes 2003) observes that the 'apprenticeship' of new recruits involves "relaying and transferring the tacit and informal knowledge...of how to get the job done in 'the real world', and the recipe knowledge, working rules, and attitudes that will facilitate this.” In MacAlister's (2004: 177-179) analysis of Canadian rank-and-file police subculture, he observes that field training is "arguably the most powerful force in teaching and reinforcing the norms of the police subculture." Along these lines, field trainers have been known to counsel, and model behaviours consistent with, the approach - 'Forget everything they told you at the academy. They do police work one way at the academy and we do it another way on the street and the street is where you will learn to be a cop' (Cordner \& Sheehan 1999; Van Maanen 1973). The following study participants' observations in this regard, and specifically in relation to de-policing, are instructive and representative of the expressed sentiments of many rank-and-file officers in the sample population, FIDO was talked about at the police college but it was my coach and most of the other officers on our shift that impressed the importance of covering your ass on me over my first year on the road. And so now after a few years making up my own mind and thinking there's got to be another way, I do FIDO often and can admit they were right (NIA185).

Fuck it, drive on. Taught when I was a recruit (RID28).

[De-policing] was presented to me by my coach, first week on the job (TOR27).

One of the first things I was told when I got out on the road with my coach [officer] was, [']You need to forget what they taught you at [the Ontario Police College]. I am going to teach you how to do your job to protect yourself, your family, and the rest of us. That means you will do like the rest of us. We do what we are obligated to do but we don't do more, we don't rock the boat and we don't take risks that can land us in a jackpot. We protect our paycheque and our career and each other [']. After I got out on my own and got some experience I saw the wisdom in what he taught me (HAM180).

I know all of us but the rookies are doing FIDO and they will after a few years of realizing what is going on now (STR05).

For self-preservation. I have been taught by veteran officers to avoid visible minorities and those with mental health problems (CAL75). 
I avoid Natives, they feel targeted by police even though they are committing a crime. I was trained to avoid such risks (CAL247).

Trained early in career 'take time getting to call' (CAL82).

It has been passed down from senior officers that it's not worth proactive policing anymore (BUF146).

I was trained this way and I see most of the officers on my platoon practicing risk-averse policing in their work, particularly with visible minorities. It's often called FIDO or CYA (OTT114).

Further, and also specifically in relation to de-policing, Harris (2016: 221, citing Bayley \& Bittner 1984; Brough et al. 2016; Chan 2007; Van Maanen 1974) finds that the "proactive behaviours...[of] inexperienced officers" are often admonished by veteran officers, who instead adopt a 'lay low and don't make waves strategy.' This notion has only intensified in recent years with heightened scrutiny of the police...It appears that officers are gradually socialized away from engaging in acts that may bring scrutiny and/or disciplinary acts against them...As such, officers become less proactive as they gain experience. ${ }^{25}$

A Buffalo officer provides a representative 'real world' example in reporting,

[I practice de-policing] daily. Senior guys will make sure younger guys understand the city and the public really doesn't care for your wellbeing, so why risk losing your job (BUF145)?

In relation to Harris' (2016) observation around intensification in citizen's scrutiny of police officers and officers' intensification of de-policing practices, Bacon (2014: 113) is instructive with his finding that, "Police culture is constantly evolving as officers adapt to accommodate new structures, experiences, and ideologies.” Harris' (2016) observations also coincide with those of Chan and colleagues (2003: 3), who find that "the conventional wisdom [related to police socialization] is that as recruits become integrated into the operational ('street cop') culture, they adopt [certain stereotypical attitudes] as well as deviant practices." 
In reflecting on Bacon's (2014) observations in the preceding paragraph, it is interesting to consider that if there exists a changing structural environment for frontline police work and if subcultural socialization is the learning of the policing habitus by rank-and-file officers, "What happens when the field of policing itself is changing" (Chan 2004: 340)? Chan and her colleagues (2003: 310) find that "social, political, and organizational conditions of policing" are changing across Western democracies and, in applying Bourdieu's framework, Chan (2004: 340) suggests that such "shifts in the field would lead to adjustments of the habitus: changes in the field create a new 'necessity' which may require the creation of new strategies for coping." In following Chan's (1996, 1997, 2004, 2007b; Chan et al. 2003) reasoning, I suggest that today's technosocial and socio-political external policing circumstances can be viewed as significant shifts in the structural environment for front-line policing ('the field') and that the depolicing results demonstrated in the present study represent a new strategy for rank-andfile officers in coping with today's 'necessity' (policing realities). Based on representations from study participants, it seems clear that today's de-policing strategy is conveyed and encouraged through the ongoing socialization process in the front-line subculture - or, as Chan $(1996,1997)$ terms it, the 'acculturation process.' The reflections of a veteran Ottawa patrol supervisor, who spoke with me in detail after a shift briefing, provide some 'real world' context to these theoretical observations,

The way this has worked here for the last [redacted] years I've been on the job [,] for most of the guys and gals that have joined, is they join with all the right ideals. They want to protect the weak and contribute to a better society. Sure, they hear about FIDO when they get on platoon with their coach officer and they see it with the other officers, but they think it won't happen to them. But after a few times being jammed up with bogus accusations and getting dragged through PSS or SIU investigations, called a racist daily regardless of what colour they are, having none of the [command] officers and no one stick up for police in media or the silent majority in the public, they get worn down and they see the light. Things have changed so much since I joined. Everyone is watching, recording, and hoping to catch an officer doing something 
people will think is wrong, even it is actually right. 'I gotcha officer! You are now on YouTube!' And most of the public that speaks up about the police hates the police. So, you learn from other officers you work with every day that have been through it [and] from getting jammed up yourself, that if you want to keep your job and have less stress, what you have to do is forget the ideals you joined with and do your job in the least confrontational way you can. You need to put the blinders on sometimes. You need to avoid people that are predisposed to complain about you. You need to avoid types of calls...You certainly don't want to stop a Black guy walking down the street no matter what he's doing. We talk about it as FIDO but I know there are other names for it. Toronto calls it NC/NC, no contact then there's no complaint. I'm sure Americans have other names for it...I estimate $80 \%$ of the 50 officers I work with every day are doing this de-policing because this is [the] new reality where we do our work out in the community. It's sad, but at some point you need to think about self-preservation...As a [rank redacted] I should be against FIDO but I can't honestly tell a young officer not to do what is [now] smart police work or not listen to the voices of experience on the platoon that will help keep you out of trouble.

In his research, Sklansky (2007) discusses similar subcultural experiences of new recruits when they first encounter the real world of police work, finding that idealist notions are quickly lost and that police officers learn to make pragmatic decisions and cover themselves and their colleagues against any criticism in the socialization process of learning how things are done in the working environment. Van Maanen (1973: 415), in a similar vein, finds that "the adjustment of a newcomer in police departments is one which follows the line of least resistance. By becoming similar in sentiment and behaviour to his peers," the new officer avoids ostracism and "censure by most importantly his brother officers."

In considering what occurs, in terms of subcultural socialization and the influence of the rank-and-file subculture, after the recruit's field training experiences and the front-line officer's advancement into the 'experienced' category, the conversation I had with two veteran Yonkers officers about de-policing is illuminating and representative of similar discussions between myself and front-line officers at many of the other study data sites throughout 2016 . 
Officer \#1: We thought we were going to be different. We would buck the trend and keep our youthful enthusiasm in our work. We heard it every day. Don't do this, don't do that, do like we do. But we have changed, no doubt about it, not all the way, but some.

Officer \#2: You call it de-policing right? There was no name for it[,] the guys we have worked with for [redacted] years have been doing it as long as I can remember and they tried every day to get us to do our work like they do. Ignoring things. Don't take unnecessary risks. Not stopping African Americans and Hispanics. That's probably not what you expected right? It's the opposite of racial profiling. Do as little proactive work as possible. Avoid certain blocks. Just take your radio calls. [Keep a] low profile.

Officer \#1: Trying to get us to change how we do our work has been going on for years and with just about every officer we have worked with, including the Sergeants. It's a lot of pressure in our group to conform to the majority. We know other officers who finally gave in to the pressure or got into serious trouble and decided they are right [about de-policing to reduce risk] and they threw in the towel.

Informing this 'real world' data, Bacon (2014: 109) finds,

The social networks officers find themselves in play a significant part in their acculturation, cultural passage and policing style. Culture is learnt, expressed and sustained in the context of social interaction, the everyday conversations between officers about the job and how to do it, the imparted words of wisdom, the narratives recounting past events and the glorified folklore.

In this vein, Goldsmith (2010: 916) advances that front-line police officers have a dual status, as both societal 'moral agents' as well as societies' front-line 'dirty workers' and, in this regard, Boivin and colleagues (2018, citing Hughes 1962; Kreiner, Ashforth \& Sluss 2006) observe, "Group identities have been found to be stronger for individuals whose occupation is a form of 'dirty work'.” In his seminal study, Skolnick (1966) found that police occupational culture develops from common strains associated with being a police officer engaged in performing societies' 'dirty work' - danger, the exercise of authority, conflict, and pressures within the agency for results and efficiencies. However, as demonstrated in the present study's results, today there are new strains, accompanying the contemporary policing landscape, in addition to the 'traditional' challenges and these are impacting on both the front-line officer (at the 
individual level) and on the rank-and-file police subculture (at the collective work group level). For example, Marier and Moule (2018) observe, "Recent protests against law enforcement have spurred claims by practitioners and editorialists that public antipathy toward the police may influence police occupational norms. A number of classic police ethnographies also suggest a link between perceived public antipathy and police culture, but limited empirical research has examined this claim."

As described throughout this dissertation, these relatively-new challenges in frontline police work are captured within the conceptual frameworks of the techno-social and socio-political transformations that characterize the external realities of today's policing landscape. Unlike Chan's (1997: 67) critique of police culture's “apparent insularity from the social, political, legal and organizations contexts of policing" in the 1990s, the present study finds that, in fact, today's widespread de-policing is both an individual and a subcultural response to the contemporary social, political, cultural, and technological contexts of front-line police work in this day and age. This is consistent with Cockcroft's (2007) counsel about the need to take into account socio-political factors in understanding contemporary police culture and Marier and Moule's (2018) observations around 'the relevance of police-public conflict for officers' cultural orientations." In relation to the latter, it is interesting to reflect on Reuss-Ianni's (1983, as cited in Marier \& Moule 2018: 4) seminal study of the American 'street cop' subculture in an earlier era, in which she observes that "public criticism and antipathy sever the policeman's bonds to the civilian world, and he retreats into fraternity, where he finds both physical and psychic security from a hostile public." In a more recent contribution, McLaughlin (2007: 40) finds similarly that "patrol officers take their cue 
not from their legal obligations or [policing executives] but from their immediate peer group. Public hostility and police solidarity are mutually reinforcing with officers 'closing rank' against the public...”

In addition to the present study, other recent research has demonstrated that police subculture affects officers' behaviours and their interactions with citizen (e.g., see Brough et al. 2016; Rose \& Unnithan 2015) and policing scholars have found that influence from the subculture is one of the most powerful determinants of an officer's conduct in the field (e.g., see Maskaly \& Donner 2015; Terrill et al. 2016). In this regard, Sklansky (2007: 20-22) advances that understanding today's rank-and-file police subculture is crucial given "its central importance in shaping the behaviour of the police" and he argues that "the police subculture schema retains explanatory power...Understanding the police still requires sensitivity to the powerful and distinctive ways in which the day-to-day experiences of law enforcement officers influence their behaviour." Similarly, Bacon (2014: 116) cautions, "If we do not understand the nature and influence of police culture, we cannot hope to understand police practices or the realities of policing in society."

\subsection{De-policing and Today's Police-Community Relations}

Ewald (2002: 278), in his analysis of the 'institionalization of precaution', as discussed in the preceding subchapter, titled one of his chapter's subsections 'A New Social Contract'. Of course, the fundamental principle of the 'social contract' (in the tradition of Hobbes, Rousseau, and Locke) is the designation, or essentially contracting out, of certain responsibilities for social control and the maintenance of order, by the citizenry in democratic societies to the state institution responsible for policing - the 
police. This assignment of power from the public to the police requires that the rankand-file police officers deployed within the community must be answerable to that community (Barton \& Johns 2014; Brown 2014; Chan 1999; Dunham \& Alpert 2010a, 2010b: Flanagan 2007; Patten et al. 1999; Reiman 1985; Shearing 2000; Walker 2005) or, as characterized by Walsh and Conway (2011: 71), to "the people on whose behalf they exercise their extraordinary powers and discharge their sensitive role." Smith (2005: 500) is instructive with his observation, "At a basic level, every police officer understands that the role of the police officer is as a public servant, accountable to the citizenry." In other words, officers are responsible to serve and protect the citizenry. Such ideas, and ideals, are often discussed in the context of the principles thought to have originated with Sir Robert Peel upon the formation of the Metropolitan Police in $1829 .{ }^{26}$ Five of the nine principles specifically relate to police responsibilities to the public, including the following, which are particularly relevant to this discussion:

To recognize always that the power of the police to fulfil their functions and duties is dependent on public approval of their existence, actions and behaviour, and on their ability to secure and maintain public respect.

To maintain at all times a relationship with the public that gives reality to the historic tradition that the police are the public and that the public are the police; the police being only members of the public who are paid to give full-time attention to duties which are incumbent on every citizen in the interests of community welfare and existence (Mayhall 1985: 425426). ${ }^{27}$

Throughout this subchapter, I discuss five interrelated considerations that are relevant to understanding today's police-community relations in the context of the depolicing phenomenon, as it has been identified within the present study: (1) the community-based policing model; (2) the expectation for proactive police work within the CBP model; (3) over-policing; (4) de-policing as a beneficial or detrimental 
outcome for communities, depending on the circumstances; and (5) discretion and depolicing.

\section{The Community-Based Policing (CBP) Model}

As discussed throughout Chapter One, CBP has become, over the past half century, adopted as the standard policing model for virtually all police agencies throughout Western democracies - both as the philosophical approach to how society should be most effectively policed and in terms of expectations for 'on the ground' front-line police practices. ${ }^{28}$ The central assumption of the CBP model is that the repudiation of the reactive emphasis of the professional model, in favour of a closer partnership between community inhabitants and their police officers, will improve the effectiveness of policing and, therefore, increase public safety (Bordua \& Reiss 1966; Fielding 2002; Manning 1988, 1993; Mastrofski et al. 1995; Reiss 1992; Reiss \& Bordua 1967; Skogan 2003, 2006b; Skolnick \& Bayley 1988). In this vein, Hawdon (2008: 192, citing Cordner 1997; Kelling \& Moore 1998; Stevens 2001, 2002) observes, “Community policing emphasizes crime prevention based on face-to-face interaction among officers and residents" and, within this model, "the community was to be a coproducer of public safety, and the priorities of the police were to be defined in consultation with the community" (Weisburd \& Braga 2019: 13).The shift to the CBP model was supposed to represent a significant restructuring of the role of the police and improve the nature of the public-police relationship, from a primarily reactive model to "more responsive, decentralized, proactive" policing of communities - in partnership with, and with the consent of, those communities (Correia 2000: 219, citing Skolnick \& Bayley 1988). ${ }^{29}$ While I do not know the reason for Weisburd and Braga (2019) 
employing the past tense in their recent analysis of $\mathrm{CBP}$, my rationale for doing so, in the preceding sentence, is that I suggest de-policing and CBP are clearly and fundamentally incompatible and, based on my observations in the field over the past nine years (as both a practitioner and an academic researcher), I advance that CBP today is more a fantastic notion espoused by police leadership and elected officials in their public sphere discourse, rather than the policing strategy applied in the 'real world' of front-line police work - in terms of rank-and-file deployments and defining expectations for the type of police work to be performed by officers in the field.

In this vein, but addressing the other constituent in the public-police relationship the citizen, throughout the literature it is well established that the effectiveness of the exercise of police authority throughout society is dependent on the public being engaged with the policing of their communities through ongoing support of, and voluntary cooperation with, the police. ${ }^{30} 31$ However, the participation of citizens, as contemplated in both the 'social contract' and the policing principles attributed to Peel, is contingent on the institution of policing, and the exercise of its awesome powers by officers in the field, being perceived by the citizens as trustworthy and legitimate. ${ }^{32}$ In this regard, Jackson and Sunshine (2007: 218) find, "Public confidence in policing has become shorthand for trust, legitimacy and consent, and there is considerable evidence that public confidence has been falling steadily over the past few decades." Trust, in the policing context, relates to the belief that an individual occupying the role of police officer will perform that role in a manner consistent with the socially-defined normative expectations associated with that role (Hawdon 2008) and, along these lines, citizens expect officers to conduct themselves in a professional manner, which includes 
performing their duties "within a set of fair, public, and accountable guidelines" (Seron et al. 2004: 666, see also Weitzer \& Tuch 2004; Deakin 1988). Reiner (2010: 34) provides an important caveat that should be considered in any such discussions, stressing that "there are inherent limits to police legitimation in any society...there is in most police actions someone who is being police against...In this sense the police can never command universal love."

That being stipulated, it is widely acknowledged that police officers more easily maintain order in society when viewed as legitimate by citizens (Callanan \& Rosenberger 2011; Hough \& Roberts 2004; McCluskey, Worden \& McLean 2014; Tyler \& Huo 2002). Therefore, citizens' subjective perceptions and assessments of police conduct are crucial considerations in terms of the effectiveness of the CBP model and in approaching ideas about trust, accountability, and legitimacy (Albrecht \& Green 1977; Bradford, Jackson \& Stanko 2009; Flanagan \& Vaughn 1995; Gallagher et al. 2001; Lee \& McGovern 2013; McCluskey et al. 2014; Tyler 2006b; Tyler \& Huo 2002; Tyler et al. 2007; Zamble \& Annesley 1987). In this regard, citizens' confidence in, and attitudes toward, front-line police officers is influenced, positively or negatively, by a range of individual and area-level characteristics; experiences with, and sources of information about, the police; and the style of policing being practiced in the relevant community. ${ }^{33}$ In relation to the last consideration, CBP was introduced, in large part, to address widespread issues with public confidence in the police.

In accordance with the CBP model, the two constituents in the public-police relationship are expected to work together to identify and address issues related to safety, crime, disagreements, and disorder. However, when a significant proportion of 
citizens lose faith in police, support can evaporate, and officers deployed within the community can be viewed as adverse to the interests of the community and as an illegitimate imposition of authority. As we have all undoubtedly observed, controversial policing interactions can have deleterious effects on public confidence in the police (Flanagan \& Vaughn 1995; Gau \& Brunson 2010; Jefferis et al. 1997; Maxson et al. 2003; Nix et al. 2018; Tyler, Fagan \& Geller 2014) and, therefore, how the community views the police is a major concern within policing, governance, and throughout the public sphere (Jefferis et al. 2011; Luna 2000).

As discussed at various junctures throughout this dissertation (particularly in subchapter 3.3), there have been recent changes across today's public sphere conditions, which have enabled substantially more widespread participation in, and critique of, rank-and-file officers' mis/conduct in the community. These techno-social and sociopolitical transformations across today's policing landscape involve more than just 'policing's new visibility' - now implicating what I argue is a significant cultural shift vis-à-vis communication technologies, the development of a 'participatory culture', 'citizen journalism', and new social movements focused on grievances with policing (i.e. Black Lives Matter). ${ }^{34}$ Tobias and Joseph (2018, citing Este, Sato \& McKenna 2017; Lindsey 2015; Love 2016; Yancy 2016) find, "Movements such as Black Lives Matter and widespread challenges to police brutality and racial profiling have reinvigorated long-standing historical positions of activism while constantly adding to the conversations and analysis." In this vein, Schneider (2015: 228) observes that "a key contemporary difference concerns the manner in which social media platforms allow users to mobilize en masse much more quickly.” Further, Doyle (2006a, 2007, 2011) 
finds that in addition to today's access to so much knowledge about policing, an unprecedented level of higher education across society has resulted in a public that thinks more critically and is skeptical of major social institutions - including, of course, the police.

In addressing endemic public discontent with policing across the USA, former president Obama observed that there are "too many communities around the country, [in which] a gulf of mistrust exists between local residents and law enforcement" (Zezima 2014). Thacher (2001: 776) finds that under such conditions, police "risk becoming irrelevant to the community." Circumstances of pervasive distrust and destabilized lawlessness, in which citizens have withdrawn from the traditional public-police relationship can be the outcome (Brants 2005; Luna 2000; Moore \& Braga 2003). Further, and as this dissertation demonstrates, police can also lose confidence in the other constituent in the public-police relationship and, therefore, front-line officers can intentionally and proactively disengage from discretionary interactions with community members - thereby unilaterally introducing modifications to traditional understandings around the 'social contract' and the fundamental tenets of the CBP model.

\section{The Expectation for Proactive Police Work within the CBP Model}

Impacting significantly on the work of rank-and-file police officers, CBP broadened the reactive crime-fighting of the professional model to include: order maintenance; conflict resolution through police-community problem-solving; social services functions; as well as a litany of other activities, not involving law and order, to be performed within, and in conjunction with, the community (Brooks 2010; Dixon 1997; Kelling \& Moore 1988; Pollock 2010; Reiner 2010; Wilson \& Kelling 1982). In 
other words, in addition to dispatched calls for service, in which "police are called upon routinely to perform a bewildering miscellany of tasks" (Reiner 2010: 7, citing Bittner 1970, 1974; Brodeur 2007), within the CBP model, front-line officers are also expected to involve themselves in a variety of proactive and/or discretionary initiatives that have come be be seen as 'police work' - including situations involving "something-thatought-not-to-be-happening-and-about-which-someone-had-better-do-something-now" (Bittner 1974: 30)! In relation to the objectives of the CBP model from the police perspective, Bayley (1988: 228) observes, "If police cannot reduce crime and apprehend more offenders, they can at least decrease fear of crime, make the public feel less powerless, less distrust between minority groups and the police, mediate quarrels, overcome the isolation of marginal groups, organize social services, and generally assist in developing 'community'."

In their seminal work, Wilson and Kelling (1982) discussed the proactive policing expectations of the community and the tactics employed, in response, by rank-and-file Chicago officers (in that era),

Sometimes what [the front-line officer] did could be described as 'enforcing the law,' but just as often it involved taking informal or extralegal steps to help protect what the neighbourhood had decided was the appropriate level of public order...[For example,] the people expect the police to 'do something' about [youth gangs that terrorize residents]. But do what? Only a tiny fraction of gang-related crimes can be solved by an arrest. What the police in fact do is to chase known gang members out the project. In the words of one officer, 'We kick ass.' Project residents both know and approve of this.

In the context of the present study, I suggest, based on data from study participants, that this is precisely the kind of front-line police work that many of today's rank-and-file officers have concluded now carries far too many risks for professional consequences 
and which, therefore, they no longer practice. In a 2016 edition of Newsweek magazine, featuring analysis of the new landscape for policing in the USA and the many issues in today's public-police relationship, Kutner (2016: 4) observes, "Not only are police increasingly having to protect people who despise them, but [the expectation is that with CBP] their jobs have expanded too." The results of the present study suggest that rather than going out of their way to protect the community ('the people who despite them') and, thereby, assuming unnecessary risk, through performing more than the basics of mandatory reactionary policing duties (i.e. proactive police work or discretionary interactions with people in the community), many of today's rank-and-file officers are simply avoiding these risks to them in this antagonistic and challenging front-line policing landscape by practicing de-policing.

\section{Over-Policing}

In the same way that communities can be de-policed, as documented throughout this dissertation, with officers intentionally avoiding certain locations, persons, and types of engagements or interactions, communities can also be over-policed, with certain neighbourhoods receiving more police attention than others. The concept of over-policing is often discussed, particularly in the American context, in association to police interactions within 'minority' racial/ethnic communities and these specific circumstances, related not only to racialization and discriminatory policing within the USA, but across Western democracies, are elaborated throughout subchapter 6.8. However, for present purposes in discussing the phenomena of over-policing, the concentration of front-line police resources to certain geographic areas is a common strategy in police deployments. Routine decisions about patrol zones and officer 
deployment are often related to volume of calls for police services and reported crime rates (Sherman, Gartin \& Buerger 1989; Withrow 2011). In addition to such decisionmaking around reactive policing, there can also be proactive strategies that involve augmenting existing police assets in particular 'high risk' neighbourhoods with more officers - often called 'hot spots policing' (Braga, Papachristos \& Hureau 2014; Sherman 1997; Sherman \& Weisburd 1995; Skogan, Steiner, Dubois, Gudell \& Fagan 2002; Taylor 1998; Weisburd \& Eck 2004; Weisburd, Eck, Hinkle \& Telep 2008). ${ }^{35}$ While these proactive initiatives are often promoted as being effective in reducing crime and disorder in affected areas (e.g. see Weisburd et al. 2008; Braga et al. 2014) there can also be detrimental effects.

Higher levels of police attention for certain neighbourhoods over others can result in inhabitants' feeling 'singled out' or 'picked on' by police (over-policed). In situations of over-policing, the higher concentration of police resources within the neighbourhood results in more opportunities for officers to scrutinize individuals and therefore to proactively observe and take discretionary actions in relation to minor infractions, which would go undetected in neighbourhoods with less police presence. ${ }^{36}$ And 'hot spots policing' initiatives can often involve not just a heightened presence of front-line officers, but also what some community members can interpret as aggressive tactics, antagonistic officer demeanour, and disrespectful methods of communicating with the residents of that particular neighbourhood. Such policing practices are often perceived as a discriminatory, confrontational, and unnecessary impositions of authority by some throughout the community and, therefore, can result in a deterioration in the relationship between police and the citizens they are sworn to serve and protect (Owens 2019). As 
discussed, and elaborated throughout subchapter 6.8, considerations around these issues often implicates issues of racialization, vis-à-vis the policing of certain minority communities.

As Rosenbaum (2019: 330-332, citing Kochel 2011; Kochel \& Weisburd 2017; Sherman 1997; Worden, Bynum \& Frank 1994) finds, "Aggressive enforcement in specific areas runs the risk of weakening police-community relations... Policing scholars have acknowledged the possibility that hot spots policing could damage policecommunity relations and undermine police legitimacy." He refers to such considerations with 'hot spots policing' as "collateral damage to the community" (Rosenbaum 2019: 334). These circumstances can be particularly problematic within 'minority' communities (Chan 1997; Cooper 2002; Herbert 2006; Skogan \& Harnett 1997; Weisburd 2016; Weisburd, Hinkle, Famega \& Ready 2011). In this regard, Mastrofski (2012: 598, citing Braga \& Weisburd 2010) observes, "By concentrating police resources in the small areas (addresses and blocks) where problems are most frequently reported by the public, this could converge police even more intensively on minority and low-income areas." In the same vein, the National Academies of Sciences, Engineering, and Medicine (2018: 11-12) find,

Proactive strategies often facilitate increased officer contact with residents and, particularly in high-crime areas, involve contacts that are often enforcement-oriented and uninvited and may allow greater officer discretion compared to standard policing models. These elements align with broad categories of possible risk factors for racially-biased behaviour by police officers.

\section{De-policing as both a Beneficial and a Detrimental Outcome for Communities}

Officers not performing proactive police work, when they have the discretionary time do so (i.e. when they are not engaged with mandatory reactive police duties), can 
be considered an undesirable or a desirable outcome - depending on the circumstances and who is situated in the role of the arbiter. I suspect that policing's leadership, elected officials, and many throughout the public sphere would consider the de-policing practices of large numbers of rank-and-file officers, as identified within the present study, as inconsistent with what is expected of police throughout Western democracies and within the prevailing CBP model, whereas those who consider their neighbourhood the victim of over-zealous 'hot spots' policing would be pleased with the relief afforded by de-policing, from circumstances in which the police are perceived as an outside and oppressive occupying force. I tend to see both sides of this issue.

On the one hand, I advance that if an officer's intervention is initiated for an illegitimate purpose (i.e. to circumvent legal requirements for probable cause or reasonable grounds or because of bias in relation to certain individuals perceived to be, for example, 'visible minorities') then curtailing such a practice, through de-policing, would be a good outcome for society. On the other hand, if an officer's proactive police work is grounded in a legitimate rationale (as is often interrogated during hearings around the admissibility of evidence in criminal trials), is in alignment with the ideals of procedural justice, and is, therefore, a good faith attempt by the officer to make the community s/he is sworn to serve and protect safer, then I suggest that officers not pursuing such discretionary initiatives, by practicing de-policing, would be a bad outcome for society. ${ }^{37}$

Similarly, Shjarback and colleagues (2017, citing Cooper 2002) have outlined both the potential 'good' and 'bad' outcomes associated with de-policing. They argue, "[structurally- disadvantaged minority] communities that tend to suffer from higher 
rates of crime and victimization to begin with," suffer even more because of the absence of police officers. However, they also advance that "there may be benefits to depolicing...particularly in minority communities, [through] alleviat[ing] the negative effects (e.g. strained community relations) of aggressive order maintenance/broken windows policing tactics (e.g. 'stop, question, and frisk') and limit[ing] highly discretionary stops and arrests, which are often viewed as controversial" (Shjarback et al. 2017: 43, citing Epp, Maynard-Moody \& Haider-Markel 2014; Morgan \& Pally 2016; White \& Fradella 2016). This dichotomy is captured by Owens (2019: 78), who observes that in the wake of high-profile police killings of Black persons in the USA, "Protesters argued that Black Lives Matter and, by implication, that those lives specifically mattered more than any additional reduction in crime generated by the aggressive broken-windows-style policing tactics perceived to have contributed to the deaths of so many people." The risk-averse behaviour reported by a Toronto officer illustrates this point,

With Black Lives Matter and anti-police hatred in Black communities we simply avoid them (no proactive interaction, enforcement, or community-based policing) (TOR300)

The following two subsections elaborate on the 'good' and the 'bad' of de-policing, in the context of community relations and the study's empirical findings.

\section{The 'good' of de-policing}

As Dewan (2017) advanced in 'Deconstructing the Ferguson effect' for The New York Times, "The idea that the police have retreated under siege will not go away. But even if it's true, is it necessarily bad?" I argue and suspect I would have near unanimous agreement (both scholarly and popular), that in circumstances of over-policing, in which proactive police work is being abused by officers, de-policing would represent a 
positive outcome for not just the affected community, but also society as a whole particular in the context of trust, legitimacy, accountability, and consent, as discussed earlier in this subsection. Along these lines, Deuchar and his colleagues (2019) note, in their recent review of the literature, that research has "highlighted that there may be benefits to de-policing...in terms of alleviating the negative effects of aggressive order maintenance and 'broken windows' police tactics." For example, Shjarback and colleagues (2017: 50) advance,

Could de-policing be a good thing? [If officers were] to retreat from aggressive tactics (e.g., frequent pedestrian stops) and high discretion arrests/citations for 'quality of life' offenses, then such a change might be beneficial from a public perception and perceived legitimacy standpoint...A substantial body of research finds that unfavourable citizen perceptions of police are, in part, the result of contentious personal and vicarious experiences with officers (Brunson 2007; Decker 1981; Webb \& Marshall 1995; Weitzer \& Tuch 2002). Often, those who view the police negatively cite harassment and unfair targeting/profiling in addition to officer demeanour characterized as discourteous and verbally abusive (Brunson 2007; Gau \& Brunson 2010; Weitzer 2000). Thus, reductions in these types of aggressive strategies might limit the opportunities for antagonistic police-citizen interactions...In this way, de-policing may be a necessary outcome of a true democratic policing function.

As observed throughout much of the literature, good outcomes of de-policing are often related to positive outcomes in the public-police relationship for racialized and/or marginalized populations (e.g., see Cooper 2002; Deuchar et al. 2019; Neyroud 2019; Owusu-Bempah \& Morgan 2018; President's Task Force on $21^{\text {st }}$ Century Policing 2015a; Rosenbaum 2019; Shjarback et al. 2017; Weisburd \& Majmundar 2017). For example, Melina Abdullah, a leader of the Los Angeles Black Lives Matter movement and chair of Pan-African studies at California State University at Los Angeles, advances, "If officers think twice about approaching people, some situations where police use of force might be avoided...If police are more cautious about making arrests 
that might be controversial, making arrests that might elicit protests, then that is a victory...We want [officers] to begin to check themselves" (Queally, Mather \& Chang 2017). Along the same lines, in their report on the effects of proactive policing on crime and communities across the USA, the National Academies of Sciences, Engineering, and Medicine (2018) included the observations of Tso (2016) and Childress (2016), which are reproduced here. Childress (2016) finds, "Practices, [such as broken windows policing, has led to aggressive over-policing of non-White communities and] can strain criminal justice systems, burden impoverished people with fines for minor offenses, and fracture the relationship between police and minorities." Along the same lines, Tso (2016) suggests,

The epidemic of police brutality, primarily affecting Black males, can be linked to the history of a technique called hot spot policing... a technique that stations many cops in areas with higher crime rates; these areas are overlapped with areas inhabited by lower-class minorities. Police initially utilized this technique to prevent crimes from happening in hot spots, but the specific measures that would be taken to prevent crime were often left unclear; there were almost no boundaries to these officers' powers as authority figures who could stop at nothing in their crime-fighting efforts, which ironically led to many officers committing brutal crimes themselves.

The contribution of a Buffalo officer in the present study is instructive on these points,

Some of the officers I have worked with over the years do not act on probable cause they act on racism. They think jacking up Black kids helps deter crime and keeps it confined to the Hood. But what it does is it makes the people in the hood hate the police even more because they know the police are not doing their job lawfully. If the police are not doing their job lawfully who are we to preach about crime? (BUF147)

\section{The 'bad' of de-policing}

I am certain that rank-and-file officers addressing only dispatched calls for their services and not practicing proactive police work, as a fundamental component of their duties and their use of discretionary time during the shift, is not what is contemplated in 
the policies of any Western police agency, along with being fundamentally antithetical to the CBP philosophy espoused, I am equally certain, within the mission statements of virtually all of these agencies. In this vein, Nix and Wolfe (2016: 14) argue,

Officers need to be held accountable for wrongdoing, but...evidence suggests that a sizeable portion of police officers are feeling the ill effects of intense public scrutiny. These are important observations not only for police agencies but the communities they serve. Ultimately, officers [de-policing] are less effective than they should be. This has direct implications for the safety of citizens and the wellbeing of communities.

This observation is consistent with Muir's (1977: 66) findings, over 40 years ago, that the community ultimately suffers when "policemen take a walk when the situation [gets] hot." He argued that the absence of effective formal social control, through engaged front-line officers working assiduously in the community, facilitates an environment in which certain "persons stood to profit from the policeman's absence the strong-arm, the bully, the vicious" (Muir 1977: 66). In this regard, some research has found that proactive policing strategies can be effective in reducing crime (e.g., see Braga et al. 2014; Braga \& Weisburd 2012; Nix et al. 2018; Weisburd et al. 2008). Along these lines, it seems likely that the absence of a routine police presence, much less the wholesale abdication of discretionary interactions and/or proactive initiatives in the neighbourhood, would not go unnoticed by inhabitants and would almost certainly, in my view, result in increases in disorderly behaviours and criminal occurrences.

In saying this, I feel somewhat like former FBI Director Comey must have when he advanced his initial thoughts on de-policing, as, despite considerable discussion over the past four years around a so-called 'Ferguson effect' and some conflicting initial analysis of serious violent crime rates in major American cities, there is no empirical 
foundation, as yet, to justify my belief in this regard. However, I suggest that the

following contribution of a Toronto officer, albeit anecdotal, speaks to this point,

I think FIDO is great. I live in a nice neighbourhood far from the shitholes I get called to over and over again when I am on shift. When I'm not called to go there, I stay far away and meet with people who actually like and respect the police. You don't want me there trying to protect you from the drug dealers and gang bangers that are shooting up the city these days? No problem. It makes my life easier but it's turning 'the hood' into a warzone with the nice people afraid to come out because there's no cops around anymore and crime is going through the roof (TOR294).

Many other study participants shared similar observations about what they believe will

be the inevitable rise in disorder and criminal occurrences in the community as a result

of officers' de-policing practices. The following is representative,

I have seen it in the last 5 years, I don't know if the [police] executive and politicians will let the statisticians show you what is happening to the public but all of us on the street know that because we are now hands off to protect ourselves, not the public, criminals are much more active, not as afraid of the police, and people getting hurt is up significantly (GAT59).

I was also presented with observations and sentiments similar to the following,

which were reported by numerous front-line officers at all of the study's 23 data sites,

If [the public and police management] wants to handcuff us in how we do our work on the streets, no problem. We will just continue to do anything in our job that is a core requirement. We can't get in shit for not doing something that is not measurable or monitorable as a core requirement of our job. That's what they are getting now from a lot of us, let's just see how they like it when some parts of the city become uninhabitable for everyone but the criminals and the nice but poor people that can't get out (HAL199).

It is sad to say but we are all doing FIDO now. It's bad for the public but we have no choice because of media and vocal minority (COR32).

Generally, for most of us, proactive work is reduced. But that is the price the public pays (ROC40).

Proactivity has decreased, streets with a lot of anti-police sentiment I patrol less (ROC173).

We don't get involved in career jeopardy situations anymore. Avoid, delay, don't do your job. (HAM50).

Today this job is about self-preservation not protecting the public (OTT68).

Policing now is all about C.Y.A.! (OTT87). 
A lot of stuff we use to do is just not worth it anymore. Why risk complaints to do a proper job of policing? Society is politically correct and soft, I hope they understand when criminals victimize them (STR24).

I no longer will look into things my senses tell me are suspicious. That's no longer in our job description. I hope the 'public' knows what they are getting into with being anti-police, I wonder if they know who they are being pro- in favour of? (TOR28).

This is the new reality in police practice. It's sad it has come to this but at the end of the day we need to protect ourselves and our families (TOR30).

There's and easy road to this job and a hard road. Many of us have figured out it's not worth the hard road even though that is what good police work involves (TOR76).

Dispersing drug dealers [is] becoming 'civil rights violations' (BUF34).

Doyle (2003) argued that the police, in the late 1990s, had the power to shape how they interacted with their new media environments. In this vein, but in the context of the contemporary de-policing phenomenon, I argue that front-line officers today have the power to shape how they interact with the community, and they are exercising this in significant numbers, as is corroborated by the results of the present study. In this regard, there does not exist an effective mechanism through which the citizens can make meaningful demands on the police regarding officers' alleged de-policing practices in the field. I emphasize the word 'alleged' here to highlight the importance of front-line officers' inherent discretion in how they perform their work. ${ }^{38}$ Consider how difficult it would be for a citizen to definitively establish that an officer was intentionally avoiding aspects of their duties that are, by their very nature, discretionary. Muir (1977: 275) is instructive on this point with his deliberations,

What conditions permit persons who have power to refuse to use it? The opportunities to avoid exercising power are available whenever the power holder is not subject to reprisal for self-limitation. Whenever inaction is likely to be condemned less severely than the effort to exercise power, avoidance is a possibility...For one thing, nonevents are harder to detect. Complainants need to have a perspective on what could have happened to see the consequences of something which never happened...inaction is hard to supervise. 
Further, and related to the idea of supervision, today, as has always been the case, it is impossible for rank-and-file supervisors to monitor the minute-by-minute activities of their officers deployed in the field. These considerations are addressed in the subsection below, but, put simply, police management and supervisors have little power and no effective mechanism to compel front-line officers to perform discretionary interactions and/or proactive initiatives in the community. As the Supreme Court of Canada, in Beaudry v The Queen (2007), affirmed,

The ability - indeed the duty - to use one's judgment to adapt the process of law enforcement to individual circumstances and to the real life demands of justice is in fact the basis of police discretion...Police discretion is an essential element of the justice system...[and it cannot be] displaced by the provisions of internal police directives.

\section{Discretion and De-Policing}

In any consideration of police powers and their exercise, or, in the context of depolicing, an officer's decision not to exercise their powers, it is important to appreciate that, as a fundamental tenet of socio-legal systems across Western democracies (as evidenced in the cited case in the preceding paragraph) and incorporated within statesanctioned models of policing, front-line officers possess inherent discretion in determining when and how to use their unique and wide-ranging powers (Bittner 1970; Brooks 2010; Cao \& Huang 2000; Cockcroft 2013; Gonzales \& Cochran 2017; Holmes \& Smith 2008; Klockars 1985; Luna 2000; Mastrofski et al. 2002; McLaughlin 2007; Muir 1977; Punch 2009; Reiner 2010; Waddington 1999; Wilson 1968). In this regard, Seron and colleagues (2004: 666) observe there exists "a strong tradition of 'street level' discretion" in the policing of society. As Waddington (1999: 287) finds, "The notion that the police possess a distinctive occupational subculture lies at the centre of 
much research and theorizing about policing and police work. It derives from the discovery that police work is rarely guided by legal precepts, but that police officers exercise extensive discretion in how they enforce the law.” Further, Reiner (2010: 27, citing Skolnick 1966; Wilson 1968; Muir 1977; Mastrofski 2004) observes, "Sociological studies of policing in action have shown that decision-making is shaped largely by the rank-and-file officers on the street, who enjoy a considerable measure of discretion." Davis (1969: 4), in one of the oft-cited definitions of police discretion, advances, "[An] officer has discretion whenever the effective limits on his power leave him free to make a choice among possible courses of action or inaction." Brooks (2010: 73, citing Davis 1969; Ericson 1982) presents an illuminating observation,

Some discretionary actions involve very subtle and perhaps minor decisions, while others involve blatant and important ones...The fewer the rules about handling incidents and situations, the more discretion officers have. Discretion involves both action and inaction. Not doing something may be equally as important as doing something. Discretion involves both having the power to decide which rules apply to a given situation and whether or not to apply these rules. Both of these decisions have potentially important implications for the community and for the police...The exercise of police discretion poses some [potential] difficulties, such as: unequal treatment of citizens, interference with due process, a reduction in deterrent effects, and the hidden or unreviewable nature of many discretionary decisions.

Similarly, Cooper (2002: 357-358) observes, "Police officers have long used their discretion to allow individual suspects to go free. Qualitative research suggests officers use that discretion when they identify with the suspect. Yet officers' senses of selfidentity and attributions of identity to others can also lead them to feel divided from particular types of suspects." In this vein, Bacon (2014: 108) finds, “The recognition that the rank-and-file exercise a considerable amount of discretion in the performance of their duties raises questions about some of the extra-legal factors influencing police 
decisions and actions." For example, a Delta officer reported, I was taught to do [depolicing] and I do it regularly for situations when I can use my discretion and avoid a complaint from anti-police citizens (DEL13). Along these lines, I suggest that technosocial and socio-political challenges in the front-line policing landscape can be considered 'extra-legal factors' and, as demonstrated in the findings of the present study, these most certainly influence rank-and-file officers' decisions and actions including those relating to de-policing and, within the de-policing phenomenon, to considerations of race/ethnicity and other 'risky' characteristics perceived throughout the population.

An interesting question that arises in the context of de-policing, from the perspective that front-line officers intentionally not engaging in proactive interventions in the community is detrimental to the effective policing of society, is - How can you stop officers from de-policing if they advance that they are simply invoking their inherent discretion? This situation implicates not only discretion but also supervision of front-line officers and our limitations, as human beings, which includes rank-and-file police supervisors, to interpret, with absolute certainty, what another person (or officer) subjectively observes, perceives, and understands. An officer can act, but also not act. An officer can police, but also de-police. Along these lines, I argue that it is impossible for an observer, even a veteran rank-and-file police supervisor, to determine whether an officer perceived what the observer perceives the officer to have perceived. As we know, perceptions are inherently subjective and distinctively individual.

For example, did the police officer perceive that a person walking down the dark alley appeared 'suspicious'? Setting aside complexities involved with the concept of 
suspicion in police work, it might very well be the case that the officer, based on their subjective understandings, did not conclude that the person was, in fact 'suspicious' and therefore, in not interdicting the person and questioning them about their activities, they were exercising their inherent discretion. The officer's supervisor, or a civilian bystander, might have perceived the situation much differently and might disagree with the officer's application of discretion, but in the 'real world' of front-line policing, no one in the field can realistically observe, supervise, nor adjudicate how discretion is mis/applied by individual front-line officers - including, in my argument, tactical and proactive applications of discretion/inaction by front-line officers through de-policing practices.

In this vein, and in my view, an important distinction between an officer's application of 'routine' discretion and the study's identified de-policing practices relates to the systematic nature of the latter and the reason for its implementation by the officer. For example, discretion is often used, to a citizen's advantage, through an officer bypassing 'going by the book' and perhaps, along these lines, the reader will have received 'a break' when stopped for a minor traffic infraction. In this vein, Reiner (2010: 19) observes, "One of the earliest findings of sociological research on policing...[is that] police routinely under-enforce the law, using their discretion to deal with incidents in a variety of other ways." I argue that de-policing, while perhaps technically a cousin to 'routine' episodic discretion (i.e. periodically giving a motorist a 'break'), is entirely different, both conceptually and as a 'real world' policing practice implemented as a sweeping and strategic application of discretion through self- 
preservation and risk-avoidance behavioural adaptations, as validated in the results of the present study.

Ultimately, it is important to appreciate that rank-and-file officers possess substantial discretion in much of their work in the field - particularly in 'proactive', 'discretionary' or 'self-initiated' actions. This, as contrasted with dispatched calls, in which officers still have considerable discretion, but less so. The limited supervision of front-line officers is another circumstance to consider in the context of this observation and in understanding the complexities involved with the de-policing phenomenon identified in the present study. The supervisor cannot simultaneously and continuously follow 5,10 , or perhaps 20 , officers deployed in the field to ensure they are meeting a subjectively-evaluated level of performance in their discretionary police work. In this regard, the same arrangement Reiss (1968: 18-19) described 50 years ago remains unchanged today,

Controlling the police in the field, where the policeman is away from direct supervision, is understandably difficult...The problem is that police contact with citizens occurs in the community, where direct supervision is not possible. Assuming our unwillingness to spend resources for almost one-to-one supervision, the problem for the police commander is to make policemen behave properly when they are not under direct supervision.

As (Wilson, 1968: 17) observed in the same era, "One of the key features of street-level policing has been the large degree of autonomy enjoyed by individual officers...in practice decisions made at street level are rarely, if ever, directly supervised." Similarly, ten years later and in the UK context, Holdaway (1979: 12) observed, "The lower ranks of the service control their own work situation and such control may well shield highly questionable practices." These realities remain as true in 2019 as they did in 1968 and 
1979 and are particularly relevant to the practice of de-policing across front-line police work.

Further, Wilson (1968, and then Reiner 2010) also found that policing is distinct from many other organizational arrangements, in that, "In the police service personal discretion increases as one moves down the hierarchy, with the effect that officers on the street exercise considerable power over the public" (Goold (2004: 178). Bayley (2011: 314), another eminent policing scholar, finds that front-line officers "bitterly resent any attempt to supervise them" and that they have virtually unmitigated discretion in their work in the field, the location of which changes with each call for service or proactive engagement (Brown 1988; Klinger 1997; Muir 1977; Reiss 1971; Wilson 1968). These spatial and temporal exigencies in front-line patrol work afford officers a high-degree of autonomy and limit the capacity for supervisory oversight (Brehm \& Gates 1994; Chan 1997; Fridell \& Scott 2010; Johnson 2012; Klinger 1997; Luna 2000; Manning 1977; Punch 2009; Shaplang \& Vagg 1980; Stenson \& Silverstone 2014; Van Maanen 1983; Weitzer 2015). Further, in terms of structural and oversight considerations, as Weitzer (2015: 479) observes, "Most officers patrol alone, unfettered by the checks that a fellow officer or supervisor might provide." These realities are significant in understanding some of the nuances in today's de-policing phenomenon.

In this regard, with the inherent, broad, and essentially unreviewable discretion afforded to front-line officers in the field (Bayley 2011; Brooks 2010; Crank 1998; Fridell \& Scott 2010; Holdaway 1983; Klockars 1985; Luna 2000; Manning 1977, 2000; Mastrofski et al. 2002; Punch 2009; Rojek, Decker \& Wagner 2010; Seron et al. 2004; Shapland \& Vagg 1980; Sherman 1974, 1984; Van Maanen 1983; Wilson 1968; 
Worden 1995a, 1995b) and the absence of any mechanism by which supervisors or the public audience can effectively delve into an officer's innermost thoughts and decisionmaking processes, the potential exists for the rank-and-file officer to simply ignore, or delay their response to, certain situations which they believe hold the opportunity for a greater risk of complications and/or the potential for unfavourable occupational consequences - contacts or circumstances that could become controversial, that might be video recorded, and/or might lead to scrutiny and criticism in the public milieu. It is important, in this context, to appreciate that discretion can apply to action or inaction (Brooks 2010; Cockcroft 2013; Davis 1969; Ericson 1982; Klockars 1985). Recall that Brooks (2010: 73) observes, "Both of these decisions have potentially important implications for the community and for the police." I argue, based on the empirical results of the present study, that many front-line officers today have come to understand that the policing of certain communities, individuals, and/or circumstances can expose them to elevated and significant professional risks and that not exposing themselves to said perceived risks, through the practice of de-policing, outweighs their sense of duty to act. In this regard, Pollock (2010: 315-316) advances,

Duty may be defined by the organization, but each individual officer also shapes and personalizes his or her own parameters of duty. To not do what one is paid to do is an obvious ethical transgression, but beyond that, there are nuanced answers for how much an officer is or should be expected to do given the complicated and difficult situations they encounter...We expect police to utilize their discretion in an ethical manner, but what does that mean?

Along these lines, Nix and colleagues (2018: 34, citing Goldstein 1960, 1963; Kubrin, Messner, Deane, Mcgeever \& Stucky 2010; Wilson \& Kelling,1982) find, "While officers are obligated to respond to 9-1-1 calls, they have much greater discretion in 
proactively stopping [or not stopping] citizens." On this subject, recall the observations of a Chicago officer from Chapter One, 'It's my job to go to work and listen to your 9-11 calls and respond. That's the basic [responsibility] of my job.'

With reactive policing, the officer responds to a set of circumstances that are reported to them by others. However, with proactive policing, if they choose to engage, the officer responds based on their subjective observations, perceptions, experiences, and judgments. As demonstrated in the present study's findings, this is often where the danger, in terms of professional consequences to the officer, and the de-policing response of self-preservation and risk aversion emerges. Following this line of reasoning, officers wonder (paraphrasing several study participants' thoughts), How can I explain, to the satisfaction of today's adversarial public audience, the decision to interdict a person walking in a dark alley because they 'look suspicious' - in a policing landscape of inherent suspicion (about the police)? Therefore, officers can develop the understanding that avoiding all but the essential tasks expected of them (i.e. responding to their dispatched calls in a reasonable time frame and doing an adequate job in addressing the issues identified in those calls) is the safest course of action in the current circumstances of front-line policing. As was explained to me repeatedly in my discussions with study participants at virtually all of the study's data sites, one does not get any extra pay, recognition, appreciation, or any other form of incentive for doing more than the minimum expected and the downside risk mitigates against doing anything more than simply answering dispatched calls. The observations of the following Calgary and Toronto officers are representative (emphasis in original), I get paid either way, I will NOT cause trouble for myself or my family for a society that doesn't want or understand policing (CAL121). 
We get paid the same to drive by and the added benefit of no stress/conflict or second guessing (TOR144).

\section{Final Thoughts Pertaining to De-Policing and Today's Police-Community Relations}

As demonstrated in the present study's empirical results, many of today's rankand-file officers have forsaken proactive police work and are, therefore, as some would argue, neglecting their sworn obligations to serve and protect the community - opting instead to disregard the purported ideals of CBP and adopt various risk-averse and selfpreservation practices, including intensive de-policing or 'fire-brigade policing'. This has major implications regarding both the core philosophy and the strategic model of Western democratic policing over the last 50 years, which involved the seismic shift from the reactive model of the professional era to CBP. In terms of police work in the field, CBP contemplates front-line officers frequently, actively, and collaboratively engaging with the citizenry - including dedicating discretionary time between dispatched calls to proactive interactions with residents, and crime prevention initiatives, throughout the community. Given that so many of today's rank-and-file officers find substantial risk in interacting with citizens, the most fundamental tenet of CBP is front-and-centre for reconsideration.

Further, with movements toward procedural justice as a strategy to rehabilitate public-police relationships and front-line officers being encouraged to shift to a more service-oriented (or 'guardian') approach in their police work, it should be clear that the widespread de-policing demonstrated in the present study is problematic. ${ }^{39}$ In an 'oped', Chicago Mayor Rahm Emanuel (2017) observed, "We will not have the level of public safety we all want without an engaged, proactive police department that has earned the trust of residents...[De-policing] is not a recipe for success in our 
communities. The only winners in that case are the gangbangers and drug dealers." Emanuel addressed the issue of de-policing in relation to the responsibilities of the police to repair damage in their relationship with the community, as is the common perspective within the academic literature and throughout public discourse. However, in considering the other constituent in the public-police relationship, Pickett and Ryon (2017) argue that improving police-community relations is "a two-way street". And, along the same lines, Nix and his colleagues (2018: 49) counsel, "The public needs to believe they can trust the police, but officers must also trust the community."

\subsection{De-Policing Implications Related to Race/Ethnicity}

Along these lines, the results of the present study demonstrate that there is clearly an issue today with front-line police officers trusting the public, including a particularly problematic situation in relation to the de-policing of racialized and marginalized individuals. These include officers' risk-oriented concerns around citizens they perceive as exhibiting a 'non-traditional' sexual orientation (i.e. members of the LGBTQ+ community) and citizens that are perceived as exhibiting overt mental health challenges. However, by a considerable margin, the most distressing circumstances in this regard, as identified in the present study, relate to officers' assessments of an elevated risk for complaints about their police work and, therefore, the potential for professional consequences in interactions with those persons perceived by officers to be 'visible minorities' - those (apparently) of a race/ethnicity different than White. These difficulties in the public-police relationship, involving strained relations between police and racialized individuals and communities, and issues therein that are, or can be, related to the identified de-policing phenomenon, are the focus of this subchapter. In 
saying this, the reader should understand that throughout the previous chapters I have, for the most part, purposely set aside addressing these issues, with the intention of doing so in this subchapter, which has always been specifically earmarked for this purpose.

In this regard, the discussion is organized around three subsections that all relate to considerations implicating race/ethnicity: (1) historical circumstances in the publicpolice relationship; (2) today's circumstances in the public-police relationship; and (3) the public-police relationship in the context of over-policing and de-policing. Informing all three, it is axiomatic to oberve that racialized and marginalized persons across Canada and the USA have been subject to discriminatory mistreatment by the police and I suspect this has likely been the case since the introduction of public policing in the two countries. As Reiner (2010: 25) finds, "Discrimination and disparity in the treatment of different groups is a perennial problem of policing. ${ }^{" 40}$ Similarly, I suggest that a resident of either country would have to be living in isolation from all forms of mediated discourse, for at least the past decade, to be oblivious to the persistent contemporary public sphere dialogue related to widespread societal concerns with police mis/conduct in officers' interactions with marginalized and racialized individuals. In this regard, Doob and Gartner (2017: A11) find, "It would be almost impossible, and inappropriate, to discuss police stops of citizens and not talk about race.” Therefore, in assuming a certain foundation of knowledge among the readership, the following three subsections are illustrative, but relatively brief.

\section{Historical Circumstances}

While the de-policing phenomenon documented in the present study may implicate a reduction in proactive police work in certain neighbourhoods, and, therefore, 
a potentially-positive outcome for racialized and marginalized individuals that have been subjected to over-policing (as discussed throughout subchapter 6.7), such would be a dramatic departure from the history of problematic over-policing of particular persons in our society. In this regard, Engel and Cohen (2014: 383) observe, "The history of race and policing in the United States is long and troubled...[and] the systematic targeting and biased treatment endured by minorities at the hands of American police has been well documented." Similarly, in his editorial introduction to the special issue of Justice Quarterly addressing policing and minority communities, Ulmer (2017: 11271128, citing Bonilla-Silva, 2014; Campbell \& Schoenfeld 2013; Muller 2012; Ward 2012), observes,

American police have had troubled relations with minority communities especially African American communities - for most of U.S. history. From enforcing runaway slave laws and serving as foot soldiers of Jim Crow oppression in the South, to less open but often just as harsh brutality and discrimination in the twentieth century Northern and Western U.S. American police have often been the face of racialized criminal justice. Thus, an essential American institution has also often been a racialized institution.

Remaining in the American context, Rosenbaum (2019:336, citing Wilkerson 2010) argues, "We must never forget the long and painful history of relations between the Black community and law enforcement, from slavery to this day." Similarly, Brunson and Gau (2014: 363, citing Bass 2001: Websdale 2001), find, "Blacks in the United States have had a long and tumultuous history of being unjustly targeted, stopped, questioned, and search by the police. Disproportionate police attention has proven especially harmful to scores of urban Black males, who consider themselves officers' primary targets, and who frequently describe their communities as having been besieged by police. ${ }^{21}$ 
While these scholars' observations are recent, they are nothing new. Over fifty years ago, the National Advisory Commission on Civil Disorders (1968: 157) raised significant questions about the relationship between police and minority communities in the USA and, in this regard, observed, "In Newark, Detroit, Watts and Harlem, in practically every city that has experienced racial disruption since the summer of 1964 , abrasive relationships between police and Negroes and other minority groups have been a major source of grievance, tension and ultimately disorder." Reflecting the policing of that era, Wilson (1968: 40-41) articulated,

The patrolman believes with considerable justification that teenagers, Negroes, and lower-income persons commit a disproportionate share of all reported crimes; being in those population categories at all makes one, statistically, more suspect than other persons; but to be in those categories and to behave unconventionally is to make oneself a prime suspect. Patrolmen believe that they would be derelict in their duty if they did not treat such persons with suspicion, routinely question them on the street, and detain them for longer questioning if a crime has occurred in the area, To the objection of some middle-class observers that this is arbitrary and discriminatory, the police are likely to answer: 'Have you ever been stopped and searched? Of course not. We can tell the difference; we have to tell the difference in order to do our job. What are you complaining about? ${ }^{42} 43$

Deuchar and colleagues (2019) find that the American situation, vis-à-vis raciallybiased policing directed towards the Black and Hispanic communities and "a racial gap in attitudes towards the police" among those minority communities, "that has persisted for decades," can be distinguished from the circumstances in other Western democracies, in terms of prominence.

Along these lines, I suggest that few, if any, policing scholars would argue that the history of racial discrimination by police toward the Black community has been more pronounced in the history of Canada than the USA. However, that being 
stipulated, many racialized Canadians have reported, and have undoubtedly experienced, racially-biased and discriminatory policing practices. In this regard, I suggest that there has existed, for many years, a diversity of racism throughout Canadian society, within various police agencies and rank-and-file police subcultures across the country and reflected in the front-line policing practices of many front-line officers. For example, in examining the 'driving while Black' phenomenon in Canada, Foster (1996) found that for decades, Blacks in major Canadian cities have been stopped frequently by police officers and searched solely based on their skin colour - a practice commonly known as 'racial profiling'. ${ }^{44}$ Several other researchers have examined issues in Canadian policing vis-à-vis the Black community (e.g., see, Fitzgerald \& Carrington 2011; Henry \& Tator 2005, 2006; MacAlister 2004; Maynard 2017; Mosher \& MahonHaft 2010; Reasons, Hassan, Ma, Monchalin, Bige, Paras \& Arora 2016; Thornhill 2008; Wortley \& Owusu-Bempah 2009, 2011; Wortley \& Tanner 2003, 2004, 2005). Maynard (2017: 3-5, citing Wortley 2006) observes, inappropriate police interactions with, and violence against,

Black persons in Canada has, by and large, remained insulated by a wall of silence and gone largely unrecognized by much of the public, outside of brief media flashpoints. Anti-Blackness in Canada often goes unspoken. When acknowledged, it is assumed to exist, perhaps, but in another time (centuries ago), or in another place (the United States). Many Canadians and attuned to the growing discontent surroung racial relations across the United States, but distance themselves from the realities surroung racial disparities at home... Many Canadians do not know that, despite being around 3 percent of the Canadian population, Black persons in some parts of the country make up around one-third of those killed by police. ${ }^{45}$

Further, Wortley \& Tanner (2003) have found that Aboriginals and South Asians in Canada have been subject to 'racial profiling' tactics for many years, leading to stops, searches and arrests. 
However, for the most part, throughout the literature, it is Canada's history of colonialism and the oppression of its Indigenous peoples, through the law and its enforcement, that is particularly well documented (e.g., see Barker, Alfred, Fleming, Nguyen, Wood, Kerr \& DeBeck 2015; Cao 2014; Jeffries \& Stenning 2014; Kitossa 2012; La Prairie 2002; La Prairie \& Stenning 2003; Monchalin 2010, 2016; OwusaBempah et al. 2015; Reasons et al. 2016; Tobias \& Joseph 2018). For example, in 2018 Prime Minister Justin Trudeau presented a public apology on behalf of all Canadians for the state's execution of six chiefs of the Tsilhqot'in community in 1864. After a confrontation with a White work crew that intruded onto their traditional lands, "colonial officials of the day...[invited] the chiefs for peace talks where they were instead arrested, tried and hanged" (Smart 2018). In the more recent past, the 1988 Aboriginal Justice Inquiry of Manitoba found that Indigenous persons have, for years, been significantly overrepresented in rates of interactions with police, arrests, convictions, and imprisonment (Smandych, Lincoln \& Wilson 1993).

Further, Goulding (2001), through a case study of the 1992 murders of three Indigenous women in Saskatoon by a serial killer, elaborated the historic and entrenched indifference of Canadian police to the Indigenous community, which has recently received considerable media and public sphere attention, given the tragic circumstances around missing and murdered Indigenous women and girls and, in my view, the shamefully inadequate police investigations into the murders of hundreds of these Canadians over the years. ${ }^{46}$ This focus on strains in the relationship between police and the Indigenous community culminated in the mobilization, by the Canadian government in 2016, of the National Inquiry into Missing and Murdered Indigenous 
Women and Girls. That inquiry is "exposing hard truths about the devastating impacts of colonization, racism and sexism - aspects of Canadian society that many Canadians are reluctant to accept" (National Inquiry into Missing and Murdered Indigenous Women and Girls Legacy Archive 2018: 1).

\section{Today's Circumstances}

I advance that today, interrelated with the complex politics of interpretation (as addressed in Endnote 15 within Chapter Three), considerations of accountability and legitimacy in policing are frequently bound up with concerns around racial/ethnic bias in the policing of society. In this regard, bias often informs and influence peoples' interpretations and perspectives on policing, with different voices, defined, and often divided, by race/ethnicity, reacting to policing events much differently. In this vein, and in returning back to the American context, the observations of Wilson (1968) and Bittner (1970), in the preceding subsection, documented manifestations of racial bias in policing a half century ago. However, as Mastrofski (2012: 593) recently observed, "Concern about racism in policing has not abated [since that era]...It is a policy matter as profoundly important today as it was then, and it is as intensely debated among scholars, policy analysts, and commentators." Along the same lines, Collins and Klahm (2019: 264) argue, "The post-Ferguson policing predicament is by no means its beginning...it is clear that, while improvements have been made [in the last 100 years], policing continues to be plagued by instances of perceived wrongdoing, bias, and discrimination." In this regard, the National Academies of Sciences, Engineering, and Medicine (2018: 11) observe, "Although in recent decades, police have often made a strong effort to address racially biased behaviour, there remain wide disparities in the 
extent to which non-White people and White people are stopped or arrested by police."

Later in their report, it is advanced that,

...the high rates at which non-Whites are stopped, questioned, cited, arrested, or injured by the police present some of the most salient criminal justice policy phenomena in the United States. Because these kinds of police contacts are associated with at least some forms of what is known as proactive policing, recognition of this reality is an important starting point for this [discussion]. Additionally, because many proactive policing strategies by design increase the volume of interactions between police and the public, such strategies may increase the overall opportunity for problematic interactions that have disparate impacts (National Academies of Sciences, Engineering, and Medicine 2018: 251, citing Kochel, Wilson \& Mastrofski 2011; Lytle 2014).

In relation to specific contemporary developments, Brunson and Gau (2014: 364, citing Fagan \& Davies 2000) argue that "it is well documented that inner-city, minority males bear the brunt of frequent, unwelcome police contacts - including disproportionate stops, frisks, and arrests" and, in the same vein, Dunham, Alpert, Stroshine and Bennett (2005: 370) note that various studies "indicate that African American suspects are more likely to be arrested and/or treated more harshly by police than are White suspects." Neyroud (2019:85, citing Weisburd \& Majmundar 2017) observes that 'stop and frisk' practices have "produced significant adverse consequences through disproportionate impacts on Black communities, breaches of constitutional rights, and reduced police legitimacy." As relate to these "differential rates of police contact," Rosenbaum (2019: 325) observes that "people of privilege rarely experience the embarrassment and humiliation associated with having their possessions dumped onto the street and search, being questioned as to where they are going, or having to 'assume the position' in front of family, friends, and bystanders... Today, we still cannot avoid the fact that policing tactics and strategies often vary by 
race and social class." Epp and colleagues (2014: 15) find such police tactics "have profound consequences for people's sense of their place and status in American society" and, in this regard, Ulmer (2017: 128) argues, "I do not think it is an exaggeration to speak of a contemporary crisis of legitimacy for the criminal justice system in the eyes of many minority communities, especially African Americans."

The prevalence of actual instances of biased policing outcomes related to race/ethnicity is empirically unascertainable and subject to contestation by those with a different perspective than that of the person making the assessment/argument. And, as demonstrated in the de-policing phenomenon investigated in this dissertation's research, in addition to tangible 'on the ground' outcomes, the attitudes and perceptions of actors in a police-citizen interaction (both the citizen and the police officer) are also important. In this vein, Sandhu and Haggerty (2015:10, citing Drake 2014; McCarthy 2014; Newport 2014), find, "At the most basic level, privileged White people tend to be more habitually supportive of the police than are poorer racialized groups. Public opinion polls have long suggested that race informs public support, trust, and confidence in policing in the United States." In this regard, editors at the Gallup polling firm observe, "The high-profile incidents in Ferguson and New York City have thrust the issue of race relations and the police into the national spotlight in a powerful way in 2014" (Gallup Editors 2014). Along these lines, I suggest that an effective (and concise) way to demonstrate the current circumstances in the public-police relationship, vis-à-vis discord in relations between police and minority racial/ethnic populations throughout the USA, is through the presentation of American public and police officer opinion data. 
The following tables present the results of a 2014 Washington Post/ABC News poll that investigated contemporary policing issues in relation to race/ethnicity. ${ }^{47}$

How confident are you that the police in [the USA] treat Whites and Blacks equally?

\begin{tabular}{|c|c|c|c|c|}
\hline & $\begin{array}{c}\text { Very } \\
\text { confident }\end{array}$ & $\begin{array}{c}\text { Somewhat } \\
\text { confident }\end{array}$ & $\begin{array}{c}\text { Not so } \\
\text { confident }\end{array}$ & $\begin{array}{c}\text { Not confident at } \\
\text { all }\end{array}$ \\
\hline Whites & $26 \%$ & $37 \%$ & $19 \%$ & $17 \%$ \\
\hline Blacks & $3 \%$ & $18 \%$ & $20 \%$ & $57 \%$ \\
\hline Hispanics & $15 \%$ & $25 \%$ & $29 \%$ & $29 \%$ \\
\hline
\end{tabular}

How confident are you that the police in [the USA] try hard enough to maintain good relations with different groups in the community?

\begin{tabular}{|c|c|c|c|c|}
\hline & $\begin{array}{c}\text { Very } \\
\text { confident }\end{array}$ & $\begin{array}{c}\text { Somewhat } \\
\text { confident }\end{array}$ & $\begin{array}{c}\text { Not so } \\
\text { confident }\end{array}$ & $\begin{array}{c}\text { Not confident at } \\
\text { all }\end{array}$ \\
\hline Whites & $31 \%$ & $43 \%$ & $15 \%$ & $10 \%$ \\
\hline Blacks & $4 \%$ & $32 \%$ & $38 \%$ & $26 \%$ \\
\hline Hispanics & $21 \%$ & $37 \%$ & $18 \%$ & $21 \%$ \\
\hline
\end{tabular}

Do you think the recent killings of unarmed African American men by police in Ferguson, Missouri and New York City are isolated incidents or a sign of broader problems in the treatment of African Americans by police?

\begin{tabular}{|c|c|c|}
\hline & Isolated incidents & A sign of broader problems \\
\hline Whites & $60 \%$ & $35 \%$ \\
\hline Blacks & $18 \%$ & $74 \%$ \\
\hline Hispanics & $45 \%$ & $51 \%$ \\
\hline
\end{tabular}

These results coincide with Gallup survey data that has, for many years, consistently demonstrated Black Americans have a significantly lower level of confidence in the police as an institution than do Whites (Newport 2014). Further, Morin and colleagues (2017) report that citizens and police officers differ substantially in their perceptions around deadly 'Black-police encounters', with $60 \%$ of Americans (54\% of Whites and $79 \%$ of Blacks) believing that recent high-profile occurrences are signs of a broader problems between Black citizens and police. This is up from $43 \%$ of Americans in 2014 (Lee et al. 2018). In contrast, 67\% of police officers believe these 
types of occurrences are isolated incidents rather than epitomizing a systemic issue (Morin et al. 2017).

Similarly, throughout the academic literature, disparities in attitudes of Americans to police along racial lines is well documented, with Blacks and Hispanics significantly less trusting than Whites, in both the socio-political institution of policing and in individual police officers deployed within the community (Bradford et al. 2009; Stein \& Griffith 2017). Brunson and Gau (2014: 363, citing Skogan 2005; Tyler 2005) observe, "A great deal of research reveals that African Americans report less favourable views of the police when compared to members of other racial groups, especially Whites" and Weitzer and Tuch (2004: 395) find, "African Americans are more likely than Whites to report negative experiences with the police and to believe that they have not been fairly treated." In their research, Callanan and Rosenberger (2011: 178) found, "Blacks were much more likely than Whites to have less confidence in the police, to believe that the police are not fair, and to believe that police use of excessive force is a problem in their neighbourhood"

Remaining in the American context, Ross (2015) found that between 2011 and 2014, the probability of being Black, unarmed, and shot by police was almost $3 \frac{1}{2}$ times the probability of being White, unarmed, and shot by a police officer. The value in relation to Hispanics was found to be more than $1 \frac{1}{2}$ times the probability of being White, unarmed, and shot by an officer. Further, Ross (2015) found that, nationwide, the average risk of being shot by a police officer was the same for unarmed Black suspects as it was for armed White suspects. ${ }^{48}$ While Black males account for $24 \%$ of the approximately 1000 persons shot and killed by police across the USA each year, 
over the past five years, they constitute only $6 \%$ of the American population (Sullivan, Thebault, Tate \& Jenkins 2017).$^{49}$ Many of these recent high-profile police use of force events involving Black suspects have showcased the racially charged nature of current police/community relations in the USA (e.g., see Benzaquen et al. 2017; Brooms 2018; Gaskew 2018; Jefferis et al. 2011; Kennedy, Otuyelu \& Graham 2018; Mack \& Mack 2018; Reed 2018; Rice \& Piquero 2005) and, in this vein, Rosenbaum (2019: 331) finds, "In this post-Ferguson world, we have learned that communities have an upper limit on their tolerance of aggressive policing tactics when real lives are at stake and when race bias by the police is perceived to be present." Perhaps better than any other representation (from the academic world or mediated observations), the contemporary experiences of a Buffalo police officer elucidate the current circumstances, I am a Black [law enforcement officer] in America at a time tensions are great on both sides. I'm damned for being a cop and damned for being Black (BUF209).

In transitioning into the Canadian context, the reader should recall the discussion earlier (in subchapters 3.3 and 6.4) regarding 'the contagion effect' (Loader \& Sparks 2015; Nix \& Wolfe 2016, 2017; Pyrooz et al. 2016; Shjarback et al. 2017; Weitzer 2015, 2018; Wolfe \& Nix 2016) and the phenomenon of 'contamination-by-association' (Weitzer 2018). Along these lines, while my understanding is that few would contest that discord in the current public-police relationship related to race/ethnicity is less acute in the USA than Canada, the American challenges in this regard permeate Canadian police, media, and public sphere discourses and, therefore, both public and police understandings of, and perspectives on, these contentious policing issues (Maynard 2017). While this cross-border transfer of information from American policing has been a reality since at least the 1960 s, I suggest it is particularly prevalent 
in today's post-Ferguson era, with pervasive social and traditional news media coverage across the USA often fixated on dramatic and disturbing video recordings and controversial and/or acrimonious police-citizen interactions - often involving Black civilians and White rank-and-file officers. In saying this, the contemporary 'crisis' in policing, involving racial injustice and questionable police conduct, is not a uniquely American phenomenon. As Heatherton and Camp (2016) observe, "Policing has become one of the urgent issues of our time, the target of dramatic movements and front-page coverage from coast to coast in the United States and across the world."

MacAlister (2004: 172) observes, "In Canada, the police in the prairies have periodically come under fire for alleged racism against Aboriginals, and in Toronto the police have recently come under considerable criticism for alleged racial profiling of Blacks." In this regard, Wortley and Tanner (2005: 582) advance,

Racial profiling has emerged as one of the most important - and controversial - issues facing the Canadian criminal justice system...This topic has been a major concern of racial minority communities for several decades... Not surprisingly, the lines are clearly drawn with respect to the racial profiling debate. Social activists and Black community organizations, on the one hand, have long maintained that the police frequently stop and search Black citizens solely because of their racial characteristics (e.g., for the 'offence' of driving while Black). Police representatives, on the other hand, consistently deny allegations of racial profiling and claim that race has absolutely no influence on their decisionmaking processes.

Along these lines, police often contend that they are inclined to focus their attention and time in areas that have greater socio-economic disadvantages and difficult social conditions, which often coincide with areas in which crime is frequently reported (Withrow 2011). Typically, in such neighbourhoods, immigrant and minority populations are disproportionately concentrated (Fitzgerald \& Carrington 2011). 
Despite the denials from within policing, "Black young men view their treatment by the police as multi-faceted, intimately tied to their status as young men in disadvantaged communities, but nonetheless ultimately, inescapably, about race" (Brunson \& Miller 2006: 634). In moving on from the discussion of 'racial profiling', Chan's (2011: 76, citing Bayley \& Mendelsohn 1969; Ericson 1982; Satzewich \& Shaffir 2009) observations, given the salience of front-line police subculture to the de-policing phenomenon, are illuminating,

Racial profiling is best understood in the context of a police subculture...There is certainly a vast literature that supports the finding that street-level police officers often form stereotypical opinions about the criminality of certain ethnic groups and use such visual cues in routine, proactive police work. These ways of seeing and acting are regarded as features of police (sub)culture that appear to be common across space and time.

Similarly, Kennedy and colleagues (2018: 153) find, "The issue of racism, community distrust, discretionary policing and racial profiling contributes to a racialized law enforcement culture."

In contrast to the USA, where official police statistics concerning race/ethnicity have been routinely collected since the 1930s, Canadian criminal justice institutions only collect information on these demographic characteristics within the corrections system - and not within policing (Moscher \& Mahon-Haft 2010; Reasons et al. 2016). ${ }^{50}$ Notwithstanding the lack of official statistics, several researchers have conducted empirical studies and presented findings in relation to Canadian police interactions with racialized and marginalized individuals. For example, Hayle, Wortley and Tanner (2016) find that 'racial minorities' are stopped and searched by Canadian police officers at a disproportionately high rate and Blacks are three times more likely than Whites to 
be stopped by a police officer in Canada (Wortley \& Owusu-Bempah 2011). Reasons and colleagues (2016) have found that Blacks and Indigenous persons are often viewed by Canadian police officers as more likely to be involved in criminality than Whites. Further, Barker and colleagues (2015) discovered that when deciding on interventions with Indigenous persons, Canadian police are more likely to rely on punitive measures than warnings, in contrast to their typical practices with the non-Indigenous population. Perhaps not unsurprisingly, Cao (2014) found that Indigenous peoples and 'visible minorities' have lower confidence in the police as compared to other demographic categories in the Canadian population. Smandych and colleagues (1993) found that Indigenous persons are arrested at a rate that is 29 times that of the non-Indigenous population. They argue that this situation is "even more egregious because it has been found that Indigenous persons are less likely than the non-Indigenous population to commit serious criminal offences, rather their offending often involves minor driving offences, petty theft, and 'drunk and disorderly' conduct” (Smandych et al. 1993: 7). As discussed in the preceding subchapter, these are typically the types of occurrences in which police officers have considerable latitude to employ discretion.

In this vein and returning to the American policing context vis-a-vis the Black population, the study completed by Shjarback and colleagues (2017), investigating a hypothesized 'racialized de-policing effect' throughout Missouri police agencies is instructive. They found that "departments serving larger African-American populations conducted fewer [traffic] stops, searches, and arrests in 2015 compared to 2014...The negative attention and increased scrutiny of law enforcement appears to have had a [significant] impact” (Shjarback et al. 2017: 42). More specifically, these policing 
scholars report, "A major finding of this study is that context - especially the racial composition of cities - shapes de-policing behaviour...De-policing may be a reaction to officers' sense of 'self-preservation' in this era of social media scrutiny...This pattern might be expected given that the national conversation and debate about policing in recent years has been focused on race" (Shjarback et al. 2017: 50). As explained throughout this dissertation, given the infancy of the social scientific study of today's de-policing phenomenon, to this point in time, no other empirical research (excepting the present study) has reported on 'real world' outcomes of de-policing practices vis-àvis race/ethnicity in police-citizen interactions (or absence thereof). My understanding is that several research projects, examining this very issue, are underway in the USA.

\section{De-Policing (and Over-Policing)}

In continuing the discussion around the concepts of de-policing and over-policing from subchapter 6.7, this subsection addresses the 'real world' implications specifically in the context of race/ethnicity. First, in relation to over-policing, in 1968, the National Advisory Commission on Civil Disorders "detailed the abuses of police power and overpolicing of Black neighbourhoods that contributed to mistrust between police and certain segments of the citizenry" (Collins \& Klahm 2019: 265). Since, various studies have found that Black persons (collectively) have been 'over-policed' for many years in Canada (e.g., see Charest 2009; Tanovich 2006; Wortley \& Oswusu-Bempah 2011), the USA (e.g., see Engel \& Tillyer 2008; Tillyer, Engel \& Cherkauskas 2010; Weitzer 2000) and, for additional context, in the UK (e.g., see Bowling 2007; Bowling \& Phillips 2002) and Australia (e.g., see Chan 1997) ${ }^{51}$ Desmond and Valdez (2012: 118, citing Fagan \& Davies 2000; Western 2006) observe that, despite the prevailing 
circumstances of over-policing in primarily urban and racialized American

neighbourhoods, this practice increased in intensity in the early 1990s, when many American police departments implemented further measures in "proactively and persistently policing disadvantaged neighbourhoods. The experience of being stopped, questioned, and frisked, cuffed, arrested, and convicted, would become [even more] commonplace for young and poor Black men."

Authors, such as Michelle Alexander (2011), who wrote The New Jim Crow: Mass Incarceration in the Age of Colorblindness, have discussed this overpolicing of Black neighbourhoods as a consequence of the 'War on Drugs', which has "created a new caste system of racial control and second-class citizens" (Rosenbaum 2019: 328). As discussed in subchapter 6.7, over-policing practices include 'hot spots policing', 'broken windows policing', 'zero tolerance policing', 'order maintenance policing', and 'stop, question, and frisk'. In this regard, records from the NYPD demonstrate that, until recently, Blacks and Hispanics were nine times more likely to be stopped by police than Whites (Hayle et al. 2016; Jones-Brown, Gill \& Trone 2010) and, a revealing study by Lynch, Omori, Rousell and Valasic (2013), documented that in relation to the San Francisco illicit drug trade, specific neighbourhoods with higher proportions of racialized individuals received a substantially disproportionate amount of police attention than predominantly White neighbourhoods. As Oliver (2017: 438, citing Unnever \& Gabbidon 2011; Weitzer 2010) finds,

Because de-policing is often a response to race-centered issues (Cooper 2003, 2006, 2009), the potential impact that de-policing may have on racially segregated areas could be profound. If the police disengage from proactive policing in these areas, this may result in a mixed reaction; a positive one from those who believe they have been harassed for too long 
[over-policed], but a negative reaction from those who believe the reduced police presence lessens their public safety.

In relation to the latter consideration, Randall Kennedy (1997: 19), who identifies as a Black male, and who is a prominent Harvard law professor and author of the influential book Race, Crime and the Law, argues that "the principal injury suffered by AfricanAmericans in relation to criminal matters is not overenforcement but underenforcement of the laws," which, the reader will note, is consistent with the 'bad' outcomes of depolicing, as discussed throughout the preceding subchapter (6.7). In a recent editorial, the Maclean's Editorial Staff (2015: 5) observed, "The protection afforded law-abiding citizens by police also varies significantly by race. According to a recent lawsuit brough by the American Civil Liberties Union, Black neighbourhoods in Chicago experience police response times that are double those for White nighbourhoods."

Along the same lines, in his seminal work on de-policing and racial minority considerations in the USA, Cooper (2002: 358) observes,

We usually associate [officers'] sense of division [from identifying with particular types of suspects] with the practice of racial profiling - the systematic over-policing of whole classes of suspects based on their racial status. Recently, [however] we have seen police officers' lack of identification with certain racial groups manifest itself in systematic underpolicing of those communities.

Similarly, Shjarback and his colleagues (2017: 50, citing Morin et al. 2017) find,

$[\mathrm{J}]$ urisdictions with higher percentages of minority residents are more prone to de-policing. Speculation about the causes of this racialized depolicing effect warrants further discussion. De-policing may be a reaction to officers' sense of 'self-preservation' in this era of social media scrutiny...The dialogue and public discourse surrounding American law enforcement over the last three years has centered on race, as Black males constitute nearly all of the recent highly-publicized cases of police deadly force and in many cases their names have become well-known to the public (see, e.g., Michael Brown, Philando Castile, Eric Garner, Tamir Rice, Freddie Gray, Walter Scott, Laquan McDonald, and Alton Sterling). 
In response, we have seen the growth of the Black Lives Matter movement, and there have been countless demonstrations across U.S. cities...Accordingly, it makes sense that officers are more likely to shy away from active police work in the very jurisdictions, and perhaps neighbourhoods, that have higher African-American populations. Indeed, $75 \%$ of officers in a nationally-representative sample agree that recent high-profile incidents have aggravated tensions between police and African- American residents in their communities.

As advanced within Chapter Two, in relation to the identified phenomenon of today's de-policing, given its relative infancy, there are only limited contributions in the literature, and even more so as pertains to an association to race/ethnicity. However, I suggest that the following representative contributions from study participants provide some instructive observations in this regard,

Fuck it. They don't like White cops or actually any cops in the hood [then] the Blacks and Hispanics can police their own problems and we will do our work protecting the public in another neighbourhood (ROC102).

The Black Lives Matter movement has been vocal in widespread criticism of police which has carried over to the general public (HAM179).

...the new racism is the blue uniform (ROC53).

[I] patrol different neighbourhoods (those that don't have race issues with White cops) (BUF113).

Because of BLM and all the controversy I seldom interact with Blacks and avoid some neighbourhoods for anything but calls sent there (BUF271).

Avoid race minorities, including First Nation's people (my own background) unless part of my call. Much more likely to complain for no reason other than they hate the police (OTT262).

With continual accusations of being a racist you learn to avoid any visible minorities, especially Natives, unless they are directly involved in a dispatched call you have to take (SAS41).

There is BLM, we have ALM (Aboriginals). Very political (SAS76).

I argue, grounded in the present study's empirical results, that de-policing practices specifically in relation to the perceived race/ethnicity of a citizen are not simply the reported response of a few renegage front-line officers - the oft-cited 'bad 
apple' characterization of police misconduct. Rather, I find that the systematic and proactive de-policing of racial/ethnic minority populations is related to the entrenchment of a risk-averse worldview throughout many of today's rank-and-file police subcultures and their socialization processes. In other words, such attitudes and practices are now, by and large, the subcultural norm and, for individual officers, aligning oneself with these subcultural perceptions of today's realities in front-line police work is encouraged. The following study participants' contributions animate these observations,

With allegations of racial profiling I was trained to avoid certain minorities for my own protection (WIN88).

I avoid Natives, they feel targeted by police even though they are committing a crime. I was trained to avoid such risks (CAL247).

I was trained this way and I see most of the officers on my platoon practicing risk-averse policing in their work, particularly with visible minorities. It's often called FIDO or CYA (OTT114).

In concluding this subchapter, I advance, for the readers's deliberation, a discussion within the rank-and-file police subculture that I have now been hearing in various jurisdictions, and quite frequently, for the last six years. This relates to the idea that, given perceptions of an inherent risk for officers in interacting with 'visible minorities', what may be occurring in 'real world' outcomes is an essential reversing of the practice of 'racial profiling' or 'Driving while Black' over-policing (e.g., see Brown 2013, 2016; Brown \& Primeau 2015; Cooper 2002, 2003, 2006; Foster, Jacobs \& Siu 2016; Kennedy 1997; McNamara \& Burns 2008; Miller 2007). In other words, the pendulum may be swinging from the over-policing of racialized and marginalized communities to the de-policing of particular neighbourhoods and individuals. In this vein, McNamara and Burns (2008: 68) find that one aspect of de-policing implicates, 
among front-line officers, a "strategy in which police avoid accusations of racial profiling by ignoring traffic violations and other petty crime committed by members of visible minorities, in a sense, de-policing is the opposite of racial profiling." As discussed in subchapter 6.7 this can be viewed, depending on the unique circumstances in each occurrence, as a 'good thing' or a 'bad thing'. The present study presents, in my argument, compelling empirical support for these observations.

In addition to representative qualitative contributions from study participants along these lines (as presented below), some unlikely corroboration emerges from the findings of the researchers that were commissioned to conduct the OPS race data traffic stop study as a result of a human rights' complaint settlement. In this regard, Foster and his colleagues (2016) found that while racialized motorists had disproportionately high incidences of traffic stops (when compared with their respective driver populations in Ottawa), in terms of the outcomes of those traffic stops, officers were considerably more lenient with individuals they perceive as belonging to a racialized community (see also Brown \& Primeau 2015). I, for one, find it interesting to consider that a collateral outcome of the widespread de-policing being practiced across today's front-line police work could actually be advantageous to racialized and marginalized communities, which were subjected to over-policing and other discriminatory policing practices for so long - at least in terms of leniency in traffic enforcement (see Shjarback et al. 2017 in relation to empirical findings of de-policing in police traffic stops throughout the State of Missouri implicating those of 'visible minorities').

Many of us limit our interaction situations with minority suspects to avoid the inevitable criticism of the public, it's sad but it's true today with how things have developed in American policing, in reality it's a reverse racial profiling with minorities now getting more breaks than Whites (SYR75). 
Black people are becoming untouchable due to media/social pressure (BUF285).

I am more likely to give a Black person a break on a ticket so they don't complain (CAL189).

Police are all racists according to much of the public and the media. So, minorities now get a free pass from many of us. If that's what the public wants we will see how they feel when a minority does something to them and the police tell them there's nothing we can do (OTT79).

\subsection{Non De-Policers}

This subchapter focuses on the $28.3 \%$ of study participants that report they are not engaging in any de-policing practices. Unlike some of the previous discussion subchapters, this is a relatively straightforward exercise. Consistent with the present study's findings that today's front-line officers are responding to their techno-social and socio-political landscape in many different ways, including 'non', 'limited', 'moderate', and 'intensive' practices of de-policing, Paoline (2004: 218-220), in his front-line policing research, also found, "Officers are responding to and coping with aspects of their occupational world in differerent ways." The one complicated aspect to the analysis and discussion in this subchapter is that it is not possible, with the present study's data, to determine precise proportions of study participants that could be classified into any of the various subcategories of non de-policers that certainly exist among the 1024 individual rank-and-file officers that do not practice any form of depolicing in their front-line police work. However, in saying this, through extrapolating from the qualitative contributions of many of these study participants, I am in a position to present what I advance is an accurate understanding around the two most prevalent rank-and-file officer archetypes that reject de-policing in how they do their work in the community and that resist the influence of their rank-and-file subculture, which, as discussed throughout subchapter 6.6, often endorses and attempts to persuade officers towards practicing de-policing. 


\section{'New' ('Rookie') Officers and Non De-Policing}

The first group, as the reader might have anticipated after reviewing subchapters 6.5 (on career stage and the de-policing continuum) and 6.6 (on the front-line police subculture and subcultural socialization), is comprised of 'New' officers, which in the present study's critera, captures those with between, quite literally, one shift in frontline policing (as with a Buffalo officer I met on roll call for their very first shift) to just shy of 6 years of front-line police experience $(\mathrm{N}=1076)$. Of these 'New' officers, $40.3 \%$ report not engaging in any de-policing practices in the field. In other words, of the 1024 study participants classified, in the present study, as non de-policers, 434 (or 42.4\%) of these are 'New' officers. As was discussed throughout Chapter Five and within earlier subchapters throughout Chapter Six, 'recruits', 'rookies', and many 'New' officers have simply not been active in the field for long enough to accumulate an inventory of negative experiences and/or to be persuaded by subcultural influences toward considering de-policing as a viable self-preservation strategy - in terms of protecting themselves from potential adverse outcomes throughout their front-line policing careers (i.e. being featured in controversial video recordings, being subject to public complaints and internal investigations, being the recipient of internal discipline, or more severe punishments for alleged misconduct, etc.).

In this regard, recall the conversation in subchapter 6.5 with two Calgary officers, who related that given typical officers' enthusiasm in their first few years 'on the job', or as they phrased it, their 'piss and vinegar', it usually takes, in their estimation, between 4 and 10 years for such junior officers to ultimately 'see the wisdom in depolicing' after being exposed to negative interactions in today's techno-social and 
socio-political policing landscape and ultimately coming to appreciate the guidance of their peers in the rank-and-file subculture. These are the newer officers that initially demonstrate strong beliefs in the altruistic ideals of front-line police work and, in this vein, a conviction that through performing hard work in their chosen vocation they can make a positive difference in the community (Benner \& Phillips 1994; Harris 2014; Labaky 2013) - the 'ideal enthusiasts' as they were by characterized by Edelwich and Brodsky (1980). Much of this ground has been covered previously, however, the observations of a selection of 'New' front-line officers is instructive in understanding this particular category of non de-policer:

I joined this job [redacted] years ago to make a difference and I have already impacted on many people's lives in a good way. I don't ever see myself becoming burned-out and disgruntled like some of the officers I work with who are doing FIDO (KIN10)

[I expect] the way I speak with people or use force may change in a negative way as I get more experience (CAL348)

I am just beginning my career and feel a lot of these questions [in the survey instrument] will apply to me as I continue to gain more experience and interact with the public more (DEL09)

So far I have been able to fend off what the older officers are trying to convince me to do and am staying active in proactive police work. Just 3 days ago I got a loaded handgun on a [traffic] stop with 2 Black gangbangers from [redacted] ...(OTT374)

In comparison, among those officers with between 6 and 10 years of front-line police experience, the percentage continuing not to practice any form of de-policing drops almost in half, from $40.3 \%$ down to $23.7 \%$. As discussed previously, particularly in the context of a de-policing continuum, this is consistent with the assessment that 'New' officers simply have not been exposed to enough of the challenges in the contemporary policing landscape to give consideration to de-policing. 


\section{Mission-Oriented Officers and Non De-Policing}

The second group of prevalent non de-policers are those rank-and-file officers that look upon their work in front-line policing as more of a calling than an occupation (Bacon 2014; Brewer, Coleman-Selden \& Facer 2000; Brough et al. 2016; Kappeler et al. 2010; Loftus 2010; Marier \& Moule 2018; McElroy et al. 1999; Newburn 2012; Oberfield 2014; Paoline 2004; Raganella \& White 2004 ; Reiner 1978, 2010; Skolnick \& Fyfe; 1993; Torres et al. 2018; Van Maanen 1978; Waddington 1999; Wallace et al. 2018; Westley 1970; White et al. 2010; Workman-Stark 2017). These are officers with a strong sense of mission - individuals intrinsically motivated to serve and protect the community through helping those in need and protecting the weak throughout society (the law-abiding majority) against the predatory (the dangerous, criminogenic minority) (Myhill \& Bradford 2013; Van Maanen 1978; Waddington 1999). I refer to this subset of non de-policers as 'mission-oriented' officers. Their philosophical outlook, vis-à-vis their front-line police work, corresponds to the concept of a 'mission orientation,' which has been advanced by various scholars as a core tenet of the 'traditional' conception of police occupation culture (Barker 1999; Campeau 2015; Kapeler et al. 2010, 2015; Loftus 2009, 2010; Reiner 1985, 2010). Along these lines, Loftus (2010: 4), in her sample population of officers in the UK, confirmed the exaggerated sense of mission towards the police role that has been identified as one of the defining features of police culture, with many officers "couching [their work] in terms of a vocation or calling." Mission-oriented officers "feel that they serve an indispensable social function, protecting society from moral and physical threats on all sides" and perceive they are "soldiers in the moral crusade of the war on crime" (Marier \& Moule 2018, citing 
Westley 1970; Skolnick \& Fyfe 1993). Similarly, Kappeler and colleagues (2015: 89) find, "The police worldview" of mission-oriented officers implicates these officers' "perception of policing as the most critical of social functions...the 'thin blue line' that stands between anarchy and order...Brave police officers patrol mean streets and are on the front lines of a war for social order and justice.” In the context of Paoline's seven typologies across the rank-and-file officer population, in contrast to 'Lay-Lows' and 'Avoiders' (that I advance bear much similarity to many de-policers identified in the present study), which he observes "are just doing their time, avoiding as much police work as possible...[doing] just enough to wear the uniform," there are, on the other hand, 'Tough- Cops' and 'Clean-Beat Crime-Fighters'... [that] have a mission”' (Paoline 2004: 212). This can be related to other scholars' observations around officers' development, over time, of a ‘working personality' (Skolnick 1966; Van Maanen 1973, 1974, 1975), which I suggest, in turn, can be related, conceptual, to the present study's understanding of a de-policing continuum. ${ }^{52}$ Officers eventually make a decision to practice, or not to practice, de-policing - as their 'working personality'. Missionoriented officers choose the latter, as to do otherwise would be inconsistent with their fundamental beliefs about the important obligation of the front-line officer to serve and protect the citizenry.

Campeau's (2015) observations, in this regard, are important in the context of the present study's findings around a de-policing continuum and front-line officers' depolicing adaptations, over time, to risks arising from today's socio-political policing landscape. She finds that 'mission-action' "as means to making sense of one's job loses ground for police in a time of intense oversight and public scrutiny. Instead, police draw 


\section{on cultural scripts about risk because these are better suited to navigating their}

current circumstances" (Campeau 2015: 682, emphasis added). I advance that this empirical determination is corroborative of one of the key de-policing findings of the present study - in that front-line officers are assessing that de-policing makes sense in today's 'risky' policing landscape, from a self-preservation perspective, and, further, that such an approach to police work has become a subcultural ethos (a 'cultural script'). Similarly, I suggest that Reiner's (2010) discussion implicating 'career disappointments' experienced, and/or perceived, by the category of officers characterized as 'uniform carriers', which I suspect would encompass negative interactions in the field and enduring citizens' critiques of, and complaints filed about, one's front-line police work, can also be viewed as implicating the concept of a depolicing continuum - in that there is observed an appreciable decline in officers' 'sense of mission' and officers are observed to become a certain 'way' over time. In this regard, he finds, “The salience of a sense of mission obviously varies between police officers...many (if not most) 'uniform carriers' ([those] who shirk the work as much as possible), with their quintessentially cynical views...became that way precisely because of the effects of career disappointment destroying a prior sense of mission" (Reiner 2010: 120-121).

In this vein, Van Hulst (2013: 624) suggests that if the transition process from officers' initial mission-orientations, as depicted throughout 'traditional' conceptions of police occupational culture, to subsequent refinements in officers' attitudes and behaviours, were to "be summarized yet again, these two sentences might do: Police officers see themselves as crime fighters on a mission in a dangerous environment. 
Gradually, they have turned into cynics who make up their own informal rules (coveryour-ass, lay low and avoid trouble), as the exciting work they thought they had and the respect they though they deserved only sporadically came their way." I argue, specifically in relation to the observed de-policing phenomenon in the present study, that consistent with the concept of a de-policing continuum, a similar process occurs with many officers, in them adapting to challenges in the techno-social and sociopolitical external front-line policing landscape - not in relation to a lack of 'excitement' in front-line police work, but rather, related to the lack of 'respect' alluded to by Van Hulst (2013, which, I argue, can be viewe as synonymous with the accumulation of negative experiences in police-citizen interactions in the field. In this regard, Van Hulst (2013) acknowledges the cynisicm inherent to de-policing and the adapatations that take the form of 'cover-your-ass, lay low and avoid trouble' - de-policing. The following observations provide some 'real world' context in demonstrating the rejection of such de-policing attidues and behaviours among mission-oriented officers:

Fuck it drive on. I assure you, if someone told me to only respond to calls and not dig up my own shit; I would politely decline and advise that I'm not a fire fighter (CAL149)

I am confident in my skill and abilities. We still have to do our job regardless of being criticized or not (CAL328).

Regardless [of public criticism of your actions] you have a job and a mission to uphold (ROC47)

I'm here to do my job. I really don't care about being filmed. I'm gonna do what has to be done (BUF11)

I will not avoid a situation. I stay true to myself and my job (OTT155)

I don't care, I have a job to do (TOR134)

Even with more risk for job consequences we still have a job to do. I have not reached the point yet where I FIDO, but many people I work with (both minorities and Whites) have reached it! (HAM140)

Police work is police work, I can't avoid people (SYR87) 
I choose to conduct myself the same in every experience and do not turn away from that difficult situation or experience (COR14)

People [are] afraid of the job or consequences [resulting from doing it] so they hide from them. These people [are] generally not fit for the job from the start (HAM167)

I will never not be proactive in my job. I love this job and I became a police officer to help people and arrest bad guys (POR03)

$F I D O=$ find another job $($ SAS55)

I refuse to work with these people [that practice de-policing] and [I] have 'outted' them (TOR288)

I do the job as it needs to be done regardless of public opinion (BUF78)

I believe de-policing relates to abandoning police services (SAS109)

Just ignore what is happening and drive on = cowardice!!! (TOR288)

Some avoid being active due to public perceptions. I stay active (BUF11)

[De-policing] is cowardly and unprofessional (OTT192)

Yes [I have observed de-policing], generally by members who are too timid and afraid of the job. I've seen it from the beginning [of my career], wrong career choice made [by these officers] (TOR288)

Most YPD officers do [de-policing] and those of us who don't think it's disgraceful and disrespectful to us who are tyring to change this department into a profession [unintelligible] (YON35)

A tangential consideration with mission-oriented officers is their inflexibility in relation to how police work in the field is to be performed. The rigid stance of a Toronto officer is representative, I am not going to change how I do my work, I don't care what anyone thinks about it (TOR40). I suggest that a problem with this attitude can arise because mission-oriented officers, while not amenable to the influence of their subculture and their challenging external techno-social and/or socio-political policing landscape, are also not likely amenable to reforms that may be initiated within the institution of policing, such as the adoption of procedural justice as the new standard for interactions with citizens. Waddington (1999: 299-300) is instructive in this regard, 
observing that mission-oriented officers' "vision of a 'thin blue line' not only places the police in the position of valiant protectors of society...[but that] criminals lie beyond the moral community of society, the suppression of whom 'serves and protects'...the remainder of the respectable citizenry."

This unequivocal rejection of not only de-policing behaviours, but also depolicing attitudes, extends, not only among 'mission-oriented' or 'New' rank-and-file officers but essentially across all non-depolicers, to the point where these officers do not even give any thought to altering their conduct in the field towards the practice of depolicing. In other words, and as demonstrated in Table 38, with the relevant portion reproduced below, not only are non de-policers not practicing any de-policing behaviours, they do not even give any thought to changing their practices in this regard - despite it being, apparently, all around them in their everyday work setting. Along these lines, $94 \%$ of study participants categorized as non de-policers report giving no consideration whatsoever to today's circumstances of visibility and/or public critique, in deciding on their operational actions in the field. Of course, within this group are 'New' officers who, for the same reasons they do not practice de-policing also do not think about practicing de-policing - for many, a lack of experience in the field. Regardless, this data demonstrates the profound sense of commitment to their vision of police work that exists among mission-oriented officers. Whatever an officers' motivation is to not practice de-policing, clearly, for the vast majority of these officers, this is not an equivocal stance, at least at that stage in their career progression, given that they apparently do not even think about de-policing. 


\begin{tabular}{|c|c|c|c|c|}
\hline & $\begin{array}{c}\text { Have thought } \\
\text { about de- } \\
\text { policing (video) }\end{array}$ & $\begin{array}{c}\text { Have not } \\
\text { thought about } \\
\text { de-policing } \\
\text { (video) }\end{array}$ & $\begin{array}{c}\text { Have thought } \\
\text { about de-policing } \\
\text { (criticism) }\end{array}$ & $\begin{array}{c}\text { Have not thought } \\
\text { about de-policing } \\
\text { (criticism) }\end{array}$ \\
\hline $\begin{array}{c}\text { Non De- } \\
\text { Policers }\end{array}$ & $5.9 \%$ & $94.1 \%$ & $6.1 \%$ & $93.9 \%$ \\
\hline
\end{tabular}

It is also noteworthy that officers working in front-line police work categorized as 'Other' (i.e. tactical, canine, gang intervention, traffic enforcement, school liaison) present considerably higher proportions of non de-policers (44.6\%) than do patrol officers $(28.8 \%)$ and even higher in relation to the other front-line categories examined in the present study, as depicted below in the relevant portion of Table 30.

\begin{tabular}{|c|c||c|c|c|c||}
\hline \multicolumn{2}{|c|}{} & $\begin{array}{c}\text { Non de- } \\
\text { policers }\end{array}$ & $\begin{array}{c}\text { Limited } \\
\text { de- } \\
\text { policers }\end{array}$ & $\begin{array}{c}\text { Moderate } \\
\text { de-policers }\end{array}$ & $\begin{array}{c}\text { Intensive } \\
\text { de-policers }\end{array}$ \\
\hline \hline \multirow{3}{*}{$\begin{array}{c}\text { Type of Front- } \\
\text { Line Police } \\
\text { Work }\end{array}$} & Patrol & $28.8 \%$ & $18.4 \%$ & $23.4 \%$ & $29.3 \%$ \\
\cline { 2 - 6 } & Community & $17.4 \%$ & $14.7 \%$ & $14.7 \%$ & $53.2 \%$ \\
\cline { 2 - 6 } & Foot/Bike & $18.8 \%$ & $20.0 \%$ & $30.6 \%$ & $30.6 \%$ \\
\cline { 2 - 6 } & Other & $44.6 \%$ & $24.3 \%$ & $18.9 \%$ & $12.2 \%$ \\
\hline \hline
\end{tabular}

There are two consideration related to this finding. First, recall that the first few years of virtually every police officers' career are spent as a general patrol officer and therefore, given the situation with 'New' officers and their relatively low de-policing proportions, as discussed throughout the first few paragraph of this subchapter, this finding is, I argue, even more compelling. This is because officers' progessions from a first assignment in general patrol to front-line duties primarily involving foot/bicycle patrol, community-based policing, tactical, canine, gang intervention, traffic enforcement, school liaison, etc. typically do not take place until an officer has several years 'on the job'. Therefore, a more apples-to-apples comparison, in terms of the impact of mission orientation on an officers' de-policing behaviours, is 'Other' officers 
versus 'Community' and/or 'Foot/Bike' officers. Along these lines, it seems likely that officers wanting to specialize within the rank-and-file policing function to work on the tactical team, the canine unit, a gang intervention task force, or in school liaison ('Other'), have, in comparison to officers working in other front-line assignments (patrol, community, foot/bike, and mixed), more of a sense that they want to accomplish certain altruistic objectives in their work, which is consistent with the mission-oriented mindset.

\section{Non De-Policers' Resistance to the Rank-and-File Police Subculture}

In relation to the impact, or lack thereof, of the rank-and-file subculture on non de-policers' views of de-policing, I argue that these study results substantiate that the front-line police subculture and individual officers' practices in the field, for that matter, are not monolithic. In fact, the findings of the present study reveal considerable diversity among individual front-line officers - in terms of their degrees of adherence to the dominant subcultural ethos around de-policing and their work practices vis-à-vis perceptions of risk in today's policing environment. As discussed within subchapters 3.4 and 6.6, characterizations of a monolithic police culture are common throughout the literature, however within the body of competing research, it is found that there is actually considerable diversity, in many respects, among the members of the rank-andfile subculture - in terms of individual officers' attitudes, behaviours, and adherence to the dictates espoused within their occupational subculture. I include the caveat 'in many respects' because, surely, no front-line officer would fail to act on the imperative within the subculture to come to the aid of a fellow officer in need of assistance in dangerous circumstances. 
In saying this, police occupational culture and the process of socialization therein is, undoubtedly, a powerful homogenizing force (Crank 2014; Gau \& Paoline 2017; Loftus 2010; Paoline 2001; Paoline \& Terrill 2014; Van Maanen 1974). However, supported by the empirical results in the present study, I agree with policing scholars (e.g., see Campeau 2015; Chan 1996, 1997, 2003; Cockcroft 2013) who find that 'traditional' representations of police culture, which assume uniformity rather than accounting for variations in culture and that downplay variations in officers' behaviours within the culture, fail to appreciate the agency of police actors in making up their own mind as to whether or not they accept the features of the prevailing occupational culture in their workplace and, furthermore, to what extent they accept them. As Chan (1996: 111) observes, "While the culture may be powerful, it is nevertheless up to individuals to accommodate or resist its influence." In this vein, not all officers are susceptible to the influence of police culture and within that culture, officers can, and do, exercise agency, including "making individual decisions about [what they consider] 'good' and 'bad' police practices" (Chan 2011: 76, citing Chan 1997; Chan et al. 2003). On this point, Foster (2003: 199, citing Chan 1996 and Dixon 1997) notes, "Recently, research has begun to acknowledge more fully the heterogenous nature of police culture, and the importance of police officers being active agents in the formation, acceptance or rejection of particular aspects of it."

Such is consistent with Cordner's (2017: 12) observation that "a series of studies since the late 1960s [Broderick 1977; Brown 1988; Muir 1977; Wilson 1968] have identified a variety of styles of police behaviour, suggesting that socialization and culture, however strong their influence, do not necessarily produce a single way of 
doing police work or a single vision of the police role." Along the same lines, Chan and colleagues (2003: 201, citing Chan 1997; Manning \& Van Maanen 1978) find,

We cannot assume that the socialization process [of rank-and-file officers] is uniform or linear: there can be many 'twists and turns' in a police career as individuals adapt to new circumstnaces and environments. Neither can we assume that within a police organization there always exists a single, dominant, stable culture to which recruits eventually become acculturated...[recruits have] to come to terms with various models of practice and decide for themselves what type of police officers they would become.

\subsection{Researcher Positionality, Participants' Trust, and Data Integrity}

This final subchapter in Chapter Six continues along the lines of the last, in terms of being relatively straightforward. However, it is also different than most other subchapters in that, while it does discuss empirical results from the present study in the context of the extant literature, given the dearth of scholarly contributions in this domain, this subchapter takes on more the role of instigating, among the readership, consideration of an important conversation that I think we, as a community of social scientists, need to have, and with some urgency. In this regard, six years ago, after completing an empirical study examining the impact of 'policing's new visibility' on front-line officers' use of force practices, which involved 231 research participants from Toronto and Ottawa, I observed, "The repeated references by interview research participants to the provision of (or [the potential] withholding of) truthful, complete and forthright data was a revelation I had not expected and something that likely warrants more investigation in considering police research generally" (Brown 2013: 186-187).

Many of the rank-and-file officers that I spoke with during those interviews told me candidly that they would not have participated in that study's surveys and/or interviews, or alternatively would have outright lied or provided 'superficial', 
'politically-correct', 'neutral', 'meaningless', 'bare minimum', or 'by-the-book'

answers to the questions, had they not determined, in their own minds, that they could trust me and my objectives in conducting the research (Brown 2013, 2016). In this regard, my standing as an academic researcher cannot be divorced from my practitioner experience as a former 28-year operational police officer - a status now commonly known as the 'pracademic' (Sherman 2013). According to many of the participants in that 2012-2013 study, my 'pracademic' status meant that those rank-and-file officers afforded me an elevated level of trust and therefore, they were more honest in their contributions of data, than would have been the case were I to have been a more traditional academic - one who has spent their career in the world of academia rather than as a practitioner in the field. In other words, the data I received was different more honest, frank, and comprehensive - than it otherwise would have been in other circumstances. I ask the reader to pause for a moment here and consider not only the implications of this apparent reality for methodological concepts like reliability, replication logic, and validity, but that research findings based on bona fide empirical data is at the core of our work, our raison d'être, in social scientific research.

\section{Context}

Before delving into the present study's empirical data related to researcher positionality, participants' trust in the researcher, and implications for data integrity, I present some framing context. In this regard, it is axiomatic to observe that the rankand-file police subculture involves a code of silence among officers, an inherent suspicion of 'civilians', and a distrust of academics (e.g., see Blake 1981; Brannick \& Coghlan 2007; Chan 2007; Chappel \& Lanza-Kaduce 2010; Ericson 1982; Loftus 2009, 
2010; Marks 2004; O’Neill et al. 2007; Reiner 2000; Rojek et al. 2015; Rosenbaum 2010; Skolnick 1966; Wortley 2006). It has also been recognized, albeit sporadically, and at a relatively muted volume, that this mindset and worldview among front-line officers can impact on the acquisition of meaningful data in studies of policing and that such challenges are even more pronounced in research probing the inner workings of police occupational subcultures, interpersonal issues among officers, and conflicts in the field (Brown 2013, 2016, 2017; Brown \& Primeau 2015; Davis et al. 2005; Goold 2004; Kutnjak-Ivkovic 2014; Rojek et al. 2015). While these challenges that "confound efforts to study the clandestine behaviour of the police" (Holmes and Smith 2008: 9) are well known to most policing scholars, very little has been written about, and even less research has been conducted regarding, the implications of researcher positionality, participants' trust, and data integrity on the social scientific investigation of front-line officers' conduct in the field (e.g., see Adler \& Adler 1987; Brown 2013, 2016, 2017; Brown \& Primeau 2015; Dawson \& Williams 2009; Loftus 2009; Marks 2004; Marks et al. 2010; Reiner 2000; Rosenbaum 2010; Van Maanen 1978; Wortley 2006).

The limited contributions to date, find that difficulties are often encountered in acquiring the trust of police officer research participants, which is, obviously, essential to the receipt of candid, complete, and honest information from this research population (e.g., see Brown 2013, 2016, 2017; Brown \& Primeau 2015; Davis et al. 2005; Fox \& Lundman 1974; Goold 2004; Ericson 1982; Loftus 2009; Marks 2004; Paoline \& Terrill 2014; Reiner 2000). Perhaps it does not require articulation, but to err on the safe side, as I am sure we all appreciate, if one does not trust someone, particularly in sensitive circumstances - as is the case with many of the issues examined in front-line policing 
research - it is unlikely that they will share $100 \%$ of their knowledge on the matter being investigated. Both Ericson (1982) and Marks (2004) have validated this observation, through their empirical findings that 'traditional' academic researchers must invest extraordinary time and energies to overcome police officers' inherent suspicion of academics and that failure to advance beyond superficial relations with this unique research population can result in the concealment of information and the introduction of illegitimate data into the study.

Specifically, Ericson (1982: 50) found that the front-line Canadian officers he studied only opened up after spending "endless hours...in the privacy of the patrol car with no shift activity." Similarly, Marks (2004: 871, emphasis added), during her investigations into rank-and-file Australian police practices, discovered, "Police members are often suspicious of outsiders. The information that researchers who are not familiar with the internal workings of the police organization may receive may be somewhat less than reliable, as police members conceal what they believe to be 'in house' knowledge." She determined that, without intensive researcher participation within the police culture, only superficial relations could be developed, which "would have rendered the [study's] interviews bald and possibly invalid' (Marks 2004: 872, emphasis added). In the same vein, Loftus (2009: 203) found that even after overcoming initial access and trustworthiness obstacles in her ethnographic research with UK rankand-file officers, "The initial stages of the research were characterized by widespread suspicion towards me and the study.” Remaining in the genre of UK policing research and, along the same lines, Brannick and Coghlan (2007) have related the observations of policing researcher Malcolm Young, who authored An Inside Job: Policing and 
Police Culture in Britain and who was a serving police officer in the Newcastle (UK) Police while he was pursuing his academic research. Young (1991) reported on the existence of "a culture of secrecy" within the police force, with "social science research [being] equated with claptrap and [any officers] engaged in it ran the risk of being labeled a traitor" (Brannick \& Coghlan 2007: 71). In this regard, American policing researcher Jacinta Gau (personal communication, 15 November 2017) observed, “The biggest hurdle in front-line police research is the attitude that, 'If you are not a cop, you don't know'."

\section{My Previous Policing Research Findings}

As eluded to earlier, in my own prior research initiatives examining various issues in contemporary policing (Brown 2013, 2016; Brown \& Primeau 2015) and policing research (Brown 2017), I have been advised by numerous research participants (from Constables to Chiefs of Police) that they have issues with trusting 'traditional' academic researchers and that this lack of trust impacts on the data they are willing to share with such researchers. While acknowledging that one could never know with certainty, I believe that such concerns have been largely mitigated in my research experiences with police officers because of my former career in operational policing and therefore, my current status as a 'pracademic'. In my 2012-2013 study with rank and file officers in Toronto and Ottawa (Brown 2013, 2016); my 2014-2015 study with 20 leaders of Canadian police agencies and 20 Canadian 'traditional' academics doing research in the policing genre (Brown 2017), and the 2015 study I undertook with a 'traditional' academic colleague, involving the interviewing of 57 front-line Ottawa officers (Brown \& Primeau 2015), many research participants advised me that they would not have 
provided truthful or complete information had they not determined that they could trust me, which they validated for themselves based on their knowledge of my biography within operational policing. In total, I conservatively estimate that approximately 75 research participants across these three studies expressed such a perspective on researcher trust and the implication for data integrity. Further, in discussing, and reviewing recordings and our notes from, our interviews with rank-and-file Ottawa officers in 2015, my research colleague (Primeau) and I arrived at the inescapable conclusion that the data that was being provided to me was considerably less guarded, in terms of forthrightness and completeness, than the data being provided to my research colleague.

Specifically, and in relation to my 2012-2013 study, "many participants advised that [my] experience within operational policing afforded them elevated levels of comfort (compared to academics without front-line policing backgrounds) as did the confidentiality covenants in the project's research ethics protocol" (Brown 2016: 300). As an example of this in operation, in a 2015 interview that I conducted with an experienced Ottawa officer who works in gang interdiction, discussing the collection of race-based data by the OPS (Brown \& Primeau 2015; Foster et al. 2016), the research participant began the interview by saying, I'm glad you have been on the job. Cops trust most other cops, but sorry to say but I wouldn't be here if you were a civilian. I can talk like myself and not sugar-coat what we do and what my thoughts are. In this vein, Professor Rick Parent (Simon Fraser University), a retired 30-year Delta police officer himself, observed that while there have been no studies that address the nature of data provided by research participants to researchers that are current or former police 
officers, "There is clearly a level of comfort and trust that allows for robust data gathering that would otherwise be non-reported or weak" (Parent, personal communication, 15 August 2013).

\section{Researcher Positionality}

Briefly, as a thorough discussion around researcher positionality was presented within subchapter 4.6, in describing Merton's observations on 'insider' versus 'outsider' research, Labaree (2002: 100, emphasis added) offers, "The insider is an individual who possesses intimate knowledge of the community and its members due to previous and ongoing association with that community and its members. The general assumption is that this intimate knowledge offers insights that are difficult or impossible to access by an outsider." In this regard, Thomas (2014: 8) observes, "Outside insiders are generally regarded as former police officers...who become academics and conduct research on policing." In assessing the value of 'insiderness', Labaree (2002: 103) observes that such researchers can have privileged access to information otherwise unobtainable and this positionality is, therefore, valuable in some research initiatives - through revealing "the unseen reality" of certain phenomena, exposing otherwise "hidden truths the public is unaware of," and presenting "a unique understanding that is not otherwise achievable by an outsider." As discussed earlier, some scholars (e.g., see Brown 2013, 2016, 2017; Brown \& Primeau 2015; Ericson 1982; Hsiung 1996; Labaree 2002; Loftus 2009; Marks 2004; Rosenbaum 2010) find that research benefits are realized because a researcher's insider status contributes to establishing initial levels of trust with participants, which can result in the sharing of more information in more open exchanges with the researcher. In this vein, Rojek and colleagues (2015: 71), find 
"Surveys that request information on more controversial topics such as use of force, emergency or pursuit driving, and use of tactics or weapons will be...more problematic to get officers to respond and to provide truthful answers...To engage in these types of projects, a sufficient amount of trust has to exist between the agency, [the officers,] and researcher." ${ }^{53}$

\section{Study Participants Conducting an 'Investigation' of the Researcher}

As the reader will have observed, in the information documents that were shared with participating police agencies and potential research participants (Appendices D and E), there is no reference to my previous status an operational police officer and, with much intention, I also steered clear from any such disclosures during my introductory remarks before survey administration on shift briefings ${ }^{54}$ In saying this, clearly all Ottawa Police Service officers would certainly have known my 'pracademic' status and likely many study participants with Le Service de police de la Ville de Gatineau, the Cornwall Community Police Service, and the Kingston Police Force (given their proximity to Ottawa) would have known of my previous operational policing career. I was also not under any illusions that it was likely that information about my policing background would come to the attention of some potential study participants at various data sites, from time to time and in a limited scope, given that several Canadian police executives knew of my research initiatives and my 'pracademic' biography from various meetings I had attended and presentations I had delivered since 2012. However, as documented in study participants' responses to Question 29 in the present study's survey questionnaire, $85 \%$ of the study's front-line research participants came to understand that I was a former operational police officer. Until I tabulated the present 
study's data, I had no idea that this was the case, although perhaps my conversations with numerous officers throughout the data collection period should have alerted me to this situation.

In this regard, many study participants spoke with me after they had completed the survey questionnaire and inquired about my policing career in front-line patrol, 'undercover' drug work, and/or homicide investigation. In turn, I often inquired of them how they had discovered that I had former policing experience. Many related, as was discussed earlier within this subchapter, in the context of my previous studies and research participants validating that they could trust me based on their knowledge of my biography within operational policing, that today's social media, communications technology, and information-sharing landscape make such 'investigations' of any researcher a straightforward matter. Indeed, a simple 'Google search' of my name accompanied by the word 'Ottawa' and/or 'Carleton University' generates a wealth of information about my police career, my media appearances discussing issues in policing, and my previous research - in which I am almost always referred to as a 'retired police officer', 'former homicide detective', etc. Police officers doing an investigation, go figure!

\section{Empirical Results in the Present Study vis-à-vis Researcher Positionality, Participants' Trust, and Data Integrity}

As I advanced in the introduction to this chapter, in suggesting that the present study's empirical results, in support of the major findings, are, in and of themselves, convincing and compelling, I will do so again here - in relation to the circumstances discussed in this subchapter. In this regard, just the fact that 1339 front-line police officers that participated in a research study (out of a potential 3660) reported that they 
were influenced in how they had answered questions in a survey instrument because of knowledge about the researcher's 'pracademic' biography, in contrast to the 'traditional' biography of a different (and hypothetical) academic policing researcher, speaks volumes about the consequential implications of researcher positionality and participants' trust therein, vis-à-vis data integrity. In relation to specifics in the present study, as depicted in Table 26, reproduced below, $43.5 \%$ of the study's research participants that knew of my policing background $(\mathrm{N}=3081)$ reported that it had influenced how they had answered one, some, or all of the questions (1 through 28) in the survey instrument.

\begin{tabular}{|c|c|c|}
\hline & $\mathbf{N}$ & Valid \% \\
\hline No & 1742 & 56.5 \\
\hline Yes & 1339 & 43.5 \\
\hline Missing & 27 & \\
\hline Totals & 3108 & 100.0 \\
\hline
\end{tabular}

The data does not allow for an analysis of how many questions were impacted for each individual research participant nor as a collective research population. However, just in, and of, itself, and without any elaboration, these results are noteworthy and thoughtprovoking.

Study participants answering 'Yes' to survey Question 30 were invited to provide a written explanation for their affirmative response and $53 \%$ of the front-line officers (706 of 1339) contributed qualitative data in this regard. These written elaborations provided context to the quantitative data. Throughout the qualitative data that study participants shared into the present study, they reported: (1) that their responses to the survey questions were more honest than they would have been if another (hypothetical) university-based researcher, who is not a 'pracademic', was conducting the study 
$(\mathrm{N}=259)$; (2) that they had an elevated level of trust in me and/or they perceived that I have more credibility, as compared to another (hypothetical) university-based researcher, who is not a 'pracademic' $(\mathrm{N}=117)$; (3) that $\mathrm{I}$, as a policing researcher, am someone they could relate to $(\mathrm{N}=108)$; (4) that they would not have participated in the survey if it was not for my policing background ('pracademic' status) and/or that they put more effort into participating in the study because of my policing background $(\mathrm{N}=102)$; that they perceive that I am fair and/or unbiased $(\mathrm{N}=36)$; and (6) that they perceive that I have more integrity, as compared to another (hypothetical) universitybased policing researcher, who is not a 'pracademic' and/or that they trust I will treat study data ethically $(\mathrm{N}=31)$.

Further, as demonstrated in Table 42, the relevant portion of which is replicated below, the influence of my 'pracademic' biography on research participants' answers in the questionnaire is even more important when considering the study participants that were most impacted.

\begin{tabular}{|c||c|c|}
\hline & Influenced & Not Influenced \\
\hline \hline Non de-policers & $21.1 \%$ & $78.9 \%$ \\
\hline Limited de-policers & $29.8 \%$ & $70.2 \%$ \\
\hline Moderate de-policers & $42.3 \%$ & $57.7 \%$ \\
\hline Intensive de-policers & $69.8 \%$ & $30.2 \%$ \\
\hline
\end{tabular}

In contrast to non de-policers (at only $21 \%$ ), $70 \%$ of intensive de-policers were influenced by my 'pracademic' biography in how they answered questions 1 through 28 in the survey instrument. It is important to appreciate that these are the study participants that reported practicing extensive risk-avoidance behaviours in their police work because of 'policing's new visibility'; and because of today's elevated societal scrutiny, and criticism, of front-line officers' actions; and because of the 'demographic' 
characteristics of some persons in the community that these officers find to be particularly problematic ('risky') - in terms of the officer's perception that such persons have a particular propensity for criticizing, or filing a complaint about, police conduct. Those are not easy things for a police officer in today's day and age, to admit, much less to an essential stranger (even one in the role of an ethical and 'pracademic' researcher) and in writing.

In this regard, disclosing that you refuse, or fail, to perform a core function in your professional responsibilities, such as engaging in proactive police work, is an important and potentially consequential admission, which, as expressed by police and/or police labour organization leadership in Winnipeg, Vancouver, Peel Region, and Yonkers, in my discussion about their officers participating in the study (as detailed in Chapter Four), can be considered a serious dereliction of a police officer's duties. And, as discussed throughout subchapter 6.8 , issues around policing and race, such as a frontline officer admitting to avoiding interactions with, for example, individuals perceived to be a 'visible minority', are 'hot button' socio-political and cultural issues that could result in grave consequences for an officer were they to be exposed (i.e. being found to be a racist in a human rights and/or internal police investigation, being publicly and professionally shamed as a result, and potentially facing disciplinary sanctions for not performing their work in accordance with established policy). Admitting to such behaviours in a research setting represents the opposite of social desirability bias, in which respondents are reluctant to admit to what many throughout society likely consider unacceptable, and, in my view, such candid revelations from a research participant substantiates that trust in the intended recipient of that related information - 
the researcher - has been established to the threshold where such sensitive information can be shared within that academic study of policing. ${ }^{55}$

In concluding this subchapter, in my view, there is no point in reproducing more study participant's written elaborations here, as a review of the representative selections presented earlier within subchapter 5.2 should suffice for the present purposes. In saying this, a complete accounting of all 706 qualitative elaborations are available to any researcher that expresses an interest. However, in transitioning into this dissertation's concluding chapter, the following observation of a Hamilton officer captures the essence of many of the study participants' contributions in this regard, I feel comfortable answering honestly because this researcher is from outside the HPS and the university 'establishment'. I trust him with the truth, which is sad because what does that say about other researchers (HAM131)? 


\section{CHAPTER SEVEN}

\section{CONCLUSION}

\subsection{Chapter Introduction}

Weber (1947, as cited in Keat \& Urry 2011: 107) advanced that sociology is "a science which attempts the interpretive understanding of social action." In this vein, and as was Banton's (1964: vii-viii) objective in his seminal research into the practices of British police officers in the community more than 50 years ago, my aim in this dissertation (minus the gendered terminology of that era) is also "to understand the policeman's occupation in its social context." Similarly, I argue that Rubinstein's (1973: ix-xi) observations almost a half century ago, as he began his landmark research into how Philadelphia's rank-and-file officers performed their front-line police work 'on the streets', remain essentially the same in 2019,

Despite the attention of countless writers, reporters and scholars, our understanding of what policemen do and what police work is remains murky...Newspapers and television are filled with stories about the police, but they tell us little of how they go about their work...Instead of studying the work, [scholars] report on [various other aspects of policing, such as its organization or administration,] instead of describing what the men do. ${ }^{1}$

As discussed in preceding chapters, empirical studies of contemporary police practices in the field, from various perspectives, including the often-neglected vantage point of policing's rank-and-file, are both necessary and valuable - some might argue imperative, given the central socio-political positioning of the front-line police officer in our Western democratic systems of governance and the fundamental importance of the public-police relationship in the context of the social contract. In this regard, Worden 
(1995b: 49) finds, "The attitudes [and behaviours] of police officers appear as central constructs in many studies of the police."

\section{The Present Study's Contributions}

The present study addresses not only demands from former FBI Director Comey and former American President Obama as detailed in Chapter One, but also various scholars' encouragement for further scientific study into the previously unascertained phenomenon of de-policing (e.g., see Campeau 2015, 2016a, 2016b; Chanin \& Sheats 2018; Deuchar et al. 2018; Lautenschlager, Capellan \& Silva 2018; Marier \& Moule 2018; Murphy 2014; Newell 2019; Nix \& Wolfe 2018; Nix et al. 2018; Oliver 2017; Pyrooz et al. 2016; Rushin \& Edwards 2017; Sandhu \& Haggerty 2017; Shjarback et al. 2017a, 2017b; Todd 2015; Wolfe \& Nix 2016). In this regard, this dissertation presents empirical data and analytical interpretations in furtherance of testing the findings of, and advancing the scholarly knowledge base around, existing work that has been undertaken in this, or closely-related, area(s) of inquiry (e.g., see Brucato 2015a, 2015b; Doyle 2003; Goldsmith 2010; Goold 2003, 2004; Haggerty \& Sandhu 2014; Heaton 2010; Newburn \& Hayman 2002; Sandhu 2016, 2019; Sandhu \& Haggerty 2015). It is my position that risk-averse practices across front-line police work, in response to recent transformations in techno-social and socio-political policing landscapes, have never before been adequately measured nor sufficiently understood - as was also observed by Oliver (2017) in his exploratory study of de-policing behaviours across American rankand-file officers.

This dissertation's presentation of, and discussion in relation to, the results of the first mixed methods, transnational, and multi-jurisdictional empirical study of de- 
policing should take a step toward addressing this research void and contribute to the developing literature in this area. In doing so, this research has also addressed scholars' appeals for more detailed empirical and micro-sociological investigations implicating various contemporary social, political, technological, and cultural phenomena - such as 'new visibility', the ubiquitous presence of surveillance throughout society, social practices of 'witnessing,' 'participatory culture,' 'citizen journalism,' and the development of 'new social movements' in our digitally-interconnected society (e.g., see Andrejevic 2007; Antony \& Thomas 2010; Ball et al. 2012; Brucato 2016; Doyle 2003; Farmer \& Sun 2016; Goold 2004; Goldsmith 2010; Greer \& McLaughlin 2010b; Haggerty 2006; Haggerty \& Sandhu 2014; Hermida and Hernández-Santaolalla 2018; Huey et al. 2006; Koskela 2003, 2006, 2011; Kurasawa 2008; Lyon 2007; Mawby 2010; McCahill \& Norris 2002; Monahan 2006, 2010; Newell 2014, 2015, 2019; Neyland \& Coopmans 2014; Sandhu 2016, 2019; Sandhu \& Haggerty 2017; Thompson 2005; Wilson \& Serisier 2010).

In relation to the front-line officer's occupational subculture, Terrill and colleagues (2018: 805) observe, "Police culture is an area of scholarship ripe for continued theoretical and empirical examination as the importance of understanding the role of culture on behaviour is perhaps at an all-time high." Various scholars suggest that the best time to study occupational culture is during moments of unsettling change (e.g., see Campeau 2015; Swidler 1986; Vaughan 1996, 2002) and, in this regard, I suspect few would contest that today's circumstances represent a period of significant upheaval for both constituents in the public-police relationship - the police and the policed. ${ }^{2}$ As discussed earlier, various observers have characterized the present state in 
the policing of society as nothing less than a 'crisis'. Ingram and colleagues (2018: 780) argue, "Although recent advancements have been made in the understanding and studying of police culture, several significant gaps remain, including deficiencies in theoretical development and the lack of research on culture's influence on police practice."

The present study has sought to contribute to both identified areas and, in this regard, this research follows in the tradition of the seminal micro-sociological works of, for example, Reuss-Ianni (1983) on the 'street cop' culture and Ericson (1982) on the officer's 'recipe of rules' and of the classic police ethnographies - those studies that examined the innermost thoughts and behaviours of the men and women who perform front-line policing in our communities and the workings of their rank-and-file subculture (e.g., see also Banton 1964; Fyfe 1988; Manning 1977; Muir 1977; Reiss 1971; Rubinstein 1973; Skolnick 1966; Van Maanen 1973, 1974, 1975; Westley 1970). In this vein, the present study sought to gain insights into the attitudes, assumptions, and behaviours of today's rank-and-file officers and those of their front-line occupational subculture, through acquiring honest, complete, and detailed data in furtherance of developing an empirically-based understanding of these officers' feelings around, and tangible reactions to, recent techno-social and socio-political modifications to the external environment in which they do their work.

\section{Distinguishing the Present Study's Findings}

I will not engage in a prolonged and detailed effort to situate the present study's findings within the extant literature - a review of Chapter Two in the context of the present study's findings will accomplish this objective. However, I 
do feel compelled to distinguish some particular results from those previously reported by Brucato (2015a, 2015b), Sandhu (2016, 2019), and Sandhu and Haggerty $(2015,2017)$. Specifically, Brucato (2015b: 39), in “[r]ecognizing the resilience of the police institution despite the new visibility of its violence," he then "challenges the presumption that increased transparency will promote institutional reform or crisis [in policing].” The present study finds that there is most certainly, by, I would argue, virtually anyone's definition, a crisis - with over 70\% of today's front-line officers practicing de-policing, many specifically in response to the 'new visibility' of officers' actions in the field. I suggest that this contradiction likely arises because Brucato's analysis was predicated largely on a relatively small data set, derived during his participant observation with, and interview data from, American anti-police activists with whom he sympathized, in contrast to the present study's wider empirical foundation. In this context, the reader is encouraged to consider the counsel offered by Greenhill (1981: 91), who, from his position as a sociologist embedded within a police agency, observed, "Nothing [is] surprising in police and sociologists evaluating each other; what is important is to encourage an awareness of the distorting effect on the perception of facts which evaluative prejudice can produce.”

On the other hand, the present study's findings are entirely consistent with the initial position advanced by Haggerty and Sandhu (2014: 11), in their article The Police Crisis of Visibility, in which they argued that today's techno-social policing landscape has fundamentally changed "the dynamics of policing on the ground... [putting police officers] in the midst of a new and quite different crisis of legitimacy." However, as 
detailed in Chapter Two, in subsequent publications these scholars (see Sandhu \& Haggerty 2015, 2017; Sandhu 2016, 2019) have advanced that "the crisis narrative is overstated and undernuanced" (Sandhu \& Haggerty 2015: 4) and that the vast majority of their study participants report being indifferent to their new occupational visibility or having learned "to adapt to cameras by engaging in "camera-friendly policing"” (Sandhu 2016: 79). ${ }^{4}$ It should be noted however that Sandhu's field research combined the experiences of Edmonton transit and university 'quasi-police' personnel, which made up the bulk of the study's 60 research participants, with those of the handful of Edmonton Police officers that took part in the research, and thus its applicability to other police contexts may be somewhat limited.

\section{De-Policing - Past and Present Contexts}

As detailed throughout earlier chapters, de-policing, as I define the phenomenon, has now been discussed within academia, across the public sphere, and inside the institution of policing itself, for at least two decades - albeit intermittently (excepting its continuous presence within the rank-and-file subculture) and, until very recently, with little social scientific investigation into the claims advanced in various, yet limited, research and discourse on the topic. In this regard, I find it confounding that more empirical work has not been done to date, given that de-policing can be considered antithetical to the most fundamental principles of contemporary policing that have been espoused over the past half century across Western democracies - including the central location of proactive police work and discretionary engagement with citizens as the prinicipal tenet of community-based policing (CBP). Even on occasions when alarm has been raised about this practice, as with the circumstances in Cincinnati and Seattle near 
the turn of the century (addressed previously within Chapter Two and in the following paragraph), in my view, and especially relative to the 'real world' importance of such mis/conduct, there has been only sporadic and largely muted reaction within policing's governance and leadership and across the public sphere. In this regard, risk-averse practices in front-line police work is not a novel idea - as described, it was certainly around when I began my police career in 1985 - nor is de-policing a phenomenon that has never before been addressed within mediated societal discourse, although, as I have argued, it is surprising that it has not attracted more attention.

For example, almost twenty years ago, the popular $A B C N e w s$ program 'Nightline' presented a full episode investigating 'Protect and serve? De-policing in America's urban neighbourhoods' - with much of the focus on the acrimonious state of public-police relationships in Cincinnati and Seattle and officers' withdrawal from engagement. ${ }^{5}$

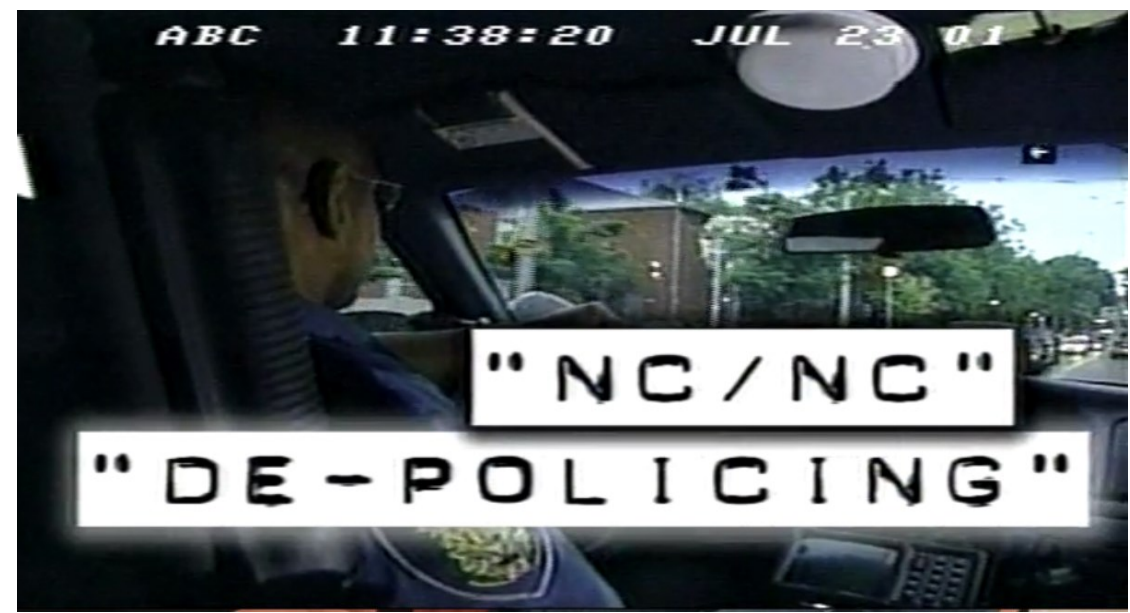

Image 11: 'Protect and serve? De-policing in America's urban neighbourhoods' ( $A B C$ News 2001; screen capture by the author)

The following are excerpts from the program's discussion:

$A B C$ News anchorperson Ted Koppel: With controversial police shootings come charges of racism and brutality [with video recorded footage of rioting and violent clashes between citizens and police in Cincinnati and Seattle scrolling in the background]... 
Many officers, Black and White, respond by dropping aggressive policing for passive policing. The cops even have code words for it - de-policing or NC/NC.

Officer Mike Solan (Seattle Police Department): No contact, no complaints. You read a book. [You] do a crossword. If you don't have any contacts on the street, you're not going to get a complaint.

Koppel: Tonight, America in Black and White: To Protect and Serve. A deadly dilemma...Look at it from the cop's point of view. He's trained to go after crime proactively. Don't wait for it to happen, try to prevent it before it happens... Traffic stops are a big part of the program. Establishing a visible presence in 'high crime areas,' another major part. But then 'high crime area' can be often just another way of saying 'minority neighbourhood.' And when the people being pulled over in those traffic stops tend disproportionately to be Black or Hispanic, before you know it you are being accused of racial profiling. So, what happens? Well, for one thing the cops are taking shelter behind a barricade of euphemisms - 'de-policing' is one of them, 'selective disengagement' is another, as is 'tactical detachment.' They all amount to the same thing. If aggressive law enforcement is being equated with racism, say some police officers, let's see what the community things of passive law enforcement. Case in point, Cincinnati...

$A B C$ News correspondent Dean Reynolds: Since riots convulsed Cincinnati in April [2001] following the fatal shooting of a Black suspect by a White policeman, the divide between the people and their police has grown dangerously wide and deep. Whatever trust there was is gone now...In this hyper critical atmosphere and with what they say is half-hearted support from civic leaders, the police here have adopted a new attitude...

Keith Fangman (President of the Cincinnati Fraternal Order of Police - the union representing rank-and-file officers): Officers have begun to throw their hands up in the air and say, 'You know what, we give up, you win, we can't do this anymore.'

Reynolds: The cops here have become more passive, more 'by-the-book', initiative is out, caution is in. That means they've dropped a lot of crime prevention tactics, like traffic stops that were seen by some as petty harassment or by others as grounds for lawsuits and investigations into racial profiling.

Sergeant Paul Broxterman (Cincinnati Police Department): It's important to put ourselves in the officers' shoes and understand that they may be somewhat tentative in 'jumping out' on a subject involved in a dice game or a drug transaction because they have seen what has happened to brother officers as far as the financial loss and the stress and everything else [they have gone through].

Reynolds: In other words, if they throw the book at your for doing your job, why bother?

Officer Jonathan Gordon (Cincinnati Police Department): Now we go somewhere, we go study our Spanish, we go read, [we] study our procedure manual. We stay out of the way until we are dispatched.

Reynolds: Cincinnati is far from the only place in this country where police feel unappreciated...The police here in Seattle are facing the same kind of racially-charged 
criticism as are the Cincinnati Police. The fact that the sergeant [Sergeant Alvin Little who is interviewed in the story] is African American means little. In his view, it's the blue uniform that turns people off... The police in Seattle are doing much the same things as their brothers in Cincinnati. They call is a policy of no contact, no complaint.

Officer Mike Solan (Seattle Police Department, emphasis added): We are going to be like the firehouses now. We are going sit in our precincts, get a 9-1-1 call, and we will respond from the precinct. That's what's going to happen, no contact, no complaint.

Reynolds: In the view of the police, the risk of being more aggressive can be too much. Even in dealing with a minor traffic violation...And to the police in Seattle, Cincinatti, and a lot of other cities, there is a supreme irony in the issue of profiling. To the men and women in uniform they are the one's being profiled - by the public...In such an atmosphere it might not be surprising to find the police withdrawing from many of the routine battles they used to fight. Perhaps with the hope that one day their absence will make the public miss them.

More recently, in this post-Ferguson era, there has been an uptick in both traditional and online media, and public sphere, attention to many aspects of contemporary policing - including further, yet still intermittent, discussions around depolicing. As with examples presented in preceding chapters, in the lead story on Fox News' national primetime broadcast of 5 May 2017, anchorperson Chris Wallace addressed an FBI report that concluded there is an ongoing and significant 'pullback' by many across American policing's rank-and-file from work that was previously considered part of an officer's routine duties (see Federal Bureau of Investigation 2016).

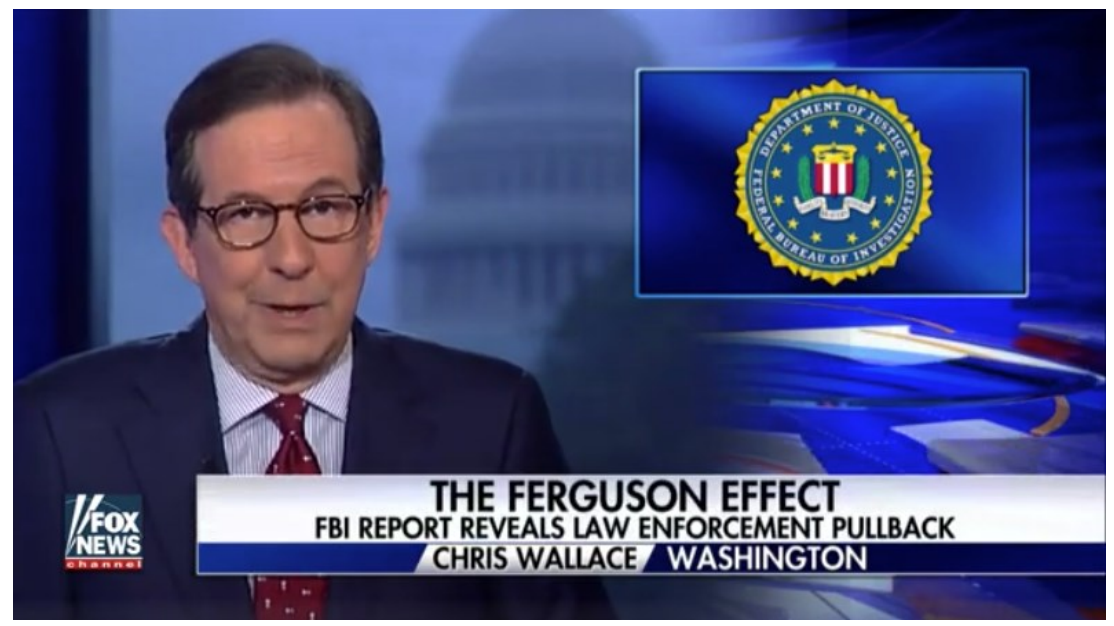

Image 12: 'The Ferguson Effect: FBI report reveals law enforcement pullback' (Wallace 2017; screen capture by the author) 
As foreshadowed earlier in this subchapter, a key question I often ponder is: Why is there, as yet, no greater reaction, across both society and policing, to today's depolicing practices? I perceive (and I follow the issue closely) a surprisingly muted response even with: (1) mediated discussions, with substantial public audiences, that have accompanied the release of preliminary information from the present study; (2) various presentations I have delivered to interested scholars at academic conferences (in Canada, the USA, and Europe), to executive command personnel of police agencies, to leadership of police oversight and governance organizations; and (3) alarming reductions in proactive police work - as documented in 'official' data originating from within the institution of policing itself. Unfortunately, I do not, as yet, have an answer to this question and being a constituent of both dimensions in the public-police relationship (as a citizen interested in being served and protected by the police I trust to ensure the safety of my family and as former rank-and-file officer myself) I am, frankly, dismayed that there has not been much more significant interest in the growing empirical validation of the current de-policing crisis - not only in the present study but also in the recent work of other scholars (e.g., Campeau 2015; Deuchar et al. 2018; Nix \& Wolfe 2016; Nix et al. 2018; Oliver 2017; Pyrooz et al. 2016; Rushin \& Edwards 2017; Sandhu \& Haggerty 2017; Shjarback et al. 2017a; Wolfe \& Nix 2016). In saying this, it is yet to be seen what reactions will accompany any possible attention this research may generate, given that I plan to continue public sociology efforts regarding my findings.

Perhaps, it is the case that, as Comey (2015b) explained to those attending his inaugural 'de-policing' speech at the University of Chicago Law School in 2015, 
“...Nobody says it on the record, nobody says it in public, but police and elected officials are quietly saying it to themselves. And they're saying it to me, and I'm going to say it to you." In other words, de-policing may be viewed by many within the institution of policing as something that is not be shared with the civilian population. As discussed previously throughout earlier chapters, perhaps this is understandable, given that failure to perform key aspects of one's occupational responsibilities is not only socially undesirable behaviour, but can constitute prima facie misconduct, which can be subject to internal disciplinary sanctions.

Or, perhaps it is the case that, as has been documented in various contributions to the literature over the years, how police officers perform their work in the field seems to have only a small impact on preventing or controlling crime and only a marginal influence on public perceptions of safety and disorder (e.g., see Braga, Welsh \& Schnell 2015; Eck \& Maguire 2000; Gottfredson \& Hirschi 1990; Kelling, Pate, Dieckman \& Brown 1974; Lee, Eck \& Corsaro 2016; Ratcliffe, Taniguchi, Groff \& Wood 2011; Tiratelli, Quinton \& Bradford 2018; Wilson \& Boland 1978). ${ }^{6}$ As Paternoster (2010: 766) observes, police tactics such as enhanced enforcement in, and surveillance of, 'high-crime neighbourhoods' are predicated on the assumption that there exists an inverse relationship between police resources and crime rates - in that would-be offenders are deterred from criminal misconduct by the actions of the police officers deployed in the community. In this regard, Bayley (1994: 3) has concluded,

The police do not prevent crime. This is one of the best kept secrets of modern life. Experts know it, the police know it, but the public does not know it. Yet the police pretend that they are society's best defense against crime...this is a myth. First, repeated analysis has consistently failed to find any connection between the number of police officers and 
crime rates. Secondly, the primary strategies adopted by modern police have been shown to have little or no effect on crime. ${ }^{7}$

Maybe de-policing is simply not that consequential in terms of the effects of this widespread practice throughout front-line police work, as it translates into routine lived experiences across the public milieu (and within the priorities of upper echelons of police leadership and governance) - although, given de-policing's fundamental tension with perhaps the most central component of the prevailing policing philosophy of the past half-century throughout Western democracies - CBP - one might think it would be considered of some significance in terms of both theoretically-oriented academic discourse and 'real world' praxis. In other words, given that not-insignificant numbers of the front-line officers, that we, as individuals and as a collective society rely on to serve and protect us, are now engaging in what amounts to the practice of 'fire-brigade policing' in response to what many officers perceive is a 'risky' contemporary technosocial and socio-political external policing landscape - avoiding proactive initiatives and discretionary interactions within the community while performing, for the most part, only reactive police response - it is surprising, at least to this interested observer, that there has not yet developed more concern and consequential discourse across all constituents in the public-police relationship.

The structure of the remainder of this concluding chapter involves informing the reader about acknowledged limitations in the present study (subchapter 7.2), presenting my suggestions for further research (subchapter 7.3), addressing potential solutions to officers' risk-averse attitudes and behaviours - in circumstances where de-policing is not considered a positive outcome (subchapter 7.4); dissemination of the present study's 
findings (subchapter 7.5); and the importance of, and my encouragement for, the practice of public sociology (subchapter 7.6).

\subsection{Study Limitations}

It is important to acknowledge limitations in any research. In this regard, the cross-sectional nature of data collection, which represents a snapshot of front-line officers' attitudes and behaviours at various points throughout 2016, is unable to measure whether, or not, these remain stable over time. In relation to the cross-sectional nature of data collection in his own work on police occupational culture, Paoline (2004: 230) advanced, "The findings here are but a snapshot of officers' occupational attitudes and fail to tell us whether these attitudes, and subgroup membership, are permanent or whether they might change over time.” Further, Myhill and Bradford (2013: 351) caution, in relation to their work, "In common with the majority of similar studies, our data are cross-sectional, thus limiting our investigation to assessment of statistical associations rather than causal effects." In this vein, Tyler and Wakslak (2004: 279) counsel, "Conclusions drawn from such data regarding causality are always tentative..."

Further, as with virtually all of the research in this genre, data in the present study originate from officers' self-reporting as opposed to objectively-measured attitudes and behaviours. It is axiomatic that self-report data are always subject to potential bias (Fowler 2002) however Tyler and Wakslak (2004: 279, citing Tyler 1990) find, "Such measures of behaviour are widely argued to be reasonable reflections of actual behaviour, but they must nonetheless be viewed with caution."

Finally, as Goold (2003) has counseled, there is always a need to be cautious when generalizing from a single empirical study. Along these lines, policing researchers 
often rely on convenience samples recruited within a single police agency in order to examine officers' attitudes and behaviours. Therefore, at least in theory, the heterogeneity of samples obtained from across a variety of agencies and various geographic locations, although not achieving random sampling, will ensure that any relationships found from the data are stronger than those found in more homogenous samples, and, as a result, those relationships can be considered more generalizable to the population (Pickett \& Ryon 2017; Shadish et al. 2002; Silver et al. 2017). In this regard, based on the level of assurance afforded by this study's substantial sample size ( $N=3660$ across 23 diverse Canadian and American police agencies) and its rigorous empirical findings, I advance that de-policing is now a very widespread phenomenon across policing's rank-and-file and, while not in the sense of strict positivist criteria, I argue that the results can, for all intents and purposes, be considered generalizable across the population of front-line officers across Canada and the USA (and likely across a number of other Western democracies).

\subsection{Suggested Areas for Further Research}

I encourage replication research with front-line participants from various police agencies in Canada, the USA, and other Western democracies. I also recommend indepth qualitative investigations, which would provide answers to any lingering questions arising from the present study and, I believe such studies would also corroborate the present study's findings, albeit from a different methodological perspective. Such confirmation across studies would undoubtedly strengthen the findings of all of the research examining the issues under consideration, including the present mixed methods study. For example, an ethnographic project involving long-term 
immersion within the rank-and-file subculture of a police agency might contribute to a better understanding as to whether, or not, there are particular organizational, or other unaccounted for, factors that explain de-policing circumstances beyond the external techno-social and socio-political considerations addressed in the present study. Also, such a research method, in alignment with much of the traditional ethnographic work investigating police occupational culture, would undoubtedly yield data that contributes to better understandings of the front-line subculture, vis-à-vis de-policing. Campeau (2016a: 9-10) is instructive on such considerations, observing,

When first explored by foundational police scholars fifty years ago (Banton 1964; Bittner 1970; Skolnick 1996; Westley 1970) policing was studied as an occupation defined by its environment, with emphasis placed on the struggles faced by officers attempting to navigate the realities of the job. Over time, however...police literature witnessed a shift away from studying policing as a broader sociological phenomenon and moved toward shortterm evaluative empirical projects...Thus, broadly, police studies seemed to lose touch with social, cultural, political and economic forces operating outside the doors of police stations, but which undeniably infiltrate the department and its practices.

As discussed throughout earlier chapters, this dissertation research focuses on two external sources of strain for many of today's rank-and-file officers - involving recently-intensified techno-social and socio-political challenges across the policing environment and the response of de-policing as an outcome. Stenning and Shearing (2005) would characterize these contemporary circumstances, arising as a result of the external environment within which front-line officers operate, as 'externalities' as opposed to 'internalities' - the latter implicating conditions originating within police institutions. Of course, it is not the case that the causes of the complex and nuanced socio-political and occupational subcultural phenomenon that is de-policing, in which myriad assumptions, understandings, influences, and behaviours, are implicated, can be 
isolated to only those external considerations (factors, issues, variables, etc.) addressed in the present study. Along these lines, Sherman (1980) advanced that police behaviour can be examined through five different units of analysis: individual, situational, organizational, community, and legal. The present study is limited to an examination of officers' de-policing behaviours at the individual and situational levels.

In this regard, scholarly contributions focused on organizational challenges confronting today's front-line officer are abundant throughout the literature and a detailed treatment of 'internalities' is obviously beyond the scope of this dissertation. That being stipulated, a concise discussion of the almost infinite range of influences on police officers' behaviours, as characterized by Wallace and colleagues (2018) in their study of 'camera-induced passivity', is instructive. These policing researchers, in following on the work of many other scholars in this area, suggest that these influences can be captured within three broad categories: (1) differences between officers (e.g., see Banton 1964; Bayley \& Mendelsohn 1969; Black 1971; Klinger 1997; Werthman \& Piliavin, 1967); (2) the environments in which policing occurs (e.g., see Bittner 1967; Brown 1988; Muir 1977); and organizational factors (e.g., see Fyfe 1988; Walker 1992). Similarly, Bishopp and colleagues (2018, citing Burke 2016; Violanti \& Aron 1995; Violanti, Fekedulegn, Hartley, Charles, Andrew, Ma \& Burchfiel 2016) observe, "Etiologically focused research has identified, inter alia, two primary sources of stress in policing: organizational and environmental. This body of research shows a strong link between stress and negative affective responses among police and that negative emotions are strong predictors of undesirable outcomes among police officers" (e.g., see also Allisey, Noblet, Lamontagne \& Houdmont 2014; Brooks \& Piquero 1998; 
Patterson, Chung \& Swan 2012; Shim, Jo \& Hoover 2015; Swatt, Gibson \& Piquero 2007).

As an example of the potential influence of 'internalities', Bishopp and colleagues (2018, citing Adams \& Buck 2010; Crank \& Caldero 1991; Kurtz 2008; Shane 2010; Violanti \& Aron 1995) explain, “Organizational structures often dictate the relationship between officers and their organization, and many of these agency/officer relationships are stressful. Departmental policies and procedures, shift work, supervisors, and the promotional process are examples of stressors noted in research on police stress." Such organizational variables may explain why, in considering data presented in Table 32, contrary to what one would expect in relation to 'New' and 'Experienced' officers within the conceptual framework of a de-policing continuum, the NRPS, with a greater proportion of 'New' officers than the OPS ( $26 \%$ to $18 \%)$, has a significantly higher proportion of moderate and intensive de-policers (61\% to $48 \%)$. Perhaps it is the case that there are unique internal circumstances in operation within the OPS and the NRPS organizations that account for this unexpected situation (further information on such observed discrepancies is situated in Endnote 16 within Chapter Six). Such is an example of a potential question still outstanding despite the present study's best efforts, as discussed in this subsection's opening paragraph.

Further, and emerging from discussions during a presentation I delivered on 19 April 2018 to attendees of a special meeting convened by the Ontario Association of Police Services Boards to discuss de-policing, I am advocating for empirical research to determine whether the de-policing phenomenon identified in the present study is an important consideration in rural policing jurisdictions. Several of the government 
appointed officials responsible for small detachments of the Ontario Provincial Police suggested they perceive that front-line officers' attitudes and behaviours in their rural communities might differ from participants in the present study - in that their citizens are more supportive of police officers than those residing in more urbanized communities. While such a study of 'small town policing,' as some in the audience characterized their jurisdictions, would be challenging, in terms of expenditures and logistics, I suggest it would be worthwhile, in contributing to understandings around the universality of the identified de-policing phenomenon. Similarly, it would be interesting to determine whether de-policing practices are prevalent among front-line officers performing policing on First Nations' reserves and/or other communities with predominant Indigenous populations.

As discussed, given inherent limitations with cross-sectional data, longitudinal designs in future research examining de-policing practices and/or front-line subcultural influence would be valuable. There has been very little longitudinal research in the area of officers' behaviours and in relation to police occupational culture (some notable exceptions are Van Maanen 1975, Fielding 1988, and Chan et al. 2003). More recently, Charman (2017) conducted a four-year longitudinal study investigating police occupational culture and police cultural socialization by following study participants from their recruit experiences in the police academy into their early years of front-line work in the community. In this regard, Ingram and colleagues (2018: 805) observe, "The theoretical framework posits that workgroup culture influences officer behaviours. This assumes a certain causal order that cannot be met through a cross-sectional research design." 
Although it is beyond the scope of this dissertation, research into more specific individual determinants of de-policing behaviours would be valuable and may be able to provide some element of prediction - in relation to those persons that would be more likely to become de-policers throughout a typical policing career. The present study has answered questions about some individual and occupational culture circumstances that can result in de-policing, however, further research could shed further light on why some rank-and-file officers are susceptible to front-line situational and subcultural influences associated with the adoption of risk-averse practices - while others are not. As Sklanksy (2007: 29-33) commends in Muir's (1977) research with Oakland police officers in the 1970s,

[Muir tried to] understand why some police officers wind up more effective and more trustworthy than others...[Muir] concluded that good police officers had democratic virtues: a comfort with moral ambiguity, an ability to see shades of grey, a broad capacity for tolerance and empathy, and, perhaps most important, 'an enjoyment of talk' - an affinity, that is to say, for conversation, argument, deliberation, advocacy, and compromise...Muir saw [effective] police officers as 'street corner politicians'.

I also advocate for research into the present study's somewhat unexpected empirical findings in regard to rank-and-file officers, not only practicing de-policing to avoid proactive initiatives and discretionary interactions in the community, but also risk-avoidance practices in situations they encounter in their reactive policing responsibilities. For example, officers responding intentionally slowly to, or avoiding altogether, certain types of dispatched calls and/or not making arrests in circumstances in which an arrest is warranted. Like the de-policing phenomenon, these risk-avoidance practices should be of concern to both constituents in today's public-police relationship. 
Finally, given that the concept of a 'Ferguson Effect' is so topical in today's discourse around the nature of police interactions in the community (or absence thereof in the case of de-policing) and criminal offending in the population, further empirical study specifically focused on whether de-policing has an appreciable influence on, or correlation with, rates of criminal occurrences (and not just homicides or serious violent crimes) would be welcomed within policing, governance, academia, and across the public sphere. Put simply, and in my assessment, having read much of the literature, the current understanding around this notion is mixed (confusing) at best. Appreciating scope restrictions, I will limit my observations, in this regard, to the policing scholar considered by most to be at the forefront of 'Ferguson Effect' research and controversy, Richard Rosenfeld, a professor at the University of Missouri-St. Louis. In the aftermath of the 2014 Michael Brown occurrence in nearby Ferguson, Missouri, Rosenfeld examined St. Louis crime data, given that there had been a 32.5\% increase in homicides year over year from 2013. Rosenfeld (2015) dismissed the notion of a post-Brown 'Ferguson Effect' on homicide occurrences, largely because the greatest increases in 2014 homicides came before Brown's death and the tumultuous aftershocks that reverberated throughout the greater St. Louis area.

However, in an expansive DOJ study the following year, looking not just at St. Louis homicide data, but data from America's 56 largest cities, Rosenfeld (2016) reached an entirely different conclusion in relation to the $17 \%$ increase in homicides throughout 2015 within those 56 cities. In changing his interpretations, Rosenfeld concluded, "preliminarily, that something like a Ferguson Effect was responsible for the 
increase" (Beckett 2016). In his most recent contribution, Rosenfeld, with his colleague Wallman (2019: 41) find,

...no association between reduced rates of arrest and elevated rates of homicide... Whatever sparked the 2015 homicide rise, it was not a reduction in arrest rates in large U.S. city police agencies...[However,] the critics...were mistaken to downplay the significance of the homicide rise. It was not a media creation. It was real, large, abrupt, and in need of explanation...The nationwide homicide rate increased by $11.4 \%$, to 4.9 homicides per 100,000 population in 2015 from 4.4 in $2014 \ldots$ That was the largest percentage change in homicide levels in a single year since 1968.

However, of particular interest to findings in the present study, they also advance,

The idea that police in the post-Ferguson period would become less inclined to confront citizens engaged in prohibited behaviour is not implausible. Concern for one's career, fear of public condemnation, and resentment at a perceived decline in citizens' regard for police work, singly or in combination, might well have engendered such a pulling back, especially from public-order offences and other minor crimes, for which officers have greater latitude in enforcement decisions...There are good reasons for assuming that police disengagements should be reflected in declining arrest rates, especially for less serious Part II offences, such as disorderly conduct, public drunkenness, vagrancy, loitering, suspicion, and vandalism...Unlike homicide or other serious violent offences, these are the kinds of law violations for which the police have some discretion when deciding whether to make an arrest. If the police have 'gone fetal,' we should expect them to make fewer such discretionary arrests (Rosenfeld \& Wallman 2019: 53-57).

Along the same lines, Wolfe and Nix (2016: 1) argue, "Although the Ferguson Effect on crime rates remains an open question, there may also be a Ferguson Effect on other aspects of police officers' jobs."

\subsection{Solutions to Address Problematic De-Policing}

As discussed in previous chapters, some practices of de-policing represent what can be considered as an improvement over the status quo - as is the case with overpolicing of certain neighbourhoods or intensive, in some instances oppressive, police tactics employed in certain communities (i.e. racialized and/or marginalized 
populations) originating in biased attitudes and practices - systemic within the institution, and/or the occupational culture, and/or manifesting in individual officers. However, given the widespread and essentially universal nature of today's de-policing, as identified in the present study, I argue that, in many, if not most, circumstances, officers engaging in these risk-averse practices is not a beneficial situation - amounting to perversion in the application of police discretion and the neglect of core police functions that have come to be expected of front-line officers - particularly given the widespread espousing of CBP principles across Western democracies. Therefore, in circumstances in which a solution to the identified de-policing phenomenon is desirable, in other words, instances of problematic de-policing practices, the following represent my thoughts on potential remedies to the situation, which have been informed by many discussions on this topic over the past three years with rank-and-file officers, police supervisors and command leadership, oversight and governance officials, and academics involved in policing research and scholarship. Given limitations related to scope and length, an in-depth analysis of each potential solution is not feasible.

However, by the end of this subchapter, the reader should possess sufficient information to give consideration to, and engage in some assessment of, these admittedly preliminary ideas.

Of course, as discussed throughout this dissertation, it is important to appreciate that any prospective reform in front-line policing requires an understanding of the central role of the rank-and-file subculture and its necessary ‘buy-in'. In this vein, Bacon (2014: 114) finds, "The key point to be taken from the available evidence is that reforms have habitually failed to override police culture when they conflict with 
established values, beliefs and assumptions." Further, the President's Task Force on $21^{\text {st }}$ Century Policing (2015: 11), observes that "culture eats policy for lunch" (a common understanding in occupational culture theory across all disciplines) and, in this regard, they advance, "Any law enforcement organization can make great rules and policies that emphasize the guardian role, but if policies conflict with the existing culture, they will not be institutionalized and behaviour will not change." I also argue that any effort to address de-policing across the rank-and-file must consider not only external influences in the front-line policing landscape, as has been the focus of this dissertation, but also internal strains that can contribute to, or further entrench, the development of a depolicing mindset among front-line officers - both 'externalities' (conditions arising through the external environments within which the police operate) and 'internalities' (conditions within police institutions) as advanced in the work of Stenning and Shearing (2005). In no particular order of importance, I submit that the following ideas/ considerations should be deliberated in efforts to address problematic de-policing across today's front-line officers' practices.

(1) In pre-employment evaluation processes, perhaps more sophisticated and comprehensive psychological screening and background investigation regimens would allow for the identification of prospective police recruits that are likely to be less prone to becoming risk-averse in their responses to the visibility and inevitable public critique they will encounter when they begin to perform front-line police work in the community and, interrelatedly, those individuals better able to resist rank-and-file subcultural dogma, which can influence officers toward initiating de-policing practices (and, therefore, into the potential for subsequent progression along the de-policing 
continuum). Further, given the strained relationships that exist between many rank-andfile officers and racialized and marginalized groups (e.g. Black, Indigenous and/or those of another 'visible minority' race/ethnicity; persons experiencing mental health challenges, including addictions and/or homelessness; and the LGBTQ+ community, and as part of such an enhanced recruit assessment protocol, a priority should be placed on the selection of candidates that are identified as unlikely to engage in biased and discriminatory policing practices throughout their front-line service. Along the same lines, within such a protocol an effort should be made to identify those prospective rank-and-file officers less likely to engage in misconduct and, therefore, at least in theory, they should have no reason to engage in de-policing behaviours - given what should be these officers' diminished concerns about their visibility and/or the potential for public criticism of their actions in the field, in comparison to officers more prone to engage in problematic behaviours.

(2) Related, is a reconsideration of how recruit training is done - in two dimensions. Perhaps most obviously, throughout the police academy experience, in terms of both the formal curriculum and recruits' informal social interactions, de-policing must be acknowledged as a significant reality in today's front-line police work and unwaveringly characterized as an inappropriate adaptation to the external and internal strains that all officers will invariably experience throughout a policing career. While not requiring adherence to the mission orientation that characterizes certain officers' repudiation of risk-averse practices, which can engender other problems, such as the insular and adversarial 'us versus them' mindset and resistance to any reform initiatives, efforts to remove de-policing as an option from the officer's 'tool box' at this formative 
career stage, can have particular traction and long-lasting impact, given the 'captive audience' realities of the police academy and the influential role modeling opportunities available through respected instructional staff. Such an experience in the infancy of one's policing career can potentially function as an inoculating effect against the accumulation of negative experiences and subcultural influences over intervening years and sustain the officer's motivation to perform all aspects of front-line police work - not just reactive response but also proactive initiatives and discretionary community interactions.

The second aspect, in relation to recruit training, was articulated in the 'real world' observations of Superintendent Stu Betts (executive officer to the Chief of the YRPS) as discussed in Endnote 22 to Chapter Five - "I wonder if our coach officers...contribute to an officer's move along the de-policing continuum by planting the seeds of that type of dissatisfaction" (personal communication, 15 February 2018). Clearly, to maintain coherence between in-field training and the lessons inculcated throughout the recruit's police academy experience it is necessary to eradicate, in the coach officer role at a minimum, the oft-referenced subcultural encouragement to 'forget everything you learned at the academy.' In this regard, Bayley and Bittner (1984: 54) are instructive with their advocacy for only "master craftsmen" to serve as coach officers. As with influencing recruit decision-making in myriad dimensions, to dissuade developing officers from considering risk-averse behaviours as an available option (i.e. as a legitimate self-preservation strategy) it is necessary to enlist coach officers that do not subscribe to de-policing and that consistently repudiate the consideration of any such attitudes and/or behaviours among recruits, regardless of 
strains experienced in the techno-social and/or socio-political external policing environment.

(3) Bacon (2014: 115) finds, "What police scholarship tells us...is that changing police culture is difficult but not impossible...How to do this begins with chief officers understanding the nature of the occupational cultures in their organizations and the way in which they can inhibit or facilitate change.” The realities of the police paramilitary structure and the protracted and arduous promotional process involved in ascending through the ranks from the entry-level position of the constable or police officer to senior command (should one aspire to do so - many do not) almost always result in the latter being decades removed from the contemporary front-line experiences of the former. Therefore, despite the frequently-expressed assertion by many police executives (chiefs, deputy chiefs, superintendents, captains, etc.) that they are 'in touch' with the current circumstances of policing's rank-and-file, they are, in fact, largely oblivious given that 20,25,30, or even more years separate the present from the last time they performed an operational front-line task, exempting the occasional 'photo op' to demonstrate a purported up-to-date nexus with what many senior commanders often refer to as 'the heart and soul of policing'.

I advance that, to understand today's circumstances in front-line police work, as the logical first step in initiating reform, there is no substitute for experience. In this regard, I have counseled several senior commanders, who have asked for my advice, to get in touch with today's front-line policing realities by experiencing them firsthand, by 'getting their hands dirty' through actually performing police work in the field. In my conception, this takes the form of police executives working shifts (perhaps once per 
month) as a rank-and-file patrol officer. To be clear, this means wearing the working uniform of the front-line officer; completing full shifts (and not just day shifts); interacting with fellow officers throughout the shift as equals, rather than as superior and subordinate; and doing police work undistinguishable from the other officers on the shift - taking dispatched calls, performing proactive duties, arresting suspects, writing reports, and even using force in situations where force is required.

As should be apparent to the reader (with information presented throughout previous chapters) trust is integral to any effort aimed at rehabilitating the public-police relationship. Similarly, in the present context, an officer's trust in their internal, as well as external, working environment is a significant consideration, vis-à-vis perceptions of occupational risk and factors underlying the decision to practice or not to practice depolicing. Having senior command descend from their offices in the 'ivory tower' of policing's leadership, to perform front-line police work in the contemporary policing environment alongside the rank-and-file, should pay significant dividends - in terms of developing greater trust relationships throughout the internal working environment. (4) Along the same lines, some scholars (e.g., see Myhill \& Bradford 2013; Novak, Cordner, Smith \& Roberg 2017; Nix \& Wolfe 2016; Wolfe \& Nix 2016) have advanced that fairness and support (often characterized as 'organizational justice') within the officer's internal working environment can "mediate against negative external uncertainty...[In this regard,] officers have felt the impact of public scrutiny, with impacts on proactive duties and public engagement" (Scott Wolfe, personal communication, 15 November 2017). In this vein, Novak and colleagues (2017: 88, citing Wolfe \& Nix 2016) find, "Reluctance [to engage with the community] is 
mitigated among officers with a strong orientation toward organizational justice and self-legitimacy."

In the present study, concerns around the fairness with which front-line officers are treated internally and the resulting deleterious effects on trust, were brought to my attention by several participants in two particular respects - (1) police leadership reacting to allegations of a potentially problematic police-citizen interaction in a 'knee jerk' and apocalyptic 'worst-case' manner and (2) police leadership 'throwing officers under the bus' through reluctance to come to the defence of front-line officers within the public milieu (in the 'court of public opinion'), following contentious police-citizen interactions, or even providing relevant materials, that may favour the officer, to the deliberating public audience - such as a video recording of the event or potentiallyinstructive use of force policy information. In applying principles of 'organizational justice', senior police management might be wise to consider officers' sensitivities around how they are treated within their internal working environment in the aftermath of potentially-controversial occurrences - as positive perceptions among the rank-andfile have been shown to have a beneficial impact on trust (e.g., see Donner, Maskaly, Fridell \& Jennings 2015; Nix \& Wolfe 2016; Reynolds, Fitgerald \& Hicks 2018; Reynolds \& Helfers 2018; Wolfe \& Nix 2016; Wolfe \& Piquero 2011) and can influence officers' assessments of risk and their deliberations on de-policing. ${ }^{8}$ (5) Within this same vein, Oliver (2017: 438) argues, "If de-policing is a reality, there is clearly a need for a response that is not punitive in nature, but one that refocuses the police officer or the entire police culture of a department." Along these lines, I suggest that any effort to address de-policing should situate such behaviour in the domain of 
training, or re-training, rather than misconduct. Such an approach is in furtherance of finding ways to vaccinate officers against what has often been characterized as the cycle of negativity in front-line career progressions, which implicates de-policing as a potential adaptation to adversity encountered in the external and/or internal work environment. Put simply, making de-policing behaviours subject to retributory discipline rather than tactfully handled remedial training runs the substantial risk of heightening officer cynicism, diminishing trust within the internal working environment, further alienating occupational subcultures, and making a bad situation even worse. As discussed throughout Chapter Six, strain from the accumulation of negative experiences, often in coordination with the influence of the rank-and-file subculture, can contribute to an officer's initial decision to engage in de-policing or his/her further progression within the de-policing continuum. Therefore, I suggest that when de-policing behaviours are identified, the officer's conduct should be addressed through recognition, remedial training, and potentially even the consideration of a motivational accommodation - in attempting to rejuvenate (or reset) the officer at this key juncture in their police career, perhaps through a different assignment for a period. (6) Further, and also in the context of training, I suggest that much more training than is currently afforded to front-line officers would be beneficial, in terms of reductions in the underlying factors that can contribute to risk-averse behaviours. Encouraging and enabling officers to be more proficient in, and confident about, their use of force techniques, through incentives such as time off and payment of training fees, and doing additional work in scenario-based training to expose officers to more potentiallyproblematic (and highly stressful) occurrences and to reinforce desired responses to a 
range of situations they may encounter in the field, would be a significant improvement over the status quo. For example, from my own experiences with in-service training (that I understand is largely representative of the regimens relied on by other police agencies across Canada and the USA), front-line officers often train in these important areas for only a day or two per year - in the case of the OPS, officers train for 1.5 days, with a half day of firearms instruction/practice, a half day of scenario-based use of force judgment training, and a half day of 'defensive tactics' instruction/practice (hand-tohand combat and use of weapons). I have observed that 'defensive tactics' training has been progressively 'watered down' over the years - in terms of intensity and degree of force applied during training. From my understanding, this is largely due to workplace injury considerations (i.e. an officer that fractures their wrist during sparring may not be deployable in the field for months). This has repercussions for police use of force in the 'real world', including incompetence (in the sense of not having the skill set), anxieties, and apprehensions that can factor into an officer's choice to engage in de-policing.

Another initiative, which has been well received in my recent discussions with police executives and governance officials, contemplates the implementation of a Bachelor of Police Sciences university degree program that would provide a far more wide-ranging educational programme and much more in-depth understandings of the breadth of contemporary considerations in policing than that which is delivered in the comparatively-abbreviated and almost entirely practitioner-focused curricula presented at police academies (for example, recruit training at the Ontario Police College now encompasses 12 weeks - reduced from the 15 weeks I experienced in 1985-1986). Successful completion of such a university degree would be a prerequisite to enter the 
police recruit application and selection processes. This would align policing with other professions that demand an occupation-specific university degree in order to practice (i.e. nursing, teaching, engineering, law, dentistry, architecture, social work, and medicine) and would undoubtedly, in my view, produce more competent, well-rounded, and enlightened recruits in areas such as procedural justice, de-escalation, racialized considerations in police-citizen interactions, mental health, drug/alcohol dependence, child welfare, etc. (e.g., see Bruns \& Bruns 2015; Bryant, Cockcroft, Tong \& Wood 2014; Punch 2007; Savage 2007; Tilley \& Laycock 2017; Wood \& Tong 2009). I argue that such a comprehensive and re-tooled police education and training regimen could ultimately mitigate against officers going on to practice de-policing once they are deployed in the field and subjected to the strains of today's front-line police work. I suggest that benefits could also be realized in terms of public trust in a more thoroughly educated complement of front-line officers. In this vein, the College of Policing (the professional body that regulates policing standards in England and Wales) enacted the Degree Holder Entry Programme as part of the Policing Educational Qualifications Framework in 2016. A requirement of the new protocol mandates that all police recruits must possess a qualifying degree in policing, beginning in 2020 (Hough \& Stanko 2019).

(7) Toch (2012: 84) recalls, "Early on [with the widespread availability of inexpensive video recording equipment], some police officials saw risk, and others potential, in these developments. The issue of perceived risk (whose invocation sometimes bordered on paranoia) was by far the most salient." I argue that this mindset is not helpful, in terms of improving the public-police relationship, and 
suggest, in fact, that police proactively and significantly increasing the visibility of officers' actions in the field, to their public audience, may have benefits in terms of rehabilitating the public's trust and therefore, given the symbiotic nature of the public-police relationship, may also lead to improvements in officers' trust of the citizenry. Within such an ethos of transparency, in addition to video recorded footage of police-citizen interactions, such disclosures by police to the citizenry should also encompass the broad dissemination of laws and policies (i.e. use of force regulations, as with Ontario's Use of Force Continuum - depicted in Endnote 15 to Chapter 6) and relevant contextual information - with the collateral benefit of a more enlightened public audience through receipt of these 'educational' materials. In this vein, Nix and Pickett (2017: 31, citing Mueller \& Singer 2015; O'Connor 2015), find,

The evidence herein suggests that it is important for police departments to take steps to counter the negative publicity in the media and ensure a more accurate depiction of policing. One approach may be for police departments to leverage different forms of media to increase both the frequency and quality of communications about policing available to the public.

(8) In a related consideration, some are suggesting that the increased visibility of police and public conduct, through the adoption of body-worn cameras (BWCs) across front-line policing, may have a mitigating effect on the development, or expansion, of distrust across both constituents in the public-police relationship. In relation to the public audience, in a 2014 Washington Post/ABC News poll 86\% of American adults indicated support for requiring patrol officers in their communities 'to wear small video cameras whenever they're on duty' to bolster trust through transparency (by race/ethnicity the percentages reported were $85 \%$ for Whites, $91 \%$ for Blacks, and $87 \%$ 
for Hispanics). On the other hand, the absence of trust in the citizenry can influence rank-and-file officers' decisions to engage in risk-averse behavioural adaptations. In this regard, Gonzales and Cochran (2017: 326) observe, "Increased use of body cameras worn by police officers appears to have the potential to reverse this disturbing trend [of de-policing] by providing police officers with an incentive to get out of their patrol cars and restoring legitimacy to police in their interactions with citizens.” They elaborate, Return again for a moment to the role of our hypothetical police officer [discussed earlier in Chapter One] and consider the effect of adding a police-worn body camera to the equation. Would this tip the balance in favour of a decision to get out of the car and talk to the young men? A bodyworn camera, unlike one held by a citizen, will show events from the perspective of the police officer, giving viewers a sense of what the officer sees and hears (or does not see or hear). This could be helpful to the officer and thus encourage the officer to take action for two reasons: (1) the officer's perspective is the legally relevant perspective, and (2) it gives context to the final frames often recorded by citizens. ${ }^{9}$

...Body cameras also may encourage police action (and thus counteract depolicing) due to their potential to have a civilizing effect on citizens in such encounters. Such an effect is explained by the theory of self-awareness, which holds that people are less likely to engage in socially undesirable behaviour if they know they are being watched. This civilizing effect also operates on the other party in the encounter - the police officer...Therefore, it appears that police-worn body cameras have the potential to counteract, and may already be counteracting, any de-policing occurring due to the 'Ferguson Effect' (Gonzales \& Cochran 2017: 308-309).

In this vein, Braga and colleagues (2018: 518) observe that only "a very small number of studies have examined the effects of BWCs on police officer work activities, such as their willingness to be proactive, problem solve, and their discretion in making arrests and citations in discretionary incidents." One such study (with the Mesa Police Department) found that officers deployed with BWCs are more proactive than officers in the control group - in terms of being more likely to issue citations and initiate discretionary contact with citizens (Ready \& Young 2015). Of relevance to this 
consideration, in another area of contemporary policing examined in the present study, $80.4 \%(\mathrm{~N}=2137)$ of Canadian and $76.3 \%(\mathrm{~N}=733)$ of American rank-and-file officers report that they would have no objection to the introduction of BWCs into their frontline police work.

(9) Trust and confidence in the police are shaped by individual subjective judgments as to whether officers are normative representatives of community values - the embodiment of idealized moral structures of integrity, legality, propriety, honesty, and ethical conduct (Bradford et al. 2014; Jackson \& Sunshine 2007; Stenson \& Silverstone 2014; Sunshine \& Tyler 2003b). In this vein, there is essential consensus among scholars that legitimacy of the police and their perceived trustworthiness increases when officers operate within appropriate ethical frameworks (Bradford et al. 2014; Jackson et al. 2012), wield their power in a fair manner (Hough 2007; Hough et al. 2010; National Research Council 2004; Tyler 2006b), and conduct themselves in a manner that promotes procedural justice (Bradford et al. 2009; Matrofski, Snipes \& Supina 1996; McCluskey et al. 2014; Paternoster, Brame, Bachman \& Sherman 1997; Sherman 1997; Stenson \& Silverstone 2014; Tyler \& Smith 1997; Tyler \& Huo 2002; Tyler \& Jackson 2013; Tyler \& Wakslak 2004). Such are communicated principally through the dignity, respect, and fairness (or absence thereof) demonstrated in the treatment of the wide variety of people with whom officers come into contact and the manner in which they exercise their authority (Hough 2007; Jackson \& Sunshine 2007; Loader 1997; McCluskey et al. 2014; Sunshine \& Tyler 2003a, 2003b; Tyler 2004; Tyler \& Huo 2002). 
Myhill and Bradford (2013: 349) observe, “The expanding literature on policing and procedural justice has focused primarily on the promise of securing public cooperation and compliance through building the legitimacy of the police. Far less attention has been focused on how police officers might be encouraged to display consistently the types of attitudes and behaviour that might foster legitimacy." In this regard, McCluskey and colleagues (2014: 10) advance that issues around legitimacy, trust, and accountability, are "to a significant degree, in the hands of the police as they relate to the public in everyday encounters." As Tyler (2011: 257) has characterized them, each police-citizen interaction "is a socializing experience that builds or undermines legitimacy."

Various scholars, discussing "the optimistic side of research on police legitimacy" (Hough et al. 2010: 203), suggest that police can repair public distrust, create legitimacy, and improve perceptions of accountability through their day-to-day practices - performances seen by the public to accord with their expectations of appropriate, professional, and ethical police conduct (Bradford et al. 2014; Callanan \& Rosenberger 2011; Hough et al. 2010; McCluskey et al. 2014; Tyler 2004, 2006a, 2006b, 2011). Studies have determined that police practices that accord with the procedural justice model produce the strongest effect on citizen's perceptions of police legitimacy and trustworthiness; even when 'demographic' variables of race/ethnicity, education, and socioeconomic status are controlled (Hawdon 2008; Sunshine \& Tyler 2003b). In this vein, the President's Task Force on $21^{\text {st }}$ Century Policing (2015a: 1) counsels, ...Law enforcement agencies should adopt procedural justice as the guiding principle for internal and external policies and practices to guide their interactions with rank and file officers and with the citizens 
they serve...Procedurally just behaviour is based on four central principles:

1. Treating people with dignity and respect

2. Giving individuals "voice" during encounters

3. Being neutral and transparent in decision making

4. Conveying trustworthy motives

Research demonstrates that these principles lead to relationships in which the community trusts that officers are honest, unbiased, benevolent, and lawful. The community therefore feels obligated to follow the law and the dictates of legal authorities and is more willing to cooperate with and engage those authorities because it believes that it shares a common set of interests and values with the police.

I endorse this recommendation and advance that the implementation of procedural justice as the model for police-citizen interaction has the potential to moderate trust issues in the public-police relationship and particularly in the strained relations between police and racialized and other minority communities (e.g. LGBT2+ and those with mental health challenges). My reading of the sentiments of today's 'public' is that many citizens want to see (largely facilitated through 'policing's new visibility') officers consistently acting in accordance with procedural justice ideals and for the institution of policing to be accountable and transparent in ensuring that officers conduct themselves in such a manner. The potential payoff for both police and for the public they serve and protect is that the more frequently citizens see their police officers exhibiting exemplary conduct (ideally $100 \%$ of the time) the more the policing of their community will be respected as legitimate. As Myhill and Bradford (2012: 418) observe, recognitions of commendable police behaviour can "induce positive change" in citizen's opinions of the police. I advance that improvements in the community's opinion of, and trust in, police can, in turn, manifest in officers' perceptions of reductions in strain that should accompany a less critical and adversarial socio-political external policing environment. 
This can impact, in a positive manner (in the context of rehabilitating the public-police relationship and working toward reductions in problematic de-policing) on risk analyses that can influence officers' decisions to practice or not practice de-policing.

(10) As demonstrated in study participants' responses presented throughout Chapter Five, one of the most prevalent frustrations expressed by rank-and-file officers is the lack of knowledge across today's public audience in relation to fundamental aspects of front-line police work. Many officers asked rhetorical questions similar to - What is 'Joe Public's' source of information/training/education in making public sphere determinations around what is, and is not lawful, appropriate, professional, and/or ethical police conduct? As with procedural justice, and other ideas advanced to address problematic de-policing outcomes, this consideration engages with trust challenges in the public-police relationship - in this particular context, officers' trust in the citizens they are sworn to serve and protect. In this regard, Shjarback and colleagues (2017a: 51) conclude, "To address [the current legitimacy crisis] we must find ways to improve community trust in the police. In doing so, however, it is necessary to help officers trust the public too. Improving police-community relations is a two-way street, and the burden of reform cannot rest squarely on one party's shoulders" (see also Nix et al. 2018; Pickett \& Ryon 2017).

In my view, with the tremendous growth in citizens' capabilities to observe the actions of their police officers in the field, and the interest of so many of today's citizens in how society is being policed, a comprehensive public education piece should be considered as a necessary development arising from today's techno-social and sociopolitical realities in front-line police work. I suggest that the majority of citizens are not 
well versed in the intricacies of police work (i.e. laws, policies, use of force rationales, legal constructs such as 'probable cause' and 'reasonable grounds') and the multitude of considerations implicated in the split-second decision-making that is often required of officers in dynamic policing occurrences. This lack of knowledge, which hinders citizens from making informed determinations in policing matters, is the source of much exasperation across policing's rank-and-file and is intertwined with officers' trust concerns vis-à-vis their public audience, arising from what they perceive as 'unjustified', 'uninformed', and/or 'overzealous' public criticisms of police actions in the field.

In addition to expressing frustration, many study participants report that the catalyst for their adoption of de-policing was the antipathy shown toward, and ignorance in relation to, the challenging tasks confronted by today's front-line officerthose who perform society's 'dirty work' (Boivin et al. 2018; Goldsmith 2010; Skolnick 1966). In this regard, numerous officers in the present study expressed that they are afraid of being judged by an ignorant adjudicator that does not understand the intricacies in police use of force policies or the distinction between a racialized policecitizen interaction, in the case of contact between an officer of a particular race/ethnicity and a citizen of an apparently different race/ethnicity, and 'racial profiling'. Along these lines, the public needs to better understand, for example, what is a lawful application of force by an officer and what is 'police brutality', as both can be equally unpleasant in terms of their physiological outcome (i.e. a broken nose) and equally unsettling for viewers of video-recorded footage. Sandhu and Haggerty (2017: 84) report similar findings, as among their study participants classified as 'camera shy', these officers, 
Regularly complained that the public was quick to judge but did not have the knowledge required to fairly interpret recordings of police actions. Officers were exasperated by the public's ignorance of the rules police are expected to follow, particularly in relation to the use of force, and routinely referred to what they characterized as biased or ignorant news media reports, political activist's claims, and comments on YouTube videos. For them, all such depictions and accounts underscore how little citizens understand the processes and legalities surrounding the use of force... Officers resented their actions being second guessed by people who were not there, did not know what the situation felt like, and assumed none of the risks officers assume.

In considering literature to inform discussion on this potential de-policing solution, implicating greater public understandings of front-line police work, I was drawn back to findings in Reiss' (1968) seminal study of rank-and-file officers in the community and the 'interesting development' he observed among the dozens of students who rode along with the police officers for hundreds of hours,

Reiss analysed the observers themselves as a collateral by-product of their work with the police. Prior to their fieldwork, the students displayed the negative views of police that were standard in campus culture of the 1960 s, seeing them as authoritarian and racist. Their observations did dutifully record many instances of the malpractices that underlay such critical views. However, after sharing the pressures of the encounters from the perspective of the patrol car, the students attenuated their condemnatory vilification of the cops. They continued to disapprove of the discriminatory and at times brutal practices they witnessed. However, they saw these as shaped - but not excused - by the pressures and problems of patrol work. They came to have an appreciation (though not an acceptance) of the police as fundamentally good people sometimes led to do bad things (Reiner 2017: 240, emphasis added).

In concluding this subchapter and while acknowledging that de-policing is clearly a complex issue, I suggest that the attitudes and behaviours underlying risk-averse behaviours across front-line police work can likely be moderated through three concurrent initiatives capable of improving trust in all facets of the contemporary 
public-police relationship (including police relations with racialized and marginalized communities). The first, as described above, implicates public education and encouragement for the pressing of a 'reset button' in the community's perceptions of their rank-and-file officers - giving changes implemented within policing, such as practices consistent with procedural justice, a fair chance. The second involves frontline officers accepting a fresh perspective in their relationship with the citizenry, which implicates greater professionalism through the entrenchment of procedural justice as the expectation in police-citizen interactions and a shift in policing philosophy from an adversarial 'thin blue line' orientation - the 'warrior', to a service-oriented model - the 'guardian', that is ready, willing, and able to serve and protect. Among the many frontline officers in the present study that indicated their response to today's techno-social and socio-political external policing landscape is to behave more professionally (as detailed throughout Chapter Five), perhaps the guidance of a Calgary officer and a Saskatoon officer are the most illuminating and instructive - Try to remain professional so that there's nothing interesting to record (CAL189) and Don't act like an asshole. Explain what you are doing (SAS43).

Finally, Wilson (1967: 162) counseled, over a half a century ago, "New ways...must be found to bring police officers and neighbourhood groups together for nonbureaucratic and meaningful communication" and as Nix and colleagues (2018: 49) observe, "This sentiment is perhaps truer now than ever before. Open dialog may help build mutual trust and deconstruct perceptions that the public is waging war on cops." As advanced by Nix and Pickett (2017: 31, citing Pickett, Baker, Metcalfe, Gertz \& Bellandi 2014), in suggesting "further steps to improve the attitudes that police officers 
and civilians hold toward each other," both constituents in the public-police relationship need to increase their positive interactions because,

Theoretically, such interventions may create a reservoir of trust and understanding that, in turn, reduces the impact of negative publicity on both officers and civilians. Indeed, evidence suggests that positive intergroup interactions can improve attitudes even in protracted conflicts with widely publicized instances of violence and high initial levels of mutual distrust (Nix \& Pickett 2017: 31, citing (Broaddus et al. 2013; Duda, Klofas \& Drake 2011; Goodrich \& Anderson 2014; Watts \& Washington 2014).

\subsection{Dissemination of Study Findings}

Rosenbaum (2010:144-145) has critiqued 'hit and run' law enforcement research, observing, "Often, it is a one-way street, with researchers receiving data but not giving back to the communities, agencies, and individuals who provided them with the information." This has not, and will not be, the case with the present study. Since beginning data collection in 2016, I have had the opportunity to discuss the phenomenon of de-policing (in conceptual/theoretical terms and also in terms of preliminary observations from empirical data), across a variety of settings/platforms and to diverse audiences in academia, governance, police leadership, police labour organizations, and across the public sphere (via mediated discussions). The following table presents these initiatives in chronological order. I would highlight (with humility) that I have presented on the de-policing identified in this dissertation, thus far, to no less than 100 Canadian and American police chiefs and several hundred additional police commanders of senior ranks; to at least 100 university-based academics (in Canada, the USA, and Europe); to more than 100 officials involved in policing governance and oversight across Canada, to more than 100 persons involved with Canadian police labour organizations; and to a public audience in Canada well in excess of half a million 
persons (with over 500,000 listening to the one-hour radio program Ontario Today and the daily circulation of the Ottawa Citizen newspaper exceeding 93,000 readers). ${ }^{10}$ An upcoming feature story on de-policing, based on the present study, by The Globe \& Mail's investigative team of journalists will be read by more than 400,000 Canadian readers when it is published later this year. ${ }^{11}$

\begin{tabular}{|c|c|c|c|}
\hline Year & Date & Title & $\begin{array}{ll}\text { Platform } \\
\end{array}$ \\
\hline 2017 & $24 \mathrm{March}$ & $\begin{array}{l}\text { Risk aversion in today's front-line } \\
\text { policing }\end{array}$ & $\begin{array}{l}\text { Presentation to the Canadian Association } \\
\text { of Chiefs of Police Research Foundation } \\
\text { annual conference (Winnipeg) }\end{array}$ \\
\hline 2017 & 18 May & $\begin{array}{l}\text { How are front-line public police } \\
\text { in Canada and the U.S. } \\
\text { responding to recent techno- } \\
\text { social and socio-political } \\
\text { modifications to the contemporary } \\
\text { policing landscape? }\end{array}$ & $\begin{array}{l}\text { Presented on my current research at a } \\
\text { networking gala to promote external } \\
\text { research partnerships between the } \\
\text { Carleton University research community } \\
\text { and key stakeholders/funders (as one of } \\
\text { seven PhD candidates chosen by the Vice } \\
\text { President - Research and International). }\end{array}$ \\
\hline 2017 & 30 May & $\begin{array}{l}\text { Practicing risk aversion in the } \\
\text { risk society: How front-line police } \\
\text { officers are responding to } \\
\text { increased visibility and societal } \\
\text { critique }\end{array}$ & $\begin{array}{l}\text { Conference paper delivered to the } \\
\text { Canadian Sociological Association annual } \\
\text { conference (Ryerson University, Toronto) }\end{array}$ \\
\hline 2017 & 20 June & $\begin{array}{l}\text { Policing the 'gotcha society': } \\
\text { Today's techno-social } \\
\text { environment for front-line police } \\
\text { work }\end{array}$ & $\begin{array}{l}\text { Presentation to attendees at the Axon } \\
\text { Accelerate annual conference (Phoenix) }\end{array}$ \\
\hline 2017 & 25 June & $\begin{array}{l}\text { Three theories to explain } \\
\text { Ottawa's violent year }\end{array}$ & $C B C$ News Ottawa (television and web) \\
\hline 2017 & 18 July & $\begin{array}{l}\text { When police pull back: De- } \\
\text { policing and the 'Ferguson Effect }\end{array}$ & $\begin{array}{l}\text { CBC News Ontario Today (radio call-in } \\
\text { program and web) }\end{array}$ \\
\hline 2017 & 15 September & $\begin{array}{l}\text { 'Inactive policing': Risk-averse } \\
\text { responses in front-line police } \\
\text { work to today's techno-social and } \\
\text { socio-political environments }\end{array}$ & $\begin{array}{l}\text { Conference paper delivered to the } \\
\text { European Society of Criminology annual } \\
\text { conference (Cardiff, Wales) }\end{array}$ \\
\hline 2017 & 16 November & $\begin{array}{l}\text { To swerve and neglect: Officers' } \\
\text { responses to increased visibility } \\
\text { and intensified societal } \\
\text { engagement with front-line police } \\
\text { work }\end{array}$ & $\begin{array}{l}\text { Conference paper delivered to the } \\
\text { American Society of Criminology annual } \\
\text { conference (Philadelphia) }\end{array}$ \\
\hline 2018 & 12 February & $\begin{array}{l}\text { Ottawa cop turned researcher } \\
\text { finds decline in proactive } \\
\text { policing. }\end{array}$ & $\begin{array}{l}\text { Ottawa Citizen (Yogaretnam) (print and } \\
\text { web) }\end{array}$ \\
\hline 2018 & 12 February & Ottawa Today with Evan Solomon & $\begin{array}{l}\text { Discussing de-policing on a radio call-in } \\
\text { program (Ottawa region) }\end{array}$ \\
\hline 2018 & 12 February & News and Views with Rob Snow & $\begin{array}{l}\text { Discussing de-policing on a radio call-in } \\
\text { program (Ottawa region) }\end{array}$ \\
\hline 2018 & 12 February & Ottawa Today with Mark Sutcliffe & $\begin{array}{l}\text { Discussing de-policing on a radio call-in } \\
\text { program (Ottawa region) }\end{array}$ \\
\hline
\end{tabular}




\begin{tabular}{|c|c|c|c|}
\hline Year & Date & Title & Platform \\
\hline 2018 & 12 February & $\begin{array}{l}\text { Officers are neglecting } \\
\text { proactive policing, } \\
\text { according to new research }\end{array}$ & CTV News Ottawa (television and web) \\
\hline 2018 & 12 February & $\begin{array}{l}\text { Police putting on 'blinders' } \\
\text { to potential crime, research } \\
\text { suggests }\end{array}$ & CTV News Ontario (television and web) \\
\hline 2018 & 12 February & $\begin{array}{l}\text { If police aren't policing - this } \\
\text { is a problem }\end{array}$ & $\begin{array}{l}\text { Ottawa Citizen (editorial board) } \\
\text { (print and web) }\end{array}$ \\
\hline 2018 & 12 February & $\begin{array}{l}\text { 'De-policing' a matter of } \\
\text { 'self-survival', police union } \\
\text { President says }\end{array}$ & $\begin{array}{l}\text { Ottawa Citizen (Crawford) (print and } \\
\text { web) }\end{array}$ \\
\hline 2018 & 13 February & Here and now & CBC News Toronto (television and web) \\
\hline 2018 & 13 February & Live at noon & CP24 News Toronto (television and web) \\
\hline 2018 & 13 February & Global news at 5:30 & Global News Ontario (television and web) \\
\hline 2018 & 14 February & The Tom McConnell Show & $\begin{array}{l}C K T B \text { St. Catharines and } C J B K \text { London } \\
\text { (radio and web) }\end{array}$ \\
\hline 2018 & 14 February & John Gormley live & $\begin{array}{l}C K O M \text { Saskatoon \& CJME Regina (radio } \\
\text { and web) }\end{array}$ \\
\hline 2018 & 15 February & $\begin{array}{l}\text { Ontario Today with Wei } \\
\text { Chen }\end{array}$ & CBC News Toronto (radio and web) \\
\hline 2018 & 15 February & $\begin{array}{l}\text { Are our police officers } \\
\text { 'de-policing?' }\end{array}$ & $\begin{array}{l}\text { CP24 News Toronto ('op/ed' by CTV } \\
\text { News public safety analyst Chris Lewis) } \\
\text { (television and web) }\end{array}$ \\
\hline 2018 & 16 February & $\begin{array}{l}\text { Proactive enforcement } \\
\text { declining as police officers } \\
\text { worry about public scrutiny, } \\
\text { Ottawa researcher says }\end{array}$ & $\begin{array}{l}\text { London Free Press (Carruthers) (print and } \\
\text { web) }\end{array}$ \\
\hline 2018 & 16 February & $\begin{array}{l}\text { New rules for street checks } \\
\text { create new problems }\end{array}$ & Ottawa Citizen (Egan) (print and web) \\
\hline 2018 & 20 February & Ottawa at 6 & CBC News Ottawa (television and web) \\
\hline 2018 & 19 April & $\begin{array}{l}\text { To swerve and neglect: De- } \\
\text { policing in today's front-line } \\
\text { police work }\end{array}$ & $\begin{array}{l}\text { Presentation to attendees of a special } \\
\text { meeting convened by the Ontario } \\
\text { Association of Police Services Boards to } \\
\text { discuss de-policing (Hawkesbury) }\end{array}$ \\
\hline 2018 & 25 April & $\begin{array}{l}\text { The police officer who } \\
\text { didn't shoot: The take down } \\
\text { in the Toronto van attack } \\
\text { and why everyone is talking } \\
\text { about Constable Ken Lam }\end{array}$ & CBC News Ontario Today (radio and web) \\
\hline 2018 & 9 May & $\begin{array}{l}\text { To swerve and neglect: De- } \\
\text { policing in today's front-line } \\
\text { police work }\end{array}$ & $\begin{array}{l}\text { Presentation to the Police Association of } \\
\text { Ontario annual conference (Kitchener) }\end{array}$ \\
\hline 2018 & 16 May & $\begin{array}{l}\text { To swerve and neglect: De- } \\
\text { policing in today's front-line } \\
\text { police work }\end{array}$ & $\begin{array}{l}\text { Presentation to the Alberta Association of } \\
\text { Chiefs of Police annual conference } \\
\text { (Banff) }\end{array}$ \\
\hline 2018 & 12 June & $\begin{array}{l}\text { To swerve and neglect: De- } \\
\text { policing in today's front-line } \\
\text { police work }\end{array}$ & $\begin{array}{l}\text { Presentation to the Ontario Association of } \\
\text { Chiefs of Police annual conference } \\
\text { (Huntsville) }\end{array}$ \\
\hline 2018 & 21 June & $\begin{array}{l}\text { Challenges in today's police work } \\
\text { on the front lines }\end{array}$ & $\begin{array}{l}\text { Webinar for the Ethics Practitioners } \\
\text { Association of Canada titled Code RED } \\
\text { (Rapid Ethical Decision-Making): Insights } \\
\text { from the Front Lines }\end{array}$ \\
\hline 2019 & 21 February & $\begin{array}{l}\text { Changing how police are } \\
\text { investigated }\end{array}$ & $C B C$ News Ontario Today (radio and web) \\
\hline
\end{tabular}




\begin{tabular}{|c|c|l|l|}
\hline Year & Date & \multicolumn{1}{|c|}{ Title } & \multicolumn{1}{|c|}{ Platform } \\
\hline 2019 & 9 May & $\begin{array}{l}\text { De-policing in today's front-line } \\
\text { police work }\end{array}$ & $\begin{array}{l}\text { Presentation to the Ontario Association of } \\
\text { Chiefs of Police Education and Training } \\
\text { Committee (Mississauga) }\end{array}$ \\
\hline 2019 & 31 October & $\begin{array}{l}\text { De-policing throughout front-line } \\
\text { police work in Ontario }\end{array}$ & $\begin{array}{l}\text { Presentation to the Ontario Association of } \\
\text { Chiefs of Police Leadership Conference } \\
\text { (Toronto) }\end{array}$ \\
\hline
\end{tabular}

\subsection{The Practice of Public Sociology}

Given my earlier comments around the intermittent and relatively muted reactions to previous discussions of de-policing, particularly when considered against the significance of this manifestation of risk aversion in today's policing, which not only strikes at the core of contemporary CBP philosophies across Western democracies but also has tangible ('real world') implications - for both our front-line officers and for us (the public that expects the officers deployed within our communities to serve and protect us), I have spent considerable time and energies (as enumerated in subchapter 7.5) in working to increase the dialogue around this important issue and the findings of the present study. My suspicion is that, similar to the rationale advanced in the dismissal of Comey's initial de-policing observations by President Obama and officials in his administration (as detailed within Chapter One), the relative dearth of discussion around, and reaction to, the serious issues presented by large numbers of policing's rank-and-file neglecting fundamental aspects of their police work, arises because of the anecdotal, rather than empirical, foundations underlying previous discourse (exceptions were presented in Chapter Two). Many of my efforts at discussing de-policing across varied audiences can be characterized as the practice of public sociology - something I feel is crucial for the discipline in this era and vitally important in encouraging further awareness, discussion, and creation of knowledge around this study's de-policing findings. ${ }^{12}$ 
Public sociology and public criminology (that are treated as interchangeable throughout this subchapter given that the distinctions between the two disciplines are, in my view, essentially irrelevant in this context) are practices about which entire books have recently been written (e.g., see Loader \& Sparks 2010; Hanemaayer \& Schneider 2014). ${ }^{13}$ Therefore, given the limited scope of this dissertation, in this subchapter I present (succinctly) my observations concerning the ongoing and unresolved debate within contemporary social sciences in relation to the role of the social scientist, vis-àvis engagement with the 'public' (e.g., see Nickel 2010) and my advocacy for the practice of public sociology.

Proponents of participation by sociologists in public discourse oppose the position that the disciplines' proper role is strictly that of producing and discussing scholarship within the academic community while, conversely, those advocating a more limited role for the social scientist, in relation to public engagement, are concerned about the potential negative effects on the legitimacy of the discipline if sociologists venture forth into public initiatives. ${ }^{14}$ Much of the recent scholarship addressing the practice of public sociology emerges in response to the designation of public sociology as the principal theme for the American Sociological Association (ASA) in 2004. Michael Burawoy served as the president of the ASA in that year and challenged his colleagues to embrace public sociology in his speech at their annual national conference, and he followed up with a series of articles in which he endorsed the practice. It should be understood that this conversation within the discipline of sociology was not new Herbert Gans had raised the topic in his 1989 presidential address to the ASA as did Alfred McClung Lee in his 1976 ASA presidential address (Burawoy 2004). But there 
emerged from Burawoy's 2004 plea, for a redoubling of efforts in the practice of public sociology, "an almost joyous collective effervescence, brought on by what seemed to many of us the consensual uniting of the discipline around the goals of public sociology" (Hays, 2007: 80). Ericson (2005: 366) interpreted Burawoy's position as seeking to appeal "to broader audiences in public spheres. The sociologist is a public intellectual, communicating to educated people outside university contexts, especially through quality news media."

My normative position aligns with Burawoy's call for action and I argue that for sociology to develop as a more relevant consideration outside of the self-referential and limiting cocoon that is academia's 'ivory tower,' the discipline must expand considerably beyond insular scholarly discourse and directly engage the vast public audience - which, for these purposes, should be considered as subsuming media (traditional, social, and other emerging online and new media technologies), policymakers, legislators, governance and oversight officials, and, in the case of the present research, perhaps most importantly, police practitioners across the rank-and-file and their command leadership. Burawoy (2004, 2005a, 2005b, 2007) captures these 'publics' within what he calls 'traditional publics' (engaging in vehicles of public discussion), 'organic publics' (engaging with organizations, groups, associations, etc.), and also constituting ourselves (sociologists) "as a public that acts in the political arena" (Burawoy 2007: 30).

Clear (2010: 722), in reflecting on the works of Burawoy and others, characterizes public criminology as that work done by social scientists "that engages the crime/justice consumer publics...Public criminologists situate their work in the so-called real 
world." ${ }^{15}$ Aronowitz's (2005: 334) observations on Burawoy's calls for more public sociology and his integrated discussion of Jacoby’s (1987) The Last Intellectuals (alongside the contributions of Dewey, Lippman, and Mills) are instructive,

For both Dewey and Mills the intellectuals had to play a key role in providing the grist for the emergence of an active public capable of making the key decisions that affected their lives. Through their interventions in books, articles, public lectures and the mass media, they can influence the discussion by articulating alternative and oppositional values and knowledge.

Aronowitz (2005: 334-338) ultimately argues that: (1) sociology has retreated from social activism; (2) there has been a retreat of intellectuals into the academy; (3) Burawoy's call does not go far enough; (4) sociology's inwardness has separated its scholars from active public engagement; and (5), that a rebirth of the public intellectual is urgently needed.

I think it is apparent that engagement with the public, broadly defined, must, by necessity, take place on their respective 'turf.' In other words, while the publication of technical, complex, and obscure journal articles with correspondingly narrow circulations among small numbers of like-minded academics has a place in the realm of sociological research and collegial discourse, such work has little relevance, and is incomprehensible, to those who have not undertaken years of studies specific to the discipline. In this regard, Bosworth and Hoyle (2011:3), in lamenting the fragmenting of criminology, worry that "the field of inquiry seems at some risk of sinking into a set of cliques where criminologists read the work of others who think like them, write for those very same people and publish only in the journals that they and their colleagues are already reading."16 To counteract this malaise in our discipline, sociologists must master the skill of effective, and intelligible, communication within the public arena. I 
advance that to successfully regain, or establish, influence where it matters most and to have our expertise recognized and embraced, as highly-qualified social scientists producing relevant research products, sociologists have to be understandable to the target audience - the public. The thoughts of bell hooks (2004: 154) are particularly instructive on this point,

We are told by gatekeepers...that it will be better for us to write and think in a more conventional way. A distinction must be made between our freedom to think and write in multiple ways and the choice to write in accepted ways because we want particular rewards. My struggle over form, content, etc. has been informed by a desire to convey knowledge in ways that make it accessible to a wide range of readers. It is not a reflection of a longing to work in ways that will enable me to have institutional power or support. This is simply not the only form of power available to writers and thinkers. There is power in having a public audience for one's work that may not be particularly academic, power that comes from writing in ways that enable people to think critically about everyday life.

The selection of a renowned feminist scholar to support my position was not random. The contemporary feminist and environmentalist social movements have excelled at the practice of public sociology (Mopas \& Moore 2012). The successes of these movements in impacting on the public consciousness and instigating policy and legislative changes provide both optimism and a framework for a more effective sociology (e.g., see Kilty \& Crépault 2016). Through the advancing of strong empirical evidence, clear messaging, and public engagement over time, these social considerations now command broad public appeal and credibility. Public and policy conversations touching on feminist and environmentalist concerns have no choice but to include these voices in the discussion. Years ago, these movements expanded outside of theory and academia into public engagement. Because of successes in demonstrating the 
validity of their positions and pressing their cases in the public milieu, their intellectuals have expertise that is not only considered but recognized and valued by other actors.

The requirement for increased public sociology in other facets of our discipline is perhaps best established through contemplating the alternatives. In reviewing the extant literature, I discovered that the two alternatives to more robust engagement by sociologists with public audiences, which have been discussed with any regularity, are either (1) the status quo or (2) the abandonment altogether of any engagement outside the academy. Ericson (2005: 369) presents the case for the status quo,

All sociology entails public knowledge. There is no such thing as 'private sociology' in the sense of self-referential practitioners who do not actively seek to publicize their ideas and research. Sociologists publicize their ideas and research in myriad institutional contexts involving various audiences and different media. The media include classrooms at various levels of education (schools, colleges, universities, and graduate schools), textbooks, research monographs, journals, government reports...

In relation to the latter position, the discipline of sociology would, therefore, consist solely of social scientists conducting research, acquiring data, liaising with academic colleagues, and publishing their work in scholarly publications - with no intention of, or efforts toward, dissemination to a public audience. Along these lines, I think it is clear that the failure to engage the public on important matters unearthed through empirical sociological research will most certainly result in even further declines in the relevance of sociology in all respects, except for within the limited realm of university-based academic scholarship.

To contrast the present state of the discipline with the past, the analysis of Chancer and McLaughlin (2007: 156) is on point in terms of relevance, and loss thereof, "[In] the immediate post-war period British criminology was an integral part of policy 
making and practice and also influenced public deliberations. An elite of academics, policy makers, senior practitioners and opinion formers synthesized criminological scholarship and policy to promote enlightened practices." I argue that no criminologist or sociologist can, with a straight face, characterize the present state of these disciplines in Canada (and perhaps other Western democracies) as bearing any resemblance to the significant and integrated social scientific contributions as described by Chancer and McLaughlin (2007).

Therefore, I advance that there is an urgent need for those who are the experts on sociological and criminological issues - sociologists and criminologists - to engage public and political discourses. Sociologists and criminologists are social scientists possessed with research skills; academic integrity; and sociological/criminological knowledge, intelligence, and context. Other actors engaged in matters of public interest implicating sociology/criminology often operate based on anecdotal information, ‘common sense,' ideology, morals, political partisanship, and/or unscientific assumptions. With sociologists and criminologists absent from public conversations on important issues in society, there is a significant expert knowledge vacuum, in which environment bad practice, misinformation, and public misconceptions thrive.

It is my contention that it is our professional responsibility to engage in public conversations on the subject matter in which we are experts. "Play[ing] with each other in our own little sandbox" is no longer acceptable in the context of future directions in sociology (Rothe, Ross, Mullins, Friedrichs, Michalowski, Barak, Kauzlarich \& Kramer 2009: 10). These authors advance that "the sociological tradition's repeated calls for the development of some form of an 'emancipatory' project of public sociology: 'the 
synthesis of theory and practice' or 'theoretically informed action"' is clear, and they conclude, "As such, we can engage in both scholarship and praxis that can make a difference" (Rothe et al. 2009: 10-11).

To facilitate this public engagement, sociological writing destined for the public must be coordinated to today's realities. Most academic journals are not accessible to the public and hooks' thoughts on this were presented earlier in this subchapter. Wilson (2007: 120) cautions that "stilted, ponderous, jargon-laden language will all but ensure that one's writings will not penetrate beyond a narrow academic field of specialization." Similarly, Gans (2011) counsels public social scientists to do work that is grounded in good sociology and give the public research "that tells people something useful or relevant and is written in non-technical English...presented in language they can understand" (e.g., see also Barak 1988, 2007; Groombridge 2007; Turner 2013). As discussed, I have been collaborating with Globe \& Mail investigative reporter Greg McArthur and his colleagues to produce a feature article that will discuss, in the public milieu and in some depth, the contemporary phenomenon of de-policing across today's front-line police work - as identified through the present study. ${ }^{17}$ This research byproduct, while being true to the findings that emerged from study data, will be written in an approachable style for readers of Canada's largest newspaper (by circulation).

In this vein, when invoking their expertise in public interventions, sociologists must bring to the discussion honest and high-quality evidence and a thorough understanding of the complexity of the issue (Clear 2010; Uggen \& Interbitzin 2010). If information provided to the public is not grounded in sound empirical practice, but rather is ideologically, morally, or politically-motivated, there can be a loss of 
legitimacy and, therefore, diminished value in that contribution and in the discipline itself. The legitimacy of public sociology is heightened by evidence, logic, and restraint - what Weber refers to as 'an ethic of responsibility' (Uggen \& Interbirtzin 2010: 743). Public sociologists doing otherwise "risk the sorry fate of many a self-styled 'public intellectual' these days - too much public, not enough intellectual" (Loader \& Sparks, 2011: 22).

Loader \& Sparks (2011: 32) also observe, “Criminology’s public value lies not only in the plurality of theoretical perspectives, focal concerns, and methodologies that comprise contemporary criminology, but also in the range of ways it can be put to work in the service of better politics of crime and its regulation." It is in respect of these scholars' latter point, and more specifically, the policing of society, that I, through the broad dissemination and public discussion of the empirical findings of this dissertation research, am hoping to inform various audiences in relation to serious and consequential issues in today's policing. Understanding the breadth, nature, and contributing factors related to de-policing is the first step for all stakeholders in the public-police relationship (in which we are all constituents) to begin to address this contemporary reality across front-line police work. In support of my position, I present the enlightened thoughts of Wilson (2007), who dismisses skeptical critics that advance that public sociology/criminology is illegitimately non-scientific and that prefer the sanctuary of the academic 'ivory tower,'

If sociologists are concerned about the present and future state of the discipline, [the skeptics] argument is short sighted. Why? Simply because the more sociology is ignored by the media and policy makers, the less attention it receives as an academic discipline and therefore the more removed it is from the decision-making arena...Accordingly, the issue is 
not whether we should be concerned about attention from the media and policy makers; the issue is how to get such attention.

I expect to continue my practice of engaging with various audiences on the issues examined in, and the findings generated from, the present study. In this regard, one of the objectives in arriving at a comprehensive and empirically-grounded understanding of how front-line officers are adapting to their new techno-social and socio-political external policing landscape is public and police education - involving the broadening of knowledge and promotion of mutual appreciations. In practicing public sociology around these 'real world' and 'on the ground' issues in today's front-line police work and the shifting nature of the contemporary public-police relationship, I seek to break away from the critique of modern sociology advanced by McAdam (2007: 426), who notes, "[Sociology today] is largely organized as a set of insular, self-referential communities of scholars who, through regular conferences, specialty journals, and tight network connections reinforce the importance...of their narrow subfield-based work, even as that work grows even more disconnected from the concerns of a host of important real-world constituencies.” In this vein, Clear (2010: 722) calls on public criminologists to "situate their work in the so-called real world."

In conducting this research, with its' 'real-world' relevance, my understanding is that engagements by reputable scholars - those who advance information grounded in empirical evidence - in arenas outside academia can influence public opinion and public policy. Such an understanding gains support from Loader and Sparks (2011: 21), who find, "Our task is to be bearers and interpreters of knowledge and to bring it to bear on matters of public concern and dispute." My belief is that the sharing of reliable information is a crucial first step in working to resolve circumstances in which 
misunderstandings form the foundation of a mistrusting relationship, which seems to characterize relations today between the public and police in several respects. This study provides an empirical understanding of the issues related to today's front-line external policing environment from the often-neglected, yet crucial, vantage point of those we expect to serve and protect us as they perform their work in our communities. This acquisition of knowledge is important in working towards a greater appreciation of the concerns and challenges in today's policing from the perspectives of all stakeholders and in working toward repairing the fractures in the contemporary public-police relationship.

In terms of the police constituent in this relationship, I suggest that, rather than retracting from service to, and protection of, the public through risk-avoidance practices and retreating into an apprehensive and insular occupational subculture, front-line officers in 2019 need to better understand their techno-social 'new visibility' circumstances and society's socio-political and cultural dynamics and, in response, practice policing that is in accord with these contemporary realities and with the expectations of today's society. Such a model of police work rejects the aggressive and confrontational 'us versus them' and 'warrior' mindsets in favour of a professional, ethical, and reflexive 'guardian' orientation toward public service, which has as its hallmarks respect for the rule of law, rights, and due process; restraint; and collaboration. In simple terms, officers who conduct themselves in this way, which can be related to a growing body of scholarship around the principles of procedural justice in policing, have little to fear from ubiquitous visibility and societal intolerance for misconduct and demands for greater scrutiny and accountability. 


\section{APPENDIX A \\ CONSENT FORM (2012-2013 STUDY)}

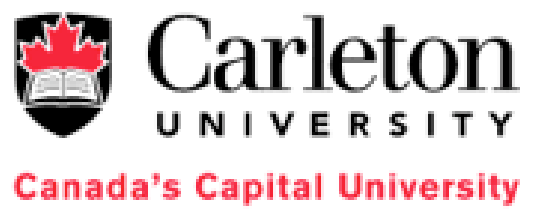

Department of Sociology and Anthropology B-750 Loeb Bullding

1125 Colonel By Drive

Ottawa, ON Canada K15 5B6

Tel: (613) 520-2582

\section{Coneant Form - Inturview Ranarch}

Title of research project:

Policing the Police in the 'Network Society': Police-Citizen Violence in the Age of 'Citizen

Journalism', Camera Phones, and YouTube

Date of ethics clearance:

August 31, 2012

Ethics Clearance for the Collection of Data Expires:

May 31, 2013

\section{Principal Investigator:}

Gregory Brown

Carleton University

Department of Sociology and Anthropology

A-708 Loeb Building

1125 Colonel By Drive

Ottawa, ON K1S 5B6

Tel: 613-327-9999

E-mail: gregoryr_brown@carleton.ca

\section{Purpose of the Study:}

One of the goals of this research is to inquire into whether the spread of mobile telephone videorecording capabilities and the distribution of recordings of police-citizen violent interactions through social media (YouTube, websites, blogs, etc.) have affected individual police officer's behaviour during the execution of their duties. The project will also inquire into impacts, if any, upon institutional policing policies and practices.

In terms of benefits arising from participation in this study the researcher suggests that this is a very important issue affecting both 'front line' police patrol officers in their chosen profession and the wider institution of policing in our society. As such, increasing the knowledge base around such an important matter as police-citizen violence and the impact of technological innovations provides a benefit to individual police officers (including those participating in the study) in terms of their day-to-day interactions with citizens and their police training in use of force considerations.

\section{Study Procedures:}

Qualitative semi-structured interviewing is one of a series of research methods being employed in the study of this phenomenon (the experiential knowledge of the researcher will form part of 
the data as will: survey sampling, Internet-based research of relevant sites, and analysis of Ontario police training literature).

\section{Participation in the Study:}

1. I volunteer to participate in an interview conducted by Gregory Brown (working under the supervision of Dr. Aaron Doyle) for a Master of Arts (Sociology) research project at Carleton University. I understand that I am one of approximately 20 police officers being interviewed in this phase of the research project.

2. I understand that this interview will last approximately one to two hours. I also understand that this interview will not be audio-recorded and that the researcher will take contemporaneous notes from time to time during the interview.

3. I understand that this Consent Form and all other research materials, including notes from this interview, will be securely and separately stored by the researcher, and that the information (data) I provide in this interview will be anonymous.

I understand that this interview is not being audio-recorded in order to alleviate any potential anxieties I might otherwise have in relation to anonymity.

I understand that no identifiers will be included in any written notes emerging from this interview and only the researcher will have access to the interview notes.

I understand that the researcher may retain the data collected for further related research, done by him, and on the same topic and for the purpose for which it was gathered.

4. I understand that there could potentially be some professional risk to me should my comments, opinions and experiences be identified with me. Therefore, I have been instructed by the researcher, prior to the commencement of the interview, to refrain from advising the interviewer of any information that could be characterized as 'sensitive' (including, but not limited to, being involved in or witnessing any form of police use of force that could be considered unlawful or inappropriate) during the interview session.

I have been instructed that should I possess any information that I consider could potentially be 'sensitive' and that I wish to convey to the researcher I am to provide this subsequent to this interview, in writing, absent any identifying information, and to deliver this by post in a sealed envelope to the researcher's university address.

As a supplementary safeguard to protect my interests, I am aware that in the event that I inadvertently disclose 'sensitive' information to the researcher, the researcher will intervene to prevent such disclosure. I understand that through the subsequent anonymous mailing of such information it would be impossible to cross-reference that information to either my pseudonym or my real name (which will appear only on this Consent Form and which will be secured separate from all other research materials in this study).

5. I understand that my participation in this project is voluntary and unpaid. I understand that I may decline to answer any question posed by the researcher. I also understand that I may withdraw my consent and participation in this research before, during, or after the interview session. If I do so during the interview, the interviewer will halt the interview, record the withdrawal in his notes (not in the interview notes proper), and forthwith destroy the interview 
notes. The interviewer will thereafter not use any of the interview content in the research process.

I further understand that should I wish to withdraw from the study following the completion of the interview, I shall send written notice to the researcher and upon receipt of my withdrawal request the data I supplied will be destroyed by the researcher and not form part of the study. I understand that I have thirty (30) days following the completion of this interview to choose to withdraw my participation. I understand that this time limit on my withdrawal recognizes the fact that withdrawal of a research participant during the data analysis stage of the project would be very difficult to manage.

\section{Research Findings:}

The research findings will be available to interested research participants and can be obtained by corresponding with the researching following the completion of this study (August 2013).

\section{Ethics Clearance:}

This project has been reviewed and has received clearance by the Carleton University Research Ethics Board. If you have any concerns or questions about your involvement in this study please feel free to contact me, my supervisor (Dr. Aaron Doyle), and/or the Research Ethics Board chair:

Professor Antonio Gualtieri, Chair

Research Ethics Board

Carleton University Research Office

Carleton University

1125 Colonel By Drive

Ottawa, ON K1S 5B6

Tel: (613) 520-2517

E-mail: ethics@carleton.ca

\section{Consent:}

Your participation in this study is entirely voluntary.

Your signature below indicates that you have read and understood these materials and you voluntarily consent to participate in this phase of the research project (semi-structured interview), as described.

Name (please print) 


\section{APPENDIX B \\ CONSENT FORM (2015-2016 STUDY)}

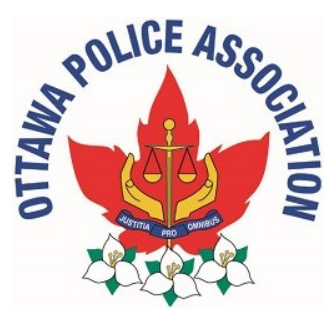

\section{Consent Form - Interview Research}

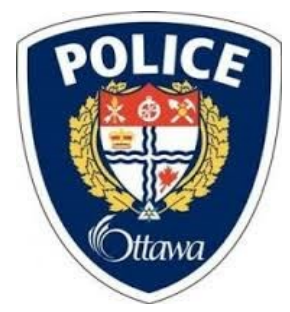

Title of research project: Implications for front-line officers emerging from the Ottawa Police Service's collection of race data on traffic stops

\section{Researchers:}

Gregory Brown, MA

Researcher and Doctoral Candidate

Tel: (613) 222-0348

E-mail: gregoryroybrown@gmail.com
Phillip Primeau, MA

Researcher and Doctoral Candidate

Tel: (514) 663-4558

E-mail: phillipprimeau@gmail.com

Study E-mail: opsfrontlinestudy@gmail.com

\section{Purpose of the Study:}

The goal of this research is to inquire into front-line officers' behaviours in response to, and sentiments around, the Ottawa Police Service's collection of race data on traffic stops since implementation of the Traffic Stop Race Data Collection Project on 27 June 2013 (resulting from the settlement agreement between the Ottawa Police Services Board and the Ontario Human Rights Commission).

In terms of benefits arising from participation in this study, the researchers and both the Ottawa Police Service and the Ottawa Police Association suggest that this is an important issue affecting policing organizations, the community, and front-line officers (individually and collectively). Increasing the knowledge base around this issue provides benefits specifically to front-line officers in that their views will be known through this study and potentially could inform practices and project next steps moving forward.

\section{Study Procedures:}

The researchers will interview a variety of front-line police officers (approximately $10 \%$ of those who serve in this capacity across the police service). Qualitative semistructured interviewing will be the research method used in this study. 


\section{Participation in the Study:}

I volunteer to participate in a qualitative semi-structured interview conducted by one of the researchers in furtherance of a study assessing front-line officers' behaviours in response to, and sentiments around, the Ottawa Police Service's collection of race data on traffic stops since implementation of the Traffic Stop Race Data Collection Project on 27 June 2013 (resulting from the settlement agreement between the Ottawa Police Services Board and the Ontario Human Rights Commission.

I understand that this interview will last approximately 30 to 60 minutes. I also understand that this interview will not be audio-recorded (unless I provide written consent for such audio-recording with the understanding that any such audio-recoding will be used strictly for the purpose of allowing the researcher to produce more accurate notes and that the researcher will then immediately destroy the recording) and that the researcher will take contemporaneous notes during the interview.

I understand that the information provided in this interview will be anonymous - in that my name will not appear in the final research product nor in the interview notes; my identity and the pseudonym substituted for the purposes of the interview notes and project will not be cross-referenced in any manner; and only the researchers will have access to the interview notes, any audio-recording (if I have consented to such audiorecording) and the Consent Form.

I understand that the researchers will securely store the Consent Form and all other research materials in separate locations that are not affiliated in any way with the Ottawa Police Service.

I understand that the researchers will destroy my Consent Form one year after the completion of the final interview in this study and that the interview notes will be retained indefinitely (until the researchers make a joint decision to destroy them at their discretion).

I understand that my participation in this project is voluntary and unpaid.

I understand that I may decline to answer any question posed by the researcher. I also understand that I may withdraw my consent and participation in this research before, during, or after the interview session. If I do so during the interview, the interviewer will halt the interview, record the withdrawal in his notes, and forthwith destroy the interview notes. The interviewer will thereafter not use any of the interview content in the research process. I further understand that should I wish to withdraw from the study following the completion of the interview, I shall send written notice to the researcher and upon receipt of my withdrawal request the data I supplied will be destroyed by the researcher and not form part of the study. I understand that I have fourteen (14) days following the completion of this interview to choose to withdraw my participation. I understand that this time limit on my withdrawal recognizes the fact that withdrawal of a research participant during the data analysis stage of the project would be very difficult to manage. 
I understand that the data I provide may be used by Gregory Brown and/or Phillip Primeau for any future academic projects they may author individually, together, or in collaboration with other researchers; restricted however to only those projects that relate to front-line officers' behaviours and/or the sentiments of police officers in contemporary policing.

\section{Research Findings:}

The research findings will be available to interested research participants and can be obtained by corresponding with the researchers following the completion of this study (May 2015). The researchers can be contacted via e-mail at opsfrontlinestudy@gmail.com.

\section{Consent:}

Your participation in this study is entirely voluntary. Your signature below indicates that you have read and understood these materials and you voluntarily consent to participate in this research project (through participating in a semi-structured interview), as described.

I consent to audio-recording of my interview under the conditions stipulated in this Consent Form.

I do not consent to audio-recording of my interview.

Signature of participant

Date

Name (please print)

Signature of researcher

Date 


\section{APPENDIX C}

\section{DATA SITE INFORMATION}

\section{Albany Police Department (Albany, New York)}

The Albany Police Department (APD) provides policing services in the City of Albany, New York. Albany is the state capital and is located within the state's Capital region, which consists of four counties with a combined population of 840,000 persons (United States Census Bureau 2017). ${ }^{1}$ Albany is the region's most populous city (with 98,000 residents), is the $6^{\text {th }}$ most populous city in the state, and the $306^{\text {th }}$ most populous city in the United States (United States Census Bureau 2017). The APD has a complement of 366 sworn officers (Federal Bureau of Investigation 2017), with roughly 200 assigned to front-line duties (Chief Brendan Cox, personal communication, 8 February 2016). The rank-and-file are deployed from two district stations with daily shift briefings in the morning, afternoon, and evening. To collect data, I attended on shift briefings on five dates, as detailed in the table below:

\begin{tabular}{|c|c|}
\hline Date & Location \\
\hline 13 June 2016 & Center Station \\
\hline 14 June 2016 & Center Station \& South Station \\
\hline 15 June 2016 & Center Station \& South Station \\
\hline 30 August 2016 & Center Station \\
\hline 1 September 2016 & South Station \\
\hline
\end{tabular}

One hundred and fifty-eight (158) surveys were administered to APD front-line officers. Of these, 156 were completed and returned to me and all were included in the study. On 15 June 2016, two officers declined to participate in the survey. Both expressed to me that they have issues with surveys (in any study) in terms of trusting that data is truly anonymous and that officers' responses will not be shared with police 
management. It should be noted that most APD front-line vehicles are equipped with a 'dashcam' that records audio and video when the vehicle's emergency equipment (lights and/or siren) are activated.

In 2016, the violent crime rate for the City of Albany was 803 occurrences per 100,000 inhabitants, which positioned Albany as having the $5^{\text {th }}$ highest rate in the state, among the 43 cities, towns, and villages with populations above 30,000 (Federal Bureau of Investigation 2017). The average median household income for Albany is $\$ 40,949$ and the average per capita income is $\$ 24,912$ (both in 2015 dollars) (United States Census Bureau 2017). Approximately $26.8 \%$ of Albany's population is living in poverty (United States Census Bureau 2017). The table below reports on the race/ethnicity of Albany's population (United States Census Bureau 2017).

\begin{tabular}{|c|c|c|}
\hline Population of Albany, NY (2010) & $\mathbf{9 7 , 8 5 6}$ & $\mathbf{1 0 0} \%$ \\
\hline White & 52,857 & $54.0 \%$ \\
\hline Black/African American & 28,479 & $29.1 \%$ \\
\hline Hispanic/Latino & 8,396 & $8.6 \%$ \\
\hline Asian & 4,850 & $5.0 \%$ \\
\hline Two or More Races & 2,740 & $2.8 \%$ \\
\hline Other Race & 296 & $0.3 \%$ \\
\hline American Indian/Alaska Native & 191 & $0.2 \%$ \\
\hline Native Hawaiian/Other Pacific Islander & 47 & $0 \%$ \\
\hline
\end{tabular}

It was brought to my attention by several Albany Police Department (APD) officers that a 2 April 2015 fatal police use of force occurrence had caused considerable friction between the APD and some in the city's African American community, which accounts for approximately 30\% of Albany's population (Jacobs 2016). Donald 'Dontay' Ivy, a 39-year-old Black man who struggled with paranoid schizophrenia and heart disease, ran from APD officers assigned to an 'anti-gun detail' and was then involved in a violent confrontation with the officers, who struck Ivy with batons and 
deployed a Taser (Carleo-Evangelist 2015; Jacobs 2016). In the days following Ivy's death, protestors mobilized in opposition to the APD, alleging police brutality and racial bias. Jacobs (2016) observed that the "tragic police encounter nearly tore apart the city of Albany" and suggested, "For many in Albany, Ivy's death was all too familiar. Allegations of widespread brutality and unfair treatment had strained the police department's relationship with the black community since the 1950's." Also writing for the local daily newspaper, McErlean (2015) advanced,

Dontay Ivy's death has been portrayed by the Albany Police Department and the district attorney as a 'tragedy,' implying that it was unpredictable, no one's fault, and 'unintentional.' According to this portrayal, no racism was involved - as if 'accidental and unintentional' mean that no racial discrimination or bias could occur...Mr. Ivy had committed no crime. No crime had been reported in the area. He was stopped based on being judged 'suspicious' for wearing a puffer coat in 26-degree weather with his hands up his sleeves...It is also of note that, according to District Attorney David Soares, of the eight police dashboard video cameras at the scene, only two were activated.

APD research participants that spoke with me expressed the view, which they suggested was essentially universal across front-line APD officers, that protesters, who carried Black Lives Matter signs, disrupted public meetings, and obstructed major thoroughfares, were political opportunists that did not appreciate the challenging and 'split-second' nature of the situation confronted by the APD officers that interacted with Ivy. Many of these APD research participants also suggested that the involved APD officers, who were cleared of wrongdoing in both a grand jury investigation and an investigation by the District Attorney's office, were being mistreated by an APD leadership that is more concerned with 'appeasing public criticism' than the morale and wellbeing of front-line APD officers. 


\section{Buffalo Police Department (Buffalo, New York)}

The Buffalo Police Department (BPD) provides policing services in the City of Buffalo, New York. With 257,000 residents, Buffalo is the second most populous city in the state, the $78^{\text {th }}$ most populous city in the country, and the city forms part of the Buffalo-Niagara census metropolitan area, which has a combined population of 1.3 million persons (United States Census Bureau 2017). The BPD has a complement of 693 sworn officers (Federal Bureau of Investigation 2017), with approximately 500 assigned to front-line duties (Lieutenant Jeff Rinaldo, personal communication, 3 February 2016). Rank-and-file operations are deployed out of five district stations across the City of Buffalo, with daily shift briefings in the morning, afternoon, and evenings. To collect data, I attended on shift briefings on ten dates, as detailed in the table below:

\begin{tabular}{|c|c|}
\hline Date & Location \\
\hline 17 May 2016 & District A \\
\hline 18 May 2016 & District B \\
\hline 19 May 2016 & District C \\
\hline 20 May 2016 & District D \\
\hline 21 May 2016 & District E \\
\hline 10 October 2016 & District A \\
\hline 11 October 2016 & District B \\
\hline 12 October 2016 & District C \\
\hline 13 October 2016 & District D \\
\hline 14 October 2016 & District E \\
\hline
\end{tabular}

Three hundred and twenty-five (325) surveys were administered to BPD frontline officers. Of these, 317 surveys were completed and returned to me and all were included in the study. On 20 May 2016 two officers had to leave the shift briefing for a priority call and I was unable to locate these officers thereafter. One officer attending a 
briefing on 20 May 2016 declined to participate. On 11 October 2016, three officers declined to participate, advising me that they have issues with trusting outside research because of undisclosed issues from previous participation in a study. Also, on the 11 October 2016 briefing, two officers took the surveys away to complete but did not return them to me.

In 2008, the United Nations evaluated the metropolitan Buffalo region as one of the worst cities in the world, in terms of economic inequality and racial bias concluding that $40 \%$ of Hispanic, Black, and ethnically-mixed households earned less than $\$ 15,000$, in comparison to only $15 \%$ of White households (World Population Review 2017). In their analysis of variables included in the United States census, Sauter, Stebbins and Comen (2017) ranked Buffalo as the $22^{\text {nd }}$ worst city in America and found, "Buffalo is the second largest city in New York, and by many measures the most economically and socially distressed." The average median household income for Buffalo is $\$ 31,918$ and the average per capita income is $\$ 20,751$ (both in 2015 dollars) (United States Census Bureau 2017). Approximately $31.4 \%$ of Buffalo's population is living in poverty (United States Census Bureau 2017).

In 2016, the violent crime rate for the City of Buffalo was 1119 occurrences per 100,000 inhabitants, which positioned Buffalo as having the $2^{\text {nd }}$ highest rate in the state, behind only Niagara Falls (Federal Bureau of Investigation 2017). This also positions Buffalo as the city with the $15^{\text {th }}$ highest violent crime rate in the country (Federal Bureau of Investigation 2017). The table below reports on the race/ethnicity of Buffalo's population (United States Census Bureau 2017). 


\begin{tabular}{|c|c|c|}
\hline Population of Buffalo, NY (2010) & $\mathbf{2 6 1 , 3 1 0}$ & $\mathbf{1 0 0} \%$ \\
\hline White & 119,801 & $45.8 \%$ \\
\hline Black/African American & 97,637 & $37.4 \%$ \\
\hline Hispanic/Latino & 27,519 & $10.5 \%$ \\
\hline Asian & 8313 & $3.2 \%$ \\
\hline Two or More Races & 5919 & $2.3 \%$ \\
\hline Other Race & 445 & $0.2 \%$ \\
\hline American Indian/Alaska Native & 1597 & $0.6 \%$ \\
\hline Native Hawaiian/Other Pacific Islander & 79 & $0 \%$ \\
\hline
\end{tabular}

In 2002, the Buffalo Police Department (BPD) entered into an agreement with the Civil Rights Division of the U.S. Justice Department for external monitoring of the BPD (often referred to as a 'consent decree'), which was modified in June 2007 and then terminated in July 2008 (Department of Justice 2017).

Several BPD officers I spoke with discussed a highly-publicized 2014 occurrence involving BPD officers and John Willet, a 22-year-old Black male, which resulted in the conviction of BPD officer John Cirulli on two federal criminal charges. Willett, who was apparently in possession of heroin, ran from BPD officers and, after being apprehended (and while immobilized on the pavement in handcuffs), he was struck and kicked by Cirulli - all of which was video recorded by a citizen using a cell phone (Hirtzel 2014; Moretti \& Ciotta 2014). In the aftermath, six BPD officers were placed on administrative leave, there was prolonged and intensive local media attention (traditional media and YouTube), and widespread public criticism of the BPD. Cirulli eventually pled guilty to the criminal charges and resigned from the BPD (Hirtzel 2014).

Of the approximately 10 BPD officers who brought this to my attention, all, but one, suggested that the 'fallout' from that occurrence had, in their assessment, forever changed how front-line BPD officers approach their work - in terms of citizen's video 
recording capabilities, the 'public throwing police under the bus' and not 'understanding how we have to deal with some criminals', and officers being less proactive as a result.

\section{Calgary Police Service (Calgary, Alberta)}

The Calgary Police Service (CPS) is responsible for municipal policing in the City of Calgary, which has a population of 1.24 million residents - the most populous city in the Province of Alberta and $3^{\text {rd }}$ most populous city in Canada (Statistics Canada 2017c). The CPS has a complement of 2172 sworn officers (Statistics Canada 2017e) with approximately 1173 assigned to front-line duties (Staff Sergeant Krista Ryan, personal communication, 4 February 2016). Some CPS officers, that do work with the Calgary Police Association, took issue with this 'official' estimate, suggesting that there would actually be perhaps 700 or 800 CPS officers engaged in 'real' front-line duties. In any event, the CPS is the largest municipal police agency in Alberta and the $3^{\text {rd }}$ largest in Canada (Statistics Canada 2017e). ${ }^{2}$ Front-line operations are deployed out of eight district facilities across the City of Calgary, with daily shift briefings in the morning, afternoon, and evenings. Within District 1, regular patrol operations are deployed out of the Ramsay station, while foot and bicycle patrol officers are deployed out of the Victoria Park station. To collect data, I attended at shift briefings on eight dates, as detailed in the table below:

\begin{tabular}{|c|c|}
\hline Date & Location \\
\hline 26 May 2016 & District 4 \\
\hline 27 May 2016 & District 3 \\
\hline 28 May 2016 & District 2 \\
\hline 29 May 2016 & District 1 (Ramsay) \\
\hline 30 May 2016 & District 7 \\
\hline 31 May 2016 & District 6 \\
\hline 1 June 2016 & District 5 \\
\hline 3 June 2016 & \\
\hline
\end{tabular}


Three hundred and sixty-two (362) surveys were administered to CPS participants and all were completed and returned to me. Of these, 358 surveys were included in the study. Four (4) were excluded because the respondent disclosed, in their response to Question 6 in the survey, that they were not currently performing front-line duties.

Many CPS front-line vehicles are equipped with a 'dashcam' and a camera in the rear prisoner compartment. The 'dashcam' records both audio and video when the vehicle's emergency equipment (lights and siren) are activated, when manually turnedon by the officer, or when collision sensors are triggered. CPS implemented a bodyworn camera program than ran between July 2015 and February 2016, which involved deployment of approximately 200 devices to rank-and-file Traffic and District 1 officers. The objective was to deploy 1300 cameras to front-line officers, however technical issues were encountered with the product and, as such, the cameras were taken out of service in February 2016 (Staff Sergeant Todd Robertson, personal communication, 28 July 2017).

For 2016, the violent crime rate for the City of Calgary (as expressed through the violent CSI) was 61.3 (Statistics Canada 2017d). As reported, the national average for 2016 was 75.3. The prevalence of low-income individuals in Calgary was $9.3 \%$ in 2016 (the national average was 14.2\%) (Statistics Canada 2017c). ${ }^{3}$ The table below reports on the race/ethnicity of Calgary's population in 2016 (Statistics Canada 2017b).

\begin{tabular}{|c|c|c|}
\hline Population of Calgary (2016) & $\mathbf{1 , 2 2 2 , 4 0 5}$ & $\mathbf{1 0 0 \%}$ \\
\hline White & 779,820 & $63.8 \%$ \\
\hline Total 'Visible Minority' Population & 442,585 & $36.2 \%$ \\
\hline South Asian & 115,795 & $9.5 \%$ \\
\hline Chinese & 87,835 & $7.2 \%$ \\
\hline
\end{tabular}




\begin{tabular}{|c|c|c|}
\hline Black & 51,515 & $4.2 \%$ \\
\hline Filipino & 67,650 & $5.5 \%$ \\
\hline Latin American & 26,265 & $2.1 \%$ \\
\hline Arab & 25,190 & $2.1 \%$ \\
\hline Southeast Asian & 21,610 & $1.8 \%$ \\
\hline West Asian & 12,610 & $1.0 \%$ \\
\hline Korean & 10,635 & $0.9 \%$ \\
\hline Japanese & 5170 & $0.4 \%$ \\
\hline Other 'Visible Minority' & 4410 & $0.4 \%$ \\
\hline Multiple 'Visible Minorities' & 13,895 & $1.1 \%$ \\
\hline Aboriginal & 35,195 & $2.9 \%$ \\
\hline
\end{tabular}

Several Calgary Police Service (CPS) front-line officers I spoke with referenced a 2008 event that was the topic of much discussion among CPS officers in May and June 2006. On 31 August 2008 CPS officers arrested Jason Arkinstall, a White male and a Hells Angels member. Unknown to all parties involved, a private citizen was video recording the events and this footage surfaced during Arkinstall's 2011 trial. Arkinstall, who had been handcuffed and was apparently insulting CPS officers as he was being ushered into an arrest van, was struck in the back of the head by CPS Constable Brant Derrick, who had denied any such actions in his sworn testimony, but which was clearly depicted in the video recording. Issues with contradictory police testimony resulted in the presiding judge concluding that the officers were 'unreliable and not credible' and, therefore, Arkinstall was acquitted on all charges (Bruser \& McLean 2012).

Following that ruling, the Calgary Criminal Defence Lawyer's Association requested that the province's police oversight agency investigate the conduct of the CPS officers involved with Arkinstall's arrest, including Constable Derrick and Sergeant Les Kaminski, who had since been elected President of the Calgary Police Association - the labour organization that represents rank-and-file CPS officers (Martin 2017). In January 
2017, Derrick and Kaminski were charged with assault causing bodily harm and Kaminski was also charged with perjury. As of the date of this writing, neither has been convicted of any crime. In July 2017, the Alberta Justice Minister initiated a provincial inquiry to "probe whether there were efforts to hinder public oversight of Calgary police officers involved in the arrest of Jason Arkinstall” (Cole 2017).

Cornwall Community Police Service (Cornwall, Ontario)

The Cornwall Community Police Service (CCPS) is responsible for policing services in the City of Cornwall, which has a population of 47,000 residents (Statistics Canada 2017c). Cornwall is located approximately 100 kilometers south-east of Ottawa and is situated on the Canada/USA (State of New York) border. The CCPS has a complement of 85 sworn police officers with 48 assigned to front-line duties (Staff Sergeant Dave Michaud, personal communication 4 February 2016). All rank-and-file are deployed from the police headquarters building and there are shift briefings each day in the morning and evening. To collect data, I attended at shift briefings on four dates, as detailed in the table below:

\begin{tabular}{|c|c|}
\hline Date & Location \\
\hline 7 March 2016 & HQ I Platoon \\
\hline 9 March 2016 & HQ P Platoon \\
\hline 9 August 2016 & HQ R Platoon \\
\hline 10 August 2016 & HQ D Platoon \\
\hline
\end{tabular}

Thirty-six (36) surveys were administered to CCPS participants and all were completed, returned to me, and included in the study.

There is no violent CSI available for Cornwall as, with its small population, it does not meet Statistics Canada's threshold for census metropolitan area (CMA) reporting. The prevalence of low-income individuals in Cornwall was $26.3 \%$ in 2016 
(Statistics Canada 2017c). The table below reports on the race/ethnicity of Cornwall's population in 2016 (Statistics Canada 2017b).

\begin{tabular}{|c|c|c|}
\hline Population of Cornwall (2016) & $\mathbf{4 6 , 1 7 5}$ & $\mathbf{1 0 0} \%$ \\
\hline White & 42,400 & $91.9 \%$ \\
\hline Total 'Visible Minority' Population & 2775 & $8.1 \%$ \\
\hline South Asian & 1500 & $3.2 \%$ \\
\hline Chinese & 170 & $0.4 \%$ \\
\hline Black & 405 & $0.9 \%$ \\
\hline Filipino & 150 & $0.3 \%$ \\
\hline Latin American & 65 & $0.1 \%$ \\
\hline Arab & 110 & $0.2 \%$ \\
\hline Southeast Asian & 70 & $0.1 \%$ \\
\hline West Asian & 155 & $0.3 \%$ \\
\hline Korean & 10 & $0 \%$ \\
\hline Japanese & 20 & $0 \%$ \\
\hline Other 'Visible Minority' & 40 & $0.1 \%$ \\
\hline Multiple 'Visible Minorities' & 80 & $0.1 \%$ \\
\hline Aboriginal & 1890 & $4.1 \%$ \\
\hline
\end{tabular}

A few Cornwall Community Police Service (CCPS) officers mentioned to me, without referring to any specific occurrences, that there exist persistent tensions between some in Cornwall's indigenous community and the CCPS. It seems, from the comments of these officers, that this is more of a generalized sentiment, and perhaps a legacy from the antiquity of the past, rather than a specific and immediate local phenomenon.

\section{Delta Police Department (Delta, British Columbia)}

The Delta Police Department (DPD) is responsible for policing services in the City of Delta, which is located within the Metro Vancouver region and has a population of 102,000 residents (Statistics Canada 2017c). Delta is the $10^{\text {th }}$ most populous city in the Province of British Columbia and the $52^{\text {nd }}$ most populous city in Canada (Statistics 
Canada 2017c). The Metro Vancouver region has a population of 2.4 million people, living in 21 municipalities and one First Nation's community (Metro Vancouver 2017). The map below (Metro Vancouver 2017) presents the relevant information about the region and the study's four data sites in the region (Delta, New Westminster, Port Moody, and Ridge Meadows).

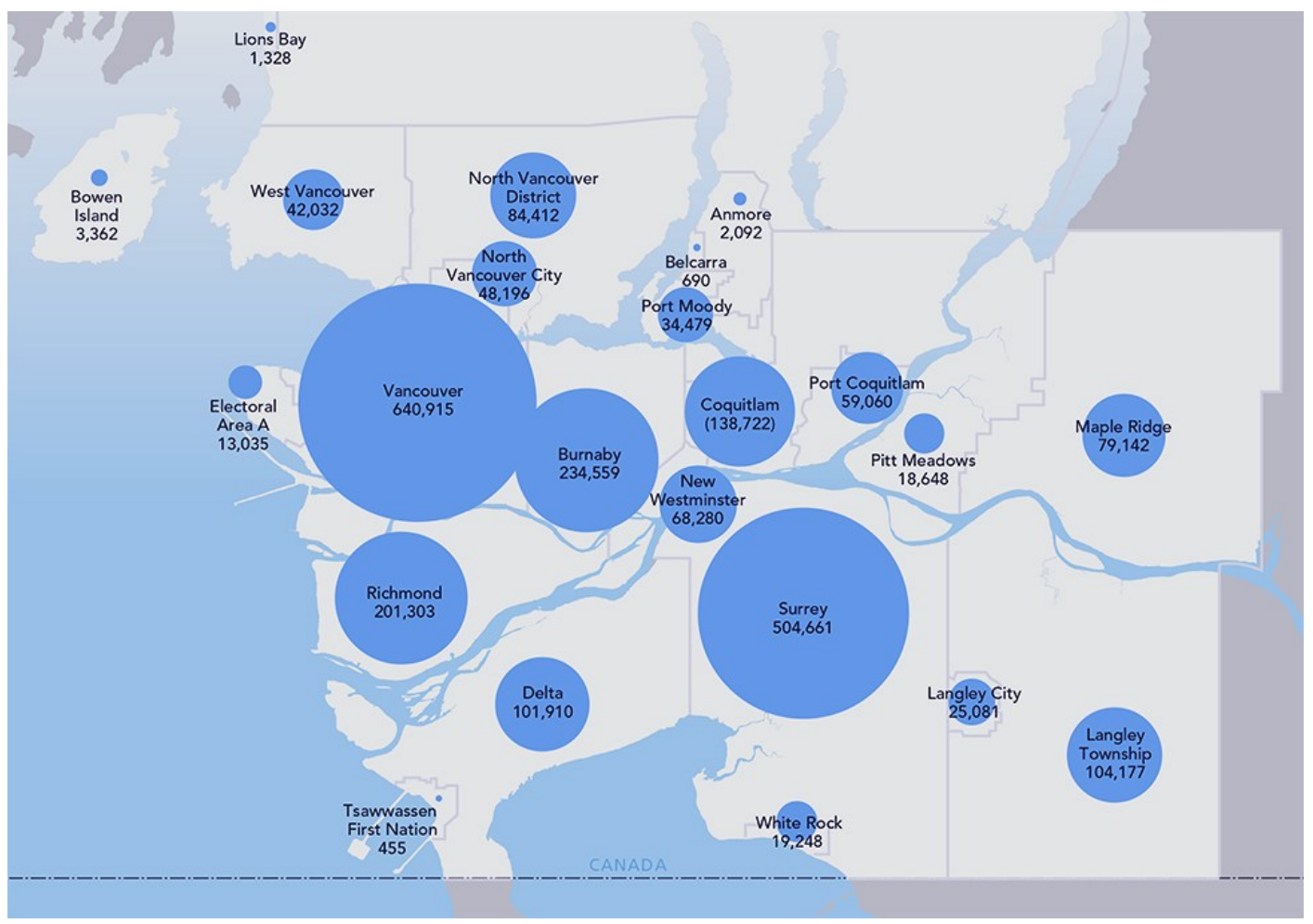

Policing across the Metro Vancouver region is complex. The RCMP provide services on a contract basis to 13 municipalities - including Surrey, Richmond, Burnaby, Coquitlam, Langley, North Vancouver, Pitt Meadows, and Maple Ridge. The municipalities of Pitt Meadows and Maple Ridge are policed by the Ridge Meadows detachment of the RCMP. Municipal police agencies across the region include Vancouver, Delta, New Westminster, Port Moody, and West Vancouver. ${ }^{4}$ The DPD has a complement of 155 sworn officers (Statistics Canada 2017e) with roughly 75 assigned to front-line duties (Chief Neil Dubord, personal communication, 11 April 2016). The 
DPD is the $47^{\text {th }}$ largest municipal police agency in Canada and the $9^{\text {th }}$ largest in British Columbia (Statistics Canada 2017e). Rank-and-file are deployed from police headquarters and there are shift briefings each day in the morning and evening. To collect data, I attended at shift briefings on three dates, as detailed in the table below:

\begin{tabular}{|c|c|}
\hline Date & Location \\
\hline 22 November 2016 & HQ D Platoon \\
\hline 23 November 2016 & HQ B Platoon \& A Platoon \\
\hline 25 November 2016 & HQ C Platoon \\
\hline
\end{tabular}

Fifty-eight (58) surveys were administered to DPD participants and all were completed, returned to me, and included in the study.

For 2016, the violent CSI for the Vancouver census metropolitan area was 72.8 (Statistics Canada 2017d). The prevalence of low-income individuals in Delta was 9.7\% in 2016 (Statistics Canada 2017c). The table below reports on the race/ethnicity of Delta's population in 2016 (Statistics Canada 2017b).

\begin{tabular}{|c|c|c|}
\hline Population of Delta (2016) & $\mathbf{1 0 0 , 8 4 0}$ & $\mathbf{1 0 0 . 0} \%$ \\
\hline White & 64,540 & $64.0 \%$ \\
\hline Total 'Visible Minority' Population & 36,300 & $36.0 \%$ \\
\hline South Asian & 20,485 & $20.3 \%$ \\
\hline Chinese & 7685 & $7.6 \%$ \\
\hline Black & 795 & $0.8 \%$ \\
\hline Filipino & 2655 & $2.6 \%$ \\
\hline Latin American & 815 & $0.8 \%$ \\
\hline Arab & 255 & $0.3 \%$ \\
\hline Southeast Asian & 585 & $0.6 \%$ \\
\hline West Asian & 260 & $0.3 \%$ \\
\hline Korean & 520 & $0.5 \%$ \\
\hline Japanese & 1115 & $1.1 \%$ \\
\hline Other 'Visible Minority' & 310 & $0.3 \%$ \\
\hline Multiple 'Visible Minorities' & 810 & $0.8 \%$ \\
\hline Aboriginal & 2710 & $2.7 \%$ \\
\hline
\end{tabular}


Several Delta Police Department (DPD) officers discussed a controversial and widely-publicized occurrence, which they suggest has impacted negatively and permanently on many police officers across the Metro Vancouver region. On 8 November 2012, DPD Constable Jordan MacWilliams (working with a Metro Vancouver integrated tactical unit as a sniper) shot and killed an armed individual during a prolonged standoff in the parking lot of a New Westminster casino. Constable MacWilliams was subsequently charged by the British Columbia Justice Department with murder. However, the charges were stayed in 2015 by the provincial prosecutor's office and the officer later returned to active duty with the DPD. Chief Neil Dubord observed, at the time, "Today, we feel an overwhelming sense of relief for Jordan MacWilliams, his family, the men and women of the Delta Police Department, and indeed all police officers across Canada...The charges laid...had the potential to deeply impact police across Canada, both operationally and psychologically" (Proctor 2015). Service de police de la Ville de Gatineau (Gatineau, Québec)

Le Service de police de la Ville de Gatineau (SPVG) or, in English, the Gatineau Police Service, is responsible for policing services in the City of Gatineau, which has a population of 276,000 residents (Statistics Canada 2017c). Gatineau is located in the Province of Québec and is immediately adjacent to the City of Ottawa - forming part of Canada's National Capital Region. Gatineau is the $4^{\text {th }}$ most populous city in Québec and the $18^{\text {th }}$ most populous city in Canada (Statistics Canada 2017c). The SPVG has a complement of 395 police officers (Statistics Canada 2017e), with approximately 175 assigned to front-line duties (Inspecteur Serge Guindon, personal communication, 4 July 2016). The SPVG is the $5^{\text {th }}$ largest municipal police agency in Québec and the $23^{\text {rd }}$ 
largest in Canada (Statistics Canada 2017e). Rank-and-file are deployed from two primary police buildings (Secteur Hull and Secteur Gatineau), with a handful of officers deployed from two substations located in outlying areas of the city. There are shift briefings each day in the morning, afternoon, and evening. To collect data, I attended at shift briefings on eight dates, as detailed in the table below:

\begin{tabular}{|c|c|}
\hline Date & Location \\
\hline 30 April 2016 & Secteur Gatineau Équipe C \\
\hline 1 May 2016 & Secteur Hull Équipe C \\
\hline 15 May 2016 & Secteur Hull Équipe E \\
\hline 11 June 2016 & Secteur Hull Équipe A \& Secteur Gatineau Équipe D \\
\hline 12 June 2016 & Secteur Hull Équipe D \& Secteur Gatineau Équipe A \\
\hline 30 June 2016 & Secteur Gatineau Équipe B \\
\hline 3 July 2016 & Secteur Hull Équipe B \\
\hline 4 July 2016 & Secteur Gatineau Équipe E \\
\hline
\end{tabular}

One hundred and forty-eight (148) surveys were administered to SPVG participants and all were completed, returned to me, and included in the study.

For 2016, the violent CSI for Gatineau was 63.8 (Statistics Canada 2017d). The prevalence of low-income individuals in Gatineau was 13.0\% in 2016 (Statistics Canada 2017c). The table below reports on the race/ethnicity of Gatineau's population in 2016 (Statistics Canada 2017b).

\begin{tabular}{|c|c|c|}
\hline Population of Gatineau (2016) & $\mathbf{2 7 1 , 8 5 0}$ & $\mathbf{1 0 0 \%}$ \\
\hline White & 235,090 & $86.5 \%$ \\
\hline Total 'Visible Minority’ Population & 36,760 & $13.5 \%$ \\
\hline South Asian & 955 & $0.4 \%$ \\
\hline Chinese & 2405 & $0.9 \%$ \\
\hline Black & 16,670 & $6.1 \%$ \\
\hline Filipino & 525 & $0.2 \%$ \\
\hline Latin American & 4170 & $1.5 \%$ \\
\hline Arab & 8305 & $3.1 \%$ \\
\hline Southeast Asian & 1280 & $0.5 \%$ \\
\hline West Asian & 875 & $0.3 \%$ \\
\hline
\end{tabular}




\begin{tabular}{|c|c|c|}
\hline Korean & 295 & $0.1 \%$ \\
\hline Japanese & 130 & $0 \%$ \\
\hline Other 'Visible Minority' & 410 & $0.2 \%$ \\
\hline Multiple 'Visible Minorities' & 745 & $0.3 \%$ \\
\hline Aboriginal & 10,420 & $3.8 \%$ \\
\hline
\end{tabular}

Halifax Regional Police (Halifax Regional Municipality, Nova Scotia)

The Halifax Regional Police (HRP) are responsible for policing the urban and more suburban communities of the Halifax Regional Municipality (including Halifax, Dartmouth, and Bedford). The outlying areas are policed by RCMP officers. The Halifax Regional Municipality has a population of 403,000 residents and it is the most populous city in the Province of Nova Scotia and the $14^{\text {th }}$ most populous city in Canada (Statistics Canada 2017c). The HRP has a complement of 512 sworn police officers (Statistics Canada 2017e) with about 250 of these officers assigned to front-line duties (Deputy Chief Robin McNeil, personal communication, 3 February 2016). The HRP is the largest municipal police agency in Nova Scotia and the $20^{\text {th }}$ largest in Canada (Statistics Canada 2017e). Rank-and-file are deployed from three divisional facilities (Central, East, and West) and there are shift briefings each day in the morning and evening. To collect data, I attended at shift briefings on six dates, as detailed in the table below:

\begin{tabular}{|c|c|}
\hline Date & Location \\
\hline 22 June 2016 & West 2 Watch \\
\hline 23 June 2016 & Central 1 Watch, West 4 Watch, East 4 Watch \\
\hline 24 June 2016 & Central 4 Watch \\
\hline 25 June 2016 & East 3 Watch, West 1 Watch \\
\hline 26 June 2016 & West 3 Watch, East 1 Watch \\
\hline 27 June 2016 & Central 2 Watch \& 3 Watch, East 2 Watch \\
\hline
\end{tabular}


Two hundred and six (206) surveys were administered to HRP participants and all were completed, returned to me, and included in the study.

For 2016, the violent CSI for Halifax was 77.3 (Statistics Canada 2017d). The prevalence of low-income individuals was 14.8\% in 2016 (Statistics Canada 2017c). The table below reports on the race/ethnicity of the Halifax population in 2016 (Statistics Canada 2017b).

\begin{tabular}{|c|c|c|}
\hline Population of Halifax (2016) & $\mathbf{3 9 7 , 3 9 5}$ & $\mathbf{1 0 0 \%}$ \\
\hline White & 352,110 & $88.6 \%$ \\
\hline Total 'Visible Minority' Population & 45,285 & $11.4 \%$ \\
\hline South Asian & 6555 & $1.6 \%$ \\
\hline Chinese & 6975 & $1.8 \%$ \\
\hline Black & 15,085 & $3.8 \%$ \\
\hline Filipino & 2575 & $0.6 \%$ \\
\hline Latin American & 1210 & $0.3 \%$ \\
\hline Arab & 7335 & $1.9 \%$ \\
\hline Southeast Asian & 865 & $0.2 \%$ \\
\hline West Asian & 1390 & $0.3 \%$ \\
\hline Korean & 1225 & $0.3 \%$ \\
\hline Japanese & 490 & $0.1 \%$ \\
\hline 'Visible Minority' & 490 & $0.1 \%$ \\
\hline Multiple 'Visible Minorities' & 1095 & $0.2 \%$ \\
\hline Aboriginal & 18,835 & $4.7 \%$ \\
\hline
\end{tabular}

Several Halifax Regional Police (HRP) officers mentioned to me that the HRP has a lengthy history of acrimonious relations with the city's relatively large Black population. However, none could cite a recent occurrence and my searches of Halifax media sources for contemporary highly-publicized events involving HRP and Black citizens were fruitless. 


\section{Hamilton Police Service (Hamilton, Ontario)}

The Hamilton Police Service (HPS) is responsible for policing services in the City of Hamilton, which has a population of 537,000 residents (Statistics Canada 2017c). Hamilton is the $5^{\text {th }}$ most populous city in the Province of Ontario and the $10^{\text {th }}$ most populous city in Canada (Statistics Canada 2017c). The HPS has a complement of 840 sworn officers (Statistics Canada 2017e) with approximately 406 assigned to frontline duties (Staff Sergeant Steven Hahn, personal communication, 26 July 2016). The HPS is the $6^{\text {th }}$ largest municipal police agency in Ontario and the $11^{\text {th }}$ largest in Canada (Statistics Canada 2017e). Rank-and-file are deployed from three divisional facilities (Central, East, and Mountain) and there are shift briefings each day in the morning, afternoon, and evening. To collect data, I attended at shift briefings on seven dates, as detailed in the table below:

\begin{tabular}{|c|c|}
\hline Date & Location \\
\hline 5 May 2016 & Central A Squad \& C Squad \\
\hline 6 May 2016 & East B Squad \& D Squad \\
\hline 21 July 2016 & Central D Squad \\
\hline 22 July 2016 & Mountain C Squad \& A Squad \\
\hline 23 July 2016 & East C Squad \\
\hline 24 July 2016 & East A Squad \\
\hline 26 July 2016 & Mountain D Squad \& B Squad, Central B Squad \\
\hline
\end{tabular}

Two hundred and ninety-five (295) surveys were administered to HPS participants and all were completed and returned to me. Of these, 289 surveys were included in the study. Six (6) completed surveys were excluded because the respondents disclosed that they were not currently performing front-line duties.

For 2016, the violent CSI for Hamilton was 66.3 (Statistics Canada 2017d). The prevalence of low-income individuals in Hamilton was 15.3\% in 2016 (Statistics 
Canada 2017c). The table below reports on the race/ethnicity of Hamilton's population in 2016 (Statistics Canada 2017b).

\begin{tabular}{|c|c|c|}
\hline Population of Hamilton (2016) & $\mathbf{5 2 7 , 9 3 0}$ & $\mathbf{1 0 0} \%$ \\
\hline White & 427,875 & $81.0 \%$ \\
\hline Total 'Visible Minority' Population & 100,060 & $19.0 \%$ \\
\hline South Asian & 22,105 & $4.2 \%$ \\
\hline Chinese & 10,070 & $1.9 \%$ \\
\hline Black & 20,245 & $3.8 \%$ \\
\hline Filipino & 8150 & $1.5 \%$ \\
\hline Latin American & 8425 & $1.6 \%$ \\
\hline Arab & 10,330 & $2.0 \%$ \\
\hline Southeast Asian & 6505 & $1.2 \%$ \\
\hline West Asian & 4800 & $0.9 \%$ \\
\hline Korean & 2090 & $0.4 \%$ \\
\hline Japanese & 1060 & $0.2 \%$ \\
\hline Other 'Visible Minority' & 2530 & $0.5 \%$ \\
\hline Multiple 'Visible Minorities' & 3745 & $0.7 \%$ \\
\hline Aboriginal & 12,135 & $2.3 \%$ \\
\hline
\end{tabular}

Several Hamilton Police Service (HPS) research participants spoke with me about a series of recent controversial events involving HPS officers, which have engendered considerable criticism of, and skepticism toward, the HPS by not insignificant numbers of Hamilton residents. Many of these officers suggested that negative public sentiment toward police officers and increased scrutiny of their work have resulted in behavioural changes among HPS officers - in terms of the 'F.I.D.O. mentality'.

Participants spoke of a 2015 occurrence involving HPS Constable Kudo Park. On 17 April 2015, HPS officers were investigating Thomas Schonberger, who "was intoxicated at the time of the incident, has a history of schizophrenia, substance abuse and trouble with the law" (O'Reilly 2017a). During the interaction, Schonberger, who was handcuffed, spat in the face of Park, who responded by immediately punching 
Schonberger in the face. A few minutes later, Park returned to where Schonberger was being held on the ground by other HPS officers and Park punched him twice more (Bennett 2017). Park was charged with assault causing bodily harm (because Schonberger had suffered fractured facial bones) but at trial in April 2017 the officer was convicted of the less severe charge of assault - with the judge excusing the first punch but finding the second and third punches were 'retribution' rather than lawful use of force (O’Reilly 2017a).

HPS research participants also spoke with me about two HPS officers, that were former partners in the 'guns and gangs enforcement unit' and that were the topic of much derision in the Hamilton media (and the broader media across the greater Toronto area). In 2012, HPS officer Robert Hansen was arrested and suspended from duty in relation to allegations that he had encouraged a police informant to plant a handgun at the home of a suspected drug dealer and then lied in obtaining a search warrant (Buist 2016). At trial in 2016, Hansen was convicted of perjury and obstructing justice, was sentenced to five years in prison, and resigned from the HPS. Hansen's former partner, Craig Ruthowsky, was arrested in 2015 as part of a Toronto Police project targeting violent street gangs and it is alleged that Ruthowsky was, himself, part of a Hamilton criminal organization that was affiliated with a Toronto gang (Buist 2016). Ruthowsky, who spent five weeks in jail before being released on bail, was charged with bribery, obstruction of justice, breach of trust, trafficking cocaine, and conspiracy and he remains suspended from duty with the HPS pending his trial (Buist 2016; O'Reilly 2017b). On 22 August 2017, Ruthowsky was charged by Toronto Police with an 
additional 16 criminal allegations related to his earlier activities while an HPS officer (O'Reilly 2017b).

Some HPS participants also spoke with me about a 2013 video recorded occurrence involving HPS Constable Mark Morelli, which developed as a viral YouTube sensation that attracted worldwide media attention. On 8 December 2013, Constable Morelli was video recorded by a bystander while the officer was wrestling on the ground with a resisting young woman who the officer was attempting to control, handcuff, and arrest in relation to a domestic assault allegation (Carter 2013). After the suspect was confined in the police cruiser, Morelli then addressed several citizens who had gathered in the area of the arrest. As described by $C B C$ journalist Adam Carter (2013), Morelli explained,

'Our domestic policy is very specific. Without going into detail, this young woman has broken the law, and we were attempting to place her under arrest and she began to resist,' he said to the people watching. 'I'm doing my best not to hurt that girl. Our mandate is to affect arrests while doing the minimum amount of damage to people as possible. And that's what I tried to do.' $\mathrm{He}$ explains that he only used 'soft hand techniques,' so no punches, kicks or forceful throws to the ground were used to subdue the suspect. 'I'm not going to get into a long wrestling match with a young girl, because the longer she fights, the more likely one of the two of us is going to get hurt,' he said. The man shooting the video questions the amount of force being used, but the officer reiterates again his approach. 'While it may appear to be very rough to you - and I apologize for you having to see that - but I have the lawful authority to arrest her and I am obligated to arrest her.'

In the days following the broad public circulation of the video recording, the HPS received thousands of telephone calls and emails from people commending the officer's handling of both the arrest and the criticism levelled by the individual who had video recorded the event (Carter 2013; White 2014). As described by Globe \& Mail reporter Patrick White (2014), "Filmed in an apparent attempt to embarrass the officers, the 
video has had the opposite effect, generating international praise for two Hamilton constables and offering a vivid example of how front-line officers are modifying everything from how they cuff suspects to where they grab lunch in the age of the camera-phone."

Kingston Police Force (Kingston, Ontario)

The Kingston Police Force (KPF) is responsible for policing services in the City of Kingston, which has a population of 124,000 residents (Statistics Canada 2017c). Kingston is the $21^{\text {st }}$ most populous city in the Province of Ontario and the $43^{\text {rd }}$ most populous city in Canada (Statistics Canada 2017c). The KPF has a complement of 201 sworn police officers (Statistics Canada 2017e) with approximately 107 assigned to front-line duties (Deputy Chief Antje McNeely, personal communication, 3 February 2016). The KPF is the $34^{\text {th }}$ largest municipal police agency in Canada and the $15^{\text {th }}$ largest in Ontario (Statistics Canada 2017e). Rank-and-file are deployed from police headquarters and there are shift briefings each day in the morning and evening. To collect data, I attended at shift briefings on four dates, as detailed in the table below:

\begin{tabular}{|c|c|}
\hline Date & Location \\
\hline 16 February 2016 & HQ A Platoon \\
\hline 19 February 2016 & HQ C Platoon \\
\hline 14 July 2016 & HQ D Platoon \\
\hline 19 July 2016 & HQ B Platoon \\
\hline
\end{tabular}

Seventy-eight (78) surveys were administered to KPF participants and all were completed, returned to me, and included in the study.

For 2016, the violent CSI for Kingston was 38.5 (Statistics Canada 2017d). The prevalence of low-income individuals in Kingston was 14.8\% in 2016 (Statistics Canada 
2017c). The table below reports on the race/ethnicity of Kingston's population in 2016 (Statistics Canada 2017b).

\begin{tabular}{|c|c|c|}
\hline Population of Kingston (2016) & $\mathbf{1 2 0 , 2 2 0}$ & $\mathbf{1 0 0 \%}$ \\
\hline White & 108,565 & $90.3 \%$ \\
\hline Total 'Visible Minority' Population & 11,655 & $9.7 \%$ \\
\hline South Asian & 2395 & $2.0 \%$ \\
\hline Chinese & 2535 & $2.1 \%$ \\
\hline Black & 1750 & $1.5 \%$ \\
\hline Filipino & 755 & $0.6 \%$ \\
\hline Latin American & 885 & $0.7 \%$ \\
\hline Arab & 1020 & $0.9 \%$ \\
\hline Southeast Asian & 545 & $0.5 \%$ \\
\hline West Asian & 460 & $0.4 \%$ \\
\hline Korean & 525 & $0.5 \%$ \\
\hline Japanese & 190 & $0.2 \%$ \\
\hline Other 'Visible Minority' & 165 & $0.1 \%$ \\
\hline Multiple 'Visible Minorities' & 425 & $0.4 \%$ \\
\hline Aboriginal & 4220 & $3.5 \%$ \\
\hline
\end{tabular}

$\underline{\text { New Westminster Police Department (New Westminster, British Columbia) }}$

The New Westminster Police Department (NWPD) is responsible for policing services in the City of New Westminster, which has a population of 71,000 residents (Statistics Canada 2017c). New Westminster is one of the municipalities making up the Metro Vancouver region, it is the $18^{\text {th }}$ most populous city in the Province of British Columbia, and the $81^{\text {st }}$ most populous city in Canada (Statistics Canada 2017c). The NWPD has a complement of 133 sworn police officers with approximately 65 assigned to front-line duties (Chief David Jones, personal communication, 7 November 2016). Rank-and-file are deployed from police headquarters and there are shift briefings each day in the morning and evening. To collect data, I attended at shift briefings on three dates, as detailed in the table below: 


\begin{tabular}{|c|c|}
\hline Date & Location \\
\hline 24 November 2016 & HQ D Platoon \& C Platoon \\
\hline 26 November 2016 & HQ A Platoon \\
\hline 27 November 2016 & HQ B Platoon \\
\hline
\end{tabular}

Forty-nine (49) surveys were administered to NWPD participants and all were completed, returned to me, and included in the study.

For 2016, the violent CSI for the Vancouver census metropolitan area was 72.8 (Statistics Canada 2017d). The prevalence of low-income individuals in New Westminster was $15.6 \%$ in 2016 (Statistics Canada 2017c). The table below reports on the race/ethnicity of New Westminster's population in 2016 (Statistics Canada 2017e).

\begin{tabular}{|c|c|c|}
\hline Population of New Westminster (2016) & $\mathbf{6 9 , 9 0 5}$ & $\mathbf{1 0 0 \%}$ \\
\hline White & 42,695 & $61.1 \%$ \\
\hline Total ‘Visible Minority' Population & 27,210 & $38.9 \%$ \\
\hline South Asian & 5790 & $8.3 \%$ \\
\hline Chinese & 7020 & $10.0 \%$ \\
\hline Black & 1740 & $2.5 \%$ \\
\hline Filipino & 5755 & $8.2 \%$ \\
\hline Latin American & 1275 & $1.8 \%$ \\
\hline Arab & 570 & $0.8 \%$ \\
\hline Southeast Asian & 795 & $1.1 \%$ \\
\hline West Asian & 730 & $1.0 \%$ \\
\hline Korean & 1500 & $2.1 \%$ \\
\hline Japanese & 945 & $1.4 \%$ \\
\hline Other 'Visible Minority' & 215 & $0.3 \%$ \\
\hline Multiple 'Visible Minorities' & 870 & $1.2 \%$ \\
\hline Aboriginal & 2295 & $3.3 \%$ \\
\hline
\end{tabular}

As discussed in the context of the Delta Police Department, a fatal police shooting occurred in New Westminster in 2012 and several NWPD officers brought to my attention their concerns with 'knee jerk' public, media, and government 'anti-police' reactions to that occurrence. 
Niagara Regional Police Service (Regional Municipality of Niagara, Ontario)

The Niagara Regional Police Service (NRPS) provides policing services across the Regional Municipality of Niagara, which has a population of 406,000 persons (Statistics Canada 2017c). The Regional Municipality of Niagara is the $6^{\text {th }}$ most populous census municipal area (CMA) in the Province of Ontario and the $12^{\text {th }}$ most populous in Canada (Statistics Canada 2017c). The regional municipality includes several cities and towns - including Niagara Falls, St. Catharines, Welland, Grimsby, and Fort Erie. The region borders on the State of New York to the east (across the Niagara River is the City of Buffalo) and the City of Hamilton to the west.

The NRPS has a complement of 706 sworn officers (Statistics Canada 2017e), with approximately 280 assigned to front-line duties (Corporate Analyst Paul Divers, personal communication, 16 February 2016). The NRPS is the $15^{\text {th }}$ largest municipal police agency in Canada and the $8^{\text {th }}$ largest in Ontario (Statistics Canada 2017e). Frontline operations are deployed out of six district stations across the region, with daily shift briefings in the morning and evening. To collect data, I attended at shift briefings on six dates, as detailed in the table below:

\begin{tabular}{|c|c|}
\hline Date & Location \\
\hline 6 December 2016 & 1 District D Platoon \& 2 District C Platoon \\
\hline 7 December 2016 & 1 District C Platoon \& 2 District D Platoon \\
\hline 8 December 2016 & 2 District B Platoon \& 3 District C Platoon \\
\hline 9 December 2016 & 1 District B Platoon \& 5 District C Platoon \\
\hline 10 December 2016 & 1 District A Platoon \& 3 District B Platoon \\
\hline 11 December 2016 & 2 District A Platoon \& 5 District B Platoon \\
\hline
\end{tabular}

Two hundred (200) surveys were administered to NRPS officers and 199 were completed and returned to me. On 7 December 2016 two officers had to leave the briefing for a priority call and took the survey documents with them. Only one was 
returned to me. Eight (8) surveys were excluded from consideration in the study because they were submitted by NRPS detectives who had attended on front-line shift briefings.

For 2016, the violent CSI for the Regional Municipality of Niagara was 37.6, which is the lowest of any Canadian census metropolitan area (Statistics Canada 2017d). The prevalence of low-income individuals across the region was $14.5 \%$ in 2016 (Statistics Canada 2017c). The table below reports on the race/ethnicity of the region's population in 2016 (Statistics Canada 2017b).

\begin{tabular}{|c|c|c|}
\hline Population of Niagara Region (2016) & $\mathbf{4 3 8 , 1 6 0}$ & $\mathbf{1 0 0 \%}$ \\
\hline White & 399,345 & $91.1 \%$ \\
\hline Total 'Visible Minority’ Population & 38,810 & $8.9 \%$ \\
\hline South Asian & 6170 & $1.4 \%$ \\
\hline Chinese & 6055 & $1.4 \%$ \\
\hline Black & 7970 & $1.8 \%$ \\
\hline Filipino & 3900 & $0.9 \%$ \\
\hline Latin American & 4620 & $1.1 \%$ \\
\hline Arab & 2570 & $0.6 \%$ \\
\hline Southeast Asian & 2050 & $0.5 \%$ \\
\hline West Asian & 860 & $0.2 \%$ \\
\hline Korean & 1445 & $0.3 \%$ \\
\hline Japanese & 730 & $0.2 \%$ \\
\hline Other 'Visible Minority' & 690 & $0.2 \%$ \\
\hline Multiple 'Visible Minorities' & 1765 & $0.4 \%$ \\
\hline Aboriginal & 12,250 & $2.8 \%$ \\
\hline
\end{tabular}

Several Niagara Regional Police Service (NRPS) officers that participated in the study mentioned to me concerns with a Fort Erie resident named Fred Bracken, a selfdescribed 'anti-police' activist who often files complaint against officers and posts his video recordings of NRPS activities on a YouTube channel. A 2016 article in the St. Catharines Standard details two lawsuits filed by Bracken, alleging that NRPS officers violated his rights by falsely arresting and unlawfully searching him (Sawchuck 2016). 
Others described the assault causing bodily harm conviction of NRPS Constable Paul Zarafonitis as a development that has negatively impacted on the psyche and behaviour of some rank-and-file NRPS officers. During a 19 May 2012 altercation with a photographer outside a Niagara Falls restaurant, the officer punched Mike Farkas in the face (resulting in fractures to the nose and orbital bone) after Farkas refused to stop taking photographs of police interactions with a large crowd of volatile and intoxicated persons (Sawchuck 2014).

\section{Ottawa Police Service (Ottawa, Ontario)}

The Ottawa Police Service (OPS) is responsible for policing services in the City of Ottawa, which has a population of 934,000 residents (Statistics Canada 2017c). Ottawa is Canada's capital city, the $2^{\text {nd }}$ most populous city in the Province of Ontario (discounting the regional municipalities of Peel and York, which are comprised of multiple cities and towns), and Canada's $4^{\text {th }}$ most populous city (Statistics Canada 2017c). The OPS have a complement of 1239 sworn officers (Statistics Canada 2017e), with approximately 450 assigned to front-line duties. ${ }^{5}$ The OPS are the $4^{\text {th }}$ largest municipal police service in Ontario and the $8^{\text {th }}$ largest in Canada (Statistics Canada 2017e). Rank-and-file are deployed out of five divisional facilities across the city, with daily shift briefings in the morning, afternoon, and evenings. To collect data, I attended at shift briefings on eighteen dates, as detailed in the table below:

\begin{tabular}{|c|c|}
\hline Date & Location \\
\hline 18 February 2016 & West Division B Platoon \\
\hline 23 February 2016 & Central Division D Platoon \\
\hline 7 April 2016 & Central Division F Platoon \\
\hline 11 April 2016 & Central Division C Platoon \\
\hline 12 April 2016 & West Division C Platoon \\
\hline 17 April 2016 & East Division C Platoon \\
\hline
\end{tabular}




\begin{tabular}{|c|c|}
\hline 29 April 2016 & Central Division E Platoon \\
\hline 30 April 2016 & West Division E Platoon \\
\hline 1 May 2016 & East Division E Platoon \\
\hline 8 May 2016 & West Division B Platoon \\
\hline 12 May 2016 & Central Division B Platoon \\
\hline 15 May 2016 & West Division F Platoon \\
\hline 3 July 2016 & East Division D Platoon \\
\hline 6 July 2016 & West Division D Platoon \\
\hline 11 July 2016 & West Division A Platoon \\
\hline 12 July 2016 & East Division A Platoon \\
\hline 15 July 2016 & Central Division A Platoon \\
\hline 25 July 2016 & East Division B Platoon \\
\hline
\end{tabular}

Three hundred and eighty-three (383) surveys were administered to OPS officers and 382 were completed and returned to me. On 11 April 2016 one officer elected not participate, advising that he/she does not trust researchers to abide by ethics protocols (in not sharing participant's names and information with management). All 382 completed surveys were included in this study.

For 2016, the violent CSI for the City of Ottawa was 62.1 (Statistics Canada 2017d). The prevalence of low-income individuals in Ottawa was 12.6\% in 2016 (Statistics Canada 2017c). The table below reports on the race/ethnicity of Ottawa's population in 2016 (Statistics Canada 2017b).

\begin{tabular}{|c|c|c|}
\hline Population of Ottawa (2016) & $\mathbf{9 1 6 , 8 6 0}$ & $\mathbf{1 0 0 \%}$ \\
\hline White & 675,610 & $73.7 \%$ \\
\hline Total 'Visible Minority' Population & 241,250 & $26.3 \%$ \\
\hline South Asian & 38,750 & $4.2 \%$ \\
\hline Chinese & 40,960 & $4.5 \%$ \\
\hline Black & 60,205 & $6.6 \%$ \\
\hline Filipino & 12,035 & $1.3 \%$ \\
\hline Latin American & 11,150 & $1.2 \%$ \\
\hline Arab & 40,920 & $4.5 \%$ \\
\hline Southeast Asian & 12,370 & $1.3 \%$ \\
\hline West Asian & 9000 & $1.0 \%$ \\
\hline
\end{tabular}




\begin{tabular}{|c|c|c|}
\hline Korean & 2955 & $0.3 \%$ \\
\hline Japanese & 2425 & $0.3 \%$ \\
\hline Other 'Visible Minority' & 2605 & $0.3 \%$ \\
\hline Multiple 'Visible Minorities' & 7865 & $0.9 \%$ \\
\hline Aboriginal & 22,960 & $2.5 \%$ \\
\hline
\end{tabular}

In conversations with me, typically after the shift briefing on which I administered surveys, numerous Ottawa Police Service (OPS) officers discussed a high-profile 2008 occurrence - in the context of negative public and media narratives ('anti-police') and the impact on OPS officers' motivation (characterized by one officer as 'the give a fuck factor') and on de-policing behaviours. That occurrence involved the arrest of a $27-$ year-old Black woman, Stacy Bonds, on 26 September 2008. Bonds was transported to the OPS cellblock after officers "observed [Bonds] to be...consuming alcohol in a public place and to be intoxicated in a public place" (Duggal 2011). In the cellblock, Bonds kicked at the officer searching her and, in response, officers applied knee strikes, pinned Bonds to the floor (where Sergeant Steve Desjourdy used scissors to cut off Bonds' shirt and bra) and then left Bonds, topless and in soiled pants, in a cell for several hours (Duggal 2011; Galloway 2011).

At Bonds' trial for assaulting police, the judge stayed the charge and ruled that the arrest was unlawful, the entire incident was 'a travesty', and that the police treatment of Bonds represented an 'indignity to a human being' (CTV News 2010c; Galloway 2011). The cellblock video recording of the interaction between Bonds and officers, which was played at the trial, was thereafter made available to a broad public audience through local media and then national media. In the wake of the video release, several Ottawa defence lawyers suggested that the Bonds' occurrence was only one example of systemic problems in the OPS cellblock (CTV News 2010b). This contention was 
validated by Acting Chief Gilles Larochelle (now Chief in Kingston), who, in a statement to media, advised that the Ontario Provincial Police had been called in to investigate other potentially problematic cellblock occurrences and observed that "the force seems to have a systemic problem with the actions of officers in cellblock" (CTV News 2010c).

Chief Vern White, in his statement after reviewing the Bonds' video, said, 'I understand that Ottawa residents will be shocked by the video' (CTV News 2010d). As White predicted, there was considerable "public outrage and questions about police abuse of power" (CTV News 2010b). White observed in this regard, “...the [OPS] has never experienced this level of concern from the public" and also suggested that “...[OPS] officers feel their integrity is being questioned" (CTV News 2010a). Ultimately, Desjourdy was charged by the provincial oversight agency with sexual assault, in relation to his actions in the cellblock, but he was acquitted at trial. Bonds has since filed a \$1.2 million civil lawsuit against the OPS, which contends that Bonds "was stopped without a valid reason and taken to the station because she questioned why officers arrested her" and alleges that "a group of vindictive officers manhandled her and tossed her in a cell half-naked in part because she is Black and female" (Galloway 2011).

Also, as discussed in Chapter One, the highly-publicized and much discussed 'racial profiling' ruling by an Ottawa judge in 2011, involving the actions of three OPS officers, was brought to my attention by many OPS participants during my data collection sessions at OPS shift briefings. Officers expressed frustration with the judge's finding, the media coverage (which included publicizing the names of the involved 
officers), and the 'knee-jerk' public 'backlash' against police. In contrast to the antagonistic reactions outside policing, Chief Vern White characterized police actions in this occurrence as 'good police work' (National Post 2016). Despite support from management, officers presented this kind of situation as a prime example of the 'shift in tone' among the judiciary, the media, and the Ottawa public, which can influence otherwise 'dedicated' and 'proactive' police officers to make the decision to adopt depolicing tactics to avoid public controversy, 'stay out of the anti-police media spotlight', and sidestep allegations of, and investigations into, professional misconduct.

More recently, the July 2016 death of a mentally-disturbed 37-year old SomaliCanadian man, Abdirahman Abdi, during a physical confrontation with OPS officers, who were trying to arrest him for a series of sexual assaults, led to considerable media and public outcry, including demonstrations in the streets of Ottawa. An OPS officer has been charged with manslaughter by the provincial oversight agency and this outcome has reverberated throughout the rank-and-file of the OPS. Rank-and-file OPS officers are supporting their colleague through fundraising and the production of bracelets - the wearing of which by on-duty OPS officers became a flashpoint between front-line OPS officers, media commentators, and some Ottawa residents.

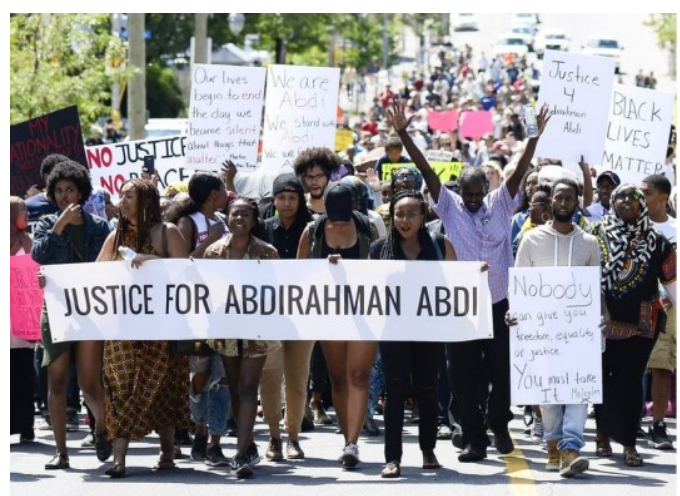

Image 13: Abdi protesters (James Park/Post Media 2017)

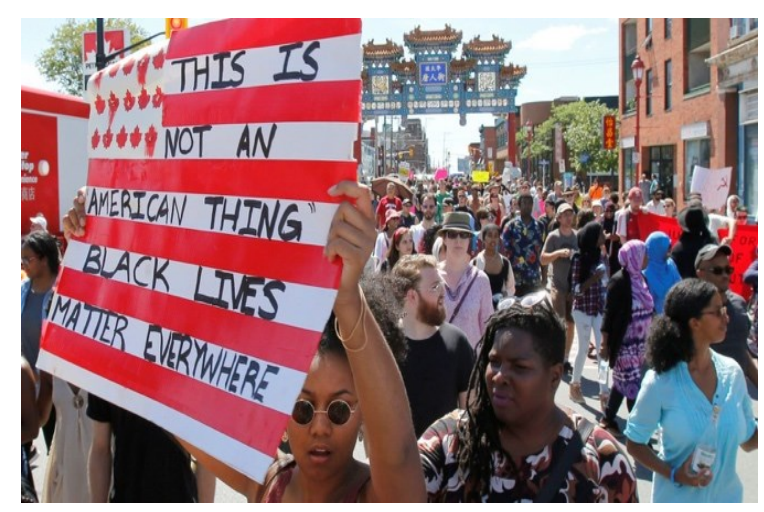

Image 14: Abdi protesters (Patrick Doyle/Reuters 2016) 
Matt Skof, an OPS Sergeant and the president of the labour organization

representing OPS rank-and-file officers, observed,

Any suggestions racism could have played a role in [this event] are inappropriate...[It is] unfortunate that conversations around the deaths of Black men by police in the United States are entering Canada...The officers were called to the scene. The officers had to attend. Race, in this case, is a fact, just like your age, your gender, your height. It doesn't have anything to do with our decision-making. Our decision-making is based on our training, and our training has nothing to do with race... We were called to a violent incident where we had to attend, and part of our mandate, as difficult as it is, is to ensure the safety of public, the accused, or the victim and/or the officers. And the use of force continuum begins, obviously, with communication, and that was occurring all the way throughout. That's always our primary focus, is to try and alleviate the situation through talking, and if that's not possible, then the laying of hands, and unfortunately a physical altercation, has to occur. And for the public to see that or digest it, it's difficult for them to see it (Nease 2016).

Port Moody Police Department (Port Moody, British Columbia)

The Port Moody Police Department (PMPD) is responsible for policing services in the City of Port Moody, which has a population of 34,000 residents (Statistics Canada 2017c). Port Moody is one of the municipalities making up the Metro Vancouver region. The PMPD has a complement of 51 sworn officers with 24 assigned to front-line duties (Chief Chris Rattenbury, personal communication, 2 November 2016). Rank-and-file are deployed from police headquarters and there are shift briefings each day in the morning and evening. To collect data, I attended at shift briefings on four dates, as detailed in the table below:

\begin{tabular}{|c|c|}
\hline Date & Location \\
\hline 25 November 2016 & HQ C Squad \\
\hline 27 November 2016 & HQ D Squad \\
\hline 29 November 2016 & HQ A Squad \\
\hline 30 November 2016 & HQ B Squad \\
\hline
\end{tabular}


Twenty-one (21) surveys were administered to PMPD participants and all were completed and returned to me. One survey was excluded from consideration because the respondent disclosed that she/he was not currently performing front-line duties.

For 2016, the violent CSI for the Vancouver census metropolitan area was 72.8 (Statistics Canada 2017d). The prevalence of low-income individuals in Port Moody was 11.5\% in 2016 (Statistics Canada 2017c). The table below reports on the race/ethnicity of Port Moody's population in 2016 (Statistics Canada 2017b).

\begin{tabular}{|c|c|c|}
\hline Population of Port Moody (2016) & $\mathbf{3 3 , 4 4 6}$ & $\mathbf{1 0 0} \%$ \\
\hline White & 23,260 & $69.5 \%$ \\
\hline Total 'Visible Minority’ Population & 10,185 & $30.5 \%$ \\
\hline South Asian & 775 & $2.3 \%$ \\
\hline Chinese & 3430 & $10.3 \%$ \\
\hline Black & 300 & $0.9 \%$ \\
\hline Filipino & 565 & $1.7 \%$ \\
\hline Latin American & 560 & $1.7 \%$ \\
\hline Arab & 70 & $0.2 \%$ \\
\hline Southeast Asian & 375 & $1.1 \%$ \\
\hline West Asian & 1265 & $3.8 \%$ \\
\hline Korean & 1770 & $5.3 \%$ \\
\hline Japanese & 510 & $1.5 \%$ \\
\hline Other 'Visible Minority' & 120 & $0.4 \%$ \\
\hline Multiple 'Visible Minorities' & 455 & $1.4 \%$ \\
\hline Aboriginal & 760 & $2.3 \%$ \\
\hline
\end{tabular}

Rochester Police Department (Rochester, New York)

The Rochester Police Department (RPD) provides policing services in the City of Rochester, which has a population of 210,000 persons (United States Census Bureau 2017). Rochester is the $3^{\text {rd }}$ most populous city in the State of New York and the $105^{\text {th }}$ most populous city in the United States (United States Census Bureau 2017). The RPD has a complement of 713 sworn police officers (Federal Bureau of Investigation 2017) 
with roughly 480 of these officers assigned to front-line duties (Captain Kevin Costello, personal communication, 17 May 2016). Rank-and-file are deployed out of five facilities across Rochester with daily shift briefings in the morning, afternoon, early evening, and late evening. To collect data, I attended at shift briefings on six dates, as detailed in the table below:

\begin{tabular}{|c|c|}
\hline Date & Location \\
\hline 18 June 2016 & Clinton Section \& Goodman Section \\
\hline 14 October 2016 & Goodman Section \\
\hline 15 October 2016 & Genesee Section \& Lake Section \\
\hline 16 October 2016 & Clinton Section \& Goodman Section \\
\hline 2 November 2016 & Clinton Section, Goodman Section, \& Central Section \\
\hline 3 November 2016 & Central Section \\
\hline
\end{tabular}

Two hundred and eighty-eight (288) surveys were administered to RPD officers and 287 were completed and returned to me. Of these, 281 were included for consideration in this study. Six (6) surveys were submitted by officers that identified they are not currently in front-line positions).

The RPD began a body-worn camera pilot in July 2016. By the end of August, 25 devices were in operation with Clinton Section officers and full implementation across the rank-and-file (involving 505 cameras) was completed in March 2017 (Lieutenant Michael Peskowski, personal communication, 2 August 2017).

In 2016, the violent crime rate for the City of Rochester was 876 occurrences per 100,000 inhabitants, which positions Rochester with the $3^{\text {rd }}$ highest rate in the State of New York (Federal Bureau of Investigation 2017). This also positions Rochester as the city with the $33^{\text {rd }}$ highest violent crime rate in the United States (Federal Bureau of Investigation 2017). The average median household income for Rochester is $\$ 30,960$ and the average per capita income is $\$ 19,158$ (both in 2015 dollars) (United States 
Census Bureau 2017). Approximately $33.5 \%$ of Rochester's population is living in poverty (United States Census Bureau 2017). As Axelson (2017) observes, "About one in three Rochester residents live in poverty, a higher poverty rate than in all but 14 other U.S. cities. Property values in Rochester are very low on average. More than half of all homes in the city are worth less than $\$ 77,000$, one of the lowest median property values in the country." In their analysis of variables from the United States census, Sauter and colleagues (2017) ranked Rochester as the $34^{\text {th }}$ worst American city. The table below reports on the race/ethnicity of Rochester's population (United States Census Bureau 2017).

\begin{tabular}{|c|c|c|}
\hline Population of Rochester, NY (2010) & $\mathbf{2 1 0 , 5 6 5}$ & $\mathbf{1 0 0} \%$ \\
\hline White & 79,178 & $37.6 \%$ \\
\hline Black/African American & 83,346 & $39.6 \%$ \\
\hline Hispanic/Latino & 34,456 & $16.4 \%$ \\
\hline Asian & 6350 & $3.0 \%$ \\
\hline Two or More Races & 6100 & $2.9 \%$ \\
\hline Other Race & 392 & $0.2 \%$ \\
\hline American Indian/Alaska Native & 666 & $0.3 \%$ \\
\hline Native Hawaiian/Other Pacific Islander & 77 & $0.0 \%$ \\
\hline
\end{tabular}

Several Rochester Police Department (RPD) officers spoke with me in October and November 2016 about a recent occurrence that they suggested exemplifies the kinds of developments that leads to de-policing frustrations among RPD front-line officers. A 17-year-old Black male, Ricky Bryant, reported that he was assaulted by multiple RPD officers on 7 August 2016 after the officers mistook him for an armed suspect being sought in the area (Hudak 2016). The allegations featured prominently in local media and became a divisive issue between the RPD and some in the city's Black community. In a federal lawsuit filed in November 2016, Bryant names 17 RPD officers as parties to the alleged assault, which Bryant claims involved a Taser, pepper spray, and kicks while 
he was held on the ground - resulting in multiple injuries, including a fractured orbital bone (Lahman 2017).

$\underline{\text { Royal Canadian Mounted Police Codiac Detachment (Moncton, New Brunswick) }}$

The RCMP is responsible for policing services in the City of Moncton and the surrounding region (including the City of Dieppe and the Town of Riverview) - known collectively as the Codiac Region. The region has a population of 145,000 residents and is the largest census municipal area (CMA) in the Province of New Brunswick and the $29^{\text {th }}$ largest CMA in Canada (Statistics Canada 2017c). The RCMP Codiac detachment has a complement of 147 sworn officers (Statistics Canada 2017e), with roughly 88 officers assigned to front-line duties (Inspector Jamie George, personal communication, 28 June 2016). The Codiac RCMP are the largest municipal police agency in New Brunswick and the $48^{\text {th }}$ largest in Canada (Statistics Canada 2017e). Rank-and-file are deployed from police headquarters in Moncton and there are shift briefings each day in the morning and evening. To collect data, I attended at shift briefings on two dates, as detailed in the table below:

\begin{tabular}{|c|c|}
\hline Date & Location \\
\hline 28 June 2016 & HQ B Watch Team 1 \& 2 and B Watch Team 3 \& 4 \\
\hline 29 June 2016 & HQ B Watch (officers absent on 28 June 2016) \\
\hline
\end{tabular}

Seventy-one (71) surveys were administered to Codiac RCMP participants and all were completed and returned to me. Two (2) were excluded from consideration because the respondents identified that they are not currently performing front-line duties. ${ }^{67}$

For 2016, the violent CSI for the City of Moncton was 79.3 (Statistics Canada 2017d). The prevalence of low-income individuals in Moncton was 15.5\% in 2016 
(Statistics Canada 2017c). The table below reports on the race/ethnicity of Moncton's population in 2016 (Statistics Canada 2017e).

\begin{tabular}{|c|c|c|}
\hline Population of Moncton (2016) & $\mathbf{1 4 1 , 5 2 5}$ & $\mathbf{1 0 0} \%$ \\
\hline White & 134,540 & $95.1 \%$ \\
\hline Total 'Visible Minority' Population & 6980 & $4.9 \%$ \\
\hline South Asian & 470 & $0.3 \%$ \\
\hline Chinese & 910 & $0.6 \%$ \\
\hline Black & 2350 & $1.7 \%$ \\
\hline Filipino & 565 & $0.4 \%$ \\
\hline Latin American & 295 & $0.2 \%$ \\
\hline Arab & 1015 & $0.7 \%$ \\
\hline Southeast Asian & 430 & $0.3 \%$ \\
\hline West Asian & 60 & $0 \%$ \\
\hline Korean & 600 & $0.4 \%$ \\
\hline Japanese & 85 & $0.1 \%$ \\
\hline Other 'Visible Minority' & 45 & $0 \%$ \\
\hline Multiple 'Visible Minorities' & 160 & $0.2 \%$ \\
\hline Aboriginal & 3515 & $2.5 \%$ \\
\hline
\end{tabular}

On 4 June 2014, three Codiac RCMP officers were shot dead and two others were shot and wounded as they engaged an armed individual in a residential neighbourhood of Moncton. Justin Bourque was observed walking through the area armed with two long guns, acting erratically, and he immediately opened fire on responding RCMP officers (CTV News 2014). Bourque was captured the following day, subsequently pleaded guilty, and is serving a minimum sentence of 75 years in prison. Significant controversy (and civil litigation) has developed in the wake of revelations that, while the RCMP understood the necessity of equipping front-line officers with carbine rifles and had purchased the weapons, none of the officers assigned to the Codiac detachment had been sent on carbine training and, therefore, none of the weapons had been deployed in the field at the time of the Bourque shooting (Thompson 2017). As a result, the RCMP 
was convicted of violating Canada's Labour Code and the widow of one of the officers killed observed that "the RCMP's decision to fight the...charges had hurt the policing community - 'I think it breaks the trust and I think the members are still very hurt and feel unsupported and I think that needs to be addressed"' (Thompson 2017).

\section{$\underline{\text { Royal Canadian Mounted Police Red Deer Detachment (Red Deer, Alberta) }}$}

The RCMP is responsible for policing services in the City of Red Deer and the surrounding region. Red Deer has a population of 100,000 residents, is the $3^{\text {rd }}$ most populous city in the Province of Alberta, and Canada's $54^{\text {th }}$ most populous city (Statistics Canada 2017c). The Red Deer RCMP detachment has a complement of 164 sworn police officers (Statistics Canada 2017e), with roughly 80 officers assigned to front-line duties (Superintendent Heidi Wild, personal communication, 3 February 2016). The Red Deer RCMP are the $3^{\text {rd }}$ largest municipal police agency in Alberta and the $41^{\text {st }}$ largest in Canada (Statistics Canada 2017e). Rank-and-file are deployed from the headquarters building in Red Deer and there are shift briefings each day in the morning and evening. To collect data, I attended at shift briefings on two dates, as detailed in the table below:

\begin{tabular}{|c|c|}
\hline Date & Location \\
\hline 29 May 2016 & HQ Watch 11 \& Watch 10 \\
\hline 2 June 2016 & HQ Watch 9 \& Watch 12 \\
\hline
\end{tabular}

Seventy-five (75) surveys were administered to Red Deer RCMP participants and all were completed, returned to me, and included in the study.

As the City of Red Deer does not meet Statistics Canada's threshold for census metropolitan area (CMA) reporting, no violent CSI is available. The prevalence of lowincome individuals in Red Deer was 10.0\% in 2016 (Statistics Canada 2017c). The table 
below reports on the race/ethnicity of Red Deer's population in 2016 (Statistics Canada 2017b).

\begin{tabular}{|c|c|c|}
\hline Population of Red Deer (2016) & $\mathbf{9 8 , 4 7 5}$ & $\mathbf{1 0 0 \%}$ \\
\hline White & 83,245 & $84.5 \%$ \\
\hline Total 'Visible Minority’ Population & 15,230 & $15.5 \%$ \\
\hline South Asian & 1665 & $1.7 \%$ \\
\hline Chinese & 1395 & $1.4 \%$ \\
\hline Black & 1735 & $1.8 \%$ \\
\hline Filipino & 6235 & $6.3 \%$ \\
\hline Latin American & 1770 & $1.8 \%$ \\
\hline Arab & 475 & $0.5 \%$ \\
\hline Southeast Asian & 660 & $0.7 \%$ \\
\hline West Asian & 440 & $0.4 \%$ \\
\hline Korean & 280 & $0.3 \%$ \\
\hline Japanese & 125 & $0.1 \%$ \\
\hline Other 'Visible Minority’ & 110 & $0.1 \%$ \\
\hline Multiple 'Visible Minorities' & 350 & $0.4 \%$ \\
\hline Aboriginal & 5185 & $5.3 \%$ \\
\hline
\end{tabular}

In my conversations with several Red Deer RCMP officers they discussed a recent controversial occurrence that received considerable publicity and these research participants suggest this may be having an impact on officers' morale and decisionmaking. In 2015, Red Deer RCMP Constable Eric Pomerleau was charged with assault in relation to his use of force during a 2012 impaired driving arrest, which was captured on the detachment's cellblock video recording system. Pomerleau was also charged with assaulting another prisoner in the cellblock, as a result of a subsequent review of the detachment's cellblock footage (CBC News 2015c). In 2016, Pomerleau was convicted of assault in relation to the first event but acquitted on the second (CBC News 2016a). 


\section{$\underline{\text { Royal Canadian Mounted Police Ridge Meadows Detachment }}$}

The RCMP is responsible for policing services in the municipalities of Maple Ridge and Pitt Meadows (and the surrounding region). This jurisdiction has a population of 101,000 residents (Statistics Canada 2017c) and is located within the Metro Vancouver region. Maple Ridge (with a population of 82,000 persons) is the $16^{\text {th }}$ most populous city in the Province of British Columbia and Canada's $71^{\text {st }}$ most populous city (Statistics Canada 2017c). The Ridge Meadows RCMP detachment has a complement of 121 sworn officers with roughly 85 assigned to front-line duties (Superintendent Dave Fleugel, personal communication, 4 February 2016). The Ridge Meadows RCMP are the $12^{\text {th }}$ largest municipal police agency in British Columbia and the $51^{\text {st }}$ largest in Canada (Statistics Canada 2017e). Rank-and-file are deployed from the headquarters building in Maple Ridge and there are shift briefings each day in the morning and evening. To collect data, I attended at shift briefings on three dates, as detailed in the table below:

\begin{tabular}{|c|c|}
\hline Date & Location \\
\hline 26 November 2016 & HQ D Watch \\
\hline 28 November 2016 & HQ B Watch \& A Watch \\
\hline 29 November 2016 & HQ C Watch \\
\hline
\end{tabular}

Seventy (70) surveys were administered to Ridge Meadows RCMP officers and all were completed and returned to me. Four (4) were excluded from consideration because the respondents disclosed that they are not currently performing front-line duties.

For 2016, the violent CSI for the Vancouver census metropolitan area was 72.8 (Statistics Canada 2017d). The prevalence of low-income individuals in the Maple 
Ridge/Pitt Meadows area was 10.5\% in 2016 (Statistics Canada 2017c). The table below reports on the race/ethnicity of the Maple Ridge/Pitt Meadows population in 2016 (Statistics Canada 2017e).

\begin{tabular}{|c|c|c|}
\hline Population of Ridge Meadows (2016) & $\mathbf{9 9 , 4 0 5}$ & $\mathbf{1 0 0 \%}$ \\
\hline White & 83,795 & $84.3 \%$ \\
\hline Total ‘Visible Minority' Population & 15,610 & $15.7 \%$ \\
\hline South Asian & 3310 & $3.3 \%$ \\
\hline Chinese & 2915 & $2.9 \%$ \\
\hline Black & 1150 & $1.2 \%$ \\
\hline Filipino & 2485 & $2.5 \%$ \\
\hline Latin American & 1070 & $1.1 \%$ \\
\hline Arab & 230 & $0.2 \%$ \\
\hline Southeast Asian & 865 & $0.9 \%$ \\
\hline West Asian & 900 & $0.9 \%$ \\
\hline Korean & 1105 & $1.1 \%$ \\
\hline Japanese & 690 & $0.7 \%$ \\
\hline Other 'Visible Minority' & 230 & $0.2 \%$ \\
\hline Multiple 'Visible Minorities' & 645 & $0.6 \%$ \\
\hline Aboriginal & 4380 & $4.4 \%$ \\
\hline
\end{tabular}

Several study participants from RCMP Ridge Meadows spoke with me about a 2016 'viral YouTube' event that involved one of their colleagues. The officer used force during the arrest of an intoxicated individual (by slamming him to the ground) and, after the release of a citizen's video recording of the event to a public audience, via social media, the officer involved was subject to an internal RCMP investigation, substantial media attention and criticism, and was taken off front-line duties for more than 6 months (Azpiri 2016). An officer who works on the same shift as the previously 'suspended' officer advised me that the 'whole detachment is now rattled', with officers hesitating in their actions and, as a result, 'some officers have been injured.' 
Saskatoon Police Service (Saskatoon, Saskatchewan)

The Saskatoon Police Service (SPS) provides policing services for the City of Saskatoon, which has a population of 246,000 persons (Statistics Canada 2017c).

Saskatoon is the most populous city in the Province of Saskatchewan and is the $19^{\text {th }}$ most populous city in Canada (Statistics Canada 2017c). The SPS has a complement of 453 sworn officers (Statistics Canada 2017e) and roughly 259 officers are assigned to front-line duties (Superintendent Mitch Yuzdepski, personal communication, 1 November 2016). The SPS is the $21^{\text {st }}$ largest municipal police agency in Canada and the largest in Saskatchewan (Statistics Canada 2017e). Front-line operations are deployed out of police headquarters, with daily shift briefings in the morning and evening. To collect data, I attended at shift briefings on three dates, as detailed in the table below:

\begin{tabular}{|c|c|}
\hline Date & Location \\
\hline 20 October 2016 & HQ D Platoon \\
\hline 21 October 2016 & HQ B Platoon \& A Platoon \\
\hline 23 October 2016 & HQ C Platoon \\
\hline
\end{tabular}

One hundred and eighty (180) surveys were administered to SPS officers and all were completed and returned to me. Five (5) of the surveys were excluded from consideration because they were completed by officers that identified they are not currently working in a front-line capacity.

For 2016, the violent CSI for the City of Saskatoon was 114.0 (Statistics Canada 2017d). The prevalence of low-income individuals in Saskatoon was 12.8\% in 2016 (Statistics Canada 2017c). The table below reports on the race/ethnicity of Saskatoon's population in 2016 (Statistics Canada 2017e). 


\begin{tabular}{|c|c|c|}
\hline Population of Saskatoon (2016) & $\mathbf{2 4 1 , 4 2 0}$ & $\mathbf{1 0 0} \%$ \\
\hline White & 193,640 & $80.2 \%$ \\
\hline Total ‘Visible Minority' Population & 47,780 & $19.8 \%$ \\
\hline South Asian & 13,335 & $5.5 \%$ \\
\hline Chinese & 7675 & $3.2 \%$ \\
\hline Black & 5390 & $2.2 \%$ \\
\hline Filipino & 10,870 & $4.5 \%$ \\
\hline Latin American & 1915 & $0.8 \%$ \\
\hline Arab & 2305 & $1.0 \%$ \\
\hline Southeast Asian & 2235 & $0.9 \%$ \\
\hline West Asian & 1170 & $0.5 \%$ \\
\hline Korean & 570 & $0.2 \%$ \\
\hline Japanese & 405 & $0.2 \%$ \\
\hline Other 'Visible Minority' & 445 & $0.2 \%$ \\
\hline Multiple 'Visible Minorities' & 1465 & $0.6 \%$ \\
\hline Aboriginal & 27,310 & $11.3 \%$ \\
\hline
\end{tabular}

Several Saskatoon Police Service (SPS) officers spoke with me about what they characterized as persistent friction between the SPS and Saskatoon's substantial indigenous population. As Comack (2012: 151) observes, "Much of police work involves the difficult and challenging job of dealing with people who are both troubled and troublesome. In a Prairie city such as Saskatoon, dealing with the troubled and troublesome means frequent encounters with Aboriginal people.” The SPS has a welldocumented history of problematic relations with the sizeable indigenous population in Saskatoon. As a result of inquiries spanning 2001 to 2003, into the freezing deaths of three indigenous men (in 1990 and 2000), through the SPS practice of 'Starlight Tours', resulted in considerable friction between the SPS and the city's indigenous community.

'Starlight Tours' involved "the police practice of detaining people, driving them to another location, and leaving them there to find their own way home...[often] raising the spectre of racism" (Comack 2012: 115). Two SPS officers were convicted of 
criminal charges for unlawfully confining an indigenous man and were imprisoned, and four officers were dismissed from the SPS. Russell Sabo, the SPS chief from 2003 through 2006, attempted to repair relations with the Aboriginal community, but was met with a non-confidence vote by $90 \%$ of SPS officers and the closing of ranks within the SPS (Comack 2012: 142). Sabo suggested publicly, that "officers may have been dumping native people outside the city for years" (CBC News 2003).

A provincial commission was empaneled in 2003 to examine the deaths of indigenous men and the practices of some SPS officers. Smith (2004: 1) reporting on the findings, wrote, "Saskatoon police took a native teenager into custody and attempted a cover-up when the 17-year-old was later discovered frozen with handcuff marks on his face, an inquiry has found. The final report of the inquiry into the death of Neil Stonechild also laments the bitter racial divide between natives and non-natives in Saskatchewan.” In this regard, Commissioner Justice David Wright observed, “As I reviewed the evidence of this inquiry, I was reminded, again and again, of the chasm that separates aboriginal and non-aboriginal people in this city and this province" (Smith 2004:1). Comack (2012: 117) finds that the revelations of 'Starlight Tours' split the Saskatoon community "into two opposing camps. Aboriginal activists and organizations declared the practice to be evidence of the racism rampant in the Saskatoon police force; [whereas] police and their supporters said Starlight Tours were a myth perpetrated by special interest groups."

\section{Stratford Police Service (Stratford, Ontario)}

The Stratford Police Service is responsible for municipal policing in the City of Stratford, which has a population of 31,000 residents (Statistics Canada 2017c). 
Stratford is located about 150 kilometers west of Toronto and 250 kilometers east of Windsor. The Stratford Police Service has a complement of 56 sworn officers with 37 assigned to front-line duties (Chief Mike Bellai, personal communication, 6 February 2016). Rank-and-file are deployed from police headquarters and there are shift briefings each day in the morning and evening. To collect data, I attended at shift briefings on two dates, as detailed in the table below:

\begin{tabular}{|c|c|}
\hline Date & Location \\
\hline 1 March 2016 & HQ C Platoon \& A Platoon \\
\hline 2 March 2016 & HQ D Platoon \& B Platoon \\
\hline
\end{tabular}

Twenty-nine (29) surveys were administered to Stratford Police Service officers and all were completed, returned to me, and included in this study.

As the City of Stratford does not meet Statistics Canada's threshold for census metropolitan area (CMA) reporting, no violent CSI is available. The prevalence of lowincome individuals in Stratford 11.9\% in 2016 (Statistics Canada 2017c). The table below reports on the race/ethnicity of Stratford's population in 2016 (Statistics Canada 2017e).

\begin{tabular}{|c|c|c|}
\hline Population of Stratford (2016) & $\mathbf{3 0 , 6 2 5}$ & $\mathbf{1 0 0} \%$ \\
\hline White & 28,800 & $94.0 \%$ \\
\hline Total 'Visible Minority' Population & 1825 & $6.0 \%$ \\
\hline South Asian & 425 & $1.4 \%$ \\
\hline Chinese & 170 & $0.6 \%$ \\
\hline Black & 335 & $1.1 \%$ \\
\hline Filipino & 130 & $0.4 \%$ \\
\hline Latin American & 165 & $0.5 \%$ \\
\hline Arab & 75 & $0.2 \%$ \\
\hline Southeast Asian & 275 & $0.9 \%$ \\
\hline West Asian & 30 & $0.1 \%$ \\
\hline Korean & 45 & $0.1 \%$ \\
\hline Japanese & 125 & $0.4 \%$ \\
\hline
\end{tabular}




\begin{tabular}{|c|c|c|}
\hline Other ‘Visible Minority' & 40 & $0.1 \%$ \\
\hline Multiple 'Visible Minorities' & 20 & $0.1 \%$ \\
\hline Aboriginal & 525 & 1.7 \\
\hline
\end{tabular}

\section{Syracuse Police Department (Syracuse, New York)}

The Syracuse Police Department (SPD) is responsible for policing services in the City of Syracuse, which has a population of 145,000 persons (United States Census Bureau 2017). Syracuse is the $5^{\text {th }}$ most populous city in the State of New York and the $182^{\text {nd }}$ most populous city in the United States (United States Census Bureau 2017).

The SPD has a complement of 414 sworn officers (Federal Bureau of Investigation 2017), with approximately 225 assigned to front-line duties (Captain David Mirizio, personal communication, 8 February 2016). Rank-and-file are deployed out of one SPD facility, with daily shift briefings in the morning, afternoon, and evening. To collect data, I attended at shift briefings over four days, as detailed in the table below:

\begin{tabular}{|c|c|}
\hline Date & Location \\
\hline 29 March 2016 & SPD patrol facility Platoon II, Platoon III \& Platoon I \\
\hline 30 March 2016 & SPD patrol facility Platoon I, Platoon II \& CRT \\
\hline 31 March 2016 & SPD patrol facility Platoon II \& Platoon III \\
\hline 2 September 2016 & SPD patrol facility Platoon I \& Platoon III \\
\hline
\end{tabular}

One hundred and sixty-eight (168) surveys were administered to SPD officers and all were completed, returned to me, and included in this study.

In 2016, the violent crime rate for the City of Syracuse was 793 occurrences per 100,000 inhabitants, which positioned Syracuse as having the $6^{\text {th }}$ highest rate in the State of New York (Federal Bureau of Investigation 2017). The average median household income for Syracuse is $\$ 31,881$ and the average per capita income is $\$ 19,558$ (both in 2015 dollars) (United States Census Bureau 2017). Approximately 34.8\% of 
Syracuse's population is living in poverty (United States Census Bureau 2017). Axelson (2017) observes, "Often indicative of less desirable places to live, property is relatively cheap in Syracuse. The typical area home is worth only $\$ 93,100$, less than a third of the state-wide median home value of $\$ 293,500$." In their analysis of variables from the United States census, Sauter and colleagues (2017) ranked Syracuse as the $31^{\text {st }}$ worst American city. The table below reports on the race/ethnicity of the Syracuse population (United States Census Bureau 2017).

\begin{tabular}{|c|c|c|}
\hline Population of Syracuse, NY (2010) & $\mathbf{1 4 5 , 1 7 0}$ & $\mathbf{1 0 0} \%$ \\
\hline White & 76,653 & $52.8 \%$ \\
\hline Black/African American & 40,672 & $28.0 \%$ \\
\hline Hispanic/Latino & 12,036 & $8.3 \%$ \\
\hline Asian & 7971 & $5.5 \%$ \\
\hline Two or More Races & 6108 & $4.2 \%$ \\
\hline Other Race & 303 & $0.2 \%$ \\
\hline American Indian/Alaska Native & 1390 & $1.0 \%$ \\
\hline Native Hawaiian/Other Pacific Islander & 37 & $0 \%$ \\
\hline
\end{tabular}

\section{Toronto Police Service (Toronto, Ontario)}

The Toronto Police Service (TPS) is responsible for policing services in the City of Toronto, which is Canada's most populous city with 2.8 million residents (Statistics Canada 2017c). The TPS has a complement of 5366 sworn police officers (Statistics Canada 2017e) with roughly 2500 of these officers assigned to front-line duties (Staff Sergeant Gordon Whealy, personal communication, 5 October 2016). The TPS is the largest municipal police agency in Canada. Rank-and-file TPS officers are deployed out of 17 divisional stations across the City of Toronto, with daily shift briefings in the morning, afternoon, and evening. To collect data, I attended shift briefings at a variety of divisions across Toronto on seven dates, as detailed in the table below: 


\begin{tabular}{|c|c|}
\hline Date & Location \\
\hline 26 April 2016 & 14 Division \\
\hline 27 April 2016 & 31 Division \\
\hline 16 August 2016 & 51 Division \\
\hline 17 August 2016 & 23 Division \\
\hline 18 August 2016 & 54 Division \\
\hline 19 August 2016 & 43 Division \\
\hline 29 September 2016 & 12 Division \\
\hline
\end{tabular}

Through a research agreement, the number of surveys I was authorized to administer to TPS front-line officers was capped at approximately 300 . Ultimately, the 314 surveys were administered and, of these, 312 were completed and returned to me. On 16 August 2016, two TPS officers declined to participate, citing distrust of management because of previous external research that apparently resulted in professional consequences for some TPS officers. Two (2) of the surveys were excluded from consideration because they were completed by officers that identified they were not presently working in front-line duties.

For 2016, the violent CSI for the City of Toronto was 70.4 (Statistics Canada 2017d). The prevalence of low-income individuals in Toronto was $20.2 \%$ in 2016 (Statistics Canada 2017c). The table below reports on the race/ethnicity of Toronto's population in 2016 (Statistics Canada 2017e).

\begin{tabular}{|c|c|c|}
\hline Population of Toronto (2016) & $\mathbf{2 , 6 9 1 , 6 6 5}$ & $\mathbf{1 0 0} \%$ \\
\hline White & $1,305,815$ & $48.5 \%$ \\
\hline Total 'Visible Minority’ Population & $1,385,850$ & $51.5 \%$ \\
\hline South Asian & 338,965 & $12.6 \%$ \\
\hline Chinese & 299,460 & $11.1 \%$ \\
\hline Black & 239,850 & $8.9 \%$ \\
\hline Filipino & 152,715 & $5.7 \%$ \\
\hline Latin American & 77,160 & $2.9 \%$ \\
\hline Arab & 36,030 & $1.3 \%$ \\
\hline Southeast Asian & 41,645 & $1.5 \%$ \\
\hline
\end{tabular}




\begin{tabular}{|c|c|c|}
\hline West Asian & 60,325 & $2.2 \%$ \\
\hline Korean & 41,640 & $1.5 \%$ \\
\hline Japanese & 13,410 & $0.5 \%$ \\
\hline Other 'Visible Minority' & 36,975 & $1.3 \%$ \\
\hline Multiple 'Visible Minorities' & 47,675 & $1.8 \%$ \\
\hline Aboriginal & 23,065 & $0.9 \%$ \\
\hline
\end{tabular}

Most TPS front-line vehicles are equipped with a 'dashcam' and a camera in the rear prisoner compartment. The 'dashcam' records both audio (officers wear compact microphones on their uniform) and video when the vehicle's emergency equipment (lights and siren) are activated, when manually turned-on by the officer, or when collision sensors are triggered. It should also be noted that TPS ran a body-worn camera pilot, involving about 80 front-line officers, from May 2015 through March 2016 (Toronto Police Service 2016).

Many Toronto Police Service (TPS) officers spoke with me about their frustrations with public and media criticism of the TPS in the wake of the fatal police shooting of Sammy Yatim. Just after midnight on 27 July 2013 Sammy Yatim, an 18year-old Syrian-Canadian man, was shot dead by TPS Constable James Forcillo. That evening, while onboard a downtown Toronto streetcar, Yatim was observed to be behaving erratically (likely due to consumption of illicit drugs) and he produced a knife, which he used to threaten passengers (Hasham 2015; National Post 2015). Passengers and the driver fled the streetcar and TPS officers responded. During the ensuing confrontation, Forcillo fatally shot Yatim. A bystander video recorded the event and, upon release of the footage to a public audience (through YouTube and traditional media), considerable criticism of police actions developed, not just across the greater Toronto region but across Canada. Substantial street protests mobilized and sit-in 
demonstrations were convened at TPS headquarters, which included Black Lives Matter activists.

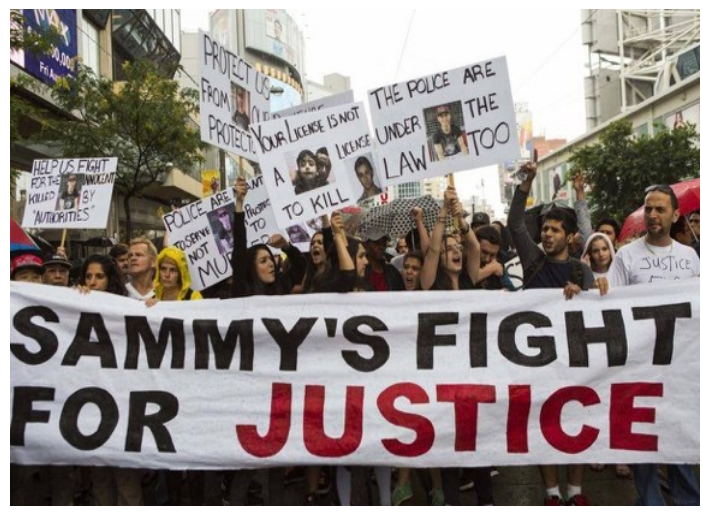

Image 16: Yatim protesters Philip Cheung/Globe \& Mail (2013)

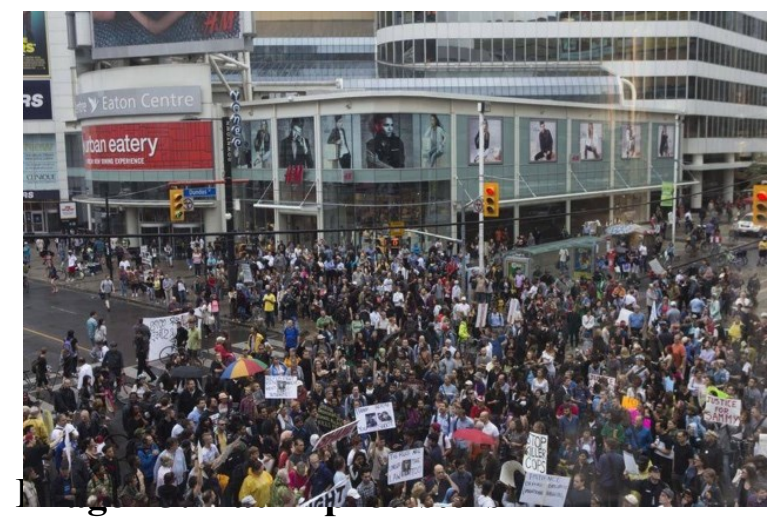

Image 17: Yatim protesters Philip Cheung/Globe \& Mail (2013)

Forcillo was charged with murder on 19 August 2013 and was convicted of attempted murder in 2016 and sentenced to six years imprisonment. Julian Falconer, a lawyer for the Yatim family, suggested, "What happened today was bad policing was sentenced. Nothing less. Bad policing was sentenced and we should see it that way. Not a sentence of police officers. At the same time, we've got to make a world in which good police officers can be supportive of what happened today" (Janus 2016). However, Peter Brauti, Forcillo's lawyer, argued that the "trial by YouTube" had compromised the jury and Toronto Police Association President Mike McCormack responded, "What kind of message does that send to our front-line officers who are out there every day dealing with violence [and] dealing with weapons?..Clearly, [this verdict] sends a chilling message to our members" (CBC News 2016b).

\section{Yonkers Police Department (Yonkers, New York)}

The Yonkers Police Department (YPD) provides policing services in the City of Yonkers, which has a population of 202,000 persons (United States Census Bureau 2017). Yonkers is the $112^{\text {th }}$ most populous city in the United States and the $4^{\text {th }}$ most 
populous city in the State of New York (United States Census Bureau 2017). Yonkers borders on the NYC borough the Bronx and, like other communities in close proximity to NYC's five boroughs, can be considered part of the NYC metropolitan area. The YPD has a complement of 620 sworn officers (Federal Bureau of Investigation 2017), with roughly 362 assigned to front-line duties (Captain Joseph Solicito, personal communication, 8 February 2016). Rank-and-file are deployed out of four precincts across the city, with daily shift briefings in the morning, afternoon, and evening. To collect data, my intention was to attend all YPD daily briefings at all four precincts, however, with the challenges experienced in this jurisdiction, I attended at only the $1^{\text {st }}$ Precinct shift briefings on 13 September 2016 and then at the two morning briefings at the $2^{\text {nd }}$ Precinct on the following day. After this, as discussed earlier, my sampling tactic changed - as suggested by a helpful, and sympathetic, YPD officer (as detailed later in this chapter's discussion of challenges). Ultimately, seventy-four (74) surveys were administered to YPD front-line officers. Of these, 51 surveys were completed, returned to me, and included in this study. Twenty-three (23) YPD officers refused to participate in the study and/or returned blank questionnaires.

In 2016, the violent crime rate for the City of Yonkers was 471 occurrences per 100,000 inhabitants. This positioned Yonkers as the city with the $15^{\text {th }}$ highest rate in the state and the $75^{\text {th }}$ highest among 112 major American cities (Federal Bureau of Investigation 2017). The average median household income for Yonkers is $\$ 59,049$ and the average per capita income is $\$ 30,263$ (both in 2015 dollars) (United States Census Bureau 2017). Approximately $16.7 \%$ of Yonkers' population is living in poverty 
(United States Census Bureau 2017). The table below reports on the race/ethnicity of Yonkers' population (United States Census Bureau 2017).

\begin{tabular}{|c|c|c|}
\hline Population of Yonkers, NY (2010) & $\mathbf{1 9 5 , 9 7 6}$ & $\mathbf{1 0 0 \%}$ \\
\hline White & 81,163 & $41.4 \%$ \\
\hline Black/African American & 31,297 & $16.0 \%$ \\
\hline Hispanic/Latino & 67,927 & $34.7 \%$ \\
\hline Asian & 11370 & $5.8 \%$ \\
\hline Two or More Races & 3065 & $1.6 \%$ \\
\hline Other Race & 714 & $0.4 \%$ \\
\hline American Indian/Alaska Native & 382 & $0.2 \%$ \\
\hline Native Hawaiian/Other Pacific Islander & 58 & $0 \%$ \\
\hline
\end{tabular}

In November 2016, the Civil Rights Division of the U.S. Justice Department (DOJ) and the City of Yonkers entered into another memorandum of agreement for external federal monitoring of the Yonkers Police Department (YPD). The agreement is intended to address "YPD's alleged use of excessive force and improper stop and search practices, unconstitutional police practices, and any other violations..." (Department of Justice 2017). As expressed in the DOJ materials explaining the history of federal concerns with the YPD,

In August 2007, the [Civil Rights] Division opened an investigation into the Yonkers Police Department (YPD) in New York. In June 2009, the Division addressed the need for reform of YPD's use of force policies, reporting uses of force, investigations of uses of force, handling of civilian complaints, officer supervision and discipline, creation of an early warning system, improved training, and expanded community engagement...(Department of Justice 2017)

In reporting on the launch of the 2007 DOJ inquiry for The New York Times, Fernanda Santos (2007) observes, "Residents here have grumbled about aggressive policing for years" and her story quotes Karen Edmonson, the president of the National Association for the Advancement of Colored People (NAACP) chapter in Yonkers, who finds that allegations of police brutality by the YPD 'have been a 
dirty little secret in this community for a long time.' Professor Randolph McLaughlin (of the Pace Law School) commented, "Yonkers has a history of needing outside intervention to fix the problems they have" (Santos 2007). In reporting on the 2009 interim DOJ report in the New York Daily News, the Association Press (2009) suggests that the Justice Department "found some fear and distrust of the police, as well as 'deep-seated' concerns of racial animus,' with 'numerous complaints of rude and discourteous conduct by officers towards citizens.' [The DOJ] applauded the goals of a police department campaign to improve relations, but said the campaign's tone was defensive, focusing on officers receiving respect rather than giving respect."

Windsor Police Service (Windsor, Ontario)

The Windsor Police Service (WPS) provides policing services for the City of Windsor, which has a population of 217,000 persons (Statistics Canada 2017c). Windsor is the $10^{\text {th }}$ most populous city in the Province of Ontario and the $23^{\text {rd }}$ most populous city in Canada (Statistics Canada 2017c). The WPS has a complement of 429 sworn officers (Statistics Canada 2017e) and roughly 210 are assigned to front-line duties (Deputy Chief Vincent Power, personal communication, 15 March 2016). The WPS is the $11^{\text {th }}$ largest municipal police agency in Ontario and the $22^{\text {nd }}$ largest in Canada (Statistics Canada 2017e). Rank-and-file are deployed out of police headquarters, with daily shift briefings in the morning, afternoon, and evening. To collect data, I attended at shift briefings on three dates, as detailed in the table below:

\begin{tabular}{|c|c|}
\hline Date & Location \\
\hline 27 April 2016 & Platoon 4 \\
\hline 28 April 2016 & Platoon 1 \& Platoon 3 \\
\hline 20 July 2016 & Platoon 5 and Platoon 2 \\
\hline
\end{tabular}


One hundred and fifty (150) surveys were administered to officers of the WPS and all were completed and returned to me. Two (2) were excluded from consideration in this study because they were completed by officers that disclosed that they were not currently performing front-line duties. ${ }^{8}$

For 2016, the violent CSI for the City of Windsor was 58.1 (Statistics Canada 2017d). The prevalence of low-income individuals in Windsor was 23.3\% in 2016 (Statistics Canada 2017c). The table below reports on the race/ethnicity of Windsor's population in 2016 (Statistics Canada 2017e).

\begin{tabular}{|c|c|c|}
\hline Population of Windsor (2016) & $\mathbf{2 1 3 , 9 8 5}$ & $\mathbf{1 0 0 \%}$ \\
\hline White & 156,380 & $73.1 \%$ \\
\hline Total 'Visible Minority' Population & 57,605 & $26.9 \%$ \\
\hline South Asian & 9640 & $4.5 \%$ \\
\hline Chinese & 7340 & $3.4 \%$ \\
\hline Black & 10,675 & $5.0 \%$ \\
\hline Filipino & 2960 & $1.4 \%$ \\
\hline Latin American & 2670 & $1.2 \%$ \\
\hline Arab & 16,115 & $7.5 \%$ \\
\hline Southeast Asian & 3365 & $1.6 \%$ \\
\hline West Asian & 1290 & $0.6 \%$ \\
\hline Korean & 315 & $0.2 \%$ \\
\hline Japanese & 110 & $0.1 \%$ \\
\hline Other 'Visible Minority' & 1295 & $0.6 \%$ \\
\hline Multiple 'Visible Minorities' & 1830 & $0.9 \%$ \\
\hline Aboriginal & 5565 & $2.6 \%$ \\
\hline
\end{tabular}

Several Windsor Police Service (WPS) officers spoke with me about two recent occurrences, which they suggest are impacting on the morale and behaviour of many WPS officers. In 2012, WPS Detective David Van Buskirk pled guilty to assault charges, was sentenced to 5 months in jail, and resigned from the WPS. This was in relation to Van Buskirk's 2010 arrest of a Windsor physician, who had been mistaken 
by the officer for a wanted suspect, and that had been video recorded by the security system of the nearby medical facility (Chen 2013; CTV News 2012). Two other WPS officers were accused of attempting to cover-up the incident, through disciplinary charges under Ontario's Police Services Act (CTV News 2012). In the aftermath, as reported in The National Post,

Embattled Windsor police Chief Gary Smith announced his immediate retirement Thursday amid allegations of police brutality and other misconduct by his officers... [Mayor Eddie Francis in announcing] Smith's abrupt departure [said], 'We are going to take steps to change the culture at the Windsor Police Service...The public is asking questions. We are asking questions' (Sacheli 2011).

The second occurrence occurred on 22 February 2012, when WPS Constable Kent Rice arrested Gladson Chinyangwa, a 22-year-old Black male, who was drunk and verbally abusive. While escorting Chinyangwa through the stairwell of the apartment building, Rice kicked the detainee twice after he had fallen to the floor (Wilhelm 2015). The interaction was video recorded by the building's security system and, when the footage surfaced to a public audience in June 2012, the officer was suspended by the WPS. Rice was subsequently convicted of assault and was also disciplined within the WPS, being demoted to a lower rank for 18 months (CTV News 2015). 


\section{APPENDIX D \\ EXPLANATION LETTER TO PROSPECTIVE AGENCIES (ENGLISH)}

\section{Carleton \\ U N I V E R I T Y \\ Canada's Capital University}

Gregory Brown is the researcher administering this survey and conducting the study Policing the 'Gotcha Society': Front-Line Police Work in Today's Techno-Social and Socio-Political Landscape. He is a doctoral candidate in the Department of Sociology and Anthropology at Carleton University in Ottawa (Canada). Dr. Aaron Doyle is his $\mathrm{PhD}$ dissertation supervisor. They can be contacted at these coordinates:

Gregory Brown

Aaron Doyle gregoryr.brown@carleton.ca

aaron_doyle@carleton.ca
(613) $520-2582$

(613) 520-2600 ext. 1914

The Research Ethics Board of Carleton University has reviewed this study's research protocol and has provided clearance to proceed.

The purpose of this research is to gain better (and empirical) understandings of how front-line police officers in Canada and in the United States are responding to the techno-social and socio-political environment in which they do their work in 2016. Some have suggested that with -1$)$ the proliferation of mobile telephones that enable videorecording; 2 ) a tendency across the population to now engage in 'citizen journalism' (by recording, disseminating, and, in some instances, providing commentary in relation to a potentially 'newsworthy' event taking place within their view);3) the ability to engage with vast public audiences through social media interconnectivity; and 4) a cultural shift across some in society toward more critical engagement with policing, increased demands for elevated scrutiny of police, and a more skeptical view of police actions in the field - some front-line officers have modified aspects of how they conduct themselves while on duty. In order to determine the prevalence of any such changes in how front-line officers do their work and to achieve a better understanding of how such changes, if any, are playing out in contemporary policing, the researcher is surveying a large sample of front-line police officers from police agencies across Canada and throughout the state of New York. Leadership of many police agencies, including yours, have provided their cooperation in allowing the researcher to attend at police facilities to present this survey questionnaire for your consideration. The researcher suggests that the benefits of an individual's research participation are through contributing to advancements in knowledge that may lead to improved conditions for the group to which the participant belongs (front-line police officers) and/or to society in general. 
To advance this study, the researcher is asking that you consider completing a survey questionnaire. This survey should only take 10 to 15 minutes of your time. Participation in this survey is voluntary. You may decline to participate. If you choose to participate, you may respond to all, some, or none of the questions. The survey is anonymous. Therefore, please do not write any identifiers on the survey document. The data you supply will not and cannot be attributed directly to you - in this study's research protocol doing so is impossible (for the researcher and for anyone who ultimately reads the final research product).

By completing all, some, or none of this survey questionnaire and subsequently submitting it to the researcher you are deemed to have consented to participation in this study and the data you provide being included in the research study's analysis of all of the data generated. Once submitted to the researcher, none of your survey data can be withdrawn from consideration in the study. Because the survey is anonymous and no identifiers are recorded, it is impossible for the researcher to subsequently locate a specific survey from a specific participant in order that some or all of the information is excised from the study. In considering consent, please also be aware that the researcher will retain the data from this study - in the format used for data analysis in this study for future academic projects that the researcher may undertake (alone or in collaboration with other researchers). The researcher is the only person who will see the questionnaires. The hardcopy questionnaires will be destroyed three months after the last survey is administered in this study. Upon completion, copies of the researcher's final dissertation will be provided to interested research participants and interested police agencies, upon request. If you would like to receive a copy, please e-mail the researcher and arrangements will be made to send you an electronic PDF file of the final dissertation.

Questions about this research can be directed to:

Dr. Louise Heslop, Chair

Dr. Andy Adler, Vice Chair

Carleton University Research Ethics Board - A

Carleton University Research Ethics and Compliance Office

511 Tory, Carleton University

1125 Colonel By Drive

Ottawa, ON K1S 5B6

613-520-2600 ext. 2517

ethics@carleton.ca

Thank you for your consideration of participation in this study. 


\section{APPENDIX E \\ EXPLANATION LETTER TO \\ PROSPECTIVE AGENCIES (FRANÇAIS)}

\section{Carleton \\ U N I V E R I T Y \\ Canada's Capital University}

Gregory Brown est le chercheur qui administre ce sondage et qui mène l'étude sur $L a$ Police de la 'Société Gotcha': Le Travail de la Police de Première Ligne, Faisant Face à l'Environnement Techno-Social et Sociopolitique d'aujourd'hui. Il est candidat au doctorat au département de sociologie et d'anthropologie à l'université Carleton d'Ottawa (Canada). Dr. Aaron Doyle est son directeur de thèse de doctorat. Pour communiquer avec un d'entre eux s'il vous plait contactez:
Gregory Brown
gregoryr.brown@carleton.ca
(613) $520-2582$
Aaron Doyle
aaron_doyle@carleton.ca
(613) 520-2600 poste 1914

Après avoir examiné le protocole de recherche de cette étude, le comité d'éthique de la recherche a donné l'autorisation de procéder avec ce sondage.

Cette recherche a pour but d'acquérir une compréhension empirique ainsi que de mieux saisir la réaction des policiers de première ligne au Canada ainsi qu'aux États-Unis faisant face à l'environnement techno-social et sociopolitique dans lequel ils ont travaillé en 2016. Certains ont suggéré que - 1) la prolifération des téléphones cellulaires permettant l'enregistrement vidéo; 2) une tendance à travers la population à s'engager dans un 'journalisme citoyen' (en enregistrant, en diffusant et, dans certains cas, commentant un événement qui, d'après eux, serait potentiellement 'digne d'intérêt' ; 3) la capacité de dialoguer avec de vastes publics grâce à l' inter connectivité des médias sociaux; et 4) un changement culturel pour certains dans la société vers un engagement plus critique avec les services de police, les demandes accrues pour un examen minutieux de la police, et une vision plus sceptique des interventions policières sur le terrain - certains agents de première ligne ont modifié certains aspects de leur conduite lorsqu'ils sont en devoir. Afin de déterminer la prévalence de tels changements dans la façon dont les agents de première ligne font leur travail et de mieux comprendre comment ces changements, le cas échéant, se produisent dans les services de police contemporains, le chercheur étudie un large échantillon d'agents de police de première ligne au sein de services de police à travers le Canada et de tout l'État de New York. La direction de nombreux services de police, y compris le vôtre, a fourni sa collaboration en permettant au chercheur d'assister aux installations policières afin de vous présenter ce sondage. Le chercheur suggère que les avantages de la participation à la recherche sont de contribuer à l'avancement des connaissances susceptibles d'améliorer les conditions du groupe auquel le participant appartient (policier de première ligne) et /ou de la société en général. 
Pour faire avancer cette étude, le chercheur vous demande d'envisager de remplir un sondage. Ceci ne devrait prendre que 10 à 15 minutes de votre temps. La participation à ce sondage est volontaire. Vous pouvez refuser de participer. Si vous choisissez de participer, vous pouvez répondre à toutes, à certaines ou à aucune des questions. Le sondage est anonyme. Par conséquent, n'écrivez aucun identificateur sur le document. Les données que vous fournissez ne seront pas et ne peuvent pas vous être attribuées directement - dans le protocole de recherche de cette étude, cela est impossible (pour le chercheur et pour toute personne qui finit par lire le produit final de la recherche).

En remplissant tout, en partie ou aucunement ce sondage et en le soumettant au chercheur, vous êtes réputé avoir accepté de participer à cette étude et consentez que les données que vous fournissez soient incluses dans l'analyse de l'étude de toutes les données générées. Une fois soumises au chercheur, aucune de vos données ne peuvent être retirées de l'étude. Étant donné que ce sondage est anonyme et qu'aucun identificateur n'est enregistré, il est impossible pour le chercheur de localiser par la suite un sondage spécifique auprès d'un participant spécifique afin qu'une partie ou la totalité de l'information soit retirée de l'étude. En tenant compte du consentement, veuillez également noter que le chercheur conservera les données de cette étude - dans le format utilisé pour l'analyse des données dans cette étude - pour les futurs projets académiques que le chercheur peut entreprendre (seul ou en collaboration avec d'autres chercheurs). Le chercheur est la seule personne à voir les questionnaires. Les questionnaires papier seront détruits trois mois après l'administration du dernier sondage dans cette étude. À la fin, des copies de la thèse finale du chercheur seront fournies, sur demande, aux participants intéressés et aux services de police intéressés. Si vous souhaitez recevoir une copie, veuillez envoyer un courriel au chercheur et des mesures seront prises pour vous faire parvenir un fichier PDF de la thèse finale.

Toutes questions concernant cette recherche peuvent être adressées à:

Dr. Louise Heslop, présidente

Dr Andy Adler, vice-président

Comité d'Éthique de la Recherche de l'Université Carleton - A

Bureau de l'Éthique de la Recherche et de la Conformité de l'Université Carleton

511 Tory, Université Carleton

1125, promenade du Colonel By

Ottawa (Ontario) K1S 5B6

613-520-2600 poste 2517

ethics@carleton.ca

Merci de votre considération pour la participation à cette étude. 
APPENDIX F

\section{RESEARCH ETHICS BOARD CLEARANCE}

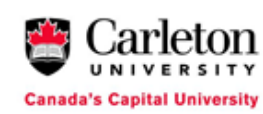

Ethics Clearance Form - New Clearance
Carleton University

Research Ethics Office

Research Ethics Board

511 Tory, 1125 Colonel By Drive

Ottawa, ON K1S 5B6 Canada

Tel: 613-520-2517, ethics@carleton.ca

This is to certify that the Carleton University Research Ethics Board has examined the application for ethical clearance. The REB found the research project to meet appropriate ethical standards as outlined in the Tri-Council Policy Statement: Ethical Conduct for Research Involving Human, 2nd edition, and the Carleton University Policies and Procedures for the Ethical Conduct of Research.

Date of Clearance: January 29, 2016

Researcher: Aaron Doyle (Primary Investigator)

Gregory Brown (Student Research: Ph.D. Student)

Department: Faculty of Arts and Social Sciences/Sociology and Anthropology (Department of)

University: Carleton University

Research Supervisor (if applicable): Prof. Aaron Doyle

Project Number: 104075

Project Title: Policing the 'Gotcha Society': Front-Line Police Work in Today's Techno-Social and SocioPolitical Landscape [Gregory Brown]

Funder (if applicable): SSHRC

Clearance Expires: August 31, 2016

\section{All researchers are governed by the following conditions:}

Annual Status Report: You are required to submit an Annual Status Report to either renew clearance or close the file. Failure to submit the Annual Status Report will result in the immediate suspension of the project. Funded projects will have accounts suspended until the report is submitted and approved.

Changes to the project: Any changes to the project must be submitted to the Carleton University Research Ethics Board for approval. All changes must be approved prior to the continuance of the research.

Adverse events: Should a participant suffer adversely from their participation in the project you are required to report the matter to the Carleton University Research Ethics Board. You must submit a written record of the event and indicate what steps you have taken to resolve the situation.

Suspension or termination of clearance: Failure to conduct the research in accordance with the principles of the Tri-Council Policy Statement: Ethical Conduct for Research Involving Humans, 2nd edition and the Carleton University Policies and Procedures for the Ethical Conduct of Research may result in the suspension or termination of the research project.

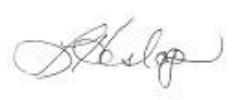

Louise Heslop

Chair, Carleton University Research Ethics Board

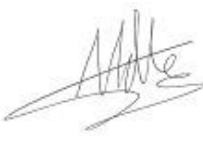

Andy Adler

Vice-Chair, Carleton University Research Ethics Board 


\section{APPENDIX G \\ SURVEY QUESTIONNAIRE (ENGLISH)}
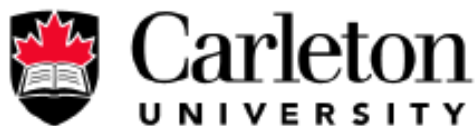

U N I V E R I T Y

Canada's Capital University

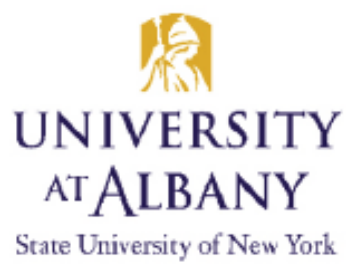

Thank you for volunteering to participate in this survey. If you are unsure about any question, please ask the researcher for clarification. If you wish to elaborate on your answer(s) do so by writing in the space allotted within the question or in the margin beside that particular question. Some questions ask for a comparison over time; it is understood that 'newer' officers may be unable to answer these questions given that they do not have enough service to do so meaningfully. This survey has 4 pages.

1. Your gender:

2. Your age:

3. Your race/ethnicity:

4. Your total years of service in policing:

5. How many years of your career have been spent in front-line police duties?

6. Presently, in what aspect of front-line policing do you work?

$\square$ primary response/general patrol $\square$ traffic enforcement

$\square$ school liaison

$\square$ foot/bicycle patrol

$\square$ community-based unit

$\square$ other (please specify, i.e. tactical, canine)

7. How would you characterize the environment where you do most of your police work?

$\square$ Rural $\square$ Suburban $\square$ Urban $\square$ Commercial

8. Do you use some form of recording device while you are on duty?

$\square$ No $\square$ Yes

If yes, please indicate the device(s) that you use:

$\square$ audio recorder $\square$ dashcam $\square$ body-worn camera $\quad \square$ other

If yes, when did you first use a recording device in your police work?

If yes, is the device you use now issued by your police agency or is it your own?

9. Do (or would) you have any objections to wearing a body camera while on duty to document (through audio and video recording) your actions and those of the public with whom you interact? $\square$ Yes $\square$ No

Please explain why? 
10. Has there been an occasion while you were on duty when you became aware that a member of the public (not a media photographer) was videorecording you using a mobile phone or similar device?
口 No
$\square$ Yes

If yes, on how many occasions has this happened in your career (if you do not know specific numbers please feel free to approximate)?

If yes, in what circumstances has the videorecording taken place?

$\square$ while making an arrest

$\square$ while talking with another officer

口 while on downtime/resting between calls

․ while stopping for a meal/coffee

$\square$ while talking with a member of the public

$\square$ other

For questions 11 through 21, 'videorecording' includes both mobile phones (and similar devices) and CCTV video surveillance systems.

11. What is your assessment as to the overall potential for videorecording of your on-duty activities in the area where you do your police work?
口 Non existent
口 Minimal
$\square$ Quite prevalent
$\square$ Video is everywhere

$\square$ Exists in some places but not in others

12. How much do you and your police colleagues speak about the potential for videorecording of police conduct?

$\square$ It is never a topic of conversation

口 It is discussed on an occasional basis

$\square$ It comes up fairly often

$\square$ It is often the primary topic of conversation

13. How much do you and your police colleagues speak about videorecordings of policing occurrences that 'go viral' (i.e. widely circulated and discussed in social and traditional media)?

$\square$ It is often the primary topic of conversation $\quad$ It comes up fairly often

$\square$ It is discussed on an occasional basis $\square$ It is never a topic of conversation

14. On a scale of 1 to 10 (with the value 1 representing it never entering your mind and the value 10 representing it is something that is always on your mind), while you are on duty what level of awareness do you have about the potential for videorecording of your actions? (Please circle the value that represents your level of awareness)

$\begin{array}{llllllllll}1 & 2 & 3 & 4 & 5 & 6 & 7 & 8 & 9 & 10\end{array}$

15. Has there been an occasion while you were doing front-line police work when you have thought about not doing something, because of a concern that your actions could be videorecorded that you otherwise would normally do?

$\square$ Yes $\square$ No

If yes, please elaborate

16. Has there been an occasion while you were doing front-line police work when you have not done something, because of a concern that your actions could be videorecorded, that you otherwise would normally have done?

$\square$ No $\square$ Yes

If yes, please elaborate 
17. In terms of frequency (as compared to when you started your police career), does the potential for videorecording of your actions influence when you use physical force?

$\square$ Yes, I use force less often now $\quad \square$ No, I use force the same

$\square$ Yes, I use force more often now

18. In terms of degree (as compared to when you started your police career), does the potential for videorecording of your actions influence the level of physical force you use (when you are in a situation in which force is required)?
$\square$ Yes, I use more force now
$\square$ No, I use force the same
$\square$ Yes, I use less force now

19. Do you do anything to avoid having your on-duty actions videorecorded?

$\square$ No $\square$ Yes

If yes, please elaborate

20. On occasions when you think your on-duty actions could be (or are being) videorecorded dol you do anything because of that videorecording potential?

$\square$ Yes $\square$ No

If yes, please elaborate

21. In terms of affecting how you perform your job, does it matter whether the camera is a police body camera worn by you or whether a citizen is recording you with their own device?"

$\square$ No $\square$ Yes

Please elaborate

22. As compared to when you started your policing career, what is your perception as to the degree of criticism of the police by the general public today in the community where you work?

$\square$ The public are less critical of police $\quad \square$ The public's criticism of police is the same

․ The public are more critical of police

23. Has there been an occasion while you were doing front-line police work when you have thought about not doing something, because of a concern that your actions could be criticized by some members of the public, that you otherwise would normally do?

$\square$ Yes $\square$ No

If yes, please elaborate

24. Has there been an occasion while you were doing front-line police work when you have not done something, because of a concern that your actions could be criticized by some members of the public, that you otherwise would normally have done?

$\square$ No $\square$ Yes

If yes, please elaborate 
25. Do you feel that there is an elevated risk of criticisms of, or complaints about, your front-line police work that is related to particular characteristics presented by individuals with whom you interact in policing occurrences?

$\square$ Yes $\quad \square$ No (proceed to Question 27)

If yes, please indicate which characteristic(s) you feel present an elevated risk of criticism or complaint about your police work, and please consider elaborating:
$\square$ gender
$\square$ race
$\square$ mental health issues
$\square$ sexual orientation
$\square$ physical disability
$\square$ other
Please elaborate

26. Do you do anything to avoid interacting with persons who present the characteristics you have identified in Question 25?

$\square$ Yes $\square$ No

If yes, please elaborate

Questions 27 and 28 relate to the concept of risk-averse police practices. Some within policing, the media, and some academics studying policing have discussed this concept using different terminologies - such as 'de-policing' or 'F.L.D.O.'

27. Are you familiar with the concept of risk-averse policing/de-policing/F.I.D.o.X

Yes $\square$ No

If yes, please explain your understanding of this concept

28. Have you ever heard of, or witnessed, risk-averse policing/de-policing/F.I.D.O. being practiced where you work?

$\square$ No $\quad$ Yes

If yes, please elaborate

29. Before answering the questions in this survey were you aware that the researcher conducting this study worked for many years as an operational police officer?

$\square$ Yes $\quad$ No (the survey is complete)

30. Has your knowledge of the policing background of this researcher influenced how you have answered questions 1 through 28 in this survey?

$\square$ No $\square$ Yes

If yes, please elaborate

Thank you for participating in this study. You have made an important contribution in increasing understanding around front-line police work in today's policing landscape. 


\section{APPENDIX H \\ SURVEY QUESTIONNAIRE (FRANÇAIS)}

Carleton

U N I V E R I T Y

Canada's Capital University

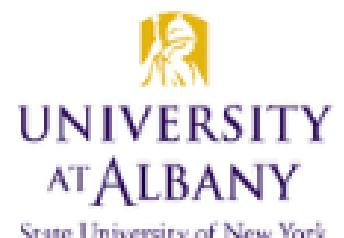

State University of New York

Merci de participer à ce sondage. Si vous n'êtes pas certain quant à une question, s.v.p. demandez au chercheur de clarifier. Si vous voulez élaborer une réponse, faites-le en écrivant dans l'espace alloué ou dans la marge à côté de la question. Certaines questions demandent une comparaison dans le temps; il est sous-entendu que les 'nouvelles recrues' peuvent être incapables de répondre à ces questions vu qu'elles n'ont pas assez d'années de service. Ce sondage comporte 4 pages.

1. Votre sexe:

2. Votre âge:

3. Votre ethnicité:

4. Total de vos années de service comme agent:

5. Combien d'années de votre carrière ont été passées à effectuer des fonctions de première ligne?

6. Présentement, comme agent de première ligne, à quelle unité êtes-vous affecté?

$\square$ premier répondant / patrouille générale $\quad \square$ règlements de la route

$\square$ personne-ressource dans les écoles

$\square$ patrouille pédestre / à vélo

$\square$ communautaire

$\square$ gang de rues

$\square$ autre (spécifiez, ex. tactique, canine)

7. Comment décririez-vous l'environnement dans lequel vous faites la majorité de votre travail d'agent?

Rural

$\square$ Suburbain

Urbain

$\square$ Commercial

8. Utilisez-vous un dispositif d'enregistrement dans le cadre de votre travail?

$\square$ Non $\square$ Oui

Si oui, indiquez le genre de dispositif d'enregistrement utilisé:

$\square$ enregistrement audio

$\square$ caméra corporelle
口 caméra de tableau de bord

$\square$ autre

Si oui, quand avez-vous commencé à utiliser un dispositif d'enregistrement dans vos fonctions d'agent?

Si oui, le dispositif d'enregistrement utilisé est-il fourni par votre organisation où c'est le vôtre?

9. Avez-vous (ou auriez-vous) des objections à porter une caméra corporelle lors de vos fonctions pour documenter (enregistrement audio et vidéo) vos actions ainsi que celles du public avec lequel vous interagissez?
ㅁoi
№n

Expliquez pourquoi 
10. Y a-t-il eu une occasion alors que vous étiez en devoir où vous vous êtes aperçu qu'un membre du public (pas un membre des médias) vous enregistrait en utilisant un cellulaire ou autre dispositif d'enregistrement?
$\square$ Non
$\square$ Oui

Si oui, combien de fois est-ce arrivé dans votre carrière? (Si vous ne savez pas le nombre exact de fois, libre à vous de faire une approximation)?

Si oui, dans quelles circonstances est-ce que l'enregistrement a-t-il eu lieu?
$\square$ en faisant une arrestation
$\square$ lors d'une conversation avec un membre du public
$\square$ durant un repas/une pause-café
$\square$ lors d'une conversation avec un autre agent
$\square$ durant un temps d'arrêt/pause entre les appels
$\square$ autre

Pour les questions de 11 à 21 inclusivement, 'enregistrement vidéo' fait référence au téléphone cellulaire (et dispositifs similaires) et vidéosurveillance en circuit fermé.

11. Comment évaluez-vous la probabilité que vos actions soient captées par enregistrement vidéo là où vous travaillez?
$\square$ Non existant
$\square$ Minime
$\square$ Existe à certains endroits
$\square$ Très répandu
Vidéo présente partout

12. Quelle est la fréquence des discussions entre vous et vos collègues de travail sur le potentiel de l'enregistrement vidéo de la conduite policière?
$\square$ Jamais
$\square$ Occasionnellement
$\square$ Souvent
$\square$ Très souvent

13. Quelle est la fréquence des discussions entre vous et vos collègues sur les enregistrements vidéos d'occurrences policières qui sont diffusés de façon virale (ex. largement diffusés et discutés dans les médias sociaux et traditionnels)
$\square$ Très souvent
$\square$ Souvent
$\square$ Occasionnellement
$\square$ Jamais

14. Sur une échelle de 1 à 10 (1 représentant que vous n'en tenez jamais compte et 10 représentant quelque chose qui vous préoccupe) alors que vous travaillez, quel niveau de conscientisation avezvous du potentiel de l'enregistrement vidéo de vos actions? (Encerclez le chiffre qui correspond à votre niveau de conscientisation]
2
34
5
6
7
89
10

15. Y a-t-il eu une occasion alors que vous travailliez où vous avez pensé ne pas faire quelque chose dâ à une inquiétude que vos actions pourraient être enregistrées, mais que vous auriez fait normalement?

$\square$ Oui $\square$ Non

Si oui, veuillez élaborer

16. Y a-t-il eu une occasion alors que vous travailliez où vous n'avez pas fait quelque chose dâ à une inquiétude que vos actions pourraient être enregistrées, mais que vous auriez fait normalement?

$\square$ Non $\square$ Oui

Si oui, veuillez élaborer 
17. En termes de fréquence (comparativement à quand vous avez commencé votre carrière d'agent) est-ce que le potentiel d'un enregistrement vidéo de vos actions a une influence sur votre utilisation de la force physique?

$\square$ Oui, j"utilise moins de force maintenant

$\square$ Non, j'utilise la même force

$\square$ Oui, j"utilise plus de force maintenant

18. En termes de deqré (comparativement à quand vous avez commencé votre carrière comme agent) est-ce que le potentiel d'enregistrement vidéo de vos actions a une influence sur le niveau de force physique que vous utilisez (lorsque vous êtes dans une situation où la force physique doit être utiliséej?

$\square$ Oui, j"utilise plus de force maintenant

on, j'utilise la même force

$\square$ Oui, j"utilise moins de force maintenant

19. Faites-vous quelque chose pour éviter que vos actions soient enregistrées lorsque vous travaillez?

$\square$ Non $\square$ Oui

Si oui, veuillez élaborer

20. Si à l'occasion vous croyez que vos actions lors de votre travail pourraient être (ou sont) enregistrées, faites-vous quelque chose à cause du potentiel de cet enregistrement vidéo?

$\square$ Oui $\square$ Non

Si oui, veuillez élaborer

21. En termes d'influence sur la façon dont vous accomplissez votre travail, accordez-vous une importance différente à une caméra corporelle portée sur vous ou à un dispositif d'enregistrement d'un membre du public qui enregistre vos actions?

$\square$ Non $\square$ Oui

Si oui, veuillez élaborer

22. Comparativement à quand vous avez commencé votre carrière, quelle est votre perception quant au degré de critique envers les agents par les membres du public aujourd'hui dans la communauté où vous travaillez?

$\square$ Le public est moins critique envers les agents

$\square$ La critique du public est la même

$\square$ Le public est plus critique envers les agents

23. Y a-t-il eu une occasion lors d'une intervention de première ligne où vous avez pensé ne pas faire quelque chose dâ à une inquiétude que vos actions pourraient être critiquées par les membres du public, mais que vous auriez fait normalement?

$\square$ Oui $\square$ Non

Si oui, veuillez élaborer 
24. $\mathrm{Y}$ a-t-il eu une occasion lors d'une intervention de première ligne où vous n'avez pas fait quelque chose dô à une inquiétude que vos actions pourraient être critiquées par les membres du public, mais que vous auriez fait normalement?

$\square$ Non $\quad \square$ Oui

Si oui, veuillez élaborer

25. Croyez-vous qu'il y a un risque élevé de critiques ou de plaintes de votre travail de première ligne relativement à des caractéristiques particulières présentes chez certains individus avec qui vous interagissez lors d'une intervention policière?

$\square$ Oui $\quad \square$ Non (passer à la question 27)

Si oui, veuillez indiquer quelles caractéristiques vous croyez représentent un risque élevé de critiques ou de plaintes de votre travail policier et soyez libre d'élaborer:
$\square$ sexe
$\square$ ethnicité
$\square$ problèmes de santé mentale
$\square$ orientation sexuelle $\quad \square$ handicap physique
$\square$ autre

Veuillez élaborer

26. Est-ce que vous évitez d'interagir avec des personnes qui présentent les caractéristiques que vous avez identifiées à la question 25 ?

$\square$ Non $\square$ Oui

Si oui, veuillez élaborer

Les questions 27 et 28 ont trait au concept 'aversion de risque' dans les pratiques policières. Certains dans l'environnement policier, les médias, et certains académiciens ont discuté ce concept en utilisant différentes terminologies telles 'dé-policiser' ou 'F.L.D.O.'

27. Êtes-vous familier avec le concept d'aversion de risque/dé-policiser/F.I.D.0?

$\square$ Oui $\square$ Non

Si oui, expliquez votre compréhension de ce concept

28. Avez-vous entendu ou été témoin de la pratique de l'aversion de risque/dé-policiser/F.I.D.o dans votre milieu de travail?

$\square$ Non $\quad \square$ Oui

Si oui, veuillez élaborer

29. Avant de répondre aux questions de ce sondage, étiez-vous conscient que le chercheur effectuant ce sondage a travaillé pendant plusieurs années comme policier opérationnel?

$\square$ Oui $\quad \square$ Non (fin du sondage)

30. Est-ce que votre connaissance de l'historique du travail de ce chercheur vous a influencé dans vos réponses aux questions 1 à 28 de ce sondage?

$\square$ Non $\square$ Oui

Si oui, veuillez élaborer 


\section{APPENDIX I \\ BIVARIATE ANALYSIS FOR QUESTIONS 16, 24, AND 26}

In analyzing the data from questions 16,24 , and 26 , in the context of de-policing, variables were recoded to correspond to those participants who have (i) modified behaviour due to the potential for the video recording of police actions in the field; (ii) modified behaviour due to the potential for public criticism of police actions in the field; and (iii) modified behaviour in reaction to the perceived elevated risk of criticism and/or complaints due to interaction with persons perceived as exhibiting identifiable demographic characteristics. The following tables present the results of the bivariate analysis for each question.

Question 16 Has there been an occasion while you were doing front-line police work when you have not done something, because of a concern that your actions could be video recorded, that you otherwise would normally have done?

Table 54: Question 16 - De-policing and video recording of police actions

\begin{tabular}{|c||c|c|c|}
\hline $\begin{array}{c}\text { Modified behaviour due to } \\
\text { video recording }\end{array}$ & No & Yes & Total \\
\hline Non de-policers & 1024 & 0 & 1024 \\
\hline Limited de-policers & 381 & 277 & 658 \\
\hline Moderate de-policers & 141 & 716 & 857 \\
\hline Intensive de-policers & 0 & 1081 & 1081 \\
\hline Count & 1546 & 2074 & 3620 \\
\hline \% 'No' and 'Yes' & $\mathbf{4 2 . 7 \%}$ & $\mathbf{5 7 . 3 \%}$ & 40 \\
\hline Missing & \multicolumn{3}{|l}{} \\
\hline Total & $\begin{array}{c}\text { Cramer's V }=.828 \\
\text { Cra }\end{array}$ \\
\hline \hline
\end{tabular}


Question 24 Has there been an occasion while you were doing front-line police work when you have not done something, because of a concern that your actions could be criticized by some members of the public, that you otherwise would normally have done?

Table 55: Question 24 - De-policing and public criticism of police actions

\begin{tabular}{|c||c|c|c|}
\hline $\begin{array}{c}\text { Modified behaviour due to } \\
\text { public criticism? }\end{array}$ & No & Yes & Total \\
\hline Non de-policers & 1024 & 0 & 1024 \\
\hline Limited de-policers & 435 & 223 & 658 \\
\hline Moderate de-policers & 72 & 785 & 857 \\
\hline Intensive de-policers & 0 & 1081 & 1081 \\
\hline Count & 1531 & 2089 & 3620 \\
\hline \%'No' and 'Yes' & $\mathbf{4 2 . 3 \%}$ & $\mathbf{5 7 . 7 \%}$ & 40 \\
\hline Missing & \multicolumn{3}{|}{} \\
\hline Total & $\begin{array}{l}\chi^{2}=2745.727 \\
\text { Cramer's V } \leq .01\end{array}$ \\
\hline
\end{tabular}

Question 26 Do you do anything to avoid interacting with persons who present the characteristics you have identified in Question 25?

Table 56: De-policing and avoidance of interaction with those perceived to present elevated risk of criticism/complaint (frequency of responses)

\begin{tabular}{|c|c|c|c|}
\hline $\begin{array}{l}\text { Modified behaviour due to risk of } \\
\text { criticism/complaint? }\end{array}$ & No & Yes & Total \\
\hline Non de-policers & 670 & 0 & 670 \\
\hline Limited de-policers & 362 & 158 & 520 \\
\hline Moderate de-policers & 526 & 213 & 739 \\
\hline Intensive de-policers & 0 & 1081 & 1081 \\
\hline Count & 1558 & 1452 & 3010 \\
\hline$\%$ 'No' and 'Yes' & $51.8 \%$ & 48.2 & \\
\hline Q.25 'No' responses & & & 621 \\
\hline Q. $25+$ Q. 26 responses (combined) & 2179 & 1452 & 3631 \\
\hline \% 'No' and 'Yes' (combined) & $60.0 \%$ & $40.0 \%$ & \\
\hline Missing & & & 29 \\
\hline Total & & & 3660 \\
\hline \multicolumn{4}{|c|}{$\begin{array}{c}\chi^{2}=1962.301 \mathrm{p} \leq .01 \\
\text { Cramer's V }=.807\end{array}$} \\
\hline
\end{tabular}




\section{NOTES FOR CHAPTER ONE}

${ }^{1}$ For the sake of consistency, I employ Canadian English spelling throughout this dissertation. As such, and for example, 'behavior' (American spelling) is written 'behaviour.' Similarly, 'neighbourhood' rather than 'neighborhood and 'colour' rather than 'color', etc.

${ }^{2}$ As Bittner has observed, the different tentacles involved in the policing of society "are consistently confused by scholars" (Brodeur 2007: 105). In this regard, Reiner (2010: 4) observes, "It is important to distinguish between the ideas of 'police' and 'policing'. 'Police' refers to a particular kind of social institution, while 'policing' implies a set of processes with specific social functions." In other words, Reiner (2000a: 205) submits that "modern police forces are but one historically specific variant of the array of policing processes found in different forms of social order..." Along these lines, Palmiotto (2010) suggests that policing mechanisms, broadly defined, have, for many years, responded to the desire for a sense of peace and social order among the citizenry, whereas the formal and institutionalized public police organizations we are familiar with today are, by comparison, a recent development (originating less than 200 years ago).

In terms of establishing this dissertation's conceptual scaffolding in this regard, I stipulate that by police (and, by extension, their work in policing society) I am referring to the particular institution of the public police - those state-funded and state-mobilized individuals (through the enactment of laws and the conferring of unique and awesome powers by the state) who are organized on a permanent basis to enforce laws and who collectively constitute a professional police force in particular geo-political jurisdictions, as differentiated from other actors involved with social control functions in society (Bittner 1970, 1974; Brants 2005; Brodeur 2007; Brown 2014; Dunham \& Alpert 2005, 2010a; Ericson 1989; Flanagan 2007; Goldsmith 2005; Jones \& Newburn 1998, 2002; Klockars 1985; Luna 2000; Manning 2007, 2010; McLaughlin 2007; Murphy 2012; Neocleous 2000; Reiner 2010; Rigakos 2002; Stenning \& Shearing 2005; Waddington 1999; Walsh \& Conway 2011; Westley 1970). Across contemporary Western democracies, the police constitute the 'specialist repositories' in applying the state's monopoly of the legitimate use of force (Barton \& Johns 2014; Ericson 1989; Klockars 1985; Loader \& Walker 2007; Manning 2010; Neocleous 2000; Reiner 2010; Westley 1951, 1970). In this vein, Ericson (1989: 155), in applying the deliberations of Marx and Weber, articulated that the police "are seen to be at the forefront of the coercive arm of the state, the embodiment of the state's monopoly on the legitimate use of force."

${ }^{3}$ The terms 'techno-social' and 'socio-political' are not intended to function as exclusionary - rather they should be understood as subsuming a range of contemporary technological, social, political, cultural, and interrelated developments.

${ }^{4}$ Reiss was appointed to President Lyndon Johnson's Commission on Law Enforcement and Administration of Justice in 1965 and he was tasked with leading a national study 
across the USA to examine how police were interacting with citizens. This commission was mobilized in the wake of several controversial policing occurrences, which came to broad public attention in the latter half of the socially and politically turbulent decade of the 1960s throughout the USA. During 1966, Reiss' thirty-six research teams conducted systematic social observation with front-line officers in Boston, Chicago, and Washington, D.C. During his career, Reiss was elected president of both the American Society of Criminology (which honoured him with its Sutherland Award) and the International Society of Criminology (which honoured him with their Prix Durkheim) (Sherman 2007).

${ }^{5}$ By the terms 'front-line' and 'rank-and-file' I am particularizing uniformed 'street cops,' who are highly visible in their movements throughout the communities in which they work and in their interactions with various members of the public (complainants, witnesses, victims, suspects, perpetrators, etc.) as part of their expected and routine duties. In their seminal works, Reuss-Ianni (1983) and Ruess-Ianni and Ianni (2005) exposed the important subcultural distinction between 'street cops' and 'management cops' - which was also elaborated by Manning (1995) in his analysis of the hierarchical structure of police work. Further discussion in this area is engaged in Chapter Three.

${ }^{6}$ The word 'public' has a variety of context-dependent meanings - for the purposes of this dissertation 'public' should be understood as being synonymous with "the members of the community collectively" (Oxford English Dictionary 2015).

${ }^{7}$ As Waddington (1999: 298, citing Klockars 1985) articulates, "Instituting a police force entails the general population licensing a specific occupational group to exercise authority over them - to intrude into their privacy, interfere in their conduct, and ultimately to use force against them."

${ }^{8}$ These two images were sourced from public-available online still images. Further, police cruisers in Chatham-Kent, Edmonton, Halifax, South Simcoe, Thunder Bay, and Toronto (in Canada) and Chicago, Denver, Huntsville, Lafayette, Los Angeles, Minneapolis, Portland, San Diego, and Winston-Salem (in the USA) are also representative examples, but should not be considered an exhaustive accounting of police jurisdictions with such expressions emblazoned on their police cruisers.

${ }^{9}$ Sherman (2007: 1110) explains, "On that basis, the American Sociological Review refused to print the article later published by the American Journal of Sociology in 1966, saying that the authors could not use the word 'proactive' because it did not exist in the English language. The Oxford English Dictionary [1989] now credits Reiss with the first printed usage of the word."

${ }^{10}$ In their front-page reporting for the New York Times, Schmidt and Apuzzo (2015: A1) observe that, in his speech at the University of Chicago, Comey suggested,

The additional scrutiny and criticism of police officers in the wake of highly publicized episodes of police brutality may have led to an increase in violent 
crime in some cities as officers have become less aggressive...With his remarks, Mr. Comey lent the prestige of the FBI, the nation's most prominent law enforcement agency, to a ['Ferguson Effect'] theory that is far from settled; [which suggests] that the increased attention on the police has made officers less aggressive and emboldened criminals. But he acknowledged that there is so far no data to back up his assertion.

In brief, the 'Ferguson Effect' can be considered,

A popular theory [that accounts for recent increases in violent crimes, because of] an alleged tendency by some police officers to second-guess themselves before responding to crime scenes for fear of getting sued or caught on video doing something perceived as nefarious. The theory was coined after a 2014 incident where [Michael Brown] an unarmed AfricanAmerican teen was fatally shot by an officer in Ferguson, [Missouri], sparking protests and national outrage (Gorner 2016a).

In this regard, in a 29 May 2015 'op/ed' piece published in The Wall Street Journal, Heather MacDonald (the Thomas W. Smith fellow at the Manhattan Institute) observes,

This incessant drumbeat against the police has resulted in what St. Louis police chief Sam Dotson last November [2014] called the 'Ferguson Effect'. Cops are disengaging from discretionary enforcement activity and the 'criminal element is feeling empowered,' Mr. Dotson reported. Arrests in St. Louis city and county by that point had dropped a third since the shooting of Michael Brown in August [2014]. Not surprisingly, homicides in the city surged $47 \%$ by early November and robberies in the county were up $82 \%$. Similar 'Ferguson Effects' are happening across the country as officers scale back on proactive policing under the onslaught of anti-cop rhetoric. Arrests in Baltimore were down 56\% in May compared with 2014. 'Any cop who uses his gun now has to worry about being indicted and losing his job and family,' a New York City officer tells me...Police officers now second-guess themselves about the use of force. 'Officers are trying to invent techniques on the spot for taking down resistant suspects that don't look as bad as the techniques taught in the academy,' says Jim Dudley, who recently retired as deputy police chief in San Francisco.

${ }^{11}$ Along these lines, my understanding of the correct application of the term 'Ferguson Effect' coincides with the idea that de-policing behaviours among front-line officers have affected the 'criminal element' in certain situations (in the sense of reducing considerations of deterrence) and the results have been significant increases in criminal behaviour - particularly violent crime (Gorner 2016a). Shjarback and colleagues (2017: 42-43) present what I suggest is the appropriate understanding of any such 'Ferguson Effect,' suggesting that, "Collectively, the connection between public scrutiny, depolicing, and crime has become known as the 'Ferguson Effect'." 
Similarly, Wallace and colleagues (2018: 481) delineate the discrete nature of the two phenomena in their observation, "Advocates of the 'Ferguson Effect' claim the decline in proactive policing increased violent crime," as do Nix and colleagues (2018: 36), who find, "Collectively, this argument - that the war on cops will lead to de-policing and de-policing will lead to increased crime - is now commonly known as the 'Ferguson Effect'."

In this regard, Chicago Police Superintendent Eddie Johnson said during a news conference on 1 January 2017 that Chicago is among many American cities that have seen a spike in violence. Johnson advanced that several factors have contributed to the increased violence, including that "anger at police... has left criminals emboldened to commit violent crimes" (Babwin 2017a). Johnson's predecessor, Garry McCarthy, has linked the surge in violence in Chicago to "a decline in street stops by cops" (Madhani 2017). Similarly, Chuck Wexler, a former Boston Police Department senior commander and current executive director of the Police Executive Research Forum, explained to then Attorney General Loretta Lynch, during a 2015 meeting, that, in trying to determine causes for the recent spike in violent crime, "'Perhaps the most difficult to calibrate, but the most significant,' he said, 'is this notion of a reduction in proactive policing" (Davis 2015). Baltimore Police union president Lieutenant Gene Ryan argues that "the 'Ferguson Effect' is the primary reason for that city's crime upsurge. 'Since the funeral of [Freddie] Gray, and the subsequent violent street protests in the city, police have pulled back on enforcement of many types of street crime,' Ryan said. 'Police are more afraid of going to jail for doing their jobs properly than they are of getting shot on duty,' he said" (Gately 2015).

${ }^{12}$ This episode of 60 Minutes was viewed by approximately $11,720,000$ persons in the United States (Associated Press 2017). The program was also aired across Canada on the CTV national television network. The website of the greater-Toronto CTV affiliate, observes, "60 Minutes, the most successful television broadcast in history, offers hardhitting investigative reports, interviews, feature segments and profiles of people in the news. The broadcast begun in 1968 and is still a hit, regularly making Nielsen's Top $10 "$ ( $\mathrm{CHCH} 2017)$.

${ }^{13}$ The year 2016 was one of the most violent in Chicago's history - with 762 murders (up from 485 in 2015 - a 57.1\% increase), 3550 shooting occurrences (up from 2426 in 2015 - a 46.3\% increase), 4331 wounded persons who survived shootings (up from 2939 in 2015 - a 47.4\% increase), and twice as many assaults on Chicago police officers as the previous year (Ansari and Flores 2017; Babwin 2017a; Gallardo \& Podesta 2017; Gorner 2016a; Madhani 2016). The final 2016 homicide total for Chicago surpassed those for the first and second most populous cities in the United States, combined - New York City (with 335 murders in 2016) and Los Angeles (with 294 murders in 2016) (Associated Press 2017). 
Figure 2: Homicide Rate in Chicago, 1985-2016

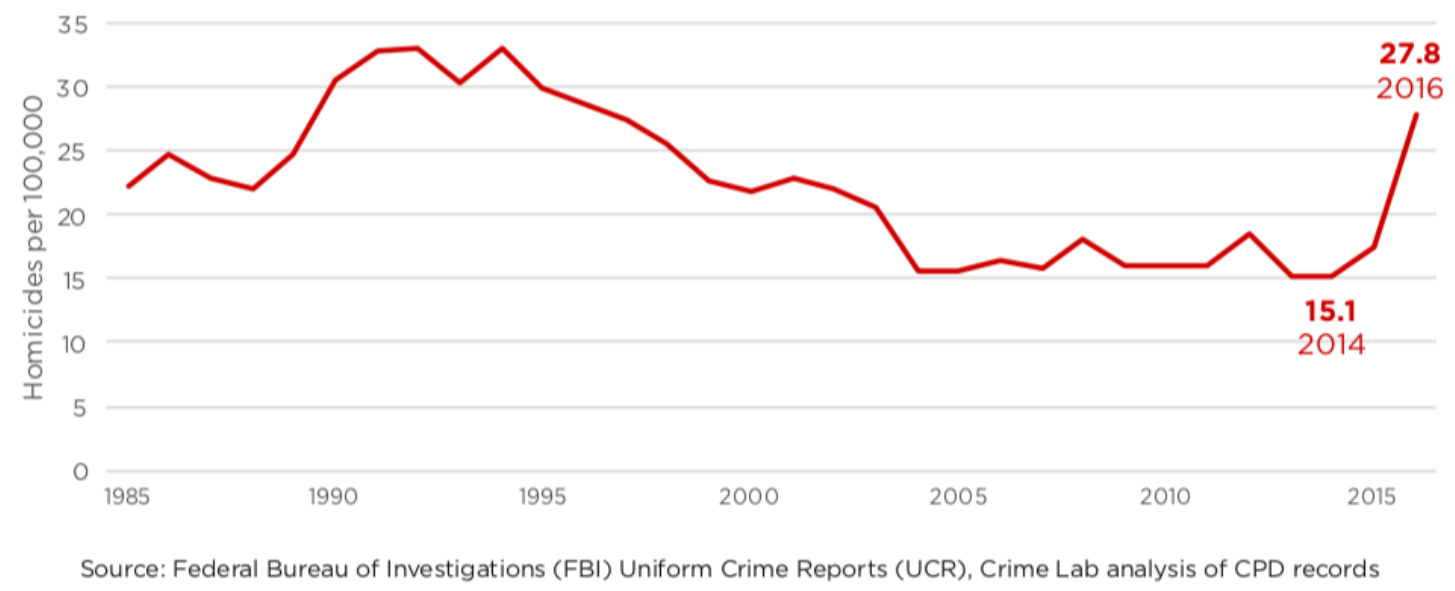

Figure 14: Homicide rate in Chicago 1985-2016

${ }^{14}$ On 20 October 2014 in Chicago, Laquan McDonald, a 17-year old Black male, who was armed with a knife and acting erratically, was shot to death by CPD officer Jason Van Dyke. CPD dashcam video cameras captured the last moments of the occurrence. The shooting was ruled as in compliance with CPD departmental policies by CPD Deputy Chief David McNaughton, however, on 15 April 2015 Chicago City Council voted 47-0 in favour of paying a \$5,000,000 settlement to McDonald's family. On 19 November 2015, Cook County Judge Franklin Valderrama ordered the City of Chicago to release the police dashcam video, which had been requested by a journalist through a Freedom of Information Act application, by no later than 24 November 2015. On that date, officer Van Dyke was charged with first-degree murder and hours later the video recording was released and then broadly disseminated to a public audience. One week later, Chicago Mayor Rahm Emmanuel terminated CPD Superintendent Garry McCarthy. On 6 December 2015 Attorney General Loretta Lynch announced that the United States Justice Department would begin an investigation into CPD practices. On 18 August 2016, CPD Superintendent Eddie Johnson recommended that seven officers involved in the McDonald shooting occurrence and its alleged cover-up be terminated. On 13 January 2017 the Justice Department released a report, which concluded that the CPD had engaged in systemic abuse against citizens, excessive force, and unfair treatment of minorities (Husain 2017).

On 24 November 2015, in addressing these Chicago developments on CNN Tonight, host Don Lemon and prominent American criminal defence attorney Mark O'Mara (who defended George Zimmerman against murder charges prosecuted by the State of Florida arising from the shooting death of Trayvon Martin in 2012) both advanced that what has now changed with questionable police conduct (as with the police shooting of Laquan McDonald) is the proliferation of video recording devices that document these occurrences - with Lemon holding aloft his smartphone to emphasize this point.

O'Mara insinuated that, until recently, the likely police construction of the event in Chicago - absent the potential for video recorded documentation - would have suggested that Mr. McDonald had advanced on the officer with his knife, thereby creating a reasonable apprehension of danger, and therefore a legal justification for the 
actions of officer Van Dyke in shooting his 'assailant.' However, Lemon and O'Mara both observed that their interpretation of the video recording suggests that $\mathrm{Mr}$.

McDonald was, in fact, moving away from officer Van Dyke's position when he was initially shot and then the video recording further depicts the officer firing multiple rounds into Mr. McDonald's prostrate (and dying) body.

Van Dyke was convicted of second-degree murder by a Chicago jury in October 2018 and he was sentenced on 18 January 2019 to a prison sentence of 63/4 years (Crepeau et al. 2019).

${ }^{15}$ Aaron Davis (2015) reported, "Participants in the discussion were told that the meeting was closed to the news media, but the mayor of [Washington] D.C. listed the event as public and a Washington Post reporter entered with her entourage and observed more than three hours of the discussion."

${ }^{16}$ In addition to discussing recent notorious American occurrences (including the fatal police shootings of Alton Sterling and Walter Scott), the episode focused on a 25 July 2015 occurrence involving Jake Anderson, a 17-year old Black male, and two front-line officers of the Chatham-Kent Police Service (the Municipality of Chatham-Kent has a population of just over 100,000 and is located approximately one hour's drive from both Toronto, to the east, and Windsor/Detroit, to the west). Much of the violent interaction between the two officers and Anderson - during which Anderson was punched in the head repeatedly and stunned by a Taser - was captured by a CCTV camera at a nearby residence (Kelly 2016). Upon disclosure of the video recording, the criminal charges filed against Anderson were withdrawn by prosecutors and the officers were disciplined (internally) because their account, as documented in their reports, did not correspond with what was depicted in the video recording (Mayor 2016).

${ }^{17}$ On 27 July 2013, 18-year old Sammy Yatim was traveling on board a Toronto streetcar in the downtown core of that city. Apparently under the influence of drugs, Yatim began acting erratically and brandished a knife while threatening some passengers. Police were called and all of the occupants of the streetcar disembarked and fled, leaving Yatim alone on the vehicle. Several TPS officers arrived in short order and approached the streetcar, instructing Yatim to disarm. After what has been characterized as a "50 second standoff," TPS Constable James Forcillo discharged nine rounds from his service pistol, in two distinct volleys, at Yatim, who died of his wounds on the floor of the streetcar. Ontario's Special Investigations Unit (SIU), a civilian police oversight agency, commenced an investigation immediately. Much of the interaction between Yatim and the TPS officers, including the fatal shooting, was video recorded by bystanders, nearby CCTV cameras, and cameras onboard the streetcar. On 29 July 2013, Forcillo was suspended from the TPS and TPS Chief Bill Blair addressed the media, saying: "Like many members of the public I have viewed the videos of this incident...I am very aware that the public is very concerned about this tragic event. They have every right to be concerned" (Pagliaro 2013). On 19 August 2013, Forcillo was charged with second-degree murder in Yatim's death. On 20 January 2016, a jury began deliberations in the case and Forcillo was convicted of attempted murder (but acquitted of second- 
degree murder) on 25 January 2016. As advanced by a Toronto Star reporter covering the proceedings, "[The] jury convicted Forcillo of attempted murder for firing the second set of shots but acquitted him of second-degree murder for the first volley, which an autopsy concluded actually caused Yatim's death (Hasham 2016). On 28 July 2016 Forcillo was sentenced to a term of incarceration of 6 years and in imposing the sentence, Justice Edward Then remarked, "When a police officer has committed a serious crime of violence by the breaking the law which the officer has sworn to uphold, it is the duty of the court to firmly denounce that conduct in an effort to repair and to affirm the trust that must exist between the community and the police to whom we entrust the use of lethal weapons within the limits prescribed by the criminal law...The shooting of Mr. Yatim was unnecessary and unreasonable and excessive from the outset of the second volley...The precipitous shooting of Mr. Yatim contrary to Officer Forcillo's training constitutes a fundamental failure to understand his duty to protect all life and not just his own" (Hasham 2016).

${ }^{18}$ Also, in a 12 April 2016 memorandum distributed to all rank-and-file TPS officers (a copy of which was later sent to me), McCormack wrote (emphasis added),

Recent weeks have seen a new provincial street check regulation combined with a broad scale lack of police support from provincial and local politicians and other public leaders. This has resulted in ambiguity and uncertainty for our members. We recognize that the absence of sound policy and procedure relating to provincial street checks exposes our members to unnecessary risk and jeopardy...

A motion passed unanimously April 1 at City Hall, requesting that police services provided by the City of Toronto and the mandate, procedures and outcomes of SIU investigations be viewed through an anti-Black and antiracism lens, adds additional challenges for our members and creates a divisive environment between the community, police officers, the SIU and the TPSB [Toronto Police Services Board]. If this motion is acted upon, officers risk judgment based on political considerations and agendas driven by special interest groups. Be aware that even if you carry out your duties lawfully, to the best of your ability and training and requirements of law, your actions may be subject to review through a subjective anti-racism lens. The Association questions how our members can effectively carry out their duties if they don't have the confidence and support from the TPSB, City Council and the Province.

Continue to do your job as best you can in accordance with the law, recognizing that legislation/policy changes may severely impact your ability to provide effective policing and expose you to unnecessary risk. Ensure that you take all precautions necessary to avoid exposing yourself to undue jeopardy. We will continue to pursue clarification from the Province and the Toronto Police Service. Until adequate detail is provided, please remain cautious in the execution of your duties. 
${ }^{19}$ Interview data provided by research participants from my MA study (Brown 2013) and the Ottawa Police Traffic Stop Race Data Collection Project - Impact on FrontLine Officers Interview Study (Brown \& Primeau 2015) are available for use in the present study through secondary use of data provisions in the consent agreements with those research participants and the study's ethics clearance (see Appendix A and B).

${ }^{20}$ Similarly, an OPS officer (in 2012) provided some insight into how scrutiny and scandals have impacted on how she/he does front-line patrol work today,

I would say that once the Marty Cardinal thing happened [in 2000] and then with the appeals by upper management to make sure the public was satisfied and got its pound of flesh I did a lot of thinking about my career. I was one of those go-getters and wasn't too worried about a Rodney King thing and this kind of stuff really never worried me or was on my mind. But when I saw the way the public freaked out over what was really a minor use of force incident and the way management bent over backwards to make sure the vocal minority who love to criticize the police got their way I changed how I do my job. I do what is required, period, and I make sure I am always aware of what is going on around me, what I am doing, and the potential for something to look bad to the public. The last thing I or my family needs is to be part of the next big incident all over YouTube or the front page of The [Ottawa] Citizen. Look at the guy in Toronto, with the bubbles...If that was me, I wouldn't say a word. Let them blow bubbles, let them disrespect the police. Let them riot. You gotta know cameras are on everything and they are trying to provoke you. Today, the motto, and I know even the young guys today who come on the job with this idea that they are going to change the world don't take long to realize, do what you are required to do on your shift but don't do anything that could be part of a controversy and get you in trouble or fired. If that means letting things go, let it go, at the end of the day no one cares, you still get paid, and you go home...If the citizens really want that kind of society,..one where gang bangers get off on technicalities and the police are the one's getting into trouble for stopping their car full of crack and guns they can have it and we will just do the bare minimum...A lot of us now don't arrest aggressive drunk people anymore because of the way the [Stacy] Bond[s'] thing was so crazy in court and then with the public bandwagon and the Chief throwing all of us who do the job under the bus. I hope the public who was apparently so upset with the police likes having these people wandering around them and the police doing nothing about it because why would we?..A lot of us don't pull over a suspicious car when we would have without a doubt years ago, who wants to be the next racial profiler?

Another OPS officer shared (in 2015),

To be honest, with the way things are today with a hyper-antagonistic public and criticism like never before from so many directions, I just try to 
get through each shift without getting into too much shit and get home with my job intact, my paycheque coming the next payday, and one day closer to pension. To do that, I do what I have to do in terms of taking my calls but the lights and sirens 100 miles an hour stuff and the discretionary stuff I used to do, like suspicious behaviour traffic stops or paying particular attention to a crack house or some particular asshole in my zone causing a lot of problems or slinging dope, well, I just don't do it anymore. It's not worth it. That stuff gets you on YouTube and in a big jackpot. That kind of stuff today can be twisted into harassment or racial profiling and a police complaint or human rights lawsuit. The public doesn't support it and neither does the Chief. I would rather spend that time reading the paper or meeting one of the guys for a coffee. I know a lot of the guys feel the same now and do the same thing. It's about us surviving our time in patrol now and staying off the news or the Internet and the bosses' radar; not about protecting the public by locking up the bad guys. I don't know if they will admit it to you but it is important for it to be said and for the public to understand what they are doing to policing today. In terms of Blacks being pulled over more than Whites, I can assure you if I can see the colour of the people in the car and they are Black they have a lot better chance of not getting pulled over than if they are White; today that's just the way it is. For example, I have [a subsidized housing project - redacted to ensure anonymity of the research participant] in my zone. It's got to be $90 \%+$ Black. I just don't go near there unless I have a specific call that is dispatched to me in there...For sure, the worst case scenario today would be to shoot a black guy and have it on video, it doesn't matter if he's cracked out and charging at you from 10 feet away with a knife yelling that he's going to kill you, some how and some way you are going to be found to have done something wrong even though that is the training we get and what the law says is right to defend yourself - racial profiling, not deescalating, not using something other than lethal force - the cop today is going to wear it. The public will go nuts and then along with SIU and some judge they bring in from Toronto for your trial they will analyze the video frame by frame and come up with 'you should have done this, you shouldn't have done that, you didn't follow policy [to the letter], this was not justified, he wasn't really going to kill you officer, he just had some mental problems you should have helped him with, so you are no longer a cop, and you can go to jail officer'. So, just survive your time in patrol and get a transfer to [a less risky section of the police service] as soon as you can.

Along the same lines, a TPS officer offered (in 2012),

It's really simple. There is no way I would accept a transfer to certain divisions. Who would want to work somewhere where a majority of the public hate the police? Why would you want to try to survive as a cop and risk your career in that situation - where everyone is suspicious of you all the time and God forbid if something goes for a shit you have 100 witnesses 
whose default position is the police were in the wrong; we used too much force, we provoked it, we should have done something different, we were racist, we were profiling. Does the public, and the courts are even worse, do they understand that if this keeps going like this, cops are not stupid...If we work somewhere else that is nice and quiet and civilized or don't get involved in the serious calls and the bullshit we still get paid and make it to pension and where does that leave them, who are they going to call when all hell is breaking lose in those neighbourhoods and the gangs are running the show? 9-1-1 will get you political correctness, social work, and the cops showing up after it's all over. I know you've heard of F.I.D.O. [the 'fuck it, drive on' principle]; well that's alive and well and every time one of these public scandals break out with the public taking default anti-police positions with video that is debatable and some officer or officers, who were trying to do their job under a microscope and in situations where Joe Public would piss his pants and run away, get charged and wind up trying to defend their actions in court...more and more guys look in the mirror and think what the hell am I doing risking my job?

${ }^{21}$ The Ottawa Police Traffic Stop Race Data Collection Project was conducted under the leadership of three York University researchers - Dr. Lorne Foster, Dr. Les Jacobs, and Dr. Bobby Siu. Additional information is available at https://www.ottawa police.ca /en/ news-and-community/Traffic-Stop-Race-Data-Collection-ProjectTSRDCP.asp.

${ }^{22}$ As explained in more detail in Chapter Four, presenting my previous police experience (the practitioner component of my 'pracademic' biography) is important both to situate the reader and also in terms of researcher reflexivity. I joined the Ottawa Police Force in 1985, after completing two years of undergraduate studies (in criminology) at the University of Ottawa. I spent the next ten years performing uniform, rank-and-file duties - primary response (general patrol), serving as a coach officer for 'rookie' officers having completed their training at the Ontario Police College, working as a member of the Emergency Response Unit, and as a member of the Crowd Control Team (the 'riot squad' as it was often called in that era - given the frequency of unruly public order events in Canada's capital city). In 1995, I began an eight-year assignment as an 'undercover' drug investigator, which included working with joint-forces units with the Ontario Provincial Police (OPP) and the Royal Canadian Mounted Police (RCMP). During this time, I was also sworn as police officer for the Province of Québec, given my work on investigations with the Sûreté du Québec and the Service de police de la Ville de Montréal in that Canadian province.

In 1999, I was promoted to the rank of Detective Sergeant and assumed supervisory duties with the Ottawa Police Service (OPS) Drug Unit. In 2002, I transferred to the Major Case Unit within the OPS, where I was engaged primarily with homicide and other serious (and often complex) criminal investigations. In 2006, I was awarded the Police Exemplary Services Medal by the Governor General of Canada. In 2010, I requested a transfer to front-line police work (in order to have the necessary time to begin postgraduate studies at Carleton University), where I served for the next three 
years as a Patrol Supervisor in the OPS Central Division (responsible for the downtown core of the City of Ottawa).

${ }^{23}$ Of note, I was familiar with the two men arrested in this occurrence, as I had interviewed both of them on 2 July 2008 (in my capacity as a homicide investigator), while they were in custody in relation to a drive-by shooting occurrence (in which a 20year old man had been shot in a hail of bullets fired at a gathering of persons in a parking lot in Ottawa's west end) and the seizure of a semi-automatic handgun (which was observed being transferred between these two men during subsequent police surveillance). As reported in the National Post (2016), "Court records show that [one of the men] has convictions for assaulting someone while in jail, robbery, carrying a concealed weapon, possession of a firearm obtained by crime, and drug trafficking."

${ }^{24}$ Coverage had not been restricted to the Ottawa region but was prominent across Ontario and Québec, with print media headlines and radio/television story introductions like, 'Judge blasts Ottawa cop for racial profiling' (Spears 2011), 'Judge says pair busted for being Black' (London Free Press 2011), and 'Judge slams Ottawa police officer for racial profiling' (1310 News 2011).

${ }^{25}$ This particular neighbourhood in Ottawa (known as the 'Beausoleil projects,' which features a densely-populated and large-scale public housing complex) has, for many years, been frequented by primarily Haitian-Canadian members of Montréal street gangs, who use addresses of allied Ottawa gang members to traffic crack cocaine and engage in human sex trafficking, robberies, and violence in support of their criminal activities.

${ }^{26}$ As another example, in his ruling on a 2011 Ottawa case (R. v Gonzales [2011]), Justice Charles Hackland of the Ontario Superior Court, found,

The reason [Constable] Hassan stopped the accused was because he associated the vehicle with gang related criminal activity. At the same time, he had no reasonable or probable cause to believe the accused was engaging in such activity, beyond mere suspicion. I disbelieve [Constable] Hassan's attempt to justify this stop as a concern to enforce the [Ontario Highway Traffic Act]. This is simply a retrospective rationalization.

Further, in the Canadian context, see also R. v. Tombs (2012), R. v. Nguyen (2012), R. v. Phengchanh (2011), R. v. Gonzales (2012), R v. Khan (2004), R. v Meerza (2013).

Interestingly, 'pretext' traffic stops are not as problematic within the American criminal justice system, as demonstrated in the 1996 unanimous ruling of the United States Supreme Court in Whren v. United States, which Dillon (1997: 739) summaries as follows, "The Court...held that pretextual traffic stops - stops where the officer pulls a motorist over for a minor violation in order to investigate a more serious offense for which the officer does not have the requisite justification - are constitutionally permissible. As long as a police officer is authorized to stop a motorist for the stated 
reason, it is immaterial that the officer had another motive for initiating the stop." In commenting on pre-text stops in the USA, Rosenbaum (2019: 325) observes, "policeinitiated contacts (often involving minor infractions) are used as a tool to identify weapons, drugs, gun, and persons with outstanding warrants." See also, the United States Supreme Court's 2014 ruling in Heien v. North Carolina, State of Kansas v. Robert Alan Wilson (2015), State of Illinois v. Jose Gaytan (2015), and State of Wisconsin v. Richard Houghton Jr. (2015).

${ }^{27}$ For the formal institution of policing in society to be effective, the police cannot rely solely on their capacity for coercive force nor on their institutionalized powers of arrest and detention, but rather, a fundamental prerequisite is the consensual engagement of the public with the policing of their communities through ongoing support of, and voluntary cooperation with, the police (Albrecht \& Green 1977; Alpert et al. 1998; Bradford et al. 2009; Callanan \& Rosenberger 2011; Cordner 1997; Dunham \& Alpert 2010b; Eck \& Rosenbaum 1994; Flanagan \& Vaughn 1995; Frank et al. 2005; Gallagher et al. 2001; Hawdon 2008; Hawdon et al. 2003; Hough et al. 2010; Jackson \& Sunshine 2007; Jefferis et al. 2011; Loader 2014; Luna 2000; Mastrofski et al. 1996; Murphy et al. 2008; Reiner 2010; Rosenbaum et al. 2005; Sampson \& Bartusch 1998; Sunshine \& Tyler 2003a, 2003b; Tyler 2004, 2006b; Tyler \& Huo 2002; Tyler et al. 2007; Zamble \& Annesley 1987). The support and participation of the populace, however, is contingent on the institution of policing (and the exercise of its powers) being perceived by the public as legitimate (Decker 1981; Frank et al. 2005; Hough \& Roberts 2004; Lee \& McGovern 2013; Sindall et al. 2012; Sunshine \& Tyler 2003a; Tyler 2004, 2006a, 2006b). Further discussion on these topics is engaged later in the dissertation within subchapter 6.7.

${ }^{28}$ While the examples presented are from American news organizations, both Time and Newsweek are widely circulated in Canada and popular cable news networks, such as $C N N$ and Fox News, are commonplace programming in most Canadian television markets. Canadian news programming has featured some stories regarding front-line policing in Canada, such as an episode of The Fifth Estate titled 'Police shootings: Caught on camera' (Kelly 2016), but, in my estimation - as a particularly-interested observer - such coverage pales in comparison to that throughout the USA in this 'postFerguson' era. However, much of that American media attention makes its way into Canadian living rooms (via television news programming) and onto the computer and mobile device screens of many Canadians (though social and traditional media platforms). This consideration is elaborated in Chapter Six.

${ }^{29}$ In their investigative reporting on "...thousands of fatal shootings at the hands of [American] police since 2005 [in which] only 54 officers have been charged, a [Washington] Post analysis found most were cleared or acquitted in the cases that have been resolved" (Kindy \& Kelly 2015). The picture below is from the front page of the publication on 11 April 2015 and depicts "an evidence photo show[ing] a Chevy Malibu that Cleveland police officers riddled with bullets after a [2012] chase that ended in the deaths of an unarmed man and woman. Officer Michael Brelo, who investigators say fired 34 shots at the car and then climbed on the hood and fired 15 more through the 
windshield, faced trial on two counts of voluntary manslaughter" (Kindy \& Kelly 2015). Officer Brelo was acquitted on 23 May 2015 (Smith \& Southall 2015).

${ }^{30}$ Oliver is a professor in the Department of Criminal Justice at Sam Houston State University, Huntsville, Texas. He is a retired military police officer and a former police officer serving in metropolitan Washington, DC.

${ }^{31}$ Todd (2015: 99-104) makes various recommendations for future research - including interviewing "larger number of officers...and officers from different areas of the country," surveying "a large sample of officers from various police departments," and "examining the impact of citizen monitoring on operational police work in both the United States and in Canada, and [making] comparisons between the two locales..."

${ }^{32}$ Alvesson (2003: 174) advances, in general terms, that 'insider' research involves, "The researcher-author [investigating] a cultural setting to which s/he has a 'natural access', [having worked or lived] in the setting and then [using] the experiences, knowledge and access to empirical material for research purposes." 'Outside insiders' are typically police officers that have become academic researchers and thereafter conduct external studies on policing (Brown 1996; Thomas 2014). Thomas (2014: 8) finds, "Individuals in this group have an intimate inside knowledge of policing." Of note, Garry Thomas was a doctoral candidate (in 2014) at the Centre for Police Sciences, University at Glamorgan (U.K.), having retired after 30 years of service as a police officer with the South Wales Police.

Reiner (2000a: 211) observes, "A significant number of police officers have in fact made the switch to becoming full-time academic specialists in police research" (referencing, for example, Waddington). Professor Jim (P.A.J.) 'Tank' Waddington is Professor of Social Policy at the University of Wolverhampton. He served as a police constable with the Birmingham City Police from 1963 to 1969 before leaving policing to pursue graduate work at the University of Leeds in 1970. In addition to the 'outside insider' moniker (Brown, 1996; Thomas 2014), academics who are also police practitioners have been characterized as 'pracademics', with Sherman (2013: 433) suggesting, "The leadership of police experiments has been moving from professors to 'pracademics' ...many are launched on a path to earn a doctoral degree, some in midcareer and some retired after 30 years in policing. Whatever their background, they are rapidly increasing the numbers of people who can produce good evidence on policing."

Reiner (2010: 10) finds, "Occasionally research projects begun by serving police officers as students have resulted in influential publications" (referencing, as examples, Holdaway 1983 and Young 1991). Professor Simon Holdaway (University of Sheffield - Centre for Criminological Research) worked for eleven years as a police officer (attaining the rank of sergeant) with the Metropolitan (London) Police before resigning to pursue an academic career. Malcolm Young worked for 25 years in operational policing roles (as a constable and also in drug enforcement) with the police service in urban Tyneside before becoming Superintendent of the rural West Mercia Constabulary. 
Throughout his 38-year policing career he engaged in a cultural anthropological investigation of the occupational culture of the police and his doctoral dissertation was the foundation for his acclaimed book An Inside Job: Policing and Police Culture in Britain. Similarly, Brown (1996: 181) finds, "A number of former police officer academics have made distinctive contributions to police research. The topics they have chosen to investigate engage issues of the informal practices of police officers and negative aspects of police occupational culture." In this vein, Heslop (2012: 525) regards Simon Holdaway as 'a pioneer' in the research on policing and describes his studies as the "most ground-breaking ethnographic studies of the occupational culture of the British police ever undertaken," particularly as Holdaway conducted his fieldwork covertly within his policing duties before leaving the police service.

The 'outside outsider' researcher is the traditional university-affiliated academic, who conducts research, on a policing topic of interest to that researcher, through the gaining of access to, and the collection of data in the possession of, police organizations (Brown 1996; Reiner 1994).

Reiner (2000a: 217-218) finds that methodological issues, "which are peculiar to, or peculiarly acute in, researching the police... arise partly out of the nature of police work and [the] organization in general... [but also, of significant importance,] according to the characteristics of the researcher vis-à-vis the police." With regard to the latter consideration, Brown (1996) has identified four categories of policing researchers, which are defined in relation to their location within, or external to, the police agency (and by extension to individual police officers in the employ of police agencies). These are the 'inside outsider,' 'outside outsider,' 'inside insider,' and 'outside insider' (Brown 1996). These classifications are accepted as authoritative throughout the policing literature (including being relied on, for example, in the works of Reiner 1994, 2000a, 200b, 2010; Sherman 2013, 2014; and Thomas 2014).

'Inside outsiders' are understood as academically-qualified 'civilians' working within, or in conjunction with, a police agency's internal research department or its research liaison officer(s) - often as external academic consultants contracted by the police agency (Brown 1996). 'Inside insiders' are those employed within a police agency to carry out in-house research. These persons most often have limited formal training and their inquiries are typically focused on internal procedural and operational issues (Brown 1996). Brown (1996: 180) advances, "Given the low level of research expertise...much of the output is parochial and very little is refereed or formally published."

${ }^{33}$ In an informal 'focus group' session that materialized unexpectedly during data collection with rank-and-file Toronto officers, a group of eight TPS officers expressed agreement with, and sentiments that were consistent to, those articulated by one particular officer during this discussion,

If those people want to live in a jungle where the laws of the jungle and not the rule of law dictates who runs the neighbourhood then let them live 
like that, why should we care or risk our lives or careers getting involved? Cordon off the area, stay away, and focus on protecting the law-abiding and supportive neighbourhoods outside the 'hood'. You see all of the supporters of the gang-bangers and drug dealers and the police haters talking about how the police racially profile and shouldn't be doing 'carding'. These people from Rosedale and other 'I grew up with a silver spoon and haven't lived a day in the real world' areas who feel sorry for some banger who got into it with the people who protect their mansions and their Ferraris; they are the first ones to go nuts if something happens to them or their property but they toe the politically-correct line of cop bashing. I'd love to see a bunch of drive-bys in their neighbourhoods and see how quick they would change their tune at their tea parties. 


\section{NOTES FOR CHAPTER TWO}

${ }^{1}$ In relation to the concept of risk aversion in front-line police work, this study presented three common terminologies (phrases synonymous across the rank-and-file subculture with risk aversion to certain policing practices) to research participants - (1) 'risk-averse policing', (2) 'de-policing', and (3) 'F.I.D.O.', which is an acronym representing the sentiment, 'Fuck it, drive on' (or 'Forget it, drive on'). Within the survey questionnaire this concept was explained to study participants as follows Questions 27 and 28 relate to the concept of risk-averse police practices. Some within policing, the media, and some academics studying policing have discussed this concept using different terminologies - such as 'de-policing' or 'F.I.D.O.' ' I argue that the term de-policing best captures the phenomenon identified in this study and, therefore, that terminology is used throughout this dissertation to characterize the identified risk-averse practices of today's front-line officers.

${ }^{2}$ Waddington (2015: 681), in discussing Muir's (1977) contribution for a series of articles themed 'revisiting the classics' (in Policing and Society: An International Journal of Research and Policy), observes that "Street Corner Politicians is an acknowledge classic in police studies...[Featuring] hard-headed political analysis in the 'realist' tradition of Hobbes, Machiavelli and Clausewitz." Banton's review, appearing on the back cover of the book, advances, "Other social scientists have observed policemen on patrol, or have interviewed them systematically. Professor Muir has brought the two together, and, because of the philosophical depth he brings to his commentaries, he has lifted the sociology of the police on to a new level."

${ }^{3}$ Other contributions within the scholarly literature include: Bradley 2007; Brown 2013, 2106; Brown \& Primeau 2015; Cooper 2002, 2003, 2006; Crank \& Caldero 2010; Davis, Henderson, Ortiz, Miller \& Massie 2002; Davis, Henderson \& Ortiz 2005; Deuchar et al. 2018; Farmer \& Sun 2016; Gonzales \& Cochran 2017; Huey, Walby \& Doyle 2006; Maguire et al. 2017; Marier \& Moule 2018; Martin 2013; Morgan \& Pally 2016; Morin et al. 2017; Murphy 2014; Nix \& Pickett 2017; Nix \& Wolfe 2016, 2017, 2018; Nix et al. 2019; Nix et al. 2018; Oliver 2017; Pyrooz et al. 2016; Roberg, Novak, Cordner \& Smith 2015; Rushin \& Edwards 2017; Sandhu 2016, 2019; Sandhu \& Haggerty 2015, 2017; Shjarback et al. 2017; Todd 2015; Torres et al. 2018; Withrow 2011; Wolfe \& Nix 2016; Wynn 2008.

Other mediated discussions include: Baker \& Goodman 2014; Berman 2016; CBC News 2016b; Davis 2015; Dewan 2017; Durkin 2014; Fenton \& George 2015; Fisher 2014; Flores 2017; Frankel 2015; Gately 2015; Graham 2015; Kelly 2016; Leo 2001; Levine \& Taylor 2015; Lichtblau 2016; MacDonald 2015, 2016; Miletich 2014; Miller 2001; Murakami 2002; Newman 2014; Reuters 2016a, 2016b; Sanchez 2015; Santori 2014; Schram, Conley \& Feuerherd 2014; Shjarback, Decker, Wolfe \& Pyrooz 2017; Sutton 2015; Whitaker 2017; Yogaretnam 2018 b.

${ }^{4}$ This does not include media coverage that developed after 12 February 2018 after my discussion of this study's preliminary findings in an interview I did with Ottawa Citizen 
police reporter Shaamini Yogaretnam (after which the newspaper published the story on the front page and it was carried across the country through the Postmedia network of publications).

${ }^{5}$ In 1994, in the aftermath of the 1991 Rodney King occurrence and ensuing controversies suggesting widespread misconduct in the LAPD, the Civil Rights Division of the U.S. Justice Department (DOJ) was granted powers (through provisions enacted in the Violent Crime Control and Law Enforcement Act) to sue state and local governments in federal court in order to correct systemic organizational practices of police misconduct. Through such actions the American federal government seeks to investigate and then intervene in what it contends are problematic police agencies. In lieu of proceeding to trial, many of these cases have been settled through agreement what is termed a 'consent decree', in which a series of reforms are agreed upon and then an independent monitoring regimen is put in place to oversee compliance. The first such agreement was implemented with the Pittsburgh Police Department in 1997 (and since with various other police agencies across the USA, such as Los Angeles, Cincinnati, Detroit, and Washington, DC). In 2001, the DOJ asked the Vera Institute to examine the effects of the first consent decree in Pittsburgh and the report was produced in 2002 (by Davis, Henderson, Ortiz, Miller \& Massie), titled Turning Necessity into Virtue:

Pittsburgh's Experience with a Federal Consent Decree. For a comprehensive overview of American consent decrees see Chapter Six in Noble and Alpert's (2008) Managing Accountability Systems for Police Conduct: Internal Affairs and External Oversight. Of particular relevance to the present study, both the Buffalo Police Department (BPD) and the Yonkers Police Department (YPD) have been subject to consent decrees and oversight as a result of problematic findings by the DOJ.

${ }^{6}$ James Warner was a nineteen-year police veteran (in 2005) who suggests that his book "tells of personal and professional obstacles that officers have and will face throughout their careers" and is "an excellent tool for discussing contemporary police ethics and the topic of de-policing in America" (https://www.therip.com/unapproved-readersubmissions/2005/10/19/book-review).

${ }^{7}$ Robert Heaton is a serving police officer with the Kent (U.K.) Police who was awarded a $\mathrm{PhD}$ in 2007.

${ }^{8}$ In the aftermath of widespread protests over the death of Eric Garner, while struggling with arresting NYPD officers in Staten Island on 14 July 2014, ensuing violence perpetrated against NYPD officers by some citizens and the perception of many in the NYPD that Mayor Bill De Blasio and certain elements of the community 'threw NYPD officers under the bus' (Durkin 2014; Santori 2014), most front-line NYPD officers, for all intents and purposes, ceased performing even routine police practices for several consecutive weeks in late 2014 and early 2015 - with staggering reductions in police interventions. In the week that can be characterized as the boiling point in the acrimony felt by NYPD officers toward Mayor de Blasio and the resentment toward those in the community who expressed criticism of the police, it become apparent that coordinated and particularly intensive de-policing had developed (Baker \& Goodman 2014; Celona 
et al. 2014; Newman 2014; Schram et al. 2014). NYPD traffic tickets and summonses for minor offenses dropped by 94\% for the week of 22-29 December 2014 (compared with the same period in 2013) - from 10,069 to 587 and from 4,831 to 300 respectively - "as officers feel betrayed...some cops [are] mounting an undeclared slowdown in protest" (Celona et al. 2014). An e-mail widely circulated among NYPD officers on 20 December 2014 "pointed to potential slowdowns in arrest and ticketing activity,

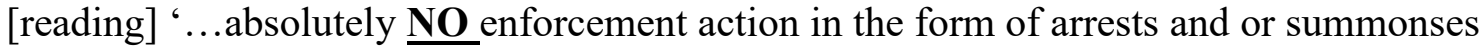
is to be taken unless absolutely necessary and an individual MUST be placed under arrest"” (Schram, Conley \& Feuerherd 2014, emphasis in original).

${ }^{9}$ Todd's (2015) method involved qualitative research, in the form of in-depth semistructured interviews conducted with 13 police officers between 29 August 2014 and 29 January 2015. The officers all worked for Vancouver-area police agencies, in various capacities and, therefore, were not all in rank-and-file patrol duties. The sample included one female officer.

${ }^{10}$ Participant \#2 in Todd's (2015: 81) study elaborated on the practice of officers intentionally neglecting to respond to their observations in certain circumstances,

Officers think that, unless they're dispatched to a call, they're not going. They see a drug deal taking place on the sidewalk and they try to forget they even saw it happen and drive past it. If you get out and deal with it, and someone makes an allegation of racism, or something, it could just go sideways on you. Then you spend the next two years of your life dealing with hearings and stuff trying to explain that 'No, I saw a drug deal taking place, that's why I stopped and checked these people.' It had nothing to do with race or gender or anything else.

${ }^{11}$ The data that Sandhu and Haggerty (2015) relied on consisted of 200 hours of 'ridealong' field observations with 'approximately 60' quasi-police (transit and university campus) officers in Edmonton (Canada) and police officers (from the Edmonton Police Service) from June 2013 to December 2013 and interviews with 20 research participants (Sandhu \& Haggerty 2015; Sandhu 2019). The focus of the research was on patrol officers, however the interview sample also included superintendents, detectives, video analysts, surveillance camera operators, dispatchers and trainers (Sandhu 2019).

12 Richard Rosenfeld is the Thomas Jefferson Professor of Criminology and Criminal Justice at the University of Missouri - St. Louis.

${ }^{13}$ Despite that their "research has shown that negative publicity surrounding the police in the aftermath of Ferguson was associated with lower levels of officer self-legitimacy" (Nix \& Wolfe 2016: 12), Wolfe and Nix (2016: 7) also suggested,

But for the most part, our findings suggest that the Ferguson Effect fearmongering may need to stop (at least for now). After we accounted for perceptions of organizational justice and self-legitimacy, the Ferguson 
Effect was no longer significant. Thus, our data reveal that reduced motivation attributable to negative publicity may be counteracted if supervisors ensure fairness among subordinates...In addition, we saw that self-legitimacy mattered. Confidence in one's authority as a police officer appears to protect against the negative effects of media coverage of highprofile incidents like Ferguson.

${ }^{14}$ Nix and Wolfe (2016: 14) go on to explain,

The problem with most media attention concerning the Ferguson Effect is that it is treated often as a singular phenomenon. The reality is that there may be many Ferguson Effects. While research suggests that a Ferguson Effect on crime rates appears to be confined to select cities in the US, there are many other consequences experienced by officers that have resulted from negative publicity...Officers need to be held accountable for wrongdoing, but this evidence suggests that a sizeable portion of police officers are feeling the ill effects of intense public scrutiny. These are important observations not only for police agencies but the communities they serve. Ultimately, officers [de-policing] are less effective than they should be. This has direct implications for the safety of citizens and the wellbeing of communities. Unfortunately, we know very little about what factors are associated with officers' sensitivity to Ferguson-related negative publicity.

${ }^{15}$ Nix and Wolfe (2017: 89-90, citing Bottoms \& Tankebe 2012) advance,

A prospective source of police self-legitimacy is perceived support from the public. According to the dialogic model of legitimacy, officers routinely engage in legitimacy dialogues with citizens who either comply with their directives or challenge their legitimacy claims. Thus, it seems likely that perceived support from the public would factor into officers' sense of self-legitimacy...Bottoms and Tankebe's (2012) dialogic model would seem to suggest that officers' self-legitimacy may be weakened if such negative publicity is seen by the police as an indication of lack of public support.

${ }^{16}$ Pyrooz and colleagues (2016: 5) did find, however, that "several cities (Baltimore, St. Louis, Newark, New Orleans, Washington, D.C., Milwaukee, and Rochester among others) experienced large increases in homicide rates following the events in Ferguson. Accordingly, the data offer preliminary support for a Ferguson Effect on homicide rates in a few select cities in the United States."

17 The survey was administered by researchers at the National Police Research Platform (NPRP) in cooperation with the Pew Research Center. A total of 91 police agencies were invited to participate in the national survey, via communication by the NPRP with chief executives of each agency. Of these, 54 
agreed to participate. Across the participating agencies, the total number of officers eligible to participate in the survey (based on the criteria established by the researchers) was 57,062, of whom 7917 completed the online survey (a response rate of $13.9 \%$ ).

${ }^{18}$ Campeau (2015: 682) shares the risk adaptations of her study participant identified as 'Constable A':

Now it's completely different. I spend my days trying to stay out of trouble, then serve the public, then look out for my partners. In fact, that's not even right. I spend...the first thing is...I don't want to get in trouble. I don't want to get nailed by somebody with a camera phone or by our own service, and then I have to take care of myself [emphasis]. And then I have to take care of my partner, and then the community...[Accountability] has swung so far in the other direction now. It's gotten to the point of ridiculousness; and I go to work and, like I said, I just spend my days not trying to get in trouble. People tell me fuck off, and I do. And it breeds... 'Oh, I don't care. Those two guys are fighting. Alright, well, I'm not getting involved because, you know what, then something will happen. I'll break it up; break a guy's arm trying to break it up, and then they'll have SIU coming down here and they'll investigate me. 


\section{NOTES FOR CHAPTER THREE}

${ }^{1}$ Parsons, in considering the positions of both Durkheim and Weber, theorized that internalization of group values and alignment of actions with colleagues within occupational contexts are often the central components in social action (Alexander 2003; Smith \& Reilly 2009).

${ }^{2}$ Stenning and Shearing (2005) discuss these in terms of 'internalities' (conditions within police institutions) and 'externalities' (conditions arising through the external environments within which the police operate).

${ }^{3}$ See also, Aas, Gundhus \& Lomell 2009; Andrejevic 2005, 2007; Ball \& Webster 2003; Bell 2009; Birchall 2011a, 2011b, 2011c, 2014; Bock 2016; Bogard 2006; Brucato 2015b; Chan 2008; Ericson 2007a; Friedman 2007; Fyfe \& Bannister 1998; Haggerty \& Ericson 2000, 2006; Koskela 2003, 2011; Lyon 1994, 2001, 2007; Marx 2002; McGrath 2012; Whitaker 1999; Yesil 2011.

${ }^{4}$ See also, Antony \& Thomas 2010; Ball et al. 2012; Brighenti 2007; Brown 2013, 2016; Campeau 2015; Chan 2007; Goldsmith \& Lewis 2000; Haggerty 2006, 2012; Haggerty \& Sandhu 2014; Jeffries 2011; Kaminski \& Jefferis 1998; Lawrence 2000; Lyon 2007; Nix \& Wolfe 2016; Ogletree et al. 1995; Phillips 2010; Potere 2012; Reiner 2010; Robinson 2012; Ross 2000; Sandhu 2016, 2019; Sandhu \& Haggerty 2015, 2017; Skolnick \& Fyfe 1993; Thompson \& Lee 2004.

${ }^{5}$ Given this new social landscape, throwing a 'veil of secrecy' around the formerly private behaviour of public figures - which, in my view, subsumes today's highlyvisible front-line police officers - is difficult (Brighenti 2007; Brown 2013, 2016; Goldsmith 2010; Thompson 2005). Increasingly sophisticated technologies "provide a powerful array of devices that can be used to increase the leakage of back-region behaviour into front regions where, coupled with [mediated dissemination], they can be turned into highly visible events" (Thompson 2005: 44). There is now "a vast array of channels, decentralized and impossible to monitor and control completely, through which images and information can flow" (Thompson 2005: 42). Thompson (2005: 38) found that "given the nature of the Internet, it is much more difficult to control the flow of symbolic content within it, and hence much more difficult for those in power to ensure that the images made available to individuals are those they would wish to see circulated."

${ }^{6}$ See also, Andrejevic 2007; Bell 2009; Brucato 2015b; Campeau 2015; Farmer, Sun \& Starks 2015; Farmer \& Sun 2016; Goldsmith 2010; Haggerty \& Sandhu 2014; Jeffries 2011; Koskela 2006, 2009, 2011; Newell 2019; Sandhu 2016, 2019; Sandhu \& Haggerty 2015, 2017; Shjarback et al. 2017; Shirky 2008; Toch 2012; Walker \& Archbold 2014; Wallace et al. 2018.

${ }^{7}$ See also, Bauman \& Lyon 2013; Brown 2013, 2016; Campeau 2015; Chan 2008; Goldsmith 2010; Lyon 2002, 2004; Lyon et al. 2012; McCahill \& Norris 2002a, 2002b; 
Newburn \& Hayman 2001; Norris \& Armstrong 1999; Sandhu \& Haggerty 2015; Töpfer, Hempel \& Cameron 2003.

${ }^{8}$ Goold, Loader and Thumala (2013: 993-994) find that today, CCTV camera surveillance is so "commonplace and unremarkable" that it can be characterized as "banal." Similarly, in terms of societal surveillance and enabling technologies in general, Parenti (2003: 78) notes, "surveillance has become more ubiquitous, automatic, anonymous, decentralized, and self-reinforcing" and Brucato (2015b: 42, citing Lyon 2007) observes, "Imaging technologies enable [citizen] oversight and are approaching ubiquity in the Global North."

${ }^{9}$ See also, Andrejevic 2007; Bock 2016; Brucato 2015b; Deuze, Bruns \& Neuberger 2007; Finn 2012; Greer \& McLaughlin 2010b; Jeffries 2011; Kies 2011; Lee \& McGovern 2014; Lievrouw 2011; Lyon 2006; McGrath 2012; Meyer 2015; Roche 2017; Sandhu 2016; Schaefer \& Steinmetz 2014; Shiffman 2008; Trottier 2015; Wall \& Linnemann 2014.

${ }^{10}$ Citizen journalism is the spontaneous adoption of the news reporter role by ordinary people who find themselves caught up in extraordinary events (Greer \& McLaughlin $2010 \mathrm{~b}$ ) and who use the tools of modern technology and the distribution capacity of the Internet to create media (Bowman \& Willis 2003; Lievrouw 2011). Various scholars have documented the development of a citizen journalist proclivity (an instinctive cultural response to, not only observe, but tangibly document, through video recording, unfolding events within view) across Western societies (e.g., see Doyle 2006a; Lyon 2006; Lee \& McGovern 2014; McGrath 2012; Trottier 2015), including "the general inclination to film the police" (Wall \& Linnemann 2014: 134). In this regard, Meyer (2015) suggests that by filming police, "unknown cameramen and women lived out high democratic ideals" through this "mode of bearing witness."

${ }^{11}$ See also, Antony \& Thomas 2010; Atton 2002; Benkler 2006; Bock 2016; Brighenti 2010a; Brown 2013, 2016; Brucato 2015a, 2015b, 2016; Campeau 2015; Goldsmith 2010; Haight, Quan-Haase \& Corbett 2014; Loader \& Sparks 2015; Roche 2017; Sandhu \& Haggerty 2015, 2017; Shjarback et al. 2017; Thompson 2005; Wallace et al. 2018; Wilson \& Serisier 2010.

${ }^{12}$ See also, Antony \& Thomas 2010; Armstrong \& McAra 2003; Bock 2016; Brucato 2015b; Doyle 2011; Goldsmith 2010; Greer \& Reiner 2012; Haggerty \& Sandhu 2014; Hartley 2011; Kahn \& Kellner 2006; Koskela 2011; Jeffries 2011; Li 2009; Newell 2019; Nix \& Wolfe 2016; Pajnik 2015; Sandhu 2016; Sandhu \& Haggerty 2015, 2017; Schneider 2015; Schwartz 2009; Shiffman 2008; Toch 2012; Trottier 2015; Yesil 2011.

${ }^{13}$ In this regard, Goldsmith (2010: 919-920) observed,

Once [an image is] captured, the Internet offers a 'generative' system that takes away a fundamental means of controlling the flow of information and images from those traditionally in charge of broadcasting... [video-sharing 
platforms like YouTube] amplify the significance of mass access to mobile camera phones, providing alternative outlets to official media for mass viewing of [user-created content].

Along these lines, Kahn and Kellner (2006: 704) observe that the Internet, as a medium, "makes more information available to a greater number of people, more easily, and from a wider array of sources, than any instrument of information and communication in history." This ties in with a developing body of literature that elaborates a cultural shift -as relates to communication technologies and the establishment of a 'participatory culture' (e.g., see Atton 2002; Benkler 2006; Bruns 2007, 2008; Burgess \& Green 2009; Castells 2009, 2011, 2012; Deuze, Bruns \& Neuberger 2007; Diamond \& Plattner 2012; Jarvis 2006; Jenkins 2008; Shiffman 2008; Shirky 2008). Jenkins (2008: 331) defines participatory culture as one "in which [ordinary individuals in society] are invited to actively participate in the creation and circulation of new content" and Burgess and Green (2009: 10) advance, "Participatory culture is a term that is often used to talk about the apparent link between more accessible digital technologies, usercreated content, and some kind of shift in the power relations between media industries and their consumers." This work augments other observations documenting the transitioning from a 'viewer society' (Mathiesen 1997) to the current 'producer-viewer' society (e.g., see Bruno 2012; Cascio 2005; Doyle 2011; Goldsmith 2010; McCahill 2012), or, as characterized by Jenkins (2008) 'prosumers' and Bruns (2008) 'produsage.' The latter recognizes "a hybrid user/producer role which inextricably interweaves both forms of participation" (Bruns 2008: 21). YouTube can be considered as exemplifying these observations, as it represents " "the convergence between commercial popular culture and community participation" (Burgess \& Green 2009: 14). Jansson and Lindell (2015) characterize this new social media environment as 'transmedia,' in which multiple platforms, as contrasted to traditional mass media, distribute knowledge and facilitate discussion throughout broad society.

In their sociological analysis of the 2009 fatal police shooting of Oscar Grant, Antony and Thomas (2010: 1290) illuminate the transformative impact of the Web on social activism, specifically, in this case, in relation to police use of force,

The Internet and YouTube [provide] a forum for voices of the oppressed and subjugated. It constitutes a democratic yet subversive platform where the citizen can reclaim power that has been usurped and wielded by corrupt authorities, either through direct physical action, or through technological artifacts. In this sense, these responses express a reversal of the typical Big Brother metaphor that characterizes the relation between state and citizen. Although surveillance technologies are increasingly used to scrutinize the actions of citizens, YouTube and cellphone cameras provide the opportunity to reverse this power dynamic and afford citizens the potential to monitor state agents. 
${ }^{14}$ See also, Bittner 1970; Campeau 2015; Ericson \& Haggerty 1997; Goldsmith \& Lewis 2000; Haggerty \& Sandhu 2014; Jeffries 2011; Marx 1985, 1988; Newburn \& Reiner 2012; Reiner 2010; Van Maanen 1973; Waddington 1999; Wilson 1968.

${ }^{15}$ In 1960, Yale law professor Joseph Goldstein characterized the police work of that era as "generally of extremely low visibility" (Goldstein 1960: 543). In the late 1980s, Marx (1988, as cited in Goold 2004: 205) observed that police were being impacted "by the emergence of new surveillance technologies that have made covert observations both cheap and easy" and then, similarly, Ericson (1989) assessed that the circumstances of police invisibility were shifting and that police were beginning to lose their privileged position to 'patrol the facts.' Six years later he observed, "While many details remain unclear, it seems highly probable that the new capacities for surveillance inherent in these technologies may increase the police's accountability to the public, while decreasing their 'account ability"' (Ericson 1995: 161). Also, in the mid-1990s, Ericson and Haggerty (1997: 56) observed a shift toward greatly-increased police visibility, advancing, "Coincident with the surveillance of suspect citizens is the surveillance of the police as a suspect population. There is probably no occupation as thoroughly scrutinized as the police."

16 Trottier (2015: 211, citing Silverstone \& Haddon 1996) explains that "social media amplify policing not because of their technological sophistication but rather because of their social saturation. Social media's saturation is a result of domestication - that is, the extent to which they are embedded in everyday life." Schneider (2015: 228) contributes, "Social media offer widely accessible public platforms that allow citizen challenges to definitional claims...Police authority has been directly challenged in the past in the form of public protests and demonstrations; however, a key contemporary difference concerns the manner in which social media platforms allow users to mobilize en masse much more quickly, and often around some form of publicly accessible empirical evidence (e.g., videos of police brutality)."

${ }^{17}$ See also, Antony \& Thomas 2010; Bonilla \& Rosa 2015; Brown 2013, 2016; Brucato 2015a, 2015b, 2016; Campeau 2015; Chan 1999; Chermak \& Weiss 2005; Fan 2012; Farmer 2016; Farmer et al. 2015; Goold 2003, 2004; Greer \& McLaughlin 2010a; Haggerty 2006; Haggerty \& Sandhu 2014; Hier 2011; Iacobucci 2014; Jennings et al. 2014; Laut 2012; Lee et al. 2018; Loftus 2009, 2010; Manning 2008a, 2008b; Newell 2019; Norris \& Armstrong 1999; Roche 2017; Sandhu 2016, 2019; Sandhu \& Haggerty 2015, 2017; Schaefer \& Steinmetz 2014; Schneider 2015, 2016; Shjarback et al. 2017; Toch 2012; Todd 2015; Trottier 2015; Victor \& McPhate 2016; Weitzer 2018; Wilson \& Serisier 2010; Wolfe \& Nix 2016.

${ }^{18}$ Goldsmith (2010: 931) observes, "Whereas, by comparison, there was once the occasional, localized performance of police impropriety achieving notoriety, we now can expect more frequent, globalized spectacles of such impropriety." Along these lines, Lee and colleagues (2018: 197, citing Goldsmith 2010; Victor \& McPhate 2016) find, "News coverage of police killings [of citizens] has increased as well, partly because of the advent of video recordings of such events, public protests after such incidents, and 
heated discussions on social media. The result is that police misconduct has experienced an unprecedented 'new visibility'."

${ }^{19}$ Doyle (2006: 218) finds that societal surveillance is "now a more public and collective phenomenon throughout a new type of 'watching public'." In this vein, Bell (2009: 206) observes that citizen's camera phones have "radically transformed the practices of photography, bringing about both a heightened pervasiveness and a heightened intimacy." In this regard, Koskela (2011: 275) advances that "bystanders equipped with camera phones become incidental witnesses in more intensive ways than before. These phones are efficiently linked to information flows, making it quick and easy to circulate images." In contrasting the actions of George Holliday, who video recorded the 1991 Rodney King occurrence out of curiosity (and was then initially unsuccessful in interesting any of the traditional Los Angeles media outlets in the footage), with those of the 'citizen journalism' exemplified by multiple individuals in the 2009 Oscar Grant occurrence, Finn (2012: 77) suggested that the latter is an example that such video recordings are no longer passive actions by citizens, but rather, "this is a conscious and overt decision to record events for public distribution. In this sense, camera surveillance is employed as a form of civic engagement or duty." Koskela (2009) observes that today's media (both traditional and online) are reinforcing citizen's 'camwitnessing' by encouraging people to record newsworthy events and publicizing their amateur video recordings.

${ }^{20}$ See also, Brucato 2015a; Farmer \& Sun 2016; Haggerty \& Sandhu 2014; Intravia et al. 2018; Lee \& McGovern 2013; Maguire et al. 2017; Newell 2019; Sandhu 2016; Sandhu \& Haggerty 2015, 2017; Schneider 2015; Stanko \& Dawson 2015; Toch 2012; Todak 2017.

${ }^{21}$ See also, Bradford et al. 2014; Campeau 2015; Candlin \& Crichton 2013; Chan 1999; Chen 2016; Cockcroft 2013; Doyle 2006a, 2007, 2011; Ericson \& Doyle 2003; Ericson \& Haggerty 1997; Garland 2003; Huggins et al. 2014; Intravia et al. 2018; Jackson, Bradford, Hough, Myhill, Quinton \& Tyler 2012; Lautt 2012; Lievrouw 2011; Loftus 2009; Maguire et al. 2017; Murphy 2012; Reiner 2010; Schneider 2015; Smith 2005; Stenning 2009; Terrill \& Paoline 2017; Todd 2015; Tulloch 2008; Uslaner 2002; Wall \& Linnemann 2014.

${ }^{22}$ In discussing Beck's work and the 'risk society thesis' (as applied to the context of police work in Ericson and Haggerty's Policing the Risk Society), Candlin and Crichton (2013: 1-2) observe, "Issues surrounding trust are foundational to people's lives in contemporary societies," and they suggest a refinement from the 'risk society' characterization to more accurately capture the present 'mistrust society.'

${ }^{23}$ The onset of risk as an important feature of contemporary culture has presented in the population as a sense of "considerable anxiety" that is related to "increased social expectations, decreased levels of trust, and new sources of insecurity" (Garland 2003: 70-71). The declining trust of experts, characteristic of 'risk society,' has consequences for public trust in governments, large-scale organizations, and major social institutions - 
including the police and traditional media (Beck 1992a, 1992b, 2009; Birchall 2011b; Doyle 2007; Garland 2003; Giddens 1990, 1994; Lievrouw 2011). Doyle (2007: 10) finds that a broad skepticism is pervasive throughout society, "including a distrust of those providing security at a time when a yearning for security is profound." In juxtaposing the works of Beck (2009) and Mathiesen (2007), Doyle (2011: 292) advances, "Rather than a passive public seduced by messages from 'the few', we have an anxious, questioning one, not knowing whom to trust."

Along these lines, Thompson (1995: 147) suggests that one of the consequences of increased visibility may be to "nourish the suspicion and cynicism" which much of the populace feel towards politics and established state institutions. In the same vein, Doyle (2006, 2007, 2011) finds that an unprecedented level of higher education and access to knowledge through the Internet, while adding to the climate of perpetual doubt, has resulted in a public that thinks more critically and is skeptical of major social institutions. Castells $(2009,2010,2011,2012)$ finds similarly in his analysis of the reconfiguration of social and institutional relations in our contemporary "network society.' In addressing the shift to the present "highly reflexive, low trust culture" (Garland 2003: 78) and the heightened social engagement and activism of today, Castells (2012: 2-3) found both to have been "provoked by the cynicism and arrogance of those in power, be it financial, political or cultural..."

${ }^{24}$ In the U.K., Newburn and Reiner (2012: 829) find, “The [earlier] process of growing acceptance of the police in Britain has been reversed... corruption and miscarriage of justice scandals... accusations of race discrimination...[and] decreasing public accountability... have plunged them into acute controversy and conflict.” Hough (2007) observes that $43 \%$ of British citizens in 1982 assessed that the police did 'a very good job,' whereas in 2002 that figure had dropped to just 14\%. In Canada, polling by Ipsos Reid found that the proportion of Canadians that have trust in police has decreased from 73\% in 2003 to 57\% in 2011, while a similar poll by Angus Reid in 2012 discovered that only $39 \%$ of Canadians have 'high confidence' in municipal police agencies (Ottawa Police Service 2012). Gallagher and colleagues (2001) discovered that from the mid-1960s to the end of the twentieth century positive views of the police have been steadily declining throughout the USA and the percentage of Americans who perceive that police brutality exits in their locale has increased threefold, now amounting to more than a third of the populace. In 2015, American's confidence in their police reached a twenty-two-year low (Jones 2015). On the other hand, and in relation to the other constituent in the public-police relationship, Cordner (2017) finds that only 58\% of American police officers believe that the citizenry respects the police.

${ }^{25}$ Various scholars (e.g., see Antony \& Thomas 2010; Barak 1994; Chibnall 1997; Doyle 2003, 2006; Doyle \& Ericson 2004; Ericson 1989, 1995; Gitlin 1980; Goldsmith 2010; Greer \& McLaughlin 2010; Hall et al. 1978; Hirschfield \& Simon 2010; Jewkes 2004; Lee \& McGovern 2014; Loader 1997; Martin 2005; Mawby 2002; McLaughlin 2007; Reiner 2000a, 2010; Schlesinger \& Tumber 1994; Surette 2011) have written about the deference of traditional media to the police and the existence of an essentially symbiotic relationship between police and media, which meant that historically police 
were better able to control the kind of information that was available in the public milieu and, until recently, how previously-infrequent video recorded imagery of questionable police actions in the field was framed - for subsequent interpretation by the public audience. This is important because most citizens never have contact with police officers in any 'meaningful' (or particularly adversarial) way (Engel 2005; Gallagher et al. 2001; Rosenbaum et al. 2005; Surette 2011; Weitzer \& Tuch 2004, 2005a) and, therefore, as Lee and colleagues (2018: 197, citing Iyengar \& Kinder 1987; McCombs \& Shaw 1972) argue, "Since most people have limited direct contact with police officers, information about the police comes largely from the media... The public does not necessarily adopt the news media's version of reality, but by setting the agenda for what is defined as news and selectively presenting content, the news media strongly influences public perceptions of events and issues."

Until recently, and along these lines, police were most often the primary controllers, and definers, of information related to their activities (Chagnon, Chesney-Lind \& Johnson 2018; Chan 1999; Chermak, McGarrell \& Gruenewald 2006; Doyle 2003; Ericson 1982, 1995; Ericson, Baranek \& Chan 1989, 1991; Greer \& McLaughlin 2010b; Hall, Critcher, Jefferson, Clarke \& Roberts 1978; Hirschfield \& Simon 2010; Lawrence 2000; McLaughlin 2007; Schneider 2015; Thompson \& Lee 2004; Tuchman 1978). In this regard, in the late 1990s Doyle (2003: 133-135) found that even with increasing availability of civilian footage of policing occurrences, television's broadcasting of video recordings of police activities in the field "often strengthened the position of those who already [held] the upper hand...TV does change things, but in ways tailored by police and similar dominant players. The medium is thus not simply the message...police most often controlled which criminal justice situations were broadcast...[And] police most often [gave] the authorized definition to these televised situations." Similarly, Chagnon and colleagues (2018: 172) conclude that, through their traditional relationship with media organizations, police were, until recently, able to "construct the issue of crime" and to, almost always, "portray the police in a positive light."

However, Reiner (2010), writing with the last decade, documented increasingly critical reporting on police matters, with reporters assiduously pursuing stories of police misconduct. In this vein, he notes that the traditional location of police misconduct within the 'one bad apple' framework has been difficult to sustain with the proliferation of scandalous policing occurrences and he observes, "The metaphor increasingly became the 'rotten barrel,' and in the late 1990s the 'poisoned trees,' from which the bad apples' came" (Reiner 2010: 181, citing Punch 2003). Further, as Schneider (2015: 241-242) observes in his more recent contribution,

Police are increasingly less able to control information that might cast officers or the institutions in an unfavourable light (e.g., citizen accusations and user-generated videos of misconduct, brutality and other forms of police deviance)...YouTube (and other social media sites) increasingly subject police work to immediate public monitoring, spotlighting brutality, misconduct and the use of questionable tactics, 
things that would have otherwise gone entirely unnoticed prior to the last decade.

${ }^{26}$ These considerations can be related to another important consideration in appreciating the historical context of front-line police actions in the field and the relative absence of public attention, concern, and criticism - the 'complex politics of interpretation' (Doyle 2003) that is engaged by all of us through our experiences, understandings, assumptions, and biases in arriving at understandings around contentious videorecorded police activities. In this regard, Doyle (2003: 72-75) finds that "surveillance cameras do not simply 'make visible' what they record; instead, those who have the power to interpret the images - who produce the authorized definition of the situation are the ones who hold the upper hand." Along these lines, Gitlin's analysis of the televised coverage of the 1968 'Chicago Police riots' is informative. He found that despite protesters' pronouncements that 'the whole world [was] watching [the misconduct of the police officers], "viewers drew wildly different conclusions from the Chicago coverage; antiwar and movement sympathizers were everlastingly horrified by the police violence, while the [political] right accused the networks of bias and rallied to the side of the police... [In other words,] polarized audiences interpreted what they saw on the screen in polar ways" (Gitlin 1980: 196). The combination of traditional media's bias in favour of the police; the technological limitations of earlier video recording devices; and 'official' constructions originating from within the policing institution (suggesting the 'proper' interpretation, contradicting others, and questioning the accuracy of the purported video recorded representation of events), often, in all but the clearest of circumstances, resulted in confusion across the public milieu as to the reality of the situation.

I argue that the infamous 1991 Rodney King occurrence is an example of this complex interpretation process. After the eventual public distribution of Holliday's videorecorded account of the event, there were discordant opinions across society about the actions of both King and police - most centering on the im/propriety of the force used by the involved officers. The meaning of the event was contested across American (and international) public audiences - as evidenced in the acquittal, by a unanimous jury verdict, of the four officers criminally charged with assaulting King and then the subsequent conviction of two of the same officers, for violating King's constitutional rights, in the unanimous verdict of a different jury. Like eyewitness testimony, video has a subjective aspect to it (Gonzales \& Cochran 2017; Mateescu et al. 2016; Otu 2016; Philips 2016), in that people with different life experiences can interpret what happened in different ways. In this regard, Mateescu and colleagues (2016:124) find that "[video] footage is only meaningful through a process of interpretation..."

Along the same lines, Sandhu and Haggerty (2015: 10), conclude,

The Rodney King case starkly demonstrated that there are no 'self-evident' images of police abuse; all such videos are open to interpretation. A video that might seem to one audience an unmistakable example of excessive force can look entirely different to an audience comprising people from a different racial or class background...videos will instead tend to confirm 
and reinforce people's existing inclinations or only minimally alter their perceptions.

Yesil (2011: 285) observes, "Camera phones...are generally conceptualized as instruments that we can believe in as neutral recorders of truth and reality and stand as symbols of neutral vision and transparency mostly because they serve as 'nonhuman witnesses' in the sense that human capacities are irrelevant to their operation. As such these devices have begun to occupy a central position within the matrix of visual documentation and the construction of truth and reality." However, as described in the preceding paragraph, despite this 'seeing is believing' property often ascribed to today's video recording, in reality the 'truth' of what people are seeing in their interpretations of the footage is often contested. It is important to appreciate that recorded imagery is not 'pure vision' and it does not produce neutral knowledge; rather, all observations are influenced by a variety of assumptions, experiences, and worldviews that each viewer engages with in making sense of what they are observing and in arriving at the ultimate meaning they will assign to the event - their socially-constructed perception of reality (Thompson 1995, 2005; Doyle 2003, 2011). In this vein, Doyle (2011: 293) advances, "People are not simply blank slates on which the synoptic media impose a message...the cultural toolkit one draws on in making...interpretations [is] crucial.” Readers should appreciate that each individual develops, through a life-long process of inculcation, a set of understandings - "a kind of implicit background knowledge" that functions as a framework for the interpretation of information (Thompson 1995: 41). Diverse political, cultural, social, and ideological considerations influence how each of us views (and interprets) events as represented in recorded imagery. This is consistent with the concept of 'confirmation bias' - the "tendencies to interpret, seek, and create information in ways that verify existing beliefs" (Brehm et al. 2002: 119).

In this vein, Newell (2019: 72, citing Boivin et al. 2017) observes, “...various studies empirically support concerns that the interpretation of video footage is subject to differing perspectives and forms of perspective bias." In general terms, communications research has demonstrated that human beings interpret information that they receive in ways that support existing beliefs and understandings and therefore they are more likely to recall such information than disconfirming information that challenges their stereotypes (Rosenbaum et al. 2005; Stone et al. 1997). In other words, as we all know from our everyday experiences, other individuals can, and do, see things much differently than we do when considering the same source of information and they are as convinced in their interpretation of the 'truth' of what they are seeing as we are of our understanding of the 'truth.' Goodwin (1994: 606) advances that "the ability to see a meaningful event is not a transparent psychological process but instead a sociallysituated activity accomplished through the deployment of a range of historically constituted discursive practices." In the specific context of policing, Weitzer (2015: 476, citing Weitzer \& Tuch 2006) finds,

Whether politicized or not, incidents of apparent police misconduct gain added significance when they resonate with other factors that condition individuals' perceptions of and experiences with the police...People are 
influenced by their 'vicarious experiences'; the narratives of friends, family members, neighbours, or remote others (as portrayed in the media) that are indirectly experienced by an individual. [As such,] Latinos and African Americans are much more likely than Whites to hear about instances of officer mistreatment from people in their social networks and to internalize these experiences.

${ }^{27}$ It is well documented across the literature that there has been a significant increase in media (both traditional and social) coverage of police actions in the field - much of this resulting from the widespread availability of bystander video recordings (e.g., see Bonilla \& Rosa 2015; Brown 2016; Farmer \& Sun 2016; Goldsmith 2010; Intravia et al. 2018; Newell 2019; Schneider 2015). However, as Bayley and Garafalo (1989) have observed, occurrences involving police use of force in their interactions with citizens are 'quite rare' and as Weitzer (2015: 479) counsels,

We should expect a growing public perception that misconduct is dramatically increasing, even if this is simply an artifact of greater reporting of altercations, including footage from video recordings. The increasing display of visual images gives the impression, as one woman at a protest in Baltimore exclaimed, that police brutality is a 'skyrocketing epidemic.' Scholars would challenge this claim by pointing out that misconduct was simply more hidden, and more prevalent, in the twentieth century.

For example, Barron (2018: 224-226, citing Brown 2016) finds that in an environment of "rapidly growing criticism of Western police forces...[this] criticism may have less to do with changing police behaviour than with perceptual and awareness-related factors such as how social media facilitates the outreach of advocacy groups critical of the police and aids in the mobilization of protest."

28 Trust is always associated with expectations about the behaviour of others and is influenced by social forces that shape both who trusts and who is seen as trustworthy (Candlin \& Crichton 2013; Giddens 1991; Hawdon 2008; Luhmann 1979). An illustration of contemporary challenges in citizens' trust of police is afforded by the recent situation with the rescinding of a Toronto Police Service (TPS) informationgathering technique known as 'carding' (otherwise known in various other jurisdictions as 'street checks' or 'person checks'). This practice (that is widespread throughout Canadian and American policing and has been for many years) involves in-the-field recording of information emerging out of an interaction between an officer and a citizen, under conditions that the officer determines merits the documentation of that person's identity (and their location, physical descriptors, vehicle, associates, circumstances, etc.), and the subsequent submission of that information to a police database. Historically, TPS Chief Mark Saunders supported 'carding' as a valuable police intelligence practice, which has solved crimes and is "keeping serious crime in check" (CBC News 2015b). However, culminating in 2015, 'carding' came under intensive criticism from some Toronto residents, the editorial staff at The Toronto Star, 
and some 30 prominent current and former politicians, civil servants, and community leaders, who advanced that the practice was discriminatory (in that it often amounted to 'racial profiling' by TPS officers of Black citizens) and was damaging the public's trust in the TPS (CBC News 2015a). Ultimately, both Toronto Mayor John Tory and Toronto Police Service Board Chair Alok Mukhergee reversed their previously-held positions on the practice and supported the prohibition of 'carding' in order to, as characterized by Tory, reverse the erosion of the publics' trust in the TPS (CBC News 2015b).

While in Toronto in August 2015, I listened to radio news programming discussing this controversial policing issue and featuring, as a contributor, popular national media columnist Christie Blatchford. Blatchford (2015a), in her companion print commentary, argued that 'carding' was being removed from the police intelligence-gathering arsenal for purely political reasons, in response to militant anti-police sentiments from some elements of society and in spite of the practice's proven successes - including being primarily responsible for the resolution of the notorious 2003 child kidnapping and murder of Cecilia Zhang and many other serious crimes throughout the greater Toronto area. In her radio contribution, Blatchford wondered aloud about how the police will respond to 'being handcuffed' through the outlawing of 'carding,' to incessant public pressure around other aspects of policing, and to persistent public demands for more and more accountability, transparency, and oversight. She challenged listeners to think about how police rank-and-file might react to the demands of today's public and the risk-averse model of policing (and individual officer's practices) that might become 'the self-preservation strategy' of front-line officers. She asked, 'Is this what we really want from our police?'

On 28 October 2015 the government of Ontario introduced regulations that came into force on 1 January 2017, which prohibit police officers across the province from stopping persons and gathering information from them absent an officer presenting a reasonable and articulable rationale for doing so (Taber 2015).

The first [legislated modification] is known as Ontario Regulation 58/16, under the Police Services Act entitled 'Collection of Identifying Information in Certain Circumstances - Prohibition and Duties,' commonly referred to as the 'Street Checks Regulation'. The second...amends the Code of Conduct under Ontario Regulation 268/10 of the Police Services Act, and provides a code of conduct violation where a police officer is found not to comply with Ontario Regulation 58/16... Ontario Regulation 58/16 provides for voluntary police-public interactions, which are designed to ensure that the regulated interactions are without bias or discrimination... The regulation applies when police are attempting to collect identifying information from an individual during 'face-to-face' encounters while: [1] investigating general criminal activity in a community; [2] inquiring into suspicious activities to detect offences; [and 3] gathering information for intelligence purposes (Bordeleau 2018: 1-2). 
As a result, the volume of officer-initiated 'street checks' ('carding') across Ontario has plummeted from traditional levels - with many officers apparently perceiving that the risk of professional consequences to them is not worth engaging in the proactive intelligence-gathering practice under the new regulations and accompanying scrutiny (some examples of reductions in 'street checks' are presented in Chapter Five).

In the American context, this discussion would implicate what are commonly referred to as 'Terry stops.' In a 1968 ruling in Terry v. Ohio, the United States Supreme Court affirmed that police officers possess the constitutional authority to stop and search individuals based on "specific reasonable inferences [the officer] is entitled to draw from the facts in light of [the officer's] experience" (392 U.S. 1 [1968]: 27).

${ }^{29}$ See also, Adams 2015; Nix \& Pickett 2017; Nix \& Wolfe 2016, 2017; Nix et al. 2018; Pickering \& Klinger 2016; Pyrooz et al. 2016; Rushin \& Edwards 2017; Shjarback et al. 2017; Silver, Roche, Bilach \& Ryon 2017; Terrill \& Paoline 2017; Wallace et al. 2018; Weitzer 2015; Wolfe \& Nix 2016.

${ }^{30}$ I suggest that it is more appropriate to address the Brown occurrence in terms of the fallout from the event rather than the event itself, because it is important to recall that Ferguson Police officer Darren Wilson was not convicted of any wrongdoing in his interaction with Brown. A comprehensive DOJ investigation (the complete report is available at https:/www. justice.gov/sites/default/files/opa/press-releases/attachments/ 2015/03/04/doj_report_on_shooting_of_michael_brown_1.pdf) found that Brown (a 6' 5, 289 pound, 18-year-old male) had attacked Wilson (a 6’4, 210 pound, 28-year-old male) as the officer was seated in his police cruiser, had attempted to forcibly take possession of the officer's handgun, and then even after being shot several times had charged at the officer in a violent rage (Harris \& Pettersson 2014). In declining to prosecute Wilson, the DOJ assessed that the officer's actions were reasonable within the legal context of self-defence and, therefore, were not unlawful.

${ }^{31}$ See also, Brown 2016; Brucato 2015a; Deuchar et al. 2018; Loader \& Sparks 2015; Maguire et al. 2017; Marier \& Moule 2018; MacDonald 2015, 2016; Nix et al. 2017; Nix \& Pickett 2017; Nix \& Wolfe 2017, 2018; Pyrooz et al. 2016; Rosenbaum 2015; Shjarback et al. 2017a, 2017b; Terrill \& Paoline 2017; Torres et al. 2018; Walker 2005; Weitzer 2015, 2018; Wolfe \& Nix 2016.

${ }^{32}$ Kutner (2016: 1) assesses that "the tension between law enforcement and large swaths of citizens has not been this high since the 1960s and 1970s... Many Americans feel the country's 18,000 police departments need major reform, especially when it comes to the use of force." In this vein, Bishopp, Piquero, Worrall \& Piquero (2018, citing Brown 2016; Chermak, McGarrell \& Gruenewald 2006; Graziano, Schuck \& Martin 2010) observe, "To be sure, stress in policing is not a new phenomenon, but politicians, citizens, and particularly the media have placed police under greater scrutiny and pressure to change." Similarly, Weitzer (2015: 475) assessed, in 2015, that "policing in America could not be a hotter topic that it is now." 
In a historical context, in an article written a half a century ago, which addressed prevailing challenges in relations between American police officers and some citizens in the latter half of the 1960s, Reiss (1968: 10) included excerpts from a 1903 article written by Frank Moss, then the New York City police commissioner, to illustrate that, while the topic of challenges in the public-police relationship have various precedents, "...never before has the issue... assumed the public urgency it has today [in 1968]." With rioting a frequent occurrence in several American cities, in response to "deep hostility between police and ghetto communities... [featuring] abrasive relationships between police and Negroes and other minority groups..[and an] increasingly bitter social debate over law enforcement" and the unprecedented exposure of a vast public audience to the 'Chicago Police riots,' few could take exception to the eminent Yale sociologist's assessment of the policing landscape of that era (National Advisory Commission on Civil Disorders 1968: 157). Now, Weitzer (2018: 29) finds that public confidence in police has been "rattled' and that the present situation is a "unique moment in American history [that is] surpassing the level of popular alarm that followed some high-profile policing incidents in the twentieth century" (Weitzer 2018: 29).

${ }^{33}$ While I stand to be corrected, my review of the literature suggests that presidential tasks forces into policing are rare. The President's Commission on Law Enforcement and Administration of Justice, ordered by President Lyndon Johnson, began its work in 1965 and issued reports in 1967. Until the most recent President's Task Force on $21^{\text {st }}$ Century Policing in 2015 none others were empanelled.

${ }^{34}$ While an extensive discussion is beyond the scope of this dissertation, it should be understood that the socio-political circumstances in the current public-police relationship is, as this chapter has sought to demonstrate, more of a multi-faceted sociological phenomenon than that which is related only to the 'new visibility' of police conduct in the field. In this regard, Davis (2015) observes, "One U.S. Department of Justice official noted that the protests and other events that occurred in and around Ferguson after the shooting of Michael Brown helped to launch 'a new civil rights movement' in the U.S." In a discussion I had with Dr. Cedric Alexander (personal communication, 4 October 2016), Director of Public Safety for Dekalb County, Georgia (serving a population of approximately 750,000 bordering, to the east, on the City of Atlanta), former Chief of Police for Rochester (New York), and frequent expert commentator on policing for cable news network $C N N$, he observed,

This is more than just Black Lives Matter, look at who the protesters are, you see more young Whites than young Blacks most of the time...Look at the demographics of the protesters, in all of these cities, Chicago, Baltimore, New York, the demographics of protesters is just like society, you had protests in Berkley, with a bunch of young White liberals...Even your typical working class mainstream are aware of it, that it's problematic, they may not be as boisterous or protest but they are aware there are serious issues in American policing... There are just a lot more people who are a lot more sensitized to these social issues with policing; everyday common 
laypeople, Hispanics, Blacks, Whites, women, gays, middle class, upper middle class. The only people that are not looking at how police do their work are the old-school conservatives. I think this is more than just police work being visible now, it is definitely a social movement. For the first time Americans across the country are seeing some of the issues with how they are policed, and they are responding by getting involved in questioning what is going on.

During a 2016 television program (Context with Lorna Dueck - in an episode titled 'Faith in the Police') that I appeared on as a guest discussant with Gerry McNeilly, the Director of Ontario's Office of Independent Police Review (one of two civilian agencies conducting overight of police in the province), he suggested that Ontario's public today has "diminished trust and confidence" in police and McNeilly observed further that, augmenting local policing scandals in various Canadian communities, Canadians are subject to the penetration of American policing controversies (through American television programs and news productions) into Canadian households and the consciousness of the Canadian public audience. In responding to a question from the show's host about 'the kind of effect' that has been observed in Canada with the aftermath of the 'major backlash against the police in the United States since Black Lives Matter,' McNeilly observed, 'Overall, it has created a sense of, in members of the public, that maybe there is something going on with the trust and confidence we should have with the police...People are paying attention to what they are seeing on TV and what they are reading in the papers, in the media, and as a result it is diminishing some trust and confidence in the police generally."

${ }^{35}$ Various other scholars have encouraged micro-sociological investigations into the implications of techno-social and socio-political developments for policing (e.g., see Andrejevic 2007; Ball et al. 2012; Goold 2004; Haggerty 2006; Haggerty \& Sandhu 2014; Koskela 2003, 2006, 2011; Kurasawa 2008; Lyon 2007; Mawby 2010; McCahill \& Norris 2002; Neyland \& Coopmans 2014; Sandhu 2016, 2019; Sandhu \& Haggerty 2017; Thompson 2005).

${ }^{36}$ Given the limited scope of this dissertation, I focused on what I assessed is the most relevant theoretical framework (front-line police occupational subculture) to inform this study's findings. Although, in saying this, I also considered that certain aspects of both social learning theory (SLT) and general strain theory (GST) could have potential in contributing to understandings of police cultural socialization and risk-averse behavioural practices (perhaps in future research or publications). I present (below) a concise snapshot of these two theoretical frameworks, which I encourage the reader to consider within this dissertation's discussion of the cultural socialization process and the practice of de-policing, as there is, in some respects, interrelated conceptual applicability. 


\section{Social Learning Theory (Differential Association)}

Akers' $(1968,1998)$ social learning theory (SLT) has been applied to research into behaviours and culture in a number of social scientific studies. This is an adaptation of Sutherland's (1947) sociologically-oriented differential association theory, which has been instrumental to understanding deviant and conforming behaviours across various research populations. The key premise of SLT is that the same learning processes can produce both types of behaviours (Akers, Krohn, Lanza-Kaduce \& Radosevich 1979). As Akers (1998: 328) argues, "Conforming and deviant behaviour are learned in the same way...[and] the general process is the same for both." In this regard, Akers (1968, 1998) finds that four variables influence attitudes toward social conduct - differential association, definitions, reinforcement, and modeling. Of these, differential association functions as the primary variable within SLT and relates to influence on the individual from those with whom one associates frequently (Akers 1968, 1998). In the context of police work, the police culture is the mechanism through which an officer learns fundamental values and norms of the occupation, including what is considered to be acceptable behaviour - which can be conduct that others (society, police management, political entities, oversight agencies, etc.) could understand as consistent with expectations or, alternatively, as misconduct (Chappell \& Piquero 2004; Kappeler et al. 1999, 2015). Burgess and Akers (1966) find that an officer's peer group presents as a strong influence as to what behaviour is acceptable or unacceptable within the prevailing occupational culture (regardless of the views of others external to that cultural entity).

While Getty and colleagues (2016: 827) observe, "To date, Chappell and Piquero (2004) are the only researchers to have formally applied Akers' social learning theory (SLT) to police deviance," I find (from my review of the literature in 2019) that a handful of other policing studies have incorporated SLT as a theoretical framework in some respect (e.g., Cochran, Maskaly, Jones \& Sellers 2017; Harris 2010; Maskaly \& Donner 2015). In this regard, I submit that it is interesting to deliberate on whether this study's findings around the practice of de-policing could be characterized as 'deviant' or 'conforming' behaviour by front-line officers. I suspect that subjective determination would very much depend on the perspective of the individual making the assessment. Some might suggest that this is reasonable 'self-preservation' behaviour (Muir 1977; Nix et al. 2018; Oliver 2017; Paoline 2003, 2004; Smith \& Alpert 2002) that is entirely understandable within the current techno-social and socio-political policing environment. Others, however, might view the abdication of the responsibility to engage in proactive policing (Broderick 1977; Brown 1988; Goldstein 1977; Van Maanen 1978) as indisputably aberrant misconduct.

\section{General Strain Theory (GST)}

General strain theory (GST), which was reformulated and expanded by Agnew (1985, 1992, 2001, 2006) from Durkheim's original work around anomie at the societal level and then Merton's refinements to the individual context, has been applied most frequently to understanding delinquent behaviours in juveniles and in relation to "the 
impact of social and systemic pressures and strains in affecting group and individual behaviours and reaction" (Arter 2007: 43). Strain refers to events or conditions found by individuals to be unpleasant (Agnew 2006; Bruhn \& Wolf 1986; Gibson, Swatt and Jolicoeur 2001; Selye 1974) and, more specifically, GST advances that there are three circumstances in which strain can manifest at the individual level - (1) the person fails to achieve positively-valued goals, (2) positively-valued stimuli have been removed from the individual, or (3), negatively-valued stimuli are presented to the individual (Agnew 1992: 51-59). In this vein, Selye (1974) found that strain in the work environment occurs when individuals are blocked from realizing psychological rewards, while undeserved criticism was identified by Terry (1981) as a major stressor encountered in police work.

Such sources of perceived strain can result in negative emotions and, in response, some individuals will employ coping strategies in attempting to moderate strain. It is theorized that maladaptive coping behaviours (i.e. delinquent, criminal, or deviant actions) can be offset by conditioning (or mediating) variables, such as, for example, certain personality traits, social supports, and/or high self-esteem. Bishopp and colleagues (2018, citing Mazerolle \& Piquero 1997, 1998; Paternoster \& Mazerolle 1994) observe, “Unlike Merton's original strain theory, Agnew's (1992) general strain theory offers an individual-level explanation for deviant conduct that is applicable to a diverse range of behaviours." As Yun and Lee (2015: 194) observe, GST has been extended, through several studies, into the adult realm in exploring crime and deviance and "although originally developed to explain the phenomena of [youth] crime and delinquency, Agnew (1992) posits that GST is a general theory that can be applied to other forms of maladaptive behaviours adopted by individuals in an attempt to cope with felt strain." These scholars further find that "there has been limited attempts to apply GST's framework to unique samples such as police officers" (Yun \& Lee 2015: 194). In this regard, Arter (2007: 46, citing Bruhn \& Wolf 1986; Seyle 1974; Terry 1981) observes, "Everyone (including police officers) attempts to achieve certain psychological rewards from the work environment. Stress results when individuals do not realize psychological rewards in the workplace, or when the rewards are removed (or threatened to be removed) from the worker's immediate enjoyment...maladaptive behavioural responses may be implemented in attempts to mediate experiential stressors."

In my review of the literature (in 2019), I found that the following studies employed GST, at least in part, in theorizing police behaviours (addressing issues such as organizational commitment, turnover intention, domestic violence, abusive behaviours, problematic alcohol use, suicide ideation): Bishopp and Boots 2014; Bishopp, Worrall and Piquero 2016; Bishopp, Worrall, Piquero and Piquero 2018; Gibson et al. 2001; Moon and Jonson 2012; Piquero 2005; Nix et al. 2018; Shim, Jo and Hoover 2015; and Swatt, Gibson and Piquero 2007). In assessing the extant literature, Bishop and colleagues (2018) found "notable gaps," which they argue are problematic in relying on GST to theorize police 'maladaptive' conduct. In my own deliberations, I found that GST's foundational requirement for analysis of mediating variables to be challenging (in the context of the present study), however the general tenets of the theory are 
instructive in considering front-line police occupational culture. Along these lines, the present study assesses whether (or not) the rank-and-file are responding to anxieties, emerging as a result of challenges presented in today's techno-social and socio-political policing landscape, by developing risk aversion that manifests in the practice of depolicing. Perhaps these anxieties could be considered as synonymous with strain and I suspect that some would suggest that the practice of de-policing would qualify as a maladaptive coping behaviour, particularly given the fundamental understanding that officers are to perform other tasks than simply responding to dispatched calls in their mandate to serve and protect. In this regard, Nix and colleagues (2018: 38, citing Agnew 1992) advance "Consistent with general strain theory...the war on cops can be viewed as a psychological strain experienced by officers who perceive it to be a reality. According to this framework, such officers likely search for ways to cope with the frustration or fear of a perceived war on cops. De-policing can be conceptualized as one such coping mechanism."

${ }^{37}$ As communication theorists Scollon, Scollon and Jones (2011: 3) find,

The biggest problem with the word culture is that nobody seems to know exactly what it means, or rather, that it means very different things to different people...Some consider culture something people think, a set of beliefs or values or mental patterns that people in a particular group share. Still others regard culture more like a set of rules that people follow, rather like the rules of game, which they can either conform to or break, and others think of it as a set of largely unconscious habits that govern people's behaviour without them fully realizing it. It is best, then to think of culture not as one thing or another, not as a thing at all, but rather as a heuristic. A heuristic is a 'tool for thinking.' ...Perhaps the best definition of culture we can settle on for now, is that culture is a way of dividing people up into groups according to some feature of these people which helps us to understand something about them and how they are different from or similar to other people.

In the policing context, Bacon (2014: 103) observes, "Despite its explanatory power, extensive usage and influence, the concept of police culture remains somewhat complicated, contested and, at times, contradictory." Similarly, Westmarland (2008: 253) suggests, "Police culture, or cultures, as many authors have commented, is difficult to define. Despite this difficulty, police culture, cultures or what is sometimes termed 'cop' or 'canteen' culture is one of the few 'academic' terms from police studies that has entered common parlance." Paoline, in the 2014 book-length analysis of police culture he co-authored with Terrill, introduced their Chapter One by explaining,

I fully understood the inherent abstract nature of concepts. In some ways, this prepared me for what I was to experience in choosing and conducting a doctoral dissertation on police culture...At the time, like many criminologists, I viewed policing as a theoretically void applied area...Following several meetings with my dissertation chair, I ultimately 
decided that I would conduct a study on police culture. In my estimation, this was the closest that I could get to my criminology roots, as culture is a prominent feature of several theories of crime. Unfortunately, when I started to conduct a review of the literature, I could not locate the theoretical assumptions, propositions, or the causal model for police culture. I quickly realized that unlike strain (Merton 1938), social disorganization (Shaw \& McKay 1942) and culture conflict (Sellin 1938) theories, a nice and tidy clear-cut foundation, with a path of empirical tests and theoretical revisions, was not available...For more than six months, I read everything and anything that mentioned police culture. This is when I fully realized the lack of cohesion and the various disparate conceptualizations (Paoline \& Terrill 2014: 3-4).

${ }^{38}$ See also, Banton 1964; Bittner 1970; Blum 2000; Brodeur 2010; Campeau 2015; Chan, Devery \& Doran 2003; Cockcroft 2013; Crank 1998, 2014; Durivage et al. 1992; Ericson 1982; Loftus 2010; MacAlister 2004; Manning 1977, 1978, 1989, 2007; Marier \& Moule 2018; Murphy \& McKenna 2007; Myhill \& Bradford 2013; Niederhoffer 1967; Paoline 2000, 2001, 2003, 2004; Paoline \& Gau 2018; Paoline, Myers \& Worden 2000; Paoline \& Terrill 2014; Regoli 1977; Reiner 2017; Silver et al. 2017; Skolnick 1966, 1996, 2011; Terrill et al. 2016; Van Maanen 1973, 1974, 1978, 1984; Van Maanen \& Barley 1982; Westley 1970; Wilson 1969.

${ }^{39}$ Reiner (2017: 238) presents an informative synopsis of early works in this regard and an important analysis around the intertwined relationship between police culture, police practice, and the external influences that impact on both,

The social structural sources of police occupational perspectives is...evident, for example, in Banton's comparison of UK and US policing (Banton 1964), Bittner's consideration of the specificities of skid-row peace-keeping (Bittner 1967), Wilson's 'varieties of police behaviour' (Wilson 1968), Cain's rural-urban contrast (Cain 1973), Manning's study of Anglo-America police work (Manning 1977), Punch's analyses of small-town Essex versus the Amsterdam inner-city (Punch 1979), and Holdaway's seminal ethnography (Holdaway 1983, 1989). The culture of police is thus not a primary cause of police practice, for good, ill or both. Cultural perspectives are mutually interdependent with practice, and structural pressures shape them both.

${ }^{40}$ See also, Barker 1999; Brown 1988; Campeau 2015; Crank 1997, 2014; Drummond 1976; Ericson 1989; Farkas \& Manning 1997; Fielding 1994; Fielding \& Fielding 1991Goldsmith 1990; Herbert 1996, 1998, 2001; Kappeler, Sluder \& Alpert 1998; Klockars 1985; Loader \& Mulcahy 2003; McNamara 1967; Niederhoffer 1967; Paoline 2003; Regoli, Crank \& Rivera 1990; Rubinstein 1973; Rumbaut \& Bittner 1979;

Skolnick 1966, 2008; Skolnick \& Fyfe 1993; Terrill et al. 2003; Van Maanen 1974; Waddington 1999; Westley 1970. 
${ }^{41}$ Some discussions across the literature contrast the competing constructs of socialization and predisposition (or selection). The socialization hypothesis advances that similar behaviours among police officers are the result of the accumulation of common experiences 'on the job'. In other words, it is the customary training, occurrences, and interactions that influences the behaviours of individual officers towards the norms of the subcultural group. On the other hand, the predisposition (or selection) hypothesis advances that it is the values and attitudes that the individual brings into the job that determines subsequent policing behaviours. Put simply, this perspective believes that there is a specific type of individual that is attracted to policing and that these individuals are the types of candidates that are then more likely to be selected in the hiring process because of their conformity to the ideal qualifications being sought by police agencies. In this vein, Boivin and colleagues (2018: 8, citing Paoline \& Terrill 2014) find,

Selection and socialization are two major processes that shape culture and values...The selection hypothesis holds that police officers are different from the start - from the moment they consider police work as a potential career...In that view, individuals who ultimately become police officers are therefore similar at the beginning of their careers. In contrast, the socialization hypothesis suggests that police officers become increasingly similar as a result of training, experience, and being with other police officers or recruits. In a metaphorical sense, this could be seen as a debate about whether police culture is 'innate' or 'acquired'.

The process of police subcultural socialization is considered later within this chapter and throughout Chapter Six and, therefore, this discussion focuses on the idea that many individuals attracted to, and successful in competing for a position within, policing are predisposed to be accepting of the tenets of existing police occupational subculture.

In this regard, Tator and Henry (2006) find that both the methods used to recruit prospective candidates and the subsequent selection processes, throughout the often lengthy and arduous application regimen, both ensure that the prevailing police culture will be sustained. Along these lines, it has traditionally been the case that selection of prospective officers into policing was based on "demonstrated conformity to dominant social norms and values," which are typically those standards common to the working middle class (Kappeler et al. 2010: 265). Further, successful candidates, chosen over other applicants in what are often highly-competitive recruitment and selection phases in pre-employment (that are purposefully designed to screen out individuals with backgrounds, characteristics, and attitudes identified as inconsistent with the soughtafter values and motivations of what is viewed, within the existing institutional order, as those exemplified by the 'ideal police officer'), are then "selected, socialized, and placed into a working environment that instills within them an ideology and shared culture that breed unprecedented conformity to the traditional police norms and values" (Kappeler et al. 2010: 265) (e.g., see Alain \& Grégoire 2008; Catlin \& Maupin 2002; Moon \& Hwang 2004; Oberfield 2012; Phillips, Sobol \& Varano 2010; Raganella \& White 2004; Twersky-Glasner 2005; White, Cooper, Saunders \& Raganella 2010). 
In his scholarship addressing Canadian police subculture, MacAlister (2004: 175, citing Bennett \& Greenstein 1975; Bonifacio 1991; Roberg \& Kuykendall 1993) articulates,

Police subculture may arise as a result of police departments hiring individual possessing personality characteristics that, when combined with like-thinking individuals in the work environment manifests itself as a police subculture. That is, police agencies may simply be hiring individual who are already committed to the norms and values that we have come to associate with police subculture... While the official goal is to find those with desirable characteristics such as 'integrity, honesty, and respect,' the inexplicit and unofficial hiring agenda of police agencies has been to select individuals who are going to fit well into the police culture...It may be that police organizations hire individuals in whom the foundation of a police personality is already present, with this being what draws them to police work.

However, MacAlister (2004: 177, citing Seagrave 1997), after describing the essence of the predisposition thesis, observes that it is "not a widely supported position today...It seems to be readily accepted in the current policing literature that the traits common to police officers do exist; however, they arise through the process of socialization rather than from pre-existing personality characteristics." Van Maanen (1973) had earlier reached the same conclusion, in finding that, while recruits enter their new career with a normative commitment to the police agency and its fundamental principles, no preexisting common personality has been identified. Similarly, Bayley and Mendelsohn (1969), Neiderhoffer (1967), and Stoddard (2006) have all similarly rejected the premise that officers have certain innate characteristics that might predetermine their police behaviour.

${ }^{42}$ Chan (1996, 1997, 1999, 2005, 2007a, 2007b, 2011; see also Chan et al. 2003) is an influential policing scholar and an important contributor to the police occupational culture and police socialization literature and she approaches these topics through a different lens than many other researchers. Given limitations of scope and space, only a concise discussion of her key arguments/findings is presented (below), however, nonetheless, this should be informative to the reader in considering the various perspectives on front-line police subculture moving forward in this dissertation.

In her Australian policing research in the 1990s, Chan $(1996,1997)$ was concerned with discovering the reasons for discriminatory ideas and practices in police work and potential strategies for changing the nature of the relationship between police and minority citizens. Chan argued that existing (primarily 'traditional') conceptions of police culture inhibited understandings as to how to reform these problematic outcomes (O’Neill 2016). On this point, Foster (2003: 199, citing Chan 1996 and Dixon 1997) notes, "Recently, research has begun to acknowledge more fully the heterogenous nature of police culture, and the importance of police officers being active agents in the formation, acceptance or rejection of particular aspects of it." In this regard, Chan 
$(1996,1997,2003)$ argues that 'traditional' and 'pessimistic' representations of police culture assume uniformity rather than accounting for variations in culture and, therefore, they are deterministic and fail to appreciate the agency of police actors in determining whether or not they accept the features of the prevailing occupational culture. Chan (1996: 111) observes, "While the culture may be powerful, it is nevertheless up to individuals to accommodate or resist its influence." Chan $(1996,1997)$ was also concerned that within 'traditional' accounts of police cultural socialization, the 'acculturation process' was understood as strictly one-way - with officers portrayed as manipulated and docile learners.

Chan's reconceptualization of cultural practice was facilitated through a Bourdieuian theoretical framework, based on relational theory (see Bourdieu 1990; Bourdieu \& Wacquant 1992). In this regard, "Chan (1996) conceptualizes police culture in terms of the interaction between 'habitus' - similar to cultural knowledge and attitudes - and 'field' - the social space in which participants interact" (Myhill \& Bradford 2013: 350). Chan argues that this understanding of police occupational culture situates practice within social and political contexts and recognizes an active role for police actors - as working within structural positions (field), officers use cultural dispositions (habitus) to change, reinforce or transform police culture and practice (Westmarland 2005). In this regard, Wacquant (1992: 16) advances, "A field consists of a set of objective, historical relations between positions anchored in certain forms of power (or capital), while habitus consists of a set of historical relations 'deposited' within individual bodies in the form of mental and corporeal schemata of perception, appreciation and action." Reiner (2010: 116) cites Chan (1992) in advancing, in his own work, "Cultures are shaped, but not determined, by the structural pressures of actors' environments."

Ewart (2014, citing Chan 1997: 73) depicts Chan's model, incorporating Bourdieu view of culture, as follows:

\begin{tabular}{|c|c|c|c|}
\hline STRUCTURAL & & CULTURAL & \\
\hline $\begin{array}{l}\text { CONDITIONS } \\
\text { (The Field) }\end{array}$ & $\rightarrow$ & $\begin{array}{l}\text { KNOWLEDGE } \\
\text { (Habitus) }\end{array}$ & $\rightarrow$ \\
\hline
\end{tabular}

In relation to police socialization, Chan argues that the process "provides officers with guidance on actions and practice, a vocabulary for justifying their choices, but still allows for individual interpretation and initiative...police actors are active interpreters of their world, using their habitus (cultural knowledge) to interpret and react to their structural conditions (the field) to produce and to modify their practice... in contrast to the usual linear model of how police practice is created: that structural conditions determine cultural knowledge which in turn dictates police practice. This omits the police actor from the process" (O’Neill 2016: 477).

${ }^{43}$ Some policing scholars (myself included) observe that today's officers are more diverse (in age, race, ethnicity, gender, and previous life experience) and better educated, and, therefore, they are less uniform in the backgrounds, philosophies, and attitudes they bring with them into their policing career. Consequently, they may be less 
likely to share 'traditional' police cultural norms than the archetypal officer within that genre of scholarship (e.g., see Cordner 2017; Punch 2007).

${ }^{44}$ In relation to the former, Bacon (2014: 112, citing Loftus 2009; Reiner 2010) observes, "The dominant culture is said to exist because the police are constantly reinventing and reproducing it in response to the common problems that arise... Whatever their later specialisms and ranks, it is important to remember that all officers have similar beginnings and shared experiences, which enable them to relate to each other on one or more cultural levels."

${ }^{45}$ See also, Bacon 2014; Campeau 2015; Chan 1996, 1997; Chan et al. 2003; Cochran \& Bromley 2003; Cockcroft 2007; Crank 1998; Farkas \& Manning 1997; Fielding 1988a, 1995; Fielding \& Fielding 1991; Fletcher 1999; Foster 2003; Haarr 1997; Heidensohn 2013; Herbert 1998; Hobbs 1988; Holdaway 1983; Ingram et al. 2013; Jefferson 1990; Jermier et al. 1991; Johnson 2012; Kappeler et al. 2010; Khatib 1996; Kraska \& Paulsen 1997; MacAlister 2004; Paoline 2004; Paoline et al. 2000; Punch 1983; Reiner 2010; Skogan \& Frydl 2004; Young 1991.

${ }^{46}$ Within the investigative subculture, many practitioners would suggest a distinction between, what I would term, 'suits' (detectives who do not often interact with suspects in the field) and 'undercover' officers (that perform an investigative function but do much of their work through interacting with those in the criminal milieu).

${ }^{47}$ See also, Albuquerque \& Paes-Machado 2004; Boivin et al. 2018; Brown 2007; Crank 2014; Holdaway 1983; Kappeler et al. 2005; Kraska \& Cubellis 1997; Lee 1981; Loftus 2007, 2008, 2009, 2010; Manning 1989, 1997; Nhan 2014; Parnaby \& Leyden 2011; Pollock 2010; Reuss-Ianni 1983; Shearing 1981; Silver et al. 2017; Skolnick 1996, 2011; Skolnick \& Fyfe 1993; Tator \& Henry 2006; Waddington 1999; Young 1991.

${ }^{48}$ See also, Bayley \& Mendelsohn 1969; Buckley \& Petrunik 1995; Cain 1973; Campeau 2015; Chan 1996, 1997, 2007; Charman 2017; Conlon 2004; Conti 2009, 2010; Ellis 1991; Ford 2003; Gallo 2001; Getty, Worrall \& Morris 2016; Innes 2003; Kappeler et al. 2010; Lundman 1980; Manning 1977, 1989; McNamara 1967; Niederhoffer 1967; Paoline \& Terrill 2014; Reuss-Ianni 1993; Rubinstein 1973; Skolnick 1966.

${ }^{49}$ Depending on the agency, some programs are run in-house, for example, Edmonton Police recruits attend the service's own William Griesbach Training Centre, while others are external, for example, all recruits from the various municipalities across British Columbia attend the Justice Institute of British Columbia. Certainly, from my own attendance at the Ontario Police College for training in 1985 (where all recruits from the various municipalities across Ontario and the Ontario Provincial Police attend), I do not dispute scholars' findings that acculturation begins immediately upon entry into the police academy. Given the limited scope of this dissertation, a lengthy discussion is not feasible, however, in brief, my experiences (that were not dissimilar from those of 
many of the $300+$ other recruits in the cohort) were consistent with the observations of Ellis (1991: 96), who found, "Police training is quite vigorous and intensive and is in many respects deliberated designed to have a direct impact on trainee attitudes, beliefs and values - strengthening some and reshaping others." The police academy presents, quite intentionally, significant mental and physical challenges (Chan 2003, Ellis 1991, Obst \& Davey 2002; Van Maanen 1973), within a paramilitary structure, culture, and model of training (Bittner 2006; Chappell \& Lanza-Kadue 2010) that facilitate the process of police cultural socialization.

In my experience, recruits were geographically and socially segregated at an isolated campus (the Ontario Police College in Aylmer, Ontario, a former military installation, is literally surrounded by nothing but thousands of acres of farmland and the closest hamlet is more than 5 kilometers away). This is consistent with the observations of Chappell and Lanza-Kaduce (2010: 190, citing Conti \& Nolan 2005), who find, "The police academy in particular is intended to isolate recruits from their outside responsibilities." And, much of the curriculum, particularly in the first weeks of training, involved military-oriented rituals (5am room inspections; marching; standing at attention; referring to all staff as 'Sir,' and 6am drill in formal dress uniform complete with a very vocal Sergeant Major, with a pace stick tucked under one arm and no tolerance for the expression of any individuality). Any such demonstration was viewed, within that training regimen, as non-conformist imperfection. Training involved a daily routine (outside of academic classes) consisting of incessant boot and leather kit polishing, uniform pressing and de-linting, meticulous and standardized grooming, exhibiting the 'appropriate' deportment at all times, running, weight lifting, self-defence sparring, firearms training, and virtually mandatory after-hours socializing and drinking at the pub centrally located within the expansive facility. The outcome of this training arrangement is that recruits spend virtually all of their approximately 18 waking hours, over 15 weeks, exclusively within the company of their classmates (classes were comprised of 32 recruits and competition between classes was very much encouraged by instructors). Group punishment for the poor performance of one recruit in the class was a standard practice. The net effect is that, for those of us that persevered through the experience (withdrawals and expulsions, while not commonplace, did impact on several recruits in my cohort), a common bond became entrenched - featuring solidarity and loyalty that is uncommon in any other occupational context (excepting the military). And, certainly now, in reflecting on the experience, I can appreciate that the process instilled, within myself and the other recruits with whom I spent much of my time, a uniform and 'traditional' understanding of the values, attitudes, and behaviours expected of a police officer - the socialization into what it means to be a police officer in accordance with the prevailing cultural tenets. My experiences accord with the observations of Chappell and Lanza-Kaduce (2010) in their ethnographic research at an American police academy. In this regard, they find,

Police academies are characterized by many of the same rituals as boot camp in the military, such as stress, an emphasis on chain of command, and group punishments and discipline...Everyone had a stake in everyone else's behaviour and conduct, and in time, recruits learned to help each 
other stay out of trouble to avoid punishment...Furthermore, group marching, 'posting' [standing at attention], uniform dressing, and group punishments reinforced group solidary and cohesion. These rituals ensured that recruits identified with each other. Indeed, the development of the police subculture began day one in the academy (Chappell \& LanzaKaduce 2010: 189-204).

Further, as Van Maanen (1973: 411) articulated,

The main result of such stress training is that the recruit soon learns it is his peer group rather than the 'brass' which will support him and which he, in turn, must support. For example, the newcomers adopt covering tactics to shield the tardy colleague, develop cribbing techniques to pass exams, and become proficient at constructing consensual ad hoc explanations of a fellow recruit's mistake. Furthermore, the long hours, new friends, and ordeal aspects of the recruit school serve to detach the newcomer from his old attitudes and acquaintances. In short, the academy impresses upon the recruit that he must now identify with a new group - his fellow officers.

${ }^{50}$ Various scholars (e.g, see Buckley \& Petrunik 1995; Chan et al. 2003; CooperThomas \& Anderson 2002; Crank 1998; Ellis 1991; Fielding 1988; Ford 2003; Haarr 2001; Lundman 1980; Obst \& Davey 2002; Paoline 2004; Reuss-Ianni 1993; Sato 2003; Van Maanen 1973) find that, even in circumstances in which recruits begin their police career with values, attitudes, and/or expectations not conforming to those that would be considered 'traditional' within the socio-political norms of the policing institution, over time during training, through the process of cultural socialization, those initial understandings are often neutralized and then modified in order to align with the homogenous worldview of colleagues (instructors and fellow students) within the dominant occupational culture. Along these lines, Oberfield (2014:58, citing Conti 2010; Chappell \& Lanza-Kaduce 2010) advances, "[Police] academy trainers convey to cadets that they are 'not normal anymore.' Rather, they are told that they are now part of an exclusive culture in which only other members of the [police] organization, their new family, truly understand them." This process of police academy socialization often implicates the transmission of culture metaphorically through 'war stories,' myths, and anecdotes (often related by veteran officers) that informally augment (and frequently contradict) the formal curriculum and explain how things 'work on the streets' (Buerger 1998; Chappell \& Lanza-Kaduce 2010; Charman 2017; Ford 2003; Reuss-Ianni 1982; Shearing and Ericson 2001; Waddington 1999). Further, as observed by MacAlister (2004: 178, citing Van Maanen 1973), "At the academy, the learning of the rules and procedures governing police work are only the beginning of the socialization process. A much more important dynamic is the development of a sense of camaraderie or esprit de corps that is shared among the recruit class."

Haarr (2005: 435, citing Van Maanen 1975) finds that officers are continually involved in the process of socialization, which begins prior to one's entry into the occupation and continues throughout the duration of their policing career. In a classic study, Savitz 
(1970) investigated whether recruits' attitudes towards police misconduct changed over time and he found that upon graduating from the academy to work on the streets, and therefore being exposed continuously to the front-line police subculture, officers' attitudes became much more permissive in relation to the behaviour of fellow officers. In a similar vein, Johnson and Lafrance (2016: 1581-1582) observe, "Career stage theory (Super 1957) suggests that employees progress through various stages during a career, each marked by work attitudes and behaviours unique to each stage... Regarding law enforcement officers, early qualitative research by Van Maanen (1973) and Bayley and Bittner (1984) suggested that experience was extremely important to the development of officer attitudes and behaviours. They suggested that experience on the job constituted the learning of the goals, tactics, and norms of the police working culture."

The influences of front-line subcultural socialization, following the recruit's graduation from the police academy through to retirement, are discussed in Chapter Six - in the context of the present study's empirical findings and police occupational culture theory (for present purposes this theoretical framework subsumes career-stage theory). Paoline and Terrill (2014: 130) are helpful with their observation that "socialization involves teaching and learning, while the substance of what is taught and what you learn is culture." 


\section{NOTES FOR CHAPTER FOUR}

${ }^{1}$ With mixed methods, the strengths of each method offset the weaknesses of other methods employed - often referred to as 'complementarity' (Hesse-Biber 2010; Onwuegbuzie \& Leech 2005; Schulenberg 2007, 2014; Small 2011).

${ }^{2}$ Given the predominantly francophone composition of the Service de police de la Ville de Gatineau and the significant numbers of bilingual (English and French) officers serving with the RCMP Codiac detachment (Moncton, New Brunswick), I felt that it was necessary to present front-line officers at those data sites with a choice of survey language. Almost all of the 148 completed Gatineau surveys and 5 of the 71 completed RCMP Codiac surveys were the French version.

${ }^{3}$ I typically advised potential study participants, in my introductory remarks, that if they felt so inclined, they could continue their written elaborations on the reverse side of the survey pages.

${ }^{4}$ Response bias, which can be considered as subsuming both acquiescence response bias and question order bias, involves the existence of factors (e.g. question phrasing and/or ordering) that might influence a respondent to answer a question incorrectly.

${ }^{5}$ In their analysis of survey mode in police research from 2008 to 2017, Nix and colleagues (2017) found that in-person surveys were the most common - with $48 \%$ administered during roll calls or at training classes (within police facilities), $25 \%$ were Web-based, and $27 \%$ were collected through the mail or by telephone.

${ }^{6}$ Given the prevalence (and prominence) of problems in contemporary front-line police work that are often framed in relation to 'demographic' characteristics identified as intrinsic to an individual, or a group of individuals (i.e. 'visible minorities') with whom officers interact, the identification of any such issues, within the present study, was thought to be essential. In furtherance of such an understanding, study participants were presented (in Question 25 of the survey) with five predetermined categories (gender, race/ethnicity, mental health challenges, sexual orientation, and physical health challenges) and the option to provide information related to 'other' demographic(s), in characterizing the public with whom they interact. Similarly, I thought it was important to receive empirical data to further understandings around whether certain officer variables (i.e. an officer's race/ethnicity, gender, age, years of police experience, and/or years of front-line police experience) impact on how individuals across the rank-and-file view, or behave in, their front-line police work.

Having said this, I recognize that requesting potential study participants (front-line officers) to self-identify into specific categories (i.e. characterizing their race/ethnicity) and to discuss their perceptions of 'demographic' characteristics, in relation to their interactions with citizens, can be problematic and controversial within the social sciences (including sociology) - given that these are fluid social constructs that some may find objectionable and that are subject to individual interpretation, bias, prejudice, 
etc. As a sociologist, I am wary of the convention of using concepts like race, ethnicity, 'demographic' characteristics, etc., which I often find offensive, however, I argue that such decisions, in social scientific research, should be made on a case-by-case basis, in which all considerations, for and against the collection of any such data, must be evaluated. In the present study, I made a determination that the solicitation of such information was, all things being considered, warranted, in that it is really the only way to identify these considerations in the minds of research participants and, as discovered, concerns around interactions with individuals perceived as non-White, mentally ill, LGBTQ+, women, physically disabled, and/or 'other' identified 'demographic' characteristics is a significant aspect of today's de-policing phenomenon. In my view, failure to investigate this aspect of officers' risk-averse behaviours would be negligent and would leave many important questions in today's policing unsanswered. Also, of course, study participants were free to choose not to respond to any questions in the survey (and several declined to characterize their demographic information).

${ }^{7}$ These considerations were informed by the insights of David Klinger, Professor of Criminology and Criminal Justice at the University of Missouri and senior research fellow at the Police Foundation (personal communication, 18 November 2015).

${ }^{8}$ This study was funded largely through research grants from the Canadian Social Sciences and Humanities Research Council (\$105,000 Cdn.), the Fulbright Foundation for Educational Exchange between Canada and the United States of America $(\$ 15,000$ U.S.), and internal university and departmental awards from Carleton University.

${ }^{9}$ It is interesting to consider whether a large-scale front-line policing study could be done successfully without institutional permission - through approaching individual officers in public locations and requesting their participation, but, given the limited scope of this dissertation, that will have to remain a topic for another discussion.

${ }^{10}$ In three instances (the Calgary Police Service, the Toronto Police Service, and the Vancouver Police Department), participation by the police agency was contingent on my entering into a formal and written 'research agreement'.

${ }^{11}$ In the case of the Service de police de la Ville de Montréal, despite my persistence, various senior officials, to whom I was directed, continually failed to respond to telephone calls and messages. Therefore, given the apparent lack of support from senior management, efforts to secure the participation of front-line Montréal officers was eventually (and unfortunately) abandoned. Chief Jennifer Evans declined to provide Peel Regional Police Service (PRPS) front-line officers the opportunity to participate in the study, citing "numerous projects on-going with personnel" (personal communication, 25 April 2015). We had earlier discussed the Chief's concerns about the diverse race/ethnicity makeup of Peel Region's population and the possibility for negative outcomes for the PRPS (i.e. unflattering media commentary) with participation in research that delves into potentially-controversial issues around policing and race/ethnicity (personal communication, 16 April 2015). Superintendent Tony Cusimano (Executive Officer to the Chief) advised me that, due to the participation of 
York Regional Police (YRP) in "several other research initiatives", management could not support YRP participating in this study due to the potential for "study fatigue" among the officers (personal communication, 4 May 2016).

${ }^{12}$ Although every effort was made, in both survey design and survey administration, to not influence survey respondents' answers (through purposeful 'impression management') the possibility exists - as with all social interaction - that a participant's interpretation of potential study objectives, could, at least in theory, influence officers' responses (a similar phenomenon is referred to in the medical literature as 'sponsor bias' and such considerations also engage the concept of 'confirmation bias'). For example, it is possible that a participant may have, for whatever reason, understood that the present study's goal (if a participant were to presume that research is undertaken with a pre-determined conclusion) was to establish that front-line officers are not engaged in de-policing (engaging concerns around 'social desirablility bias') or, alternatively, officers are engaging in de-policing - and therefore, such participants, if any, may have answered some survey questions disingenuously in an effort to contribute to what they understood as the researcher's goal. However, as detailed within Chapter Six, given (1) the unsolicited and spontaneous disclosures of relevant information, which were made by many officers outside the formal research setting and (2) the present study's findings around trust in the researcher and its impact on data integrity, I think it is reasonable to conclude that this would have been the case in only a small number of cases - in either underreporting or overreporting de-policing. Further, while it is one thing to answer a 'Yes' or 'No' question disingenuously (for whatever purpose one might do so), it is another matter to carry that deception through into a detailed qualitative elaboration of that initial quantitative response.

${ }^{13}$ In situations where an officer had not yet read the document, I provided them with a copy and sufficient time to inform themselves and consider consent before providing them with the survey document.

${ }^{14}$ Both the 'shuffling' and sealing of the envelope procedures were intentional and intended to demonstrate (in a highly visible and transparent fashion) my commitment to the tenets of anonymity protection in ethical research and my trustworthiness in protecting officers' anonymity and confidentiality.

${ }^{15}$ As discussed, some particular challenges developed with data collection in Yonkers. As a result, most of the surveys considered in this study that originated from Yonkers Police Department (YPD) front-line officers, were administered to individual officers I met away from YPD police facilities. To protect the anonymity of the 51 YPD officers that ignored the non-participation directive of their labour organization (the YPBA), I do not present any identifying information in relation to their location/assignment or whether that participant was met in a YPD facility or elsewhere.

${ }^{16}$ This index is calculated based on occurrences involving four specific criminal offence categories: murder and non-negligent manslaughter, rape, robbery, and aggravated assault, and in 2016, an estimated 1,250,000 of these violent crimes were reported to 
police across the USA (Federal Bureau of Investigation 2016). Of these, aggravated assaults accounted for $64.3 \%$ of the reports, robberies $26.6 \%$, rapes $7.7 \%$, and murders $1.4 \%$ (Federal Bureau of Investigation 2016). The city with the highest violent crime rate was Detroit, with 13,705 violent occurrences reported by its 669,673 residents (a violent crime rate of 2046.5 per 100,000 inhabitants).

${ }^{17}$ It is important for the reader to appreciate that the five participating American police agencies (data sites) are all from the State of New York, which is only one of the 50 states that comprise the USA.

${ }^{18}$ This race/ethnicity information (and that presented in Appendix C for Albany, Buffalo, Rochester, Syracuse, and Yonkers) is from the most recent self-identification data reported by the United States Census Bureau (from the 2010 Census demographic profile).

${ }^{19}$ In British Columbia, RCMP officers account for $61.2 \%$ of that province's police personnel (5378 of 8761); in New Brunswick, 54.1\% (695 of 1285); in Nova Scotia, $43.3 \%$ (789 of 1822); and in Alberta, RCMP officers represent $36.1 \%$ of the police personnel (2640 of 7312) (Statistics Canada 2017e).

${ }^{20}$ These Canadian offences are: homicide, 'other violations causing death,' attempted murder, sexual assault, sexual violations against children, robbery, and 'major assault' which includes aggravated assault, assault with a weapon, and assault causing bodily harm (Statistics Canada 2017d).

${ }^{21}$ However, it should be noted that, given the differences in statutory interpretations and applications of 'aggravated assault' in American criminal justice contexts, I have included, for the purposes of the calculation, those Canadian offences captured within the terminology 'major assault' by Statistics Canada. In some instances, these 'major assault' offences in Canada would correspond with the American interpretation of 'aggravated assault,' while in other occurrences they would not. Therefore, it is likely that the Canadian violent crime rate of 286.8 would be lower (it is not possible to present an accurate estimate of how much lower) if a Canadian category of 'aggravated assault,' identical to the American definition, were available. In considering this, it should be understood that the national rate for the Statistics Canada category 'major assault' (in 2016) was reported as 143.0 occurrences per 100,000 persons.

22 "A census metropolitan area (CMA) consists of one or more neighbouring municipalities situated around a major urban core...To be included in the CMA, other adjacent municipalities must have a high degree of integration with the central urban area...A CMA typically comprises more than one police service." (Statistics Canada 2017c)

${ }^{23}$ For the four greater Vancouver region data sites (Delta, New Westminster, Port Moody, and RCMP Ridge Meadows) refer to Vancouver CMA data. Data is not 
available for Cornwall, Red Deer, and Stratford as they do not reach Statistics Canada's population threshold for CMA's.

${ }^{24}$ Differences between the number of surveys administered (column 3 ) and the number of surveys received (column 4) occurred in situations when front-line officers declined to participate in completing the survey (either advising me of their intention not to complete the survey or returning it blank) or when officers took the survey from the shift briefing and failed to return it to me. Differences between the numbers of surveys received (column 4) and those subsequently included in this study (column 5) occurred in circumstances where police officers, who are not considered 'front-line' for the purposes of this study, completed and submitted the survey to me (e.g. officers working primarily in investigative, administrative, or support functions). Despite the advanced circulation of the information document (refer to Appendices D and E), situations arose during the administering of the surveys where officers not currently performing frontline functions attended shift briefings, received the survey when administered, completed the survey, and submitted it to me. These surveys, which were identified based on the participants' responses to Question 6, were set aside and not included in the present study. This accounts for the difference between completed surveys received (3706) and surveys included in the study (3660). In other words, 46 police officers who submitted a completed survey were subsequently determined not to be working in a front-line capacity, based on their identification of their current assignment.

${ }^{25}$ For example, in the present study's sample, 660 participants self-identified as female (18.0\% of the total sample or $18.2 \%$ of Canadian and $17.5 \%$ of American respondents). As reported, on a national level, women comprise approximately $12 \%$ of American police officers (Federal Bureau of Investigation 2016) and 21\% of Canadian police officers (Statistics Canada 2017e). Similarly, in this study's sample of police officers from across the State of New York, $12.2 \%$ of respondents identified their race/ethnicity as 'Black,' which is similar to the national reported rate of $12.0 \%$ (Bekiempis 2015). I argue that such slight differences serve as confirmation that systematic sampling bias in the present study is unlikely.

${ }^{26}$ The following are some examples from other policing studies I encountered in the literature or through meeting with the researcher(s): (1) In their study examining officers' perceptions around the use of body-worn cameras, Jennings and colleagues (2014) solicited the participation of 400 Orlando officers to complete an online survey. Of these, 95 officers agreed to participate (a response rate of 23.8\%); (2) In a 2016 study examining the opinions of Buffalo and Rochester officers regarding body-worn video, BPD Captain Joe Gramaglia (who was completing the study as part of his Master of Public Administration degree) administered an online survey to the approximately 700 sworn officers within each department. Of these, 256 surveys were completed -105 from RPD officers and 151 from BPD officers - which represents a response rate of 18.3\%; (3) In a study conducted by Buckley and Petrunik (1995), a questionnaire was disseminated under the auspices of the Canadian Police College to a random sample of 250 police constables from two mid-sized Canadian regional police forces. Of these, 156 officers completed the questionnaire, yielding a $62.4 \%$ response rate; (4) In their 
study examining officers' attitudes toward their work, McElroy and colleagues (1999) administered surveys to recruit officers $(\mathrm{N}=79)$ and veteran officers $(\mathrm{N}=85)$ that were in attendance at a police academy in the midwestern United States. Response rates were $66 \%$ for recruits and $100 \%$ for veterans. The researchers suggest that the difference in response rate can be attributed to "the fact that the recruits were allowed to leave the classroom setting with the survey for later completion while the veterans completed the survey in class" (McElroy et al. 1999: 510); (5) In their research examining police culture and adaptations to the strains of the job, similar to the present study's sampling protocol, Paoline and Terrill (2014), attended, in person, on roll calls at seven participating police agencies. Through this sampling procedure, response rates ranged from $95.1 \%$ (Portland, Oregon) to $99.4 \%$ (Knoxville, Tennessee).

${ }^{27}$ In terms of a research participant's race/ethnicity, a decision had to be made as to the categories that would be used to describe sample 'demographic' characteristics in this study. As the reader will have noticed, even between Statistics Canada and the United States Census Bureau there are significantly different categorizations - the former uses 12 while the latter relies on 8. Given that this study involves both Canadian and American front-line officers, I decided to create a seven-category measurement that amalgamated commonalities between the two countries' categories and that recognized respondents' self-report information - 'White,' 'Black,' 'Hispanic,' 'Asian,' 'Aboriginal,' 'mixed races,' and 'other' race/ethnicity. This is a similar approach to that of a York University research team, with whom I did some work as a research consultant on behalf of the Ottawa Police Service's Traffic Stop Race Data Collection Project (they created a six-category measurement).

Also, given the small numbers of participants that indicated they are primarily engaged in traffic enforcement, school liaison, or gang intervention, it was decided to merge these participants with those who identified 'other' types of front-line police work (predominantly tactical and police canine) into a single 'other' category. Further, for the purposes of this study, the demarcation between eastern Canada and western Canada was considered as the Ontario/Manitoba provincial boundary, which is a common geographic and cultural understanding.

As with differences across the various regions of the USA, as discussed in relation to the study's 5 data sites in the State of New York, there are significant regional differences across Canada - including throughout 'Eastern Canada' and 'Western Canada'. The reader should understand that these are analytical categories and that in 'Eastern Canada', for example, there are significant distinctions between the Atlantic provinces (Newfoundland, Prince Edward Island, Nova Scotia, and New Brunswick), Québec, and Ontario.

The following tables present the information for Canada and the United States (separately). 
Table 57: Sample characteristics (Canada)

\begin{tabular}{|c|c|c|c|}
\hline & & $\mathbf{N}$ & $\%$ \\
\hline \multirow{4}{*}{ Gender } & Male & 2194 & $81.7 \%$ \\
\hline & Female & 490 & $18.2 \%$ \\
\hline & Total & 2684 & $100 \%$ \\
\hline & Missing & 2 & \\
\hline \multirow{9}{*}{ Race/Ethnicity } & White & 2204 & $82.1 \%$ \\
\hline & Black & 104 & $3.9 \%$ \\
\hline & Hispanic & 21 & $0.8 \%$ \\
\hline & Asian & 93 & $3.5 \%$ \\
\hline & Aboriginal & 67 & $2.5 \%$ \\
\hline & Mixed & 71 & $2.6 \%$ \\
\hline & Other & 109 & $4.1 \%$ \\
\hline & Total & 2669 & $100 \%$ \\
\hline & Missing & 17 & \\
\hline \multirow{9}{*}{ Age } & $21-25$ & 128 & $4.8 \%$ \\
\hline & $26-30$ & 530 & $19.7 \%$ \\
\hline & $31-35$ & 580 & $21.6 \%$ \\
\hline & $36-40$ & 586 & $21.8 \%$ \\
\hline & $41-45$ & 437 & $16.3 \%$ \\
\hline & $46-50$ & 257 & $9.6 \%$ \\
\hline & Older than 50 & 163 & $6.1 \%$ \\
\hline & Total & 2681 & $100 \%$ \\
\hline & Missing & 5 & \\
\hline \multirow{8}{*}{ Years of police service } & $1-5$ & 715 & $26.6 \%$ \\
\hline & $6-10$ & 842 & $31.3 \%$ \\
\hline & $11-15$ & 531 & $19.8 \%$ \\
\hline & $16-20$ & 295 & $11.0 \%$ \\
\hline & $21-25$ & 138 & $5.1 \%$ \\
\hline & 26 or more & 159 & $5.9 \%$ \\
\hline & Total & 2680 & $100 \%$ \\
\hline & Missing & 6 & \\
\hline \multirow{8}{*}{ Years of front-line policing } & $1-5$ & 796 & $29.6 \%$ \\
\hline & $6-10$ & 1067 & $39.7 \%$ \\
\hline & $11-15$ & 498 & $18.5 \%$ \\
\hline & $16-20$ & 207 & $7.7 \%$ \\
\hline & $21-25$ & 66 & $2.5 \%$ \\
\hline & 26 or more & 44 & $1.6 \%$ \\
\hline & Total & 2678 & $100 \%$ \\
\hline & Missing & 8 & \\
\hline \multirow{3}{*}{ Agency location (region) } & Western Canada & 801 & $29.8 \%$ \\
\hline & Eastern Canada & 1885 & $70.2 \%$ \\
\hline & Total & 2686 & $100 \%$ \\
\hline \multirow{3}{*}{ Size of police agency } & Small (<100 front-line) & 6991 & $25.7 \%$ \\
\hline & Medium (100-399 front-line) & 945 & $35.2 \%$ \\
\hline & Large (400+ front-line) & 1050 & $39.1 \%$ \\
\hline
\end{tabular}




\begin{tabular}{|c|c|c|c|}
\hline & Total & 2686 & $100 \%$ \\
\hline \multirow{7}{*}{$\begin{array}{c}\text { Policing Environment } \\
\left({ }^{*} \text { more than } 1 \text { response }=\text { mixed }\right)\end{array}$} & Rural & 97 & $3.6 \%$ \\
\hline & Suburban & 383 & $14.3 \%$ \\
\hline & Urban & 1794 & $66.8 \%$ \\
\hline & Commercial & 16 & $0.6 \%$ \\
\hline & Mixed* & 388 & $14.4 \%$ \\
\hline & Total & 2678 & $100 \%$ \\
\hline & Missing & 8 & \\
\hline \multirow{7}{*}{$\begin{array}{c}\text { Type of police work } \\
\left({ }^{*} \text { more than } 1 \text { response }=\text { mixed }\right)\end{array}$} & Primary Response/General Patrol & 2348 & $87.4 \%$ \\
\hline & Community-Based & 79 & $2.9 \%$ \\
\hline & Foot/Bicycle Patrol & 77 & $2.9 \%$ \\
\hline & $\begin{array}{l}\text { Other (gang intervention, traffic } \\
\text { enforcement, school liaison, tactical, } \\
\text { canine, etc.) }\end{array}$ & 61 & $2.3 \%$ \\
\hline & Mixed* & 117 & $4.4 \%$ \\
\hline & Total & 2682 & $100 \%$ \\
\hline & Missing & 4 & \\
\hline
\end{tabular}

Table 58: Sample characteristics (USA)

\begin{tabular}{|c|c|c|c|}
\hline & & $\mathbf{N}$ & $\%$ \\
\hline \multirow{4}{*}{ Gender } & Male & 803 & $82.5 \%$ \\
\hline & Female & 170 & $17.5 \%$ \\
\hline & Total & 973 & $100 \%$ \\
\hline & Missing & 1 & \\
\hline \multirow{9}{*}{ Race/Ethnicity } & White & 706 & $73.0 \%$ \\
\hline & Black & 118 & $12.2 \%$ \\
\hline & Hispanic & 89 & $9.2 \%$ \\
\hline & Asian & 9 & $0.9 \%$ \\
\hline & Aboriginal & 1 & $0.1 \%$ \\
\hline & Mixed & 27 & $2.8 \%$ \\
\hline & Other & 17 & $1.8 \%$ \\
\hline & Total & 967 & $100 \%$ \\
\hline & Missing & 7 & \\
\hline \multirow{9}{*}{ Age } & $21-25$ & 35 & $3.6 \%$ \\
\hline & $26-30$ & 206 & $21.3 \%$ \\
\hline & $31-35$ & 253 & $26.1 \%$ \\
\hline & $36-40$ & 176 & $18.2 \%$ \\
\hline & $41-45$ & 124 & $12.8 \%$ \\
\hline & $46-50$ & 123 & $12.7 \%$ \\
\hline & Older than 50 & 51 & $5.3 \%$ \\
\hline & Total & 968 & $100 \%$ \\
\hline & Missing & 6 & \\
\hline \multirow[t]{5}{*}{ Years of police service } & $1-5$ & 275 & $28.3 \%$ \\
\hline & $6-10$ & 286 & $29.5 \%$ \\
\hline & $11-15$ & 142 & $14.6 \%$ \\
\hline & $16-20$ & 155 & $16.0 \%$ \\
\hline & $21-25$ & 72 & $7.4 \%$ \\
\hline
\end{tabular}




\begin{tabular}{|c|c|c|c|}
\hline & 26 or more & 41 & $4.2 \%$ \\
\hline & Total & 971 & $100 \%$ \\
\hline & Missing & 3 & \\
\hline \multirow{8}{*}{ Years of front-line policing } & $1-5$ & 280 & $28.8 \%$ \\
\hline & $6-10$ & 303 & $31.2 \%$ \\
\hline & $11-15$ & 161 & $16.6 \%$ \\
\hline & $16-20$ & 139 & $14.3 \%$ \\
\hline & $21-25$ & 62 & $6.4 \%$ \\
\hline & 26 or more & 27 & $2.8 \%$ \\
\hline & Total & 972 & $100 \%$ \\
\hline & Missing & 2 & \\
\hline \multirow{4}{*}{ Size of police agency } & STmall ( $<100$ front-line $)$ & 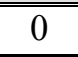 & $0 \%$ \\
\hline & Medium (100-399 front-line) & 375 & $38.5 \%$ \\
\hline & Large (400+ front-line) & 599 & $61.5 \%$ \\
\hline & Total & 974 & $100 \%$ \\
\hline \multirow{7}{*}{$\begin{array}{c}\text { Policing environment } \\
\left({ }^{*} \text { more than } 1 \text { response }=\text { mixed }\right)\end{array}$} & Rural & 29 & $3.0 \%$ \\
\hline & Suburban & 18 & $1.9 \%$ \\
\hline & Urban & 829 & $85.7 \%$ \\
\hline & Commercial & 12 & $1.2 \%$ \\
\hline & Mixed* & 79 & $8.2 \%$ \\
\hline & Total & 767 & $100 \%$ \\
\hline & Missing & 7 & \\
\hline \multirow{7}{*}{$\begin{array}{c}\text { Type of police work } \\
\left({ }^{*} \text { more than } 1 \text { response }=\text { mixed }\right)\end{array}$} & Primary Response/General Patrol & 814 & $83.7 \%$ \\
\hline & Community-Based & 30 & $3.1 \%$ \\
\hline & Foot/Bicycle Patrol & 10 & $1.0 \%$ \\
\hline & $\begin{array}{c}\text { Other (gang intervention, traffic } \\
\text { enforcement, school liaison, tactical, } \\
\text { canine) }\end{array}$ & 13 & $1.3 \%$ \\
\hline & Mixed* & 106 & $10.9 \%$ \\
\hline & Total & 973 & $100 \%$ \\
\hline & Missing & 1 & \\
\hline
\end{tabular}

${ }^{28}$ In addition, at many of the data sites, some research participants (ranging from one to perhaps 20 in a group) engaged me with general comments/observations about the topical nature of this study and their views about today's public criticism of police, video recording of police actions, lack of support from 'the community,' and (in some instances) lack of support from police management. In this regard, at several sites, participants directed my attention to policing occurrences that have generated significant controversy, substantial media coverage, and, in some instances, serious morale issues among the rank-and-file. A brief description of each of these events (by jurisdiction) are outlined in Appendix C. Readers interested in more information should review the often-extensive media coverage of each occurrence - widely available through online search engines. 
${ }^{29}$ Similarly, 3, 11, 9, and 10 study participants (respectively) did not indicate their gender (Question 1), age (Question 2), total years of service in policing (Question 4), and/or years of front-line policing experience (Question 5). Given conversations with several study participants, it is my understanding that such omissions were likely intentional and related to concerns with trust in the researcher, trust in the participating police agency, and/or trust in research anonymity protections - in that, as discussed in subchapter 4.6, such information could potentially narrow the field of participants that could be responsible for survey responses, if one (either the researcher or someone in the police agency's administration that might come into possession of data in violation of the study's ethics protocols) were to analyze this data in comparison to demographic information about personnel that is maintained by police agencies.

${ }^{30}$ For example, WIN94 (a Windsor officer), answered every question in the survey (and presented written elaborations for almost all questions), however for Question 25, this participant recorded a check mark in the 'Yes' box, but did not particularize any of the demographic options presented in the survey, present any 'Other' characteristic(s), or provide any qualitative elaboration. WIN94 also left Question 26 blank. Similarly, BUF207 (a Buffalo officer) answered every question in the survey except Question 26. This participant provided affirmative answers to Questions 16, 24, and 25 and written elaborations to every other question inviting further explanation throughout the survey.

${ }^{31}$ For example, Statistics Canada uses twelve 'visible minority' (those persons that selfidentity as other than 'White') race categories for the national census: 'South Asian,' 'Chinese,' 'Black,' 'Filipino,' 'Latin American,' 'Arab,' 'Southeast Asian,' 'West Asian,' 'Korean,' 'Japanese,' 'Other Visible Minority,' and 'Multiple Visible Minorities.' Canadians who identify as Indigenous peoples are captured (as 'Aboriginal') within another section of the census, which reports on ethnicity. On the other hand, the United States Census Bureau uses eight race/ethnicity categories: 'White,' 'Black/African American,' 'Hispanic/Latino,' 'Asian,' 'Two or More Races,' 'Other Race,' 'American Indian/Alaska Native,' and 'Native Hawaiian/Other Pacific Islander.' Within policing research, for example, the 2016 Ottawa Police study of race data on traffic stops traffic, conducted by a multidisciplinary team of professors from York University, organized data for the purposes of analysis, into 6 race/ethnicity categories ('White,' 'Black,' 'East/Southeast Asian,' 'South Asian,' 'Middle Easterners,' and 'Other Racialized Minorities') rather than the Ottawa Police Service's existing 7 race classifications or Statistics Canada's 12 'visible minority' classifications (Foster et al. 2016).

${ }^{32}$ Less than $10 \%$ of research participants in the study recorded an answer that required interpretation. In this regard, the vast majority of 'White,' 'Black,' and 'Asian' officers recorded those exact words in their write-in responses to Question 3. As indicated, some assumptions (which, I acknowledge could be incorrect in individual circumstances) were made in assigning other (more ambiguous) race/ethnicity responses. For example, I assumed, for the purposes of data organization, that a 'Polish' individual is more likely to be 'White' than one of the other race/ethnicity categories. In saying this, I recognize that it is possible I got a few of these wrong (for example, there are obviously 'non- 
White' Irish and 'White' Jamaicans), but, in the 'big picture,' I submit that this consideration is inconsequential. The table below illustrates where I positioned some Question 3 responses within the present study's seven race/ethnicity categories.

\begin{tabular}{|l|l|}
\hline Aboriginal & 'First Nation'; 'native'; 'indigenous'; 'Metis'; 'Status Indian' \\
\hline Asian & 'Chinese'; 'Korean'; 'Vietnamese'; 'oriental'; 'Japanese'’ \\
\hline Black & $\begin{array}{l}\text { 'African-American'; 'Haitian'; 'Nigerian'; 'Black, Non-Hispanic'; } \\
\text { 'Caribbean'; 'East African'; 'Somali', 'Afro-Canadian'; 'Jamaican' }\end{array}$ \\
\hline Hispanic & $\begin{array}{l}\text { 'Guatemalan'; 'Peruvian'; 'Mexican'; 'Latino'; 'White, Hispanic'; 'Black, } \\
\text { 'Hispanic'; 'Puerto Rican'; 'South American Hispanic'; 'Guyanese'; }\end{array}$ \\
\hline Other & $\begin{array}{l}\text { 'Eny response with multiple categories identified by the participant } \\
\text { Lankan'; 'Tamil'; 'Arabic'; 'Brown'; 'Desi'; 'Pakistani'; 'European'; } \\
\text { 'Indian'; 'Lebanese'; 'Persian'; 'Mulato', 'Muslim' }\end{array}$ \\
\hline White & $\begin{array}{l}\text { 'Caucasian'; 'White, Non-Hispanic'; 'Polish'; 'British'; 'Scottish'; 'Irish'; } \\
\text { 'White Boy'; 'Ukrainian' }\end{array}$ \\
\hline
\end{tabular}

${ }^{33}$ Applying some judgment was also required in organizing data in relation to Question 10 of the survey. A small number of participants, in answering how many times they have been video recorded while on duty, provided non-numeric responses - for example, a popular response was 'too many to count.' These types of responses were captured within the qualitative data analysis process and grouped by common themes. Some other participants used ranges to express their answer (i.e. '20 to 25'), other representations ('100+', or 'dozens', or 'more than 100'), or qualifiers ('approximately 10 times'). In these instances, the values $25,100,12,100$, and 10 (from these examples) were entered into the quantitative data management software.

${ }^{34}$ As a further hypothetical illustration, consider that with a small police agency that participated in the present study (like the Port Moody Police Department or the Stratford Police Service), attributing information to a female, non-White officer with 5 years of front-line police experience would be tantamount to explicitly naming that participant. Similarly, a participant belonging to an uncommon race or ethnicity within their policing jurisdiction, might be distinguished because there are only one (or only a few) officers of that race/ethnicity. This holds even with large agencies like the Toronto Police Service (with over 5,0000 officers). Consider that an officer from that agency's 31 Division (TOR191) shared, in their survey responses, that he/she practices depolicing daily because 'the community hates us in 31 Div.' Presenting demographic characteristics particular to TOR191 might have the effect of inadvertently identifying that otherwise anonymous research participant, who remains, otherwise, undistinguishable across the 30 or 40 TPS officers that participated in this study from 31 Division.

${ }^{35}$ These numbers were assigned randomly, in the sense that they were assigned after a 'shuffling' process that, therefore, ensured the order of input did not correspond to the date or location of sampling with each police agency. Also, for potential downstream 
analytical considerations, four 'demographic' characteristics for each participant (when available from their survey responses) were also recorded in the qualitative analysis documents alongside the individual alpha-numeric coding: (1) self-identified gender (for this purpose either 'female' or 'male'), (2) identified race/ethnicity (for this purpose either 'White' or 'non-White'), (3) number of years of front-line police experience, and (4) the type of area policed by the participant (for this purpose 'rural', 'suburban', 'urban', and/or 'commercial').

${ }^{36}$ As played out in my experiences with leadership of the Vancouver Police Department (VPD), maintaining initial approval is also an important consideration, and one that is not often discussed across the policing research literature. The specific circumstances encountered with leadership of the VPD and the Vancouver Police Union (VPU) are elaborated below in Endnote 37.

${ }^{37}$ For a review of issues around anonymity/confidentially in survey administration and candor in research participants' responses please consult Harnett and Seligsohn (1967); Klein, Maher and Dunnington (1967); Moore and Ames (2002); Olson, Stander and Merrill (2004); O’Malley, Johnston, Bachman and Schulenberg (2000); Ong and Weiss (2000); Singer, von Thurn and Miller (1995); and Tourangeau and Yan (2007).

\section{${ }^{38}$ Vancouver Police Department and Vancouver Police Union}

In relation to Vancouver, on 4 April 2015 I was contacted by then VPD Deputy Chief Doug LePard, the officer-in-charge of the VPD's Operations Division, who was following up on the discussion I had initiated on 11 March 2015 (LePard left VPD in March 2016 to assume the position of Chief of Metro Vancouver's Transit Police). LePard advised me that he was recommending participation of VPD in the study. As he requested, I then engaged with Drazen Manojlovic, VPD's Director of Planning, Research and Audit. On 27 May 2015 Manojlovic advised me that he supports the research, and, at his direction, I completed an Application for Research document. On 6 August 2015 Manojlovic advised me, via e-mail, that VPD was confirmed as a participant in the study. On 29 January 2016 I sent Manojlovic a copy of the REB ethics clearance and we agreed to discuss, in the coming weeks, tentative scheduling for data collection with VPD front-line officers later in the year.

On 16 July 2016 I sent Manojlovic e-mail suggesting a week in November 2016 for data collection with the VPD. On 3 August 2016 VPD Sergeant Michael Tsok contacted me and explained that he had been assigned to coordinate logistics for data collection with VPD in November 2016. The following day, Tsok advised me that, at the direction of VPD Deputy Chief Warren Lemcke, the research was to be discussed further at a VPD executive meeting on 9 August 2016. Shortly thereafter, I received a message to call Manojlovic and, in our subsequent telephone conversation, he explained that concerns had developed with some in VPD leadership, given opposition to the research by Tom Stamatakis, the president of the Vancouver Police Union (VPU), and 'concerns over the possible interpretation of some of the [survey] questions.' 
In e-mail correspondence sent to me on 5 August 2016, Lemcke had advised, 'I am speaking with Tom Stamatakis about this as well to see if there are any concerns from the Vancouver Police Union.' On 9 August 2016 I spoke with Lemcke by telephone and he advised me that he and others in VPD leadership have concerns with participation in the study and that VPD Deputy Chief Steve Rai would be raising those concerns at a meeting of the CACP Research Foundation on 11 August 2016 in Ottawa. Lemcke went on to explain that, should the study find that VPD officers are, in fact, practicing depolicing in response to their present techno-social and socio-political working environment, there could be negative reactions across the 'adversarial media landscape in Vancouver,' accompanied by public concern and criticism of VPD officers, in general, and VPD leadership, specifically.

On 12 August 2016 I spoke, as part of a three-person 'expert' panel discussing police body-worn video, at the Canadian Association of Police Governance (CAPG) annual conference in Ottawa and Stamatakis, who was a fellow panelist, declined my invitation to discuss his opposition to study participation by rank-and-file VPD officers. As I walked out of the CAPG venue to attend another nearby facility to deliver a presentation to the Canadian Association of Chiefs of Police (CACP) Ethics Committee (on the present study), I encountered Rai and we had a brief conversation. He encouraged me, in all of the circumstances, to abandon efforts at pursuing participation of VPD frontline officers in the study. This echoed the advice of Manojlovic, who, in a 10 August 2016 e-mail, following up on my exasperation at this turn of events, wrote, 'Deep down I believe your research will be valuable and I suggest you consider turning the page with this unfortunate experience and focus on those police departments who are eager to be involved.' With opposition from both VPD and VPU leadership, in my assessment, continuing with VPD front-line officers' participation was impossible - on both ethical and logistical grounds.

\section{Winnipeg Police Association}

In relation to Winnipeg, on 10 March 2015 I spoke with Chief Devon Clunis of the Winnipeg Police Service (WPS) about the participation of WPS rank-and-file in this study and on 16 March 2015 I received correspondence from WPS Superintendent Bruce Ormiston, who wrote, 'Thank you very much for your letter of March 10, 2015 in which you requested the participation and support of the Winnipeg Police Service in your research project into increased visibility of front-line policing as it relates to the perceptions of elevated societal scrutiny and critique of the police, and the potential influence on police officer response. Chief Clunis agrees that this topic of research is timely and relevant, and is pleased to have the Winnipeg Police Service as a participant in this research...'

After REB ethics clearance for the study was received on 29 January 2016, efforts to coordinate logistics for data collection with WPS officers began in February 2016. As part of this process, on 29 March 2016 I corresponded with Inspector Brian Miln, who had been designated as my WPS contact, and he indicated, 'We normally have our Association review the study to ensure there are no concerns from their perspective. I 
have forwarded a copy to them and I am waiting to hear back. Once that is confirmed, we can finalize some dates that work for you.' On 7 April 2016 I was notified by Miln that Maurice Sabourin, the president of the Winnipeg Police Association (WPA), did, indeed, have issues with participation of rank-and-file WPS officers in the study.

To discuss this, I contacted Sabourin by telephone on 7 April 2016 and he advised that he has concerns about 'several questions' in the study. He observed that 'members are being asked to admit violations of various regulations in their duties' and that his issues are not alleviated by the study's anonymity protection for participants. Sabourin explained that 'the Winnipeg Police Association has a good reputation in Winnipeg with the public and if information came out from this survey about some of the things officers are being questioned about it could damage that reputation.' Sabourin concluded by telling me that the WPA had contacted Tom Stamatakis, who, in addition to being president of the VPU, is the president of the Canadian Police Association. Given these developments with leadership of the WPA, I notified the WPS that I could not include WPS front-line officers in the study - given my concerns around research ethics and participants' consent and voluntariness in such circumstances.

\section{$\underline{\text { Yonkers Police Benevolent Association }}$}

In relation to Yonkers, on 30 October 2015 Yonkers Police Department (YPD) Deputy Chief John Mueller wrote to me in response to my inquiry to YPD Commissioner Charles Gardner on 17 October 2015, 'The YPD is in receipt of your request to participate in your research. Your topic, to determine the impact of, as you say, the elevated socio-political critique and its impact on how our officers do their jobs, is a very important one. I have spoken with our Police Commissioner Charles Gardner in regard to your research and he is willing to have our officers participate in your important work. I have cc'd Captain Joseph Solicito, of our Training Unit, who will be your contact person to facilitate the survey process and you can begin when ready to reach out him to discuss logistics.'

In February 2016 I provided Captain Solicito with the ethics clearance from the Carleton University REB and we began discussions to organize data collection logistics. In an e-mail on 31 March 2016, Solicito advised, 'We are looking forward to working with you and will be of as much assistance as we possibly can. Once we get a little closer I will put out a memo to the precincts, so you have their cooperation. Sometimes cops are uneasy about surveys...' Ultimately, we settled on a week in September 2016 and Solicito provided me with a proposed data collection schedule that would have me attending, over five days, at all four YPD precincts for daily shift briefings at 07:00, 08:00, 15:00, 16:00, 23:00, and 24:00.

As planned, on 13 September 2016 I attended at YPD Precinct 1 and was greeted by some of the front-line officers on duty with a significantly less-welcoming reception that I had, to that point, been accustomed at other police agencies. In retrospect, with the explanation that follows, this now makes sense. A few YPD officers at Precinct 1 on this date refused to participate in completing the survey, others returned blank surveys, 
and some took the survey document away and never returned it to me. The following morning, I attended at YPD Precinct 2 for the 07:00 roll call. My reception was even cooler, as I was told wait in the vestibule before being allowed into the precinct to speak with the on-duty YPD officers about 20 minutes later. When the Sergeant came out to escort me into the building, he said, 'I'm not sure how much cooperation you are going to get.' At this time, I learned that a directive had been issued the previous week to all YPD officers that belong to the labour organization representing the rank-and-file, the Yonkers Police Benevolent Association (YPBA), suggesting they not participate in the research.

As I spoke with YPD officers present on the 07:00 and 08:00 shift briefings at Precinct 2 , some ignored me (one stared at the ceiling and another turned his back on me) while others were visibly uncomfortable in declining to take a survey document or in returning it blank. One particularly rude YPD officer seemed to be functioning in an oversight role, through applying pressure on the officers to comply with the YPBA directive. Another officer, who I encountered upon leaving the building, explained in a sympathetic tone, 'This is Yonkers, we don't really do what they do other places; we don't participate in things like this.' I left a message with the receptionist at the YPBA office, requesting that YPBA president Keith Olson get in contact with me, and I also notified YPD leadership of these developments.

Olson contacted me about six hours later and explained that he and legal counsel for the YPBA have concerns with some of the questions in the survey - particularly as the YPBA is in the midst of an ongoing disagreement with city administration over the potential implementation of police body-worn cameras. Olson also advised that the YPBA only found out about the research study the previous week, via a 'leaked' copy of information distributed to commanding officers at the four YPD precincts.

Fortunately, I had some success the previous day in encountering a few YPD officers about the city in my travels between roll calls at Precinct 1 . Those officers were noticeably friendlier and expressed no reservations in reviewing the information document, expressing their voluntary consent, and completing a survey. Another YPD officer I met after the 08:00 roll call at Precinct 2 gave me excellent advice. That officer was pleased to be able to complete a survey and suggested that I abandon attendance on shift briefings and instead try to meet with individual YPD officers away from the scrutiny and peer pressure that is present in the precinct buildings. As this officer explained, many YPD officers are frustrated with YPBA leadership on many issues, including with the YPBA position on participation in this research. The officer suggested that many front-line YPD officers see positive aspects of having their observations on the contemporary police work environment considered in a scientific study.

As a result, I spent much of my time after the 08:00 shift briefing at Precinct 2 traveling throughout Yonkers (which, fortunately, is a densely-populated urban city of only 18.3 square miles) in search of situations where I could speak with front-line YPD officers one-on-one - to have them review the information document and consider completion 
of a survey. Through this revised sampling technique, I was able to meet with many YPD officers, from all four precincts, and almost all of those interactions were respectful and friendly. While I would have liked to achieve a higher proportion of front-line YPD officers in the study, I was pleased, in the circumstances, to receive 51 completed surveys, which represents $14.1 \%$ of the potential YPD sample frame.

Ultimately, I left Yonkers on 16 September 2016 after a disconcerting conversation with a YPD officer I met in a coffee shop. That officer explained that there are 'longstanding rifts' among members of the YPBA, which have been aired publicly and that have led to discord and 'open hostility' within the membership. I was counseled to 'be careful' in my travels around Yonkers as some of the 'more militant union-oriented' YPD officers could become upset at me continuing with the research effort and their behaviour might be 'unpredictable.' As I reported to YPD leadership, as a former police officer, hearing such cautionary words from a police officer, about fellow police officers, was more than a little unsettling. In e-mail correspondence I sent to Olson on 17 September 2016, I outlined my experiences in Yonkers, requested a copy of the union information circulated about the study, and invited Olson to 'explain, in writing, the specific issues your PBA had with the research.' No further communication has been received from Olson to date. 


\section{NOTES FOR CHAPTER FIVE}

${ }^{1}$ For Theme 1 (officers' awareness of 'policing's new visibility') and Theme 3 (officer's use of force practices in relation to 'policing's new visibility') study participants were not invited to contribute a qualitative response (a written elaboration) to their quantitative response. However, a small number of participants recorded comments in the margin adjacent to these questions in the hardcopy survey questionnaire document.

${ }^{2}$ All qualitative contributions (numbering 15,118) from anonymized study participants have been retained in a comprehensive working document and are available through discussion with the author for ethically-appropriate scholarly purposes.

${ }^{3}$ In addition to the information presented by journalists in relation to Edmonton, Toronto, and Peel Region, it has been reported (Carruthers 2018) that the approximately 582 police officers of the London Police Service (Statistics Canada 2017e), serving the 392,000 persons inhabiting this Ontario city (Statistics Canada 2016c), recorded just six person checks (or 'street checks') in 2017, down from more than 8400 three years earlier.

Also, in their analysis for the Los Angeles Times, Queally, Mather and Chang (2017) found that arrests by LAPD officers dropped by 25\% from 2013 to 2015 despite "persistent increases in crime, which began in 2014" and this development mirrored similar "significant drops in arrests" by officers serving with the Los Angeles County Sherrif's Department and the San Diego Police Department. The reporters observe, "Two other measures of police productivity, citations and field interviews, have also declined significantly" (Queally et al. 2017). LAPD Chief Charlie Beck acknowledges,

Nationwide criticism of police stoked by the 2014 fatal shooting of Michael Brown in Ferguson, Mo., and other highly publicized law enforcement killings has had an effect on officers' mindsets - but not to the detriment of crime fighting...suggesting 'I'd be denying human nature if I didn't say police are very cautious about what they do now because of the scrutiny...[However,] I don't really see things that make me think that the workforce as a body is retreating' (Queally et al. 2017).

In interviews with Los Angeles County Sheriffs' deputies the reporters were told,

It is inevitable that some officers will pull back, taking care of necessary work while not engaging in the 'proactive policing' that could lead to more arrests - and to more encounters that turn violent. 'Not to make fun of it, but a lot of guys are like, "Look I'm just going to act like a fireman." I'm going to handle my calls for service and the things that I have to do', said George Hofstetter, a motorcycle deputy in Pico Rivera and former president of the union representing L.A. County sheriffs' deputies. 'But going out there and 
making traffic stops and contacting persons who may be up to something nefarious? "I'm not going to do that anymore"” (Queally et al. 2017).

Analysis of official records document, "Field interviews conducted by Los Angeles sheriff's deputies have also been in steep decline, falling by $67 \%$ from 2012 to $2016 . .$. The number of citations, which includes traffic violations and other types of tickets, issued by LAPD officers also fell sharply, from almost 600,000 in 2010 to about 269,5000 five years later" (Queally et al. 2017).

${ }^{4}$ For this question, study participants were free to choose more than one of the listed items and to also particularize 'Other' circumstances - including an invitation to provide a written elaboration of such 'Other' circumstances. Percentages calculated (within the table) are in relation to both those participants who answered 'Yes' to Question 10 (column 3) and to the entire sample population in the study (column 4).

${ }^{5}$ It should be noted that these subthemes are broadly defined, as described in Chapter Four, and, throughout data analysis, I applied subjective (but consistent and, in my view, fair) decision-making in assigning certain responses within the identified classifications. Also, many participant's written responses relate to more than just one identified subtheme. For example, an Ottawa Police participant (OTT38) wrote, Use of force. Blunt language. Stopping minorities (I get accused of racial profiling even though I am Black), which engages three of the identified subtheme categories. The reader should understand that these considerations hold true across much of the qualitative data presented throughout this chapter.

${ }^{6}$ The reader should understand that the instructions provided to potential study participants requested that they complete the 30 questions, within the four-page survey document, in the order in which the questions were presented. Therefore, officers that reference the terms 'F.I.D.O.' and/or 'de-policing' in their responses to questions preceding Question 27 did so without the benefit of the explanation presented on page 4 immediately prior to Question 27, which reads: Questions 27 and 28 relate to the concept of risk-averse police practices. Some within policing, the media, and some academics studying policing have discussed this concept using different terminologies such as 'de-policing' or 'F.I.D.O.'

${ }^{7}$ A further subtheme was identified by only six (6) study participants, related to officers using video recording to their advantage in their police work. Their qualitative elaborations are included below - given the relevance to the previous research findings of Sandhu $(2016,2019)$ and Sandhu and Haggerty $(2015,2017)$ around 'camerafriendly policing':

Giving more loud directions to cultivate witnesses (DEL08).

I allow people all the time to argue with me, essentially making them look like a dolt, rather than argue back (HAL169).

Explaining the arrest to the suspect in detail, not for them but for the video (SAS03). 
Try to use more 'key words' loudly!! Such as 'stop resisting!' (ALB34).

Usually when cameras are out I keep the interaction brief, or I overdo how nice I am/how professional I am while they are recording (ALB120).

I will let a suspect run on a little more or his actions to gain additional evidence of his/her behaviour (ROC45).

${ }^{8}$ Sheptycki (2018: 26) defines a 'street check' as “stopping, questioning and documenting individuals for later use in intelligence analysis - a procedure known colloquially on the street (at least in Toronto) as "carding'." An American terminology characterizing similar police practices is 'stepping out' - as in 'stepping out with a group of suspicious guys on a street corner.'

${ }^{9}$ A small number of Rochester and Toronto officers had limited experience with police body-worn cameras (BWCs) at the time of data collection (in 2016) given their participation in RPD and TPS pilot projects with the technology. On the other hand, many rank-and-file Calgary officers had experience, albeit quite limited in duration, with police BWCs as CPS had rolled out the devices for front-line deployment, however they were taken out of circulation shortly thereafter due to technical issues with the equipment.

${ }^{10}$ Kappeler, Sluder \& Alpert (2010: 283), observe that one of the enduring ethos of traditional front-line subculture is the "minimizing [of] public scrutiny of police activities and behaviour." Throughout data collection in the present study, no example stood out any clearer, in this regard, than my observations at a TPS division one day. While attended on a shift briefing to administer the survey instrument, for the first time I noticed several officers wearing an elongated piece of equipment with a yellow screen (or lens) on the front of the otherwise black device, which was mounted on the front of their body armour carrier (bullet proof vest) facing outwards. Having been a front-line supervisor myself only a few years earlier and, at that time, having to update my uniform equipment (with a telescoping baton instead of the old oak nightstick, a Taser, extra pistol magazines and holders, an LED flashlight, a pouch to carry protective latex gloves, a multi-tool and pouch, a device that functions as a seatbelt cutter and a leg restraint, and, of course, a mobile device carrying holster), I was curious about this piece of equipment that I was unfamiliar with and had not noticed previously on any other research participants. I was told that this is a bright LED light that can be activated by the officer and which projects a bright beam outward from the officer in a forward direction to illuminate the area immediately in front of them. Over the years, I had observed that officers often configure a small LED flashlight on their body armour carrier near the shoulder to illuminate the area in front of them for the purpose of writing in their duty notebook when it is dark and I assumed this was just a newer version of this idea.

However, after the shift briefing, several officers approached me and explained that, in addition to providing illumination of things in front of them from time to time, these lights are also effective in casting a bright beam of light from the officer toward anyone who might be videorecording them, which creates issues for all but the most skilled 
photographers - typically resulting in blurring of detail and creating a silhouetting effect in any footage. These officers explained that this was, in fact, the primary purpose of these lights and the rationale advanced was that officers were not fond of having their images on social media without their consent. After this initial exposure to these devices, I began to notice them more and more on officers' equipment at various data sites. Below is the picture that I took at that initial shift briefing. Beside it is a photograph published in the Toronto Star on 8 August 2017, accompanying a story about the depicted officer, TPS Constable Niran Jeyanesa, buying clothes for a shoplifter who stole the clothing for job interview (McQuigge 2018). The reader should note the device on the officers' uniform (to the right of the picture just under the ' $E$ ' in 'POLICE').

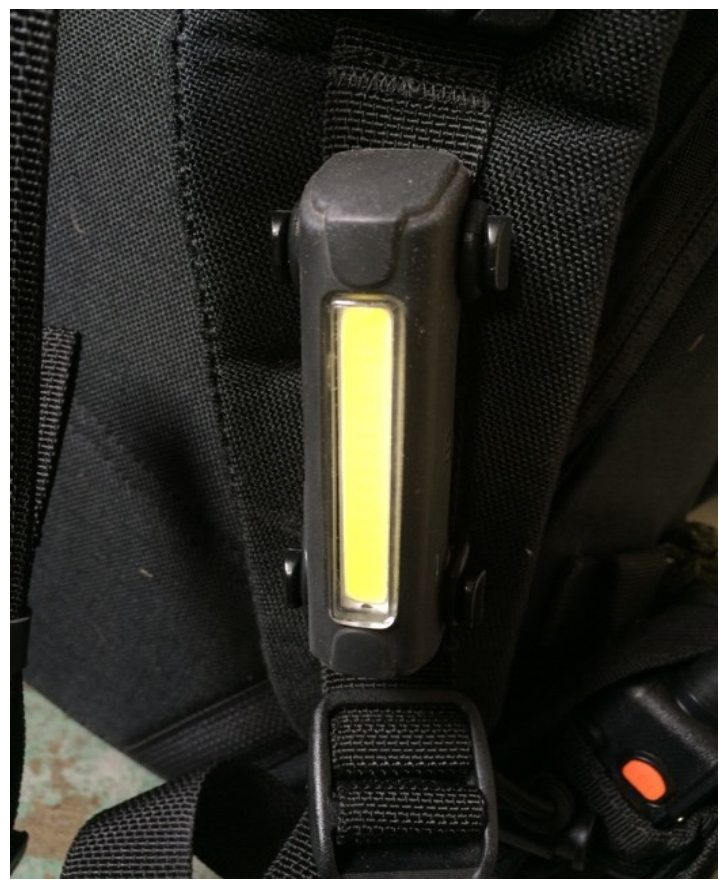

Image 17: LED light device (Brown 2016)

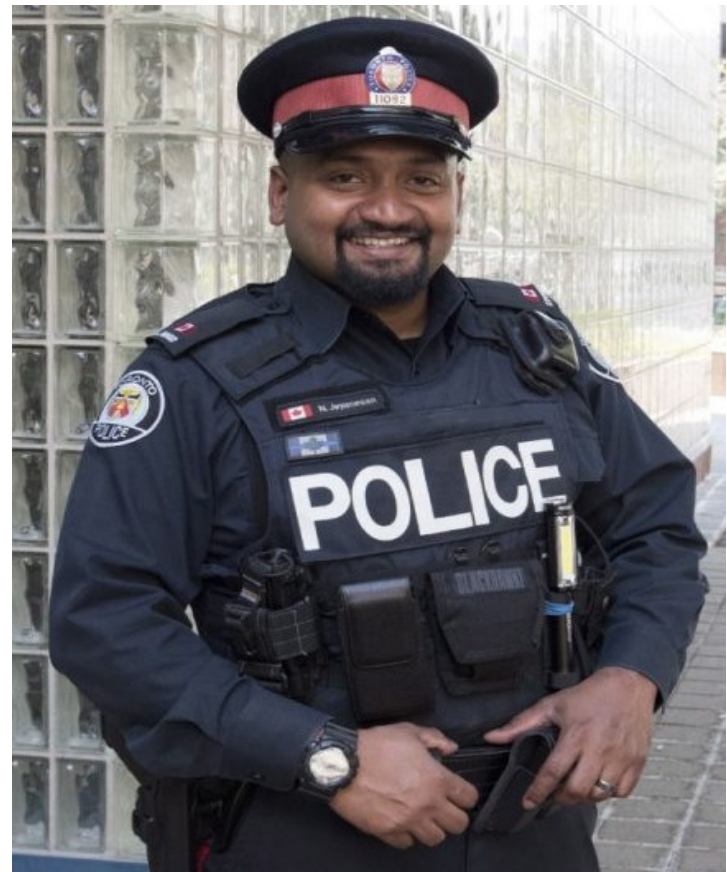

Image 18: Officer wearing LED light device on uniform (McQuigge 2017)

${ }^{11}$ Interestingly, of the 20 respondents in this identified subtheme ('wear uniform hat'), 13 are officers serving with the Hamilton Police Service. I suspect my former patrol Sergeant Mike Dunn will chuckle if he reads this dissertation, as on countless occasions he yelled at me (while I was working in uniform patrol duties in the community), 'Brown, put on your [insert one or more expletives] hat!'

${ }^{12}$ For this question, study participants were free to choose more than one of the listed items and to also particularize 'Other' circumstances - including an invitation to provide a written elaboration of such 'Other' circumstances. Percentages calculated (within the table) are in relation to those participants who answered 'Yes' to Question $25(\mathrm{~N}=3027)$ (column 3$)$ and also to the entire sample population in the study $(\mathrm{N}=3660)$ (column 4). 
${ }^{13}$ During qualitative data analysis it became apparent that officers serving with certain participating police agencies have more pronounced concerns around interacting with persons belonging to a specified 'minority community' than do officers serving with other police agencies - as demonstrated by their written elaborations to Question 25(a). The table below presents the circumstances that stand out in this regard (those with double-digit numbers in terms of percentage across participants from each police agency and in ascending order).

Table 59: Concerns around interacting with 'minorities' (by police agency percentages of qualitative responses)

\begin{tabular}{|c|c|c|c|}
\hline Participating Police Agency & 'Minority' Group & N & \% of officers \\
\hline Halifax Regional Police Service & Black persons & 24 (of 206) & 11.7 \\
\hline RCMP Red Deer & Indigenous persons & 10 (of 75) & 13.3 \\
\hline Rochester Police Department & Black persons & 38 (of 281) & 13.5 \\
\hline Windsor Police Service & Black persons & 20 (of 148) & 13.5 \\
\hline Syracuse Police Department & Black persons & 23 (of 168) & 13.7 \\
\hline Toronto Police Service & Black persons & 43 (of 310) & 13.9 \\
\hline Albany Police Department & Black persons & 22 (of 156) & 14.1 \\
\hline Buffalo Police Department & Black persons & 47 (of 317) & 14.8 \\
\hline Saskatoon Police Service & Indigenous persons & 26 (of 175) & 14.9 \\
\hline Yonkers Police Department & Black persons & 13 (of 51) & 25.4 \\
\hline
\end{tabular}

14 The value 3019 represents those participants who answered 'Yes' to Question 25 $(\mathrm{N}=3027)$ with the missing value for Question $26(\mathrm{~N}=8)$ subtracted.

${ }^{15}$ Similarly, the value 3640 (for the entire sample population) represents the total sample population $(\mathrm{N}=3660)$ with the missing value for Question $25(\mathrm{~N}=12)$ and Question $26(\mathrm{~N}=8)$ subtracted.

${ }^{16}$ Some other interesting observations shared by study participants are worth considering in this context (outside of the identified principal subthemes), such as:

It's the way of policing now (KIN41).

Avoiding conflict even though sometimes it's a necessary part of the job (KIN77).

Mistakes will happen as you learn a job - in policing with current trends it can be life changing (RID62).

It just exists as normal course of duty. Officers are smart and rarely take unnecessary risks today (SYR33).

${ }^{17}$ To appropriately characterize the nature of associations, in augmenting the reader's examination of study data represented in the tables (the raw frequencies) and the results of Pearson's chi square independence tests, Cramer's V was relied on as a measure of statistical power, which indicates the strength of an association between variables (how closely variables are related) (Cohen 1988; Cramer 1946). Cramer's V is based on measures of association ranging between 0 and 1 - with 0.0 corresponding to no 
association between the variables and 1.0 corresponding to a complete association between the variables (when the two variables are equal to each other). It is generally accepted, in terms of established cut points, that when the measure of $\mathrm{V}$ is between (a) 0 to .10, a weak relationship exists between the variables; (b) .11 to .29, a moderate relationship exists between the variables; and (c) .30 to 1, a strong relationship exists between the variables (Cohen 1988; Gall, Borg \& Gall 1996; Healey \& Prus 2016; Rutgers University 2003).

${ }^{18}$ In this regard, McElroy and colleagues (1999: 508) observe, "Defining career stage has engendered controversy in that some researchers advocate the use of age to define career stage, while others advocate occupational tenure, organizational tenure, or positional tenure." In the context of correctional officers, Lambert and colleagues (2017: 418) find, "Given that people can enter into the field of institutional corrections at different ages, tenure with the correctional agency, rather than age of the staff member or institutional tenure, was used to establish career stages (similar findings were advanced by Cohen 1991; Grifin et al. 2014; Ornstein et al. 1989...).”

${ }^{19}$ A contributing factor among 'rookie' non de-policers may relate to this group's familiary with the tremendous proliferation of camera phones throughout society, relative to officers with more seniority. In this regard, the impact of 'policing's new visibility' may not be as profound given that these officers are much more used to the presence of camera phones and the cultural practices associated with them (i.e. 'citizen journalism', 'participatory culture,' etc.). See Brown's $(2013,2016)$ study of 'policing's new visibility' in relation to police use of force and Sandhu's (2016, 2019; Sandhu \& Haggerty 2017) discussion of more junior officers' familiarity with the presence of cameras in their work environment and their adaptation through 'camera-friendly policing'.

${ }^{20}$ The following are further representations (but in no way should be interpreted as an exhaustive accounting) of de-policers in the study who, based on their responses to Question 28 in the questionnaire, do not appreciate that the behaviours they have reported in responding to other questions (primarily 16, 24 and/or 26) constitute the practice of de-policing. For example, ROC122 (a Rochester officer) who answered 'Yes' to questions 16 and 24 but 'No' to questions 27 and 28 and another Rochester officer (ROC134) who answered 'Yes' to questions 16, 24, and 26 but 'No' to questions 27 and 28. Also, an Ottawa officer (OTT211) responded 'Yes' to Question 24 and reported that she/he does not conduct traffic stops of racial minorities because of a concern that her/his actions could be criticized by some members of the public, however, this officer then answered questions 26, 27, and 28 in the negative. Based on their own response (as reported in Question 24) this officer is clearly engaging in depolicing behaviour (in not conducting traffic stops with person perceived to be a 'racial minority'), however, they apparently do not realize this amounts to the de-policing practices being interrogated in Question 26. Further representative examples include: 
Never heard of these terminologies (ALB14). This officer reported 'Yes' to questions $15,16,23,24$, and 26 (which, according to this study's classification protocol, categorizes this officer as an intensive de-policer), but 'No' to questions 27 and 28 .

I'm not sure what that refers to (HAM115). This officer reported 'Yes' to Question 26, but 'No' to questions 27 and 28.

Less proactive policing, more likely to just respond to calls (NIA114). As with ALB14 (above), this officer reported 'Yes' to questions 15, 16, 23, 24, and 26 (therefore, they are an intensive de-policer), but 'No' to questions 27 and 28.

I don't stop Black males, traffic or person stops (HAL66). Yet this officer answered 'No' to questions 27 and 28.

Only deal with people when required. Radio calls only (OTT160). Yet this officer answered 'No' to questions 27 and 28.

No proactive work with minorities (OTT270). Yet this officer answered 'No' to questions 27 and 28.

To survive in this anti-police environment today you cannot do certain things with visible minorities: proactive street checks, traffic enforcement, arrests unless for serious in-progress call. Just leave them alone and don't impact your career (OTT340). Yet this officer answered 'No' to questions 27 and 28.

In responding to Question 28, an Ottawa officer (OTT351) reported, Guys on my shift do, but I personally don't subscribe to it. However, this officer reported (in response to Question 24), [I don't conduct] street checks with visible minorities.

If you are White and do something to a Black person you are considered racist, so I avoid them unless necessary as part of a call I am sent on (OTT377). Yet this officer answered 'No' to questions 27 and 28.

Unless part of a reactive dispatched call we try to avoid anyone who is a visible minority, not White (RED13). Yet this officer answered 'No' to questions 27 and 28.

I will not do any proactive policing but avoid any contact with minorities, due to propensity to make false complaints against police because of their own racism versus police (TOR278). Yet this officer answered 'No' to questions 27 and 28.

Unless a radio call I am dispatched on I avoid minority races and EDP's acting out in public (TOR284). Yet this officer answered 'No' to questions 27 and 28.

Minority races and EDPs, dangerous for your job to do any extra interactions with these-ignore whenever possible (TOR297). Yet this officer answered 'No' to questions 27 and 28.

I avoid Blacks because of BLM crap. I want to keep my job and not be accused of racism (TOR269). Yet this officer answered 'No' to questions 27 and 28. 
No more stopping Black people in high risk areas (TOR301). Yet this officer answered 'No' to questions 27 and 28.

${ }^{21}$ Other than the standard application fee (in the range of $\$ 5$ to $\$ 25$ ), the WPS initially requested I pay additional costs associated with labour for the retrieval of the requested information, however upon my application for a waiver, pursuant to a provision in the applicable Manitoba FOI statute, they waived any additional costs. After more than six months of waiting on the disclosure of information requested from the TPS, on 11 June 2018 I received correspondence from 'Mr. P. McGee, Coordinator, Access and Privacy Section,' advising, 'Further to your access request under the Municipal Freedom of Information and Protection of Privacy Act, section 45 of the Act allows fees to be charged...the estimated fee for the records requested is $\$ 480.00$.' Begrudgingly, I paid these fees (that were reduced on my appeal to $\$ 240.00$ ).

${ }^{22}$ In terms of further confirmatory information, the following represent what I characterize as eight (8) corroborative observations in relation to the de-policing thesis I am advancing in this dissertation - in addition to those previously presented in Chapter One and those contained within the literature reviewed, as presented throughout Chapter Two. The sources of information are varied and, in my view, represent compelling endorsements of the validity and reliability of the present study's research and this study's key findings around de-policing in today's front-line police work. These are presented, as follows, in no particular order and should be understood by the reader as examples, rather than an exhaustive accounting.

(1) On 18 July 2017, I appeared as a guest for an episode of the $C B C$ Radio in-studio discussion and listener call-in program Ontario Today, which was addressing the theme, 'When police pull back: De-policing and the Ferguson effect.' Another guest on the program was Bruce Chapman, the president of the Police Association of Ontario, which is "the official voice and representative body for Ontario's front-line police personnel... provid[ing] representation, resource[s] and support for 53 police associations...

membership is comprised of over 18,000 police and civilian members of [Ontario's] police services" (Police Association of Ontario 2018). After listening to my comments on de-policing earlier in the program (discussing the preliminary analysis of data in this study) and being asked by the host, 'What are police officers telling you?', Chapman replied, "I am hearing the same...that officers are disengaging or not engaging when they should be." The host later asked Chapman, 'Do the findings of Greg Brown's analysis resonate with you, in particular police officers saying that they are trying to avoid getting on YouTube, avoiding confrontations that could involve public scrutiny, what are you finding?' His response was, "I'm hearing it, and it's startling when a study and surveys back those findings up. I believe that this is the new reality..."

(2) Peter Kirkham, a retired Chief Inspector with London's Metropolitan Police and frequent media commentator on policing in the U.K., observed, in a 1 March 2018 tweet in response to an ongoing international dialogue on Twitter in the wake of media reporting on this de-policing study, "Interesting research by @ gregoryroybrown on impact of excessive, overbearing scrutiny of cops in Canada...He finds there is a 'FIDO' 
effect...Fuck it, Drive on'...We are hearing of the same here...Maybe someone should replicate the research!"

(3) Superintendent Stu Betts, executive officer to the Chief of the York Regional Police Service (YRPS), Canada's fifth largest police agency - with 1586 officers serving a population of about 1.2 million (Police Resources in Canada 2017) and bordering the City of Toronto (to the north), wrote to me (via e-mail) after media coverage of this study's preliminary de-policing findings and he observed, "We here in York Region, typically enjoy a favourable public perception, but I am very aware that many of the sentiments you describe have infiltrated our ranks (why would we be any different). We are also contemplating at what stage the idealism that is present in all new recruits makes that all too predictable shift to the cynicism that is appearing with increasing certainty, earlier in an officer's career" (personal communication, 13 February 2018). Betts followed-up (after I sent him some preliminary and general study information), suggesting, "Your findings certainly support our anecdotal observations regarding that point at which an officer starts to exhibit signs of de-policing. I wonder if our (policing) coach officers (who are increasingly junior officers, and certainly with no more than 15 years on) contribute to an officer's move along the de-policing continuum by planting the seeds of that type of dissatisfaction" (personal communication, 15 February 2018).

(4) Martin Kaste (2015), host of the American National Public Radio program 'All Things Considered' advanced in his introduction to the discussion on de-policing, "When people use the term de-policing, it usually refers to inaction on the job." The show's guest, retired Seattle Police Department officer Mike Severance, presented his observations, "In the simplest terms...officers aren't doing proactive police work. They'll respond to their calls, you know, if something heinous happens ... if they observe an armed robbery in progress, an officer's going to do what needs to be done. But you're not going out looking for the bad guys" (Kaste 2015).

(5) Similarly, Peter Moskos, a former Baltimore police officer who is now an assistant professor at John Jay College of Criminal Justice, observed, "From cops I've spoken to [in Baltimore], they are saying, 'Yeah, we're not frisking people and we're not making discretionary arrests"” (Gately 2015).

(6) On 12 June 2018 I appeared as one of three 'academics' on an 'expert panel', at the Ontario Association of Chiefs of Police annual conference, discussing contemporary issues in policing that are impacting on today's police leadership. After my presentation on de-policing and a lively interactive discussion with the audience (of hundreds of Ontario police commanders), I was engaged in conversation by London (Ontario) Police Chief John Pare, who was interested in the research and who validated the study's preliminary de-policing findings - based on his own observations of the phenomenon among his front-line officers (personal communication, 12 June 2018). 
(7) In a 7 July 2018 guest column written for a national chain of Canadian newspapers (Post Media), Julian Fantino (Toronto's police chief from 2000 to 2005 and then Commissioner of the Ontario Provincial Police from 2006 to 2010) wrote,

Ironically, one of the most fundamental but hugely effective tools police use in the detection, prevention and solving of crimes is the proactive gathering of street level intelligence lawfully generated by frontline officers who are the eyes and ears on what's going on at the street level.

As expected, the over exercised and erroneously portrayed 'carding' situation is now having the predictable results, more political hand wringing and the rush to further hamper and discourage frontline police officers from engaging in anything proactive especially with members of the racialized community.

To better appreciate the reality of the current situation and to better understand the direct outflow of the hysterical 'carding' controversy, all that matters is numbers; negligible street contacts by frontline officers versus the escalation of gun play in the community. In essence, a chilling effect on frontline police officers - an emboldened upsurge of gunplay in the public spaces.

Some will discount the direct relationship that exists between proactive and passive policing about which I happen to know a little. I also speak with current serving officers, especially those on the front lines who speak volumes about their frustration and who feel unfairly maligned and erroneously portrayed as villains. So, while police street checks have dramatically decreased gun play has predictably gone the other way (Fantino 2018, emphasis added).

(8) Between 19 May 2016 and 14 August 2016, researchers from the (American) National Police Research Platform (with Professor Dennis Rosenbaum as principal investigator and Stephen Mastrofski, Susan Harnett, and Wesley Skogan as coinvestigators) interviewed, through an online survey instrument, 7,917 officers serving with 54 police and sheriff's departments across the United States - in collaboration with researchers at the Pew Research Center (see Morin et al. 2017). In their final report, the authors observed that the study was conducted "at a crisis point in America's relationship with the men and women who enforce its laws, precipitated by a series of deaths of Black Americans during encounters with the police that have energized a vigorous national debate over police conduct and methods" (Morin et al. 2017: 4). The most relevant findings of that study, vis-à-vis corroboration of the present de-policing study, are presented as follows.

Within America's police and sheriff's departments, the survey finds that the ramifications of these deadly encounters have been less visible than the public protests, but no less profound. Three-quarters [of participating 
officers] say the incidents have increased tensions between police and Blacks in their communities. About as many (72\%) say officers in their department are now less willing to stop and question suspicious persons. Overall, more than eight-in-ten $(86 \%)$ say police work is harder today...(Morin et al. 2017: 4, emphasis added).

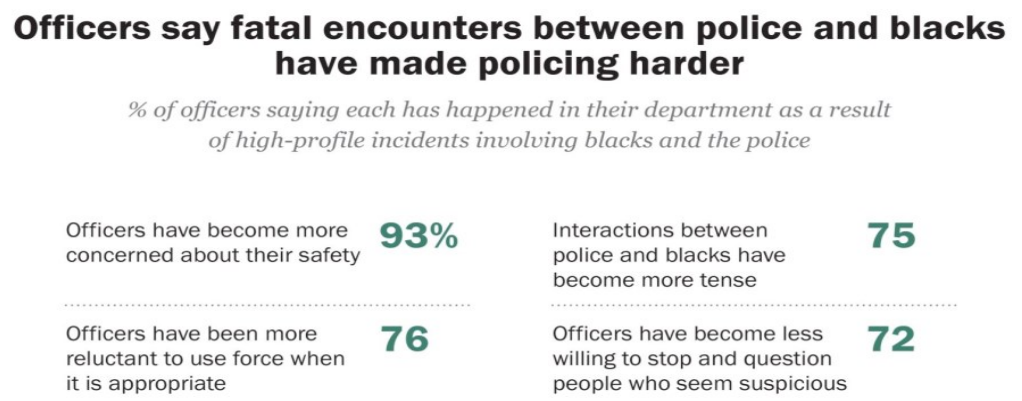

Source: Survey of law enforcement officers conducted May 19-Aug. 14, 2016.

Police work is a hard job that most officers say has become harder as a result of deaths of Blacks who died during encounters with police. Across every major demographic group analyzed for this survey, about eight-inten officers or more say these high-profile incidents have made policing more challenging and more dangerous. Overall, fully $86 \%$ of officers say their job is harder now as a result of these deadly encounters. Even officers from smaller departments that typically serve smaller communities say it's harder to be a police officer now; $84 \%$ of police in departments with fewer than 300 sworn officers say their job is more difficult now - and so do $89 \%$ of officers in big-city departments with 2,600 or more police (Morin et al. 2017: 64).

About seven-in-ten officers $(72 \%) \ldots$.. [report that]officers in their department [are now] less willing to stop and question people who seem suspicious. This finding raises the possibility that many officers are responding to [recent controversial] incidents by de-policing - that is, by not fully carrying out their law enforcement responsibilities out of fear of becoming involved in a high-profile incident. A larger share of White police officers (73\%) than Black officers (64\%) say their colleagues are now more hesitant to question suspicious people. In particular, White male officers are significantly more likely to say this (74\%) than White female or Black male officers (65\% for both) (Morin et al. 2017: 65, emphasis added).

Officers also are more likely on average than employed Americans overall to say their jobs frequently make them feel frustrated and somewhat less likely to feel fulfilled by their work. Half (51\%) of officers say their job 
nearly always or often frustrates them, compared with $29 \%$ of all workers...At the same time, about four-in-ten officers ( $42 \%)$ say their work frequently makes them feel fulfilled, compared with half of employed adults (52\%) who feel that way (Morin et al. 2017: 79-80).

${ }^{23}$ As written by Captain Jeff Rinaldo, the officer in charge of HQ Administration for the BPD, in his correspondence dated 21 December 2017,

The City of Buffalo Police Department is in receipt of your Freedom of Information Law request. Please allow this letter to serve as an acknowledgment of your request pursuant to New York State Public Officer's Law, Section 89, entitled Freedom of Information Law.

Although the Freedom of Information Law ("FOIL") is based upon the presumption of access to agency records, FOIL requests must reasonably describe the records sought so as to enable the agency to perform a diligent search. See Public Officers Law $\S 89(3)$. The Court of Appeals held that a request reasonably describes the record sought when agency staff has the ability, with reasonable effort, to locate and identify the records sought. See Konigsberg v. Coughlin, 68 N.Y.2d 245 (1986).

Your attached FOIL request has failed to satisfy the standard set under Konigsberg. The request fails to identify or reasonably describe the records sought, including the type of information that you are requesting and a time frame for our search. We are therefore unable to assist you with your request. Moreover, although your request does not reasonably describe the record sought, it appears likely that this department does not maintain some of the records that you seek...

${ }^{24}$ Should any reader have a concern around 'cherry picking' of data throughout subchapter 5.4, the following table presents data, in its entirety, as provided by the CPS pursuant to my FOI request. Readers can assess for themselves, but my observation is that for almost every category of officer-initiated (proactive) occurrences, the last year's (2016, or for some data 2014) numbers represent historic lows.

Table 60: All CPS officer-generated calls data

\begin{tabular}{|l||c|c|c|c|c|c|c|c|c|c|c|}
\hline & $\mathbf{2 0 0 6}$ & $\mathbf{2 0 0 7}$ & $\mathbf{2 0 0 8}$ & $\mathbf{2 0 0 9}$ & $\mathbf{2 0 1 0}$ & $\mathbf{2 0 1 1}$ & $\mathbf{2 0 1 2}$ & $\mathbf{2 0 1 3}$ & $\mathbf{2 0 1 4}$ & $\mathbf{2 0 1 5}$ & $\mathbf{2 0 1 6}$ \\
\hline Check on welfare & 347 & 336 & 356 & 327 & 371 & 383 & 337 & 406 & 425 & 355 & 379 \\
\hline Abandoned auto & 153 & 199 & 259 & 199 & 259 & 141 & 106 & 89 & 95 & 80 & 73 \\
\hline Disturbance & 610 & 653 & 628 & 668 & 718 & 661 & 577 & 505 & 460 & 429 & 300 \\
\hline Driving suspension & 1301 & 1308 & 1402 & 1407 & 1235 & 1246 & 1035 & 794 & 785 & 588 & 562 \\
\hline Drunk & 1146 & 1140 & 1308 & 1494 & 1713 & 1707 & 1505 & 1175 & 1030 & 633 & 535 \\
\hline Juvenile complaint & 343 & 284 & 300 & 276 & 233 & 182 & 140 & 84 & 60 & N/A & N/A \\
\hline Property damage & 278 & 224 & 307 & 287 & 283 & 235 & 255 & 195 & 149 & N/A & N/A \\
\hline Subject wanted & 5503 & 4986 & 5741 & 5682 & 5514 & 6054 & 5237 & 4613 & 3718 & N/A & N/A \\
\hline Impaired driver & 538 & 496 & 486 & 666 & 641 & 735 & 728 & 565 & 467 & 343 & 263 \\
\hline Noise party & 28 & 40 & 57 & 57 & 40 & 40 & 28 & 19 & 14 & 6 & 10 \\
\hline Prostitution & 242 & 183 & 326 & 776 & 773 & 468 & 443 & 431 & 371 & 65 & 33 \\
\hline Stop-traffic & 64848 & 61837 & 60959 & 70479 & 69917 & 63304 & 62912 & 53433 & 64143 & 65264 & 73977 \\
\hline Suspicious person & 9391 & 8718 & 9790 & 11141 & 10891 & 8192 & 6236 & 5215 & 4621 & 3842 & 4653 \\
\hline
\end{tabular}


25 The Toronto Police Service figures for 'street checks' relate to officers' submissions of documents (often termed 'carding' - the filing of a TPS208 Field Information Card) in relation to an officer's proactive observations of, or interaction with, an individual that, in the officer's opinion, should be documented for police intelligence purposes. 


\section{NOTES FOR CHAPTER SIX}

${ }^{1}$ I suggest that I am in the position to argue, with a solid empirical foundation in support, that de-policing is a largely universal phenomenon across today's rank-and-file police community. Even within the police agency with the lowest proportion of identified de-policers among the study's participating police agencies (the Red Deer RCMP) over half of the front-line officers (55.4\%) are practicing de-policing in one form or another. At the other end of the spectrum, eight in ten Buffalo (79.5\%) and Toronto officers (78.2\%) are de-policers and, across the entire research population, $71.7 \%$ of study participants are engaging in de-policing behaviours.

${ }^{2}$ I suspect that before they considered the materials presented throughout the preceding chapter, some readers may have been wondering about the selection of the rank-and-file police subculture as the primary theoretical framework to inform this study's empirical de-policing results. This, rather than what might have seemed like the more obvious choice - the 'risk society thesis' or one of myriad scholarly contributions that fall within the broad category of 'risk theory'. In reviewing the Handbook of Risk Theory (2012), which, over its 1187 pages, provides a good overview of the broad range of topics within what has come be considered 'risk theory', Doorn (2013: 1409) observes,

Risk is a central notion in a broad range of fields, varying from engineering to medicine, from life sciences to economics. It is also a topic being studied from different perspectives and by people with different disciplinary backgrounds. This raises not only questions related to the nature of risk - a question which may be answered differently by different people - but also fundamental questions related to the study of risks, risk perception, risk and society, and the acceptability of risks. These and other questions related to risks could be captured under the general heading of risk theory.

Briefly, and in relation to the role of theory in social research (in addition to the earlier discussion in subchapter 3.4), I suggest that, perhaps beginning with Parsons' (1938) foundational work, a theoretical framework, within social scientific research, has come to be understood as a theory or concept that is relied on by the researcher(s) to provide further explanation, interpretation, illumination, and in certain situations, prediction, in relation to empirical results (Abend 2008; Ravitch \& Riggan 2017; Weick 2014). In other words, theories provide explanations, or perhaps more nuanced understandings, of social phenomena beyond that which can be gleaned strictly from empirical data.

In this regard, it is clear from reviewing the literature, that risk, as a potential theoretical framework, can mean many different things to many different people in relation to the policing of society - including complex and abstract applications of various aspects of risk. For example, risk, as applied by Ericson \& Haggerty (1997) and risk, in its straightforward everyday application in the present study, are conceptually very different. As described throughout this endnote, risk as applied by Ericson and Haggerty (1997) and risk as applied in the present study are conceptually very different. Perhaps 
to confuse things even more, 'risk-based policing', which is now a popular consideration within police strategy discussions, is also a different conceptual application. As advanced by Kennedy, Caplan and Piza (2018),

Risk-based policing uses risk governance as a way of applying evidencebased practice to resource allocation and problem-solving...Risk-based policing uses the theory of risky places to identify locations where environmental factors interact to raise vulnerability and exposure to crime...High-risk places are forecast to experience the most crime, so they are the targets of police patrols and intervention activities.

My rationale in the choice of theoretical framework for this dissertation - front-line police subculture over risk - is two-fold and explained as follows.

First, the Oxford English Dictionary (2019) defines risk "as a chance or possibility of danger, loss, injury or other adverse consequences." In the context of the present study, the dimension of risk that is involved with front-line officers' perceptions of risk in their external techno-social and socio-political work environments, and their risk-averse attitudes and behaviours in response to those perceptions, relates to the potential for 'adverse consequences' to their policing career (i.e. the filing of complaints about their behaviour, investigations into their actions, allegations of misconduct, being subject to disciplinary sanctions, public humiliation, demotions, dismissal, etc.). Therefore, I suggest that the present study's understanding of risk, is, frankly, uncomplicated and not in need of elaborate explanations - certainly not in comparison to abstract risk considerations relied on as theoretical frameworks in informing other research findings, as evidenced throughout the social sciences literature. In other words, relying on a familiar and straightforward understanding of risk - a concern about the potential for 'adverse consequences' - as a principal theoretical framework, to inform research data and provide context and nuance to the results of an empirical study, would not add much to the present study's findings of widespread de-policing. In terms of such an understanding of risk, the identified de-policing phenomenon involves a majority of today's rank-and-file officers perceiving the possibility for 'adverse (occupational) consequences' in their external work environment and, therefore, these front-line officers adapt to these 'risky' circumstances by engaging in risk-averse practices (depolicing) to mitigate against the possibility of undesirable occupational consequences.

And, second, it became clear throughout the research process that the rank-and-file police subculture is a crucial consideration in appreciating the full picture of the depolicing phenomenon as it exists today. As demonstrated throughout this dissertation, de-policing is not just a front-line officer's individual adaptation to 'risky' circumstances that s/he perceives in their external work environment. Rather, in addition to each officer's individual risk considerations, the officer's interactions with colleagues in their close-knit work group and the influence of their rank-and-file subculture is, as described throughout subchapter (6.6), found to play a significant role in today's de-policing phenomenon - at the individual, group, and organizational units of analysis In this vein, in his analysis of significant reductions in police activity in 
Cincinnati following a fatal police shooting and subsequent rioting in 2001, Shi (2005:

19) suggested that "de-policing probably does not result from orders from above but rather from officer's own assessments of risk." In this regard, I should explain that at no point throughout data collection in the present study was it conveyed to me from any study participant, nor was it observed during my considerable time spent at the data sites, that any police administration mandates, or even tacitly sanctions, the practice of de-policing - excepting for some front-line supervisors (Sergeants and Lieutenants) that counsel their rank-and-file officers to, and/or informally endorse the practice of, depolicing. A veteran Buffalo Lieutenant typified the kind of acceptance of de-policing among front-line supervisory ranks I encountered at most of the study's data sites, observing,

Officers generally just do the minimum to stay out of trouble with our department (BUF157).

Therefore, as a theoretical framework, I argue that the rank-and-file police subculture is most ideal to inform this study's empirical results.

Moving on, as a sociologist educated and currently residing in Canada, scholarly discourse involving risk and the policing of society brings to most minds, in this country, Ericson and Haggerty's (1997) Policing the Risk Society. In what is widely acknowledged as a classic in the sociological and criminological literature, these Canadian policing scholars found, "Policing and the society in which it takes place are best understood in terms of the model of risk communications" (Ericson \& Haggerty 1997: 1). The central thesis of their book advanced that the function of police, in that era, prioritized collaboration and the sharing of knowledge (information gathered by the police with surveillance and data collection technologies) with other major institutions in the management of risk and establishment of security (Ericson \& Haggerty 1997). While I find their scholarship fascinating, Ericson and Haggerty's reliance on the 'risk society thesis' (Beck 1992a, 1992b, 1994; Beck, Giddens \& Lash 1994; Giddens 1990, 1991 ) as a theoretical lens, through which to advance understandings of the policing of Western democracies in the mid-1990s, is, as explained, a much different genus of the concept of risk than as it is understood within the present study.

In this regard, Beck and Giddens found that late modernity - embodying risk, reflexive modernization, individualization, and a broad societal disenchantment with major institutions throughout society, is

...characterized by a critique of the processes of modernity...now seen to produce many of the dangers or 'bads' from which we feel threatened. The central institutions of late modernity - government, industry and science are singled out as the main producers of risk. An emphasis on risk...is thus an integral feature of a society which has come to reflect on itself, to critique itself (Lupton 1999: 4).

The climate of doubt and fear that characterizes 'risk society', as described by Beck and Giddens, has resulted in an "increasingly endemic sense of insecurity" (Garland 2003:

78). Lupton (2006: 19) advanced "While most sociological research on risk has thus far 
focused on environmental, health and technological risks, a number of intriguing studies conducted by sociologists have examined other aspects of risk."

Along these lines, in their introduction to Risk and Morality, Ericson and Doyle (2003: 14) observe that the substantial social changes associated with the concept of risk, as it is often understood in the context of the traditional "risk society thesis', "perhaps" justifies the use of the term "risk society', "albeit in a way markedly different from Beck's use of it." This observation is just one example demonstrating the various dimensions of risk across differing conceptual applications. Further in this regard, Brown (2013: 119, citing Doyle 2007; Ericson \& Doyle 2003, Ericson \& Haggerty 1997; Garland 2003; Zinn 2008) finds,

Numerous scholars, from diverse theoretical and disciplinary positions, have conducted analyses of the sweeping social, political, and cultural dimensions of the 'risk society' and in doing so have applied a multiplicity of conceptions related to risk, which have become integral to a wide range of socio-political considerations (and sociological theorizing) in the contemporary world.

Further, from the front materials of Policing the Risk Society,

Richard Ericson and Kevin Haggerty contend that the police have become information brokers to institutions, such as insurance companies and health and welfare organizations whose operations are based on a knowledge of risk. These institutions influence the ways in which police officers think and act... These institutions are part of an emerging 'risk society' where knowledge of risk is used to control danger (Ericson \& Haggerty 1997: iv).

In her analysis of the book, O’Malley (2016) advances,

Policing the Risk Society remains a classic of criminological theory and has been of considerable importance to both risk and policing studies. Despite its influence, there has been no systematic research program established that has followed up its leads. Even its authors immediately moved to different fields and seemingly abandoned the 'risk society thesis' that was its theoretical core. In practice, most of its characterisation of contemporary police in the risk society has proven problematic: police have not become creatures of the insurance industry...Rather, the influence of risk - primarily in the massive field of traffic detection and enforcement regarding traffic offending - has been to shift much policing away from police per se, and on to electronic and computerised surveillance technologies.

Similarly, Rigakos (2002: 17) challenges the veracity of the claims of Ericson and Haggerty (1997) in relation to the impact of risk narratives on police actions and 
decision-making power in society and he takes issue with the characterization of the police as "automatons enslaved by risk information demands."

Therefore, despite the present study's finding that risk is a constituent element of the observed de-policing phenomenon, this dissertation does not borrow from Ericson and Haggerty's (1997) work, as I argue that while 'risk society' conditions are still a significant consideration in contemporary societal surveillance, visibility, scrutiny, and broader and more critical public engagement with policing (and other major institutions in society), the key aspect of the risk concept, for the purposes of the present study, is much different than that which Ericson and Haggerty applied to their analysis of policing more than 20 years ago. They found that risk was the principal driver of a transformative shift in policing from the pursuit of more traditional goals of crime control and order maintenance to circumstances in which the redefined primary role of the police was to leverage their strategic and privileged socio-political and legal positioning - as the preeminent institution in amassing security-related knowledge and disseminating this knowledge of risk to other institutions concerned with security - to function as the key facilitators of information in furtherance of the insatiable demands brought about by late modernity's preoccupation with security and the identification, prediction, and management of risk (Ericson \& Haggerty 1997; Hood 1997; Mythen 2014; O’Malley 2015).

In contrast, and in terms of risk as it should be understood in relation to the present study, it is in the sense of officers' assessments of the potential for professional consequences as a result of their occupation (i.e. being investigated for alleged misconduct resulting from a citizen's complaint about the officer's actions and/or a video recording purported to depict an alleged indiscretion by the officer), which might be visited upon them because of how they perform their front-line police work within today's external policing environment - or more accurately, how their performance of police work is subjectively interpreted by today's public audience, featuring unprecedented visibility of police officers' actions in the field and heightened societal scrutiny and critique of policing. Waddington (1992: 301-302, citing Bayley \& Bittner 1984; Chatterton 1979, 1983; Norris 1989; Van Maanen 1973, 1974, 1975; Waddington 1994) assists in furthering this understanding with his observations in this regard,

Exercising coercive authority over fellow citizens may be injurious to the individual officer...the truly ever-present danger [as opposed to the relatively remote possibility of an adversary inflicting injury] lies in taking action that is judged improper. Policing is a "punishment-centred bureaucracy' in which officers are rarely praised for good practice, often because it is invisible to the organization, but face draconian penalties if they are deemed to have behaved improperly...Police officers are acutely aware of this, hence the observed preoccupation with avoiding and minimizing 'in the job trouble'. It gives rise to the defensive solidarity of the lower ranks who perceive every action they take to be fraught with danger and hence adopt the cynical posture of 'lay low and don't make waves.' 
Along the same lines, risk in the present study should be understood in terms of the hazards of exposure and detection of misconduct (actual or alleged) and of the danger of misunderstandings or mischaracterizations of officers' behaviour. This includes the existential sense of insecurity these potential outcomes may invoke in the mindset of today's front-line officers and the precautionary logic and responses to perceived threats that dominate contemporary approaches to risk management. In this regard, Ewald (2002: 286-287) is instructive with his findings that,

The precautionary hypothesis...implies that in a context that is always relative, it will be necessary to take into account what one can only imagine, suspect, presume, or fear...The institutionalization of precaution invites one to consider the worst hypothetical (defined as the serious and irreversible consequence) in any decision...Precaution designates, first and foremost, a logic of decision to act or not to act, to undertake or not to undertake. It complicates this by introducing the dimension of uncertainty, based on the worst hypothetical.

In this same vein, Furedi (2009: 197) suggests this form of risk assessment can "encourage the dramatization of uncertainty...[and instill] a precautionary response to threats." In this risk context, I advance that the de-policing practices of today's rankand-file officers can be situated, on both empirical and theoretical planes, as 'precautionary responses' to 'dimensions of uncertainty' in today's techno-social and socio-political police landscape, involving the 'logic of decision' not to undertake certain discretionary actions in one's front-line police work.

Beck (1992a, 1992b, 1994) found, and various sociologists have since theorized, that the population's heightened awareness of risk now constitutes an important feature of contemporary society and this development has instigated significant social changes. This risk awareness across the population has presented as a sense of "considerable anxiety" that is related to "increased social expectations, decreased levels of trust, and new sources of insecurity" (Garland 2003: 70-71). Like these macro-level transformational developments throughout society, implicating diminished trust in, and elevated skepticism of, major institutions - including policing - that are often discussed as consequences arising from the onset of late modernity and within the framework of the 'risk society' (Chan 1999; Cockcroft 2013; Ericson \& Haggerty 1997), I argue that the present study's empirical results demonstrate that front-line police officers are now responding to the public with less trust and more skepticism, through various behavioural adaptations to societies' increased social expectations, decreased levels of trust, and new sources of insecurity.

In this regard, societies' major institutions have come under increasing scrutiny from a more demanding and less compliant public (Cockcroft 2013; Loftus 2009), that exhibit a broad disenchantment with, and pervasive distrust of, those institutions - including the police, which has led to demands for, among other things, more accountability and transparency from the state and its institutions (Bradford et al. 2014; Cockcroft 2013; Doyle 2006a, 2007, 2011; Ericson \& Doyle 2003, Ericson \& Haggerty 1997; Garland 
2003; Ivkovic 2008; Jackson et al. 2012a; Lievrouw 2011; Tulloch 2008; Uslaner 2002). Along the same lines, it seems clear that today's rank-and-file officers are concerned that some aspects of their job hold the potential for scrutiny, misinterpretation, and public complaints and therefore, they are engaging in risk-averse policing (various manifestations of de-policing).

In support of this argument, I took the photograph reproduced below on 30 March 2016 while in the patrol briefing room of the Syracuse Police Department. I suggest that not only does it demonstrate rank-and-file officers' appreciation for the significance of 'policing's new visibility' and today's techno-social landscape for front-line police work, but I advance that it is also interesting from a risk perspective - with 'Try[ing] not to end up on YouTube' in only a slightly subordinate position to the paramount risk consideration for any police officer - to 'get home safely' at the end of shift - and to the exclusion of any other imperative concerns that might arise throughout the shift.

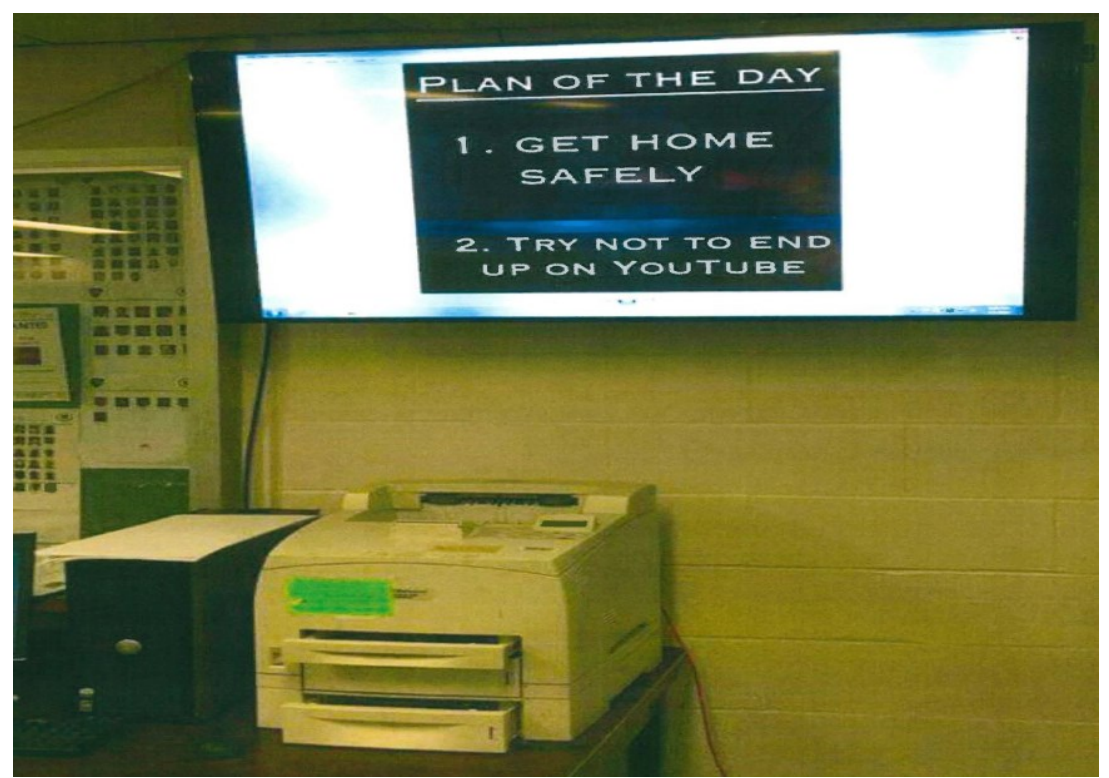

\section{Image 19: Video display in Syracuse Police briefing room} (Brown 2016)

Along these lines, and in employing a rhetorical play on Garland's (2003) words, I argue that awareness of, and concerns about, risk in today's techno-social and sociopolitical external policing landscape has presented as a sense of 'considerable anxiety' across the population of rank-and-file officers that is related to 'increased social expectations, decreased levels of trust, and new sources of insecurity' and many frontline officers have adapted through the adoption of various de-policing practices. In addition to the present study's empirical findings along these lines, Campeau (2016) also observes,

Officers sense they are very much alone in this work...another way in which intensifying forms of oversight and accountability are influencing how officers interpret their work is through the emphasis it forces officers to place on managing risk. And I don't mean in the usual sense of 
managing crime and disorder but rather in the sense of managing themselves and their own vulnerability in navigating certain situation. So, officers reported general feelings of wanting to disengage to avoid possible reprisals.

In further reflecting on today's techno-social and socio-political circumstances in relation to front-line policing, it is interesting to consider Furedi's (2009) observation that one of the by-products of knowledge is risk. Similarly, both Beck (1992a, 1992b, 1994) and Giddens (1994) find a close association between apprehension of risk and an increase in knowledge. In this regard, Giddens (1994: 185) finds, "Many of the uncertainties which face us today have been created by the very growth of knowledge" and Beck (1992a: 183) argues, "The sources of danger are no longer ignorance but knowledge." In other words, knowledge creates both new hazards and an awareness of their risks (Furedi 2009: 201). In the context of the present study's empirical findings, I advance that today's knowledge regarding mis/conduct of police officers in the field, now possessed by many across the public sphere and engaging each individual's subjectivities - through contemporary developments in visibility, 'citizen journalism', social networking and communications interconnectivity, 'participatory culture', external scrutiny and oversight, and new social movements - present to many individual front-line officers, and, more broadly to the collective rank-and-file subculture, as potentially threatening or 'risky' knowledge, which, therefore, encourages precautionary responses in the form of de-policing practices.

In this regard, while not a large percentage of the population is active, in terms of direct participation in new socio-political movements focused on policing issues (such as Black Lives Matter, CopWatch, Campaign Zero, Cop Block), with today's unprecedented exposure to mediated accounts of controversial policing occurrences (through traditional and social media, the 24/7 news cycle, and online interconnectivity - discussed in detail below in Endnote 33), many more citizen than only a few years ago are now aware of issues (real, apparent, possible, or alleged) in police-citizen interactions and the views of activist groups in this regard. Such developments are considered within the 'participatory culture' literature, which was discussed previously in Endnote 3 to Chapter Three. In other words, it is now the case that a vast audience across the contemporary public sphere is frequently exposed to negative discourse about the police and the propagation of the idea that society is witnessing a groundswell of opposition to the police. Hence, why so many front-line officers $(94 \%$ of the participants in the present study) find that the public is more critical of the police today than ever before.

As described throughout this dissertation, I argue that this risk aversion across today's front-line police work (manifested through practices of de-policing) implicates the convergence of two contemporary phenomena in the external techno-social and sociopolitical policing landscape - principally, the increased visibility of rank-and-file officers' actions in the field and today's more pervasive societal concern with, and criticism of, police practices. 
${ }^{3}$ As discussed in Chapter Five and depicted in Table 32, even $60 \%$ of 'New' officers (those with 1 to 5 years of front-line experience) report engaging in de-policing. The proportion of de-policers increases thereafter (to between $76 \%$ and $79 \%$ of study participants) in relation to front-line service spanning from 6 years to 25 years. Interestingly and inexplicably, among officers with more than 25 years of front-line experience, only $68 \%$ report engaging in de-policing practices in their police work.

${ }^{4}$ Subsection 34(1) of Ontario's Liquor Licence Act makes it an offence for a person to be intoxicated in a public place or common area and arrest powers are granted to police officers in enforcing this statute.

${ }^{5}$ The following is excerpted from the OPS Review of Cellblock Operations (White 2011):

Dealing with people who are found drunk in a public place and who pose a safety concern, is detailed in Intoxicated Persons, Policy No. 10.05. The new changes give officers clearer guidelines when dealing with possible intoxicated persons, and alternatives to taking the person into custody are given. A section has been added that outlines four criteria for arrest, namely that: the person is intoxicated; the person is in a public place; there is a safety concern; and less intrusive means of ensuring their safety are not available. Behavioural and physical indicators are listed and defined, to assist officers in assessing these cases.

${ }^{6}$ This did not go over well with one citizen who summoned me, as the on-duty patrol supervisor, to a location where a severely-intoxicated individual had vomited on the windshield and then laid across the hood of his new BMW automobile and he wanted answers as to why the attending officer had explained that he could not convey the individual to jail but rather would caution the person to try to refrain from such conduct in the future, which was obviously going to fall on deaf ears as the individual in question is a chronic homeless alcoholic, who engages in such behaviours daily in that neighbourhood. In terms of less anecdotal data, throughout 2010 Ottawa Police Service (OPS) officers made 1777 arrests for public intoxication, however for 2011 this number dropped to 560 arrests (CBC News 2012). In a 2014 conversation on this topic with OPS Superintendent Mike Flanagan (now retired), who was the executive command officer in charge of cellblock operations, he advised that "drunk arrests" coming into the OPS cellblock continue to be "way down" from historic 'pre-Bonds' numbers because officers do not want to take the risk of being accused of misconduct. As Flanagan explained, it is easier and far less risky for an officer to simply conclude (in many instances disingenuously) that the individual does not meet one of the prescribed criteria in the new policy - determinations of 'intoxication' and 'safety concern' are obviously subjective in nature.

${ }^{7}$ I take some issue with Waddington's (1999: 289) reliance on Holdaway's (1983) insider research observations around the exaggeration often accompanying officer's relating of 'stories' (in my observation a common phenomenon regardless of 
occupation) as "evidence" to support his finding around a "gap between canteen chatter and the reality it purports to depict." Similarly, I find the contribution of Silver and her colleagues (2017: 1297, citing Engel \& Worden 2003), in this regard, to be ambiguous and not particularly helpful in resolving the debate, "Although it generally seems reasonable to expect behaviour to conform to attitudes, this is not always the case among police.'What does 'not always the case' mean? To what population are police being compared?

${ }^{8}$ The reader should understand that I consider my review of this genre in the literature thorough, but not exhaustive, and therefore there could be other studies that inform this topic that are not captured here.

${ }^{9}$ Professor Scott Phillips is a former Houston police officer and he is co-editor of the book Change and Reform in Law Enforcement: Old and New Efforts from Across the Globe (2017).

${ }^{10}$ Levinson's theory advances the existence of four distinct stages between ages 20 and 45 - each with their own developmental tasks and related behaviours and attitudes. "The central thesis of this model is that people...no matter what occupation or background, will grow through specific life stages during where there are different crucial activities and psychological adjustments that must be completed" (Ornstein, Cron \& Slocum 1989: 118).

${ }^{11}$ More recently, Savickas, Briddick, Watkins \& Edward (2002) advanced similar findings to those of Super. In relation to the duration of career stages in policing, while subject to fluctuation in 'real world' applications, they are often conceptualized in 5year increments of work experience (e.g., see Barker 1999; Johnson \& Lafrance 2016; McElroy et al. 1999).

12 In saying this, previous research found that police officers' attitudes and personality characteristics vary as a function of time spent 'on the job' (e.g., see Evans, Coman \& Stanley 1992; Hillgren \& Bond 1975; Rabinowitz \& Hall 1981; Rogers 1991; Symonds 1972; Violanti \& Marshall 1983).

${ }^{13}$ Barker (1999), who engaged in 18 years of ethnographic research within the LAPD, found that both officers attitudes and behaviours evolved as they progressed through career stages, with arrest productivity declining significantly after the 'rookie' stage. Brandle, Stroshine and Frank (2001), Brown (2005), Johnson (2006), and Mastrofski, Snipes, Parks and Maxwell (2000) found similarly, with officer tenure being negatively associated with arrest productivity.

14 This involved a small number of officers engaged in field training at their departments but no recruits in attendance at the police academy were sampled.

${ }^{15}$ In relation to the 'continuum of compromise', Gilmartin and Harris (1998: 25) find, 
A problem occurs when a sense of victimization and over-identification with the job sets into motion the dilemma of loyalty versus integrity. Here is where an officer called in to Internal Affairs and asked questions about another officer might find himself/herself lying to the investigator, often about a minor issue. An officer who develops a significant sense of victimization and intense resentment toward supervisors and administrators for controlling his/her job-role may be confronted with another loyalty dilemma: a sense of entitlement; entitlement suggests that a police officer, both on-duty and off-duty, acts as if the law and standards of ethical behaviour do not apply to him/her. In the final stage of the continuum of compromise, an officer may rationalize criminal behaviour.

In sociology, the conceptual framework of a continuum is often advanced in discussions around agency and structure, which are "poles bracketing a continuum, with social entities moving along this continuum over time" (Fuchs 2001: 24). In criminology, Moyer (1996) relied on Cavan's (1961) conceptual model of the "continuum of behaviour' to broaden contributions to criminological theory.

Within Canadian and American policing, the concept of continuum often involves guidelines around officers' use of force, which is to be considered on a continuum. For example, Ontario's Police Use of Force Continuum is depicted graphically below.

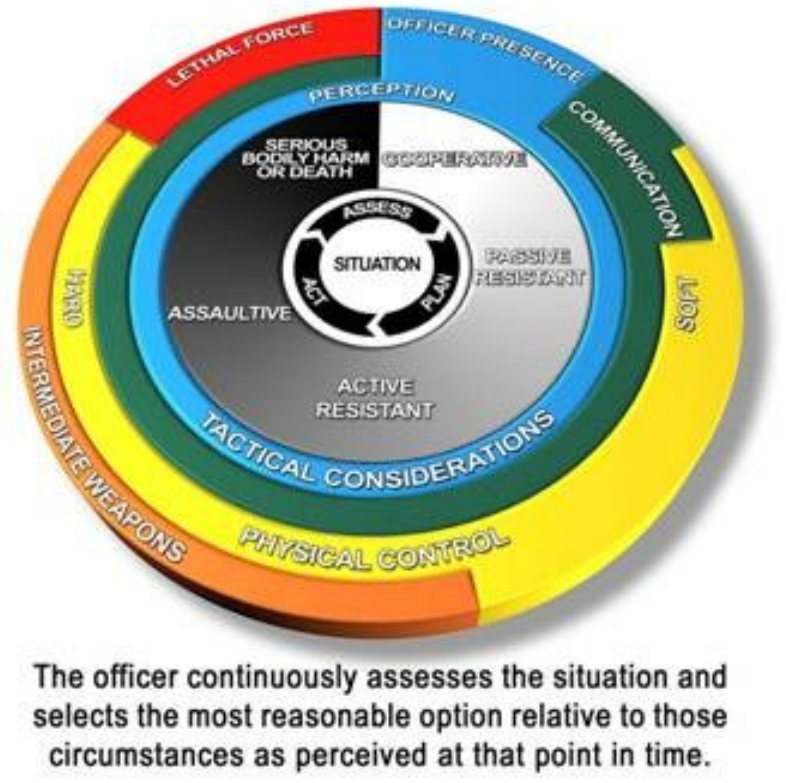

Figure 15: Ontario's Police Use of Force Continuum

${ }^{16}$ Table 32 demonstrates the variability in de-policing practices among front-line officers, when considered in relation to the various police agencies involved in the present study. If the de-policing continuum was a precise model, rather than an instructive conceptual framework, one would expect to see consistent correlations between the degrees of de-policing behaviour (non + limited de-policers versus 
moderate + intensive de-policers) and the levels of front-line police experience (the ratio of 'New' to 'Experienced' officers). In other words, it would be the case that police agencies with higher proportions of 'New' officers than the average across all participating agencies (30\%) should exhibit less de-policing, in terms of degree, as represented by the grouped variable 'moderate + intensive de-policers,' than the average across all participating agencies (54\%). Conversely, agencies with higher proportions of 'Experienced' officers should experience more officers practicing moderate + intensive de-policing (rather than none + limited de-policing).

This 'model' holds true across 13 of the study's 23 participating police agencies. For example, Delta Police deploys 'New' officers as $45 \%$ of their front-line complement (versus the study's average of $30 \%$ ) and only $36 \%$ of Delta officers practice moderate or intensive de-policing (versus the study's average of 54\%). However, in the case of 10 participating agencies there are contradictions observed in relation to such a 'model.' Four (Albany, Buffalo, Calgary, and Saskatoon) deploy higher proportions of 'New' officers than the study average, but higher proportions of officers in those agencies are practicing moderate or intensive de-policing than the study average. On the other hand, six (Cornwall, Kingston, Ottawa, RCMP Codiac, Stratford, and Yonkers) deploy higher proportions of 'Experienced' officers than the study average but lower proportions of officers in those agencies are practicing moderate or intensive de-policing than the study average.

${ }^{17}$ Of course, there are always exceptions and I have met a few officers that have done exactly this during my interactions with officers during this study and throughout my own policing career.

${ }^{18}$ As depicted in Table 34, while 83\% of all study participants feel that there exists an elevated risk of criticisms of, or complaints about, their police work that is related to particular 'demographic' characteristics presented by individuals with whom officers might interact in the community, as one would expect within the conceptual framework of a de-policing continuum, non-depolicers are represented at $65 \%$, limited de-policers at $79 \%$, moderate de-policers at $86 \%$, and all intensive de-policers $(100 \%)$ perceive such a risk. Similarly, as depicted in Table 37, only $45 \%$ of non-depolicers are familiar with the concept of de-policing whereas $56 \%$ of limited de-policers, $62 \%$ of moderate de-policers, and $82 \%$ of intensive de-policers understand de-policing. Also, consistent with the understanding that de-policing attitudes and behaviours develop with rank-andfile experience, while less than half of the officers with five years or less of front-line experience $(47 \%)$ are familiar with the concept of de-policing, seven in ten of their colleagues with six or more years of front-line experience are knowledgeable in this regard.

${ }^{19}$ Paoline and Gau (2018: 671, citing Paoline \& Terrill 2014) find that early ethnographic studies of front-line police culture (e.g. Skolnick 1966; Westley 1953, 1970) documented the monolithic model, which advanced that "officers uniformly endorse a single way of adapting to the strains of the occupation," whereas "subsequent typology research (e.g. Brown 1988; Worden 1995b) and more recent quantitatively- 
driven classification schemes (e.g. Cochran \& Bromley 2003; Jermier et al. 1991; Paoline 2001, 2004) have informed the [cultural heterogeneity model]."

In this regard, the 'classic' police culture studies and many contemporary investigations into the inner workings of officers' occupational culture(s) typically involve(d) ethnography and qualitative methods. While taking nothing away from those research methods, I advance that a mixed methods approach to developing greater understandings around the influence of contemporary rank-and-file subculture on frontline officers' behaviour in the field is equally appropriate. As described within subchapter 4.2, mixed methods allow for a degree of measurement, in terms of the breadth (or prevalence) of the phenomenon, in this case, de-policing, through the use of quantitative methods, while also benefitting from the in-depth and descriptive data produced through qualitative methods. Along these lines, Johnson and colleagues (2007: 123) describe mixed methods as, "[Research that] combines elements of qualitative and quantitative research approaches...for the broad purposes of breadth and depth of understanding and corroboration."

${ }^{20}$ Eugene Paoline III is Professor of Criminal Justice and graduate programs director at the University of Central Florida. In 2000, he completed a PhD in criminal justice at the University at Albany, where he was supervised by Professor Robert Worden. It is interesting to note that I also worked with Professor Worden during my Fulbright visiting researcher fellowship at the University at Albany throughout 2016-2017. From my review of the contemporary police occupational culture literature, it seems clear that Paoline is almost certainly the most prolific scholar in this area of research in the past twenty years. Professor Paoline and I have had the opportunity to discuss our mutual research interests on a few occasions, most recently in person at the November 2018 annual meeting of the American Society of Criminology in Philadelphia and since via email correspondence.

For a detailed appreciation of Paoline's empirical and theoretical contributions to contemporary understandings of police occupational culture, which is well beyond the scope of this dissertation, the reader should refer to Paoline's dissertation research work (2000), the book-length presentation of his doctoral research (2001), and his various other published works on the topic (e.g., see Ingram et al. 2013, 2018; McCluskey et al. 2005; Paoline 2003, 2004; Paoline \& Gau 2018; Paoline et al. 2000; Paoline \& Terrill 2005, 2014; Terrill \& Paoline 2015; Terrill et al. 2003, 2016).

${ }^{21}$ Waddington (1999: 288, citing Manning 1989) finds, "In common with general sociological and anthropological definitions of culture," police occupational culture "refers to the accepted practices, rules, and principles of conduct that are situationally applied, and generalized rationales and beliefs." As presented earlier, in applying various scholars' definitions to the present study, I suggest that front-line police subculture should be understood as the shared values and attitudes, common understandings, agreed upon informal 'craft rules,' and principles of conduct that are situationally applied and that inform officers' actions in the field. 
${ }^{22}$ Within the literature, similar observations around diversity in officers' coping mechanisms, in terms of attitudes and behaviours, have been found by other policing scholars (e.g., Campeau 2015; Chan 1996, 1997; 2007; Chan et al. 2003; Cochran \& Bromley 2003; Jermier et al. 1991; Paoline 2001, 2004; Paoline et al. 2000; and Terrill et al. 2016).

${ }^{23}$ Paoline and Terrill (2014: 130) assess that Van Maanen's two-and-a-half-year longitudinal and participant observation study, which found four distinct stages of socialization for police officers, is the "most eloquent study of police socialization." Van Maanen (1974) identified four stages a police officer goes through as he or she is initiated into policing and continues in this career through the years. The pre-entry 'choice' stage is the period when the individual selects policing as their career choice and applies to become a police officer. The second stage is the 'introduction', when the recruit attends the police academy for formal institutionalized training. The 'encounter' is stage three and involves the the initial teachings of the recruit's field training officer and his or her patrol officer colleagues. During this stage, the new officer experiences what police work is really like. The final stage, termed 'metamorphosis', is the period when the officer adjusts to policing having discovered the true realities of police work.

${ }^{24}$ Paoline and Terrill (2014: 8, citing Skolnick 1966; Van Maanen 1973), in considering the police officer's 'working personality', observe that "the police personality develops as a function of the conditions that occupational members confront on the job." In this regard, Marier and Moule (2018) find, "Prior research on police culture suggests that officers' perceptions of public support influence the policeman's working personality."

${ }^{25}$ In relation to de-policing and those I have characterized as 'lazy' officers from an earlier era in front-line police work, Van Maanen (1973: 415) observed almost fifty years ago, "The young patrolman discovers that the most satisfying solutions to the labyrinth [of challenges in police work] is to adopt the group norm stressing staying out of trouble. And the best way in which he can stay out of of trouble is to minimize the set of activities he pursues." Also, in this vein, Ericson (2007b: 377, emphasis added) advanced,

Consider a police officer in her patrol car who sees another car being driven along the road. What does she do about it? The possibilities are many: nothing, follow the car and check for speed; run a computerized check on the vehicle and its owner...stop the car and question the driver; search the car; search the driver; check the driver's documents; run a computerized check on the driver; give the driver a Breathalyzer test; ask the driver for information about other suspects; and so on. These are possibilities she learns on the job. Initially, a training officer accompanies her to a variety of situations to help her learn from different experiences. When the training period is over, she continues to share experiences with colleagues. 
${ }^{26}$ Emsley (2014: 11) takes exception to the attribution of these policing principles to Peel, arguing,

There is no evidence to suggest that they were written in 1829 , let along by Peel, or by either of the first two Commissioners of the Metropolitan Police. Rather, they were given their first significant formulation in the work of Charles Reith, writing more than a hundred years after the first Metropolitan Police constables took to the streets of London.

${ }^{27}$ The following are the nine 'Peelian principles' of policing (Myhall 1985: 425-426):

1. To prevent crime and disorder, as an alternative to their repression by military force and severity of legal punishment.

2. To recognize always that the power of the police to fulfil their functions and duties is dependent on public approval of their existence, actions and behaviour, and on their ability to secure and maintain public respect.

3. To recognize always that to secure and maintain the respect and approval of the public means also the securing of the willing cooperation of the public in the task of securing observance of the law.

4. To recognize always that the extent to which the cooperation of the public can be secured diminishes, proportionately, the necessity of the use of physical force and compulsion for achieving police objectives.

5. To seek and preserve public favour, not by pandering to public opinion, but by constantly demonstrating absolutely impartial service to law, in complete independence of policy, and without regard to the justice or injustice of the substance of individual laws, by ready offering of individual service and friendship to all members of the public without regard to their wealth or social standing; by ready exercise of courtesy and good humour; and by ready offering of individual sacrifice in protecting and preserving life.

6. To use physical force only when the exercise of persuasion, advice and warning is found to be insufficient to obtain public cooperation to an extent necessary to secure observance of law or restore order; and to use only the minimum degree of physical force which is necessary on any particular occasion for achieving a police objective.

7. To maintain at all times a relationship with the public that gives reality to the historic tradition that the police are the public and that the public are the police; the police being only members of the public who are paid to give full-time attention to duties which are incumbent on every citizen in the interests of community welfare and existence.

8. To recognize always the need for strict adherence to police- executive functions, and to refrain from even seeming to usurp the powers of the judiciary of avenging individuals or the state, and of authoritatively judging guilt and punishing the guilty. 
9. To recognize always that the test of police efficiency is the absence of crime and disorder and not the visible evidence of police action in dealing with them.

${ }^{28}$ Citations in support of this observation are located on pages 8 and 9 in Chapter One.

${ }^{29}$ As an example, the current organizational motto of my former primary police agency, the Ottawa Police Service, recognizes the idealized fundamentals of the CBP philosophy, with the police situated as, 'A trusted partner in community safety'.

${ }^{30}$ Citations demonstrating scholarly support for these assertations are located within Endnote 27 for Chapter One.

${ }^{31}$ There is essential consensus among policing researchers that citizen perceptions of police trustworthiness and legitimacy correspond to the willingness of residents to cooperate with police and comply with the law (Bradford et al. 2009; Callanan \& Rosenberger 2011; Frank et al. 2005; Gallagher et al. 2001; Hawdon 2008; Hawdon et al. 2003; Hough et al. 2010; Loader 2014; McCluskey et al. 2014; Stoutland 2001; Sunshine \& Tyler 2003b; Tyler 2004, 2006b; Tyler \& Huo 2002).

${ }^{32}$ Research has demonstrated a hierarchical power imbalance between the police and citizens (e.g., see Doyle 2003; Ericson 1982, 1993; Reiner 2010; Newburn \& Reiner 2012) and as Goldsmith (2005: 445) suggests, "[Policing powers] are not distributed evenly across the population, hence the privileged position and power of the police. This places them in a position of formal trust, whether or not their actions accord with their official responsibilities." As characterized by Hough (2003: 146-147), "The police function depends critically on the authority that the police can command, rather than the force that they can deploy as a last resort." In this vein, Rosenbaum and his collaborators (2005: 344 ) suggest that, "The public's trust and confidence in the police is considered the cornerstone for public cooperation and the basis for police legitimacy in a democratic society."

Legitimacy is a political construct (Loader \& Sparks 2013) that rests on recognition by the governed in the legality of enacted rules, the right of those in authority to issue commands (Bottoms \& Tankebe 2012; Bradford et al. 2014; Hawdon 2008; Luna 2000; Tankebe \& Leibling 2013; Tyler \& Jackson 2013) and also on people's perceptions that rules and commands are appropriate and ought to be followed (Tyler 2006b; Tyler et al. 2007; Zelditch 2001). In this regard, the subjective perceptions and understandings of individuals are integral to the concepts of legitimacy and accountability (Bradford et al. 2014; Hough et al. 2010; McCluskey et al. 2014; National Research Council 2004; Tyler 2006b; Tyler et al. 2007). Along the same lines, Bradford and colleagues (2014: 80 ) find, "Any consideration of policing policy and practice must encompass the legitimacy of the police in the eyes of the policed... Individuals in society confer authority to institutions, believe they are morally and normatively justified to hold power, and judge that institutions respect the rule of law. Theorists from Weber onwards have viewed legitimacy as a vital component of social institutions." Police 
agencies are public institutions (Crank 2003; Vitale 2005), which compel them to seek legitimacy in the arena of public opinion. This makes them subject to deliberative contestations, emanating from the public sphere, over the legitimacy of their practices (Jefferson 2013; McCluskey et al. 2014).

Policing's legitimacy is closely aligned with the concept of public trust (Bottoms \& Tankebe 2012; Bradford et al. 2009; Hawdon 2008; Lee \& McGovern 2013; Luna 2000; Mawby 2002; Miller et al. 2014; Seron et al. 2004; Toronto Police Service 2013; Tyler 2006a, 2006b; Tyler \& Huo 2002). In this vein, Hugh Orde, President of the United Kingdom's Association of Chief Police Officers, suggests, "Legitimacy in policing is built on trust" (Miller et al. 2014: 14). Legitimacy involves the "belief about the moral right of the police to possess and exercise power and influence, while trust is a belief about how individual police officers perform their roles" (Tyler \& Jackson 2013: 94). Hawdon (2008: 186) finds, "The role is legitimate; the individual is trusted." To be trusted police officers must demonstrate moral authority, embodying a shared sense of right and wrong with those being policed (Beetham 1991; Bradford \& Jackson 2010). Bradford and colleagues (2014: 81) suggest, "When people do not feel a duty of deference toward the police, do not feel that police share their values, and when they believe the police do not abide by the rules, legitimacy is fragile and may, in certain circumstances, be effectively absent." McCluskey and his collaborators (2014: 7) observe that while policing's legitimacy is "intrinsically important", it is important also "because it is consequential...Legitimacy affects citizens' acceptance of police decisions, their cooperation with the police, and even their compliance with the law more generally."

I suggest that policing's legitimacy can be best understood as the police being perceived by those being policed as legitimate - in that they are thought to be entitled to the public's confidence and trust and to have their authority accepted and reciprocated with obedience and consent (e.g., see Coicaud 2013; Hough et al. 2010; McCluskey et al. 2014; Reiner 2010; Stenson \& Silverstone 2014; Sunshine \& Tyler 2003a, 2003b; Tyler 2006b). Similarly, Bradford and colleagues (2014: 85) find, "A legitimate police service has the right to exercise power; it commands consent (a sense of obligation to obey) grounded in legality and moral alignment."

${ }^{33}$ Citizens' confidence in, and attitudes toward, front-line police officers is influenced, positively or negatively, by various factors - related to demographics, ecological factors, direct and vicarious experiences, mediated treatments of policing, and policing practices. Each category identified throughout the literature is particularized in the subsections below, excepting policing practices, which are addressed in various locations throughout the dissertation, including within this subchapter (6.7). Further, it should be understood that research has determined the existence of an asymmetric effect in relation to citizens' trust and police legitimacy, with the effects of negative experiences far outweighing those of positive ones in contact experiences, of any type, between the public and police officers (e.g., see Bradford et al. 2009; Gallagher et al. 2001; Mastrofski et al. 2002; McCluskey et al. 2014; Myhill \& Bradford 2012; Reisig \& Parks 2000; Rosenbaum et al. 2005; Skogan 2006). 


\section{Demographic Considerations}

Much of the demographic research inquiring into citizens' perceptions of the police emerges from the USA and the UK. Dating back into the 1960s, various studies have found that opinions of the police, and citizens' evaluations of policing, are shaped by (and differ along) a variety of demographic characteristics such as race/ethnicity, age, gender, education, and/or socioeconomic status (Chermak et al. 2005; Bridenball \& Lesilow 2008; Decker 1981; Gallagher et al. 2001; Greenfield et al. 1997; Henderson et al. 1997; Miller et al. 2005; Reisig \& Parks 2000, 2002; Rosenbaum et al. 2005; Sampson \& Bartusch 1998; Weitzer \& Tuch 1999; Wortley et al. 1997). While results examining the impact of age, gender, education, and socioeconomic status are not clearly universal or definitive (perhaps being best characterized as generally ambiguous), a preponderance of the American literature on race/ethnicity factors finds that this consideration is one of the most salient predictors of attitudes toward the police - with non-White individuals (Blacks and Hispanics) expressing significantly lower levels of satisfaction with policing as compared to Whites (for example, see Ackerman et al. 2001; Cao et al. 1996; Cheurprakobkit 2000; Decker 1981; Flanagan and Vaughn 1995; Huang and Vaughn 1996; Jefferis et al. 2011; Rosenbaum et al. 2005; Tyler 2004; Weitzer 1999, 2002; Weitzer and Tuch 2004, 2005a, 2005b). Several studies find that the effect of race on perceptions of police misconduct exists even when controlling for other social factors such as income, education, and employment (Jefferis et al. 2011; Rice \& Piquero 2005).

However, as articulated by Rosenbaum and his collaborators (2005: 344), "To suggest that attitudes toward the police are uniform within racial or ethnic groups would be to ignore evidence that attitudes are influence by a variety of factors." Brown and Benedict (2002: 563) note, as have the other scholars they cite, one or two variables cannot adequately predict differences in how individuals view the police (Cao et al. 1996; Hayes \& Brewer 1997; Poister \& McDavid 1978; Reisig \& Parks 2000, 2002). The role of situational and structural (non-demographic) factors - microlevel influences, such as personal experience with the police or knowledge gleaned from others' experiences, and macrolevel factors, such as neighbourhood conditions and media reports on policing, complicate our understandings of the complexities involved in determining what considerations or determinants are involved in establishing citizen's views on policing. Weitzer and Tuch (2005a: 280) suggest, "When [such] variables are factored into the equation, race may remain a significant predictor or may disappear; the studies in this genre are not sufficient to reach definitive conclusions (see Sampson \& Bartusch 1998; Tyler \& Huo 2002; Weitzer 1999; Weitzer \& Tuch 2002).”

\section{Ecological Variables}

Ecological variables, such as neighbourhood class position, the type of neighbourhood, the degree of social capital present in the community, and environments/situations of concentrated economic disadvantage have also been shown to be important in shaping some citizens' perceptions of police misconduct (Decker 1981; Flanagan \& Vaughn 1995; Reisig \& Parks 2000, 2002; Sampson \& Bartusch 1998; Stoutland 2001; Weitzer 
1999, 2002). Decker (1981) found that community-level predictors in relation to individual attitudes toward the police include considerations of 'neighbourhood culture' and general community beliefs about the police. Stoutland (2001) supports Decker's (1981) conclusions, as well as those of Flanagan and Vaughn (1996) and Sampson and Bartusch (1998), in her assertion that, "Research has found that the residents from areas of concentrated disadvantage have a much lower regard for the police than the general public."

\section{Direct and Vicarious Experiences with Policing}

Outside of demographic and ecological variables in the development of an individual's perceptions about the police, one must also give consideration to the different ways in which the public acquires and internalizes their impressions of the police, or, in other words, what experiences influence a person's subjective understandings of policing? Certainly, direct personal experience is one particularly powerful mechanism through which people acquire an impression of policing Cheurprakobkit 2000; Frank et al. 2005; Miles-Johnson \& Pickering 2018; Weitzer \& Tuch 2005b). Citizen's direct interactions with police officers have been found to influence individual's general attitudes toward, and levels of satisfaction with, the police - with experiences viewed as 'negative' having a more profound effect in negatively impacting attitudes than do 'positive' experiences' in improving attitudes and reducing antipathy toward the police (Correia et al. 1996; Klockars 1985, 1996; Reisig \& Parks 2000, 2002; Rosenbaum et al. 2005; Tyler \& Huo 2002; Weizter \& Tuch 2004, 2005a; Wortley et al. 1997). In other words, unpleasant personal experiences with the police have a stronger effect in forming or reinforcing negative opinions of the police than do pleasant experiences in creating or affirming positive impressions of policing (Gallagher et al. 2001). Weitzer and Tuch (2005a: 283) find that, "when police treat individuals in a discourteous, brusque, or unfair manner, this experience colours not only their assessment of the immediate encounter but also their overall opinion of the police."

Research has also found that being a victim of a crime (Apple \& O'Brien 1983; Cao et al. 1996; Koenig 1980; Smith \& Hawkins 1973) and having a past arrest (Smith \& Hawkins 1973) significantly reduce citizen's positive attitudes towards the police (Callanan \& Rosenberger 2011). Witnessing apparent police misconduct directed at another individual has also been found to have a significant adverse impact on an individual's opinion of the police (Son et al. 1997; Weitzer \& Tuch 2004). Ivkovic (2008: 412, citing Skogan 1996; Yeo \& Budd 2000, emphasis in original) bluntly asserts, "Although certain types of contacts are more likely than other to generate dissatisfaction, it seems that having any contact with the police results in more negative attitudes, because the chances of a negative contact and/or unsatisfactory contact increase."

Most citizens, however, seldom have contact with police officers in any meaningful way (Engel 2005; Gallagher et al. 2001; Rosenbaum et al. 2005; Surette 2007, 2011; Weitzer \& Tuch 2004, 2005a), which implies that most people draw heavily on other sources of information in terms of their perceptions of the police and also that fundamental 
attitudes toward policing are shaped by mechanisms other than strictly personal experiences. Trust in the police is about beliefs and/or expectations, not only about observations and conclusions made on the basis of personal experience (Callanan \& Rosenberger 2011; Kaarianinen 2008). Studies have demonstrated that a person's preexisting attitudes toward the police influences how they interpret a police action they have experienced or witnessed - either as a confirmatory representation of their inculcated and generalized positive or negative view of policing (Brandl et al. 1994; Brown \& Benedict 2002; Reisig \& Chandek 2001). Weitzer and Tuch (2005a: 283) summarize this genre of research findings effectively, "People who have a good encounter with the police may still hold very critical views of the police, and experiences themselves may be coloured by pre-existing opinions of the police. In short, personal contact clearly affects some individuals' attitudes toward the police, but other people are obviously influenced by other factors." Rosenbaum and colleagues (2005) suggest sources such as the media, family, friends, and other social networks as significant sources of such non-personal policing experiences.

Vicarious policing experiences, which implicate the internalization of the experiences of other persons, such as family members, neighbours, friends, acquaintances, co-workers, with the police, have been found to be one such important factor in shaping individual's perceptions about the police (Miller et al. 2005; Rosenbaum et al. 2005; Weitzer \& Tuch 2004, 2005a). Given that beliefs about police misconduct are shaped not only by personal direct experience but also through shared experiences, via communications from others, a police-citizen occurrence featuring perceived misconduct has been found to present the capacity to engender a far-reaching 'ripple effect' (Callanan \& Rosenberger 2011). The knowledge of other persons' experiences with the police has also been found to have the possibility of functioning as 'a multiplier effect' on existing cultural or neighbourhood beliefs about the police (Harris 2002; Weitzer \& Tuch 2004). As Rosenbaum and his collaborators found (2005: 360$)$, in confirming the previous findings of Brandl et al. (1994), "Encounters with the police can be conditioned by prior attitudes about the police rather than the other way around...one implication is that changing people's attitudes about the police will require more than changing their direct experience with the police." In this regard, Chermak and his colleagues (2005: 274) determined, "Media coverage might strike a chord with viewers, but their interpretation and reactions to the coverage will depend on other factors, [including] existing perceptions of the police." Such observations are consistent with the notion of 'confirmation biases' - the "tendencies to interpret, seek, and create information in ways that verify existing beliefs" (Brehm et al. 2002: 119). In general terms, communications research has demonstrated that people interpret information that they receive in ways that support existing beliefs or stereotypes and that they are more likely to recall such information than disconfirming information that challenges their entrenched stereotypes (Rosenbaum et al. 2005; Stone et al. 1997). The individual's interpretation of the portrayal of police through media is thought to be of fundamental importance in establishing, and/or changing, citizens' attitudes toward the police (Dowler \& Zawilski 2007; Doyle 2003, 2006b; Doyle \& Ericson 2004; Ericson et al. 1987; Huang \& Vaughn 1996; Lawrence 2000; Surette 2007, 2011). 


\section{Mediated Treatments of Policing}

It is axiomatic that exposure to mediated accounts of police actions influence individual's perceptions of the police (e.g., see Beckett \& Sasson 2000; Doyle 2003, 2006b; Flanagan \& Vaughn 1995; Leishman \& Mason 2003; Mawby 2002; Surette 2007, 2011; Weitzer 2002; Weitzer \& Tuch 2004). Media reporting on police brutality and/or corruption occurrences has been correlated with documented increases in audience's negative perceptions of, and negative fundamental attitudes toward, the police (Kaminski \& Jefferis 1998; Hirschfield \& Simon 2010; Lasley 1994; Lawrence 2000; Weitzer 2002; Weitzer \& Tuch 2004, 2005a). Weitzer and Tuch (2004, 2005a) have determined that repeated exposure to different incidents of police misconduct is responsible for increases in the levels of negative attitudes toward the police and increases in the perceived frequency and prevalence of such misconduct. Weitzer and Tuch (2004: 320) conclude, "People who take this view may see the entire police department as rotten, which can have important implications for the overall legitimacy of a police agency and for the people's willingness to cooperate with its officers." Weitzer and Tuch (2004: 321) find, "Net of other factors, repeated exposure of media reports on police abuse is one of the strongest predictors of citizens' perceptions of misconduct." In locations in society where such reporting on police misconduct coincides with prevailing perceptions of reality, the combined effect of the two can be powerful (Weitzer \& Tuch 2004).

As discussed earlier (particularly within Endnote 14 in Chapter Three), until recently, media's deferential (Gitlin 1980; Hall et al. 1978; Loader 1997) and essentially symbiotic (Antony \& Thomas 2010; Doyle \& Ericson 2004; Ericson 1989; Reiner 2010) relationship with policing functioned to keep police misconduct away from public discourse and, with only sporadic exceptions (i.e. the 'Chicago Police riots', the beating of Rodney King, and the fatal RCMP interaction with Robert Dziekański), reinforced the dominant ideology in society, thereby legitimizing the historical social order and maintaining the status quo (Gitlin 1979; Hall 1977; Murdock 1973). This void in the provision of information to the public about the police and alleged misconduct, via the media, was problematic given the lack of comparative materials and resources available to citizens in order to engage in any critical analysis (Chermak et al. 2005; Ericson et al. 1987; Gitlin 1980; Jewkes 2004). As a result, most throughout society subscribed to the hegemonic perception that police officers seldom behaved improperly and that "police business' was no place for civilian intrusions or demands from the citizenry for more information or increased accountability. One of the central tenets of mass communication theory, as expressed by McQuail (1972: 13), advances that, "[The media are integral] in shaping the individual collective consciousness by organizing and circulating the knowledge which people have of their own everyday life and of the more remote contexts of their lives."

As discussed at various locations throughout this dissertation, today the mediated environment for policing is significantly different. Seismic shifts within television and print journalism have, for the most part, relegated formerly congenial relations with the police to the past; now replaced with a 'highly contentious' (Doyle 2006b), 'critical' 
(Reiner 2010), 'sensational' (Frost 2008) and 'adversarial' (Greer \& McLaughlin 2010a, $2010 \mathrm{~b}, 2012)$ co-existence in which there is no privileging of the police perspective nor deference to their authority (Fox 2007; Greer \& McLaughlin 2012; McLaughlin 2007). The rise of social media has amplified this transformation and the contemporary increase in sensational and negative 'tabloid-style' journalism, in mediated treatments of policing, has been linked in some studies with reductions in people's perceptions around the legitimacy of the police (Fox \& Van Sickel 2001; Gallagher et al. 2001).

${ }^{34}$ Citations in support of these observation are located throughout subchapter 3.3 and within endnote 6 in Chapter Three.

${ }^{35}$ In this regard, Withrow (2011: 85-86) advances,

The deployment of patrol resources is among the most important decisions made by police administrators. Crime is not equally distributed throughout a community. Some neighbourhoods have more crime and therefore deserve more attention from the police department. Ideally patrol resources should be apportioned to optimize response time and increase the deterrence effect created by police officer visibility. In other words, the police are deployed where they are need the most... When a police administrator decides when and where to concentrate policing resources, particularly patrol resources, he also inadvertently decides who is most likely to be stopped. Here's why. Drivers do most of their driving within the patrol beat where they happen to live. A short trip to the grocery store or a cross-country drive both start and end in the patrol beat where the traveler lives. Also, drivers who are familiar with their neighbourhoods may be more likely to ease through sparsely used stop intersections or drive faster on well-known roads. In doing so, these drivers put themselves at higher risk of being observed violating the traffic law by a police officer. As a result, drivers are more likely to be stopped within or very near the patrol beat in which they reside. All things being equal...the residents of a high crime patrol beat (that is assigned more policing resources) have a higher probability of being observed by a police officer.

Similarly, Bittner (1970: 9-10) observes,

The ecological distribution of police work at the level of departmentally determined concentrations of deployment, as well as in terms of the orientations of individual police officers, reflects a whole range of public prejudices. That is, the police are more likely to be found in places where certain people live or congregate than in other parts of the city. Though this pattern of manpower allocation is ordinarily justified by reference to experientially established needs for police service, it inevitably entails the consequence that some persons will receive the dubious benefit of extensive police scrutiny merely on account of their membership in those social groupings which invidious social comparisons locate at the bottom 
of the heap. Accordingly, it is not a paranoid distortion to say that police activity is as much directed to who a person is as to what he does. As is well known, the preferred targets of special police concern are some ethnic and racial minorities, the poor living in urban slums.

${ }^{36}$ It is axiomatic to observe that typically neighbourhoods with high rates of violent crime also struggle with social disorganization and a lack of informal social control/lack of collective efficacy (Sampson, Raudenbush \& Earls 1997). As articulated by Kochel and Weisburd (2018), "In disadvantaged neighbourhoods, prior research has found reduced social cohesion and less willingness among residents to address disruptive behaviours and violations of social norms. This deficiency is commonly associated with higher levels of disorder and crime."

${ }^{37}$ Procedural justice, as applied to policing, can be considered strategies and officers' behaviours that further the fair, equal, and professional treatment of all parties to policecitizen interactions and that can engender the perception among citizens that, for example, police treat everyone throughout the community with respect (Bradford et al. 2009; McCluskey et al. 2014; Sindall et al. 2012; Sunshine \& Tyler 2003; Tyler 2004). The strategy focuses on communicating messages of status and worth to those being policed through demonstrating that the individuals concerned in interactions with officers are valued and respected members of the social group the police represent - all of society (Bradford et al. 2014; Sunshine \& Tyler 2003). When applied in policing practices, procedural justice tends to positively influencing citizen's perceptions of how people are treated by police regardless of the policing outcomes - including officers' effectiveness in responding to, or reducing crime (Brown \& Benedict 2002; Fagan 2002; Gallagher et al. 2001; Hough et al. 2010; Jackson \& Sunshine 2007; McCluskey et al. 2014; Myhill \& Quinton 2011; Stoutland 2001; Tyler \& Wakslak 2004). Policing in accordance with procedural justice principles involves officers giving citizens a voice in police-citizen interactions, behaving in a way that leads citizens to perceive that the officer is neutral in such interactions, treating citizens with dignity, and behaving in ways that lead citizens to assess the officer's motives as trustworthy and fair. As Weisburd and Braga (2019: 15) find in this regard, "In this policing model, changes in how police behave in encounters with citizens have the potential to alter not only the perceptions of the police of those directly affected but, through them, the community's perceptions of police legitimacy."

${ }^{38}$ Reiner (2017: 237, citing Newburn and Reiner 2012) advances,

One of the key discoveries of early empirical research was the extent of discretion about whether and how to invoke legal powers and processes, and more broadly how to behave or misbehave, that was held by operational police officers. Whether or not this was sanctioned de jure, police inevitably had considerable de facto discretion.

${ }^{39}$ In their 2015 report, the President's Task Force on 21 st Century Policing presented a host of recommendations "aimed at repairing the mistrust between police officers and citizens that has deepened to the point of crisis in communities across the country" 
(Berman 2015). Two of the key suggestions, featured prominently on the first page of the report, are a fundamental shift in the mindset guiding the work of police in the field and in how officers interact with citizens in the community, as follows,

Building trust and nurturing legitimacy on both sides of the police/citizen divide is the foundational principle underlying the nature of relations between law enforcement agencies and the communities they serve. Decades of research and practice support the premise that people are more likely to obey the law when they believe that those who are enforcing it have authority that is perceived as legitimate by those subject to the authority. The public confers legitimacy only on those whom they believe are acting in procedurally just ways. In addition, law enforcement cannot build community trust if it is seen as an occupying force coming in from outside to impose control on the community...Law enforcement culture should embrace a guardian - rather than a warrior - mindset to build trust and legitimacy both within agencies and with the public (President's Task Force on 21st Century Policing 2015a: 1).

${ }^{40}$ Reiner (2010: 25) continues, "Policing is regularly blighted by inequality, injustice, and discrimination in its operation. Groups that are low in power and status, such as the poor and unemployed, ethnic minorities, young men (and underclass young women), gays, and lesbians, become 'police property' (Lee 1983), disproportionately likely to be treated as suspects at each stage of the criminal justice process: stop - search, arrest, detention, charge, prosecution."

${ }^{41}$ Along the same lines, the National Academies of Sciences, Engineering, and Medicine (2018: 251-252, citing Campbell 2012; Lichtenstein 1996; Loewen 2005) find,

It is important to note that the origins of policing in the United States are intimately interwoven with the country's history of discrimination against non-White people, particularly toward Black people. From the tracking and kidnapping of enslaved Black people to the regulation of Black movement and the criminalization of Black bodies for the purpose of economic exploitation police officers have often been the enforcement arm of both explicitly racist and tacitly discriminatory norms and laws. Although some of the more egregious historical practices ended a long time ago, others ended later and within the living memory of many Americans - and all are remembered as part of the collective history shared by Black and other non-White communities. From this perspective, it is easy to see how the nation's history is intrinsically linked to misgivings some non-White Americans continue to have about possible police animus and racial bias.

${ }^{42}$ To provide some context around this concept of 'suspicion' (from my own experiences as a practitioner), when I began my police career in 1985, it was my 
understanding from my training and the work of those veteran officers I respected that in addition to reactive policing - responding to calls for service generated from members of the public, via 9-1-1 or otherwise - front-line police work involved proactive work. In fact, one's skills in proactively discovering crimes in progress is what separated the outstanding front-line officer from the mediocre. As it was understood, anyone can (reactively) respond to a 9-1-1 call and address the issues involved in such an occurrence. However, the expectation of the police agency, the public, and respected peers within the front-line subculture was that an officer was to patrol their assigned zone, when not engaged on a call, looking for 'suspicious' activities and checking in on the locations of known offenders or crime 'hot spots' in the area. The exercise of acting on one's 'suspicions' was advanced as one of the core functions of the front-line patrol officer - relying on one's training and both interpersonal and policing experiences to determine what appeared to be potentially out of the ordinary and looking into it. It was my understanding that being inquisitive (or 'suspicious') was part of the individual's personality characteristics that was assessed in the hiring process to be a police officer and was fundamental to the police role in society. In other words, we (front-line police officers) were supposed to be inherently 'suspicious' in any of our observations or interactions and it was this 'suspicious' nature (sometimes referred to as 'a cop's sixth sense' or 'spidey senses') that made for good cops. This is a qualitative characteristic that is hard to describe; it relates to the ability to rely on one's personal and policing experiences and senses to detect when something is not quite right ('suspicious').

I respected and tried to model those officers who were very active in their work and who used their time between calls to do proactive work. Many other officers, as discussed earlier in relation to traditional 'lazy' officers, counseled me to 'slow down' and to be cautious in how many cars I stopped or how many people I interdicted in acting on my understanding of the police function and inquiring into what I felt were potentially 'suspicious' activities. It was pointed out by several veteran officers that it was in this aspect of policing that danger lurked - not only physical danger but the danger of having a complaint initiated against oneself by a member of the public. In relation to acting on one's 'suspicions' as a police officer, I recognized then, and I do even more so now, that the biases we all have in our individual makeup play a significant role in determinations around what is 'suspicious' for each of us.

These biases are often discussed in the context of the rank-and-file police subculture and 'racial profiling', in that prejudiced attitudes within the subculture can, and often do, influence individual officers' behaviours in the field. These considerations relate to how front-line officers make operational decisions around 'suspicion' and who, in the community, needs to be surveilled and investigated. Satzewich and Shaffir (2009: 14) observe that officers "develop a perceptual shorthand to identify certain kinds of people as symbolic assailants...In this view, the police officer responds to the indication of danger [and 'suspicion'] suggested by appearance.” Hayle, Wortley and Tanner (2016) address this in terms of the use of race in rank-and-file officers' determinations around who is deserving of 'suspicion', however, while often a focus on race, this also 
subsumes perceptions of characteristics implicating ethnicity and nationality (Glaser 2006; Hackney \& Glaser 2013).

${ }^{43}$ Also writing in that era, Bittner (1970: 10-11) observed,

As is well known, the preferred targets of special police concern are some ethnic and racial minorities, the poor living in urban slums, and young people in general. On the face of it, this kind of focusing appears to be, if not wholly ullobjectionable, not without warrant. Insofar as the abovementioned segments of society contribute disproportionately to the sum total of crime and are more likely than others to engage in objectionable conduct, they would seem to require a higher degree of surveillance. In fact, this kind of reasoning was basic to the very creation of the police; for it was not assumed initially that the police would enforce laws in the broad sense, but that they would concentrate on the control of individual and collective tendencies towards transgression and disorder issuing from what were referred to as the 'dangerous classes.' What was once a frankly admitted bias is, however, generally disavowed in our times. That is, in and of itself, the fact that someone is young, poor, and dark-complexioned is not supposed to mean anything whatsoever to a police officer. Statistically considered, he might be said to be more likely to run afoul of the law, but individually, all things being equal, his chances of being left alone are supposed to be the same as those of someone who is middle aged, well-to-do, and fair-skinned. In fact, however, exactly the opposite is the case. All things being equal, the young-poor-Black and the old-richWhite doing the very same things under the very same circumstances will almost certainly not receive the same kind of treatment from policemen. In fact, it is almost inconceivable that the two characters could ever appear or do something in ways that would mean the same thing to a policeman.

Doob and Gartner (2017: A2-A3, emphasis in original) are instructive in this regard, with their observation,

One fact about crime that no one questions is that it is not evenly (or even randomly) distributed across people, groups of people, or neighbourhoods in our society. Young males, for example, are disproportionately more likely to be involved in a variety of different kinds of crime than other people. People who live in certain kinds of neighbourhoods are more likely to commit offences than people in other neighbourhoods. But some neighbourhoods themselves appear to have characteristics that make them more likely to be the sites for crime above and beyond the characteristics of the individuals who live in them...In this context, a policing perspective that did not consider any other concerns could justify focusing surveillance resources on certain neighbourhoods or types of people (e.g. young males). The problem is that there almost always are other concerns, and concerns 
that could easily have the effect of undermining the crime control goal of proactive policing activities, such as police stops.

${ }^{44}$ Satzewich and Shaffir (2009: 200) define racial profiling as "heightened scrutiny based solely or in part on race, ethnicity, Aboriginality, place of origin ancestry, or religion, or on stereotypes associated with any of these factors." Engel and Cohen (2014: 383) observe, "A current concern in American society remains the use of race or ethnicity by police as reason for some form of coercive action. This police practice often referred to as racial profiling - is widely recognized by politicians, the public, and even the police themselves as inherently problematic." Racial profiling can result from the formation of stereotypes among officers around certain characteristics and their association with elevated criminality, sometimes the result of institutionalized bias but, in my view, certainly today, more through the organizational culture within police agencies (Smith \& Alpert 2007). Lever (2005: 97) discussed racial profiling and its underlying implication that links Black persons with a predisposition to more criminality than other individuals in the population, which serves to "remind Blacks, all too painfully, that odious claims about their innate immorality and criminality justify their subordination" and to "perpetrate, as well as reflect, White tendencies to draw invidious and complacent racial distinctions, and exacerbate unmerited indifference and hostility to the legitimate interests of Black people."

Reed (2018: 39, citing Harris 2002) is instructive with the observation,

There is a difference between criminal profiling and racial profiling. Criminal profiling, or offender profiling, is a technique used by law enforcement to identify likely suspects for a specific crime. In this instance, of course, they can use race as onof the factors in profiling the offender That process is distinguished from racial profiling where the race is the only or primary criterion. The Fourth Amendment to the US Constitution, which provides protection against unreasonable search and seizure and the equal protection provisions of the Fourteenth Amendment, make racial profiling illegal.

${ }^{45}$ For an in-depth and book-length analysis of the Canadian context, vis-à-vis Policing Black Lives (the title of her publication), I would refer the reader to this important work by Maynard (2017). As described in Maynard's website (https://robynmaynard.com),

The book is a CBC national bestseller, currently in its third printing, designated as one of the 'best 100 books of 2017' by the Hill Times, listed in The Walrus's 'best books of 2018', shortlisted for an Atlantic Book Award, the Concordia University First Book Prize and the Mavis Gallant Prize for Nonfiction, and the winner of the 2017 Annual Errol Morris Book Prize. This work received a starred review in Publishers Weekly as well as glowing coverage in the Toronto Star, the Globe and Mail, Now Toronto, Maclean's, and the Ottawa Citizen. In the words of the Winnipeg Free Press: 'Every Canadian Black, White, Indigenous or otherwise - could benefit from reading Maynard's frank and thorough assessment of racism in Canada'. 
${ }^{46}$ A 2014 report, ordered by the Commissioner of the RCMP, found that 1017 Indigenous women were murdered across the country between 1980 and 2012 and another 164 are missing (RCMP 2014).

${ }^{47}$ Among a random national sample of 1000 adults, with a 3.5\% margin of sampling error. The breakdown of responses by participants' self-reported race/ethnicity to the various questions are presented in the corresponding tables (Washington Post/ABC News 2014).

${ }^{48}$ However, Menifield, Shin, and Strother (2018), in experimental studies with American police officers find, "Despite clear evidence of implicit bias against Black suspects, officers were slower to shoot armed Black suspects than armed White suspects and less likely to shoot unarmed Black suspects than unarmed white suspects" (e.g. see James, Vila \& Daratha 2013; James, James \& Vila 2016). Further,

While African Americans are disproportionately killed by police, they are killed at much higher rates by non-White officers than by White officers... Our research contributes to the perspective that persistent racial disparities in police killings are driven primarily by prior disparities in racial policing generally: disproportionate killing is a function of disproportionate police contact among members of the African American community (Menifield, Shin \& Strother 2018).

${ }^{49}$ In relation to American corrections, Harris (2009) finds that $29 \%$ of Black males will be incarcerated during their lifetime in comparison to $5 \%$ of White males. Wacquant (2001) reached similar, but even more disturbing, findings, suggesting that Black males are eight times more likely to be sent to prison than White males.

${ }^{50}$ As observed, correctional data in Canada includes race and therefore it is known that certain 'visible minority' populations are overrepresented in federal penitentiaries. For example, Blacks comprise approximately $2 \%$ of the Canadian population, but $6 \%$ of those in federal custody (Linden 2012). In this regard, Maynard (2017: 5, citing OwusuBempah \& Wortley 2014) observes, "It is not yet uncommon knowledge that African Canadians are incarcerated in federal prisons at a rate three times higher than the number of Blacks in the Canadian population, a rate comparable to the United States... Fewer still are aware that in many provincial jails, the rate is even more disproportionate that it is at the federal level."

However, most disproportionate are Indigenous persons, who comprise roughly $4 \%$ of the Canadian population, but $18 \%$ of federal prisoners (Linden 2012). Further, Indigenous adults account for $24 \%$ of admissions to provincial/territorial custody (Reasons et al. 2016). In some provinces, they are grossly overrepresented. While making up $16 \%$ of the Manitoba population, Indigenous persons accounted for $71 \%$ of correctional admissions in 2005/2006 (Reasons et al. 2016: 78, citing (Perry 2011). In Saskatchewan, where Indigenous persons comprise $12 \%$ of the population, they account for $78 \%$ of the incarcerated population (Manikis 2016). On a national scale, the 
Indigenous population is incarcerated at ten times the rate of the non-Indigenous population (Barker et al. 2015) and while Aboriginal youth "account for $5 \%$ of the total youth population, [they] make up 24\% of total admissions to custody" (Hogeveen 2004: 296). Finally, Owusa-Bempah and colleauges (2015) find that in the years 2005 through 2015 in Canada, the Indigenous prison population rose $40 \%$ whereas the nonIndigenous prison population only increased by $2 \%$.

${ }^{51}$ In a chapter titled 'Over-policing: Unfair targeting and harassment of minorities,' Chan (1997: 21-23) observes,

One form of discriminatory prolicing practice is the unfair targeting and harassment of certain minority groups...The concept of over-policing encompasses both the degree (e.g. the number of officers station in areas with high concentrations of Aboriginals) and the nature of police intervention... [which can include] the discriminatory use of particular legislation (for example, the use of public order offences) [and] regular foot or vehicle patrols which create an atmosphere of surveillance and tension...It has been argued that over-policing is responsible for the gross over-representation of Aborgines in the criminal justice system... Whether or not police actually or routinely discriminate against minority groups, the perception of such discriminatory practices by minority groups must be taken seriously. Since fear and distrust of the police are common among some groups, any evidence of unfair treatment can only add ot the distance between police and minorities.

${ }^{52}$ I advance this is why the present study's data demonstrates substantially less changes in rank-and-file officers' reported adoption of de-policing practices after the first major transitioning of many officers toward de-policing - after graduating from 'New' officers (with 1 to 5 years of front-line police experience) into officers occupying the 6 to 10 year category. As the relevant portion of Table 31, represented below, validates, there is far less movement from non de-policer status to de-policer status (in any of the three iterations) in later stages of an officers' front-line tenure. Therefore, consistent with the observations in this subchapter around both 'mission-oriented' and 'New' rank-and-file officers, and various observations around a de-policing continuum throughout this dissertation, I argue that many officers' 'working personality' (Skolnick 1966; Van Maanen 1973, 1974, 1975) becomes entrenched after the first few years of performing front-line police work and the various experiences therein.

\begin{tabular}{|c|c|c|c|c|c|}
\hline \multirow{2}{*}{} & $\begin{array}{c}\text { Non de- } \\
\text { policers }\end{array}$ & $\begin{array}{c}\text { Limited } \\
\text { de-policers }\end{array}$ & $\begin{array}{c}\text { Moderate de- } \\
\text { policers }\end{array}$ & $\begin{array}{c}\text { Intensive } \\
\text { de-policers }\end{array}$ \\
\hline \multirow{3}{*}{$\begin{array}{c}\text { Years of } \\
\text { Front-Line } \\
\text { Police } \\
\text { Experience }\end{array}$} & $1-5$ & $40.3 \%$ & $19.5 \%$ & $24.1 \%$ & $16.1 \%$ \\
\cline { 2 - 6 } & $6-10$ & $23.7 \%$ & $19.1 \%$ & $26.6 \%$ & $30.6 \%$ \\
\cline { 2 - 6 } & $11-15$ & $22.9 \%$ & $14.8 \%$ & $19.1 \%$ & $43.1 \%$ \\
\cline { 2 - 6 } & $16-20$ & $21.6 \%$ & $15.5 \%$ & $21.1 \%$ & $41.8 \%$ \\
\hline
\end{tabular}


${ }^{53}$ While being a 'pracademic' researcher can have advantages, as described throughout this paragraph, there can also be a number of challenges (potential downsides) to this researcher positionality. For example, the 'pracademic' researcher has to be much more conscious of reflexivity, given their almost inevitable bias (if not conscious, almost certainly subconscious) in relation to the research question(s) being investigated. Also, given familiarity with the research setting, the study population, and/or the research question(s) being investigated, the 'pracademic' researcher can take certain things for granted that would not likely be overlooked by an outside researcher. There is also a greater possibility that the 'pracademic' researcher, as compared to an outside researcher, might be presented with disingenuous data because study participants want to help by telling the researcher what they think s/he wants to hear.

${ }^{54}$ I do not think you can discount the relational aspect of in-person survey administration, which not only typically results in a higher response rate than other modes (as described in Chapter Four) but there is likely almost always some element of rapport building, even if the research participant just simply appreciates the human interaction and effort in the researcher attending in person. Although, as a researcher, it is my understanding that it is important to try to limit the potential influence of this rapport building. In this regard, I proactively limited my interactions with officers prior to their shift briefings and kept my introductory remarks short and businesslike. My intention was to remain relatively aloof until after the survey administration was completed. Thereafter, I was able to interact more freely with the officers and there were many discussions that took place after the surveys had been completed. Given the interactions that officers often sought out after the survey administration was completed it seems apparent to me that an element of trust had been established.

${ }^{55}$ A good example of social desirability bias in operation emerges from Goold's (2004) study of UK officers' responses to the presence of CCTV cameras throughout their external work environment. Goold (2004: 180-182, emphasis added) found, "Typically, officers initially responded by stating that the presence of CCTV had not had any impact on the way in which they carried out their duties or exercised their powers...[However,] although the majority of officers interviewed initially claimed to be unconcerned or unaffected by the presence of CCTV cameras, over time it became apparent that many harboured private concerns about the possibility of their actions being exposed to external scrutiny." Goold (2004) observed that officers participating in the study had to be 'pressed' over time to provide the truth about the impact of the CCTV cameras on how they performed their duties. In other words, the initial data reported to the researcher was not accurate, although it did conform to societies expectations of how a police officer should respond to the presence of CCTV cameras.

In relation to the present study, as opposed to the potential that my 'pracademic' status may have resulted in some research participants reporting de-policing behaviour because they may have somehow inferred that is what I, as a researcher, wanted to establish in this study - essentially the inverse of social desirability bias - many officers I met after the formal survey process had been completed or later during my stays in their community told me, to the contrary, that they had not felt comfortable being $100 \%$ 
candid in writing (with their survey responses and written elaborations) despite my 'pracademic' credentials and assurances of ethical research and anonymity. In fact, many of the rank-and-file officers that subsequently approached me advised that they had been more conservative in their responses and comments in relation to their depolicing activities and those they observe within their contemporary occupational subculture. Others advised that their understanding was that the rationale of the study was to disprove that de-policing practices were occurring to any pronounced degree. That assumption was, as several explained, grounded in the social undesirability of depolicing practices - not only among the citizenry, but also within policing's leadership and among rank-and-file officers that are vehemently opposed to any such approach to the important work of the front-line officer in society (as discussed in subchapter 6.9 in the context of 'mission-oriented' officers). Apparently many study participants inferred that, based on my policing and now academic biography, I was likely a 'missionoriented' officer, and therefore, my bias was likely toward disproving the de-policing phenomenon, which is a frequent topic throughout the rank-and-file subculture and in sporadic mediated public discourse. Therefore, it may be the case that de-policing is an even more pronounced phenomenon than as it is represented in the present study's results, which emerge from the data provided by research participants. 


\section{NOTES FOR CHAPTER SEVEN}

${ }^{1}$ I suspect that Reiner would agree with Rubinstein's assessment, however he would likely exempt the early investigations of Banton (1964), Bittner (1967b), Reiss (1971), and Skolnick (1966) from such a critique - given that Reiner (2015: 309) characterizes the work of these scholars, into unchartered areas of police work and police occupational culture, as representing "theoretically sophisticated and nuanced analyses of rich empirical data, gathered through sensitive fieldwork" and, in doing so, Reiner finds that their research "[plays] some part in influencing wider debate and policy" as "public sociologists avant la lettre" (a discussion of public sociology is engaged throughout subchapters 7.5 and 7.6 within this chapter). Similarly, Hudson (1973: 1568) assessed that Reiss' study, which contributed to publication of The Police and the Public (1971), introduced "a rich array of data on the day-to-day working milieu of the policeman" into the popular, scholarly, and policy discourses of that era.

In this vein, I advance that the present empirical study of contemporary police work can be considered as introducing a novel social theory of risk - specifically as relates to risk-averse adaptations in occupations and occupational subcultures in response to workers' perceptions. Along similar lines, Michael Hayden, former director of the USA's Central Intelligence Agency (CIA) suggested, in the wake of the 2014 public release of information about 'sensitive' activities of the CIA, that the outcome resulting from scrutiny by, and denunciation from, the public would be an intelligence organization in which agents were 'timid' and 'risk-averse' (Wong 2014). Hayden believes 'uninformed' and 'partisan' criticism will have a significant impact on the effectiveness and behaviour of CIA agents, much to the detriment of the most fundamental objectives of the CIA. Hayden complains, "What's happening now [is] these folks [are] having the rug pulled out from under them, people who thought they were doing what [the public] wanted them to do" (Associated Press 2014a).

As such, I argue that the present study functions as an empirical and conceptual test of a risk aversion thesis as applied to front-line police behaviour -similar to Doyle's (2003) thesis on policing in front of cameras, Ericson and Haggerty's (1997) thesis on the risk society as applied to shifts in contemporary policing, and Thompson's (2005) new visibility thesis and its applicability to today's police work (e.g., see Brown 2013, 2016; Brucato 2015a, 2015b; Goldsmith 2010; Haggerty \& Sandhu 2014; Sandhu 2016, 2019; Sandhu \& Haggerty 2015, 2017).

${ }^{2}$ Along these lines, as it was in subchapter 6.7, it is worthwhile to reflect on the foundational principles of Western democratic policing, as contemplated in the social contract and as presented in the instructions to inaugural members of the Metropolitan Police in 1829, which recognized, "The power of the police to fulfill their functions and duties is dependent on public approval of their existence, actions and behaviour, and on their ability to secure and maintain public respect" (Flanagan 2007: 4). In this regard, Banton (1964: 268) presented an argument that retains its relevance today, "Both the police and the public would benefit if there were more informed and independent 
opinion in the universities and among the public at large about the police and their duties."

${ }^{3}$ In a slightly different, yet equally applicable, context, Paoline and Terrill (2014: 170) counsel,

A creative study of police culture will undoubtedly include getting into a variety of police departments and digging around...Researchers should continue to search for empirical evidence of past notions, but more important keep your eyes peeled for new ideas. In short, challenge traditional police culture thoughts...expand the environments, elements of the environments, coping mechanisms, and outcomes. Policing has undergone numerous changes since the $1950 \mathrm{~s}$, and additions to the culture model are needed.

${ }^{4}$ As presented in Endnote 7 for Chapter Five, in responding to Question 16, only six participants in the present study identified using video recording to their advantage similar to the 'camera-friendly' manner suggested by Sandhu $(2016,2019)$ and Sandhu and Haggerty $(2015,2017)$. In a related consideration within Question 16, among the handful of participants that reported discounting any influence of video recording on their police actions in the field, the response of a Gatineau officer is largely representative, At the beginning of the video recordings phenomenon I did [change my behaviours], but now it happens so frequently I am accustomed to it and don't worry about changing my behaviour for the camera (GAT40). In relation to the first identified subtheme within Question 20, most participants, that reported doing something because they think their on-duty could be video recorded, did so in order to stage any resulting narrative rather than behaving in a more 'camera-friendly' manner. For example, a Saskatoon officer reports, If having to use force I voice 'stop resisting, etc.' to create witnesses on my side (SAS160). In other words, rather than officers becoming 'camerafriendly', most participants in the present study that discussed using their new vibility to their advantage in certain situations spoke of the tactical consideration of controlling the narrative (the framing of what is depicted in video footage) - putting the officer's version of the interaction 'on the record' and 'out in front' of any potential controversy.

5 This image is a screen capture I produced during my viewing of the episode, which is available for viewing online at https://archive.org/details/ABC-Nightline-2001-07-23.

${ }^{6}$ See also, Braga et al. 2014; Chaiken 1978; Correia 2000; Gill et al. 2014; Evans \& Owens 2007; Jackson et al. 2012; Jones \& Newburn 2002; Kelling \& Sousa 2001; Klick \& Tabarrok 2005; Levine 1975; Lum \& Nagin 2017; National Academies of Sciences, Engineering \& Medicine 2018; National Research Council 2004; President's Commission on Law Enforcement \& Administration of Justice 1967; Rosenbaum 2006; Rosenfeld, Fornango \& Rengifo 2007; Sherman \& Weisburd 1995; Spelman \& Brown 1984; Weisburd \& Braga 2006a, 2006b; Weisburd \& Eck 2004; Weisburd, Wyckoff, Ready, Eck, Hinkle \& Gajewski 2006. 
${ }^{7}$ In an interrelated line of scholarship, various policing researchers (e.g., see Braga et al. 2014; Braga \& Weisburd 2010; Nagin, Solow \& Lum 2015; Neyroud 2019; Sherman \& Weisburd 1995; Weisburd \& Majmundar 2017) have argued that the tactic commonly known as 'hot spots' policing, which involves the saturation of a specific geographic area with many more police resources than are typically deployed in that particular community, can "generate statistically significant crime-reduction impacts without simply displacing crime into areas immediately surrounding the targeted locations" (National Academies of Sciences, Engineering \& Medicine 2018: 129).

${ }^{8}$ Numerous study participants (across virtually every participating police agency) complained that police leadership seems to have no issues in jumping to the worst-case outcome in their immediate (hasty) assessments and initial public comments in the aftermath of controversial police-citizen interactions. For example, as discussed earlier within Endnote 17 in Chapter One, on 29 July 2013 TPS officer James Forcillo was suspended from the TPS in the aftermath of the fatal shooting of Sammy Yatim on a Toronto streetcar and, on that same date, TPS Chief Bill Blair addressed the media, saying: "Like many members of the public I have viewed the videos of this incident...I am very aware that the public is very concerned about this tragic event. They have every right to be concerned" (Pagliaro 2013). However, thereafter (even in situations where there is the availability of exculpatory information or materials that would provide important context to the 'court of public opinion') most often the organization takes the position that there can be 'no further comment' as, for example, 'the matter is under investigation' or 'the matter is now before the courts'. Therefore, the narrative is often seized by those with no restrictions on commentary or opinion (lawyers, family members, witnesses, the person alleging police misconduct, etc.). This was the source of much frustration, as was conveyed to me by many officers that participated in the present study.

Perhaps such complaints have found a sympathetic audience as, while I do not know the motivations of the government, on 19 February 2019, Bill 68 (the Comprehensive Ontario Police Services Act) was introduced in the Ontario legislature. In my analysis of the proposed legislation, I note that section 177 of the Community Safety and Policing Act (2019) and section 29 of the Special Investigations Unit Act (2019) will allow both the Complaints Director and/or the SIU Director to issue public statements regarding an ongoing investigation if, '(a) the statement is aimed at preserving public confidence; and (b) the benefit of preserving public confidence clearly outweighs any detriment to the integrity of the investigation.'

${ }^{9}$ The next paragraph in Gonzales and Cochran's (2017) article reads,

First, legally, the officer's perspective is the one that matters if an allegation of excessive force is made. in determining whether an officer used excessive force, the courts will look to whether the action was reasonable, and " $[\mathrm{t}] \mathrm{he}$ 'reasonableness' of a particular use of force must be judged from the perspective of a reasonable officer on the scene, rather than with the 20/20 vision of hindsight" [citing the 1989 decision of the United States Supreme 
Court in Graham v Connor]. Accordingly, a police body camera video will provide a court with the view that legally matters. Moreover, in the court of public opinion, the public's ability to see what the officer saw is critical to understanding the officer's actions. It is the view most capable of putting the public in the officer's position and giving them the context needed to determine whether or not the actions taken were reasonable. Additionally, the presence of a body camera that the officer controls can ensure that the public does not see only the provocative piece of the encounter. If operated properly, such cameras will guarantee that the entire interaction is recorded and preserved so that the focus is not solely on the final frame of the incident, and a reviewing court or the public will be able to see the events leading up to the final part of the encounter.

${ }^{10}$ CBC Radio's Ontario Today, is listened to daily (Monday to Friday) by over 500,000 Ontarians (https://www. cbc.ca/listen/shows/ontario-today). The daily circulation for The Ottawa Citizen exceeds 93,000 newspapers (http://www.cision.ca/trends/canadastop-20-daily-newspapers).

${ }^{11}$ The Globe \& Mail is Canada's largest newspaper (by daily circulation) with a Saturday circulation of approximately 403,000 newspapers (https://www.theglobe andmail.com/news/national/new-newspaper-circulation-figures/article1022927).

${ }^{12}$ Some content in this subchapter has been informed by, and modified from, materials previously presented in the form of a conference paper, titled Indecent Exposure or Academic Masturbation? Public Criminology and the Role of the Criminologist in Canada delivered on 5 May 2012 to an audience at the Critical Perspectives: Criminology and Social Justice annual conference at Carleton University (Ottawa).

${ }^{13}$ As relates to the interchangeable treatment of sociology and criminology for this purpose, I note that across the extant literature various scholars advance that both sociology and criminology share many of the same considerations in relation to public engagement and have followed a similar trajectory in relation to diminishing relevance outside of, and a retreat into, academia. Along these lines, Burawoy's (2004, 2005a) calls for increasing the practice of public sociology, including his 2004 presidential address to the American Sociological Association, are referenced by many criminologists as the catalyst for the subsequent discussion engaged in the discipline of criminology.

${ }^{14}$ The following five paragraphs present the thoughts of oft-cited scholars (Brady, Tittle, Nielsen, Carlen, and Light) on the topic of public engagement by sociologists often in the context of Burawoy's call for enhanced public sociology.

Brady (2004) applauded the fundamental objectives implicit in public sociology, which are reaching a public audience (gaining a broader and larger reception for research and theories) and serving to improve the public's wellbeing (contributing to the betterment of society). And he argued that "all sociologists are either in agreement with these goals 
or should be" (Brady 2004: 1630). However, Brady (2004) feared the failure of Burawoy's initiative because of shortcomings in the operationalization of the concept. Unlike Gans' previous efforts, which included proposals for implementation and practice (among them, "the discipline should recruit and encourage public intellectuals; we should revitalize social criticism; and we need to get more sociology into the media"), Burawoy advanced no concrete proposals for practice (Brady 2004: 1631). Brady (2004) also pointed to the lack of incentives in academia for social scientists to deviate from the current practice of scholarship through emerging from their 'academic cocoon' into the public milieu.

Tittle (2004: 1640) dismissed public sociology as antithetical to social scientific practice, it being "incompatible with good 'professional sociology'." He elaborated that, in his opinion, sociologists and criminologists have "shaky" knowledge in their subject matters and that assuming expert knowledge that can be projected into the public, and assuming superiority in knowledge, is "really quite arrogant" (Tittle 2004: 1640). Tittle (2004: 1641) believes that the pursuit of public scholarship poses a danger to "what little legitimacy sociology currently has."

Nielsen's (2004) stated opposition to a more public sociology involves his discomfort with engaging people rather than things and ideas (confessing his introversive nature and temperamental incompatibility). "Public sociology is not for me. I will happily remain in my professional cell" (Nielsen 2004: 1619).

Carlen (2011: 97) is troubled by evangelical tendencies in academic criminology (including public criminology) and "bending it to assuage a variety of differently located and variously manifested institutional or public appetites." Carlen (2011: 104) also expresses a particularly defeatist view (from her perspective within British criminology) in observing the nature of current public criminology and any potential results moving forward, "All this has not achieved much, but it is wishful thinking (or arrogance) to assume that if criminologists wrote in more easily understandable prose, or talked in the media more frequently, or took jobs as government advisors, that either the public at large or politicians would interpret their research findings in the way the critical or cultural or public criminologists might hope or expect."

Light (2005) references a recurring problem discussed at the 2004 ASA thematic sessions on public sociology - the derivative character of public sociology as a central problem to establishing its academic legitimacy. Light (2005: 1647) assures critics, "Public sociology can be practiced so that it makes original contributions to scholarship and theory." Light (2005: 1650, citing Burawoy 2004) concurs with Burawoy's contention that "professional sociology becomes stronger, more vital and less selfreferential from continuous contact with its publics." Light (2005: 1648) also refutes Tittle's (2004) concerns with legitimacy, arguing, "Good public sociology can actually strengthen and broaden that legitimacy."

15 Todd Clear is the Dean of the School of Criminal Justice at Rutgers University and past president of the American Society of Criminology. He is the founding editor of the journal Criminology and Public Policy. 
${ }^{16}$ Herbert Gans (retired Robert S. Lynd professor emeritus of sociology at Columbia University and past-president of the American Sociological Association) provides observations in regard to the decline of influence and diminished recognition of qualifications from the perspective of an eminent sociologist who has lived through the transformations in the discipline. Gans (2011), in an interview, offered his view that the discipline "made public contributions in the 1950s and 1960s and was rising both in scholarly and public status. However, since then, it seems to me to have lost that status and it may even be on a downwardly mobile path."

Similarly, in Sociology in America: A History, McAdam (2007: 411) laments the present and palpable disregard for sociology outside academia. He notes that this was not always the case. Until the 1970s "sociology enjoyed a public presence and policy resonance that far exceeds its influence today." Now, sociology is "largely organized as a set of insular, self-referential communities of scholars who, through regular conferences, specialty journals, and tight network connections reinforce the importance... of their narrow subfield-based work, even as that work grows even more disconnected from the concerns of a host of important real-world constituencies" (McAdam 2007: 426).

Loader and Sparks $(2008,2010 a, 2010 b, 2011)$ have written prolifically on public criminology. A 2008 article discussed criminology's "successful failure." A situation in which academic criminology is highly successful while criminological knowledge, influence, and attempts at policy intervention are largely marginalized in the public sphere (Loader \& Sparks 2008: 18-19).

${ }^{17}$ Greg McCarthur's “reporting has been recognized by all of the major journalism awards in Canada: he has won two National Newspaper Awards, several National Magazine Awards and the top prize awarded by the Canadian Association of Journalists" (https:// www.theglobeandmail.com/authors/greg-mcarthur/). 


\section{NOTES FOR APPENDICES}

${ }^{1}$ All population figures presented throughout these appendices are approximations (as obtained from the cited source) and have been rounded to the nearest value of 1,000.

${ }^{2}$ These Canadian rankings include RCMP and provincial police detachments performing municipal policing.

${ }^{3}$ Based on the low-income measure after tax (LIM-AT) as calculated by Statistics Canada.

${ }^{4}$ Integrated operations combine personnel from various Metro Vancouver police agencies for specialized tasks, such as tactical operations, forensic identification, homicide investigations, illicit drug interdiction, and crowd control (demonstrations, protests, and civil disorder)., gangs, drug trafficking, human trafficking, firearms trafficking, financial crimes, etc.

${ }^{5}$ This estimation is based on my experience as a former OPS front-line platoon supervisor (2010-2013) and the information obtained during a 2015 study I conducted for the OPS as a research consultant.

${ }^{6}$ All front-line officers in this detachment are assigned to one of four teams on two watches (A Watch or B Watch). Due to a scheduling miscommunication with RCMP management, I was unable to meet with officers on A Watch while in Moncton on 28 June 2016 and 29 June 2016. Therefore, a supply of surveys (both English and French) was left with Inspector George to be administered by him (on my behalf) at the next shift briefings for A Watch. Inspector George informed me that he did so over the next three days (including a few B Watch officers that had been off work on 28 June 2016 and 29 June 2016) and he subsequently forwarded me the completed survey documents in three sealed envelopes. Prior to my departure from Moncton, Inspector George and I met, and he was instructed on this study's protocol for survey administration (to ensure participant anonymity) and he assured me, subsequently, that he had followed the protocol. In this regard, Inspector George ensured that all potential participants on the shift briefings had reviewed the study's introductory document. All officers that agreed to complete a survey, on each of three briefings, deposited that document into an envelope, which was sealed and validated jointly by the last officer to place his/her survey into the envelope and Inspector George. These sealed envelopes were then couriered to me and I received them intact.

${ }^{7}$ As potentially relates to issues around trust, mode of administration, and/or researcher positionality, and as discussed further in subchapter 4.6, four (4) participants of the 69 from this police agency omitted certain potentially-identifying demographic information in the surveys submitted. Participants COD09, COD53, and COD68 did not disclose their years of front-line policing experience and COD39 did not share information about their race/ethnicity. 
${ }^{8}$ Illustrative of some participants' (and potential participants that declined to participate) distrust in policing research and/or researchers, are officers such as WIN06 (a veteran Windsor officer), who provided gender and age information but left the race/ ethnicity question (Question 3) blank and presented a range response (' $10+$ ') to Questions 4 and 5 in the survey, which invited an answer in the form of a continuous variable.

${ }^{9} \mathrm{Mr}$. Stamatakis, who joined the VPD as a police officer in 1990, has served as the president of the VPU since 2000 and also currently serves as the president of the British Columbia Police Association (since its inception in 2008) and president of the Canadian Police Association (since 2011).

${ }^{10}$ Ironically, both the Canadian Association of Chiefs of Police (CACP) Research Foundation and the CACP Ethics Committee had endorsed the present study. 


\section{LIST OF CASES}

Beaudry v. The Queen [2007] 1 SCR 190

Brown v. Durham Regional Police Force [1998] 131 C.C.C. (3d)

Heien v. North Carolina 135 S. Ct. 530 (2014)

R. v. Brown [2003] O.J. No. 1251

R. v. Gonzales [2011] O.J. No. 395

R. v. Gonzales [2012] ONCA 861

R. v. Khan [2004] O.J. No. 3819

R. v. Ladouceur [1990] 1 S.C.R. 1257

R. v. Nguyen [2012] ABQB 199

R. v. Phengchanh [2011] BCSC 484

R. v. Tombs [2012] BCSC 1826

Terry v. Ohio 392 U.S. 1 (1968)

Whren v. United States 517 U.S. 806 (1996) 


\section{REFERENCES}

1310 News (2011) Judge slams Ottawa Police officer for racial profiling. 1310 News. 7 July. [Online] http://www.1310news.com/2011/07/07/judge-slams-ottawa-policeofficer-for-racial-profiling/.

Aas, K., Gundhus, H. \& Lomell, H. (2009) Technologies of (In)Security: The Surveillance of Everyday Life. London: Routledge.

ABC News (2001) Protect and serve? De-Policing in urban neighborhoods. Nightline. 23 July [Online] https://archive.org/details/ABC-Nightline-2001-07-23.

Abend, G. (2008) The meaning of theory. Sociological Theory, 26: 173-199.

Ackerman, G., Anderson, B., Jensen, S., Ludwig, R., Montero, D., Plante, N., et al. (2001) Crime rates and confidence in the police: America's changing attitudes toward crime and police, 1972-1999. Journal of Sociology \& Social Welfare, 28: 43-55.

Adams, G. \& Buck, J. (2010) Social stressors and strain among police officers: It's not just the bad guys. Criminal Justice \& Behaviour, 37(9): 1030-1040.

Adams, K. (2015) What we know about police use of force. In (Dunham, R. \& G. Alpert, eds.) Critical Issues in Policing, 7th ed.: 532-566. Long Grove, IL: Waveland Press.

Adler, P. \& Adler, P. (1987) Membership Roles in Field Research. Thousand Oaks, CA: Sage.

Adler, S., \& Aranya, N. (1984) A comparison of the work needs, attitudes, and preferences of professional accountants at different career stages. Journal of Vocational Behaviour, 25(1): 45-57.

Agnew, R. (1985) A revised strain theory of delinquency. Social Forces, 64(1): 151167.

Agnew, R. (1992) Foundation for a general strain theory of crime and delinquency. Criminology, 30: 47-87.

Agnew, R. (2001) Building on the foundation of general strain theory: Specifying the types of strain most likely to lead to crime and delinquency. Journal of Research in Crime \& Delinquency, 38(4): 319-361.

Agnew, R. (2006) Pressured into Crime: An Overview of General Strain Theory. Los Angeles: Roxbury Publishing.

Ajzen, I. \& Fishbein, M. (1980) Understanding Attitudes and Predicting Social Behavior. Englewood Cliffs, NJ: Prentice-Hall.

Ajzen, I. \& Fishbein, M. (2005) The influence of attitudes on behaviours. In (D. Albarracin, B. Johnson \& M. Zanna, eds.) The Handbook of Attitudes: 173-211. Mahwah, NJ: Erlbaum. 
Akers, R. (1998) Social Learning and Social Structure: A General Theory of Crime and Deviance. Boston: Northeastern University Press.

Akers, R., Krohn, M., Lanza-Kaduce, L. Radosevich, M. (1979) Social learning and deviant behavior: A specific test of a general theory. American Sociological Review, 44(4): 636-655.

Alain, M., \& Grégoire, M. (2008). Can ethics survive the shock of the job? Québec's police recruits confront reality. Policing \& Society, 18(2): 169-189.

Albrecht, S. \& Green, M. (1977) Attitudes toward the police and the larger attitude complex: Implications for police-community relationships. Criminology, 15: 67-86.

Albuquerque, C. \& Paes-Machado, E. (2004) The hazing machine: The shaping of Brazilian military recruits. Policing \& Society, 14(2): 175-192.

Aldridge, A. \& Levine, K. 2001. Surveying the Social World: Principles and Practice in Survey Research. Buckingham, UK: Open University Press.

Alexander, J. (2003) The Meanings of Social Life: A Cultural Sociology. New York: Oxford University Press.

Alexander, M. (2011) The New Jim Crow: Mass Incarceration in the Age of Colourblindness. New York: The New Press.

Alise, M. \& Teddlie, C. (2010) A continuation of the paradigm wars? Prevalence rates of methodological approaches across the social/behavioral sciences. Journal of Mixed Methods Research, 4(2): 103-126.

Allan, S. (2013) Citizen Witnessing: Revisioning Journalism in Times of Crisis. Cambridge: Polity Press.

Allen, N. \& Meyer, J. (1993) Organizational commitment: Evidence of career stage effects? Journal of Business Research, 26(1): 49-61.

Allisey, A., Noblet, A., Lamontagne, A \& Houdmont, J. (2014) Testing a model of officer intentions to quit: The mediating effects of job stress and job satisfaction. Criminal Justice \& Behavior, 41(6): 751-771.

Alpert, G., Dunham, R. \& Piquero, A. (1998) On the study of neighborhoods and the police. In (Alpert, G. \& Piquero, A., eds.) Community Policing: Contemporary Readings: 309-326. Prospect Heights, IL: Waveland Press.

Alpert, G., Rojek, J. \& Porter, L. (2012) Measuring the Impact of Organisational Culture and Climate on Police Officers' Decisions and Behaviour. Brisbane, Australia: Centre of Excellence in Policing and Security.

Amaranto, E., Steinberg, J., Castellano, C. \& Mitchell, R. (2003) Police stress interventions. Brief Treatment \& Crises Intervention, 3(1), 47-53.

Anderson, E. (1990) Streetwise: Race, Class, and Change in an Urban Community. Chicago: University of Chicago Press. 
Andrejevic, M. (2005) The work of watching one another: Lateral surveillance, risk, and governance. Surveillance \& Society, 2(4): 479-497.

Andrejevic, M. (2007) iSpy: Surveillance and Power in the Interactive Era. Lawrence, KS: University Press of Kansas.

Andrew-Gee, E. (2014) YouTube video had huge impact on Sammy Yatim case.

Toronto Star. 27 July. [Online] Retrieved on 9 August 2014 from http://www.thestar .com/news/crime /2014/07/27/youtube_video_had_huge_impact_on_sammy_ yatim_case.html.

Ansari, A. \& Flores, R. (2017) Chicago's 762 homicides in 2016 is highest in 19 years. CNN. 2 January. [Online] https://www.cnn.com/2017/01/01/us/chicago-murders-2016/ index.html.

Antony, M. \& Thomas, R. (2010) This is citizen journalism at its finest: YouTube and the public sphere in the Oscar Grant shooting incident. New Media \& Society, 12(8): 1280-1296.

Apple, N. \& O'Brien, D. (1983) Neighborhood racial composition and residents' evaluation of police performance. Journal of Police Science and Administration, 11: 7684.

Ariel, B., Farrar, W. \& Sutherland, A. (2014) The effect of police body-worn cameras on use of force and citizens' complaints against the police: A randomized controlled trial. Journal of Quantitative Criminology, 31(3): 509-535.

Armstrong, S. \& McAra, L. (2003) Audiences, borders, architecture: The contours of control. In (S. Armstrong \& L. McAra, eds.) Perspectives on Punishment: The Contours of Control: 1-30. Oxford: Oxford University Press.

Arnold, L. (2014) Marrying Mixed Methods and Critical Realism. [Online] https:// lydiaarnold. files.wordpress.com/2014/06/mixed-methods.pdf.

Aronowitz, S. (2005) Comments on Michael Burawoy's 'The critical turn to public sociology'. Critical Sociology, 31(3): 333-338.

Arter, M. (2007) Stress and deviance in policing. Deviant Behavior, 29(1): 43-69.

Aryee, S., Chay, Y. \& Chew, J. (1994) An investigation of the predictors and outcomes of career commitment in three career stages. Journal of Vocational Behavior, 44(1):116.

Asher-Shapiro, A. (2015) The FBI Director says cops are 'under siege' from viral videos. Vice News. 27 October [Online] https://news.vice.com/article/the-fbi-directorsays-cops-are-under-siege-from-viral-videos.

Associated Press (2009) Justice dept. reports on Yonkers' police. New York Daily News. 21 June. [Online] http://www.nydailynews.com/new-york/brox/justce-dept-reportsyonkers-olice-article-1.375938. 
Associated Press (2014a) Former CIA director Michael Hayden: 'I didn't lie' about torture. Huffington Post. 10 December. [Online] http://www.huffingtonpost.com/ 2014/12/10/michael-hayden-cia-torture-lie_n_6300656 .html.

Associated Press (2014b) St. Louis officers 'quickly' release video of deadly police shooting after 'lessons learned from Ferguson'. The National Post. 21 August. [Online] http://news.nationalpost.com/2014/08/21/st-louis-police-quickly-release-video-ofdeadly-police-shooting-after-lessons-learned-from-ferguson/.

Associated Press (2017) Football carried NBC, ESPN to big wins in audience last week. The Daily Mail. 4 January. [Online] http://www.dailymail.co.uk/wires/ap/article4088632 /Football-carried-NBC-ESPN-big-wins-audience-week.html.

Association of Chief Police Officers (2008) ACPO Response to the Green Paper: From the Neighbourhood to the National. London: Association of Chief Police Officers.

Atton, C. (2002) Alternative Media. London: Sage.

Austin, R. (2014) Response to your petition on the use of body-worn cameras. The White House. [Online] https://petitions.whitehouse.gov/petition/mike-brown-lawrequires-all-state-county-and-local-police-wear-camera/8tlS5czf.

Axelson, B. (2017) Syracuse, Rochester, Buffalo all rank among the worst U.S. cities to live in. NYup.com. 17 June. [Online] http://www.newyorkupstate.com/news/ 2017/06/ syracuse_rochester_buffalo_all_rank_among_the_worst_us_cities_to_live_in $2 . \mathrm{html}$.

Azpiri, J. (2016) Officers involved in Maple Ridge takedown video being investigated. Global News. 26 January. [Online] https:/globalnews.ca/news/2476976/exclusivewitness-captures-rcmp-takedown-in-maple-ridge-on-video/.

Babbie, E., (1992) The Practice of Social Research. New York: Macmillan.

Babwin, D. (2017a) 1 of Chicago's Bloodiest Years Ends With 762 Homicides. The Associated Press. 2 January 2017. [Online] https://apnews.com/aec6bc156b594589 a6ef5ecf9657b1b2.

Babwin, D. (2017b) Murder city: Chicago finishes 2016 with 762 homicides - more than New York and Los Angeles combined. The National Post. 1 January 2017. [Online] https://nationalpost.com/news/world/murder-city-chicago-finishes-2016-with762-homicides-more-than-new-york-and-los-angeles-combined.

Bacon, M. (2014) Police culture and the new policing context. In (J. Brown, ed.), The Future of Policing: 133-149. New York: Routledge.

Baker, A. \& Goodman, D. (2014) Arrest statistics decline sharply; Police unions deny an organized slowdown. The New York Times. 31 December. [Online] http://www. nytimes.com /2015/01/01/nyregion/arrest-statistics-decline-sharply-police-unions-denyan-organized-slowdown.html?_r=0.

Ball, K. \& Webster, F. (2003) The Intensification of Surveillance: Crime, Terrorism and Warfare in the Information Age. London: Pluto Press. 
Ball, K., Haggerty, K. \& Lyon, D. (2012) Introducing surveillance studies. In (K. Ball, K. Haggerty \& D. Lyon, eds.) Routledge Handbook of Surveillance Studies. New York: Routledge.

Banton, M. (1964) The Policeman in the Community. London: Tavistock.

Barak, G. (1988) Newsmaking criminology: Reflections on the media, intellectuals and crime. Justice Quarterly, 5(4): 565-585.

Barak. G. (2007) Doing newsmaking criminology from within the academy. Theoretical Criminology, 11(2): 191-207.

Barker, B., Alfred, G., Fleming, K., Nguyen, P., Wood, E., Kerr, T. \& DeBeck, K. (2015) Aboriginal street-involved youth experience elevated risk of incarceration.

Public Health, 129(12): 1662-1668.

Barker, J. (1999) Danger, Duty, and Disillusion: The Worldview of Los Angeles Police Officers. Prospect Heights, IL: Waveland Press.

Barrett, P. (2014) The verdict from Ferguson: Put video cameras on cops. Business Week. 25 November. [Online]. http://www.businessweek.com/ articles/2014-11-25/theverdict-from-ferguson-put-video-cameras-on-cops.

Barton, A. \& Johns, N. (2014) Engaging the citizen. In (J. Brown, ed.) The Future of Policing: 417-428. New York: Routledge.

Bass, S. (2001) Policing space, policing race: Social control imperatives and police discretionary decisions. Social Justice, 28: 156-176.

Bauman, Z. (2000) Social issues of law and order. British Journal of Criminology, 40: 205-221.

Bauman, Z. (2006) Liquid Fear. Cambridge, UK: Polity Press.

Bauman, Z. \& Lyon, D. (2013) Liquid Surveillance: A Conversation. Hoboken, NJ: Wiley-Blackwell.

Bayley, D. (1983) Accountability and control of the police: Lessons from Britain. In (T. Bennett, ed.) The Future of Policing. Cambridge: Cambridge University Press.

Bayley, D. (1985) Patterns of Policing: A Comparative International Analysis. New Brunswick, NJ: Rutgers University Press.

Bayley, D. (1988) Community policing: A report from the devil's advocate. In (J. Greene \& S. Mastrofski, eds.) Community Policing: Rhetoric or Reality? 225-238. New York: Praeger.

Bayley, D. (1994) Police for the Future. New York: Oxford University Press.

Bayley, D. (1995) Getting serious about police brutality. In (P. Stenning, ed.) Accountability for Criminal Justice: Selected Essays. Toronto: University of Toronto Press. 
Bayley, D. (2005) What do the police do? In (T. Newburn, ed.) Policing: Key Readings: 141-149. Cullompton: Willan.

Bayley, D. (2011) Et tu brute: Are police agencies managed better or worse than universities? Police Practice \& Research, 12(4): 313-316.

Bayley, D. (2013) Policing the world stage. In (R. Mawby, ed.) Policing Across the World: Issues for the Twenty-First Century: 3-12. New York: Routledge.

Bayley, D. \& Bittner, E. (1984). Learning the skills of policing. Law and Contemporary Problems, 47(4), 35-59.

Bayley, D. \& Garafalo, J. (1989) The management of violence by police patrol officers. Criminology, 27(1): 1-27.

Bayley, D. \& Mendelsohn, H. (1969) Minorities and the Police: Confrontations in America. New York: Free Press.

Bayley, D. \& Shearing, C. (1996) The future of policing. Law \& Society Review, 30(3): 585-606.

Bayley, D. \& Shearing, C. (2003) The future of policing. In (Newburn, T., ed.) Policing: Key Readings. Cullompton: Willan.

Bazeley, P. (2015) Writing up multimethod and mixed research for diverse audiences. In (C. Hesse-Biber \& R. Johnson, eds.) The Oxford Handbook of Multimethod and Mixed Method Research Inquiry. Pp 296-315. New York: Oxford University Press.

BBC News (2005) Transcript of Sir Ian Blair's Speech. [Online] Retrieved on 17 February 2014 from http://news.bbc.co.uk/2/hi/uk_news/4443386.stm.

BBC News (2014) New St. Louis shooting 'unlike Ferguson', says mayor. BBC News. 25 December. [Online] http://www.bbc.com/news/world-us-canada- 30601305.

Beck, K. \& Wilson, C. (2000) Development of affective organizational commitment: A cross-sequential examination of change with tenure. Journal of Vocational Behaviour, 56(1): 114-136.

Beck, U. (1992a) Risk Society: Toward a New Modernity, trans. M. Ritter. London: Sage.

Beck, U. (1992b) Modern society as risk society. In (N. Stehr \& R. Ericson, eds.) The Culture and Power of Knowledge: Inquiries into Contemporary Societies: 199-214. New York: Walter de Gruyter.

Beck, U. (1994) The reinvention of politics: Towards a theory of reflexive modernization. In (Beck, U., Giddens, A. \& Lash, S., eds.) Reflexive Modernization: Politics, Tradition and Aesthetics in the Modern Social Order: 1-55. Stanford: Stanford University Press.

Beck. U., Giddens, A. \& Lash, S. (1994) Reflexive Modernization: Politics, Tradition and Aesthetics in the Modern Social Order. Stanford: Stanford University Press. 
Beckett, K. \& Sasson, T. (2004) The Politics of Injustice: Crime and Punishment in America, $2^{\text {nd }}$ ed. Thousand Oaks, CA: Sage.

Beckett, L. (2016) Is the 'Ferguson effect' real? Researcher has second thoughts. The Guardian. 13 May. [Online] https:/www.theguardian.com/us-news/2016/may/13/ ferguson-effect-real-researcher-richard-rosenfield-second-thoughts.

Bedeian, A., Pizzolatto, A., Long, R. \& Griffith, R. (1991) The measurement and conceptualization of career stages. Journal of Career Development, 17: 153-166.

Bedi, S. (2017) Chicago incentivizes abusive policing through overtime policy. The Chicago Reporter. 16 October. [Online] https://www.chicagoreporter.com/chicagoincentivizes-abusive-policing-through-overtime-policy/.

Bekiempis, V. (2015) The new racial makeup of U.S. police departments.

Newsweek. 14 May. [Online] http://www.newsweek.com/racial-makeup-policedepartments-331130.

Bell, D. (2009) Surveillance is sexy. Surveillance \& Society, 6(3): 203-212.

Benazquen, M., Cave, D. \& Oliver, R. (2017) The raw videos that have sparked outrage over police treatment of Blacks. The New York Times. 19 August. [Online] https://www. nytimes.com/interactive /2017/08/19/us/police-videos-race.html.

Benkler, Y. (2006) The Wealth of Networks: How Social Production Transforms Markets and Freedom. New Haven, CT: Yale University Press.

Benner, P. \& Phillips, S., eds. (1994) The Crisis of Care: Affirming and Restoring Caring Practices in the Helping Professions. Washington, DC: Georgetown University Press.

Bennett, K. (2017) Hamilton police officer found guilty of assault. CBC News. 26 April. [Online] http://www.cbc.ca/news/canada/hamilton/hamilton-police-officer-foundguilty-of-assault-1.4086320.

Bennett, R. (1984) Becoming blue: A longitudinal study of police recruit occupational socialization. Journal of Police Science \& Administration, 12: 47-58.

Bennett, R. \& Greenstein, T. (1975) The police personality: A test of the predisposition model. Journal of Police Science \& Administration, 3: 439-445.

Bennis, W., Goleman, D. \& O’Toole, J., eds. (2008) Transparency: How Leaders Create a Culture of Candor. San Francisco, CA: Jossey-Bass.

Berg, B. (2009) Qualitative Research Methods for the Social Sciences, $7^{\text {th }}$ ed. Boston: Allyn \& Bacon.

Berman, M. (2016) Chicago has had more homicides this year than New York and Los Angeles combined. The Washington Post. 1 September. [Online] https://www. washingtonpost.com/news/post-nation/wp/2016/09/01/chicago-has-seen-morehomicides -this-year-than-new-york-and-los-angeles combined/?utm_term $=.89 \mathrm{e} 5 \mathrm{c}$ $829 \mathrm{a} 730$. 
Berman, M. \& Lowery, W. (2014) Ferguson police say Michael Brown was a robbery suspect, identify Darren Wilson as officer who shot him. The Washington Post 15 August. [Online] http://www.washingtonpost.com/news/post-nation/wp/2014/08/15/ ferguson-police-releasing-name-of-officer-who-shot-michael-brown/.

Berman, R. (2015) Obama's missing police-reform proposal. The Atlantic. 18 May. [Online] https://www.theatlantic.com/politics/archive/2015/05/the-limits-of-policereform $/ 393530 /$.

Berry, J. (2010). Reducing Bureaucracy in Policing: Final Report. London: Home Office.

Better Regulation Commission (2006) Risk, Responsibility, Regulation: Whose Risk Is It Anyway? London: Stationery Office.

Biemer, P. \& Lyberg, L. 2003. Introduction to Survey Quality. Hoboken, NJ: Wiley.

Birchall, C. (2011a) The politics of opacity and openness. Theory, Culture \& Society, 28(7-8): 7-25.

Birchall, C. (2011b) There's been to much secrecy in this city: The false choice between secrecy and transparency in US politics. Cultural Politics, 7(1): 133-156.

Birchall, C. (2011c) Transparency interrupted: Secrets of the left. Theory, Culture \& Society, 28(7-8): 60-84.

Birchall, C. (2014) Radical Transparency? Cultural Studies \& Critical Methodologies, 14(1): 77-88.

Birkinshaw, P. (2006) Transparency as a human right. In (Hood, C. \& Heald, D., eds.) Transparency: The Key to Better Governance? 47-58. Oxford: Oxford University Press.

Bishopp, S. \& Boots, D. (2014) General strain theory, exposure to violence, and suicide ideation among police officers: A gendered approach. Journal of Criminal Justice, 42(6): 538-548.

Bishopp, S., Piquero, N., Piquero, A. \& Worrall, J. (2018) Police stress and race: Using general strain theory to explain racial differences in police suicide ideation and misconduct. Conference paper presented at the American Society of Criminology annual meeting, Atlanta, GA, 14 November.

Bishopp, S., Piquero, N., Worrall, J. \& Piquero. A. (2018) Negative affective responses to stress among urban police officers: A general strain theory approach. Deviant Behaviour. DOI: 10.1080/01639625.2018.1438069.

Bishopp, S., Worrall, J. \& Piquero, N. (2016) General strain and police misconduct: The role of organizational influence. Policing: An International Journal of Police Strategies \& Management, 39(4): 635-651.

Bisman, J. (2010) Postpositivism and accounting research: A (personal) primer on critical realism. Australasian Accounting, Business and Finance Journal, 4(4): 3-25. 
Bittner, E. (1967) The police on skid-row: A study of peacekeeping. American Sociological Review, 32(5): 699-715.

Bittner, E. (1970) The Function of the Police in Modern Society: A Review of Background Factors, Current Practices, and Possible Role Models. Washington: National Institute of Mental Health.

Bittner, E. (1974). Florence Nightingale in pursuit of Willie Sutton: A theory of the police. In (H. Jacob, ed.) The Potential for Reform of Criminal Justice: 17-44. Beverly Hills, CA: Sage.

Bittner, E. (1990) Aspects of Police Work. Boston: Northeastern University Press.

Bittner, E. (2006) The quasi-military organization of the police. In (V. Kappeler, ed.) The Police \& Society: Touchstone Readings, 3rd ed.: 190-200. Long Grove, IL: Waveland Press.

Black, D. (1971) The social organization of arrest. Stanford Law Review, 23(6): 1087-1111.

Black, D. (1982) Manners and Customs of the Police. New York: Academic Press.

Black, D. \& Reiss, A. (1967) Patterns of behavior in police and citizen transactions. Studies of Crime and Law Enforcement in Major Metropolitan Areas. Washington: U.S. Government Printing Office.

Blair, J., Czaja, R. \& Blair, E. (2014) Designing Surveys: A Guide to Decisions and Procedures, $3^{\text {rd }}$ ed. Thousand Oaks, CA: Sage.

Blake, J. (1981) The role of the police in society. In (W. Mcgrath \& M. Mitchell, eds.) Police Function in Canada: 77-84. Toronto: Methuen.

Blatchford, C. (2015a) Controversial 'carding' practice is an invaluable source of intelligence for police, if done right. The National Post. 5 June: 2.

Blatchford, C. (2015b) Canada's citizens aren't the enemy, and neither are the cops. The National Post, 3 December: 3.

Blum, L. (2000). Force Under Pressure: How Cops Live and why they Die. New York, NY: Lantern.

Blumberg, M. (1981) Race and police shootings: Analysis in two cities. In (J. Fyfe, ed.) Contemporary Issues in Law Enforcement: 152-166. Beverly Hills, CA: Sage.

Bock, M. (2016) Film the police! Cop-watching and its embodied narratives. Journal of Communication, 66: 13-34.

Bogard, W. (2006) Surveillance assemblages and lines of flight. In (Lyon, D., ed.) Theorizing Surveillance: The Panopticon and Beyond: 97-121. Cullompton, UK: Willan.

Boivin, R., Faubert, C., Gendron, A. \& Poulin, B. (2018) The 'us vs them' mentality: A comparison of police cadets at different stages of their training, Police Practice \& Research, DOI: 10.1080/15614263.2018.1555480 
Bond, M. (2019) Toronto police cruiser: Daytime close up. 680 News. 7 July. [Online] https://www.680news.com/2019/07/07/2-men-arrested-after-car-crashes-into-etobicokehome/toronto-police-cruiser-daytime-closeup-jpg-6/.

Bonifacio, P. (1991) The Psychological Effects of Police Work: A Psychodynamic Approach. New York: Plenum Press.

Bonilla, Y. \& Rosa, J. (2015) \#Ferguson: Digital protest, hashtag ethnography, and the racial politics of social media in the United States. American Ethnologist, 42(1): 4-17.

Bonilla-Silva, E. (2014) Racism without Racists: Color-Blindness and the Persistence of Racial Inequality in America. Lanham, MD: Rowman \& Littlefield.

Bonkiewicz, L. (2017) Shooting stars: Estimating the career productivity trajectories of patrol officers. Police Quarterly, 20(2): 164-188.

Bordeleau, C. (2018) Report to Ottawa Police Services Board - 29 January 2018.

Ottawa: Ottawa Police Service.

Bordua, D. \& Reiss, A. (1966) Command, control and charisma: Reflections on police bureaucracy. American Journal of Sociology, 72(1): 68-76.

Bosworth, M. \& Hoyle, C. (2011) What is criminology? An introduction. In (M. Bosworth \& C. Hoyle, eds.) What is Criminology? 1-9. New York: Oxford University Press.

Bottoms, A. \& Tankebe, J. (2012) Beyond procedural justice: A dialogical approach to legitimacy in criminal justice. Journal of Criminal Law and Criminology, 102(1): 119170.

Bottoms, A. \& Tankebe, J. (2013) A voice within: Power-holders perspectives on authority and legitimacy. In (Tankebe, J. \& and Leibling, A., eds.) Legitimacy and Criminal Justice: An International Exploration: 60-82. Oxford: Oxford University Press.

Bourdieu, P. (1990) The Logic of Practice. Stanford, CA: Stanford University Press.

Bourdieu, P. \& Wacquant, L. (1992) An Invitation to Reflexive Sociology. Cambridge, UK: Polity.

Bowling, B. (2007) Fair and effective police methods: Towards 'good enough' policing. Scandinavian Studies in Criminology \& Crime Prevention, 21(4): 395-407.

Bowling, B., Iyer, S., Reiner, R. \& Sheptycki, J. (2016) Policing: Past, present and future. In (R. Matthews, ed.) What is to be Done About Crime and Punishment? 123158. London, Palgrave Macmillan.

Bowling, B. \& Phillips, C. (2002) Racism, Crime and Justice. New York: Pearson Education.

Bowman, S. \& Willis, C. (2003) We Media: How Audiences are Shaping the Future of News and Information. Reston, VA: The Media Centre at the American Press Institute. 
Bradford, B. (2011) Convergence, not divergence? Trends and trajectories in public contact and confidence in the police. British Journal of Criminology, 51(1): 179-200.

Bradford, B. \& Jackson, J. (2010) What is trust and confidence in the police? Policing: A Journal of Policy and Practice, 4(3). 241-248.

Bradford, B., Jackson, J. \& Hough, M. (2014) Police futures and legitimacy: Redefining 'good policing'. In (J. Brown, ed.) The Future of Policing: 79-99. New York:

Routledge.

Bradford, B., Jackson, J. \& Stanko, E. (2009) Contact and confidence: Revisiting the impact of public encounters with the police. Policing \& Society, 19(1): 20-46.

Bradford, B. \& Quinton, P. (2014) Self-legitimacy, police culture and support for democratic policing in an English constabulary. British Journal of Criminology, 54(6): 1023-1046.

Bradley, D. (2007). Black \& Blue: Understanding Modern Law Enforcement in your America. Bloomington, IN: Author House.

Bradley, D., Nixon, C. \& Marks, M. (2006) What works, what doesn't work and what looks promising in police research networks. In (J. Fleming \& J. Wood, eds.) Fighting Crime Together: The Challenges of Policing and Security Networks. Sydney: University of New South Wales Press.

Brady, D. (2004) Why public sociology may fail. Social Forces, 82(4): 1629-1638.

Braga, A., Kennedy, D., Waring, E. \& Piehl, A. (2001) Problem-oriented policing, deterrence, and youth violence: An evaluation of Boston's operation ceasefire. Journal of Research in Crime \& Delinquency, 38(3): 195-225.

Braga, A, Papachristos, A. \& Hureau, D. (2014) The effects of hot spots policing oncrime: An updated systematic review and meta-analysis. Justice Quarterly, 31(4): 633-663.

Braga, A., Sousa, W., Coldren, J. \& Rodriguez, D. (2018) The effects of body-worn cameras on police activity and police-citizen encounters: A randomized controlled trial. Journal of Criminal Law \& Criminology, 108(3): 511-538.

Braga, A. \& Weisburd, D. (2010) Policing Problem Places: Crime, Hot Spots, and Effective Prevention. Oxford: Oxford University Press.

Braga, A. \& Weisburd, D. (2012) The effects of 'pulling levers': Focused deterrence strategies on crime. Campbell Systematic Reviews, 8(6): 1-88.

Braga, A., Welsh, B. \& Schnell, C. (2015). Can policing disorder reduce crime? A systematic review and meta-analysis. Journal of Research in Crime \& Delinquency, 52(4): 567-588.

Brandl S., Frank, J., Worden, R. \& Bynum, T. (1994) Global and specific attitudes toward the police: Disentangling the relationship. Justice Quarterly, 11: 119-134. 
Brandle, S., Stroshine, M. \& Frank, J. (2001) Who are the complaint prone officers? An examination of the relationship between police officers' attributes, arrests activity, assignment, and citizens' complains about excessive force. Journal of Criminal Justice, 29: $521-529$.

Brannick, T. \& Coghlan, D. (2007) In defence of being 'native': The case for insider academic research. Organizational Research Methods, 10(1): 59-74.

Brants, C. (2005) Human rights: A state of mind - Policing and the criminal law in a democratic society. In (N. Uildriks, ed.) Police Reform and Human Rights: 15-25. Antwerp: Intersentia.

Brehm, J. \& Gates, S. (1993) Donut shops and speed traps: Evaluating models of supervision on police behaviour. American Journal of Political Science, 37(2): 555581.

Brehm, J. \& Gates, S. (1994). When supervision fails to induce compliance. Journal of Theoretical Politics, 6: 323-343.

Brehm, J. \& Gates, S. (1999) Working, Shirking, and Sabotage: Bureaucratic Response to a Democratic Public. Ann Arbor: University of Michigan Press.

Brent, J. \& Kraska, P. (2012) Moving beyond our methodological default: A case for mixed methods. In (H. Copes, ed.) Advancing Qualitative Methods in Criminology and Criminal Justice: 25-43. London: Routledge.

Brewer, G., Coleman-Selden, S. \& Facer, R. (2000) Individual conceptions of public service motivation. Public Administration Review, 60: 254-264.

Bridenball, B. \& Jesilow, P. (2008) What matters? The formation of attitudes toward the police. Police Quarterly, 11(2): 151-181.

Bridges, G \& Steen, S. (1998) Racial disparities in official assessment of juvenile offenders: Attributional stereotypes as mediating mechanisms. American Sociological Review, 63: 554-570.

Brighenti, A. (2007) Visibility: A category for the social sciences. Current Sociology, 55(3): 323-342.

Brighenti, A. (2010a) Democracy and its visibilities. In (Haggerty, K. \& Samatas, M., eds.) Surveillance and Democracy: 51-68. New York: Routledge-Cavendish.

Brighenti, A. (2010b) Visibility in Social Theory and Social Research. Basingstoke, UK: Palgrave Macmillan.

Broderick, J. (1977) Police in a Time of Change. Morristown, NJ: General Learning Press.

Brodeur, J. (2005) Trotsky in blue: Permanent policing reform. Australian \& New Zealand Journal of Criminology, 38(2): 254-267.

Brodeur, J. (2007) An encounter with Egon Bittner. Crime, Law \& Social Change, 48(3): 105-132. 
Brodeur, J. (2010) The Policing Web. Oxford, UK: Oxford University Press.

Brogden, M. \& Nijhar, P. (2005) Community Policing. Cullompton: Willan.

Brogden, M., Jefferson, T. \& Walklate, S. (1988) Introducing Policework. London: Unwin Hyman.

Brooks, L. (2010) Police discretionary behavior: A study of style. In (R. Dunham \& G. Alpert, eds.) Critical Issues in Policing: Contemporary Readings, ${ }^{\text {th }}$ ed.: 71-89. Long Grove, IL: Waveland Press.

Brooks, L. \& Piquero, N. (1998) Police stress: Does department size matter? Policing: An International Journal of Police Strategies and Management, 21(4): 600-617.

Brooms, D. (2018) 'They don't really care about us': Policing Black and Brown lives and futures. In (S. Weissinger \& D. Mack, eds.) Law Enforcment in the Age of Black Lives Matter: Policing Black and Brown Bodies: 137-152. New York: Lexington Books.

Brough, P., Chataway, S., \& Biggs, A. (2016) You don't want people knowing you're a copper!' A contemporary assessment of police organisational culture. International Journal of Police Science \& Management, 18(1): 28-36.

Brown, B. \& Benedict, W. (2002) Perceptions of the police. Policing, An International Journal of Police Strategies and Management, 25: 543-580.

Brown, G. (2013) The Blue Line on Thin Ice: Police Use of Force in the Era of Cameraphones, 'Citizen Journalism', and YouTube. Unpublished MA thesis. Carleton University.

Brown, G. (2016) The blue line on thin ice: Police use of force modifications in the era of cameraphones and YouTube. British Journal of Criminology, 56(2): 293-312.

Brown, G. (2017) A dialogue of collaboration: Cooperation in Canadian policing research today. Police Practice \& Research, 18(6): 528-543.

Brown, G. \& Primeau, P. (2015) Traffic Stop Race Data Collection Project: Impacts on Front-Line Officers (Draft Report). Unpublished research report. Ottawa: Ottawa Police Service.

Brown, J. (1996) Police research: Some critical issues. In (F. Leishman, B. Loveday \& S. Savage, eds.) Core Issues in Policing: 177-190. London: Longman.

Brown, J. (2007) From cult of masculinity to smart macho: Gender perspectives on police occupational culture. In (M. O’Neil, M. Marks \& A. Singh, eds.) Police Occupational Culture: New Debates and Directions: 205-226. Oxford: Elsevier.

Brown, J., ed. (2014) The Future of Policing. New York: Routledge.

Brown, L. (2004). Officer or overseer? Why police desegregation fails as an adequate solution to racist, oppressive, and violent policing in Black communities. NYU Review of Law \& Social Change, 29: 757-795. 
Brown, M. (1988) Working the Street: Police Discretion and the Dilemmas of Reform. New York: Sage.

Brown, R. (2005) Black, white, and unequal: Exmaining situational determinants of arrest decisions from police-suspect encounters. Criminal Justice Studies, 18: 51-68.

Brucato, B. (2015a) Policing made visible: Mobile technologies and the importance of point of view. Surveillance \& Society, 13(3/4): 455-473.

Brucato, B. (2015b) The new transparency: Police violence in the context of ubiquitous surveillance. Media \& Communications, 3(3): 39-55.

Brucato, B. (2016) Standing by police violence: On the constitution of the ideal citizen as sousveiller. American Studies Journal. [Online] http://www.asjournal.org/61-2016/ standing-police-violence-constitution-ideal-citizen-sousveiller.

Bruno, F. (2012) Surveillance and participation on Web 2.0. In (K. Ball, K. Haggerty \& D. Lyon, eds.) Routledge Handbook of Surveillance Studies, 343-351. New York: Routledge.

Bruns, A. (2007) Produsage, Generation C, and Their Effects on the Democratic Process. [Online] https://snurb.info/files/Produsage,GenerationC,andTheirEffects ontheDemocraticProcess.pdf.

Bruns, A. (2008) Blogs, Wikipedia, Second Life and Beyond. New York: Peter Lang.

Bruns, D. \& Bruns, J. (2015) Assessing the worth of the college degree on selfperceived police performance. Journal of Criminal Justice Education, 26(2): 121-146.

Brunson, R. \& Gau, J. (2014) Race, place, and policing the inner city. In (M. Reisig \& R. Kane, eds.) The Oxford Handbook of Police and Policing: 362-382. Oxford: Oxford University Press.

Brunson, R. \& Miller, J. (2006) Young Black men and urban policing in the United States. British Journal of Criminology, 46: 613-640.

Bruser, D. \& McLean, J. (2012) Police who lie: How officers thwart justice with false testimony. The Toronto Star. 26 April [Online] https://www.thestar.com/news/ canada/ 2012/04/26/police_who_lie_how_officers_thwart_justice_with_false_testimony.html

Bryant, R., Cockcroft, T., Tong, S. \& Wood, D. (2014) Police training and education: Past, present and future. In (J. Brown, ed.) The Future of Policing: 383-397. New York: Routledge.

Bryman, A. (2012) Social Research Methods, $4^{\text {th }}$ ed. Oxford: Oxford University Press.

Buckley, L \& Petrunik, M. (1995) Socio-demographic factors, reference groups and the career aspirations and career satisfaction of Canadian police officers. American Journal of Police, 14(2): 107-148.

Buerger, M. (1998) Police training as a Pentecost: Using tools singularly ill-suited to the purpose of reform. Police Quarterly, 1: 27-63. 
Buist, S. (2016) Crossing the thin blue line: Police misconduct in Hamilton. The Hamilton Spectator. 10 September. [Online] https:/www.thespec.com/news-story/ 6849680-crossing-the-thin-blue-line-police-misconduct-in-hamilton/.

Bulmer, M., Gibbs, J. \& Hyman, L., eds. (2010) Social Measurement through Social Surveys: An Applied Approach. Burlington, VT: Ashgate.

Burawoy, M. (2004) Public sociologies: Contradictions, dilemmas, and possibilities. Social Forces, 82: 1603-1618.

Burawoy, M. (2005a) For public sociology. British Journal of Sociology, 56(2): 259-294.

Burawoy, M. (2005b) Response: Public sociology: Populist fad or path to renewal? British Journal of Sociology, 56: 417-432.

Burawoy, M. (2007) For public sociology. In (D. Clawson et al., eds.) Public Sociology: Fifteen Eminent Sociologists Debate Politics and the Profession in the Twenty-first Century: 23-64. Berkeley, CA: University of California Press.

Burawoy, M. (2012) Foreword. In (P. Nyden, L. Hossfeld \& G. Nyden, eds.) Public Sociology: Research, Action, and Change: x-xi. Thousand Oaks, CA: Sage.

Bureau of Justice Statistics (2015) Local Police Departments 2013: Personnel, Policies and Practices. Washington, DC: Department of Justice.

Bureau of Justice Statistics (2016) Program Report: National Sources of Law Enforcement Employment Data. Washington: Department of Justice.

Burgess, J. \& Green, J. (2009) YouTube: Online Video and Participatory Culture, $1^{\text {st }}$ ed. Cambridge, UK: Polity Press.

Burke, R. (1989). Career stages, satisfaction, and well-being among police officers. Psychological Reports, 65: 3-12.

Burke, R., ed. (2016) Stress in Policing: Sources, Consequences and Interventions. New York: Routledge.

Burke, R. \& Mikkelsen, A. (2005) Career stage and police cynicism. Psychological Reports, 96(3): 989-992.

Burke, R. \& Mikkelsen, A. (2006) Police officers over career stages: Satisfaction and well being. Europe's Journal of Psychology, 2(1) [Online] https://ejop.Psychopen.eu/ article/view/314/html.

Buzawa, E., Austin, T. \& Bannon, J. (1994) The role of selected socio-demographic and job-specific variables in predicting patrol officer job satisfaction: A reexamination ten years later. American Journal of Police, 13: 51-75.

Byers, C. (2014a) Forensic evidence shows teen shot at St. Louis officer, police say. St. Louis Post-Dispatch. 15 October. [Online] http://www.stltoday.com/ news/local/crimeand-courts/forensic-evidenc...icle_3dafbce7-22b0-5c9a-b7e5-98460279f135.html. 
Byers, C. (2014b) St. Louis police seek no charges in officer's killing of VonDerrit Myers, but prosecution review pending. St. Louis Post-Dispatch. 5 December. [Online] http://www.stltoday.com/news/local/crime-and-courts/st-louis-police-seek-no-chargesin-officer-s-killing/article_613ee14a-5c6f-50a9-9566-009df039d49e.html.

Byers, C. (2014c) St. Louis region on edge in wake of fatal police shooting in Berkeley. St. Louis Post-Dispatch. 24 December. [Online] http://www.stltoday.com/news/local/ crime-and- courts/st-louis-region-on-edge-in-wake-of-fatal-police/article.

Byrne, D. (2000) Common reasons for rejecting manuscripts at medical journals: A survey of editors and peer reviewers. Science Editor, 23(2): 39-44.

Byrne, J. (2017) Emanuel avoids fight with McCarthy over '60 Minutes'. The Chicago Tribune. 4 January. [Online] http://www.chicagotribune.com/news/local/politics/ctemanuel-johnson-cpd-met-0105-20170105-story.html.

Cain, M. (1973) Society and the Policeman's Role. London: Routledge.

Cain, M. (1979) Trends in the sociology of police work. International Journal of the Sociology of Law, 7: 143-167.

Caldero, M. \& Crank, J. (2011) Police Ethics: The Corruption of Noble Cause, 3rd ed. Burling, MA: Anderson.

Calgary Herald (2016) Red Deer RCMP charge armed man wounded in police-involved shooting. Calgary Herald. 8 June. [Online] http://calgaryherald.com/news/crime/reddeer-rcmp-charge-armed-man-wounded-in-police-involved-shootingcalifornia-achampion-for-police-cameras.html?pagewanted $=\mathrm{all} \& \_r=0$.

Callanan, V. \& Rosenberger, J. (2011) Media and pubic perception of the police:

Examining the impact of race and personal experience. Policing \& Society, 21(2): 167189.

Campbell, B., Nix, J. \& Maguire, E. (2018) Is the number of citizens fatally shot by police increasing in the post-Ferguson era? Crime \& Delinquency, 64(3): 398-420.

Campbell, M., \& Schoenfeld, H. (2013) The transformation of America's penal order: A historicized political sociology of punishment. American Journal of Sociology, 118(5): 1375-1423.

Campbell, S. (2012) The Slave Catchers: Enforcement of the Fugitive Slave Law, 1850 1860. Chapel Hill, NC: UNC Press Books.

Campeau, H. (2015) Police culture at work: Making sense of police oversight. British Journal of Criminology, 55(4): 669-687.

Campeau, H. (2016a) Policing in unsettled times: An analysis of culture in the police organization. Unpublished $\mathrm{PhD}$ dissertation. University of Toronto.

Campeau, H. (2016b) What police governance bodies need to know about managing risk from the inside out. Canadian Association of Police Governance. Webinar. 15 December. 
Campeau, H. (2017) Institutional myths and generational boundaries: Cultural inertia in the police organization. Policing \& Society: DOI: 10.1080/10439463.2017.1371718.

Candlin, C. \& Crichton, J. (2013) From ontology to methodology: Exploring the discursive landscape of trust. In (C. Canlin \& J. Crichton, eds.) Discourse of Trust: 120. New York: Palgrave Macmillan.

Cannizzo, T. \& Liu, P. (1995) The relationship between levels of perceived burnout and career stage among sworn police officers. Police Studies, 18: 53-68.

Canter, D. (2004) A tale of two cultures: A comparison of the cultures of the police and academia. In (P. Villiers \& R. Adlam, eds.) Policing a Safe, Just and Tolerant Society: 109-121. Winchester, UK: Waterside Press.

Cao, L. (2014) Aboriginal peoples and confidence in the police. Canadian Journal of Criminology \& Criminal Justice, 56(5): 499-525.

Cao, L. \& Huang, B. (2000) Determinants of citizen complaints against police abuse of power. Journal of Criminal Justice, 28(3): 203-213.

Cao, L., Frank, J. \& Cullen, F. (1996) Race, community context, and confidence in the police. American Journal of Police, 15(1): 3-22.

Carlen, P. (2011) Against evangelism in academic criminology: For criminology as a scientific art. In (M. Boswoth \& C. Hoyle, eds.) What is Criminology? 95-108. New York: Oxford University Press.

Carleo-Evangelist, J. (2015) Albany police on Donald Ivy: 'This is a night we didn't want to happen'. Times Union. 30 October. [Online] http://www.timesunion.com/local /article/ Albany-police-to-show-video-on-Donald-Ivy-6600838.php.

Carley-Baxter, L. et al. (2009) Does response rate matter? Journal editors' use of survey quality measures in manuscript publication decisions. Survey Practice. [Online] http://www.surveypractice.org/index.php/SurveyPractice/article/view/192/html.

Carruthers, D. (2018) Proactive enforcement declining as police officers worry about public scrutiny, Ottawa researcher says. The London Free Press. 16 February. [Online] http://www.lfpress.com/2018/02/16/proactive-enforcement-declining-aspolice-officers-worry-about-public-scrutiny-ottawa-researcher-says.

Carter, A. (2013) Hamilton police arrest video goes viral. CBC News. 12 December. [Online] http://www.cbc.ca/news/canada/hamilton/news/hamilton-police-arrest-videogoes-viral-1.2461046.

Castells, M. (2000) Materials for an exploratory theory of the network society. British Journal of Sociology, 51(1): 5-24.

Castells, M. (2004) Informationalism, networks, and the network society: A theoretical blueprint. In (M. Castells, ed.) The Network Society: A Cross-Cultural Pespective: 3-45. Cheltenham, UK: Edward Elgar.

Castells, M. (2009) Communication power. Oxford: Oxford University Press. 
Castells, M. (2010) The Rise of the Network Society, 2nd ed. West Sussex, UK: WileyBlackwell.

Castells, M. (2011) A network theory of power. International Journal of Communication, 5: 773-787.

Castells, M. (2012) Networks of Outrage and Hope: Social Movements in the Internet Age. Cambridge, UK: Polity.

Catlin, D. \& Maupin, J. (2002) Ethical orientations of state police recruits and one-year experienced officers. Journal of Criminal Justice, 30: 491-498.

Cave, D. \& Oliver, R. (2015) The videos that are putting race and policing in sharp relief. The New York Times. 30 July. [Online] http://www.nytimes.com/interactive /2015/07/30 /us/police-videos-race.

CBC News (2003) Saskatoon police chief says drop-offs happened 'more than once'. $C B C$ News. 9 June. [Online] http://www.cbc.ca/news/canada/saskatoon-police-chiefsays-drop-offs-happened-more-than-once-1.380299.

CBC News (2012) Cellblock video blamed for drop in Ottawa drunk arrests. CBC News. 28 February. [Online] https://www.cbc.ca/news/canada/ottawa/cellblock-videoblamed-for-drop-in-ottawa-drunk-arrests-1.1182538.

CBC News (2013) Police worry about being caught on video, researcher says. Canadian Broadcasting Corporation. 6 November. [Online] http://www. cbc.ca/ news/ canada/ ottawa/police-worry-about-being-caught-on-video- researcher-says-1.2417618.

CBC News (2014a) Kajieme Powell shooting video released by St. Louis police. CBC News. 21 August. [Online] http://www.cbc.ca/news/world/kajieme-powell-shootingvideo-released-by-st-louis-police-1.2742838.

CBC News (2014b) Man killed by off-duty St. Louis officer was unarmed, mother says. CBC News. 9 October. [Online] http://www.cbc.ca/news/world/man-killed-by-off-dutyst-louis-officer-was-unarmed-mother-says-1.2793795.

CBC News (2014c) The National. CBC News. 11 June. [Online] Retrieved on 29 August 2014 from http://www.cbc.ca/player/News/ID/2464288010/.

CBC News (2015a) 'End carding now,' says coalition of prominent Torontonians. CBC News. 4 June. [Online] https://www.cbc.radio/thecurrent/the-current-for-june-4-2015.

CBC News (2015b) John Tory calls for end to 'illegitimate, disrespectful' practice of carding. $C B C$ News. 7 June. [Online] http://www.cbc.ca/news/canada/toronto/john-torycalls-for-end-to-illegitimate-disrespectful-practice-of-carding-1.3103855.

CBC News (2015c) Red Deer RCMP officer racks up another assault charge. $C B C$ News. 15 July. [Online] http://www.cbc.ca/news/canada/edmonton/red-deer-rcmpofficer-racks-up-another-assault-charge-1.3154065.

CBC News (2016a) Alberta RCMP officer acquitted of punching teen but guilty of separate assault. $C B C$ News. 13 June. [Online] http://www.cbc.ca/news/canada 
/edmonton /alberta-rcmp-officer-acquitted-of-punching-teen-but-guilty-of-separateassault-1.3633782.

CBC News (2016b) James Forcillo guilty of attempted murder in streetcar shooting of Sammy Yatim. 25 January. CBC News. [Online] http://www.cbc.ca/news/canada/toronto /james-forcillo-verdict-1.3414974.

CBC Radio (2018) Police shootings of unarmed Black men are a Canadian problem too, says author. CBC Radio. 23 March. [Online] https://www.cbc.ca/news/health/policekillings-unarmed-black-americans-worsen-mental-health-1.4715000.

Celona, L., Cohen, S. \& Golding, B. (2014) Arrests plummet 66\% with NYPD in virtual work stoppage. New York Post. 29 December. [Online] http://nypost.com/2014/12/29/ arrests-plummet-following-execution-of-two-cops/.

Centre for Police Accountability (2014) Website for the Centre for Police Accountability (C4PA). [Online] www.c4pa.ca.

Chagnon, N., Chesney-Lind, M. \& Johnson, D. (2018) Cops, lies, and videotape: police reform and the media in Hawaii. Crime, Media, Culture, 14(2): 171-190.

Chaiken, J. (1978). What is known about deterrent effects of police activities? In (J. Cromer, ed.) Preventing Crime. Beverly Hills, CA: Sage

Chan, J. (1996) Changing police culture. British Journal of Criminology, 36(1): 109134.

Chan, J. (1997) Changing Police Culture: Policing in a Multicultural Society. New York: Cambridge University Press.

Chan, J. (1999) Governing police practice: Limits of the new accountability. British Journal of Sociology, 50(2): 251-279.

Chan, J. (2003) Police and new technologies. In (T. Newburn, ed.) Handbook of Policing, $1^{\text {st }}$ ed.: 655-679. Cullompton, UK: Willan.

Chan, J. (2004) Using Pierre Bourdieu's framework for understanding police culture. Droit et Société, 56/57: 327-346.

Chan, J. (2005) Changing police culture. In (T. Newburn, ed.) Policing: Key Readings: 338-363. Cullompton: Willan.

Chan, J. (2007a) Making sense of police reforms. Theoretical Criminology, 11(3): 32345 .

Chan, J. (2007b) Police stress and occupational culture. In (M. O’Neill, M., Marks \& A. Singh, eds.) Police Occupational Culture: New Debates and Directions: 129-151.

Oxford: Elsevier.

Chan, J. (2008) The new lateral surveillance and a culture of suspicion. In (Deflem, M., ed.) Surveillance and Governance: Crime Control and Beyond. Pp. 223-239. Bingley, UK: JAI Press. 
Chan, J. (2011) Racial profiling and police subculture. Canadian Journal of Criminology \& Criminal Justice, 53(1): 75-78.

Chan, J. \& Doran, S. (2009) Staying in the Job: Job satisfaction among mid-career police officers. Policing: A Journal of Policy and Practice, 3(1): 66-77.

Chan, J., Devery, C. \& Doran, S. (2003) Fair Cop: Learning the Art of Policing. Toronto: University of Toronto Press.

Chancer, L. \& McLaughlin, E. (2007) Public criminologies: Diverse perspective on academia and policy. Theoretical Criminology, 11: 155-73.

Chang, A., Neugebauer, S. \& Birmingham, D. (2017) A critical race theory analysis of post-Ferguson critical incidents across ecological levels of academia. Journal of Educational Controversy, 12(1): 1-21.

Chanin, J. \& Sheats, B. (2018) Depolicing as dissent shirking: Examining the effects of pattern or practice misconduct reform on police behavior. Criminal Justice Review, 43(2): 105-126.

Chappell, A. \& Lanza-Kaduce, L. (2010) Police academy socialization: Understanding the lessons learned in a paramilitary-bureaucratic organization. Journal of Contemporary Ethnography, 39(2): 187-214.

Chappell, A. \& Piquero, A. (2004). Applying social learning theory to police misconduct. Deviant Behavior, 25(2): 89-104.

Charest, M. (2009) Mécontentement Populaire et Pratiques d'Interpellations Du SPVM Depuis 2005: Doit-on Garder Le Cap Après La Tempête. Montreal: SPVM.

Charman, S. (2017) Police Socialization, Identity and Culture. London: Palgrave MacMillan.

Charmaz, K. (2004) Grounded theory. In (S. Hesse-Biber \& P. Leavy, eds.) Approaches to Qualitative Research: A Reader on Theory and Practice: 496-521. New York:

Oxford University Press.

Chatterton, M. (1979) The supervision of patrol work under the fixed points system. In (S. Holdaway, ed.) The British Police. London: Edward Arnold.

Chatterton, M. (1983) Police work and assault charges. In (M. Punch, ed.) Control in the Police Organization. Cambridge, MA: MIT Press.

Chen, D. (2013) Hearing continues on alleged coverup of Windsor police brutality. The Windsor Star. 19 February. [Online] https://windsorstar.com/news/local-news/hearingcontinues-on-alleged-coverup-of-windsor-police-brutality.

Chen, Z. (2016) Measuring Police Subcultural Perceptions: A Study of Frontline Police Officers in China. New York: Springer.

Chermak, S. \& Weiss, A. (2005) Maintaining legitimacy using external communication strategies: An analysis of police-media relations. Journal of Criminal Justice 33(5): 501-512. 
Chermak, S., McGarrell, E. \& Gruenewald, J. (2005) Media coverage of police misconduct and attitudes toward police. Policing, An International Journal of Police Strategies and Management 29(2): 261-281.

Cheurprakobkit, S. 2000. Police-citizen contact and police performance. Journal of Criminal Justice, 28(4): 325-336.

Chibnall, S. (1975) The crime reporter: A study in the production of commercial knowledge. Sociology, 9: 49-66.

Chibnall, S. (1977) Law and Order News: An Analysis of Crime Reporting in the British Press. London: Tavistock Publications.

Childress, S. (2016). The problem with 'broken windows' policing. Frontline.June 28. [Online] http://www.pbs.org/wgbh/frontline/article/the-problem-with-broken-windowspolicing.

Christensen, W. \& Crank, J. (2001) Police work and culture in a nonurban setting: An ethnographic analysis. Police Quarterly, 4(1): 69-98.

Clear, T. (2010) Policy and evidence: The challenge to the American Society of Criminology: 2009 Presidential Address to the American Society of Criminology. Criminology, 48: 701-725.

CNN (2015) Police under fire: An AC 360 special report. CNN. 4 May. [Television \& Online] https://archive.org/details/CNNW_20150505_010000_Police_Under Fire_An_AC360_Special_Report/start/0/end/60.

CNN Tonight (2015) Police shooting of a Black teenager in Chicago. CNN. 24 November. [Online] http://transcripts.cnn.com/TRANSCRIPTS/1511/24/cnnt.02.html.

Cochran, J. \& Bromley, M. (2003) The myth (?) of the police sub-culture. Policing: An International Journal of Police Strategies and Management, 26: 88-117.

Cockcroft, T. (2007). Police culture(s): Some definitional, contextual, and analytical considerations. In (M. O'Neill, M. Marks, \& A. Singh, eds.) Police Occupational Culture: New Debates and Directions: 85-104. New York: Elsevier.

Cockcroft, T. (2013) Police Culture: Themes and Concepts. New York: Routledge.

Cohen, A. (1991). Career stage as a moderator of the relationships between organizational commitment and its outcomes: A meta-analysis. Journal of Occupational Psychology, 64(3): 253-268.

Cohen, J. (1988) Statistical Power Analysis for the Behavioral Sciences, $2^{\text {nd }}$ ed. Hillsdale, NJ: Lawrence Erlbaum Associates.

Coicaud, J. (2013) Crime, justice, and legitimacy: A brief theoretical inquiry. In (J. Tankebe, J. \& A. Leibling eds.) Legitimacy and Criminal Justice: An International Exploration: 37-59. Oxford: Oxford University Press.

Cole, Y. (2017) Inquiry to examine handling of perjury, assault allegations involving two Calgary officers. Calgary Herald. 26 July [Online] http://calgaryherald.com 
/news/local-news/inquiry-to-examine-handling-of-perjury-assault-allegations-involvingtwo-officers.

Collins, T. \& Klahm, C. (2019) Police accountability and ethics. In (M. Deflem, ed.) The Handbook of Social Control: 263-275. Oxford: Wiley.

Comack, E. (2012) Racialized Policing: Aboriginal People's Encounters with the Police. Winnipeg: Fernwood Publishing.

Comey, J. (2015a) Keeping our Communities Safe Through Shared Perspectives: Information and Understanding. Text of speech delivered 26 October to the International Association of Chiefs of Police Conference, Chicago. [Online] https://www.fbi.gov/news/ speeches /keeping-our-communites-safe-through-sharedperspectives-information-and-understanding.

Comey, J. (2015b) Law Enforcement and the Communities We Serve: Bending the Lines Toward Safety and Justice. Text of speech delivered 23 October to the University of Chicago Law School. [Online] https://www.fbi.gov/news/speeches/law-enforcementand-the-communites-we-serve-bending-the-lines-toward-safety-and-justice.

Conlon, E. (2004) Blue Blood. New York: Riverhead Books.

Conti, N. (2009) A visigoth system: Shame, honor, and police socialization. Journal of Contemporary Ethnography, 38(3): 409-432.

Conti, N. (2010) Weak links and warrior hearts: A framework for judging self and others in police training. Police Practice \& Research, 12(5): 410-423.

Conti, N. \& Nolan, J. (2005) Policing the platonic cave: Ethics and efficacy in police training. Policing \& Society, 14: 99-117.

Conway, E. (2004) Relating career stage to attitudes towards HR practices and commitment: Evidence of interaction effects? European Journal of Work and Organizational Psychology, 13(4): 417-446.

Cooper, F. (2002) Understanding depolicing: Symbiosis theory and critical cultural theory. University of Missouri-Kansas City Law Review, 71(2): 355-376.

Cooper, F. (2003). Understanding depolicing: Symbiosis theory and critical cultural theory. Villanova University School of Law Working Paper \#17. DOI: 10.219/SSRN.380022.

Cooper, F. (2006) The 'seesaw effect' from racial profiling to depolicing: Toward a critical cultural theory. In (B. Fleury-Steiner \& L. Nielson, eds.) The New Civil Rights Research: A Constitutive Approach: 139-156. Aldershot, UK: Ashgate.

Cooper-Thomas, H. \& Anderson, N. (2002) Newcomer adjustment: The relationship between organizational socialization tactics, information acquisition and attitudes. Journal of Occupational \& Organizational Psychology, 75(4): 423-437.

Cooper, W. (1982) Police officers over career stages. Canadian Police College Journal, 6: 93-112. 
Cordner, G. (1997) Community policing: Elements and effects. In (R. Dunham \& G. Alpert, eds.) Critical Issues in Policing: Contemporary Readings, $3^{\text {rd }}$ ed.: 451-468. Prospect Heights, IL: Waveland Press.

Cordner, G. (2017) Police culture: Individual and organizational differences in police officer perspectives. Policing: An International Journal of Police Strategies \& Management, 40(1): 11-25.

Cordner, G. \& Sheehan, R. (1999) Police Administration, 4th ed. Cincinatti, OH: Anderson Publishing.

Correia, M. (2000) The conceptual ambiguity of community in community policing. PolicIng: An International Journal of Police Strategies \& Management, 23(2): 218-232.

Correia, M., Reisig, M. \& Lovrich, N. (1996) Public perceptions of state police; An analysis of individual-level and contextual variables. Journal of Criminal Justice, 24: $17-28$.

Cosette, M. (2018) Critics doubt new police stats on street checks. CBC News. 29 January. [Online] http://www.cbc.ca/news/canada/ottawa/street-check-2017-report1.4506005 .

Cramer, H. (1946) Mathematical Methods of Statistics. Princeton, NJ: Princeton University Press.

Crank, J. (1997) Celebrating agency culture: Engaging a traditional cop's heart in organizational change. In (Q. Thurman \& E. McGarrell, eds.) Community Policing in a Rural Setting: 49-57. Cincinnati: Anderson Publishing.

Crank, J. (1998) Understanding Police Culture. Cincinnati: Anderson Publishing.

Crank, J. (2003) Institutional theory of police: A review of the state of the art. Policing: An International Journal of Police Strategies \& Management, 26(2): 186-207.

Crank, J. (2014) Understanding Police Culture, $2^{\text {nd }}$ ed. London: Routledge.

Crank, J. \& Caldero, M. (1991) The production of occupational stress in medium-sized police agencies: A survey of line officers in eight municipal departments. Journal of Criminal Justice, 19(4): 339-349.

Crank, J. \& Caldero, M. (2010) Police Ethics: The Corruption of Noble Cause, 3rd ed. New Providence, NJ: Bender \& Company.

Crawford, B. (2018) De-policing a matter of 'self-survival', police union president says. Ottawa Citizen. 12 February: 6.

Crepeau, M., Gutowski, C., Meisner, J. \& St. Clair, S. (2019) How the day unfolded: Inside the courtroom for former Chicago police Officer Jason Van Dyke's sentencing. Chicago Tribune. 18 January. [Online] https://www.chicagotribune.com/news/local/ breaking/ct-met-laquan-mcdonald-jason-van-dyke- sentencing-20190118-story.html.

Creswell, J. \& Plano-Clark, V. (2007) Designing and Conducting Mixed Methods Research. Thousand Oaks, CA: Sage. 
Cron, W. (1984). Industrial salesperson development: A career stages perspective. Journal of Marketing, 48(4), 41-52.

Cron, W., \& Slocum, J. (1986). The influence of career stages on salespeople's job attitudes, work perceptions, and performance. Journal of Marketing Research, 23(2): 119-129.

Crotty, M. (1998) The Foundation of Social Research: Meaning and Perspectives in the Research Process. London: Sage.

CTV News (2010a) Chief battles internal grumbling over Bonds case. CTV News. 29 November. [Online] https://ottawa.ctvnews.ca/chief-battles-internal-grumbling-overbonds-case-1.579947.

CTV News (2010b) Lawyer calls police misconduct 'systemic' problem. CTV News. 30 November. [Online] https://ottawa.ctvnews.ca/lawyer-calls-police-misconductsystemic-problem-1.580686.

CTV News (2010c) Ottawa police chief offered to resign: Report. CTV News. 9 December [Online] https://www.ctvnews.ca/ottawa-police-chief-offered-to-resignreport-1.584181.

CTV News (2010d) Police chief says public will be 'shocked' by video. CTV News. 25 November. [Online] https://ottawa.ctvnews.ca/police-chief-says-public-will-beshocked-by-video-1.578765.

CTV News (2012) Windsor police beating caught on tape. CTV News Windsor. 27 April. [Online] http://www.cbc.ca/news/canada/windsor/windsor-police-beating-caughton-tape-1.1226902.

CTV News (2014) A timeline of the shootings in Moncton, N.B. 6 June. [Online] http://www.ctvnews.ca/canada/a-timeline-of-the-shootings-in-moncton-n-b-1.1856021.

CTV News (2015) Windsor police Const. Rice demoted for 18 months, loses $\$ 14 \mathrm{~K}$ in salary. CTV News. 18 September. [Online] https://windsor.ctvnews.ca/windsor-policeconst-rice-demoted-for-18-months-loses-14k-in-salary-1.2570033.

CTV News (2018) Officers are neglecting proactive policing, according to new research. CTV News. 12 February. [Television].

Culhane, S., Boman, J. \& and Schweitzer, K. (2016) Public perceptions of the justifiability of police shootings: The role of body cameras in a pre- and post-Ferguson experiment. Police Quarterly, 19(3): 251-274.

Currie, E. (2007) Against marginality: Arguments for a public criminology.

Theoretical Criminology, 11(2): 175-190.

Currier, J. (2014) St. Louis police release video, audio of deadly police shooting. St. Louis Post-Dispatch. 20 August. [Online] https://www.stltoday.com/news/local/crimeand-courts/st-louis-police-release-video-audio-of-deadly-police-shooting/article_9326 e02a-2a60-5baa-b63a-9c77b8b81bab.html. 
Curtis, B. (2014) Data provenance, metadata, and reflexivity: Comments on method. Encounters on Education, 15: 43-61.

Curtis, C. (2014) Require Ferguson and St. Louis County and City police officers to wear body cameras. change.org [Online] https://www.change. org/p/police-chief-jonbelmar-require-ferguson-and-st-louis-county-and-city-police-officers-to-wear-bodycameras.

Dabney, D., Teasdale, B., Ishoy, G., Gann, T. \& Berry, B. (2017) Policing in a largely minority jurisdiction: The influence of appearance characteristics associated with contemporary hip-hop culture on police decision-making. Justice Quarterly, 34(7): 1310-1338.

Dalton, G., Thompson, P., \& Price, R. (1977) The four stages of professional careers: A new look at performance by professionals. Organizational Dynamics, 6(1): 19-42.

Dalton, G. (1996) Developmental views of careers in organizations. In (M. Arthur, D. Hall \& B. Lawrence) Handbook of Career Theory: 89-109. New York: Cambridge University Press.

Dantzker, M. (1992). An issue for policing: Educational level and job satisfaction: A research note. American Journal of Police, 12: 101-118.

Davis, A. (2015) 'YouTube effect' has left police officers under siege, law enforcement leaders say. The Washington Post. 8 October. [Online] https://www.washingtonpost. $\mathrm{com} /$ news/post-nation/wp/2015/10/08/youtube-effect-has-left-police-officers-undersiege-law-enforcement-leaders-say/?utm_term $=.5 \mathrm{~b} 07 \mathrm{~d} 94 \mathrm{e} 0044$.

Davis, K. (1969) Discretionary Justice: A Preliminary Inquiry. Baton Rouge: Louisiana State University Press.

Davis, R. (2015). Letter from the director. Institute for Intergovernmental Research: After-Action Assessment of the Police Response to the August 2014 Demonstrations in Ferguson, Missouri: ix-x. Washington, DC: Department of Justice.

Davis, R., Henderson, N. \& Ortiz, C. (2005) Can Federal Intervention Bring Lasting Improvement in Local Policing: The Pittsburgh Consent Decree. New York: Vera Institute of Justice.

Davis, R., Henderson, N., Ortiz, C., Miller, J. \& Massie, M. (2002) Turning Necessity into Virtue: Pittsburgh's Experience with a Federal Consent Decree. New York: Vera Institute of Justice.

Dawson, P. \& Williams, E. (2009) Reflections from a police research unit: An inside job. Policing, 3(4), 373-380.

De Angelis, J. (2016) What do citizens think about police accountability measures? Lessons from community attitudinal surveys. Criminal Justice Policy Review, 27(5): 520-536.

Decker, S. (1981) Citizen attitudes toward the police: A review of past findings and suggestions for future policy. Journal of Police Science \& Administration, 9(1): 80-87. 
Deflem, M., ed. (2019) Summary: The Handbook of Social Control. [Online] https://onlinelibrary.wiley.com/doi/10.1002/9781119372394.ch19.

DeJong, C. (2004) Gender differences in officer attitudes and behaviour: Providing comfort to citizens. Women \& Criminal Justice, 15: 1-32.

Deleuze, G. \& Foucault, M. (1977) Intellectuals and power. In (D. Bouchard, ed.) Language, Counter-Memory, Practice: Selected Essay and Interviews: 205-217. Ithica, NY: Cornell University Press.

Denzin, N. (2004) The art and politics of interpretation. In (S. Hesse-Biber \& P. Leavy, eds.) Approaches to Qualitative Research: A Reader on Theory and Practice: 447-472. New York: Oxford University Press.

Denzin, N. \& Giardina, M. (2009) Qualitative inquiry and social justice: Toward a politics of hope. In (N. Denzin \& M. Giardina, eds.) Qualitative Inquiry and Social Justice: Toward a Politics of Hope: 11-50. Walnut Creek, CA: Left Coast Press.

Denzin, N. \& Lincoln, Y. (2005) Introduction (In N. Denzin \& Y. Lincoln, eds.) The Sage Handbook of Qualitative Research, $3^{\text {rd }}$ ed.: 1-32. Thousand Oaks, CA: Sage.

Department of Justice (2003) Principles of Good Policing: Avoiding Violence Between Police and Citizens. Washington, DC: U.S. Department of Justice.

Department of Justice (2017) An Interactive Guide to the Civil Rights Division's Police Reforms. [Online] Accessed on 21 October 2017. https://www.justice.gov/crt/page/file /922456/download.

Desmond, M. \& Valdez, N. (2012) Un-policing the urban poor: Consequences of thirdparty policing for inner-city women. American Sociological Review, 78(1): 117-141.

Deuchar, R., Crichlow, V.\& Fallik, S. (2019): Cops in crisis? Ethnographic insights on a new era of politicization, activism, accountability, and change in transatlantic policing, Policing \& Society, DOI: 10.1080/10439463.2019.1584197.

Deuchar, R., Fallik, W. \& Crichlow, V. (2018) Despondent officer narratives and the 'post-Ferguson' effect: Exploring law enforcement perspectives and strategies in a southern American state. Policing \& Society. DOI: 10.1080/10439463.2018.1480020.

Deuze, M. (2005) What is journalism? Journalism, 6(4): 442-464.

Deuze, M., Bruns, A. \& Neuberger, C. (2007) Preparing for an age of participatory news. Journalism Practice, 1(3): 322-338.

Dewan, S. (2017) Deconstructing the 'Ferguson Effect'. The New York Times. 29 March. [Online] https://www.nytimes.com/interactive/2017/us/politics/fergusoneffect.html.

Diamond, L. \& Plattner, M. (2012) Liberation Technology: Social Media and the Struggle for Democracy. Baltimore, MD: Johns Hopkins University Press. 
Dillman, D. et al. (2002) Survey nonresponse in design, data collection, and analysis. In (R. Groves, D. Dillman, J. Eltinge \& R. Little, ed.) Survey Nonresponse. 3-26. New York: Wiley.

Dillman, D., Smyth, J. \& Christian, L. (2014). Internet, Mail, and Mixed-Mode Surveys: The Tailored Design Method, $4^{\text {th }}$ ed. Hoboken, NJ: Wiley.

Dillon, C. (1997) Whren v United States and pretextual traffic Stops: The Supreme Court declines to plumb collective conscience of police. Boston College Law Review, 38(4): 737-770.

DiManno, R. (2017) Why bad cops usually get away with brutish behaviour. Toronto Star. 16 August. [Online] https://www.thestar.com/news/gta/2017/08/16/why-bad-copsusually-get-away-with-brutish-behaviour-dimanno.html.

Dixon, D. (1997) Law in Policing: Legal Regulation and Police Practices. Oxford: Clarendon Press.

Dixon, D. (2005) Beyond zero tolerance. In (T. Newburn, ed.) Policing: Key Readings, 483-507. Cullompton: Willan.

Donner, C. \& Jennings, W. (2014). Low self-control and police deviance: Applying Gottfredson and Hirschi's general theory to officer misconduct. Police Quarterly, 17(3): 203-225.

Donner, C., Maskaly, J., Fridell, L. \& Jennings, W. (2015) Policing and procedural justice: A state-of-the-art review. Policing: An International Journal of Police Strategies \& Management, 38(1): 153-172.

Doob, A. \& Gartner, R. (2017) Understanding the Impact of Police Stops. Toronto: Toronto Police Services Board.

Doucette, C. (2018) Killing carding: The deadly cost of putting street checks on ice. Toronto Sun. 29 September. [Online] https://torontosun.com/news/local-news/killingcarding-the-deadly-toll-of-putting-street-checks-on-ice.

Dowler, K. \& Zawilski, V. (2007) Public perceptions of police misconduct and discrimination: Examining the impact of media consumption. Journal of Criminal Justice, 35: 193-203.

Doyle, A. (2003) Arresting Images: Crime and Policing in Front of the Television Camera. Toronto: University of Toronto Press.

Doyle, A. (2006a) An alternative current in surveillance and control: Broadcasting surveillance footage of crimes. In (K. Haggerty \& R. Ericson, eds.) The New Politics of Surveillance and Visibility: 199-224. Toronto: University of Toronto Press.

Doyle. A. (2006b) How not to think about crime in the media. Canadian Journal of Criminology \& Criminal Justice, 48(6): 867-885.

Doyle, A. (2007) Introduction: Trust, citizenship and exclusion in the risk society. In (Law Commission of Canada, ed.) Risk and Trust: Including or Excluding Citizens? 722. Black Point, NS: Fernwood Publishing. 
Doyle, A. (2011) Revisiting the synopticon: Reconsidering Mathiesen's 'The Viewer Society' in the age of Web 2.0. Theoretical Criminology, 15(3): 283-299.

Doyle, A., Lippert, R., Lyon, D., eds. (2012) Eyes Everywhere: The Global Growth of Camera Surveillance. London: Routledge.

Drake, B. (2014) Ferguson highlight deep divisons between Blacks and Whites in America. Fact Tank: News in the Numbers. Pew Research Center. [Online] http://www.pewresearch.org/fact-tank/2014/11/26/ferguson-highlights-deep-divisionsbetween-blacks-and-whites-in-america/.

Drummond, D. (1976) Police Culture. Beverly Hills, CA: Sage.

Dubar, C. (1995) La Socialisation Professionnelle: Construction des Identités Sociales et Professionnelles. Paris: Armand Colin.

Duggal, S. (2011) Police file defence in Stacy Bonds case. Ottawa Citizen. 1 February. [Online] http://www.pressreader.com/canada/ottawa-citizen/20110201/2828021 22807998.

Dunham, R. \& Alpert, A., eds. (2005) Critical Issues in Policing: Contemporary Readings, $5^{\text {th }}$ ed. Long Grove, Ill: Waveland Press.

Dunham, R. \& Alpert, A., eds. (2010a) Critical Issues in Policing: Contemporary Readings, $6^{\text {th }}$ ed. Long Grove, Ill: Waveland Press.

Dunham, R. \& Alpert, G. (2010b) The foundation of the police role in society. In (R. Dunham \& G. Alpert, eds.) Critical Issues in Policing: Contemporary Readings, $6^{\text {th }}$ ed.: 1-19. Long Grove, Ill: Waveland Press.

Dunham, R., Alpert, G., Stroshine, M. \& Bennett, K. (2005) Transforming citizens into suspects: Factors that influence the formation of police suspicion. Police Quarterly, 8(3): 366-393.

Dunn, W. (1996) Boot: An L.A.P.D. Officer's Rookie Year. New York: William Morrow.

Dunphy, J. (2005) Betrayal, not bullets, is what cops fear. Los Angeles Times. 4 September: 7.

Durivage, A. et al. (1992) Analysis of organizational culture: The key to strategic planning. Canadian Police College Journal, 16(2): 94-134.

Durkin, E. (2014) NYPD union chief slams Bill de Blasio for comments made about cops. New York Daily News. 4 December. [Online] http://www.nydaily news.com/newyork/nypd-union-boss-knocks-de-blasio-comments-cops-article-1.2034128.

Eck, J. \& Maguire, E. (2000) Have changes in policing reduced violent crime? In (A. Blumstein and J. Wallman, eds.) The Crime Drop in America: 207-265. New York: Cambridge University Press. 
Eck, J. \& Rosenbaum, D. (1994) The new police order: Effectiveness, equity, and efficiency in community policing. In (D. Rosenbaum, ed.) The Challenge of Community Policing: Testing the Promises: 3-23. Thousand Oaks, CA: Sage.

Edelwich, J. \& Brodsky, A. (1980) Burn-out: Stages of Disillusionment in the Helping Professions. New York: Human Sciences Press.

Elias, N. (1994) The Civilizing Process: The History of Manners and State Formation and Civilization. Oxford: Blackwell.

Ellis, R. (1991) Perceptions, attitudes and beliefs of police recruits. Canadian Police College Journal, 15(2): 95-117.

Emanuel, R. (2017) Mayor: 4,368 shootings have neither one cause nor one cure. Chicago Sun Times. 11 January. [Online] https://chicago.suntimes.com/opinion /opinion-4368-shootings-have-neither-one-cause-nor-one-cure.

Emsley, C. (2014) Peel's principles, police principles. In (J. Brown, ed.) The Future of Policing, 11-22. London: Routledge.

Enciso, G., Maskaly, J. \& Donner, C. (2017) Organizational cynicism in policing: Examing the development and growth of cynicism among new police recruits. Policing: An International Journal of Police Strategies \& Management, 49(1): 86-98.

Engel, R. (2005) Citizens' perceptions of procedural and distributive injustice during traffic stops with police. Journal of Research in Crime and Delinquency, 42(4): 445481.

Engel, R. \& Cohen, D. (2014) Racial profiling. (In (M. Reisig \& R. Kane, eds.) The Oxford Handbook of Police and Policing: 383-408. Oxford: Oxford University Press.

Engel, R. \& Tillyer, R. (2008) Searching for equilibrium: The tenous nature of the outcome test. Justice Quarterly, 25(1): 54-71.

Engel, R. \& Worden, R. (2003) Police officers' attitudes, behaviour, and supervisory influence: An analysis of problem solving. Criminology, 41(1): 131-166.

Engelson, W. (1999) The organizational values of law enforcement agencies: The impact of field training officers in the socialization of police recruits to law enforcement organizations. Journal of Police \& Criminal Psychology, 14(2): 11-19.

Epp, C., Maynard-Moody, S. \& Haider-Markel, D. (2014) Pulled Over: How Police Stops Define Race and Citizenship. Chicago. University of Chicago Press.

Ericson, R. (1981). Making Crime: A Study of Detective Work. Toronto: University of Toronto Press.

Ericson, R. (1982) Reproducing Order: A Study of Police Patrol Work. Toronto: University of Toronto Press.

Ericson, R. (1989) Patrolling the facts: Secrecy and publicity in police work. The British Journal of Sociology, 40(2): 205-226. 
Ericson, R. (1993) Making Crime: A Study of Detective Work, 2nd ed. Toronto: University of Toronto Press.

Ericson, R. (1995) The news media and account ability in criminal justice. In (P. Stenning, ed.) Accountability for Criminal Justice: Selected Essays: 135-161. Toronto: University of Toronto Press.

Ericson, R. (2005) Publicizing sociology. British Journal of Sociology, 56(3): 365372.

Ericson, R. (2007a) Crime in an Insecure World. Cambridge: Polity Press.

Ericson, R. (2007b) Rules in policing: Five perspectives. Theoretical Criminology, 11(3): 367-401.

Ericson, R. \& Doyle, A. (2003) Risk and morality. In (R. Ericson \& A. Doyle, eds.) Risk and Morality: 1-10. Toronto: University of Toronto Press.

Ericson, R. \& Haggerty, K. (1997) Policing the Risk Society. Toronto: University of Toronto Press.

Ericson, R., Baranek, P. \& Chan, J. (1987) Visualizing Deviance: A study of News Organizations. Toronto: University of Toronto Press.

Ericson, R., Baranek, P. \& Chan, J. (1989) Negotiating Control: A Study of News Sources. Toronto: University of Toronto Press.

Ericson, R., Baranek, P. \& Chan, J. (1991) Representing Crime: Crime, Law and Justice in the News Media. Milton Keynes, UK: Open University Press.

Erikson, E. (1959) Identity and the Life Cycle. New York: International Universities Press.

Erikson, E. (1963) Childhood and society, 2nd ed. New York: Norton

Eschholz, S., Blackwell, B., Gertz, M., \& Chiricos, T. (2002) Race and attitudes toward the police: Assessing the effects of watching 'reality' police programs. Journal of Criminal Justice, 30: 327-241.

Este, D., Sato, C. \& McKenna, D. (2017) The coloured women's club of Montreal, 1902-1940: African-Canadian women confronting anti-black racism. Canadian Social Work Review, 34: 81-99.

Etzioni, A. (2010) Is transparency the best disinfectant? Journal of Political Philosophy, 18 (4): 389-404.

Evans, R. (2015) 'The footage is decisive': Applying the thinking of Marshall McLuhan to CCTV and police misconduct. Surveillance \& Society, 13(2): 218-232.

Evans, W. \& Owens, E. (2007) COPS and crime. Journal of Public Economics, 91(1-2): 181-201.

Ewald, F. (2002) The return of Descartes' malicious demon: An outline of a philosophy of precaution, trans. S. Utz. In (T. Baker \& J. Simon, eds.) Embracing Risk: The 
Changing Culture of Insurance and Responsibility: 273-302. Chicago: University of Chicago Press.

Ewart, D. (2014) The Enduring Quality of Police Canteen Culture/Subculture in a Changing Police Landscape. [Online] https://www.academia.edu/11393467/ The_endur ing_quality_of_Police_canteen_culture_subculture_in_a_changing_Police_landscape.

Fagan, J. (2002), Law, social science and racial profiling. Justice Research and Policy, 4 (December): 104-129.

Fagan, J. \& Davies, G. (2000) Street stops and broken windows: Terry, race and disorder in New York City. Fordham Urban Law Journal, 28: 457-504.

Fagan, J. \& Tyler, T., eds. (2006) Legitimacy, Criminal Justice, and the Law. New York; Russell Sage Foundation Press.

Fagan, M. \& Ayers, K. (1982) The life of a police officer: A developmental perspective. Criminal Justice and Behavior, 9: 273-285.

Fallik, S., Deuchar R. \& Crichlow, V. (2018) Body-worn cameras in the post-Ferguson era: An exploration of law enforcement perspectives. Journal of Police \& Criminal Psychology. DOI: 10.1007/s11896-018-9300-2.

Fan, M. (2012) Panopticism for police: Structural reform bargaining and police regulation by data-driven surveillance. Washington Law Review, 87: 92-138.

Fantino, J. (2018) In support of our police. Toronto Sun. 7 July. [Online] https://torontosun.com/opinion/columnists/fantino-in-support-of-our-police

Farkas, M. \& Manning, P. (1997) The occupational culture of corrections and police officers. Journal of Crime \& Justice, 20(2): 51-68.

Farmer, A. (2016) Copwatchers: Citizen Journalism and the Changing PoliceCommunity Dynamic. Unpublished $\mathrm{PhD}$ dissertation. University of Delaware.

Farmer, A. \& Sun, I. (2016) Citizen journalism and police legitimacy: Does recording the police make a difference? In (M. Deflem, ed.) The Politics of Policing: Between Force and Legitimacy: 239-256. Bingley: Emerald Group Publishing Limited.

Farmer, A., Sun, I. \& Starks, B. (2015) Willingness to record police-public encounters: The impact of race and social and legal consciousness. Race \& Justice, 5(4): 356-377.

Farrar, A. (2013) Self-Awareness to Being Watched and Socially-Desirable Behavior: A Field Experiment on the Effect of Body-Worn Cameras on Police Use-of-Force. Washington, DC: The Police Foundation.

FBI National Press Office (2016) Director Comey Remarks During May 11 'Pen and Pad' Briefing with Reporters. [Online] https://www.fbi.gov/news/pressrel/pressreleases/director-comey-remarks-during-may-11-2018pen-and-pad2019-briefing-withreporters.

Federal Bureau of Investigation (2016) The Assailant Study: Mindsets and Behaviours. Washington, DC: Office of Partner Engagement. 
Federal Bureau of Investigation (2017) 2016: Crime in the United States [Online] https:// ucr. fbi.gov/crime-in-the-u.s/2016/crime-in-the-u.s.-2016.

Fenton, J. \& George, J. (2015) Violence surges as Baltimore police officers feel hesitant. The Baltimore Sun. 8 May: 1.

Ferguson, Missouri (City of) (2014) City of Ferguson Issues Call for Nightime Quite and Reconciliation. Media Release. 19 August. [Online] Retrieved from on 19 August 2014 from The Wire at http://www.thewire.com/national/2014/08/city-of-fergusonpledges-to-foster-diverse-police-force-and-vest-cameras/378778/.

Fielding, N. (1988a) Competence and culture in the police. Sociology, 22: 45-64.

Fielding, N. (1988b) Joining Forces: Police Training, Socialization and Occupation Competence. London: Routledge.

Fielding, N. (1994) Cop canteen culture. In (T. Newburn \& E. Stanko eds.) Just Boys Doing Business: Men, Masculinities and Crime. London: Routledge.

Fielding, N. (1995) Community Policing. Oxford: Oxford University Press.

Fielding, N. (2002) Theorizing community policing. British Journal of Criminology, 42(1): 147-163.

Fielding, N. \& Fielding, J. (1987) A study of resignation during British police training. Journal of Police Science \& Administration, 15(1): 24-36.

Fielding, N. \& Fielding, J. (1991) Police attitudes to crime and punishment. British Journal of Criminology, 31: 39-53.

Findlay, L. (2003) The reflexive journey: Mapping multiple routes. In (L. Findlay \& B. Gough, eds.) Reflexivity: A Practical Guide for Researchers in Health and Social Sciences. Oxford: Blackwell.

Finn, J. (2012) Seeing surveillantly: Surveillance as social practice. In (A. Doyle, R. Lippert \& D. Lyon, eds.) Eyes Everywhere: The Global Growth of Camera Surveillance: 83-96. New York: Routledge.

Fisher, M. (2014) Cincinnati still healing from its riots and has lessons to share with Ferguson. The Washington Post. 5 September. [Online] https://www.washingtonpost .com/politics/cincinnati-still-healing-from-its-riots-and-has-lessons-to-share-withferguson /2014/09/05/2ff8b944-34a1-11e4-9e92-0899b306 bbea_story.html.

Fisk, C. \& Song-Richardson, L. (2017) Police unions. George Washington Law Review, 85: 712-799.

Fiske, J. (1998) Surveilling the City: Whiteness, the Black Man and Democratic Totalitarianism. Theory, Culture and Society, 15(2): 67-88.

Fitzgerald, R. \& Carrington, P. (2011) Disproportionate minority contact in Canada: Police and visible minority youth. Canadian Journal of Criminology \& Criminal Justice, 53(4): 449-486. 
Flaherty, K. \& Pappas, J. (2002) Using Career Stage Theory to Predict Turnover Intentions among Salespeople, Journal of Marketing Theory and Practice, 10(3): 48-57.

Flanagan, R. (2007) Interim Report of the Independent Review of Policing. London: Home Office.

Flanagan, R. (2008) Review of Policing: Final Report. London: Home Office.

Flanagan, T. \& Vaughn, M. (1995) Public opinion about police abuse of force. In (W. Geller \& H. Toch eds.) And Justice for All: Understanding and Controlling Police Abuse of Force: 113-131. Washington, DC: Police Executive Research Forum.

Fleming, J. (2011) Qualitative encounters in policing research. In (L. Bartels \& K. Richards, eds.) Qualitative Criminology: Stories from the Field: 13-24. Leichhardt, NSW: Federation Press.

Fletcher, C. (1996) The 250lb man in an alley: Police storytelling. Journal of Organizational Change Management, 9: 36-42.

Flores, R. (2017) Deadly Year. CNN. 2 January. [Television].

Flyvbjerg, B. (2001) Making Social Sciences Matter. New York: Cambridge University Press.

Foley, P., Guameri, C., \& Kelly, M. (2008) Reasons for choosing a police career: Changes over two decades. International Journal of Police Science and Management, 10: $2-8$.

Ford, M. (2017) What's causing Chicago's homicide spike? The Atlantic. 24 January [Online] https://www.theatlantic.com/politics/archive/2017/01/chicago-homicide-spike2016/514331/.

Ford, R. (2003) Saying one thing and meaning another: The role of parables in police training. Police Quarterly, 6(1): 84-110.

Form, W. \& Miller, D. (1949) Occupational career pattern as a sociological instrument. American Journal of Sociology, 54: 317-329.

Foster, C. (1996). A Place Called Heaven: The Meaning of being Black in Canada. Toronto: Harper-Collins.

Foster, J. (2003) Police culture. In (T. Newburn, ed.) Handbook of Policing, $1^{\text {st }}$ ed.: 196227. Cullompton, UK: Willan.

Foster, L., Jacobs, L. \& Siu, B. (2016) Race Data and Traffic Stops in Ottawa, 20132015: A Report on Ottawa and the Police Districts. Ottawa: Ottawa Police Service.

Fowler, F (1995) Improving Survey Questions: Design and Evaluation. Thousand Oaks, CA: Sage.

Fowler, F. (2002). Survey Research Methods. Thousand Oaks, CA: Sage.

Fox, J. \& Lundman, R. (1974) Problems and strategies in gaining research access in police organizations. Criminology, 16: 187-198. 
Fox, J. \& Tracy, P. (1986) Randomized Response: A Method for Sensitive Surveys. Beverly Hills, CA: Sage.

Fox, N. (2003) Practice-based evidence: Towards collaborative and transgressive research. Sociology, 37(1): 81-102.

Fox News (2017) Cautious cops: NYPD cops handcuffed by new stop-and-frisk policies. Tucker Carlson Tonight. 17 November. [Television \& Online] https://video.foxnews. com/v/_5650753085001/\#sp=show-clips.

Fox, R. \& Van Sickel, R. (2001) Tabloid Justice: Criminal Justice in an Age of Media Frenzy. Boulder, CO: Lynne Rienner Publishing.

Frank, J., Smith, B. \& Novak, K. (2005) Exploring the basis of citizens' attitudes toward the police. Police Quarterly, 8(2): 206-228.

Frankel, T. (2015) DEA chief joins FBI chief in giving credence to 'Ferguson effect'. The Washington Post. 4 November. [Online] https://www.washingtonpost.com/news/post-nation/wp/2015/11/04/dea-chief-joins-fbichief-in-giving-credence-to-ferguson-effect/? utm_term=.27c4a261a710.

Freeman, M., de Marrais, K., Preissie, J., Roulston, K. \& St. Pierre, E. (2007) Standards of evidence in qualitative research: An incitement to discourse. Educational Researcher, 36(1): 25-32.

Freidman, T. (2007) The whole world is watching. The New York Times. 27 June: A23.

Friedrich, R. (1980) Police use of force: Individuals, situations, and organizations. The Annals of American Academy Political and Social Science, 452: 82-97.

Fridell, L. \& Scott, M. (2010) Law enforcement agency responses to racially biased policing and the perceptions of its practice. In (R. Dunham \& G. Alpert, eds.) Critical Issues in Policing: Contemporary Readings, $6^{\text {th }}$ ed.: 343-360. Long Grove, IL: Waveland Press.

Friedersdorf, C. (2014) Video killed trust in police officers. The Atlantic. [Online] http://www. theatlantic.com/national/archive/2014/08/police-officers-havent-earnedour-instinctive-trust $/ 378657$.

Furedi, F. (2007). The Culture of Fear Revisited. London: Continuum Press.

Furedi, F. (2009) Precautionary culture and the rise of possibilistic risk assessment. Erasmus Law Review, 2(2): 197-220.

Fyfe, J. (1981) Who shoots? A look at officer race and police shooting. Journal of Police Science \& Administration, 9: 367-382.

Fyfe, J. (1988) Police use of deadly force: Research and reform. Justice Quarterly, 5: 165-205.

Fyfe, J., Klinger, D. \& Flavin, J. (1997) Differential police treatment of male-on-female spousal violence. Criminology, 35: 455-473. 
Fyfe, N. \& Bannister, J. (1996) City watching: Closed circuit television surveillance in public spaces. Area, 28: 37-46.

Fyfe, N. \& Bannister, J. (1998) The eyes upon the street: Closed-circuit television surveillance and the city. In (N. Fyfe, ed.) Images of the Street: Representation, Experience and Control in Public Space: 254-267. London: Routledge.

Gall, M., Borg, W. \& Gall, J. (1996) Educational Research: An Introduction, $6^{\text {th }}$ ed. White Plains, NY: Longman Publishing.

Gallagher, C., Maguire, E., Mastrofski, S. \& Reisig, M. (2001) The Public Image of the Police: Final Report to the International Association of Chiefs of Police. Manassas, VA: George Mason University.

Gallagher, E., Kim, J., Markovic, J. \& Spence, D., eds. (2016). The State of Policing in the United States, Volume 1. Washington, DC: Department of Justice.

Gallardo, M. \& Podesta, L. (2017) 762 murdered in Chicago in 2016, CPD releases plan to curb violence. $A B C$ News. 1 January. [Online] http://abc7chicago.com/1681356/.

Gallo, G. (2001) Armed and Dangerous: Memoirs of a Chicago Policewoman. New York: Forge Books.

Galloway, G. (2011) Ottawa police officer charged with sexual assault after strip search. The Globe and Mail, 15 March [Online] https:/www.theglobeandmail.com/news/ national/ottawa-police-officer-charged-with-sexual-assault-after-strip search/ article572349/.

Gallup Editors (2014) Gallup review: Black and White differences in views on race. Gallup. 12 December. [Online] https://news.gallup.com/poll/180107/gallup-reviewblack-white-differences-views-race.aspx.

Gans, H. (2011) How to be a public intellectual: An interview with Nikki Jones. Public Intellectual. 31 May. [Online] http://thepublicintellectual.org/wpcontent/ uploads/ 2011/05/gans_lead.jpg.

Garland, D. (2003) The rise of risk. In (R. Ericson \& A. Doyle, eds.). Risk and Morality: 48-86. Toronto: University of Toronto Press.

Garland, D. (2004) Beyond the culture of control. Critical Review of International Social and Political Philosophy, 7(2): 160-189.

Garner, R. (2005). Police attitudes: The impact of experience after training. Applied Psychology in Criminal Justice, 1: 56-70.

Gaskew, T. (2018) Unfriending the police culture: The reawakening Black consciousness. In (S. Weissinger \& D. Mack, eds.) Law Enforcment in the Age of Black Lives Matter: Policing Black and Brown Bodies: 187-200. New York: Lexington Books.

Gately, G. (2015) Why is violent crime surging in many cities? The Crime Report. [Online] https://thecrimereport.org/2015/07/13/2015-07-why-is-violent-crime-surgingin-many-cities/. 
Gau, J. \& Brunson, R. (2010) Procedural justice and order maintenance policing: A study of inner-city young men's perceptions of police legitimacy. Justice Quarterly, 27(2): 255-279.

Gau, J. \& Paoline, E. (2017) Officer race, role orientations, and cynicism toward citizens. Justice Quarterly, 34(7): 1246-1271.

Gau, J., Corsaro, N., Stewart, E. \& Brunson, R. (2012) Examining macro-level impacts on procedural justice and police legitimacy. Journal of Criminal Justice, 40(4): 333343.

Gaub, J., Choate, D., Todak, N., Katz, C. \& White, M. (2016) Officer perceptions of body-worn cameras before and after deployment: A study of three departments. Police Quarterly, 19(3): 275-302.

Gee, M. (2016) Forcillo trial a warning: Police can't hide behind badges. The Globe \& Mail. 25 January: A5.

Geertz, C. (1973) The Interpretation of Cultures. New York: Basic Books.

Getty, R., Worrall, J, \& Morris, R. (2016) How far from the tree does the apple fall? Field training officers, their trainees, and allegations of misconduct. Crime \& Delinquency, 62(6): 821-839.

Gibson, C., Swatt, M. \& Jolicoeur, J. (2001) Assessing the generality of general strain theory: The relationship among occupational stress experienced by male police officers and domestic forms of violence. Journal of Crime \& Justice, 24(2): 29-57.

Giddens, A. (1990) Consequences of Modernity. Cambridge, UK: Polity Press.

Giddens, A. (1991) Modernity and Self Identity. Cambridge: Polity Press.

Giddens, A. (1994) Risk, trust, reflexivity. In (U. Beck, A. Giddens, \& S. Lash, eds.) Reflexive Modernization: Politics, Tradition and Aesthetics in the Modern Social Order: 184-197. Cambridge, UK: Polity Press.

Gilgun, J. (2011) Coding in deductive qualitative analysis. Current Issues in Qualitative Research: An Occasional Publication for Field Researchers from a Variety of Disciplines, 2(1): 1-4.

Gill, C., Weisburd, D., Telep, C., Vitter, Z. \& Bennett, T. (2014) Community-oriented policing to reduce crime, disorder and fear and increase satisfaction and legitimacy among citizens: A systematic review. Journal of Experimental Criminology, 10(4) 399428.

Gilliom, J. (2006) Struggling with surveillance: Resistance, consciousness and identity. In (K. Haggerty \& R. Ericson, eds.) The New Politics of Surveillance and Visibility: 111-129. Toronto: University of Toronto Press.

Gilmartin, K. \& Harris, J. (1998) Continuum of compromise. Police Chief, 65(1): 2528. 
Gitlin, T. (1980) The Whole World is Watching: Mass Meida in the Making \& Unmaking of the New Left. Berkeley: University of California Press.

Glaser, B. (1978) Theoretical Sensitivity. Mill Valley, CA: Sociology Press.

Glaser, B. \& Strauss, A. (1967) The Discovery of Grounded Theory: Strategies for Qualitative Research. Chicago: Aldine.

Glaser, J. (2006) The efficacy and effect of racial profiling: A mathematical simulation approach. Journal of Policy Analysis \& Management, 25(2): 395-416.

Glawe, J. (2014) St. Louis shooting is the anti-Ferguson. The Daily Beast. 24 December. [Online] http://www.thedailybeast.com/articles/2014/12/24/.

Globe and Mail Editorial Board. (2013) How citizen video has changed the Ontario SIU. Globe and Mail. 1 October. [Online] http://www.theglobeandmail.com /news/news-video/ video-how-citizen-video-has-changed-the-special-investigationunit/article14621083.

Globe and Mail Editorial Staff (2014) With the First Nations Financial Transparency Act, seeing is believing. The Globe and Mail. 17 August. [Online] http://www.The globeand mail.com/globe-debate/editorials/with-the-first-nations-financialtransparency-act-seeing-is-believing/article20082359/.

Glomseth, R. \& Gottschalk, P. (2009) Police personnel cultures: A comparative study of counter terrorist and criminal investigation units. Criminal Justice Studies, 22(1): 3-15.

Goffman, E. (1959) The Presentation of Self in Everyday Life. Garden City, NY: Anchor Books.

Goldsmith, A. (1990) Taking police culture seriously: Police discretion and the limits of law. Policing \& Society, 1(2): 91-114.

Goldsmith, A. (2005) Police reform and the problem of trust. Theoretical Criminology, 9(4): 443-470.

Goldsmith, A. (2010) Policing's new visibility. British Journal of Criminology, 50: 914934.

Goldsmith, A. \& Lewis, C. (2000) Civilian oversight of policing: Governance, Democracy and Human Rights. Oxford: Hart Publishing.

Goldstein, H. (1963) Police discretion: The ideal versus the real. Public Administration Review, 23(1): 140-148.

Goldstein, H. (1977) Policing a Free Society. Cambridge, MA: Ballinger.

Goldstein, H. (1987). Toward community-oriented policing: Potential, basic requirements, and threshold questions. Crime \& Delinquency, 33(1): 6-30.

Goldstein, J. (1960) Police discretion not to invoke the criminal process: Low-visibility decisions in the administration of justice. Yale Law Journal, 69(4): 543-594. 
Gonzales, A. \& Cochran, D. (2017) Police-worn body cameras: An antidote to the 'Ferguson Effect'? Missouri Law Review, 82(2): 299-337.

Goodman, A. (1990). A model for police officer burnout. Journal of Business and Psychology, 5(1), 85-99.

Goodman, D. (2014) New York police officers to start using body cameras in a pilot program. The New York Times. 4 September. [Online] http://www.nytimes.com/2014/ 09/05/nyregion/new-york-police-officers-to-begin-wearing-body-cameras-in-pilotprogram.html.

Goodenough, W. (1976) Multiculturalism as the normal human experience. Anthropology \& Education Quarterly, 7: 7-18.

Goold, B. (2003) Public area surveillance and police work: The impact of CCTV on police behaviour and autonomy. Surveillance \& Society, 1(2): 191-203.

Goold, B. (2004) CCTV and Policing: Public Area Surveillance and Police Practices in Britain. Oxford: Oxford University Press.

Goold, B., Loader, I. \& Thumala, A. (2013) The banality of security: The curious case of surveillance cameras. British Journal of Criminology, 53: 977-996.

Gorner, J. (2016a) Chicago violence, homicides and shootings up in 2015. Chicago Tribune. 2 January. [Online] www.chicagotribune.com/.../ct-chicago-police-violence2015-met1-20160101-story.

Gorner, J. (2016b) Morale, policing suffering in hostile climate cops say. Chicago Tribune. 27 November: 1.

Gorner, J. (2016c) Police: Morale still low, emboldening criminals and contributing to violence. Chicago Tribune. 25 November. [Online] http://www.chicagotribune.com/ news/ct-chicago-police-morale-met-20161123-story.html.

Gottfredson, M. \& Hirschi, T. (1990) A General Theory of Crime. Stanford, CA: Stanford University Press.

Gould, J. \& Mastrofski, S. (2004) Suspect searches: Assessing police behaviour under the U.S. Constitution. Criminology \& Public Policy, 3: 315-361.

Gould, S. \& Hawkins, B. (1978) Organizational career stage as a moderator of the satisfaction-performance relationship. Academy of Management Journal, 21: 434-450.

Goulding, W. (2001) Just Another Indian: A Serial Killer and Canada's Indifference. Markham, ON: Fitzhenry \& Whiteside.

Graham, D. (2015) The White House smacks down its DEA chief on the 'Ferguson Effect. The Atlantic. 9 November. [Online] https://www.theatlantic.com/politics/ archive/2015/11/white-house-dea-chuck-rosenberg-ferguson-effect/414993/.

Gramaglia, J. (2016) Police officers' perceptions of body-worn cameras in the Buffalo and Rochester Police Departments. Unpublished MPA thesis. State University of New York College at Buffalo. 
Gray, D. (2014) Doing Research in the Real World, $3^{\text {rd }}$ ed. London: Sage.

Graziano, L., Schuck, A. \& Martin, C. (2010) Police misconduct, media coverage, and public perceptions of racial profiling: An experiment. Justice Quarterly, 27(1): 52-76.

Greene, J. (1998) Evaluating planned change strategies in modern law enforcement: Implementing community-based policing. In (J. Brodeur, ed.) How to Recognize Good Policing: Problems and Issues: 141-160. Thousand Oaks, CA: Sage.

Greene, J. (2000) Community policing in America: Changing the nature, structure, and function of the police. Criminal justice, 3(3): 299-370.

Greene, J. (2007) Mixed Methods in Social Inquiry. San Francisco: Josey-Bass.

Greene, J. (2014) New directions in policing: Balancing prediction and meaning in police research. Justice Quarterly, 31(2): 193-228.

Greene, J. \& Hall, J. (2010) Dialectics and pragmatism: Being of consequence. In (A. Tashakkori \& C. Teddlie, eds.) The Sage Handbook of Mixed Methods in Social and Behavioral Research: 119-143. Thousand Oaks, CA: Sage.

Greene, J. \& Mastrofski, S. (1988) Community Policing: Rhetoric or Reality? New York: Praeger.

Greene, J., Bergman, W. \& McLaughlin, E. (1994) Implementing community policing: Cultural and structural change in police organizations. In (D. Rosenbaum, ed.) The Challenge of Community Policing: 92-109. Thousand Oaks, CA: Sage.

Greenhaus, J. (1987) Career Management. Orlando, FL: Dryden Press.

Greenhaus, J., Callanan, G., \& Godshalk, V. (2000) Career Management, $3^{\text {rd }}$ ed. Thousand Oaks, CA: Sage.

Greenhill, N. (1981) The value of sociology in policing. In (D. Pope \& N. Weiner, eds.) Modern Policing: 90-109. London: Croom Helm.

Greer, C. \& McLaughlin, E. (2010a) 'Trial by media': Policing, the 24/7 mediasphere and the 'politics of outrage'. Theoretical Criminology, 15(1): 23-46.

Greer, C. \& McLaughlin, E. (2010b) We predict a riot? Public order policing, new media environments and the rise of the citizen journalist. British Journal of Criminology, 50(6): 1041-1059.

Greer, C. \& McLaughlin, E. (2012) This is not justice: Ian Tomlinson, institutional failure and the press politics of outrage. British Journal of Criminology, 52(2): 274-293.

Greer, C. \& Reiner, R. (2012) Mediated mayhem: media, crime, criminal justice. In (M. Maguire, R. Morgan \& R. Reiner, eds.) The Oxford Handbook of Criminology: 245278. Oxford: Oxford University Press.

Griffin, C., \& Ruiz, J. (1999) The sociopathic police personality: Is it a product of the 'rotten apple' or the 'Rotten Barrel'? Journal of Police and Criminal Psychology, 14(1): 28-37. 
Griffin, M., Hogan, N. \& Lambert, E. (2014) Career stage theory and turnover intent among correctional officers. Criminal Justice \& Behavior, 41(1): 4-19.

Grimmelikhuijsen, S. \& Meijer, A. (2014) Effects of transparency on the perceived trustworthiness of a government organization: Evidence from an online experiment. Journal of Public Administration Research \& Theory, 24(1): 137-157.

Groombridge, N. (2007) Criminologists say...: An analysis of UK national press coverage of criminology and criminologists and a contribution to the debate on public criminology. The Howard Journal, 46: 459-475.

Groves, R., Fowler, F., Couper, M., Lepkowski, J., Singer, E. \& Tourangeau, R. (2009) Survey Methodology, $2^{\text {nd }}$ ed. Hoboken, NJ: Wiley.

Guba, E. \& Lincoln, Y. (2005) Paradigmatic controversies, contradictions, and emerging confluences. In (N. Denzin \& Y. Lincoln, eds.) The Sage Handbook of Qualitative Research, $3^{\text {rd }}$ ed.: 191-216. Thousand Oaks, CA: Sage.

Guest, G., Bunce, A. \& Johnson, L. (2006) How many interviews are enough? An experiment with data saturation and variability. Field Methods, 18: 59-82.

Haarr, R. (1997) Patterns of integration in a police patrol bureau: Race and gender barriers to integration. Justice Quarterly, 14: 53-85.

Haarr, R. (2001) Making of a community police officer: The impact of basic training and occupational socialization on police recruits. Police Quarterly, 4(4): 402-433.

Haarr, R. (2005) Factors affecting the decision of police recruits to drop out of police work. Police Quarterly, 8(4): 431-453.

Haberfeld, M. (2013) Critical Issues in Police Training, 3rd ed. Upper Saddle River, NJ: Pearson.

Hackney, A. \& Glaser, J. (2013) Reverse deterrence in racial profiling: Increasing transgressions by non-profiled Whites. Law \& Human Behaviour, 37(5): 348-353.

Haggerty, K. (2006) Tear down the wall: On demolishing the Panopticon. In (D. Lyon, ed.) Theorizing Surveillance: The Panopticon and Beyond: 23-45. Cullompton, UK: Willan.

Haggerty, K. (2012) Surveillance, crime and the police. In (K. Ball, K. Haggerty \& D. Lyon, eds.) Routledge Handbook of Surveillance Studies: 235-243. New York: Routledge.

Haggerty, K. \& Ericson, R. (2000) The surveillant assemblage. The British Journal of Sociology, 51(4): 605-622.

Haggerty, K. \& Ericson, R. (2006) The new politics of surveillance and visibility. In (K. Haggerty \& R. Ericson, eds.) The New Politics of Surveillance and Visibility: 3-25.

Toronto: University of Toronto Press.

Haggerty, K. \& Sandhu, A. (2014) The police crisis of visibility. Technology \& Society, summer: 9-12. 
Haight, M., Quan-Haase, A. \& Corbett, B. (2014) Revisiting the digital divide in Canada: The impact of demographic factors on access to the internet, level of online activity, and social networking site usage. Information, Communication \& Society, 17(4): 503-519.

Hall, D. (1976). Careers in Organizations. Santa Monica, CA: Gonageor.

Hall, J. (2009) Heard this police acronym? FIDO: 'Forget it, drive on'. Edmonton Journal. 24 March. [Online] https://www.canada.comstory.html?id=d702510e-b2354073-819e-783a2f59765d.

Hall, S., Critcher, C., Jefferson, T., Clarke, J. \& Roberts, B. (1978) Policing the Crisis: Mugging, the State, and Law and Order. London: Macmillan.

Han, B. (2012). Transparenzgesellschaft. Berlin: Matthes \& Seitz.

Hanemaayer, A. \& Schneider, C., eds. (2014) The Public Sociology Debate: Ethics and Engagement. Vancouver: University of British Columbia Press.

Harnett, R. T., \& Seligsohn, H. C. (1967) The effects of varying degrees of anonymity on responses to different types of psychological questionnaires. Journal of Educational Measurement, 4: 95-103.

Harris, A. \& Pettersson, E. (2014) Ferguson officer compared Brown to Hulk Hogan. Bloomberg News. 25 November [Online] http://www.bloomberg.com/news/2014-1125/ferguson-cop-told-grand-jury-he-feared-for-his-life.html.

Harris, C. (2010) Pathways of Police Misconduct: Problem Behavior Patterns and Trajectories from Two Cohorts. Durham, NC: Carolina Academic Press.

Harris, C. (2014) The onset of police misconduct. Policing: An International Journal of Police Strategies \& Management, 37(2): 285-304.

Harris, C. (2016) Towards a career view of police misconduct. Aggression \& Violent Behaviour, 31, 219-228.

Harris, D. (1994) Factors for reasonable suspicion: When Black and poor means stopped and frisked. Indiana Law Journal, 69: 659-687.

Harris, D. (2002) Profiles in Injustice: Why Racial Profiling Cannot Work. New York: New Press.

Harris, F. (2012) Holding police accountability theory to account. Policing: An International Journal of Police Strategies \& Management, 6(3): 240-249.

Harrison, C. (2006) Tip sheet on survey sampling, Coverage and nonresponse. Harvard University Program of Survey Research. [Online] http://psr.iq.harvard.edu/files/psr/ files/PSRTipSheetSamplingCoverageNonresponse_1_0.pdf.

Harrison, C. (2007) Tip Sheet on question wording. Harvard University Program of Survey Research. [Online] http://psr.iq.harvard.edu/files/psr/files/PSRQuestionnaire TipSheet0.pdf. 
Harrison, S. (1998) Police organizational culture: Using ingrained values to build positive organizational improvement. Public Administration \& Management, 3(2): 3250.

Hartley, J. (2011) Digital Futures for Cultural and Media Studies. Malden, MA:

Hasham, A. (2015) Stand-off between Const. James Forcillo and Sammy Yatim took less than 50 seconds, court hears. Toronto Star. 20 October. [Online] https://www.the star.com/ news/crime /2015/10/20/murder-trial-begins-for-const-james-forcillo-insammy-yatim-shooting.html.

Hasham, A. (2016) Const. James Forcillo sentenced to 6 years in Sammy Yatim shooting. Toronto Star. 28 July. [Online] https://www.thestar.com/news/crime/ 2016/07/28/const-james-forcillo-sammy-yatim-shooting-sentence.html.

Hawdon, J. (2008) Legitimacy, trust, social capital and policing styles: A theoretical statement. Police Quarterly, 11(2): 182-201.

Hawdon, J. \& Ryan, J. (2003) Police-resident interactions and satisfaction with police: An empirical test of community policing assertions. Criminal Justice Policy Review, 14: $55-74$.

Hawdon, J., Ryan, J. \& Griffin, S. (2003) Policing tactics and perceptions of police legitimacy. Police Quarterly, 6(4): 469-491.

Hawkins, H. (2001). Police officer burnout: A partial replication of Maslach's Burnout Inventory. Police Quarterly, 4(3), 343-360.

Hayle, S., Worley, S. \& Tanner, J. (2016) Race, street life, and policing: Implications for racial profiling. Canadian Journal of Criminology and Criminal Justice, 58(3): 322353.

Hays, S. (2007) Stalled at the altar? Conflict, hierarchy, and compartmentalization in Burawoy's public sociology. In (D. Clawson et al., eds.) Public Sociology: Fifteen Eminent Sociologists Debate Politics and the Profession in the Twenty-first Century: 79-90. Berkeley, CA: University of California Press.

Healey, J. \& Prus, S. (2016) Statistics: A Tool for Social Research, $3^{\text {rd }}$ ed. Toronto: Nelson.

Heath, B. (2018) Baltimore police stopped noticing crime after Freddie Gray's death: A wave of killings followed. USA Today. 12 July. [Online] https://www.usatoday.com/ story/news/nation/2018/07/12/baltimore-police-not-noticing-crime-after-freddie-graywave-killings-followed/744741002/.

Heatherton, C. \& Camp, J., eds. (2016) Policing the Planet: Why the Policing Crisis Led to Black Lives Matter. New York: Verso.

Heaton, R. (2010) We could be criticized! Policing and risk aversion. Policing: A Journal of Policy \& Practice, 5(1): 75-86. 
Hedberg, E., Katz, C. \& Choate, D. (2017) Body-worn cameras and citizen interactions with police officers: Estimating plausible effects given varying compliance levels. Justice Quarterly, 34(4): 627-651.

Heidensohn, F. (2003) Gender and policing. In (T. Newburn, ed.) Handbook of Policing. Cullompton, UK: willan.

Henderson, M., Cullen, F., Cao, L., Browning, S. \& Kopache, R. (1997) The impact of race on perceptions of criminal injustice. Journal of Criminal Justice, 25: 447-462.

Henderson, N. (2014) With Eric Garner, Obama's body camera argument just took a big hit. The Washington Post. 3 December [Online http://www.washingtonpost com/blogs/ the-fix/wp/2014/12/03/obamas-body-camera-argument-just-took-a-big-hit/.

Henry, F., \& Tator, C. (2005). Racial Profiling in Toronto: Discourses of Domination, Mediation, and Opposition. Toronto, ON: Canadian Race Relations Foundation.

Henry, F., \& Tator, C. (2006). Racial Profiling in Canada: Challenging the Myth of a Few Bad Apples. Toronto, ON: University of Toronto Press.

Henry, F. \& Tator, C. (2011) Rejoinder to Satzewich and Shaffir on 'Racism versus professionalism: Claims and counterclaims about racial profiling'. Canadian Journal of Criminology \& Criminal Justice, 53: 65-74.

Herbert, S. (1996) The geopolitics of the police: Foucault, disciplinary power and the tactics of the Los Angeles Police Department. Political Geography, 15: 47-59.

Herbert, S. (1998) Police subculture reconsidered. Criminology, 36(2): 343-369.

Herbert, S. (2001) From spy to okay guy: Trust and validity in fieldwork with the police. Geographical Review, 91(1/2): 304-310.

Herbert, S. (2006) Citizens, Cops, and Power: Recognizing the Limits of Community. Chicago: University of Chicago Press.

Hermer, J., Kempa, M., Shearing, C., Stenning, P. \& Wood, J. (2005) Policing in Canada in the twenty-first century: Directions for law reform. In (D. Cooley, ed.) Reimagining Policing in Canada. Toronto: University of Toronto Press.

Hermida, A. \& Hernández-Santaolalla, V. (2018) Twitter and video activism as tools for counter-surveillance: The case of social protests in Spain. Information, Communication \& Society, 21(3): 416-433.

Hesse-Biber, S. (2010) Mixed Methods Research: Merging Theory with Practice. New York: Guilford Press.

Hesse-Biber, S. \& Leavy, P., eds. (2004) Approaches to Qualitative Research: A Reader on Theory and Practice. New York: Oxford University Press.

Hier, S. (2011) Panoptic Dreams: Streetscape Video Surveillance in Canada. Vancouver: UBC Press.

Hirchfield, P. \& Simon, D. (2010) Legitimating police violence: Newspaper narratives of deadly force. Theoretical Criminology, 14: 155-182. 
Hirtzel, A. (2014) Former Buffalo police officer receives probation for assault caught on video. WBFO NPR News. 1 December. [Online] http://news.wbfo.org/post/formerbuffalo-police-officer-receives-probation-assault-caught-video.

Ho, T. (1997) Police use of deadly force and experience: Rookie v. veteran. The Justice Professional, 10(2): 127-14.

Hobbs, D. (1988) Doing the Business: Entrepreneurship, the Working Class and Detectives in the East End of London. Oxford: Clarendon Press.

Holdaway, S. (1979) Introduction. In (S. Holdaway, ed.) The British Police: 1-13. London: Edward Arnold.

Holdaway S. (1983) Inside the British Police. Oxford: Blackwell.

Holdaway S. (1989) Discovering structure: Studies of the British police occupational culture. In (M. Weatheritt, ed.), Police Research: 55-75. Aldershot: Avebury.

Holgersson, S. (2015) An inside job: Managing mismatched expectations and unwanted findings when conducting police research as a police officer. In (E. Cockbain \& J. Knutsson, eds.) Applied Police Research: Changes and Opportunities: 106-116. New York: Routledge

Holmes, M. \& Smith, B. (2008) Race and Police Brutality: Roots of an Urban Dilemma. Albany, NY: SUNY Press.

Holmwood. J. (2007) Sociology as a public discourse and professional practice: A critique of Michael Burawoy. Sociological Theory, 25(1): 46-66.

Home Office (2008) From the Neighbourhood to the National: Policing our Communities Together. Green Paper. London: Stationery Office.

Home Office (2009). Protecting the Public: Supporting the Police to Succeed. White Paper. London: Stationery Office.

Hood, C. (2006) Transparency in historical perspective. In (C. Hood \& D. Heald, eds.) Transparency: The Key to Better Governance? 3-23. Oxford: Oxford University Press.

Hood, C. \& Heald, D., eds. (2006) Transparency: The Key to Better Governance? Oxford: Oxford University Press.

Hoogeboom, B. (2009) Bringing the Police Back In: Notes on the Lost and Found Character of the Police in Police Studies. Manuscript provided by the author.

hooks, b. (2004) Culture to culture: Ethnography and cultural studies as critical intervention. In (S. Hesse-Biber \& P. Leavy, eds.) Approaches to Qualitative Research: 149-158. New York: Oxford University Press.

Hopper, T. (2018) Why Canadian police are so good at not shooting people. The National Post. 24 April: 3.

Hough, M. (2003) Modernization and public opinion: Some criminal justice paradoxes. Contemporary Politics, 9(2): 143-155. 
Hough, M. (2007) Policing, new public management and legitimacy. In (T. Tyler, ed.) Legitimacy and Criminal Justice: 63-83. New York: Sage.

Hough, M. \& Roberts, J. (2004) Confidence in Justice: An International Review. London: Home Office.

Hough, M., Jackson, J., Bradford, B., Myhill, A. \& Quinton, P. (2010) Procedural justice, trust and institutional legitimacy. Policing: A Journal of Policy and Practice, 4(3): 203-210.

Hough, M. \& Stanko, E. (2019) Designing degree-level courses for police recruits in England and Wales: Some issues and challenges. Policing. DOI:10.1093/police/pay096.

Howard, G., Donofrio, H. \& Boles, J. (2004) Inter-domain work-family, family work conflict and police work satisfaction. Policing: An International Journal of Police Strategies \& Management, 27: 380-395.

Hsiung, P. (1996) Between bosses and workers: The dilemmas of a keen observer and a vocal feminist. In (D. Wolf, ed.) Feminist Dilemmas in Fieldwork: 122-137. Boulder, CO: Westview Press.

Huang, W. \& Vaughn, M. (1996) Support and confidence: Public attitudes toward the police. In (T. Flanagan \& D. Longmire, eds.) America's View of Crime and Justice: A National Opinion Survey: 31-45. Thousand Oaks, CA: Sage.

Hudak, A. (2016) Young man claims RPD used excessive force in mistaken identity case. WHAM. 23 August. [Online] http://13wham.com/news/top-stories/teen-claimsrpd-beat-used-pepper-spray-on-him-after-puerto-rican-festival.

Hudson, D. (2014) Excerpts from President Obama's BET interview on race relations, and the progress we still have to make. The White House Blog. 10 December. [Online] http://www.whitehouse.gov/blog/2014/12/10/excerpts-president-obamas-bet-interviewrace-relations-and-progress-we-still-have-ma.

Hudson, J. (1973) Book review: The Police and the Public. American Journal of Sociology, 78(6): 1568-1570.

Huey, L. \& Broll, R. (2015) 'I don't find it sexy at all': Views of media glamorization of police 'dirty work'. Policing \& Society, 25(2): 236-247.

Huey, L., Walby, K. \& Doyle, A. (2006) Cop watching in the downtown eastside: Exploring the use of (counter) surveillance as a tool of resistance. In (T. Monahan, ed.) Surveillance and Security: Technological Politics and Power in Everyday Life: 149165. New York: Routledge.

Huggins, R., Wright, C. \& Murphy, J. (2014) National Policing Research Symposium: Outcomes Report. Ottawa: Public Safety Canada.

Hughes, E. (1962) Good people and dirty work. Social Problems, 10(1): 3-11.

Hunt, R. \& McCadden, K. (1985) A survey of work attitudes of police officers: Commitment and satisfaction. Police Studies, 8: 17-25. 
Hurley, M. (2012) Police sued over racial profiling. Ottawa Citizen. 10 August. [Online] https://www.pressreader.com/canada/ottawacitizen/20120810/28189588 5390372.

Husain, N. (2017) Laquan McDonald timeline: The shooting, the video and the fallout. The Chicago Tribune. 20 October. [Online] http://www.chicagotribune.com/news/ laquanmcdonald/ct-graphics-laquan-mcdonald-officers-fired-timeline-htmlstory.html.

Iacobucci, F. (2014) Police Encounters with People in Crisis: An Independent Review Conducted by the Honourable Frank Iacobucci for Chief of Police William Blair. Toronto: Toronto Police Service.

Ingram, J. \& Terrill, W. (2014) Relational demography and officer occupational attitudes: The influence of workgroup context. Journal of Criminal Justice, 42(4): 309320.

Ingram, J., Paoline, E. \& Terrill, W. (2013) A multilevel framework for understanding police culture: The role of the workgroup. Criminology, 51(2): 365-397.

Ingram, J., Terrill, W. \& Paoline, E. (2018) Police culture and officer behaviour: Application of a multilevel framework. Criminology, 56(4): 780-811.

Innes, M. (2003) Investigating Murder: Detective Work and the Police Response to Criminal Homicide. Oxford: Oxford University Press.

Intravia, J., Wolff, K. \& Piquero, A. (2018) Investigating the effects of media consumption on attitudes toward police legitimacy. Deviant Behavior, 39(8): 963-980.

Ivkovic, S. (2008) A comparative study of public support for the police. International Criminal Justice Review, 18(4): 406-434.

Jackson, J. \& Sunshine, J. (2007) Public confidence in policing: A neo-Durkheimian perspective. British Journal of Criminology, 47: 214-233.

Jackson, J., Bradford, B., Hough, M., Myhill, A., Quinton, P. \& Tyler T. (2012) Why do people comply with the law? Legitimacy and the influence of legal institutions. British Journal of Criminology, 52(6): 1051-1071.

Jackson, J., Bradford, B., Stanko, E. \& Hohl, K. (2012) Just authority? Trust in the police in England and Wales. Oxon: Routledge.

Jacobs, H. (2016) How New York's capital avoided becoming 'a flaming city' after police killed a mentally ill, unarmed black man. Business Insider. 19 November. [Online] http://www.businessinsider.com/albany-community-policing-reforms-anddontay-ivy-police-death-2016-10.

James, L., James, S. \& Vila, B. (2016) The reverse racism effect: Are cops more hesitant to shoot Black than White suspects? Criminology \& Public Policy, 15(2): 457479.

James, L., Vila, B. \& Daratha, K. (2013) Results From experimental trials testing participant responses to White, Hispanic and Black suspects in high-fidelity deadly 
force judgment and decision-making simulations. Journal of Experimental Criminology, 9(2): 189-212.

James, M. \& Madhani, A. (2018) Video in Toronto killings shows a divide between U.S. and Canada on deadly force. USA Today. 26 April. [Online] https://www. usatoday. com /story/news/2018/04/26/video-toronto-killings-shows-divide-between-us-and-canada-deadly-force/551798002/.

Jamieson, A. (2018) A cop union lashed out at a tweet informing people of their fourth amendment rights. BuzzFeed News. 5 May 2018. [Online] https://www.buzzfeed.com /amberjamieson/nypd-sba-sergeants-benevolent-association-policefourth?utm_term $=$. eg8dB4roq\#.uqwr2jPJ3.

Jansson, A. \& Lindell, J. (2015) News media consumption in the transmedia age. Journalism Studies, 16(1): 79-96.

Janus, A. (2016) Yatim family lawyers deny Forcillo had 'trial by YouTube' after fatal streetcar shooting. CBC News. 28 July. [Online] https://www.cbc.ca/news/canada/ toronto/lawyers-react-to-forcillo-sentencing-1.3699509.

Jayaratne, S. (1993) The antecedents, consequences, and correlates of job satisfaction. In (R. Golembiewski, ed.) Handbook of Organizational Behaviour: 111-140. New York: Dekker.

Jefferis, E., Butcher, F. \& Hanley, D. (2011) Measuring perceptions of police use of force. Police Practice \& Research, 12(1): 81-96.

Jefferis, E., Kaminski, R., Holmes, S. \& Hanley. D. (1997) The effect of a videotaped arrest on public perceptions of police use of force. Journal of Criminal Justice, 25: 381395.

Jefferson, T. (1990) The Case Against Paramilitary Policing. Milton Keynes: Open University.

Jefferson, T. \& Grimshaw, R. (1984) Controlling the Constable. London: Muller.

Jeffries, J. (2011). Democracy for the few: How local governments empower cops at citizens' expense. Journal of Law and Conflict Resolution, 3(5): 71-75.

Jeffries, S. \& Stenning, P. (2014) Sentencing Aboriginal offenders: Law, policy, and practice in three countries. Canadian Journal of Criminology \& Criminal Justice, 56(4): 447-494.

Jenkins, H. (2008) Convergence Culture: Where Old and New Media Collide. New York: New York University Press.

Jenkins, M. (2015) The use of qualitative methods and practitioners-as-authors in journal publications of police research. Police Practice \& Research, 16(6): 499-511.

Jennings, W., Fridell, L. \& Lynch, M. (2014) Cops and cameras: Officer perceptions of the use of body-worn cameras in law enforcement. Journal of Criminal Justice, 42(6): $549-556$. 
Jermier, J., Slocum, J., Fry, L. \& Gaines, J. (1991) Organizational subcultures in a soft bureaucracy: Resistance behind the myth and façade of an official culture.

Organizational Science, 2: 170-194.

Jewkes, Y. (2004) Media and Crime. London: Sage.

Jick, T. (1979). Mixed qualitative and quantitate methods: Triangulation in action. Administrative Science Quarterly, 24(4): 602-611.

Johnson, B. \& Gray, R. (2010) A history of philosophical and theoretical issues for mixed methods research. In (A. Tashakkori \& C. Teddlie, eds.) The Sage Handbook of Mixed Methods in Social and Behavioral Research, $2^{\text {nd }}$ ed. Thousand Oaks, CA: Sage.

Johnson, D. \& Wayland, K. (2010) Surveillance and transparency as sociotechnical systems of accountability. In (K. Haggerty \& M. Samatas, eds.) Surveillance and Democracy: 19-33. New York: Routledge-Cavendish.

Johnson, D., \& Regan, P. (2014) Transparency and Surveillance as Sociotechnical Accountability: A House of Mirrors. New York: Routledge.

Johnson, K. (2010) For cops, citizen videos bring increased scrutiny. USA Today. 15 October. [Online] http://usatoday30.usatoday.com/news/nation/2010-10-151Avideocops 15_CV_N.htm.

Johnson, K. (2014) Police body cameras offer benefits, require training. USA Today. 12 September. http://www.usatoday.com/story/news/nation/2014/09/ 12/police-bodycameras/15522059/.

Johnson, R. (2006) Management influences on officer traffic enforcement activity. International Journal of Police Science \& Management, 8: 205-217.

Johnson, R. (2012) Police officer job satisfaction: A multidimensional analysis. Police Quarterly, 15(2): 157-176.

Johnson, R. \& Lafrance, C. (2016) The influence of career stage on police officer work behavior. Criminal Justice \& Behavior, 43(11): 1580-1599.

Johnson, R. \& Onwuegbuzie, A. (2004) Mixed methods research: A research paradigm whose time has come. Educational Researcher, 33(7): 14-26.

Johnson, R., Onwuegbuzie, A. \& Turner, L. (2007) Toward a definition of mixed methods research. Journal of Mixed Methods Research, 1(2): 112-133.

Johnston, R. \& Smith, S. (2010) How critical realism clarifies validity issues in theorytesting research: Analysis and case. In (D. Hart \& S. Gregor, eds.) Information Systems Foundations: The Role of Design Science. Acton, Australia: Australian National University Press.

Jones-Brown, D., Gill, J. \& Trone, J. (2010) Stop, Question and Frisk Policing Practices in New York City: A Primer. New York: John Jay College of Criminal Justice.

Jones, J. (2015) In U.S. confidence in police lowest in 22 years. Gallup. 19 June. [Online] http://www.gallup.com/poll/183704/confidence-police-lowest-years.aspx. 
Jones, T. (2008) The accountability of policing. In (T. Newburn, ed.) Handbook of Policing. Cullompton: Willan.

Jones, T. \& Newburn, T. (1998) Private Security and Public Policing. Oxford: Clarendon Press.

Jones, T. \& Newburn, T. (2002) The transformation of policing? Understanding current trends in policing systems. British Journal of Criminology, 42(1): 129-146.

Joyce, P. \& Wain, N. (2010) A Dictionary of Criminal Justice. London: Routledge.

Kaariainen, J. (2008) Why do the Finns trust the police? Journal of Scandinavian Studies in Criminology and Crime Prevention, 9(2): 141-159.

Kadushin, C., Hecht, S., Sasson, T. \& Saxe, L. (2008) Triangulation and mixed methods designs: Practicing what we preach in the evaluation of an Israel experience program. Field Methods, 20(1): 46-65.

Kahn, R. \& Kellner, D. (2006) Oppositional politics and the Internet: A critical/ reconstructive approach. In (M, Durham \& D. Kellner, eds.) Media and Cultural Studies: Key Works: 703-725. Oxford: Blackwell.

Kaminski, R. \& Jefferis, E. (1998) Effect of a violent televised arrest on public perceptions of the police: A partial test of Easton's theoretical framework. Policing: An International Journal of Police Strategies \& Management 21(4): 683-706.

Kane, R. (2002) The social ecology of police misconduct. Criminology, 40(4): 867-896.

Kappeler, V., Sluder, R. \& Alpert, G. (1998) Forces of Deviance: Understanding the Dark Side of Policing, 2nd ed. Prospect Heights, IL: Waveland Press.

Kappeler, V., Sluder, R. \& Alpert, G. (2005) Breeding deviant conformity: Police ideology and culture. In (V. Kappeler, ed.) The Police and Society, $3^{\text {rd }}$ ed. Long Grove, IL: Waveland Press.

Kappeler, V., Sluder, R. \& Alpert, G. (2010) Breeding deviant conformity: The ideology and culture of police. In (R. Dunham \& G. Alpert, eds.) Critical Issues in Policing: Contemporary Readings, $6^{\text {th }}$ ed.: 265-291. Long Grove, IL: Waveland Press.

Kappeler, V., Sluder, R. \& Alpert, G. (2015) Breeding deviant conformity: The ideology and culture of the police. In (R. Dunham \& G. Alpert, eds.) Critical Issues in Policing, 7th ed.: 79-105. Long Grove, IL: Waveland Press.

Kapustin, M., Ludwig, J., Punkay, M., Smith, K. Speigel, L. \& \& Welgus, D. (2017) Gun Violence in Chicago, 2016. Chicago: University of Chicago Crime Lab.

Kaste, M. (2015) When morale dips. Some cops walk the beat - but do the minimum. National Public Radio. 8 January. [Online] https://www.npr.org/2015/01/08/

375914022/when-morale-dips-some-cops-walk-the-beat-but-do-the-minimum.

Kaye, R (2015) Caught on camera: Cellphone video vs police. CNN. 11 April. [Televison \& Online] https://www.cnn.com/videos/tv/2015/04/11/ac-kaye-cops-andcell-phone-video.cnn. 
Kearns, E. (2017) Why are some officers more supportive of community policing with minorities than others? Justice Quarterly, 34(7): 1213-1245.

Keat, R. \& Urry, J. (2010) Social Theory as Science. London: Routledge.

Kelling, G. (1997) Crime control, the police, and culture wars: Broken windows and cultural pluralism. Perspectives on Crime and Justice: 1997-1998 Lecture Series: 1-28. Washington, DC: National Institute of Justice.

Kelling, G. \& Coles, C. (1996) Fixing Broken Windows: Restoring Order and Reducing Crime in Our Communities. New York: The Free Press.

Kelling, G. \& Moore, M. (1998) From political to reform to community: The evolving strategy of police. In (J. Greene \& S. Mastrofski, eds.) Community Policing: Rhetoric or Reality? New York: Praeger.

Kelling, G. \& Sousa, W. (2001) Do Police Matter? An Analysis of the Impact of New York City's Police Reforms. Civic Report No. 22. New York: Manhattan Institute for Policy Research.

Kelling, G., Pate, A., Dieckman, D. \& Brown, C. (1974) The Kansas City Preventive Patrol Experiment: Technical Report. Washington, DC: Police Foundation.

Kelly, D. (2014) Accountability by Camera: Online Video's Effects on Police-Civilian Interactions. El Paso, TX: LFB Scholarly Publishing.

Kelly, M. (2016) Police shootings: Caught on camera. The Fifth Estate. 4 November. [Television].

Kennedy, R. (1997) Race, Crime, and the Law. New York: Pantheon.

Kennedy, S., Otuyelu, F. \& Graham, W. (2018) To protect and serve: Examing race, law enforcement culture and socia work practice. In (S. Weissinger \& D. Mack, eds.) Law Enforcment in the Age of Black Lives Matter: Policing Black and Brown Bodies: 153-169. New York: Lexington Books.

Khatib, T. (1996) Organizational Culture, Subcultures, and Organizational Commitment. Unpublished $\mathrm{PhD}$ dissertation. Iowa State University.

Kiedrowski, J., Melchers, R., Riddell, R. \& Petrunik, M. (2015) The Civilianization of Police in Canada. Ottawa: Public Safety Canada.

Kies, M. (2011) Policing the police: Freedom of the press, the right to privacy, and civilian recordings of police activity. The George Washington Law Review, 80: 274310.

Kilty, J. \& Crépault, C. (2016) Institutionalizing public scholarship: Lessons from feminism and symbolic interactionism. Symbolic Interaction, 39(4): 615-633.

Kindy, K. \& Kelly, K. (2015) Thousands dead, few prosecuted. The Washington Post. 11 April 2015. [Online] https://www.washingtonpost.com/sf/investigative/2015/04/11/ thousands-dead-few-prosecuted/?utm_term=.e9299fb09acc. 
King, M. (2013) 'Broken windows,' urban policing, and the social contexts of race and neighborhood (dis-)empowerment. Critical Criminology, 21(4): 533-538.

King, W. \& Dunn, T. (2004) Dumping: Police-initiated transjurisdictional transport of troublesome persons. Police Quarterly, 7(3): 333-358.

Kirby, S., Greaves, L. \& Reid, C. (2006) Experience Research Social Change: Methods Beyond the Mainstream. Peterborough, ON: Broadview Press.

Kirkham, G. (1974) From professor to patrolman. Journal of Police Science \& Administration, 2(2): 127-137.

Kitossa, T. (2012) Criminology and colonialism: Counter colonial criminology and the Canadian context. Journal of Pan African Studies, 4(10), 204-226.

Klein, S., Maher, J. \& Dunnington, R. (1967) Differences between identified and anonymous subjects in responding to an industrial opinion survey. Journal of Applied Psychology, 51: 152-160.

Klick, J. \& Tabarrok, A. (2005). Using Terror Alert Levels to Estimate the Effect of Police on Crime. Journal of Law \& Economics 48(1): 267-279.

Klinger, D. (1997) Negotiating order in patrol work: An ecological theory of police response to deviance. Criminology, 35: 277-306

Klockars, C. (1983) The Dirty Harry problem. In (C. Klockars, ed.) Thinking About Police: Contemporary Readings. New York: McGraw-Hill.

Klockars, C. (1985) The Idea of Police. London: Sage.

Klockars, C. (1996) A theory of excessive force and its control. In (W. Geller \& H. Toch, eds.) Police Violence: Understanding and Controlling Police Abuse of Force. New Haven, CT: Yale University Press.

Knapp, A. (2016) Walter Scott effect: North Charleston traffic stops cut in half after shooting, but is it the lasting change critics seek? The Post \& Courier. 2 April. [Online] http://www.postandcourier.com/article/20160402/PC16/ 160409955.

Kochel, T. (2011) Constructing hot spots policing: Unexamined consequences for disadvantaged populations and for police legitimacy. Criminal Justice Policy Review, 22(3): 350-374.

Kochel, T. \& Weisburd, D. (2017) Assessing community consequences of implementing hot spots policing in residential areas: Findings from a randomized field trial. Journal of Experimental Criminology, 13: 143-170.

Kochel, T., Wilson, D. \& Mastrofski, S. (2011) The effect of suspect race on police officers' arrest decisions. Criminology, 49(2): 473-512.

Koenig, D. (1980) The effect of crime victimization and judicial or police contacts on public attitudes toward the local police. Journal of Police Science \& Administration, 8: 243-249. 
Koskela, H. (2003) 'Cam Era' - The contemporary urban Panopticon. Surveillance \& Society, 1(3): 292-313.

Koskela, H. (2006) The other side of surveillance: Webcams, power and agency. In (D. Lyon, ed.) Theorizing Surveillance: The Panopticon and Beyond: 163-181. Cullompton: Willan Publishing.

Koskela, H. (2009) Hijacking surveillance? The new moral landscapes of amateur photographing. In (K. Aas, H. Gundhus \& H. Lomell, eds.) Technologies of (In)Security: The Surveillance of Everyday Life: 147-168. London: Routledge/ Cavendish.

Koskela, H. (2011) Hijackers and humble servants: Individuals as camwitnesses in contemporary controlwork. Theoretical Criminology, 15(3): 269-282.

Kowalski, B. \& Lundman, R. (2007) Vehicle stops by police for driving while Black: Common problems and some tentative solutions. Journal of Criminal Justice, 35(2): 165-181.

Kraska, P. \& Cubellis, L. (1997) Military Mayberry and beyond. Making sense of American paramilitary policing. Justice Quarterly, 14: 607-629.

Kraska, P. \& Paulsen, D. (1997) Grounded research into US paramilitary policing: Forging the iron fist inside the velvet glove. Policing \& Society, 7(4): 253-270.

Kreiner, G., Ashforth, B. \& Sluss, D. (2006) Identity dynamics in occupational dirty work: Integrating social identity and system justification perspectives. Organization Science, 17(5): 619-636.

Krumpal, I. (2013) Determinants of social desirability bias in sensitive surveys: A literature review. Quality \& Quantity, 47(4): 2025-2047.

Krupanski, M. (2012) Policing the police: Civilian video monitoring of police activity. The Global Journal. [Online] http://theglobaljournal. net/articles /view/643.

Kubrin, C., Messner, S., Deane, G., Mcgeever, K. \& Stucky, T. (2010) Proactive policing and robbery rates across US cities. Criminology, 48, 57-97.

Kuokkanen, R. (2008) Globalization as racialized violence: The case of Indigenous women. Internaitonal Feminist Journal of Politics, 10(2): 216-233.

Kurasawa, F. (2008) A message in a bottle: Bearing witness as a mode of transnational practice. Theory, Culture \& Society, 26(1): 92-111.

Kurtz, D. (2008) Controlled burn: The gendering of stress and burnout in modern policing. Feminist Criminology, 3(3): 216-238.

Kusow, A., Wilson, L. \& Martin, D. (1997) Determinants of citizen satisfaction with the police. Policing: An International Journal of Police Strategies \& Management, 20(4): 655-664.

Kutner, M. (2016) The new American cop: Smarter, more diverse, better equipped and scared. Newsweek. 11 August: 1-4. 
Kutnjak-Ivkovic, S. (2014) Police misconduct. In (M. Reisig \& R. Kane, eds.) The Oxford Handbook of Police and Policing: 302-338. New York: Oxford University Press.

Labaky, E. (2013) Women in Policing: Their Disillusion Phase at Work. Unpublished MA thesis. Ottawa: University of Ottawa.

Labaree, R. (2002) The risk of going 'observationalist': Negotiating the hidden dilemmas of being an insider participant observer. Qualitative Research, 2(1): 97-122.

Lafrance, T. \& Day, J. (2013) The role of experience in prioritizing adherence to SOPs in police agencies. Public Organization Review, 13: 37-48.

Lahman, S. (2017) 17 RPD officers named in lawsuit alleging assault of city teen. Democrat and Chronicle. 7 August. [Online] http://www.democratandchronicle.com/ story/news/2017/08/07/17-rpd-officers-named-lawsuit/543910001/

Lambert, E. Hogan, N. \& Griffin, M. (2017) Research note: A career-stage analysis of correctional staff outcomes. The Prison Journal, 97(4): 411-430.

Lambert, E. \& Paoline, E. (2012) Exploring potential antecedents of job involvement: An exploratory study among jail staff. Criminal Justice \& Behaviour, 39: 264-286.

La Prairie, C. (2002) Aboriginal over-representation in the criminal justice system: A tale of nine cities. Canadian Journal of Criminology, 44(2): 181-208.

La Prairie, C. \& Stenning, P. (2003) Exile on Main Street: Some thoughts on Aboriginal over-representation in the criminal justice system. In (D. Newhouse \& E. Peters, eds.) Not Strangers in These Parts: Urban Aboriginal Peoples: 179-193. Ottawa: Policy Research Initiative.

Lasley, J. (1994) The impact of the Rodney King incident on citizen attitudes toward police. Policing \& Society, 3(4): 245-255.

Lather, P. (1994) Fertile Obsession: Validity after poststructuralism. In (A. Gitlin, ed.) Power and Method: Political Activism and Educational Research: 36-60. Routledge: New York.

Laucius, J. (2017) A turning point': Police legislation will provide more transparency, say Abdi supporters. Ottawa Citizen. 3 November. [Online] https://ottawacitizen.com/ news/ local-news/a-turning-point-police-legislation-will-provide-more-transparencysay-abdi-supporters.

Lautenschlager, R., Capellan, G. \& Silva, J. (2018) Deconstructing the Ferguson Effect: A Multilevel Mediation Analysis of Public Scrutiny, De-policing, and Crime.

Conference paper presented at the American Society of Criminology annual meeting, Atlanta, GA, 14 November.

Lautt, S. (2012) Sunlight is still the best disinfectant: The case for a first amendment right to record the police. Washburn Law Journal, 51(2): 349-381.

La Vigne, N., Fontaine, J. \& Dwivedi, A. (2017) How do people in high- crime, lowincome communities view the police? Urban Institute. [Online] https://www.urban.org/ 
sites/default/files/publication/88476/how_do_people_in_high-crime_view_the_police. pdf.

Lawrence, R. (2000) The Politics of Force: Media and the Construction of Police Brutality. Berkeley: University of California Press.

Lawson, T. (1997) Economics and Reality. London: Routledge.

LeCount, R. (2017) More Black than blue? Comparing the racial attitudes of police to citizens. Sociological Forum, 32(1): 1051-1072.

Lee, A., Weitzer, R. \& Martinez, D. (2018) Recent police killings in the United States: A three-city comparison. Police Quarterly, 21(2): 196-222.

Lee, H., Lim, H., Moore, D. \& Kim, J. (2013) How police organizational structure correlates with frontline officers' attitudes toward corruption: A multilevel model. Police Practice \& Research, 14: 386-401.

Lee, J. (1981) Some structural aspects of police deviance in relations with minority groups. In (C. Shearing, ed.) Organizational Police Deviance. Toronto: Butterworth.

Lee, M. \& McGovern, A. (2013) Force to sell: Policing the image and manufacturing public confidence. Policing \& Society, 23(2): 103-124.

Lee, M. \& McGovern, A. (2014) Policing and Media: Public Relations, Simulations and Communications. New York: Routledge.

Lee, Y., Eck, J. \& Corsaro, N. (2016) Conclusions from the history of research into the effects of police force size on crime - 1968 through 2013: A historical systematic review. Journal of Experimental Criminology, 12(3): 431-451.

Legewie, J. (2016) Racial profiling and use of force in police stops: How local events trigger periods of increased discrimination. American Journal of Sociology, 122(2): 379-424.

Lenzo, K. (1995) Validity and self-reflexivity meet poststructuralism: Scientific ethos and the transgressive self. Educational Researcher, 24(4): 17-23.

Leo, J. (2001) Riot ideology in the aftermath. The Washington Times. 25 July: A15.

Lersch, K. \& Mieczkowski, T. (2005) Violent police behavior: Past, present, and future research directions. Aggression \& Violent Behaviour, 10: 552-568.

Lever, A. (2005) Why racial profiling is hard to justify: A response to Risse and Zeckhauser. Philosophy \& Public Affairs, 33(1): 94-110.

Levi, M. (2013) Legitimacy, crimes, and compliance in the city: De maximis non curat lex? In (Tankebe, J. \& and Leibling, A., eds.) Legitimacy and Criminal Justice: An International Exploration: 157-177. Oxford: Oxford University Press.

Levi, M., Sacks, A. \& Tyler, T. (2009) Conceptualizing legitimacy, measuring legitimating beliefs. American Behavioral Scientist, 53(3): 354-375. 
Levine, J. (1975) Ineffectiveness of adding police to prevent crime. Public Policy, 23(4): 523-545.

Levine, M. \& Taylor, A. (2015) DEA Chief Backs FBI Director on Whether 'Ferguson Effect' May Be Real. ABC News. 4 November. [Online] http://abcnews.go.com/Politics/ dea-chief-backs-fbi-director-ferguson-effect-real/story?id=34971183.

Levine, S. (2014) White house petition for 'Mike Brown Law' quickly earning support. Huffington Post. 21 August. [Online] http://www.huffingtonpost. com/2014/08/20/ mike-brown-law_n_5696585.html.

Levinson, D. (1986) A conception of adult development. American Pscvchologist, 41: 3-13.

Levinson, D., Darrow, C., Klein, E., Levinson, M. \& McKee, B. (1978) The Seasons of a Man's Life. New York: Alfred A. Knopf.

Lewis-Beck, M., Bryman, A. \& Liao, T. (2003) The Sage Encyclopedia of Social Science Research Methods. London: Sage.

Lewis, C. (2017) OpEd: Stand-out public safety issues for 2017. CP24. 26 December. [Online] https://www.cp24.com/news/a-view-from-the-top/oped-stand-out-publicsafety-issues-for-2017-1.3734607.

Lewis, C. (2018a) Are our police officers de-policing? CP24 Toronto News. 15 February. [Online] https://www.cp24.com/news/a-view-from-the-top/oped-are-ourpolice-officers-de-policing-1.3805477.

Lewis, C. (2018b) Is de-policing alive and not so well? Blue Line: Canada's Law Enforcment Magazine. 15 June: 30.

Li, H. (2009) The turn to the self: From big character posters to YouTube videos. Chinese Journal of Communication, 2: 50-60.

Lichtblau, E. (2016) F.B.I. director says 'viral video effect' blunts police work. The New York Times. 11 May. [Online] https://www.nytimes.com/2016/05/12/us/comeyferguson-effect-police-videos-fbi.html.

Lievrouw, L. (2011) Alternative and Activist New Media. Cambridge: Polity.

Light, D. (2005) Contributing to scholarship and theory through public sociology. Social Forces, 83(4): 1647-1653.

Lindsey, T. (2015) Post-Ferguson: A 'herstorical' approach to Black violability. Feminist Studies, 41(1): 232-237.

Lippert, R. \& Walby, K. (2012) Municipal corporate security and the intensification of urban surveillance. Surveillance \& Society, 9(3): 310-320.

Lipset, S. (1990) Continental Divide: The Values and Institutions of the United States and Canada. New York: Routledge.

Loader, I. (1997) Policing and the social: Questions of symbolic power. The British Journal of Sociology, 48(1): 1-18. 
Loader, I. (2008) Social movements and new media. Sociology Compass, 2(6): 19201933.

Loader, I. (2014) Why do the police matter? Beyond the myth of crime-fighting. In (J. Brown, ed.) The Future of Policing: 40-51. New York: Routledge.

Loader, I. (2016) Changing climates of control: The rise and fall of police authority in England and Wales. In (M. Bosworth, C. Hoyle \& L. Zedner, eds.) Changing Contours of Criminal Justice. Oxford: Oxford University Press.

Loader, I. \& Mulcahy, A. (2003) Policing and the Condition of England: Memory, Politics and Culture. Oxford: Oxford University Press.

Loader, I. \& Sparks, R. (2008) What are we gonna do now? Revisiting the public roles of criminology. Criminal Justice Matters, 72: 18-19.

Loader, I. \& Sparks, R. (2010a) Public criminology? London: Routledge.

Loader, I. \& Sparks, R. (2010b) What is to be done with public criminology?

Criminology \& Public Policy, 9(4): 771-781.

Loader, I. \& Sparks, R. (2011) Criminology's public role: A drama in six acts. In (M. Boswoth \& C. Hoyle, eds.) What is Criminology? 17-34. New York: Oxford University Press.

Loader, I. \& Sparks, R. (2013) Unfinished business: Legitimacy, crime control, and democratic politics. In (J. Tankebe \& and A. Leibling, eds.) Legitimacy and Criminal Justice: An International Exploration: 105-126. Oxford: Oxford University Press.

Loader, I. \& Sparks, R. (2015) Ideologies and crime: Political ideas and the dynamics of crime control. Global Crime, 17(3-4): 314-330.

Loewen, J. (2005) Sundown Towns: A Hidden Dimension of American Racism. New York: The New Press.

Loftus, B. (2007) Policing the 'irrelevant': Class, diversity and contemporary police culture. In (M. O’Neill, M. Marks \& A. Singh, eds.) Police Occupational Culture: New Debates and Directions: 181-204. Oxford: Elsevier.

Loftus, B. (2008) Dominant culture interrupted: Recognition, resentment and the politics of change in an English police force. British Journal of Criminology, 48: 756777.

Loftus, B. (2009) Police Culture in a Changing World. Oxford: Oxford University Press.

Loftus, B. (2010) Police occupational culture: Classic themes, altered times. Policing \& Society, 20(1): 1-20.

Loftus, B., Goold, B. \& MacGiolllabhui, S. (2016) From a visible spectable to an invisible presence: The working culture of covert policing. British Journal of Criminology, 56(4): 629-645. 
London Free Press (2011) Judge says pair busted for being Black. London Free Press. 7 July. [Online] http://www.lfpress.com/news/canada/2011/07/07/18386796.html.

Love, B. (2016) Anti-Black state violence, classroom edition: The spirit murdering of Black children. Journal of Curriculum \& Pedagogy, 13: 22-25.

Loveday, B. (2000) New directions in accountability. In (F. Leishman et al., eds.) Core Issues in Policing: 213-231. Harlow: Longman.

Lovett, I. (2013) In California, a champion for police cameras. The New York Times. 21 August. [Online] http://www.nytimes.com/2013/08/22/us/in-california-a-champion-forpolice-cameras.html.

Lum, C. \& Nagin, D. (2017). Reinventing American policing. Crime and Justice, 46(1): 339-393.

Lum, C., Koper, C., Merola, L., Scherer, A. \& Reioux, A. (2015) Existing and Ongoing Body Worn Camera Research: Knowledge Gaps and Opportunities. Report for the Laura and John Arnold Foundation. Fairfax, VA: George Mason University.

Luna, E. (2000) Transparent policing. Iowa Law Review, 85(4): 1107-1194.

Lundman, R. (1980) Police and Policing: An Introduction. New York: Holt, Rinehart \& Winston.

Lynch, M., Omori, M., Roussell, A. \& Valasik, M. (2013) Policing the 'progressive' city: The racialized geography of drug enforcement. Theoretical Criminology, 17(3): 335-357.

Lynn, S., Cao, L. \& Horn, B. (1996) The influence of career stage on the work attitudes of male and female accounting professionals. Journal of Organizational Behavior, 17(2): 135-149.

Lyon, D. (1994) The Electronic Eye: The Rise of Surveillance Society. Cambridge, UK: Polity Press.

Lyon, D. (2001) Surveillance Society: Monitoring Everyday Life. Buckingham, UK: Open Univesity Press.

Lyon, D. (2002) Surveillance as social sorting: computer codes and mobile bodies. In (D. Lyon, ed.) Surveillance as Social Sorting: Privacy, Risk and Digital Discrimination: 13-30. London: Routledge.

Lyon, D. (2004) Globalizing surveillance: Comparative and sociological perspectives. International Sociology, 19(2): 135-149.

Lyon, D. (2006) The search for surveillance theories. In (D. Lyon, ed.) Theorizing Surveillance: The Panopticon and Beyond: 3-20. Portland, OR: Willan.

Lyon, D. (2007) Surveillance Studies: An Overview. Cambridge, UK: Polity.

Lyon, D. (2013) The Culture of Surveillance. Outstanding Contribution Award Lecture. Canadian Sociological Association Annual Meeting. University of Victoria, BC. 7 June. 
Lyon, D., Doyle, A. \& Lippert, R. (2012) Introduction. In (A. Doyle, R. Lippert \& D. Lyon, eds.) Eyes Everywhere: The Global Growth of Camera Surveillance: 1-19. New York: Routledge.

Lytle, D. (2014) The effects of suspect characteristics: A meta-analysis. Journal of Criminal Justice, 42(6): 589-597.

MacAlister, D. (2004) Canadian police subculture. In (S. Nancoo, ed.) Contemporary Issues in Canadian Policing. Toronto: Canadian Educators' Press.

MacDonald, H. (2005) Depolicing. In (L. Sullivan, M. Rosen. D. Schulz \& M. Haberfeld, eds.) Encyclopedia of Law Enforcement. Thousand Oaks, CA: Sage.

MacDonald, H. (2015) The new nationwide crime wave. The Wall Street Journal. 29 May. [Online] https://www-wsj-com./articles/the-new-nationwide-crime-wave1432938425.

MacDonald, H. (2016). Ferguson effect detractors are wrong. Quillette [Online]. https:/quillette.com/2016/03/21/ferguson-effect-detractors-are-wrong/.

Mack, D. \& Mack, F. (2018) Policing with impunity: Racialized policing in the $21^{\text {st }}$ century. In (S. Weissinger \& D. Mack, eds.) Law Enforcment in the Age of Black Lives Matter: Policing Black and Brown Bodies: 13-35. New York: Lexington Books.

Maclean's Editorial Staff (2015) The Editorial: Race relations in the U.S. are at their worst in recent memory. It's not too late for Obama to lead. Maclean's. 5 January: 5.

Madhani, A. (2016) Chicago's murder rate soars 72\% in 2016; shootings up more than 88\%. USA Today. 1 April. [Online] https:/www.usatoday.com/story/news/2016/04/01/ murders-shootings-soar-chicago-through-first-three-months-2016/82507210/.

Madhani, A. (2017) 'Ferguson effect': 72\% of U.S. cops reluctant to make stops. USA Today. 11 January. [Online] https://www.usatoday.com/story/news/2017/01/11/ ferguson-effect-study-72-us-cops-reluctant-make-stops/96446504.

Maguire, E., Nix, J. \& Campbell, B. (2017) A war on cops? The effects of Ferguson on the number of US police officers murdered in the line of duty. Justice Quarterly, 34(5): 739-758.

Mallin, A. (2015) White House Pushes Back on 'Ferguson Effect' Comments by Top Officials. $A B C$ News. 6 November. [Online] http://abcnews.go.com/Politics/whitehouse-pushes-back-ferguson-effect-comments-top/story?id=35032579.

Manikis, M. (2016) Towards accountability and fairness for Aboriginal people: The recognition of Gladue as a principle of fundamental justice that applies to prosecutors. Canadian Criminal Law Review, 21: 173- 194.

Manning, P. (1977) Police Work: The Social Organization of Policing. Cambridge, MA: MIT Press.

Manning, P. (1978) Rules, colleagues, and situationally justified actions. In (P. Manning \& J. Van Maanen, eds.) Policing: A View from the Street. Santa Monica, CA: Goodyear. 
Manning, P. (1988) Community policing as a drama of control. In (J. Greene \& S. Mastrofski, eds.) Community Policing: Rhetoric or Reality? 27-46. New York: Praeger.

Manning, P. (1989) Occupational culture. In (W. Bailey, ed.) The Encyclopaedia of Police Science. London: Garland.

Manning, P. (1993) Community-based policing. In (R. Dunham, \& G. Alpert, eds.) Critical Issues in Policing, $2^{\text {nd }}$ ed.: 421-431. Prospect Heights, IL, Waveland Press.

Manning, P. (1995) The police occupational culture in Anglo-American societies. In (W. Bailey, ed.) The Encyclopedia of Police Science: 472-475. London: Garland.

Manning, P. (1997) Police Work; The Social Organization of Policing, 2nd ed. Prospect Heights, IL: Waveland Press.

Manning, P. (2003) Policing Contingencies. Chicago: University of Chicago Press. Manning, P. (2005a) Impression management. In (G. Ritzer, ed.) The Encyclopedia of Social Theory: 397-399. Thousand Oaks, CA: Sage.

Manning, P. (2005b) The police mandate, strategies, and appearances. In (T. Newburn, ed.) Policing: Key Readings, 191-214. Cullompton: Willan.

Manning, P. (2005c) The study of policing. Police Quarterly, 8: 23-43.

Manning, P. (2007) A dialectic of organizational and occupational culture. In (M. O’Neill, M. Marks \& A. Singh, eds.) Police Occupational Culture: New Debates and Directions: 47-83. Oxford: Elsevier.

Manning, P. (2008a) Performance rituals. Policing: A Journal of Policy \& Practice, 2(3): 284-293.

Manning, P. (2008b) The Technology of Policing: Crime Mapping, Information Technology, and the Rationality of Crime Control. New York: New York University Press.

Manning, P. (2010) Democratic Policing in a Changing World. Boulder, CO: Paradigm Publishers.

Manning, P. (2014a) Ethnographies of policing. In (M. Reisig \& R. Kane, eds.) The Oxford Handbook of Police and Policing: 518-547. Oxford: Oxford University Press.

Manning, P. (2014b) Review of police culture: Themes and concepts. Howard Journal of Criminal Justice, 53(1): 111-112.

Manning, P. \& Van Maanen, J., eds. (1978) Policing: A View from the Street. Chicago: Goodyear Publishing Company.

Marier, C. \& Moule, R. (2018) Feeling blue: Officer perceptions of public antipathy predict police occupational norms. American Journal of Criminal Justice. DOI:

10.1007/s12103-018-9459-1. 
Marks, M. (2004) Researching police transformation: The ethnographic imperative. British Journal of Criminology, 44(6), 866-888.

Marks, M. (2007) Police unions and their influence: Subculture or counter-culture? In (M. O’Neill, M. Marks \& A. Singh, eds.) Police Occupational Culture: New Debates and Directions: 229-251. Oxford: Elsevier.

Marks, M., Wood, J., Ali, F., Walsh, T. \& Witbooi, A. (2010) Worlds apart? On the possibilities of police/academic collaborations. Policing, 4(2), 112-118.

Marks, P. (2013) Body-worn cameras put police evidence beyond doubt. New Scientist. 23 October. [Online] http://www.newscientist.com/article/mg22029404.400-bodyworncameras-put-police-evidence-beyond-doubt.html.

Marshall, G. (2005) Police accountability revisited. In (Newburn, T., ed.) Policing: Key Readings. New York: Routledge.

Martin, A., Rosamunde, E. \& Bernhard, D. (2009) Understanding resistance to digital surveillance: Towards a multi-disciplinary, multi-actor framework. Surveillance \& Society, 6(3): 213-232.

Martin, B. (2005) The beating of Rodney King: The dynamics of backfire. Critical Criminology, 13: 307-326.

Martin, D. (2013) The Politics of Sorrow: Families, Victims, and the MicroOrganization of Youth Homicide. Aldershot, UK: Ashgate.

Martin, D., Tong, S. et al. (2016) Conclusion: Challenges and changes in policing research. In (M. Brunger, S. Tong, D. Martin, et al., eds.) Introduction to Policing Research: Taking Lessons from Practice: 217-223. New York: Routledge.

Martin, K. (2017) Officer's case may affect gun trial. 17 February. Calgary Herald [Online] https://www.pressreader.com/canada/calgary-herald/20170217/28303834 9302895.

Martinussen, M., Richardsen, A. \& Burke, R. (2007) Job demands, job resources, and burnout among police officers. Journal of Criminal Justice, 35: 239-249.

Marx, G. (1985) The surveillance society: The threat of 1984 style techniques. Futurist, 19(3): 21-26.

Marx, G. (1988) Undercover: Police Surveillance in America. Berkeley: University of California Press.

Marx, G. (2002) What's new about the 'new surveillance'? Classifying for change and continuity. Surveillance \& Society, 1(1): 9-29.

Marx, G. (2003) A tack in the shoe: Neutralizing and resisting the new surveillance. Journal of Social Issues, 59(2): 369-390.

Marx, G. (2005) The new surveillance. In (T. Newburn, ed.) Policing: Key Readings, 761-785. Cullompton, UK: Willan. 
Marx, G. (2016) Windows into the Soul: Surveillance and Society in the Age of High Technology. Chicago: University of Chicago Press.

Maskaly, J. \& Donner, C. (2015) A theoretical integration of social learning theory with terror management theory: Towards an explanation of police shootings of unarmed suspects. American Journal of Criminal Justice, 40(2): 205-225.

Mason, J. \& Dale, A. (2011) Creative tensions in social research: Questions of method. In (J. Mason \& A. Dale, eds.) Understanding Social Research: Thinking Creatively about Method. London: Sage.

Mason, M. (2010) Sample size and saturation in Ph.D. studies using qualitative interviews. Forum: Qualitative Social Research, 11(3): n.p.

Mastrofski, S. (1993) Varieties of community policing. American Journal of Police, 12: 65-77.

Mastrofski, S. (1998) Community policing and police organization structure. In (J. Brodeur, ed.) How to Recognize Good Policing: Problems and Issues: 161-189. Thousand Oaks, CA: Sage.

Mastrofski, S. (2004) Controlling street-level police discretion. Annals of the American Academy of Political \& Social Science, 593(1): 100-118.

Mastrofski, S. (2012) Race, policing, and equity. Criminology \& Public Policy, 11(4): 593-600.

Mastrofski, S. (2019) Community policing: A skeptical view. In (D. Weisburd \& A. Braga, eds.) Police Innovation: Contrastic Perspectives, $2^{\text {nd }}$ ed.: 45-70. New York: Cambridge University Press.

Mastrofski, S. \& Greene, J. (1993) Community policing and the rule of law. In (D. Weisburd, C. Uchida, \& L. Green, eds.) Police Innovation and Control of the Police: 80-102. New York: Springer-Verlag.

Mastrofski, S. \& Ritti, R. (2000) Making sense of community policing: A theoretical perspective. Police Practice \& Research, 1(2): 183-210.

Mastrofski, S., Reisig, M. \& McCluskey, J. (2002) Police disrespect toward the public: An encounter-based analysis. Criminology, 40: 519-552.

Mastrofski, S., Snipes, J. \& Supina, A. (1996) Compliance on demand: The public's response to specific police requests. Journal of Research in Crime \& Delinquency 33: 269-305.

Mastrofski, S., Willis, J. \& Snipes, J. (2002) Styles of patrol in a community policing context. In (M. Morash \& J. Ford, eds.) The Move to Community Policing: 81-111. Thousand Oaks, CA: Sage.

Mastrofski, S., Worden, R. \& Snipes, J. (1995) Law enforcement in a time of community policing. Criminology, 33(4): 539-563. 
Mateescu, A., Rosenblat, A. \& Boyd, D. (2016) Dreams of accountability, guaranteed surveillance: The promises and costs of body-worn cameras. Surveillance \& Society, 14(1): 122-127.

Mathiesen, T. (1997) The viewer society: Michel Foucault's 'Panopticon' revisited. Theoretical Criminology, 1(2): 215-232.

Mauthner, N. \& Doucet, A. (2003) Reflexive account and accounts of reflexivity in qualitative research. Sociology, 37(3): 413-431.

Mawby, R. (2002) Policing Images: Policing, Communication, and Legitimacy. Cullompton, UK: Willan.

Mawby, R. (2010) Police corporate communications, crime reporting and the shaping of policing news. Policing \& Society, 20(1): 124-39.

Mawby, R. (2013) Approaches to comparative analysis: The impossibility of becoming an expert on everywhere. In (R. Mawby, ed.) Policing Across the World: Issues for the Twenty-First Century: 13-22. New York: Routledge.

Maxson, C., Hennigan, K. \& Sloane, D. (2003) Factors that influence public opinion of the police. National Institute of Justice: Research for Practice. Washington: National Institute of Justice.

Maxwell, J. \& Mittapalli, K. (2010) Realism as a stance for mixed methods research. In (A. Tashakkori \& C. Teddlie, eds.) The Sage Handbook of Mixed Methods in Social and Behavioral Research, $2^{\text {nd }}$ ed.: 145-167. Thousand Oaks, CA: Sage.

Maynard, R. (2017) Policing Black Lives: State Violence in Canada from Slavery to the Present. Halifax: Fernwood Publishing.

Mayor, L. (2016) Black Ontario teen cleared in encounter with police says officers should have body cameras. CBC News. 7 November. [Online] http://www.cbc.ca/ news/canada/jake-anderson-police-video-body-cameras-1.3836882.

McAdam, D. (2007) From relevance to irrelevance: The curious impact of the 1960s on public sociology. In (C. Calhoun, ed.) Sociology in America: A History: 411-426. Chicago: University of Chicago Press.

McCahill, M. \& Norris, C. (2002a) Analysing the Employment of CCTV in European Cities and Assessing its Social and Political Impacts. Berlin: Technical University of Berlin.

McCahill, M. \& Norris, C. (2002b) CCTV in London. Urban Eye Working Papers No 6. [Online] http://www.urbaneye.net/results/ue_wp6.pdf.

McCarthy, C. (2015) Loretta Lynch: No data to support 'Ferguson effect' policing theory. The Guardian. 17 November. [Online] https://www.theguardian.com/usnews/ 2015/nov/17/loretta-lynch-no-data-ferguson-effect-policing-theory.

McCarthy, J. (2014) Nonwhites less likely to feel police protect and serve them. Gallup. 17 November. [Online] https://news.gallup.com/poll/179468/nonwhites-less-likely-feelpolice-protect-serve.aspx. 
McCarthy, N. (2017) How much do U.S. cities spend every year on policing? Forbes Magazine. 7 August. [Online] https://www.forbes.com/sites/niallmccarthy/2017/08/07/ how-much-do-u-s-cities-spend-every-year-on-policing.

McCarty, W. \& Skogan, W. (2012) Job-related burnout among civilian and sworn police personnel. Police Quarterly, 16: 66-84.

McCarty, W. \& Lawrence, D. (2016) Coping, confidence, and change within the academy: A longitudinal look at police recruits. Police Practice \& Research, 17(3): 263-278.

McCarty, W., Zhao, J. \& Garland, B. (2007) Occupational stress and burnout between male and female police officers. Policing: An International Journal of Police Strategies \& Management, 30: 672-691.

McCluskey, J. \& Terrill, W. (2005) Department and citizen complaints as predictors of police coercion. Policing: An International Journal of Police Strategies \& Management, 28(3): 513-529.

McCluskey, J., Terrill, W. \& Paoline, E. (2005) Peer group aggressiveness and the use of coercion in police-suspect encounters. Police Practice \& Research, 6: 19-37.

McCluskey, J., Worden, R. \& McLean, S. (2014) Police legitimacy and police encounters. Encyclopedia of Criminology \& Criminal Justice: 3665-3675. New York: Springer.

McElroy, J., Morrow, P. \& Wardlow, T. (1999) A career stage analysis of police officer work commitment. Journal of Criminal Justice, 27(6): 507-516.

McElroy, J., Rodriguez, J., Griffin, G., Morrow, P. \& Wilson, M. (1993) Career stage, time spent on the road, and truckload driver attitudes. Transportation Journal, 33(1): 514.

McErlean, J. (2015) Dontay Ivy was killed by the system. Times Union. 13 November. [Online] http://www.timesunion.com/tuplus-opinion/article/Dontay-Ivy-was-killed-bythe-system-6630369.php.

McEvoy, P. \& Richards, D. (2006) A critical realist rationale for using a combination of quantitative and qualitative methods. Journal of Research in Nursing, 11(1): 66-78.

McGinnis, J. (1985) Career development in municipal policing. Canadian Police College Journal, 9: 254-294.

McGrath, J. (2012) Performing surveillance. In (K. Ball, K. Haggerty \& D. Lyon, eds.) Routledge Handbook of Surveillance Studies: 83-90. New York: Routledge.

McElroy, J., Morrow, P. \& Wardlow, T. (1999) A career stage analysis of police officer work commitment. Journal of Criminal Justice, 27(6): 507-516.

McLaughlin, E. (2007) The New Policing. London: Sage. 
McLaughlin, S. \& Prendergast, J. (2001). Police frustration brings slowdown: Arrests plummet from 2000. The Cincinnati Enquirer. 30 June. [Online] http://www.enquirer. $\mathrm{com} /$ editions/2001/06/30/ loc_police_frustration.html.

McNamara, J. (1967) Uncertainties in police work: The relevance of police recruits' background and training. In (D. Bordua, ed.) The Police: Six Sociological Essays: 163252. New York: John Wiley \& Sons.

McNamara, R. (1999) The socialization of the police. Police \& Policing: Contemporary Issues, 2: 1-12.

McNamara, R. \& Burns, R. (2008) Multiculturalism in the Criminal Justice System. New York: McGraw Hill.

McQuigge, M. (2017) Toronto cop buys clothes for alleged shoplifter who needed an outfit for job interview. Toronto Star. 8 August. [Online] https://www.thestar.com/ news/gta/2017/08/08/toronto-cop-buys-clothes-for-alleged-shoplifter-who-needed-anoutfit-for-job-interview.html.

Menifield, C., Shin, G. \& Strother, L. (2018) Do White law enforcement officers target minority suspects? Public Administration Review, 79(1): 56-68.

Mercer, J. (2007) The challenges of insider research in educational institutions: Wielding a double-edged sword and resolving delicate dilemmas. Oxford Review of Education, 33(1): 1-17.

Merton, R. (1972) Insiders and outsiders: A chapter in the sociology of knowledge. American Journal of Sociology, 78: 9-47.

Metro Vancouver (2017) Regional Services. [Online] http://www.metrovancouver. org/about/municipalities/Pages/default.aspx.

Meyer, R. (2015). The courage of bystanders who press 'record'. The Atlantic. 8 April. [Online] http:// www.theatlantic.com/technology/archive/2 015/04/the-courage-ofbystanders-who-pressrecord/389979.

Michalka, A. \& Maher, T. (2018) The Ferguson Effect: A Qualitative Study of Police Officers in the Epicenter of Change. Conference paper presented at the American Society of Criminology annual meeting, Atlanta, GA, 14 November.

Miles-Johnson, T. \& Pickering, S. (2018) Police recruits and perceptions of trust in diverse groups. Police Practice \& Research: An International Journal, 19(4): 311-328.

Miles, M. \& Huberman, A. (1994) Qualitative Data Analysis: An Expanded Sourcebook. Thousand Oaks, CA: Sage.

Miles, M., Huberman, A. \& Saldaña, J. (2014) Qualitative Data Analysis: A Methods Sourcebook, 3rd ed. Thousand Oaks, CA: Sage.

Miletich, S. (2014) Report cites plunge is SPD enforcement of low-level crime. Seattle Times. 14 May. [Online] https://seattletimes.com/seattle-news/report-cites-plunge-inspd-enforcement-of-low-level-crime. 
Miller, J. \& Davis, R. (2008) Unpacking public attitudes to the police: Contrasting perceptions of misconduct with traditional measures of satisfaction. International Journal of Police \& Management, 10(1): 9-22.

Miller, J., Davis, R., Henderson, N., Markovic, J. \& Ortiz, C. (2005) Measuring influences on public opinion of the police using time-series data: Results of a pilot study. Police Quarterly, 8(3): 394-401.

Miller, K. (2007) Racial profiling and postmodern society police responsiveness, image maintenance, and the left flank of police legitimacy. Journal of Contemporary Criminal Justice, 23(3): 248-262.

Miller, S. (2001) Cincinnati in grip of a crime wave months after riots: Profiling fears cited as shootings rise. The Washington Times. 18 July: A6.

Mills, C.W. (1959) The Sociological Imagination. New York: Oxford University Press.

Monahan, T. (2006) Counter-surveillance as political intervention? Social Semiotics, 16(4): 515-534.

Monahan, T. (2010) Surveillance as governance: Social inequality and the pursuit of democratic surveillance. In (K. Haggerty \& M. Samatas, eds.) Surveillance and Democracy: 91-110. New York: Routledge-Cavendish.

Monchalin, L. (2010) Canadian Aboriginal peoples victimization, offending, and its prevention: Gathering the evidence. Crime Prevention \& Community Safety: An International Journal, 12(2), 119-132.

Monchalin, L. (2016) The Colonial Problem: An Indigenous Perspective on Crime and Injustice in Canada. Toronto: University of Toronto Press.

Moon, B. \& Jonson, C. (2012) The influence of occupational strain on organizational commitment among police: A general strain theory approach. Journal of Criminal Justice, 40: 249-258.

Moore, M. \& Braga, A. (2003) The 'Bottom Line' of Policing: What Citizens Should Value (and Measure) in Police Performance. Washington: Police Executive Research Forum.

Mopas, M. \& Moore, D. (2012) Talking heads and bleeding hearts: Newsmaking, emotion and public criminology in the wake of a sexual assault. Critical Criminology, 20(2): 183-196.

Morabito, M. (2014) American police unions: A hindrance or help to innovation? International Journal of Public Administration, 37(11): 773-780.

Morash, M. \& Ford, J. (eds.) (2002) The Move to Community Policing. Thousand Oaks, CA: Sage.

Morash, M. \& Haarr, R. (1995) Gender, workplace problems and stress in policing. Justice Quarterly, 12(1): 113-135. 
Moretti, L. \& Ciotta, R. (2014) Man speaks out after alleged police brutality. WIVB News. 28 April. [Online] http://wivb.com/2014/04/28/man-speaks-out-after-allegedpolice-brutality/.

Morgan, D. (2007) Paradigms lost and pragmatism regained: Methodological implications of combining qualitative and quantitative methods. Journal of Mixed Methods Research, 1(1): 48-76.

Morgan, S. \& Pally, J. (2016) Ferguson, Gray, and Davis: An analysis of recorded crime incidents and arrests in Baltimore City, March 2010 through December 2015. A report written for the 21st Century Cities Initiative at Johns Hopkins University. [Online] http://socweb.soc.jhu.edu/faculty/morgan/papers/MorganPally2016.pdf.

Morin, R., Parker, K., Stepler, R. \& Mercer, A. (2017) Behind the Badge: Amid Protests and Calls for Reform, How Police View their Jobs, Key Issues and Recent Fatal Encounters between Blacks and Police. Washington, D.C.: The Pew Research Centre.

Morrow, P. \& McElroy, J. (1987) Work commitment and job satisfaction over three career stages. Journal of Vocational Behavior, 30: 330-346.

Morse, J. (1995) The significance of saturation. Qualitative Health Research, 5(3): 147149.

Morse, J. (1998) Designing funded qualitative research. In (N. Denzin \& Y. Lincoln, eds.) Strategies of Qualitative Inquiry: 56-85. Thousand Oaks, CA: Sage.

Morse, J. (2000) Determining sample size. Qualitative Health Research, 10(1): 3-5.

Mosher, C. \& Mahon-Haft, T. (2010) Race, crime, and criminal justice in Canada. In (A. Kalunta-Crumpton, ed.) Race, Crime and Criminal Justice: International Perspectives: 242-269. London: Palgrave MacMillan.

Mueller, B. \& Baker, A. (2014) 2 NYPD officers killed in Brooklyn ambush: Suspect commits suicide. The New York Times. 20 December. [Online] https://www.nytimes. com/2014/12/21/ nyregion/two-police-officers-shot-in-their-patrol-car-in-brooklyn. html.

Muir, W. (1977) Police: Streetcorner Politicians. Chicago: University of Chicago Press.

Muller, C. (2012) Northward migration and the rise of racial disparity in American incarceration, 1880-1950. American Journal of Sociology, 118(2): 281-326.

Munley, P. (1977) Erikson's theory of psychosocial development and career development. Journal of Vocational Behaviour, 10(3): 261-269.

Murakami, K. (2002) Racial profiling: A fine line. Seattle Post-Intelligencer. 29 April: A1.

Murphy, C. (2012) Canadian police and policing policy post-9/1. In (K. Ismaili, J. Sprott \& K. Varna, eds.) Canadian Criminal Justice Policy: Contemporary Perspectives: 5-29. New York: Oxford University Press. 
Murphy, C. \& McKenna, P. (2007) Rethinking Police Governance, Culture \& Management: Report Prepared for the Task Force on Governance and Cultural Change in the RCMP. Ottawa: Public Safety Canada.

Murphy, J. (2014) Beyond a Split-Second: An Exploratory Study of Police Use of Force and Use of Force Training in Canada. Unpublished MA thesis. Simon Fraser University.

Murphy, K., Hinds, L. \& Fleming, J. (2008) Encouraging public cooperation and support for the police. Policing \& Society, 18(2): 136-155.

Murray, K. \& Harkin, D. (2017) Policing in cool and hot climates: Legitimacy, power and the rise and fall of mass stop and search in Scotland. British Journal of Criminology, 57(4): 885-905.

Myers, S. (2011) How citizen journalism has changed since George Holliday's Rodney King video. Poynter Institute. 24 August. [Online] http://www.poynter.org/latestnews/top-stories/121687/how-citizen-journalism-has-changed-since-george-hollidaysrodney-king-video.

Myhill, A. \& Bradford, B. (2012) Can police enhance public confidence by improving quality of service? Results from two surveys in England and Wales. Policing \& Society, 22(4): 397-425.

Myhill, A. \& Bradford, B. (2013) Overcoming cop culture? Organizational justice and police officers' attitudes toward the public. Policing: An International Journal of Police Strategies \& Management, 36(2): 338-356.

Myhill, A. \& Quinton, P. (2011) It's a Fair Cop? Police Legitimacy, Public Cooperation and Crime Reduction: An Interpretive Evidence Commentary. London: National Policing Improvement Agency.

Mythen, G. (2014) Understanding the Risk Society: Crime, Security and Justice. London: Palgrave Macmillan.

Natapoff, A. (2006) Underenforcement. Fordham Law Review, 75(3): 1715-1776.

National Academies of Sciences, Engineering, and Medicine (2018) Proactive Policing: Effects on Crime and Communities. Washington, DC: The National Academies Press.

National Advisory Commission on Civil Disorders (1968) Report of the National Advisory Commission on Civil Disorders. Washington, DC: US Government Printing Office.

National Inquiry into Missing and Murdered Indigenous Women and Girls (2019) Our Women and Girls are Sacred. [Online] http://www.mmiwg-ffada.ca.

National Inquiry into Missing and Murdered Indigenous Women and Girls Legacy Archive (2018) Call for Expression of Interest. [Online] https://www.mmiwgffada.ca/wp-content/uploads/2018/08/Contest-bid-for-Legacy-Archive.pdf. 
National Post (2015) A violent end: Tracing Sammy Yatim's standoff with Toronto police. The National Post. 30 November. [Online] http://nationalpost.com/news/toronto/ a-violent-end-tracing-sammy-yatims-standoff-with-toronto-police.

National Post (2016) Man who successfully sued Ottawa police over racial profiling faces human trafficking charges. National Post. 1 November. [Online] http://nationalpost.com/ news/canada/man-who-successfully-sued-ottawa-police-overracial-profiling-faces-human-trafficking-charges.

National Post Editorial Board. (2012) Smashing the Cone of Silence. The National Post. 30 May. [Online] http://fullcomment.nationalpost .com/2012/05/30/national-posteditorial-board-smashing-the-cone-of-silence.

National Research Council (2004) Fairness and effectiveness in policing: The evidence. In (W. Skogan \& K. Frydl, eds.) Committee to Review Research on Police Policy and Practices. Washington: National Academies Press.

Nease, K. (2016) Ottawa police union president calls racism speculation in fatal arrest 'inappropriate'. CBC News. 26 July. [Online] http://www.cbc.ca/news/canada/ottawa/ matt-skof-abdirahman-abdi-amran-ali-1.3695349.

Neocleous, M. (2000) The Fabrication of Social Order: A Critical Theory of Police Power. Sterling, VA: Pluto Press.

Newburn, T. (2012) Police and crime commissioners: The Americanization of policing or a very British reform? International Journal of Law, Crime \& Justice, 40(1): 31-46.

Newburn, T. \& Hayman, S. (2001) Policing, Surveillance and Social Control: CCTV and Police Monitoring of Suspects. Cullompton, UK: Willan.

Newburn, T. and Reiner, R. (2012) Policing and the police. In (M. Maguire, R. Morgan $\&$ R. Reiner, eds.) The Oxford Handbook of Criminology, $5^{\text {th }}$ ed: 806-837. Oxford: Oxford University Press.

Newell, B. (2014) Crossing lenses: Policing's new visibility and the role of 'smartphone journalism' as a form of freedom-preserving reciprocal surveillance. Journal of Law, Technology \& Policy, 1: 59-104.

Newell, B. (2015) Transparent Lives and the Surveillance State: Policing, New Visibility, and the Information Policy. Unpublished $\mathrm{PhD}$ dissertation. University of Washington.

Newell, B. (2019) Context, visibility, and control: Police work and the contested objectivity of bystander video. New Media \& Society, 21(1): 60-76.

Newman, A. (2014) Updates on fatal shooting of two NYPD officers. The New York Times. 20 December. [Online] from http://cityroom.blogs.nytimes. com/2014/12/20/ brooklyn-police-shooting-live-blog/? $\mathrm{r}=0$.

Newport, F. (2014) Gallup review: Black and White attitudes toward police. Gallup. 20 August. [Online] https://news.gallup.com/poll/175088/gallup-review-black-whiteattitudes-toward-police.aspx. 
Neyland, D. \& Coopmans, C. (2014) Visual accountability. The Sociological Review, 62: $1-23$.

Neyroud, P. (2019) De-policing and homicide. Criminology \& Public Policy, 18(1): 8188.

Neyroud, P. \& Sherman, L. (2013) Dialogue and dialectic: Police legitimacy and the new professionalism. In (J. Tankebe \& A. Leibling, eds.) Legitimacy and Criminal Justice: An International Exploration: 293-307. Oxford: Oxford University Press.

Nhan, J. (2014) Police culture. In (J. Albanese, ed.) The Encyclopedia of Criminology and Criminal Justice. Wiley Online Library.

Nickel, P. (2010) Public Sociology and the public turn in the social sciences, Sociology Compass, 4(9): 694-704.

Niederhoffer, A. (1967) Behind the Shield: The Police in Urban Society. New York: Anchor Books.

Nix, J. \& Pickett, J. (2017) Third-person perceptions, hostile media effects, and policing: Developing a theoretical framework for assessing the Ferguson Effect. Journal of Criminal Justice, 5: 24-33.

Nix, J., Pickett, J., Baek, H. \& Alpert, G. (2019) Police research, officer surveys, and response rates, Policing \& Society, 29(5): 530-550.

Nix, J. \& Wolfe, S. (2016) Sensitivity to the Ferguson effect: The role of managerial organizational justice. Journal of Criminal Justice, 47: 12-20.

Nix, J. \& Wolfe, S. (2017) The impact of negative publicity on police self-legitimacy. Justice Quarterly, 34(1): 84-108.

Nix, J. \& Wolfe, S. (2018) Management-level officers' experiences with the Ferguson Effect. Policing, 41(2): 262-275.

Nix, J., Wolfe, S. \& Campbell, B. (2018) Command-level police officers' perceptions of the 'War on Cops' and de-policing. Justice Quarterly, 35(1): 33-54.

Nix, J., Wolfe, S., Rojek, J. \& Kaminski, R. (2014) Trust in the police: The influence of procedural justice and the perceived collective efficacy. Crime \& Delinquency, 60(3): 131.

Noaks, L. \& Wincup, E. (2006) Criminological Research: Understanding Qualitative Methods. London: Sage.

Noordin, F., Rahim, A., Ibrahim, A. \& Omar, M. (2011) Career stages and organizational commitment: A case of Malaysian managers. International Journal of Humanities and Social Science, 1(8): 105-112.

Norris, C. (1989) Avoiding trouble: The patrol officer's perception of encounters with the public. In (M. Weatheritt, ed.) Police Research: Some Future Prospects. Aldershot, UK: Avebury. 
Norris, C. \& Armstrong, G. (1999) The Maximum Surveillance Society: The Rise of $C C T V$. New York: Berg.

Norris, C., McCahill, M. \& Wood, D. (2002) The growth of CCTV: A global perspective on the international diffusion of video surveillance in publicly accessible space. Surveillance \& Society, 2/3: 110-135.

Norris, G. \& Armstrong, G. (1999) The Maximum Surveillance Society: The Rise of CCTV. Oxford: Berg Publishers.

Northey, M., Tepperman, L. \& Albanese, P. (2012) Making Sense: A Student's Guide to Research and Writing in Social Sciences. Oxford: Oxford University Press.

Novak, K., Cordner, G., Smith, B. \& Roberg, R. (2017) Police \& Society, $7^{\text {th }}$ ed. New York: Oxford University Press.

Novak, K., Smith, B. \& Frank, J. (2003) Strange bedfellows: Civil liability and aggressive policing. Policing: An International Journal of Police Strategies and Management, 26(2): 352-368.

O'Connel, B., Holzman, H. \& Armandi, B. (1986) Police cynicism and the modes of adaption. Journal of Police Science and Administration, 14: 307-313.

O'Donnell, E. (2018) Why cops are standing down all across America. New York Post. 30 May. [Online] https://nypost.com/2018/05/30/why-cops-are-standing-down-allacross-america/.

O'Malley, P. (2015) Revisiting the classics: 'Policing the Risk Society' in the twentyfirst century. Policing \& Society, 25(4): 426-431.

O'Malley, P. (2016) 'Policing the Risk Society' in the 21st Century. Sydney Law School Research Paper No. 16/11.

O’Malley, P., Johnston, L., Bachman, J. \& Schulenberg, J. (2000) A comparison of confidential versus anonymous survey procedures: Effects on reporting drug use and related attitudes and beliefs in a national survey of students. Journal of Drug Issues, 30(1): 35-54.

O'Neill, M. (2016) Revisiting the classics: Janet Chan and the legacy of 'Changing Police Culture'. Policing \& Society, 26(4): 475-480.

O'Neill, M. \& Singh, A. (2007) Introduction. In (M. O'Neill, M. Marks \& A. Singh, eds.) Police Occupational Culture: New Debates and Directions: 1-16. Oxford: Elsevier.

O'Reilly, N. (2017a) Hamilton police officer found guilty of assault. The Hamilton Spectator. 27 April. [Online] https://www.thespec.com/news-story/7262643-hamiltonpolice-officer-found-guilty-of-assault/.

O'Reilly, N. (2017b) Suspended Hamilton officer arrested by Toronto police is facing more criminal charges. Toronto Star. 22 August. [Online] https://www.thestar.com/ news/gta/2017/08/22/suspended-hamilton-police-officer-arrested-by-toronto-police-isfacing-more-criminal-charges.html. 
Oberfield, Z. (2012) Socialization and self-selection: How police develop their views about using force. Administration \& Society, 44(6): 702-730.

Oberfield, Z. (2014) Motivation, change, and stability: Findings from an urban police department. The American Review of Public Administration, 44: 210-232.

Obst, P. \& Davey, J. (2003) Does the police academy change your life? A longitudinal study of changes in socializing of police recruits. International Journal of Police Science \& Management, 5(1): 31-40.

Office of the Press Secretary (2014) Strengthening community policing. The White House. 1 December [Online] http://www.whitehouse.gov/the-press-office/2014/12/01/ fact-sheet-strengthening-community-policing.

Ogletree, C., Prosser, M., Smith, A. \& Talley, W. (1995) Beyond the Rodney King Story: An Investigation of Police Misconduct in Minority Communities. Boston: Northeastern University Press.

Oliver, W. (2017) Depolicing: Rhetoric or reality? Criminal Justice Policy Review, 28(5): 437-461.

Olson, K. (2011) Essentials of Qualitative Interviewing. Walnut Creek, CA: Left Coast Press.

Ong, A. \& Weiss, D. (2000) The impact of anonymity on responses to sensitive questions. Journal of Applied Social Psychology, 30: 1691-1708.

Ontario Today (2017) When police pull back: De-policing and the 'Ferguson Effect'. CBC News. 18 July. [Online] http://www.cbc.ca/player/play/1002630211890.

Onwuegbuzie, A. \& Teddlie, C. (2003) A framework for analyzing data in mixed methods research. In (A. Tashakkori \& C. Teddlie, eds.) The Sage Handbook of Mixed Methods in Social and Behavioural Research: 351-383. Thousand Oaks, CA: Sage.

Ornstein, S., Cron, W. \& Slocum, J. (1989) Life stage versus career stage: A comparative test of the theories of Levinson and Super. Journal of Organizational Behavior, 10(2): 117-133.

Oxford University Press. (2014) Oxford English Dictionary. [Online] http://www.oed. com.proxy.library.carleton.ca.

Owens, E. (2019) Economic approach to 'de-policing'. Criminology \& Public Policy, 18(1): 77-80.

Owusa-Bempah, A., Kanter, S., Druyts, E., Toor, K., Muldoon, K., Farquhar, J. \& Mills, E. (2015) Years of life lost to incarceration: Inequities between Aboriginal and non-Aboriginal Canadians. BMC Public Health, 14(1): 585-591.

Owusu-Bempah, A. \& Morgan, A. (2018) A hard truth: Canada's policing style is very similar to the U.S. The Globe \& Mail. 16 May. [Online] https://www.theglobeandmail. com/opinion/a-hard-truth-canadas-policing-style-is-very-similar-to-the us/article 30942053 . 
Pagliaro, J. (2013) Sammy Yatim: Timeline of events after teen shot dead. The Toronto Star. 19 August [Online] https://www.thestar.com/news/crime/2013/08/19/sammy_ yatim_timeline_of_events_after_teen_shot_dead.html.

Pajnik, M. (2015) Changing citizenship: Practicing (alternative) politics. The Routledge Companion to Alternative and Community Media: 113-121. London: Routledge.

Paoline, E. (2000) Attitudes of Police: Implications for Police Culture. Unpublished $\mathrm{PhD}$ dissertation, State University of New York at Albany.

Paoline, E. (2001) Rethinking Police Culture: Officers' Occupational Attitudes. New York: LFB Publishing.

Paoline, E. (2003) Taking stock: Toward a richer understanding of police culture. Journal of Criminal Justice, 31: 199-214.

Paoline, E. (2004) Shedding light on police culture: An examination of officers' occupational attitudes. Police Quarterly, 7(2): 205-236.

Paoline, E. \& Gau, J. (2018). Police occupational culture: Testing the monolithic model. Justice Quarterly, 35(4), 670-698.

Paoline, E., Myers, S. \& Worden, R. (2000) Police culture, individualism, and community policing: Evidence from two police departments. Justice Quarterly, 17: 575605 .

Paoline, E. \& Terrill, W. (2005) The impact of police culture on traffic stop searches: An analysis of attitudes and behavior. Policing: An International Journal of Police Strategies \& Management, 28(3): 455-472.

Paoline, E. \& Terrill, W. (2014) Police Culture: Adapting to the Strains of the Job. Durham, NC: Carolina Academy Press.

Paperman, P. (2003) Surveillance underground: The uniform as an interaction device. Ethnography, 4(3): 397-419.

Parnaby, P. \& Leyden, M. (2011) Dirty Harry and the station queens: A Mertonian analysis of police deviance. Policing \& Society 21(3): 249-264.

Parsons, C. \& Hennessey, K. (2014) Obama seeks more police training, avoids addressing Ferguson head-on. Los Angeles Times. 1 December: A1.

Parsons, T. (1938) The role of theory in social research. American Sociological Review, 3(1): 13-20.

Paternoster, R. (2010) How Much Do We Really Know about Criminal Deterrence? Journal of Criminal Law \& Criminology, 11(3): 765-823.

Paternoster, R., Brame, R., Bachman, R. \& Sherman, L. (1997) Do fair procedures matter? The effect of procedural justice on spouse assault. Law \& Society Review, 31(1): 163-204. 
Patten, C., Hayes, M., Lynch, G., O’Toole, K., Shearing, C. et al. (1999) A New Beginning: Policing in Northern Ireland. Norwich, UK: Independent Commission on Policing for Northern Ireland.

Patterson, G., Chung, I. \& Swan, P. (2012) The effects of stress management interventions among police officers and recruits. Campbell Systematic Reviews, 8(7): 154.

Patton, M. (2002) Qualitative Research and Evaluation Methods, $3^{\text {rd }}$ ed. Thousand Oaks, CA: Sage.

Payne, S. 1980. The Art of Asking Questions. Princeton, NJ: Princeton University Press.

Peak, K. (1990) A research note on successful criminal justice survey research: A 'personal touch' model for enhancing rates of return. Criminal Justice Policy Review, 4(3): 268-277.

Peak, K. (1997) Policing America: Methods, Issues, Challenges. Upper Saddle River, NJ: Prentice-Hall.

Perez, D. and Shtull, P. (2002) Police research and practice: An American perspective. Police Practice \& Research, 3(3): 169-187.

Perkel, C. (2013) Police interactions with public should be on video, outgoing SIU head says. The Globe \& Mail. 1 October. http://www.theglobeandmail.com/news/national/ police-interactions-with-public-should-be-recorded-outgoing-siu-headsays/article $14628173 /$.

Perrott, S. (2018) Progress towards more culturally and gender-sensitive policing: Perceptions from a Canadian sample of police officers. The Police Journal: Theory, Practice \& Principles, 91(3): 224-238.

Peytchev, A. (2013) Consequences of survey nonresponse. The Annals of the American Academy of Political and Social Science, 645(1): 88-111.

Phillips, S. (2010) Police officers' opinions on the use of unnecessary force by other officers. Police Practice \& Research, 11(4): 197-210.

Phillips, S., Sobol, J. \& Varano, S. (2010) Work attitudes of police recruits: Is there a family connection? International Journal of Police Science \& Management, 12(3): 460479.

Pickering, J. \& Klinger, D. (2016) Enhancing police legitimacy by promoting safety culture. In (M. Deflem, ed.) Sociology of Crime, Law and Deviance. The Politics of Policing: Between Force and Legitimacy: 21-39. London: Emerald.

Pickett, J. (2017) Methodological myths and the role of appeals in criminal justice journals: The case of response rates. ACJS Today, 41(3): 61-69.

Pickett, J., Baker, T., Metcalfe, C., Gertz, M. \& Bellandi, R. (2014) Contact and compromise: Explaining support for conciliatory measures in the context of violent intergroup conflict. Journal of Research in Crime and Delinquency, 51(5): 585-619. 
Pickett, J. \& Ryon, S. (2017) Procedurally just cooperation: Explaining support for due process reforms in policing. Journal of Criminal Justice, 48: 9-20.

Pickler, N. (2014a) Obama proposes body-worn cameras for police. Associated Press. 1 December [Online] http://www.pbs.org/newshour/rundown/obama-proposes-bodyworn-cameras-police/.

Pickler, N. (2014b) Obama wants to avoide 'militarized' police culture. Associated Press. 2 December. [Online] http://abcnews.go.com/Politics/wireStory/obama -holdwhite-house-meetings-ferguson-27272768.

Pieterman, R. (2001) Culture in the risk society: An essay on the rise of a precautionary culture. Zeitschrift fur Rechtsoziologie, 22(2): 145-168.

Piquero, N. (2005) Understanding police stress and coping resources across gender: A look toward general strain theory. In (H. Copes, ed.) Policing and Stress: 126-139. Upper Saddle River, NJ: Prentice Hall.

Pogarsky, G. \& Piquero, A. (2004) Studying the reach of deterrence: Can deterrence theory help explain police misconduct? Journal of Criminal Justice, 32(4): 371-386.

Pogrebin, M., ed. (2003) Qualitative Approaches to Criminal Justice: Perspectives from the Field. Thousand Oaks, CA: Sage.

Pogson, C., Cober, A., Doverspike, D. \& Rogers, J. (2003) Differences in self-reported work ethic across three career stages. Journal of Vocational Behavior, 62(1): 189-201.

Pollock, J. (2010) Ethics and law enforcement. In (R. Dunham \& G. Alpert, eds.) Critical Issues in Policing: Contemporary Readings, $6^{\text {th }}$ ed.: 313-342. Long Grove, Ill: Waveland Press.

Pomper, S. (2018) De-Policing America: A Street Cop's View of the Anti-Police State. New York: Post Hill Press.

Porta, D. \& Keating, M. (2008) Approaches and Methodologies in the Social Sciences. Cambridge, UK: Cambridge University Press.

Porter, A. (2010) Tories want 'in your face' street policing: Election 2010 justice shakeup would stop early release. The Daily Telegraph. 5 April: 1.

Porter, L. \& Prenzler, T. (2016) The code of silence and ethical perceptions: Exploring police officer unwillingness to report misconduct. Policing: An International Journal of Police Strategies \& Management, 39(2): 370-386.

Potere, M. (2011) Who will watch the watchers? Citizens recording police conduct. Northwestern University Law Review, 106(1): 273-316.

Prat, A. (2006) The more closely we are watched, the better we behave. In (C. Hood \& D. Heald, eds.) Transparency: The Key to Better Governance? 91-103. Oxford: Oxford University Press.

President's Task Force on 21st Century Policing (2015a) Final Report. Washington, DC: Office of Community Oriented Policing Services. 
President's Task Force on 21st Century Policing (2015b) Interim Report. Washington, DC: Office of Community Oriented Policing Services.

Proctor, J. (2015) Delta Const. Jordan MacWilliams' police colleagues supported stay of murder charge. CBC News. 14 July. http://www.cbc.ca/news/canada/british-columbia/ delta-const-jordan-macwilliams-police-colleagues-supported-stay-of-murder-charge1.3151816 .

Punch M. (1979) Policing the Inner City. London: Macmillan.

Punch, M. (1983) Officers and men: Occupational culture, inter-rank antagonism and the investigation of corruption. In (M. Punch, ed.) Control in the Police Organization. Cambridge, MA: MIT Press.

Punch, M. (1989) Researching police deviance: A personal encounter with the limitations and liabilities of field work. British Journal of Sociology, 40(2): 177-204.

Punch, M. (2003) Rotten orchards: 'Pestilence', police misconduct and system failure. Policing \& Society, 13(2): 171-196.

Punch, M. (2007) Cops with honours: University education and police culture. In (M. O’Neill, M. Marks \& A. Singh, eds.) Police Occupational Culture: New Debates and Directions: 105-128. Oxford: Elsevier.

Punch, M. (2009) Police Corruption, Deviance, Accountability and Reform in Policing. Cullompton, UK: Willan.

Pyrooz, D., Decker, S., Wolfe, S. \& Shjarback, J. (2016) Was there a Ferguson Effect on crime rates in large US cities? Journal of Criminal Justice, 46: 1-8.

Queally, J., Mather, K. \& Chang, C. (2017) Police arrests are plummeting across California, fueling alarm and questions. Los Angeles Times. 1 April. [Online] http://www.latimes.com/local/lanow/la-me-In-police-slowdown-20170401-story.html.

Rabinowitz, S. \& Hall, D. (1981) Changing concepts of job involvement in three career stages. Journal of Vocational Behavior, 18: 128-144.

Raganella, A. \& White, M. (2004) Race, gender, and motivation for becoming a police officer: Implications for building a representative police department. Journal of Criminal Justice, 32(6): 501-513

Ratcliffe, J., Taniguchi, T., Groff, E. \& Wood, J. (2011) The Philadelphia foot patrol experiment: A randomized controlled trial of police patrol effectiveness in violent crime hotspots. Criminology, 49(3): 795-831.

RCMP (2014) Missing and Murdered Aboriginal Women: A National Operational Overview. Ottawa: RCMP.

Rea, L. \& Parker, R. (1992) Designing and Conducting Survey Research: A Comprehensive Guide. San Francisco: Josey-Bass. 
Ready, J. \& Young, J. (2015) The impact of on-officer video cameras on police-citizen contacts: Findings from a controlled experiment in Mesa, AZ. Journal of Experimental Criminology, 11(3): 445-458.

Reasons, C., Hassan, S., Ma, M., Monchalin, L., Bige, M., Paras, C. \& Arora, S. (2016) Race and criminal justice in Canada. International Journal of Criminal Justice Sciences, 11(2): 75-99.

Redline, C. \& Dillman, D. (2002) The influence of alternative visual designs on respondents' performances with branching instructions in self-administered questionnaires. In (R. Groves, D. Dillman, J Eltinge \& R. Little, eds.) Survey Nonresponse. New York: Wiley.

Reed, W. (2018) Police use force: Practices, policies, and the law. In (S. Weissinger \& D. Mack, eds.) Law Enforcment in the Age of Black Lives Matter: Policing Black and Brown Bodies: 37-51. New York: Lexington Books.

Regoli, R. (1977) Police in America. Washington, DC: University Press of America.

Regoli, R., Crank, J. \& Rivera, G. (1990) The construction and implementation of an alternative measure of police cynicism. Criminal Justice \& Behavior, 17(4): 395-409.

Reilly, R. (2015) Eric Holder weighs in on Black Lives Matter and the 'Ferguson effect.' Huffington Post. 28 October. [Online] https://www.huffingtonpost.ca/entry/ericholder- black-lives-matter-ferguson-effect_us_56312137e4b00aa54a4c6681.

Reiman, J. (1985) The social contract and the police use of deadly force. In (F. Ellsion \& M. Feldberg, eds.) Moral Issues in Police Work. Savage, MD: Rowman \& Littlefield.

Reiner, R. (1978) The Blue-Coated Worker: A Sociological Study of Police Unionism. Cambridge, UK: Cambridge University Press.

Reiner, R. (1985) The Politics of the Police, 1st ed. Brighton, UK: Wheatsheaf.

Reiner, R. (1992a) Policing a postmodern society. Modern Law Review, 55(6): 761-781.

Reiner, R. (1992b) The Politics of the Police, 2nd ed. London: Harvester Wheatsheaf.

Reiner, R. (2000a) Police research. In: Roy D. King, and Emma Wincup, ed. Doing research on crime and justice. New York: Oxford University Press, 205-235.

Reiner, R. (2000b) The Politics of the Police, 3rd ed. Oxford: Oxford University Press.

Reiner, R. (2006) Out of the blue: The shifting contours and causes of the politicisation of policing. London School of Economics: Working Paper.

Reiner, R. (2010) The Politics of the Police, 4th ed. Oxford: Oxford University Press.

Reiner, R. (2012) In Praise of Fire Brigade Policing: Contra Common Sense Conceptions of the Police Role. London: The Howard League for Penal Reform.

Reiner, R. (2015) Revisiting the classics: Three seminal founders of the study of policing: Michael Banton, Jerome Skolnick and Egon Bittner. Policing \& Society, 25(3): 308-327. 
Reiner, R. (2017) Is police culture cultural? Policing: A Journal of Policy and Practice, 11(3): 236-241.

Reisig, M. \& Parks, R. (2000) Experience, quality of life, and neighbourhood context: A hierarchical analysis of satisfaction with the police. Justice Quarterly, 17(3): 607-630.

Reiss, A. (1968) Police brutality: Answers to key questions. Trans-Action, 5(8): 10-19.

Reiss, A. (1971) The Police and the Public. New Haven, CT: Yale University Press.

Reiss, A. (1983) The policing of organizational life. In (M. Punch, ed.) Control in the Police Organization: 78-97. Cambridge, MA: MIT Press.

Reiss, A. (1992) Police organizations in the twentieth century. In (M. Tonry \& N. Morris, eds.) Modern Policing: 51-97. Chicago: University of Chicago Press.

Reiss, A. \& Bordua, D. (1967) Environment and organization. In (D. Bordua, ed.) The Police: Six Sociological Essays. New York: Wiley.

Reuss-Ianni, E. (1983) Two Cultures of Policing: Street Cops and Management Cops. New Haven, CT: Yale University Press.

Reuss-Ianni, E. \& Ianni, F. (2005) Street cops and management cops: The two cultures of policing. In (T. Newburn, ed.) Policing: Key Readings, 297-314. Cullompton: Willan.

Reuters (2016a) FBI Director James Comey links 'Viral Video Effect' to spike in crime rates. Reuters/Vice News. 12 May. [Online] https://news.vice.com/article/fbi-directorjames-comey-links-viral-video-effect-to-spike-in-crime-rates.

Reuters (2016b) Hundreds in Canada protest against police brutality after death of black man. The Guardian. 31 July. [Online] https://www.theguardian.com/world/2016/jul/31/ hundreds-in-canada-protest-against-police-brutality-after-death-of-black-man.

Reynolds, P., Fitzgerald, B. \& Hicks, J. (2018) The expendables: A qualitative study of police officers' responses to organizational injustice. Police Quarterly, 21(1): 3-29.

Reynolds, P. \& Helfers, R. (2018) Differences in perceptions of organizational fairness based on job characteristics among police officers. American Journal of Criminal Justice, 43(2): 371-388.

Rice, S. \& Piquero, A. (2005) Perceptions of discrimination and justice in New York City. Policing: An International Journal of Police Strategies and Management, 28(1): 98-117.

Rice, S. \& White, M., eds. (2010) Race, Ethnicity, and Policing: New and Essential Readings. New York: New York University Press.

Rigakos, G. \& Frauley, J. (2011) The promise of critical realism: Toward a postempiricist criminology. In (A. Doyle \& D. Moore, eds.) Critical Criminology in Canada: New Voices, New Directions: 243-268. Vancouver: UBC Press.

Riksheim, E. \& Chermak, S. (1993) Causes of police behavior revisited. Journal of Criminal Justice, 21: 353-382. 
Risk and Regulation Advisory Council (2009) Response with Responsibility: PolicyMaking for Public Risk in the 21st Century. London: Department for Business, Enterprise and Regulatory Reform.

Ritchie, J., Lewis, J. \& Elam, G. (2003) Designing and selecting samples. In (J. Ritchie $\&$ J. Lewis, eds.) Qualitative Research Practice. A Guide for Social Science Students and Researchers: 77-108. Thousand Oaks, CA: Sage.

Roberg, R., Novak, K., Cordner, G. \& Smith, B. (2015) Police and Society, 6th ed. New York: Oxford University Press.

Roberts, J. \& Hough, M. (2005) Understanding Public Attitudes to Criminal Justice. Maidenhead, UK: Open University Press.

Robinette, H. (1987) Burnout in Blue: Managing the Police Marginal Performer. New York: Praeger.

Robinson, D. (2012) Bad footage: Surveillance laws, police misconduct, and the Internet. Georgetown Law Journal, 100: 1399-1435.

Robson, C. (2011) Real World Research: A Resource for Social Scientists and Practitioner-Researchers, $3^{\text {rd }}$ ed. London: Wiley.

Roche, S. (2017) Cops and Cells: Theorizing and Assessing the Implications of Smartphone Surveillance for Policing. Unpublished PhD dissertation. State University of New York at Albany.

Rojek, J., Decker, S. \& Wagner, A. (2010) Addressing police misconduct: The role of citizen complaints. In (R. Dunham \& G. Alpert, eds.) Critical Issues in Policing: Contemporary Readings, $6^{\text {th }}$ ed.: 292-312. Long Grove, Ill: Waveland Press.

Rojek, J., Rosenfeld, R., \& Decker, S. (2004) The influence of driver's race on traffic stops in Missouri. Police Quarterly, 7: 126-147.

Rose, T. \& Unnithan, P. (2015) In or out of the group? Police subculture and occupational stress. Policing: An International Journal of Police Strategies \& Management, 38(2): 279-294.

Rosenbaum, D., ed. (1996) Community Policing. Thousand Oaks, CA: Sage.

Rosenbaum, D. (1998) The changing role of the police: Assessing the current transition to community policing. In (J. Brodeur, ed.) How to Recognize Good Policing: Problems and Issues: 3-29. Thousand Oaks, CA: Sage.

Rosenbaum, D. (2006) The limits of hot spots policing. In (D. Weisburd \& A. Braga, eds.) Police Innovation: Contrasting Perspectives: 245-266. New York: Cambridge University Press.

Rosenbaum, D. (2010) Police research: Merging the policy and action research traditions. Police Practice \& Research, 11(2): 144-49.

Rosenbaum, D. (2015) Welcome from the Chair. American Society of CriminologyDivision of Policing News, 1(1): 1. 
Rosenbaum, D. (2019) The limits of hot spots policing. In (D. Weisburd \& A. Braga, eds.) Police Innovation: Contrastic Perspectives, $2^{\text {nd }}$ ed.: $314-346$. New York: Cambridge University Press.

Rosenbaum, D., Schuck, A., Costello, S., Hawkins, D. \& Ring, M. (2005) Attitudes toward the police: The effects of direct and vicarious experience. Police Quarterly, 8(3): 343-365.

Rosenfeld, R. (2015) Was there a 'Ferguson Effect' on Crime in St. Louis? Washington, DC: Sentencing Project.

Rosenfeld, R. (2016) Documenting and Explaining the 2015 Homicide Rise: Research Directions. Washington, DC: U.S. Department of Justice.

Rosenfeld, R. (2018) The 2017 Sutherland address. Studying crime trends: Normal science and exogenous shocks. Criminology, 56: 5-26.

Rosenfeld, R., Fornango, R. \& Rengifo, A. (2007) The impact of order maintenance policing on New York City robbery and homicide rates: 1988-2001. Criminology, 45(2): 355-384.

Rosenfeld, R. \& Wallman, J. (2019) Did de-policing cause the increase in homicide rates? Criminology \& Public Policy, 18(1): 51-75.

Ross, C. (2015) A multi level Bayesian analysis of racial bias in police shootings at the county level in the United States, 2011-2014. PLOS ONE 10(11): 1-34

Ross, J. (2000) Making News of Police Violence: A Comparative Study of Toronto and New York City. Westport, CT: Praeger.

Rossman, G. \& Wilson, B. (1985) Numbers and words: Combining quantitative and qualitative methods in a single large-scale evaluation study. Evaluation Review, 9: 627643.

Rothe, D., Ross, J., Mullins, C., Friedrichs, D., Michalowski, R., Barak, G., Kauzlarich, D. \& Kramer, R. (2009) That was then, this is now, what about tomorrow? Future directions in state crime studies. Critical Criminology, 17(1): 3-13.

Roziere, B. \& Walby, K. (2019) Special weapons and tactics teams in Canadian policing: Legal, institutional, and economic dimensions. Policing \& Society, doi.org/10.1080/ 10439463.2019.1586899.

Rubenstein, J. (1973) City Police. New York: Farrar, Straus \& Giroux.

Rumbaut, R. \& Bittner, E. (1979) Changing conceptions of the police role: A sociological review. In (N. Morris \& M. Tonry, eds.) Crime and Justice: An Annual Review of Research: 239-288. Chicago: University of Chicago Press.

Rushin, S. \& Edwards, G. (2017) De-policing. Cornell Law Review, 102(3): 721-782.

Rutgers University (2003) Table/graph Formation in Interpretation. [Online] http://sociology.camden.rutgers.edu/curriculum/format.html. 
Rynes, S., Bartunek, J. \& Daft, R. (2001) Across the great divide: Knowledge creation and transfer between practitioners and academics. Academy of management Journal, 44(2): 340-355.

Sacheli, S. (2011) Windsor police chief quits amid officer brutality allegations. The National Post. 23 December. [Online] http://nationalpost.com/news/canada/windsorpolice-chief-quits-amid-officer-brutality-allegations.

Saldaña, J. (2013) The Coding Manual for Qualitative Researchers, 2nd ed. Los Angeles: Sage.

Sanchez, R. (2015) Chicago mayor: Anti-police backlash makes officers 'fetal'. CNN. 16 October. [Online] https:/www.cnn.com/2015/10/16/us/rahm-emanuel-policecomments/index.html.

Sandelowski, (1995) Sample size in qualitative research. Research in Nursing and Health, 18: 179-183.

Sandhu, A. (2016) Camera-friendly policing: How the police respond to cameras and photographers. Surveillance \& Society, 14(1): 78-89.

Sandhu, A. (2019) 'I'm glad that was on camera': A case study of police officers' perceptions of cameras. Policing \& Society, 29(2): 223-235.

Sandhu, A. \& Haggerty K. (2015) High-visibility policing: Policing on camera and the crisis of police legitimacy. Oxford Handbooks Online. [Online] http://www.oxfordhand books. com/view/10.1093/oxfordhb/9780199935383.001.0001/oxfordhb978019993538 -e-80.

Sandhu, A. \& Haggerty, K. (2017) Policing on camera. Theoretical Criminology, 21(1): 78-95.

Santori, M. (2014) Mayor de Blasio announces retraining of New York Police. The New York Times. 4 December. [Online] http://www.nytimes.com/2014/12 /05/nyregion/ mayor-bill-de-blasio-retraining-new-york-police-dept-eric-garner.html? _r=0.

Santos, F. (2007) Inquiry into police opens old wounds in Yonkers. The New York Times. 31 August. [Online] http://www.nytimes.com/2007/03/31/yonkers.html?_r=0.

Sato, M. (2003) Police recruits' training and the socialization process: From the network perspective. The Police Journal, 76(4): 289-303.

Satzewich, V.\& Shaffir, W. (2009) Racism versus professionalism: Claims and counter-claims about racial profiling. Canadian Journal of Criminology \& Criminal Justice, 51(2): 199-226.

Sauter, M., Stebbins, S. \& Comen, E. (2017) 50 Worst American Cities to Live In. 24/7 Wall Street. 16 June. [Online] http://247wallst.com/special-report/2017/06/16/50-worstcities-to-live-in/7/.

Savage, L. (2014) America can't breathe: Why a new racial crisis has exploded despite big gains under Barack Obama. Maclean's, 127(51): 16-17. 
Savage, S. (2007) Police Reform: Forces for Change. Oxford: Oxford University Press.

Savage, S. (2013) Seeking 'civilianness': Police complaints and the civilian control model of oversight', British Journal of Criminology, 53: 886-904.

Sawchuck, B. (2014) Officer convicted of assault pleads guilty to police act charges. St. Catharines Standard. 4 February. [Online] http://www.stcatharinesstandard.ca/ 2014/02/04/officer-convicted-of-assault-pleads-guilty-to-police-act-charges.

Sawchuck, B. (2016) Fort Erie man suing police, town and regional officials. St. Catharines Standard. 13 January. [Online] http://www.stcatharinesstandard.ca /2016/01/13/fort-erie-man-suing-police-town-and-regional-officials.

Schaefer-Morabito, M. (2014) Policing vulnerable populations. In (M. Reisig \& R. Kane, eds.) The Oxford Handbook of Police and Policing: 195-213. Oxford: Oxford University Press.

Schaefer, B. \& Steinmetz, K. (2014) Watching the watchers and McLuhan's tetrad: The limits of cop-watching in the Internet age. Surveillance \& Society, 12(4): 502-515.

Schaefer, Z. (2012) Secretly recording the police: The confluence of communication, culture, and technology in the public sphere. Communication Teacher, 26: 199-202.

Schaible, L. \& Gecas, V. (2010) The impact of emotional labor and value dissonance on burnout among police officers. Police Quarterly, 13: 316-341.

Schein, E. (1978). Career Dynamics. Reading, MA: Addison-Wesley.

Schein, E. (1996) Career anchors revisited: Implications for career development in the 21 st century. Academy of Management Executives, 10(4): 80-89.

Schein, E. (2010) Organizational Culture and Leadership, 4th ed. San Francisco: Jossey-Bass.

Schmidt, M. \& Apuzzo, M. (2015) F.B.I. Chief links scrutiny of police with rise in violent crime. The New York Times. 23 October, A1.

Schneider, C. (2015) Police 'image work' in an era of social media: YouTube and the 2007 Montebello Summit protest. In (D. Trottier \& C. Fuchs, eds.) Social Media, Politics and the State: Protests, Revolutions, Riots, Crime and Policing in the Age of Facebook, Twitter and YouTube: 227-246. New York: Routledge.

Schneider, C. (2016) Policing and Social Media: Social Control in an Era of New Media. Lanham, MD: Lexington Books

Schram, J., Conley, K. \& Feuerherd, B. (2014) Email circulated among cops: 2 cars respond to every call. The New York Post. 21 December. [Online] http://nypost.com/ 2014/12/21/email-circulated-among-cops-2-cars-respond-to-every-call/.

Schulenberg, J. (2007) Analyzing police decision-making: Assessing the application of a mixed-method/mixed-model research design. International Journal of Social Research Methodology, 10(2): 99-119. 
Schulenberg, J. (2014) Systematic social observation of police decision-making: The process, logistics, and challenges in a Canadian context. Quality \& Quantity, 48(1): 297-315.

Schulenberg, J. (2016) The Dynamics of Criminological Research. Oxford: Oxford University Press.

Schwartz, L. (2009). Mechanical Witness: A History of Motion Picture Evidence in U.S. Courts. New York: Oxford University Press.

Scollon, R., Scollon, S. \& Jones, R. (2011) Intercultural Communication: A Discourse Approach, $3^{\text {rd }}$ ed. London: Wiley.

Scott, C. and Medaugh, M. (2017) Axial coding. The International Encyclopedia of Communication Research Methods. DOI: 10.1002/9781118901731.iecrm0012.

Scrivener, L. (2010) When police are guilty of FIDO - forget it, drive on. Toronto Star. 26 November. [Online]https://thestar.com/news/gta/2010/11/26/when_police_are_ guilty_of_fido_forget_it_drive_on. html.

Seron, C., Pereira, J. and Kovath, J. (2004) Judging police misconduct: 'Street level' versus professional policing. Law and Society Review, 38(4): 665-710.

Sessions, J. (2017) Remarks at National Association of Attorneys General Annual Winter Meeting. Office of the Attorney General. [Online] https://www.justice.gov/ opa/speech/ attorney- general-jeff-sessions-delivers-remarks-national-associationattorneys-general.

Shadish, W., Cook, T. \& Campbell, D. (2002) Experimental and Quasi-Experimental Designs for Generalized Causal Inference. Belmont, CA: Wadsworth.

Shane, J. (2010). Organizational stressors and police performance. Journal of Criminal Justice, 38(4): 807-818.

Shane, J. (2011). Daily work experiences and police performance. Police Practice \& Research, 13(3): 1-19.

Shapland, J. \& Vagg, J. (1980) Policing by the Public. London: Routledge.

Shearing, C. (1981) Subterranean processes in the maintenance of power. Canadian Review of Sociology \& Anthropology, 18(3): 283-298.

Shearing, C. (2000) A new beginning for policing. Journal of Law \& Society, 27(3): 386-393.

Shearing, C. \& Ericson, R. (1991) Culture as figurative action. British Journal of Sociology, 42(4): 481-506.

Sheley, J. \& Nock, S. (1979) Determinants of police job satisfaction. Sociological Inquiry, 49: 49-55.

Sheptycki, J. (1993) Innovations in Policing Domestic Violence: Evidence from Metropolitan London. Brookfield, CT: Avebury. 
Sheptycki, J. (2002) Accountability across the policing field: Towards a general cartography of accountability for post-modern policing. Policing \& Society, 12(4): 323338.

Sheptycki, J. (2018) What is police research good for? Reflections on moral economy and police research. European Journal of Policing Studies, 5(3): 16-35

Sherman, L. (1974) The sociology and the social reform of the American police: 19501973. Journal of Police Science \& Administration, 2: 255-263.

Sherman, L. (1980) Causes of police behavior: The current state of quantitative research. Journal of Research in Crime \& Delinquency, 17: 69-100.

Sherman, L. (1984) Experiments in police discretion: Scientific boon or dangerous knowledge. Law \& Contemporary Problems, 47(4): 61-81.

Sherman, L. (1997) Communities and crime prevention. In (L. Sherman, D. Gottfredson, D. MacKenzie, J. Eck, P. Reuter \& S. Bushway, eds.), Preventing Crime: What Works, What Doesn't, What's Promising: 58-109. Washington, DC: Department of Justice.

Sherman, L. (2007) Reiss, Albert J. In (J. Greene, ed.) Encyclopedia of Police Science, $3^{\text {rd }}$ ed.: 1110-1112. New York: Routledge.

Sherman, L. (2013) The rise of evidence-based policing: Targeting, testing and tracking. Crime and Justice, 42: 377-431.

Sherman, L., Gartin, P. \& Buerger, M. (1989) Hot spots of predatory crime: Routine activities and the criminology of place. Criminology, 27(1): 27-56.

Sherman, L. \& Weisburd, D. (1995) General deterrent effects of police patrol in crime 'hot spots': A randomized, controlled trial. Justice Quarterly, 12(4): 625-648.

Shi, L. (2005) Does Oversight Reduce Policing? Evidence from the Cincinnati Police Department after the April 2001 Riot. Unpublished manuscript. [Online] https://www. aeaweb.org/assa/2006/0106_0800_09 03pdf.

Shi, L. (2009) The limit of oversight in policing: Evidence from the 2001 Cincinnati riot. Journal of Public Economics, 93(1): 99-113.

Shiffman, D. (2008) The Age of Engage: Reinventing Marketing for Today's Connected, Collaborative, and Hyperinteractive Culture. Ladera Ranch, CA: Hunt Street Press.

Shim, H., Jo, Y. \& Hoover, L. (2015) A test of general strain theory on police officers' turnover intention. Asian Journal of Criminology, 10(1): 43-62.

Shirky, C. (2008) Here Comes Everybody: The Power of Organizing without Organizations. New York: Penguin.

Shjarback, J. (2016) Cops, Culture, and Context: The Integration of Structural and Cultural Elements for Explanations of Police Use of Force. Unpublished PhD dissertation. Arizona State University. 
Shjarback, J., Decker, S., Wolfe, S. \& Pyrooz, D. (2017) De-policing, crime and the 'Ferguson Effect' in Missouri. St. Louis Post-Dispatch. 3 October. [Online] http://www. stltoday.com/opinion/columnists/de-policing-crime-and-the-ferguson-effect-inmissouri/article_c660258e-05a0-58c8-8b7a-0a9edfd1d2ac.html.

Shjarback, J., Pyrooz, D., Wolfe, S. \& Decker, S. (2017) De-policing and crime in the wake of Ferguson: Racialized changes in the quantity and quality of policing among Missouri police departments. Journal of Criminal Justice, 50: 42-52.

Sigelman, L., Welch, S., Bledsoe, T., \& Combs, M. (1997). Police brutality and public perceptions of racial discrimination: A tale of two beatings. Political Research Quarterly, 50(4): 777-791.

Silver, J. \& Pickett, J. (2015) Toward a better understanding of politicized policing attitudes: Conflicted conservatism and support for police use of force. Criminology, 53(4): 650-676.

Silver, J., Roche, S., Bilach, T. \& Ryon, S. (2017) Traditional police culture, use of force, and procedural justice: Investigating individual, organizational, and contextual factors. Justice Quarterly, 34(7): 1272-1309.

Silverman, D. (2006) Interpreting Qualitative Data: Methods for Analyzing Talk, Text, and Interaction, $3^{\text {rd }}$ ed. London: Sage.

Simon S. (2012) Suspicious encounters: Ordinary preemption and the securitization of photography. Security Dialogue, 43: 157-173.

Simonson, J. (2016). Copwatching. California Law Review, 104(2): 391-445.

Sindall, K., Sturgis, P. \& Jennings, W. (2012) Public confidence in the police: A timeseries analysis. British Journal of Criminology, 52(4): 744-764.

Sklansky, D. (2007) Seeing blue: Police reform, occupational culture, and cognitive burn-in. In (M. O’Neill, M. Marks \& A. Singh, eds.) Police Occupational Culture: New Debates and Directions: 19-45. Oxford: Elsevier.

Sklansky, D. (2008) Democracy and the Police. Stanford: Stanford University Press.

Sklansky, D. (2014) The promise and perils of police professionalism. In (J. Brown, ed.) The Future of Policing: 343-354. New York: Routledge.

Skogan, W. (2003) Community Policing: Can it Work? Belmont, CA: Wadsworth.

Skogan, W. (2005) Citizen satisfaction with police encounters. Police Quarterly, 8(3): 298-321.

Skogan, W. (2006a) Asymmetry in the impact of encounters with the police. Policing \& Society, 162: 99-126.

Skogan, W. (2006b) Police and Community in Chicago. Oxford: Oxford University Press.

Skogan, W. (2008) Why reforms fail. Policing \& Society, 18(1): 23-34. 
Skogan, W. (2015) Surveying police officers. In (M. Maltz \& S. Rice, eds.) Envisioning Criminology: Researchers on Research as a Process of Discovery: 109-115. New York: Springer.

Skogan, W. (2019) Community policing. In (D. Weisburd \& A. Braga, eds.) Police Innovation: Contrastic Perspectives, second edition: 27-44. New York: Cambridge University Press.

Skogan, W. \& Frydl, K., eds. (2004) Fairness and Effectiveness in Policing: The Evidence. Washington: National Academies Press.

Skogan, W. \& Harnett, S. (1997) Community Policing: Chicago Style. New York: Oxford University Press.

Skogan, W., Steiner, L., Dubois, J., Gudell, J. \& Fagan, A. (2002) Taking Stock: Community Policing in Chicago. Washington, DC: National Institute of Justice.

Skolnick J. (1966) Justice Without Trial: Law Enforcement in a Democratic Society. New York: Wiley.

Skolnick, J. (1994) Justice Without Trial: Law Enforcement in a Democratic Society, $3^{\text {rd }}$ ed. New York: Macmillan.

Skolnick, J. (1996) A sketch of the police officer's 'working personality.' In (B. Hancock \& P. Sharp, eds.) Criminal Justice in America: Theory, Practice, and Policy: 89-113. Upper Saddle River, NJ: Prentice-Hall.

Skolnick, J. (2008) Enduring issues of police culture and demographics. Policing \& Society, 18(1): 35-45.

Skolnick, J. (2011) Justice without Trial. Law Enforcement in Democratic Societies, $4^{\text {th }}$ ed. New Orleans: Qui Pro Books.

Skolnick, J. \& Bayley, D. (1988) Community Policing: Issues and Practices around the World. Washington, DC: National Institute of Justice.

Skolnick, J. \& Fyfe, J. (1993) Above the Law: Police and the Excessive Use of Force. New York: The Free Press.

Skolnick, J. \& Fyfe, J. (2005) The beating of Rodney King. In (T. Newburn, ed.) Policing: Key Readings: 568-580. Cullompton, UK: Willan.

Slocum, J. \& Cron, W. (1985) Job attitudes and performance during three career stages. Journal of Vocational Behaviour, 26(2): 126-145.

Small, M. (2011) How to conduct a mixed methods study: Recent rends in a rapidly growing literature. Annual Review of Sociology, 37: 57-86.

Smandych, R., Lincoln, R. \& Wilson, P. (1993) Toward a cross-cultural theory of Aboriginal crime: A comparative study of the problem of Aboriginal overrepresentation in the criminal justice systems of Canada and Australia. International Criminal Justice Review, 3(1): 1-24. 
Small, M. (2011) How to conduct a mixed methods study: Recent trends in a rapidly growing literature. Annual Review of Sociology, 37: 57-86.

Smart, A. (2018) Trudeau apologizes to B.C. Indigenous community for 1864 hanging of chiefs. The Globe \& Mail. 2 November [Online] https://www.theglobeandmail.com/ canada/article-trudeau-to-apologize-to-bc-indigenous-community-for-1864-hanging-of/.

Smith, D. (2007a) New challenges to police legitimacy. In (Henry, A. \& Smith, D., eds.) Transformations of Policing: 273-305. Aldershot, UK: Ashgate.

Smith, D. (2007b) The foundations of legitimacy. In (T. Tyler, ed.) Legitimacy and Criminal Justice: International Perspectives: 30-58. New York: Sage.

Smith, D. \& Gray, J. (1983) Police and People in London: The Police in Action. London: PSI.

Smith, G. (2004) Stonechild report lashes Saskatoon Police. The Globe \& Mail. 26 October: 1 .

Smith, G. (2009) Why don't more people complain against the police? European Journal of Criminology, 6(3): 249-266.

Smith, M. \& Alpert, G. (2002) Searching for direction: Courts, social science, and the adjudication of racial profiling claims. Justice Quarterly, 19: 673-703.

Smith, M. \& Alpert, G. (2007) Examining police bias: A theory of social conditioning and illusory correlation. Criminal Justice \& Behaviour, 34(10): 1262-1283.

Smith, P. \& Riley, A. (2009) Cultural Theory: An Introduction, 2nd ed., Oxford: Blackwell.

Smith, S. (2013) Determining Sample Size: How to Ensure You Get the Correct Sample Size. [Online] http://www.qualtrics.com/blog/determining-sample-size/.

Smith, T. et al. (2002) Developing nonresponse standards. In (R. Groves, D. Dillman, J. Eltinge, R. Little et al., eds.) Survey Nonresponse: 27-40. New York: Wiley.

Smith, W. (2005) Police operational management: Rethinking the legal shell game. In (R. Dunham \& G. Alpert, eds.) Critical Issues in Policing: Contemporary Readings, $5^{\text {th }}$ ed.: 492-505. Long Grove, Ill: Waveland Press.

Sobol, J. (2010) The social ecology of police attitudes. Policing: An International Journal of Police Strategies \& Management, 33(2): 253-269.

Sousa, W., Miethe, T. \& Sakiyama, M. (2017) Inconsistencies in public opinion of body-worn cameras on police: Transparency, trust, and improved police-citizen relationships. Policing: A Journal of policy and Practice, 12(1): 100-108.

Spangenthal-Lee, J. (2011) SPD disputes rumors of de-policing within the department. Seattle Metropolitan. [Online] http://www.seattlemet.com/news-and-profiles/publicola/ articles/spd-disputes-rumors-of-de-policing-within-the-department. 
Spears, T. (2011) Judge blasts Ottawa cop for 'racial profiling'. Kingston Whig Standard. 7 July. [Online] http://www.thewhig.com/2011/07/07/judge-blasts-ottawacop-for-racial-profiling.

Speiser, M. (2015) NYPD union head's letter lambastes reporters, say they aren't 'qualified to judge the actions of police'. The National Post. 18 September: 5.

Spelman, W. \& Brown, D. (1984) Calling the Police: Citizen Reporting of Serious Crime. Washington, DC: Police Executive Research Forum.

Squires, P. (2016) Beyond contrasting traditions in policing research? In (M. Brunger, S. Tong \& D. Martin, eds.) Introduction to Policing Research: Taking Lessons from Practice: 9-28. New York: Routledge.

Stake, R. (2005) Qualitative Case Studies. In (N. Denzin \& Y. Lincoln, eds.) The Sage Handbook of Qualitative Research: 443-466. Thousand Oaks, CA: Sage.

Stalcup, M. \& Hahn, C. (2016) Cops, cameras and the policing of ethics. Theoretical Criminology, 20(4): 482-50.

Stanfield, J. (2011) Black Reflexive Sociology: Epistemology, Theory and Methodology. Walnut Creek, CA: Left Coast Press.

Stanko, E. \& Dawson, P. (2015) Police Use of Research Evidence: Recommendations for Improvement. New York: Springer.

Stanko, E. \& Dawson, P. (2016) What should be the balance between 'craft experience' and 'scientific evidence'? In (E. Stanko \& P. Dawson, eds.) Police Use of Research Evidence: 63-83. London: Springer.

Statistics Canada (2017a) Aboriginal Population Profile, 2016 Census. [Online] https:// www12.statcan.gc.ca/censusrecensement/2016/dppd/abpopprof/index.cfm?Lang=E.

Statistics Canada (2017b) Census of Population, 2016 Immigration and Ethnocultural Diversity Highlight Tables [Online] https://www12.statcan.gc.ca/ census-recensement/ 2016/dp-pd/hlt-fst/imm/Table.cfm?Lang=E\&T=31\&Geo=01.

Statistics Canada (2017c) Census Profile, 2016 Census. [Online] https://www12.statcan. gc.ca/census-recensement/2016/dp-pd/prof/index.cfm?Lang=E.

Statistics Canada (2017d) Police-Reported Crime Severity Indexes, by Census Metropolitan Area, 2016 [Online] https://doi.org/10.25318/3510002601-eng.

Statistics Canada (2017e) Police Resources in Canada 2016. [Online] https://www. statcan.gc.ca/pub/85-002-x/2017001/article/14777-eng.html.

Stein R. \& Griffith C. (2017) Resident and police perceptions of the neighborhood: Implications for community policing. Criminal Justice Policy Review, 28(2): 139-154.

Stenning, P. (2009) Governance and accountability in a plural policing environment: The story so far. Policing: A Journal of Policy and Practice, 3(1): 22-33.

Stenning, P. \& Shearing, C. (2005) Reforming police: Opportunities, drivers and challenges. Australian \& New Zealand Journal of Criminology, 38(2): 167-180. 
Stenson, K. \& Silverstone, D. (2014) Making police accountable: Governance and legitimacy. In (J. Brown, ed.) The Future of Policing: 429-445. New York: Routledge.

Stevens, D. (2001) Case Studies in Community Policing. Upper Saddle River, NJ: Prentice Hall.

Stevens, D. (2002) Community policing and police leadership. In (D. Stevens, ed.) Policing and Community Partnerships: 185-210. Upper Saddle River, NJ: Prentice Hall.

Stoddard, E. (2006) The informal code of police deviancy: A group approach to bluecollar crime. In (V. Kappeler, ed.) The Police \& Society: Touchstone Readings, 3rd ed.: 201-222. Long Grove, IL: Waveland Press.

Stone, C., Fogelsong, T. \& Cole, C. (2009) Policing Los Angeles under a Consent Decree: The Dynamics of Change at the LAPD. Cambridge, MA: Harvard Kennedy School.

Stoughton, S., Alpert, G. \& Noble, J. (2015) Why police need constructive criticism. The Atlantic. 23 December. [Online] http://www.theatlantic.com/politics/archive/2015/ 12/officer-porter-mistrial-police-culture/421656/\#article-comments.

Strauss, A. \& Corbin, J. (1998) Basics of Qualitative Research: Techniques and Procedures for Developing Grounded Theory. Thousand Oaks, CA: Sage.

Stumpf, S. \& Rabinowitz, S. (1981) Career stage as a moderator of performance relationships with facets of job satisfaction and role perceptions. Journal of Vocational Behaviour, 18(2): 202-218.

Sturges, P. \& Cooke, L. (2008) Finding the grey in the blue: Transparency and disclosure in policing. The Grey Journal, 4(1): 21-28.

Sullivan, J., Thebault, R., Tate, J. \& Jenkins, J. (2017) Number of fatal shootings by police is nearly identical to last year. The Washington Post. 1 July. [Online] https:// www.washingtonpost.com/investigations/number-of-fatal-shootings-by-police-isnearly-identical-to-last-year/2017/07/01/98726cc6-5b5f-11e7-9fc6-c7ef4bc58d13 story. html? utm_term $=.994 \mathrm{~d} 293 \mathrm{~d} 6 \mathrm{dd} 7$.

Sullivan, J., Anthony, Z., Tate, J. \& Jenkins, J. (2018) Nationwide, police shot and killed nearly 1,000 people in 2017. The Washington Post. 6 January. [Online] https:// www.washingtonpost.com/investigations/nationwide-police-shot-and-killed-nearly1000-people-in-2017/2018/01/04/4eed5f34-e4e9-11e7-ab50-621fe0588340_story.html? noredirect $=$ on\&utm_term $=. \mathrm{d} 1$ eea061 $\mathrm{c} 09 \mathrm{f}$.

Sun, I. (2002) Police officer attitudes toward peers, supervisors, and citizens: A comparison between field training officers and regular officers. American Journal of Criminal Justice, 27: 69-83.

Sunahara, D. (2004) A model of unethical and unprofessional police behaviour. The Canadian Review of Policing Research [Online] http://crpr.icaap.org/index.php/crpr/ article/view/17/16. 
Sunshine, J. \& Tyler, T. (2003a) Moral solidarity, identification with the community, and the importance of procedural justice: The police as prototypical representatives of a group's moral values. Social Psychology Quarterly, 66: 153-165.

Sunshine, J. \& Tyler, T. (2003b) The role of procedural justice and legitimacy in shaping public support for policing. Law \& Society Review, 37(3): 513-548.

Super, D. (1953) A theory of vocational development. American Psychologist, 8: 185190.

Super, D. (1957). The Psychology of Careers: An Introduction to Vocational Development. New York: Harper Row.

Super, D. (1963) The definition and measurement of early career behavior: A first formulation. Personnel and Guidance Journal, 41: 775-779.

Super, D. (1980) A life span, life space approach to career development. Journal of Vocational Behavior, 16(3): 282-298.

Super, D. (1984). Career and life development. In (D. Brown \& L. Brooks, eds.) Career Choice and Development. San Francisco: Jossey-Bass.

Super, D. (1990). A life-span, life-space approach to career development. In (D. Brown $\&$ L. Brooks, eds.), Career choice and development: Applying contemporary theories to practice, $2^{\text {nd }}$ ed.: 197-261. San Francisco, CA: Jossey-Bass.

Super, D. (1992). Toward a comprehensive theory of career development. In (D. Montross \& C. Shinkman, eds.), Career development: Theory and Practice: 35-64. Springfield, IL: Charles C. Thomas.

Super, D., Zelkowitz, R. \& Thompson, A. (1981) Career Concerns Inventory: Adult Form 1 Preliminary Manual. New York: Columbia University.

Surette, R. (2011) Media, Crime, and Criminal Justice: Images, Reality and Policies $4^{\text {th }}$ ed. Belmont, CA: Thomson.

Susswein, R. (2008) Eradicating Racial Profiling Companion Guide. Trenton, NJ:

Division of Criminal Justice.

Sutton, R. (2015). The dangers of de-policing: Will cops just stand down? New York Post. 5 May. [Online] http://nypost.com/2015/05/05/ the-dangers-of-de-policing-willcops-just-stand-down/.

Swatt, M., Gibson, C. \& Piquero, N. (2007) Exploring the utility of general strain theory in explaining problematic alcohol consumption by police officers. Journal of Criminal Justice, 35(6): 596-611.

Swidler, A. (1986) Culture in action: Symbols and strategies. American Sociological Review, 51: 273-286.

Swidler, A. (2001) Talk of Love: Why Culture Matters. Chicago: Chicago University Press. 
Tankebe, J. (2013) Viewing things differently: Examining the dimensions of public perceptions of police legitimacy. Criminology, 51(1): 103-135.

Tankebe, J. \& Leibling, A., eds. (2013) Legitimacy and Criminal Justice: An International Exploration. Oxford: Oxford University Press.

Tanovich, D. (2006) The Colour of Justice: Policing Race in Canada. Toronto: Irwin Law.

Tashakkori, A. \& Teddlie, C. (2003) The past and future of mixed methods research: From data triangulation to mixed model designs. In (A. Tashakkori \& C. Teddlie, eds.) The Sage Handbook of Mixed Methods in Social and Behavioural Research: 671-701. Thousand Oaks, CA: Sage.

Tator, C. \& Henry, F. (2006) Racial Profiling in Canada: Challenging the Myth of 'A Few Bad Apples'. Toronto: University of Toronto Press.

Tatum, J. (2011) Crown withdraws charges after court raises concerns about racial profiling. Windsor Law LEAP Blog. 10 July. [Online] http://windsorlaw-leap.blogspot. ca/2011/07/crown-withdraws-charges-after-court.html.

Taylor, I. (1981) Law and Order: Arguments for Socialism. London: MacMillan.

Taylor, R. (1998) Crime and small-scale places: What we know, what we can prevent, and want else we need to know. Crime and Place: Plenary papers of the 1997 conference on criminal justice research and evaluation. Washington, DC: National Institute of Justice.

Taylor, S. (1981) A categorization approach to stereotyping. In (D. Hamilton, ed.) Cognitive Processes in Stereotyping and Intergroup Behaviour: 83-114. Hillsdale, NJ:

L. Erlbaum Associates.

Teddlie, C. \& Tashakkori, A. (2003) Major issues and controversies in the use of mixed methods in the social and behavioral sciences. In (A. Tashakkori \& C. Teddlie, eds.) The Sage Handbook of Mixed Methods in Social and Behavioral Research: 51-89. Thousand Oaks, CA: Sage.

Teddlie, C. \& Tashakkori, A. (2010) Overview of contemporary issues in mixed methods research. In (A. Tashakkori \& C. Teddlie, eds.) The Sage Handbook of Mixed Methods in Social and Behavioral Research, $2^{\text {nd }}$ ed.:1-41. Thousand Oaks, CA: Sage.

Telep, C. \& Weisburd, D. (2018) Crime concentration at place. In (S. Johnson \& G. Bruinsma, eds.) Oxford Handbook of Environmental Criminology. Oxford: Oxford University Press.

Terrill, W. \& Mastrofski, S. (2002) Situational and officer-based determinants of police coercion. Justice Quarterly, 19: 215-248.

Terrill, W. \& Paoline, E. (2015) Citizen complaints as threats to police legitimacy: The role of officers' occupational attitudes. Journal of Contemporary Criminal Justice, 31: 192-211. 
Terrill, W. \& Paoline, E. (2017) Police use of less lethal force: Does administrative policy matter? Justice Quarterly, 34(2): 193-216.

Terrill, W., Paoline, E. \& Gau, J. (2016) Three pillars of police legitimacy: Procedural justice, use of force, and occupational culture. In (M. Deflem, ed.) Sociology of Crime, Law and Deviance. The Politics of Policing: Between Force and Legitimacy: 59-76. London: Emerald.

Terrill, W., Paoline, E. \& Manning, P. (2003) Police culture and coercion. Criminology, 41(4): 1003-1034.

Teurlings, J, \& Stauff, M. (2014) Introduction: The transparency issue. Cultural Studies \& Critical Methodologies, 14(1): 3-10.

Thacher, D. (2001) Conflicting values in community policing. Law \& Society Review, 35: 765-799.

Thomas, G. (2014) Research on policing: Insights from the literature. The Police Journal: Theory, Practice \& Principles, 87(1): 5-16.

Thompson, A. (2017) RCMP found guilty of violating Labour Code in 2014 Moncton shooting. The Toronto Star. 29 September. [Online] https:/www.thestar.com/news/ canada /2017/09/29/rcmp-found-guilty-of-violating-labour-code-in-2014-monctonshooting.html.

Thompson, B. \& Lee, J. (2004) Who cares if police become violent? Explaining approval of police use of force using a national sample. Sociological Inquiry, 74(3): 381-410.

Thompson, J. (2005) The new visibility. Theory, Culture \& Society, 22(6): 31-51.

Thomson, B. (2004) Qualitative Research: Grounded Theory-Sample Size and Validity [Online] http:// www.buseco.monash.edu.au/research/studentdocs/mgt.pdf.

Thornhill, E. A. (2008). So seldom for us, so often against us: Blacks and law in Canada. Journal of Black Studies, 38(3): 321-337.

Tilley, N. \& Laycock, G. (2017) The why, what, when and how of evidence-based policing. In (J. Knutsson \& L. Tompson, eds.) Advances in Evidence Based Policing. London: Routledge.

Tillyer, R., Engel, R. \& Cherkauskas, J. (2010) Best practices in vehicle stop data collection and analysis. Policing: An International Journal of Police Strategies \& Management, 33(1): 69-92.

Tiratelli, M., Quinton, P. \& Bradford, B. (2018) Does stop and search deter crime? Evidence from ten years of London-wide data. The British Journal of Criminology, 58(5): 1212-1231.

Tittle, C. (2004) The arrogance of public sociology. Social Forces, 82: 1639-1643. 
Tizon, A. \& Forgrave, R. (2001) Wary of racism complaints, police look the other way in black neighborhoods. The Seattle Times. 26 June. [Online] http://community.seattle times.nwsource.com /archive/?date $=20010626 \&$;slug=police $26 \mathrm{~m}$.

Tobias, H. \& Joseph, A. (2018) Sustaining systemic racism through psychological gaslighting: Denials of racial profiling and justifications of carding by police utilizing news media. Race \& Justice. DOI: 10.1177/2153368718760969.

Toch, H. (2012) Cop Watch: Spectators, Social Media, and Police Reform. Washington: American Psychological Association.

Todak, N. (2017) The decision to become a police officer in a legitimacy crisis. Women \& Criminal Justice, 27(4): 250-270.

Todd, H. (2015) The Effects of Citizen Monitoring on the Police: An Examination of Citizen Monitoring and Police Use of Justified Force. Unpublished MA thesis. Simon Fraser University.

Töpfer, E., Hempel, L. \& Cameron, H. (2003) Watching the Bear: Networks and the Islands of Visual Surveillance in Berlin. Berlin: Technical University Berlin.

Toronto Police Service (2013) The Police and Community Engagement Review: Phase II - Internal Report and Recommendations. Toronto: Toronto Police Service.

Toronto Police Service (2016) Body-Worn Cameras: A Report on the Findings of the Pilot Project to Test the Value and Feasibility of Body-Worn Cameras for Police Officers in Toronto. Toronto: Toronto Police Service.

Torres, J., Reling, T. \& Hawdon, J. (2018) Role conflict and the psychological impacts of the post-Ferguson period on law enforcement motivation, cynicism, and apprehensiveness. Journal of Police \& Criminal Psychology, 33(4): 358-374.

Tourangeau, R. \& Plewes, T. (2013) Nonresponse in Social Science Surveys: A Research Agenda. Washington, DC: National Academies Press.

Tourangeau, R. \& Yan, T. (2007) Sensitive questions in surveys. Psychological Bulletin, 133(5): 859-883.

Trottier, D. (2015) Vigilantism and power users: Police and user-led investigations on social media. In (D. Trottier \& C. Fuchs, eds.) Social Media, Politics and the State: Protests, Revolutions, Riots, Crime and Policing in the Age of Facebook, Twitter and YouTube: 209-226. New York: Routledge.

Tso, G. (2016). Police brutality is not invisible. The Hill, Congress Blog, January 14. [Online] http://thehill.com/blogs/congress-blog/civil-rights/265795-police-brutality-isnot-invisible.

Tuchman, G. 1978. Making News: A Study in the Construction of Reality. New York: Free Press.

Tulloch, J. (2008) Culture and risk. In (J. Zinn, ed.) Social Theories of Risk and Uncertainty: An Introduction: 138-167. Oxford, UK: Blackwell. 
Turner, E. (2013) Beyond 'facts' and 'values: Rethinking some recent debates about the public role of criminology. British Journal of Criminology, 53(2): 149-166.

Turner, J. (2013) Theoretical Sociology: 1830 to the Present. Los Angeles: Sage.

Tyler, T. (2004) Enhancing police legitimacy. Annals of the American Academy of Political and Social Science, 593: 84-99.

Tyler, T. (2005) Policing in Black and White: Ethnic group difference sin trust and confidence in the police. Police Quarterly, 8(3): 322-342.

Tyler, T. (2006a) Legitimacy and legitimation. Annual Review of Psychology, 57: 375400.

Tyler, T. (2006b) Why People Obey the Law: Procedural Justice, Legitimacy, and Compliance. Princeton, NJ: Princeton University Press.

Tyler, T. (2011) Trust and legitimacy: Policing in the USA and Europe. European Journal of Criminology, 8: 254-266.

Tyler, T., Braga, A., Fagan, J., Meares, T., Sampson, R. \& Winship, C. (2007)

Legitimacy and criminal justice: International perspectives. In (T. Tyler, ed.) Legitimacy and Criminal Justice: International Perspectives: 9-29. New York: Sage.

Tyler, T. \& Fagan, J. (2008) Legitimacy and cooperation: Why do people help the police fight crime in their communities? Ohio State Journal of Criminal Law, 6(1): 231275.

Tyler, T., Fagan, J. \& Geller, A. (2014) Street stops and police legitimacy: Teachable moments in young urban men's legal socialization. Journal of Empirical Legal Studies, 11(4): 751-785.

Tyler, T. \& Huo, Y. (2002) Trust in the Law: Encouraging Public Cooperation with The Police and Courts. New York: Sage.

Tyler, T. \& Jackson, J. (2013) Future challenges in the study of legitimacy and criminal justice. In (J. Tankebe \& and A. Leibling, eds.) Legitimacy and Criminal Justice: An International Exploration: 83-104. Oxford: Oxford University Press.

Tyler, T. \& Smith, H. (1997) Social justice and social movements. In (D. Gilbert, S. Fiske \& G. Lindzey, eds.) Handbook of Social Psychology, $4^{\text {th }}$ ed. New York: McGrawHill.

Tyler, T. \& Wakslak, C. (2004) Profiling and police legitimacy: Procedural justice, attributions of motive, and acceptance of police authority. Criminology, 42(2): 253-281.

Uchida, C. (2005) The development of American police: An historical overview. In (R. Dunham \& G. Alpert, eds.) Critical Issues in Policing: Contemporary Readings, $5^{\text {th }}$ ed.: 20-40. Long Grove, IL: Waveland Press.

Uggen, C. \& Inderbitzin, M. (2010) Public criminologies. Criminology \& Public Policy, 9(4): $725-750$. 
Ulmer, J. (2017) Special issue: Policing and minority communities. Justice Quarterly, 34(7): 1127-1128.

United States Census Bureau (2015) QuickFacts from the U.S. Census Bureau. [Online] www.census.gov/quickfacts.

United States Census Bureau (2017) Population and Housing Estimates. [Online] https://www.census.gov/programs-surveys/popest.html.

Unnever, J. \& Gabbidon, S. (2011). A Theory of African American Offending: Race, Racism, and Crime. New York: Routledge.

Uslaner, E. 2002. The Moral Foundation of Trust. Cambridge: Cambridge University Press.

Van Hulst, M. (2013) Storytelling at the police station: The canteen culture revisited. British Journal of Criminology, 53: 624-642.

Van Maanen, J. (1973) Observations on the making of policemen. Human Organization, 32(4): 407-418.

Van Maanen, J. (1974) Working the street: A developmental view of police behavior. In (H. Jacob, ed.) The Potential for Reform of Criminal Justice: 83-130. New York: Sage.

Van Maanen, J. (1975) Police socialization: A longitudinal examination of job attitudes in an urban police department. Administrative Science Quarterly, 20(2): 207-228.

Van Maanen, J. (1978) The asshole. In (P. Manning \& J. Van Maanen, eds.) Policing: A View from the Street: 221-237. Santa Monica, CA: Goodyear.

Van Maanen, J. (1984) Making rank: Becoming an American police sergeant. Urban Life, 13: 155-176.

Van Maanen, J. (1988) Tales of the Field: On Writing Ethnography. Chicago: University of Chicago Press.

Van Maanen, J. \& Barley, S. (1982) Occupational Communities: Culture and Control in Organizations. Cambridge, MA: Alfred P. Sloan School of Management.

Van Maanen, J. \& Schein, E. (1979) Toward a theory of organizational socialization. Research in Organizational Behaviour, 1: 209-264.

Vaughan, D. (1996) The Challenger Launch Decision: Risky Technology, Culture, and Deviance at NASA. Chicago: University of Chicago Press.

Vaughan, D. (2002) Signals and interpretive work: The role of culture in a theory of practical action. In (K. Cerulo, ed.) Culture in Mind: Toward a Sociology of Culture and Cognition: 28-54. New York: Routledge.

Vick, K. (2015) What it's like to be a cop in America: One year after Ferguson. Time. 24 August. [Print \& Online] https://time.com/magazine/us/3995775/august-24th-2015vol-186-no-7-u-s/. 
Victor, D. \& McPhate, M. (2016) Critics of law enforcement embrace Facebook Live and other video tools. New York Times, 8 July: A18.

Violanti, J. \& Aron, F. (1995) Police stressors: Variations in perception among police personnel. Journal of Criminal Justice, 23(3): 287-294.

Violanti, J., Fekedulegn, D., Hartley, T., Charles, L., Andrew, M., Ma, C. \& Burchfiel, C. (2016) Highly rated and most frequent stressors among police officers: Gender differences. American Journal of Criminal Justice, 41(4): 645-662

Vitale, A. (2005) Innovation and institutionalization: Factors in the development of 'quality of life' policing in New York City. Policing \& Society, 15(2): 99-124.

Waddington, P. (1999) Police (canteen) sub-culture: An appreciation. British Journal of Criminology, 39(2): 287-309.

Waddington, P. (2014) Policing Citizens: Police, Power and the State. London: Routledge.

Waddington, P. (2015) Revisiting the classics: Police: streetcorner politicians: William K. Muir. Policing \& Society: An International Journal of Research \& Policy, 25(6): 681-686.

Wagner, L. (2015) FBI director doubles down on linking scrutiny of police with rise in violent crime. National Public Radio. 26 October. [Online] https://npr.org/sections/ thetwo-way/2015/10/26/451992173.

Waisbord, S. (2014) Citizen journalism, development and social change: Hype and hope. In (S. Allan \& E. Thorson, Eds.) Citizen Journalism: Global Perspectives: 185197. New York: Peter Lang.

Wakefield, J. (2018) 'Carding' stops by Edmonton police drop 30 per cent in one year. The Edmonton Journal. 23 June. [Online] http://edmontonjournal.com/news/crime/ carding-stops-by-edmonton-police-drop-30-per-cent-in-one-year.

Walker, S. (1992) The Police in America, $2^{\text {nd }}$ edition. New York: McGraw-Hill

Walker, S. (2005) The New World of Police Accountability. Thousand Oaks, CA: Sage.

Walker, S. (2008) The neglect of police unions: Exploring one of the most important areas of American policing. Police Practice \& Research, 9(2): 95-112.

Walker, S. (2010) Police accountability and the central problem in American criminal justice. In (C. McCoy, ed.) Holding Police Accountable: 1-25. Washington: The Urban Institute Press.

Walker, S. \& Archbold, C. (2014). The New World of Police Accountability. Los Angeles, CA: Sage.

Walker, S. \& Katz, C. (2008) The Police in America: An Introduction, $6^{\text {th }}$ ed. New York: McGraw-Hill.

Walker, S., Spohn, C. \& DeLone, M. (2000) The Color of Justice: Race, Ethnicity and Crime in America. Belmont, CA: Wadsworth. 
Wall, T. \& Linnemann, T. (2014) Staring down the state: Police power, visual economics, and the 'war on cameras'. Crime, Media, Culture, 10(2): 133-149.

Wallace, C. (2017) The Ferguson Effect: FBI report reveals law enforcement pullback. Fox News. 5 May. [Television].

Wallace, D., White, M., Gaub, J. \& Todak, N. (2018) Body-worn cameras as a potential source of depolicing: Testing for camera-induced passivity. Criminology, 56(3): 481509.

Walliman, N. (2016) Social Research Methods, $2^{\text {nd }}$ ed. Los Angeles: Sage.

Walsh, D. \& Conway, V. (2011) Police governance and accountability: Overview of current issues. Crime, Law and Social Change, 55(2-3): 61-86.

Ward, G. (2012) The Black Child Savers: Racial Democracy and Juvenile Justice. Chicago: University of Chicago Press.

Waring, E. \& Weisburd, D., eds. (2002) Advances in Criminological Theory: Crime \& Social Organization. New York: Routledge.

Warner, B. (2005) 101 Reasons Why You Should Not Become a Cop. Lincoln, NE: iUniverse.

Washington Post (2014) Full transcript: Obama's remarks on Ferguson, Mo. and Iraq. The Washington Post. 18 August. [Online] http://www.washingtonpost.com/politics/ running-transcript-obamas-remarks-on-ferguson-mo-and-iraq/2014/08/18/ed29d07a2713-11e4-86ca-6f03cbd15c1a_story.html.

Washington Post/ABC News (2014) Poll, December 11-14. https://www.washington post.com/politics/on-racial-issues-america-is-divided-both-black-and-white-and-redand-blue/2014/12/26/3d2964c8-8d12-11e4-a085-34e9b9f09a58_story.html?utm_term 1/4. f7133a91ac3e.

Waters, E. (2014) Transparency in law enforcement: The trend towards officer body cameras. Richmond Journal of Law and Technology, 21(1). [Online] http:// jolt. richmond. edu/index.php/volume-xxi-issue-i/.

Weber, M. (1947) The Theory of Social and Economic Organization. New York: Oxford University Press.

Websdale, N. (2001) Policing the Poor: From Slave Plantation to Public Housing. Boston: Northeastern University Press.

Weick, K. (2014) The work of theorizing. In (R. Swedberg, ed.) Theorizing in Social Science: The Context of Discovery: 177-194. Stanford, CA: Stanford University Press.

Weisburd, D. (2015) The law of crime concentration and the criminology of place. Criminology, 54: 133-157.

Weisburd, D. (2016) Does hot spots policing inevitably lead to unfair and abusing policing practices or can we maximize both fairness and effectiveness in the new 
proactive policing? The University of Chicago Legal Forum, 16: 661-689. [Online] http://chicagounbound.uchicago.edu/uclf/vol2016/iss1/16.

Weisburd, D. \& Braga, A. (2006a) Introduction: Understanding police innovation. In (D. Weisburd \& A. Braga eds.) Police Innovation: Contrasting Perspectives: 1-26. New York: Cambridge University Press.

Weisburd, D. \& Braga, A., eds. (2006b) Police Innovation: Contrasting Perspectives. Cambridge, UK: Cambridge University Press.

Weisburd, D. \& Braga, A. (2019) Understanding police innovation. In (D. Weisburd \& A. Braga, eds.) Police Innovation: Contrastic Perspectives, second edition: 3-26. New York: Cambridge University Press.

Weisburd, D. \& Eck, J. (2004) What can police do to reduce crime, disorder, and fear? The Annals of the American Academy of Political and Social Science, 593(1): 4265.

Weisburd, D. Eck, J., Hinkle, J. \& Telep, C. (2008) Effects of problem-oriented policing on crime and disorder. Campbell Collabroation Library of Systematic Reviews. [Online] www.campbellcollaboration.org/library.php.

Weisburd, D., Hinkle, J., Famega, C. \& Ready, J. (2011) The possible' backfire' effects of hot spots policing" An experimental assessment of impacts on legitimacy, fear and collective efficacy. Journal of Experimental Criminology, 7(4): 297-320.

Weisburd, D. \& Majmunder, M. (2017) Proactive Policing: Effects on Crime and Communities. Washington, DC: National Academies Press.

Weisburd, D. \& White, C. (2019) Hot spots of crime are not just hot spots of crime: Examining health outcomes at street segments. Journal of Contemporary Criminal Justice, 35(2): 142-160.

Weisburd, D., Wyckoff, L., Ready, J., Eck, J., Hinkle, J. \& Gajewski, F. (2006). Does crime just move around the corner? A controlled study of spatial displacement and diffusion of crime control benefits. Criminology, 44(3): 549-591.

Weitzer, R. (1999) Citizens' perceptions of police misconduct: Race and neighborhood context. Justice Quarterly, 16(4): 819-846.

Weitzer, R. (2002) Incidents of police misconduct and public opinion. Journal of Criminal Justice, 30: 397-408.

Weitzer, R. (2010) Race and policing in different ecological contexts. In (S. Rice \& M. White, eds.) Race, Ethnicity, and Policing: New and Essential Readings: 118-139. New York: New York University Press.

Weitzer, R. (2015) American policing under fire: Misconduct and reform. Social Science \& Public Policy, 52(5): 475-480.

Weitzer, R. (2017) Theorizing racial discord over policing before and after Ferguson, Justice Quarterly, 34(7): 1129-1153. 
Weitzer, R. (2018) Recent trends in police-citizen relations and police reform in the United States. In (D. Oberwittler \& S. Roché, eds.) Police-Citizen Relations Across the World: 29-45. London: Routledge.

Weitzer, R. \& Tuch, S. (2002) Perceptions of racial profiling: Race, class and personal experience. Criminology, 40: 435-456.

Weitzer, R. \& Tuch, S. (2004) Reforming the police: Racial differences in public support for change. Criminology, 42(2): 391-416.

Weitzer, R. \& Tuch, S. (2005a) Determinants of public satisfaction with the police. Police Quarterly, 8: 279-297.

Weizter, R. \& Tuch, S. (2005b) Racially biased policing: Determinants of citizen perceptions. Social Forces, 83: 1009-1030.

Weitzer, R. \& Tuch, S. (2006) Race and Policing in America: Conflict and Reform. New York: Cambridge University Press.

Werthman, C. \& Piliavin, I. (1967) Gang members and the police. In (D. Bordua, ed.) The Police: Six Sociological Essays. New York: Wiley.

Western, B. (2006) Punishment and Inequality in America. New York: Sage.

Westley, W. (1951) The Police: A Sociological Study of Law, Custom and Morality. Unpublished PhD dissertation. Chicago: University of Chicago.

Westley, W. (1953) Violence and the police. American Journal of Sociology, 49(1): 3441.

Westley, W. (1955) Secrecy and the police. Social Forces, 34: 254-257.

Westley, W. (1970) Violence and the Police: A Sociological Study of Law, Custom, and Morality. Cambridge, MA: MIT Press.

Westmarland L. (2008) Police cultures. In (T. Newburn, ed.) Handbook of Policing, $2^{\text {nd }}$ ed.: 253-280. Cullompton, UK: Willan.

Westmarland, L. (2014) Ethics and policing. In (J. Brown, ed.) The Future of Policing: 463-475. New York: Routledge.

Wexler, M. (1974) Police culture: A response to ambiguous employment. In (C. Boydell, P. Whitehead \& C. Grinstaff, eds.) The Administration of Criminal Justice in Canada: 126-151. Toronto: Holt, Rinehart \& Winston.

Whitaker, B. (2017) Crisis in Chicago. 60 Minutes. 1 January. [Television].

Whitaker, R. (1999) The End of Privacy: How Total Surveillance is Becoming a Reality. New York: The New Press.

White, M. \& Fradella, H. (2016) Stop and Frisk: The Use and Abuse of a Controversial Police Tactic. New York: NYU Press. 
White, M., Cooper, J., Saunders, J. \& Raganella, A. (2010) Motivations for becoming a police officer: Re-assessing officer attitudes and job satisfaction after six years on the street. Journal of Criminal Justice, 38: 520-530.

White, P. (2014) Hamilton's 'Honest Cops' video helps renew faith in police. The Globe \& Mail. 19 January. [Online] https:/www.theglobeandmail.com/news/national/ hamiltons-honest-cops-video-helps-renew-faith-in-police/article16403040/.

White, S. (1972) A perspective on police professionalization. Law \& Society Review, 7: 61-85.

White, V. (2011) Review of Cellblock Operations. Ottawa: Ottawa Police Service.

Whittemore, R., Chase, S. \& Mandle, C. (2001) Validity in qualitative research. Qualitative Health Research, 11(4): 522-537.

Wilhelm, T. (2015) Windsor police Const. Kent Rice pleads guilty to police act charges. Windsor Star. 10 September. [Online] http://windsorstar.com/news/windsor-policeconst-kent-rice-pleads-guilty-to-police-act-charges.

Wilkerson, I. (2010) The Warm of Other Suns: The Epic Story of America's Great Migration. New York: Vintage Books.

Williams, S., Kyle, J., Hegarty, T., Lockett, L. \& Grube, J. (2019) The arc of procedural justice in a U.S. non-metro community: Traffic stops and policing in the age of disruption. Police Practice \& Research. DOI: 10.1080/15614263.2019.1658580.

Wilson, D. \& Serisier, T. (2010) Video activism and the ambiguities of countersurveillance. Surveillance \& Society, 8(2): 166-180.

Wilson, J. (1968) Varieties of Police Behaviour: The Management of Law and Order in Eight Communities. Cambridge, MA: Harvard University Press.

Wilson, J. \& Boland, B. (1978) The effect of the police on crime. Law \& Society Review, 12(3): 367-390.

Wilson, J. \& Kelling, G. (1982) Broken windows: The police and neighborhood safety. Atlantic Monthly, 249: 29-38.

Wilson, R. (2015) Police accountability measures flood state legislatures after violent events. Washington Post. 8 February. [Online] https://www.washingtonpost.com/blogs/ govbeat/wp/2015/02/04/police-accountability-measures-flood-state-legisla- tures-afterferguson-staten-island/? utm_term1/4.363720ec5617.

Wilson, S. \& Buckler, K. (2010) The debate over police reform: Examining minority support for citizen oversight and resistance by police unions. American Journal of Criminal Justice, 35: 184-197.

Wilson, W. (2007) Speaking to publics. In (D. Clawson et al., eds.) Public Sociology: Fifteen Eminent Sociologists Debate Politics and the Profession in the Twenty-first Century: 117-144. Berkeley, CA: University of California Press. 
Winston, R. \& Mather, K. (2014) L.A. will buy 7,000 body cameras for police officers. The Los Angeles Times. 16 December. [Online] http://www. latimes. com/local/lanow/ la-me-ln-mayor-to-announce-onbody-camera-rol-lout-for-lapd-20141216-story.html.

Withrow, B. (2011) The Racial Profiling Controversy. Flushing, NY: Looseleaf Law Publications.

Wolfe, S. \& Nix, J. (2016) The alleged 'Ferguson Effect' and police willingness to engage in community partnership. Law \& Human Behavior, 40(1): 1-10.

Wolfe, S. \& Piquero, A. (2011) Organizational justice and police misconduct. Criminal Justice \& Behaviour, 38(4): 332-353.

Wong, K. (2014) Hayden: Senate report will make CIA officers 'more timid'. The Hill. 11 December. [Online] http://thehill.com/policy/defense/226863-hayden-senate-reportwill-make-cia-officers-more-timid.

Wood, D. \& Bryant, R. (2015) Researching police professionalism. In (M. Brunger, S. Tong \& D. Martin, eds.) Introduction to Policing Research. Taking Lessons from Practice: 87-100. Abingdon, UK: Routledge.

Wood, D., Cockcroft, T., Tong, S. \& Bryant, R. (2018) The importance of context and cognitive agency in developing police knowledge: Going beyond the police science discourse. The Police Journal, 91(2): 173-187.

Wood, D. \& Tong, S. (2009) The future of initial police training: A university perspective. International Journal of Police Science \& Management, 11(3): 294-305.

Woodside, A. (2010) Case Study Research: Theory, Methods, Practice. Bingley, UK: Emerald.

Worden, R. (1989) Situational and attitudinal explanations of police behavior: A theoretical reappraisal and empirical assessment. Law \& Society Review, 23(4): 667711.

Worden, R. (1995a) Police officers' belief systems: A framework for analysis. American Journal of Police, 14: 49-81.

Worden, R. (1995b) The 'causes' of police brutality: Theory and evidence on police use of force. In (W. Geller \& H. Toch, eds.) And Justice for All: Understanding and Controlling Police Abuse of Force. Washington, D.C.: Police Executive Research Forum.

Worden, R. (1996) The causes of police brutality: Theory and evidence on police use of force. In (W. Geller \& H. Toch, eds.) Police Violence: Understanding and Controlling Police Abuse of Force. New Haven, CT: Yale University Press.

Worden, R., Bynum, T. \& Frank, J. (1994) Police crackdowns on drug trafficking. In (D. McKenzie \& C. Uchida, eds.) Drugs and Crime: Evaluating Public Police Initiatives. Thousand Oaks, CA: Sage.

Worden, R. \& McLean, S. (2017) Mirage of Police Reform: Procedural Justice and Police Legitimacy. Oakland, CA: University of California Press. 
Workman-Stark, A. (2017) Inclusive Policing from the Inside Out. New York: Springer. World Population Review (2017) Buffalo Population 2017. [Online] http://world populationreview.com/us-cities/buffalo-population/.

Wortley, S. (2006) Police Use of Force in Ontario: An Examination of Data from the Special Investigations Unit. [Online] http://www.attorneygeneral.jus.gov.on.ca/ inquiries/ipperwash/policy_part/projects/pdf/AfricanCanadianClinicIpperwashProject _SIUStudybyScotWortley.pdf.

Wortley, S. \& Marshall, L (2005) Race and Police Stops in Kingston, Ontario: Results of a Pilot Project. Kingston, ON: Kingston Police Services Board.

Wortley, S., \& Owusu-Bempah, A. (2009) Unequal before the law: Immigrant and minority perceptions of the Canadian criminal justice system. Journal of International Migration and Integration, 10(4), 447-473.

Wortley, S., \& Owusu-Bempah, A. (2011) The usual suspects: Police stop and search practices in Canada. Policing \& Society, 21(4): 395-407.

Wortley, S., \& Tanner, J. (2003) Data, denials, and confusion: The racial profiling debate in Toronto. Canadian Journal of Criminology \& Criminal Justice, 45(3): 367389.

Wortley, S., \& Tanner, J. (2004) Discrimination or good policing? The racial profiling debate in Canada. Our Diverse Cities, 1: 197-201.

Wortley, S., \& Tanner, J, (2005) Inflammatory rhetoric? Baseless accusations? A response to Gabor's critique of racial profiling research in Canada. Canadian Journal of Criminology \& Criminal Justice, 47(3) 581-610.

Wyatt, S. \& Bell, N. (2014) Perspectives on police training and education: The Canadian experience. In (P. Stanislas, ed.) International Perspectives on Police Education and Training: 72-89. New York: Routledge.

Wynn, D. \& Williams, C. (2012) Principles for conducting critical realist case study research in information systems. MIS Quarterly, 36(3): 787-810.

Wynn, M. (2008) Rising Through the Ranks: Leadership Tools and Techniques for Law Enforcement. New York: Kaplan Publishing.

Yancy, G. (2016) Black Bodies, White Gazes: The Continuing Significance of Race in America. New York: Rowman \& Littlefield.

Yesil, B. (2011) Recording and reporting: Camera phones, user-generated images and surveillance. In (R. Firmino, F. Duarte \& C. Ultramari, eds.) ICTs for Mobile and Ubiquitous Urban Infrastructures: Surveillance, Locative Media, and Global Networks: 272-293. Hershey, PA: IGI Global.

Yin, R. (2014) Case Study Research: Design and Methods, $5^{\text {th }}$ ed. Los Angeles: Sage

Yin, R. (2018) Case Study Research and Applications: Design and Methods. Los Angeles: Sage. 
Yogaretnam, S. (2018a) Gun violence a result of understaffed force, loss of street checks: police union. The Ottawa Citizen. 29 January. [Online] http://ottawacitizen. com/news/ local-news/gun-violence-a-result-of-understaffed-force-loss-of-streetchecks-police-union.

Yogaretnam, S. (2018b) Ottawa cop turned researcher finds decline in proactive policing. Ottawa Citizen. 12 February. [Online] https://ottawacitizen.com/news/localnews/to-swerve-and-neglect-ottawa-cop-turned-researcher-finds-decline-in-proactivepolicing.

Yokum, D., Ravishankar, A. \& Coppock, A. (2017) Evaluating the Effects of Police Body-Worn Cameras: A Randomized Controlled Trial. Washington, DC: Government of the District of Columbia.

Young, M. (1991) An Inside Job: Policing and Police Culture in Britain. Oxford: Clarendon Press.

Yun, I. \& Lee, C. (2015) Hazardous alcohol use among South Korean police officers: Examining predictions from general strain theory. International Journal of Law, Crime \& Justice, 43(2): 194-213.

Zaccaria, J. (1965) Developmental tasks: Implications for the goals of guidance. Personnel \& Guidance Journal, 44: 372-375.

Zamble, E. \& Annesley, P. (1987) Some determinants of public attitudes toward the police. Journal of Police Science \& Administration, 15: 285-290.

Zezima, K. (2014) Obama: Attorney General Holder to go to Ferguson Wednesday. The Washington Post. 18 August. [Online] http://www.washingtonpost. com/blogs/postpolitics/wp/2014/08/18/white-house-takes-stock-of-the-situation-in-ferguson/.

Zhao, J., Ren, L. \& Lovrich, N. (2010) Budgetary support for police services in U.S. municipalities: Comparing political culture, socioeconomic characteristics and incrementalism as rival explanations for budget share allocation to police. Journal of Criminal Justice, 38(3): 266-275.

Zhao, J., Thurman, Q. \& He, N. (1999) Sources of job satisfaction among police officers: A test of demographic and work environment models. Justice Quarterly, 16(1): 153-174.

Zinn, J., ed. (2008) Social Theories of Risk and Uncertainty: An Introduction. Oxford: Blackwell. 